NBS

PUBLICATIONS

A I10? 210915

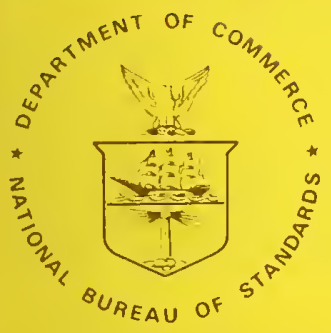

NBS MONOGRAPH 25_SECTION 20

U.S. DEPARTMENT OF COMMERCE/National Bureau of Standards

\title{
Standard X-ray Diffraction Powder Patterns
}

QC

100

.4556

No. 25

SECT, 20

1934 c.2 


\section{NATIONAL BUREAU OF STANDARDS}

The National Bureau of Standards' was established by an act of Congress on March 3, 1901. The Bureau's overall goal is to strengthen and advance the Nation's science and technology and facilitate their effective application for public benefit. To this end, the Bureau conducts research and provides: (1) a basis for the Nation's physical measurement system, (2) scientific and technological services for industry and government, (3) a technical basis for equity in trade, and (4) technical services to promote public safety. The Bureau's technical work is performed by the National Measurement Laboratory, the National Engineering Laboratory, and the Institute for Computer Sciences and Technology.

THE NATIONAL MEASUREMENT LABORATORY provides the national system of physical and chemical and materials measurement; coordinates the system with measurement systems of other nations and furnishes essential services leading to accurate and uniform physical and chemical measurement throughout the Nation's scientific community, industry, and commerce; conducts materials research leading to improved methods of measurement, standards, and data on the properties of materials needed by industry, commerce, educational institutions, and Government; provides advisory and research services to other Government agencies; develops, produces, and distributes Standard Reterence Materials; and provides calibration services. The Laboratory consists of the following centers:

\section{Absolute Physical Quantities ${ }^{2}$ - Radiation Research - Chemical Physics - Analytical Chemistry - Materials Science}

THE NATIONAL ENGINEERING LABORATORY provides technology and technical services to the public and private sectors to address national needs and to solve national problems; conducts research in engineering and applied science in support of these efforts; builds and maintains competence in the necessary disciplines required to carry out this research and technical service; develops engineering data and measurement capabilities; provides engineering measurement traceability services; develops test methods and proposes engineering standards and code changes; develops and proposes new engineering practices; and develops and improves mechanisms to transfer results of its research to the ultimate user. The Laboratory consists of the following centers:

Applied Mathematics - Electronics and Electrical Engineering ${ }^{2}$ - Manufacturing Engineering - Building Technology - Fire Research - Chemical Engineering ${ }^{2}$

THE INSTITUTE FOR COMPUTER SCIENCES AND TECHNOLOGY conducts research and provides scientific and technical services to aid Federal agencies in the selection, acquisition, application, and use of computer technology to improve effectiveness and economy in Government operations in accordance with Public Law 89-306 (40 U.S.C. 759), relevant Executive Orders, and other directives; carries out this mission by managing the Federal Information Processing Standards Program, developing Federal ADP standards guidelines, and managing Federal participation in ADP voluntary standardization activities; provides scientific and technological advisory services and assistance to Federal agencies; and provides the technical foundation for computer-related policies of the Federal Government. The Institute consists of the following centers:

Programming Science and Technology-Computer Systems Engineering.

'Headquarters and Laboratories at Gaithersburg, MD, unless otherwise noted;

mailing address Washington, DC 20234.

${ }^{2}$ Some divisions within the center are located at Boulder, CO 80303. 


\section{Standard X-ray Diffraction \\ Powder Patterns \\ Section 20 - Data for 71 Substances}

Marlene C. Morris, Howard F. McMurdie, Eloise H. Evans, Boris Paretzkin, Harry S. Parker, and Nikos P. Pyrros

International Centre for Diffraction Data

1601 Park Lane

Swarthmore, PA 19081

and

Camden R. Hubbard

National Measurement Laboratory

National Bureau of Standards

Washington, DC 20234
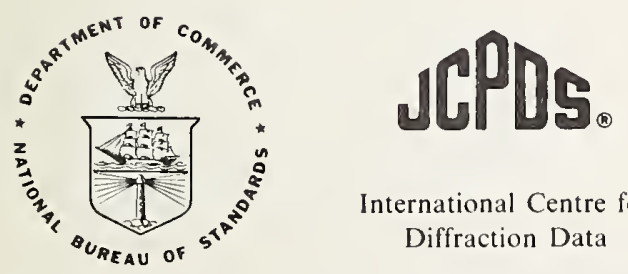

International Centre for

Diffraction Data

U.S. DEPARTMENT OF COMMERCE, Malcolm Baldrige, Secretary

NATIONAL BUREAU OF STANDARDS, Ernest Ambler, Director

Issued January 1984 
Library of Congress Catalog Card Number: 53-61386

National Bureau of Standards Monograph 25

Section 20-Data for 71 Substances

Natl. Bur. Stand. (U.S.), Monogr. 25-Sec. 20, 149 pages (Jan. 1984) CODEN: NBSMA6 
Introduction

Experimental patterns:

Barium cadmium phosphate,

$\operatorname{BaCd}\left(\mathrm{PO}_{3}\right)_{4} \ldots \ldots \ldots \ldots \ldots \ldots \ldots$

Barium titanium oxide, $\mathrm{BaTiO}_{3} \ldots \ldots$.

Barium titanium oxide, $\mathrm{BaTi}_{2} \mathrm{O}_{5} \ldots \ldots$

Barium titanium oxide, $\mathrm{BaTi}_{4} \mathrm{O}_{9} \ldots \ldots$

Barium titanium phosphate,

$\mathrm{BaTi}_{4}\left(\mathrm{PO}_{4}\right)_{6} \ldots \ldots \ldots \ldots \ldots \ldots$

Barium zirconium phosphate,

$\mathrm{BaZr}_{4}\left(\mathrm{PO}_{4}\right)_{6} \ldots \ldots \ldots \ldots \ldots \ldots$

Bismuth germanium oxide,

$\mathrm{Bi}_{4}\left(\mathrm{GeO}_{4}\right)_{3} \ldots \ldots \ldots \ldots \ldots \ldots \ldots \ldots$

Bismuth germanium oxide,

$\mathrm{Bi}_{12} \mathrm{GeO}_{20} \ldots \ldots \ldots \ldots \ldots \ldots \ldots \ldots$

Bismuth titanium oxide, $\mathrm{Bi}_{12} \mathrm{TiO}_{20} \ldots$

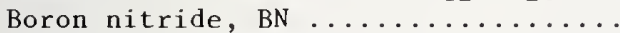

Boron phosphate, $\mathrm{BPO}_{4} \ldots \ldots \ldots \ldots \ldots$

Cadmium aluminum oxide, $\mathrm{CdAl}_{2} \mathrm{O}_{4} \ldots$.

Cadmium zinc phosphate,

$\mathrm{Cd}_{0.9} \mathrm{Zn}_{2.1}\left(\mathrm{PO}_{4}\right)_{2} \ldots \ldots \ldots \ldots \ldots \ldots$

Calcium iron silicate (kirschsteinite),

$\mathrm{CaFeSiO}_{4} \ldots \ldots \ldots \ldots \ldots \ldots \ldots \ldots \ldots \ldots \ldots$

Calcium magnesium silicate (monticel-

lite), $\mathrm{CaMgSiO}_{4} \ldots \ldots \ldots \ldots \ldots \ldots$.

Calcium magnesium silicate (akermanite),

$\mathrm{Ca}_{2} \mathrm{MgSi}_{2} \mathrm{O}_{7} \ldots \ldots \ldots \ldots \ldots \ldots \ldots \ldots$

Calcium magnesium silicate (merwinite),

$\mathrm{Ca}_{3} \mathrm{Mg}\left(\mathrm{SiO}_{4}\right)_{2} \ldots \ldots \ldots \ldots \ldots \ldots$

Calcium manganese phosphate fluoride,

$\mathrm{Ca}{ }_{8} \mathrm{Mn}_{2}\left(\mathrm{PO}_{4}\right)_{6} \mathrm{~F}_{2} \ldots \ldots \ldots \ldots \ldots \ldots$

Calcium zirconium phosphate,

$\mathrm{CaZr}_{4}\left(\mathrm{PO}_{4}\right)_{6} \ldots \ldots \ldots \ldots \ldots \ldots \ldots$

Cerium oxide (cerianite), $\mathrm{CeO}_{2} \ldots \ldots$

Cesium hydrogen phosphate,

$\mathrm{CsH}_{5}\left(\mathrm{PO}_{4}\right)_{2} \ldots \ldots \ldots \ldots \ldots \ldots \ldots \ldots$

Cesium zirconium phosphate,

$\mathrm{CsZr}_{2}\left(\mathrm{PO}_{4}\right)_{3} \ldots \ldots \ldots \ldots \ldots \ldots \ldots$

Chromium tungsten oxide, $\mathrm{CrWO}_{4} \ldots \ldots$

Cobalt telluride, CoTe ...........

Copper chromium oxide, $\mathrm{CuCr}_{2} \mathrm{O}_{4} \ldots \ldots$

Copper iron oxide, $\mathrm{CuFe}_{2} \mathrm{O}_{4} \ldots \ldots \ldots \ldots$

Copper mercury iodide, $\beta-\mathrm{Cu}_{2} \mathrm{HgI}_{4} \ldots$.

Europium oxide, $\mathrm{Eu}_{2} \mathrm{O}_{3}$ (cubic) .......

Europium oxide, $\mathrm{Eu}_{2} \mathrm{O}_{3}$ (monoclinic) ..

Hafnium oxide, $\mathrm{HfO}_{2} \ldots \ldots \ldots \ldots \ldots$.

Iron niobium oxide, $\mathrm{Fe}\left(\mathrm{NbO}_{3}\right)_{2} \ldots \ldots \ldots$

Iron niobium oxide, $\mathrm{Fe}_{4} \mathrm{Nb}_{2} \mathrm{O}_{9} \ldots \ldots \ldots$

Iron silicate (fayalite), $\mathrm{Fe}_{2} \mathrm{SiO}_{4} \ldots$

Iron titanium oxide (ulvöspinel),

$\mathrm{Fe}_{2} \mathrm{TiO}_{4} \ldots \ldots \ldots \ldots \ldots \ldots \ldots \ldots \ldots$

Lanthanum boride, $\mathrm{LaB}_{6} \ldots \ldots \ldots \ldots$

Lithium thorium molybdenum oxide,

$\mathrm{Li}_{4} \mathrm{Th}_{7}\left(\mathrm{MoO}_{4}\right)_{16} \ldots \ldots \ldots \ldots \ldots$

Lithium thorium tungsten oxide,

$\mathrm{Li}_{4} \mathrm{Th}_{7}\left(\mathrm{WO}_{4}\right)_{16} \ldots \ldots \ldots \ldots \ldots \ldots$

Lithium titanium oxide, $\mathrm{Li}_{2} \mathrm{Ti}_{3} \mathrm{O}_{7} \ldots$

Magnesium manganese zinc iron sulfate

hydroxide hydrate, zincobotryogen,

$(\mathrm{Zn}, \mathrm{Mg}, \mathrm{Mn}) \mathrm{Fe}\left(\mathrm{SO}_{4}\right)_{2}(\mathrm{OH}) \cdot 7 \mathrm{H}_{2} \mathrm{O} \ldots \ldots$

Magnesium silicate (clinoenstatite),

$\mathrm{MgSiO}_{3} \ldots \ldots \ldots \ldots \ldots \ldots \ldots \ldots$
Magnesium silicate (forsterite),

$\mathrm{Mg}_{2} \mathrm{SiO}_{4} \ldots \ldots \ldots \ldots \ldots \ldots \ldots \ldots$

Potassium barium niobium oxide,

$\mathrm{KBa}_{2}\left(\mathrm{NbO}_{3}\right)_{5} \ldots \ldots \ldots \ldots \ldots \ldots \ldots \ldots$

Potassium strontium niobium oxide,

$\mathrm{KSr}_{2}\left(\mathrm{NbO}_{3}\right)_{5} \ldots \ldots \ldots \ldots \ldots \ldots \ldots$

Potassium titanium phosphate,

$\mathrm{KTi}_{2}\left(\mathrm{PO}_{4}\right)_{3} \ldots \ldots \ldots \ldots \ldots \ldots \ldots$

Potassium zinc phosphate, $\mathrm{KZnPO}_{4} \ldots$

Rubidium hydrogen phosphate,

$\mathrm{RbH}_{2} \mathrm{PO}_{4} \ldots \ldots \ldots \ldots \ldots \ldots \ldots \ldots \ldots$

Rubidium phosphate, $\mathrm{RbPO}_{3} \ldots \ldots \ldots$.

Rubidium strontium niobium oxide,

$\operatorname{RbSr}_{2}\left(\mathrm{NbO}_{3}\right)_{5} \ldots \ldots \ldots \ldots \ldots \ldots$

Sodium antimony fluoride, $\mathrm{NaSbF}_{4} \ldots$

Sodium barium niobium oxide,

$\mathrm{NaBa}_{2}\left(\mathrm{NbO}_{3}\right)_{5} \ldots \ldots \ldots \ldots \ldots$

Sodium germanium phosphate,

$\mathrm{NaGe}_{2}\left(\mathrm{PO}_{4}\right)_{3} \ldots \ldots \ldots \ldots \ldots \ldots \ldots$

Sodium iron silicate (acmite),

$\mathrm{NaFe}\left(\mathrm{SiO}_{3}\right)_{2} \ldots \ldots \ldots \ldots \ldots \ldots \ldots$

Sodium molybdenum oxide hydrate,

$\mathrm{Na}_{2} \mathrm{MoO}_{4} \cdot 2 \mathrm{H}_{2} \mathrm{O} \ldots \ldots \ldots \ldots \ldots \ldots \ldots$

Sodium strontium niobium oxide,

$\mathrm{NaSr}_{2}\left(\mathrm{NbO}_{3}\right)_{5} \ldots \ldots \ldots \ldots \ldots \ldots \ldots$

Strontium aluminum oxide, $\mathrm{SrAl}_{2} \mathrm{O}_{4} \ldots$

Strontium silicate, $\alpha-\mathrm{SrSiO}_{3} \ldots \ldots$.

Strontium zirconium phosphate,

$\mathrm{SrZr}_{4}\left(\mathrm{PO}_{4}\right)_{6} \ldots \ldots \ldots \ldots \ldots \ldots \ldots$

Tantalum tungsten oxide, $\mathrm{Ta}_{2} \mathrm{WO}_{8} \ldots$.

Thorium molybdenum oxide,

$\alpha-\mathrm{Th}\left(\mathrm{MoO}_{4}\right)_{2} \ldots \ldots \ldots \ldots \ldots \ldots$

Thorium molybdenum oxide,

$\beta-\mathrm{Th}\left(\mathrm{MoO}_{4}\right)_{2} \ldots \ldots \ldots \ldots \ldots \ldots \ldots$

Thorium silicate (huttonite),

$\beta-\mathrm{ThSiO}_{4} \ldots \ldots \ldots \ldots \ldots \ldots \ldots \ldots$

Thorium tantalum oxide, $\mathrm{Th}_{2} \mathrm{Ta}_{2} \mathrm{O}_{9} \ldots$

Thorium tungsten oxide, $\alpha-\mathrm{Th}\left(\mathrm{WO}_{4}\right)_{2} \ldots$

Vanadium oxide (karelianite), $\mathrm{V}_{2} \mathrm{O}_{3} \ldots$

Ytterbium fluoride, $\mathrm{YbF}_{3} \ldots \ldots \ldots \ldots$

Zinc vanadium oxide, $\mathrm{Zn}_{3}\left(\mathrm{VO}_{4}\right)_{2} \ldots \ldots$

Zirconium boride, $\mathrm{ZrB}_{2} \ldots \ldots \ldots \ldots$

Zirconium hydrogen phosphate hydrate,

$\mathrm{Zr}\left(\mathrm{HPO}_{4}\right)_{2} \cdot \mathrm{H}_{2} \mathrm{O} \ldots \ldots \ldots \ldots \ldots \ldots$

Zirconium titanium oxide, $\mathrm{ZrTiO}_{4} \ldots$.

Zirconium titanium oxide,

$\mathrm{Zr}_{5} \mathrm{Ti}_{7} \mathrm{O}_{24} \ldots \ldots \ldots \ldots \ldots \ldots \ldots$

Calculated pattern:

Molybdenum oxide (molybdite), $\mathrm{MoO}_{3} \ldots$

Cumulative indices

(Circular 539, Volumes 1-10 and Monograph 25, Sections 1-20 inclusive)

1. Inorganic

2. Organic formula

3. Organic name

4. Mineral 
Publications Available.

Previous work has been published as a book entitled Powder Diffraction Data from the Joint Committee on Powder Diffraction Standards Associateship at the National Bureau of Standards (1976) (obtainable from the publisher: JCPDS--International Centre for Diffraction Data, 1601 Park Lane, Swarthmore, PA 19081 , price furnished on request). The volume is sold with an accompanying search manual, and contains 949 card images of patterns of experimental data, published originally as Circular 539 (vols. 1-10) and Monograph 25, Sections 1-12, and most of Section 13.

Individual copies of the Circular and Monograph are still available and may be obtained from the National Technical Information Service, 5285 Port Royal Road, Springfield, VA 22161. If a publication listed below is identified with a number, use this number in ordering. All are available in photocopy or microfiche; the price is not fixed and will be furnished on request.

NBS Publication

Order Number

Circular 539, Volume 1.........PB 178902

Volume 2..........PB 178903

Volume $3 \ldots \ldots \ldots$......PB 178904

Volume $4 \ldots \ldots \ldots \ldots$.....PB 178905

Volume $5 \ldots \ldots \ldots . .$. PB 178906

Volume $6 \ldots \ldots \ldots$......PB 178907

Volume $7 \ldots \ldots \ldots \ldots$.......PB 178908

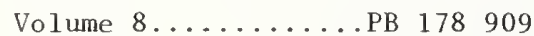

Volume $9 \ldots \ldots \ldots \ldots$.................. 178910

Volume $10 \ldots . . . .$. .... 178911
NBS Publication

Order Number

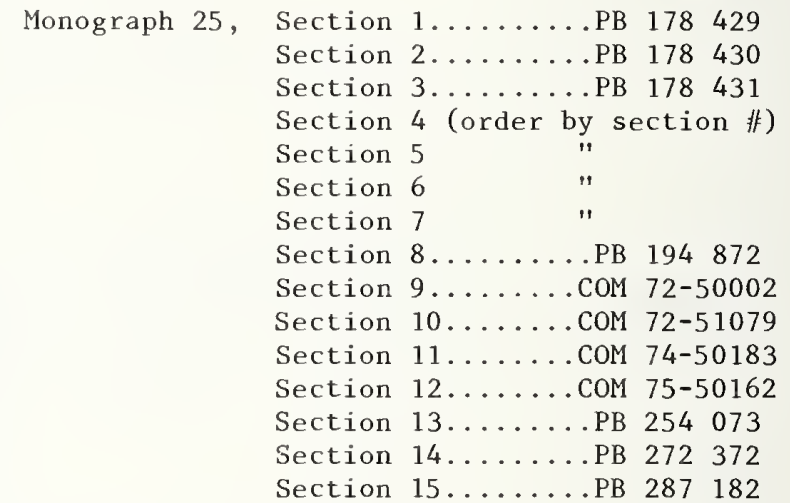

Also, until the present supply is exhausted, the following issues are for sale from the Superintendent of Documents, U.S. Government Printing Office, Washington, DC 20402. Order by given catalog number, and add $25 \%$ to the price, for other than U.S. mailing.

\section{Catalog Number Price}

Section $12 \ldots \ldots \ldots \ldots \ldots \ldots \ldots \ldots$. . . $003-003-01376-5$

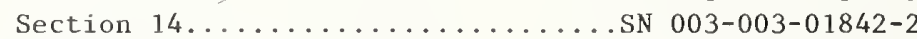

Section 15............................. 003-003-01986-1

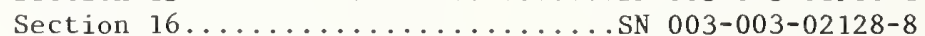

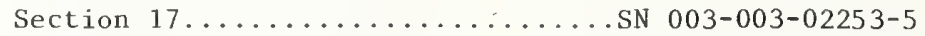

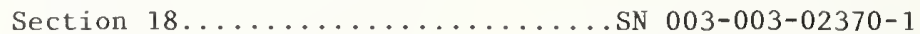

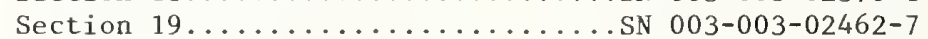

ERRATA

Monograph 25

Section 7, p. 160: The space group should be $\mathrm{P} 2{ }_{1} / \mathrm{a}$ (14).

Section 19, p. 24: The date for the Mitchell reference (3 locations) should be 1956.
$\$ 1.50$

2.75

4.00

5.00

4.50

5.50

5.50 
STANDARD X-RAY DIFFRACTION POWDER PATTERNS

Section 20 -.- Data for 71 Substances

by

Marlene C. Morris, Howard F. McMurdie, Eloise H. Evans,

Boris Paretzkin, Harry S. Parker, and Nikos P. Pyrros

JCPDS--International Centre for Diffraction Data

and

Camden R. Hubbard

National Bureau of Standards

\begin{abstract}
Standard x-ray powder diffraction patterns are presented for 71 substances. These patterns, useful for identification, were obtained by manual or automated diffractometer methods, or were calculated from published crystal structure data. The lattice constants from the experimental work were refined by least-squares methods, and reflections were assigned Miller indices consistent with space group extinctions. Relative intensities, calculated densities, literature references, and other relevant data are included.
\end{abstract}

Key words: Crystal structure; densities; lattice constants; powder patterns; reference intensities; standard; $x$-ray diffraction.

\title{
INTRODUCTION
}

The Powder Diffraction File (PDF) is a continuing compilation of diffraction patterns gathered from many sources. Produced and published by the JCPDS--International Centre for Diffraction Data ${ }^{1}$, the PDF is used for identification of crystalline materials by matching d-spacings and diffraction intensity measurements. Under the partial sponsorship of the JCPDS, the program at the National Bureau of Standards contributes new or improved data to the PDF. Our work also aids in the development of diffraction techniques. This report presents information for one calculated and 70 experimental patterns, and is the thirtieth of the series of Standard X-ray Diffraction Powder Patterns ${ }^{2}$.

\section{EXPERIMENTAL POWDER PATTERNS}

Names. The nomenclature follows the current practice of the PDF. A mineral name in ( ) indicates a synthetic sample.

CAS registry number. The Chemical Abstracts Service Registry Number is included, when available, to help identify the sample. This number forms the basis for computer aided searching of Chemical Abstracts [Chemical Abstracts Service Registry Handbook-Number Section, 1974]

${ }^{1}$ JCPDS--International Centre for Diffraction Data, 1601 Park Lane, Swarthmore, PA 19081. This Pennsylvania non-profit corporation functions in cooperation with the American Ceramic Society, the American Crystallographic Association, the American Society for Testing and Materials, The Clay Minerals Society, The Institute of Physics, the Mineralogical Association of Canada, the Mineralogical Society of America, The Mineralogical

Society of Great Britain and Ireland, the National Association of Corrosion Engineers, and the Société Française de Minéralogie et de Cristallographie.

${ }^{2}$ See previous page for other published volumes.
Sample. The samples used to make NBS patterns were obtained from a variety of sources or were prepared in small quantities in our laboratory. Appropriate annealing or recrystallization of the samples improved the quality of many of the patterns. A check of phase purity was provided by indexing the $x$-ray pattern and by optical examination.

Color. The names of the sample colors are selected from the ISCC-NBS Centroid Color Charts [1965].

Interplanar spacings. All spacing determinations were made using one or more internal standards mixed with the sample, packed in a shallow holder. Choice of the standard was determined by the need for low angle and unobstructed reflections. The amount of standard was estimated so that the intensity of its strongest peak would be about equal to the intensity of the strongest peak of the sample. The internal standards used were of high purity $(99.99 \%)$. The calculated $2 \theta$ values used for them at $25{ }^{\circ} \mathrm{C}$ are given in Tables 1 and 2 ; the $2 \theta$ angles were computed using cell dimensions uncorrected for index of refraction.

Standard Reference Material 640a [1982], Si powder $(a=5.430825 \AA)$, was used for many patterns. The SRM 640a lattice constant for Si was refined from multiple powder data measurements made with tungsten and silver as internal standards. Single crystal cell parameter data were also collected. The lattice parameters from the two methods agreed within three parts in $10^{5}$ [Hubbard, 1982]. Dspacing results using SRM 640 a will be in agreement with patterns recorded in this series of Monographs since 1966.

Another internal standard, fluorophlogopite (FP), is available as Standard Reference Material 675 [1982]. The d(001) spacing was refined from multiple powder data measurements using SRM 640a (Si) and tungsten as internal standards [Hubbard, 
1983]. The calculated $2 \theta$ values of the $00 \ell$ lines are given in Table 2 .

Table 1

\begin{tabular}{|c|c|c|c|}
\hline \multicolumn{4}{|c|}{ Calculated $2 \theta$ Angles, $\operatorname{CuK} \alpha_{1} \lambda=1.540598 \AA$} \\
\hline $\mathrm{h} \ell$ & $\begin{aligned} \mathrm{a}= & 3.16524 \AA \\
& \pm .00004\end{aligned}$ & $\begin{array}{c}\mathrm{Ag} \\
\mathrm{a}=4.08651 \AA \\
\pm .00002\end{array}$ & $\begin{array}{c}\mathrm{Si} \\
\mathrm{a}=5.430825 \AA \\
\pm .000011 \\
(\mathrm{SRM} 640 \mathrm{a})\end{array}$ \\
\hline 110 & 40.262 & & \\
\hline 111 & & 38.112 & 28.443 \\
\hline 200 & 58.251 & 44.295 & \\
\hline 211 & 73.184 & & \\
\hline 220 & 86.996 & 64.437 & 47.304 \\
\hline 310 & 100.632 & & \\
\hline 311 & & 77.390 & 56.124 \\
\hline 222 & 114.923 & 81.533 & \\
\hline 321 & 131.171 & & \\
\hline 400 & 153.535 & 97.875 & 69.132 \\
\hline 331 & & 110.499 & 76.378 \\
\hline 420 & & 114.914 & \\
\hline 422 & & 134.871 & 88.033 \\
\hline $511 / 333$ & & 156.737 & 94.955 \\
\hline 440 & & & 106.712 \\
\hline 531 & & & 114.096 \\
\hline 620 & & & 127.550 \\
\hline 533 & & & 136.900 \\
\hline 444 & & & 158.644 \\
\hline
\end{tabular}

Table 2

\begin{tabular}{|c|c|}
\hline \multirow{2}{*}{\multicolumn{2}{|c|}{ Calculated $2 \theta$ Angles, $\operatorname{CuK} \alpha_{1} \lambda=1.540598 \AA^{\circ}$}} \\
\hline & \\
\hline $\begin{array}{c}\frac{00 \ell}{1} \\
2 \\
3 \\
4 \\
5 \\
6 \\
7 \\
8 \\
10 \\
11 \\
12\end{array}$ & $\begin{array}{r}2 \theta \\
8.853 \\
17.759 \\
26.774 \\
35.962 \\
45.397 \\
55.169 \\
65.399 \\
76.255 \\
101.025 \\
116.193 \\
135.674\end{array}$ \\
\hline
\end{tabular}

All data were collected at room temperature on a diffractometer equipped with a focusing graphite crystal monochromator located between the sample and the scintillation counter. Pulse height discrimination was used as well. The data were collected using copper radiation: $\lambda\left(\mathrm{CuK} \alpha_{1}\right.$, peak $)$ $=1.540598 \AA$ [Deslattes and Henins, 1973].
The majority of the patterns reported in this monograph were measured with a computer controlled diffractometer. Digital data were measured on one of two diffractometers controlled by the AUTO program [Snyder et al., 1981]. All the patterns were measured in step-scan mode with a step width of 0.01 degrees and counting times at each point greater than or equal to $3 \mathrm{sec}$.

The data were processed with the JCPDS-NBS POWDER-PATTERN system of programs [Pyrros and Hubbard, 1983]. First the raw data were processed by the program POWDER. PATTERN that locates peaks with the second derivative algorithm of Savitzky and Golay [1964]. A three point Newton-Gregory interpolation [Daniels, 1978] was used to locate the derivative minimum. For some patterns, weak peaks were read from strip chart recordings or were located with the interactive graphics program PLOT.PATTERN/INT. This program displays the spectrum on a Tektronix graphic terminal. The user can locate peaks by positioning a cursor at the peak. The peak position is defined either as the position of the cursor or as the minimum of the second derivative nearest to the cursor. The $\mathrm{K} \alpha_{2}$ peaks were occasionally read to assist in establishing a $K \alpha_{1}$ peak position, but $K \alpha_{2}$ peaks are not reported.

All patterns were plotted on paper with the plot program PLOT.PATTERN/HRD on a scale of one degree per inch and were visually inspected. The program POWDER.CALIBR was used to calculate a polynomial correction curve from the expected and observed $2 \theta$ peak positions of the internal standard reflections and to correct the observed $2 \theta$ values of the sample. At low angles, $\mathrm{K} \alpha_{1}$ and $\mathrm{K \alpha}_{2}$ peaks were unresolved for both the sample and the internal standard. Internal standard corrections were established from the theoretical values for $K \alpha_{1}$ and were applied to the unresolved low angle peaks, as well as to the resolved $\mathrm{K} \alpha_{1}$ peaks in the higher angle regions. The program POWDER.EDTPKS was used to flag reflections to be used in the least-squares cell parameter refinement. Reflections due to $\mathrm{CuK \alpha} \alpha_{2}$ radiation were excluded from the refinement.

Structure, lattice constants. The space group symbols are given in the short Hermann-Mauguin notation. Also given are the space group numbers listed in the International Tables for X-ray Crystallography, Vol. I [1965]. When the space group symbol is not known, the lattice centering symbol or the diffraction aspect for the Laue class may be given [Donnay and Kennard, 1964; Mighell et al., 1981].

Orthorhombic cell dimensions are arranged according to the Dana convention $b>a>c$ [Palache et al., 1944]. Monoclinic and triclinic lattice constants are transformed if necessary in order to follow the convention of Crystal Data [1973]. The lattice constant ratios, a/b, $\mathrm{c} / \mathrm{b}$, and $\mathrm{c} / \mathrm{a}$, also follow the conventions used for the determinative ratios in Crystal Data [1973].

In most cases, preliminary lattice constants were available in the literature, and were used for the initial indexing and refinement. In cases where such data were not available, other methods were tried. If suitable single crystals were 
available, the lattice constants were obtained by use of a four-circle diffractometer. Axial ratios and densities from Groth [1908] were sometimes useful. Cell constants were also found in some instances by use of Visser's computer program, Ito 9 [Visser, 1969].

A least squares program, JCPDS-NBS*LSQ82, derived from the program of Evans, Appleman and Handwerker [1963], assigned hkl's and refined the lattice constants. The cell refinement was based only upon corrected $2 \theta$ values $\left(2 \theta_{\text {corr }}\right)$ which could be indexed without ambiguity. The program minimized the value $\Sigma\left(\theta_{c_{c r r}}{ }^{-}{ }_{c a l c}\right)^{2}$. Generally, when two or more calculated $2 \theta$ 's were within 0.03 degrees of the corrected $2 \theta$, unique indices were not assigned. The possible multiple indices are reported. A plus sign ( + ) indicates more than 2 possible indices. Multiple hkl's were not utilized or reported in indexing cubic patterns. Instead, a single appropriate index was used.

The estimated standard deviations (e.s.d.'s) of the reciprocal cell parameters were determined from the inverse matrix of the normal equations. The program calculated the e.s.d.'s of the direct cell constants by the method of propagation of errors. Since 1973, the e.s.d.'s derived by the computer program have been increased by $50 \%$ in order to reflect more truly the uncertainty in the lattice constants. A similar increase should also be applied to all lattice constants published in this series of NBS publications prior to 1973. The e.s.d.'s in the least significant figures are given in parentheses following the lattice constants.

For each d-value, the number of significant figures was derived from the average error in $\left|2 \theta_{\operatorname{cor} r}-2 \theta_{\operatorname{cal} c}\right|$ and the equation $\Delta \mathrm{d} / \mathrm{d}=-(\cot \theta) \overline{\Delta \theta}$. With these conditions, the rounded value of $d$ agrees with its appropriate $2 \theta$ within the average error in $2 \theta$. The value of $\overline{\Delta \theta}$ varies with the symmetry and crystallinity of each sample.

Densities. These were calculated from the specified lattice constants, the Avogadro number $6.0220943 \times 10^{23}$ [Deslattes et al., 1974] and the 1977 atomic weights published by the International Union of Pure and Applied Chemistry [1979].

Figures of merit. Several figures of merit ratings are available for assessing indexed powder data. $M_{20}$ [de Wolff, 1968] is a criterion for the reliability of the unit cell and indexing. $M_{20}$ is defined by:

$$
\mathrm{M}_{20}=\frac{\mathrm{Q}_{20}}{2 \bar{\varepsilon} \mathrm{N}_{20}}
$$

where $Q_{20}$ is the value of $1 / d^{2}$ for the 20 th observed line (not counting unexplained lines), $\mathrm{N}_{20}$ is the number of different calculated $Q$ values up to $Q_{20}$, and $\bar{\varepsilon}$ is the average magnitude of the discrepancy in $Q$ for these 20 lines. The number of unindexable lines occuring up to the 20th observed and indexed line is $X_{20}$. A value of $M_{20}>$ 10 will guarantee the essential correctness of the indexing provided there are not more than 2 spurious lines $\left(X_{20} \leq 2\right)$ [de Wolff, 1968]. In general, patterns reported in this publication had $\mathrm{M}_{20}>20$ and $X_{20}=0 . \quad M_{20}$ is reported if a cell was derived only through computer indexing from powder data, without further confirmation.

The accuracy and completeness of the measured interplanar spacings is conveniently reported using the format:

$$
F_{N}=\text { overall value }\left(\mid \overline{\Delta 2 \theta}, N_{\text {poss }}\right)
$$

The "overall" value is the figure of merit of Smith and Snyder [1979] defined by:

$$
\frac{1}{|\Delta 2 \theta|} \cdot \frac{\mathrm{N}}{\mathrm{N}_{\text {poss }}}
$$

$\mathrm{N}$, the number of observed reflections, was chosen as 30 or as the maximum number of lines of the pattern if the pattern had fewer than 30 lines. $\triangle \Delta 2 \theta$ is the average absolute magnitude of the discrepancy between observed and calculated $2 \theta$ values for each reported hkl. When multiple indices are reported for an observed reflection, then each possible $\Delta 2 \theta$ is included in the $\overline{\Delta 2 \theta}$. $N_{\text {poss }}$ is the number of diffraction lines allowed in the space group, up to the Nth observed and indexed line. Co-positional lines such as the cubic 221 and 300 are counted as one possible line.

Intensity measurements. The intensities of the diffraction lines were measured as peak heights above background and were expressed in percentage of the strongest line. It has been found that samples which give satisfactory intensity patterns usually have an average particle size smaller than $10 \mu \mathrm{m}$, as recommended by Alexander et al. [1948]. In order to avoid the orientation effects which occur when powdered samples are packed or pressed, a sample holder was made that had in its top face a rectangular cavity which extended to one end of the holder. To prepare the sample, a glass slide was clamped over the top face to form a temporary cavity wall (see Figure 1), and the powdered sample was allowed to drift into the end opening while the holder was held in a vertical position.

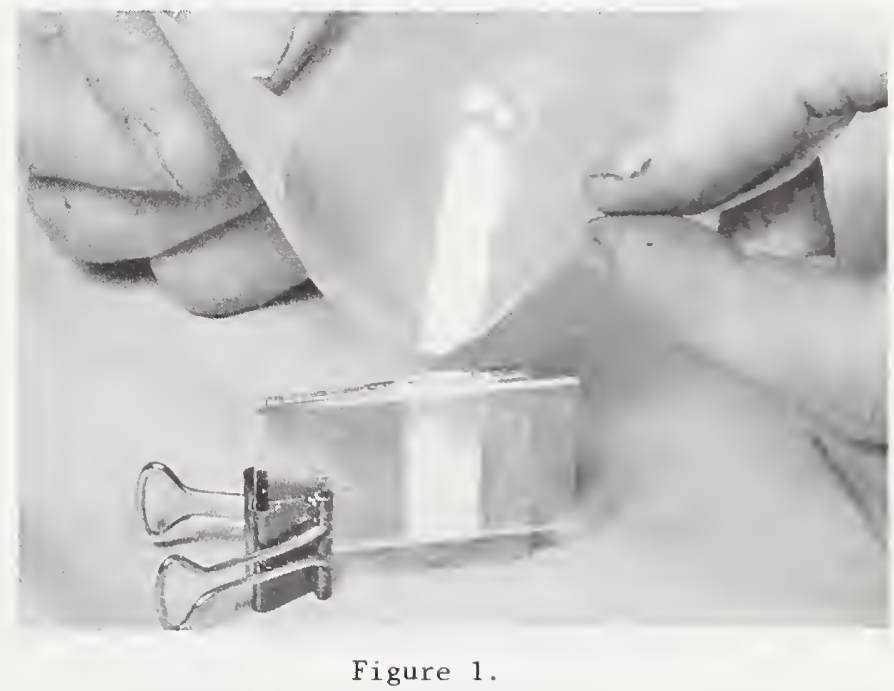

With the sample holder returned to a horizontal position, the glass slide was carefully removed so that the sample could be exposed to the $x$-ray beam (see Figure 2). 


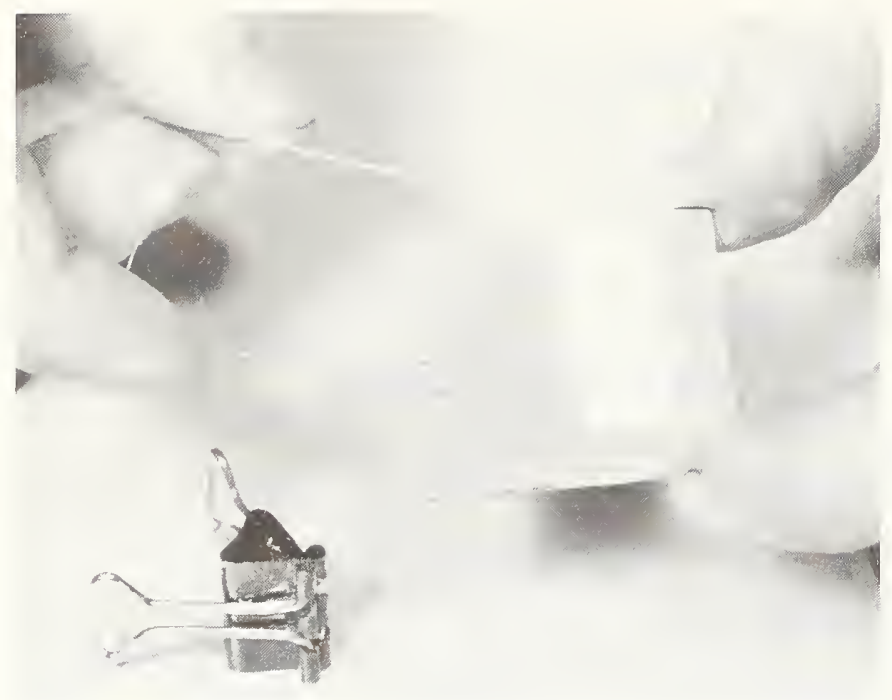

Figure 2.

As a general practice, approximately 50 volume percent of finely ground silica gel was added as a diluent. Occasionally, a rotating sample holder was used.

As a check on reproducibility, each sample was mounted at least 3 times. The intensity values were cietermined for each of the mountings. The reported Irel value for each observed spacing is the average of 3 or more observations and is rounded to the nearest integer. Theta-compensating (variable divergence) slits were sometimes used to gather the intensity data. In that case, the average I(comp) for each spacing was converted to an equivalent fixed slit value, using the approximate equation:

$$
I(\text { fixed })=\frac{I(\text { comp })}{\sin \theta}
$$

The estimated standard deviation, $\sigma$, in the relative intensity values was calculated from the values of the five strongest lines, excluding the line with Irel=100.

$$
\sigma_{i}^{2}=\frac{1}{n-1} \sum_{k=1}^{n}\left(I_{i}^{r e l}(k)-\langle I\rangle_{i}\right)^{2}
$$

and

$$
\sigma=\left\{\frac{1}{m} \sum_{i=1}^{m} \quad \sigma_{i}^{2}\right\}^{\frac{1}{2}}
$$

where

$m$ is the number of strong lines (usually 5), and

$\mathrm{n}$ is the number of independent observations $i$, per line.

Where conversion of intensities for effects of theta-compensating slits was required, each $\sigma_{i}$ was multiplied by the conversion factor

$$
\mathrm{f}=\frac{\mathrm{I}(\mathrm{comp})}{\mathrm{I}(\mathrm{fixed})}
$$

Format of tables. The printing of the data has been computerized. Superimposed reflections are treated in one of two ways. If a d-spacing has only two possible indices, an $M$ is added to the d-spacing which is repeated on the next line, but with the second index. However, if there are more than two possible indices, a plus sign is used in like manner. In both cases, the composite intensity is printed only once and aligned with the first reflection. The symbol " $1 L^{"}$ in the intensity column is used to indicate "less than 1 ".

\section{UNITS}

In this publication the Angström unit $(1 \AA$ $100 \mathrm{pm}$ ) was selected for presentation of the d-spacings and lattice parameters. This maintained consistency with (a) the earlier publications of Standard X-ray Diffraction Powder Patterns [1982] (b) the publications of the International Union of Crystallography, and (c) the continuing publication of cards and search manuals of the PDF (now consisting of over 40,000 entries). The PDF search manuals are based on the d-spacings in $\AA$ of the 3 strongest lines. Consistent with the choice of the $\AA$ unit for length, the volume of the unit cell is expressed in $\AA^{3}\left(1 \AA^{3}=1 \times 10^{-30} \mathrm{~m}^{3}\right)$. Densities are reported in $\mathrm{g} / \mathrm{cm}^{3}\left(1 \mathrm{gm} / \mathrm{cm}^{3}=10^{3} \mathrm{~kg} / \mathrm{m}^{3}\right)$.

\section{ACKNOWLEDGMENTS}

We would like to thank Carolyn Wingo, Donna Gladhill, and Tracy Olert of the JCPDS Associateship for their assistance in keyboarding the data, proofreading of this manuscript, measuring intensities, and preparing compounds. Appreciation is also expressed to the Text Editing Facility of the National Measurement Laboratory of NBS for typing the manuscript.

\section{REFERENCES}

Alexander, L., Klug, H. P., and Kummer, E. (1948). J. App 1. Phys., 19, No. 8, 742.

Chemical Abstracts Service Registry Handbook Number Section (1974), (The American Chemical Society, Chemical Abstracts Service, The Ohio State Univ., Columbus, OH 43210).

Crystal Data (1973). (3rd Ed. Published jointly by the U.S. Dept. of Commerce, Nat1. Bur. of Stand., Washington, D.C. 20234, and the Joint Committee on Powder Diffraction Standards, 1601 Park Lane, Swarthmore, PA 19081).

Daniels, R. W. (1978). An Introduction to Numerical Methods and Optimization Techniques, (Elsevier North-Holland Inc., New York), 78.

Deslattes, R. D. and Henins, A. (1973). Phys. Rev. Lett. 31, 972.

Deslattes, R. D., Henins, A., Bowman, H. A., Schoonover, R. M., Carroll, C. L., Barnes, I. L., Machlan, L. A., Moore, L. J., and Shields, W. R. (1974). Phys. Rev. Lett. 33, 463.

Donnay, J. D. H. and Kennard, O. (1964). Acta Crystallogr. 17, 1337.

Evans, H. T., Jr., Appleman, D. E., and Handwerker, D. S. (1963). Report 非B216188, U.S. Dept. of Commerce, National Technical Information Center, 5285 Port Royal Rd., Springfield, VA 22151, $\$ 3.50$.

Groth, P. (1908). Chemische Kristallographie II, (Engelmann, Leipzig, Germany). 
Hubbard, C. R. (1982). J. Appl. Cryst. 16, 285.

Hubbard, C. R. (1983). Submitted for Publication.

International Tables for X-ray Crystallography, I (1965), (The Kynoch Press, Birmingham, Eng.).

International Union of Pure and Applied Chemistry (1979). Pure Appl. Chem. 51, No. 2, 407.

ISCC-NBS Centroid Color Charts, SRM 2106 (1965), or Color Kit, SRM 2107 (1977) which contains both SRM 2106 and Color, an NBS Special Publication 非P 440 . These can be obtained from the Nat1. Bur. of Stand., Office of Standard Reference Materials, Washington, DC 20234. Current prices will be quoted on request.

Mighell, A. D., Hubbard, C. R., and Stalick, J. K. (1981). NBS Technical Note 1141, U. S. Dept. of Commerce, Natl. Bur. Stand., Washington, DC 20234.

Palache, C., Berman, H., and Frondel, C. (1944). Dana's System of Mineralogy I, (John Wiley and Sons, New York, 7th Ed.), 6 .

Pyrros, N. P. and Hubbard, C. R. (1983). In Advances in X-ray Analysis, (Plenum Publishing Corporation, New York, NY), 26, 63.

Savitzky, A. and Golay, M. J. E. (1964). Anal. Chem. 36, 1627.

Smith, G. S. and Snyder, R. L. (1979). J. Appl. Crystallogr. 12, 60 .

Snyder, R. L., Hubbard, C. R., and Panagiotopoulos, N. C. (1981). Auto: A Real Time Diffractometer Control System Report, NBSIR 81-2229, U. S. Dept of Commerce, Natl. Bur. Stand., Washington, DC 20234).

Standard Reference Material 640a (1982), Silicon Powder X-ray Diffraction Standard, obtainable from the Natl. Bur. of Stand., Office of Standard Reference Materials, Washington, DC 20234. Current price will be quoted on request.

Standard Reference Material 675 (1982), Fluorophlogopite Powder X-ray Diffraction Standard. To obtain, see procedure above for SRM 640a.

Standard X-ray Diffraction Powder Patterns (1982), see page iv of this Monograph.

Visser, J.W. (1969). J. Appl. Crystallogr. 2, 89.

Wolff, de, P. M. (1968). J. Appl. Crystallogr. 1, 108 . 

Barium Cadmium Phosphate, $\mathrm{BaCd}\left(\mathrm{PO}_{3}\right)_{4}$

Synonym

Barium cadmium metaphosphate

Sample

The sample was made by heating $\mathrm{Ba}(\mathrm{OH})_{2} \cdot 8 \mathrm{H}_{2} \mathrm{O}$, $\mathrm{CdCO}_{3}$, and $\left(\mathrm{NH}_{4}\right)_{2} \mathrm{HPO}_{4}$ at $500{ }^{\circ} \mathrm{C}$ overnight. It was reground, heated at $700{ }^{\circ} \mathrm{C}$ for 2 days followed by heating at $800{ }^{\circ} \mathrm{C}$ overnight.

Color

Colorless

Structure

Monoclinic, $\mathrm{P} 2{ }_{1} / \mathrm{n}$ (14). The structure of $\mathrm{BaCd}\left(\mathrm{PO}_{3}\right)_{4}$ was determined by Averbuch-Pouchot et al. (1975). This phase is isostructural with other phases with $\mathrm{Mn}, \mathrm{Ca}$, and $\mathrm{Ag}$ in place of $\mathrm{Cd}$.

Crystallographic constants of this sample

$a=14.954(2) \AA$

$\mathrm{b}=9.2066(11)$

$c=7.2290(11)$

$\beta=90.82(2)^{\circ}$

$\mathrm{a} / \mathrm{b}=1.6243$

$c / b=0.7852$

$Z=4$

$\begin{array}{ll}Z & =4 \\ V & =995.15 \AA^{3}\end{array}$

Density $(\mathrm{calc})=3.775 \mathrm{~g} / \mathrm{cm}^{3}$

Figure of merit

$F_{30}=53.6(0.0087,64)$

Additional patterns

PDF card 29-152 (Averbuch-Pouchot, 1975)

Majling et al. (1979) (calculated pattern)

References

Averbuch-Pouchot, M.-T. (1975). J. Appl.

Crystallogr. 8, 389 .

Averbuch-Pouchot, M.-T., Durif, A., and Guitel, J. C. (1975). Acta Crystallogr., B31, 2453.

Majling, J., Raninec, 亡̌., and Ďurovič, S. (1979). Calculated Powder Diffraction Patterns for Anhydrous Phosphates (VEDA, Bratislava, Czechoslovakia).

\begin{tabular}{|c|c|c|c|c|c|}
\hline \multicolumn{6}{|c|}{$\begin{array}{c}\text { CuKa }_{1} \lambda=1.540598 \AA \text {; mean } \mathrm{T}=25.8{ }^{\circ} \mathrm{C} \\
\text { Internal standards Si, SRM } 640 \mathrm{a} \\
\text { Fluorophlogopite, SRM } 675\end{array}$} \\
\hline $\mathrm{d}(\stackrel{\circ}{\mathrm{A}})$ & $\begin{array}{c}\text { rel } \\
\sigma= \pm 2\end{array}$ & & hke & & $2 \theta\left(^{\circ}\right)$ \\
\hline 7.84 & 8 & 1 & & 0 & 11.280 \\
\hline 7.48 & 3 & 2 & 0 & 0 & 11.821 \\
\hline 5.801 & 38 & 2 & 1 & 0 & 15.262 \\
\hline 5.686 & 8 & 0 & 1 & 1 & 15.573 \\
\hline 5.333 & 3 & -1 & 1 & 1 & 16.609 \\
\hline 4.604 & 14 & 0 & 2 & 0 & 19.262 \\
\hline 4.497 & 4 & 2 & 1 & 1 & 19.727 \\
\hline 4.397 & 32 & 1 & 2 & 0 & 20.179 \\
\hline 4.135 & $1 \mathrm{~L}$ & -3 & 0 & 1 & 21.471 \\
\hline 3.919 & 8 & 2 & 2 & 0 & 22.674 \\
\hline 3.754 & 69 & 1 & 2 & 1 & 23.681 \\
\hline 3.737 & 68 & 4 & 0 & 0 & 23.789 \\
\hline 3.615 & 5 & 0 & 0 & 2 & 24.608 \\
\hline 3.462 & 100 & 4 & 1 & 0 & 25.709 \\
\hline 3.456 & 98 & -2 & 2 & 1 & 25.761 \\
\hline 3.432 & 20 & 2 & 2 & 1 & 25.943 \\
\hline 3.291 & 16 & -1 & 1 & 2 & 27.070 \\
\hline $3.270 \mathrm{M}$ & 15 & 1 & 1 & 2 & 27.249 \\
\hline $3.270 \mathrm{M}$ & & -2 & 0 & 2 & 27.249 \\
\hline 3.109 & 18 & 4 & 1 & 1 & 28.688 \\
\hline 3.074 & 58 & -3 & 2 & 1 & 29.022 \\
\hline $3.053 \mathrm{M}$ & 70 & 2 & 1 & 2 & 29.233 \\
\hline $3.053 \mathrm{M}$ & & 3 & 2 & 1 & 29.233 \\
\hline 3.006 & 11 & 1 & 3 & 0 & 29.694 \\
\hline 2.845 & 22 & 5 & 1 & 0 & 31.414 \\
\hline 2.825 & 20 & 0 & 3 & 1 & 31.647 \\
\hline 2.805 & 22 & -3 & 1 & 2 & 31.874 \\
\hline $2.778 \mathrm{M}$ & 54 & -1 & 3 & 1 & 32.192 \\
\hline $2.778 \mathrm{M}$ & & -5 & 0 & 1 & 32.192 \\
\hline $2.648 \mathrm{M}$ & 9 & -2 & 3 & 1 & 33.827 \\
\hline $2.648 \mathrm{M}$ & & 2 & 2 & 2 & 33.827 \\
\hline 2.616 & 10 & -4 & 0 & 2 & 34.253 \\
\hline 2.580 & 13 & 4 & 0 & 2 & 34.742 \\
\hline 2.517 & 11 & -4 & 1 & 2 & 35.637 \\
\hline 2.509 & 6 & 5 & 2 & 0 & 35.763 \\
\hline 2.491 & 5 & 6 & 0 & 0 & 36.020 \\
\hline 2.464 & 9 & -3 & 3 & 1 & 36.427 \\
\hline 2.458 & 10 & 3 & 2 & 2 & 36.528 \\
\hline 2.452 & 6 & 3 & 3 & 1 & 36.621 \\
\hline 2.405 & 10 & 6 & 1 & 0 & 37.359 \\
\hline $2.372 \mathrm{M}$ & 6 & 1 & 0 & 3 & 37.893 \\
\hline $2.372 \mathrm{M}$ & & 4 & 3 & 0 & 37.893 \\
\hline 2.331 & 12 & 0 & 1 & 3 & 38.601 \\
\hline $2.308 \mathrm{M}$ & 4 & -1 & 1 & 3 & 38.995 \\
\hline $2.308 \mathrm{M}$ & & 1 & 3 & 2 & 38.995 \\
\hline
\end{tabular}


Barium Cadmium Phosphate, $\mathrm{BaCd}\left(\mathrm{PO}_{3}\right)_{4}$ - (continued)

\begin{tabular}{|c|c|c|c|c|c|}
\hline$d(\stackrel{\circ}{A})$ & $I^{\text {rel }}$ & & hke & & $2 \theta\left(^{\circ}\right)$ \\
\hline $2.2743^{+}$ & 16 & -4 & 2 & 2 & 39.596 \\
\hline $2.2743+$ & & 1 & 4 & 0 & 39.596 \\
\hline 2.2597 & 10 & -4 & 3 & 1 & 39.862 \\
\hline 2.2481 & 6 & 4 & 3 & 1 & 40.077 \\
\hline 2.1679 & 12 & 1 & 4 & 1 & 41.626 \\
\hline 2.1168 & 4 & -1 & 2 & 3 & 42.680 \\
\hline 2.1046 & 14 & -6 & 2 & 1 & 42.939 \\
\hline $2.0906 \mathrm{M}$ & 5 & 6 & 2 & 1 & 43.240 \\
\hline $2.0906 \mathrm{M}$ & & 3 & 4 & 0 & 43.240 \\
\hline 2.0723 & 12 & -5 & 2 & 2 & 43.643 \\
\hline $2.0589 \mathrm{M}$ & 23 & -2 & 2 & 3 & 43.941 \\
\hline $2.0589 \mathrm{M}$ & & -5 & 3 & 1 & 43.941 \\
\hline $2.0484 M$ & 28 & 5 & 2 & 2 & 44.178 \\
\hline $2.0484 M$ & & 5 & 3 & 1 & 44.178 \\
\hline 2.0110 & 19 & -3 & 4 & 1 & 45.045 \\
\hline 2.0046 & 15 & 3 & 4 & 1 & 45.196 \\
\hline $1.9914 \mathrm{M}$ & 12 & 7 & 1 & 1 & 45.512 \\
\hline $1.9914 \mathrm{M}$ & & -4 & 3 & 2 & 45.512 \\
\hline 1.9712 & 6 & -3 & 2 & 3 & 46.005 \\
\hline 1.9602 & 3 & 4 & 4 & 0 & 46.279 \\
\hline 1.9268 & 4 & -1 & 4 & 2 & 47.130 \\
\hline $1.8947 \mathrm{M}$ & 3 & -4 & 4 & 1 & 47.976 \\
\hline $1.8947 \mathrm{M}$ & & 0 & 3 & 3 & 47.976 \\
\hline $1.8833 \mathrm{M}$ & 8 & -1 & 3 & 3 & 48.285 \\
\hline $1.8833 \mathrm{M}$ & & -2 & 4 & 2 & 48.285 \\
\hline $1.8775 \mathrm{M}$ & 18 & -7 & 2 & 1 & 48.446 \\
\hline $1.8775 \mathrm{M}$ & & 1 & 3 & 3 & 48.446 \\
\hline 1.8652 & 10 & 7 & 2 & 1 & 48.785 \\
\hline $1.8325 \mathrm{M}$ & 8. & 2 & 3 & 3 & 49.715 \\
\hline $1.8325 \mathrm{M}$ & & 8 & 1 & 0 & 49.715 \\
\hline 1.8276 & 6 & 1 & 5 & 0 & 49.856 \\
\hline $1.8130 \mathrm{M}$ & 3 & -7 & 1 & 2 & 50.285 \\
\hline $1.8130 \mathrm{M}$ & & -3 & 4 & 2 & 50.285 \\
\hline 1.8074 & 4 & 0 & 0 & 4 & 50.452 \\
\hline 1.7841 & 5 & 0 & 5 & 1 & 51.158 \\
\hline $1.7724 \mathrm{M}$ & 10 & -1 & 5 & 1 & 51.522 \\
\hline $1.7724 \mathrm{M}$ & & -5 & 4 & 1 & 51.522 \\
\hline
\end{tabular}


Barium Titanium Oxide, $\mathrm{BaTiO}_{3}$

Synonym

Barium titanate

CAS registry no. 12047-27-7

Sample

The sample was prepared by heating $\mathrm{BaTiO}_{3}$ at $1550{ }^{\circ} \mathrm{C}$ for one hour and quenching it in water.

Color

Light gray

Structure

Hexagonal, $\mathrm{P}_{3} / \mathrm{mmc}$ (194). The structure was determined by Burbank and Evans (1948).

Crystallographic constants of this sample

$\mathrm{a}=5.72481(11) \AA$

$c=13.9673(3)$

$c / a=2.4398$

$Z=6$

$\begin{array}{ll}Z & =6 \\ V & =396.43 \AA^{3}\end{array}$

Density $(\mathrm{calc})=5.862 \mathrm{~g} / \mathrm{cm}^{3}$

Figure of merit

$F_{30}=105.1(0.0071,40)$

Polymorphism

Blattner et al. (1947) reported the existence of two polymorphs, cubic and tetragonal. The rhombohedral form reported by Megaw (1946) is the same as the phase being reported here.

Additional patterns

PDF card 8-372 (Rase and Roy, 1955)

Dickson et al. (1961)

Arend and Kihlborg (1969)

\section{References}

Arend, H. and Kihlborg, L. (1969). J. Am.

Ceram. Soc. 52, No. 2, 63.

Blattner, H., Matthias, B., and Merz, W.

(1947). Helv. Phys. Acta 20, 225.

Burbank, R. D. and Evans, H. T., Jr. (1948).

Acta Crystallogr. 1,330 .

Dickson, J. G., Katz, L., and Ward, R. (1961). J. Am. Chem. Soc. $83,3026$.

Megaw, H. D. (1946). Proc. Phys. Soc., London, 58,133 .

Rase, D. E. and Roy, R. (1955). J. Am. Ceram. Soc. 38,108 .

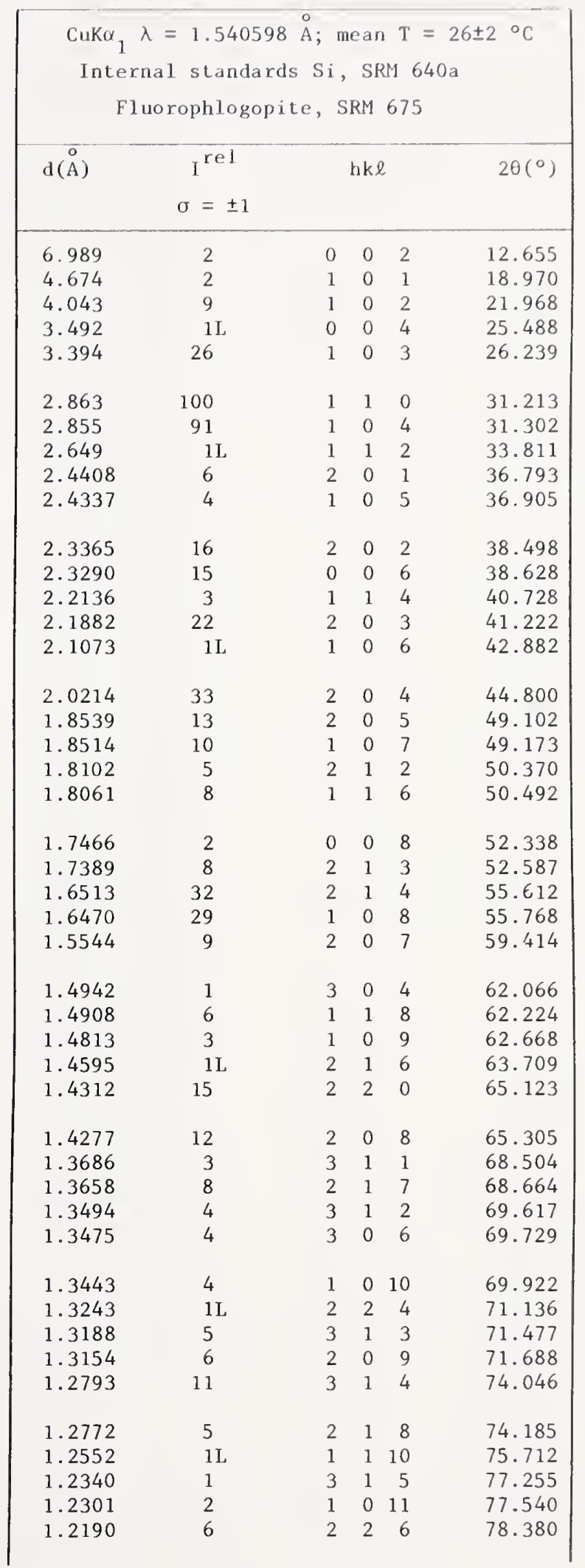


Barium Titanium 0xide, $\mathrm{BaTiO}_{3}$ - (continued)

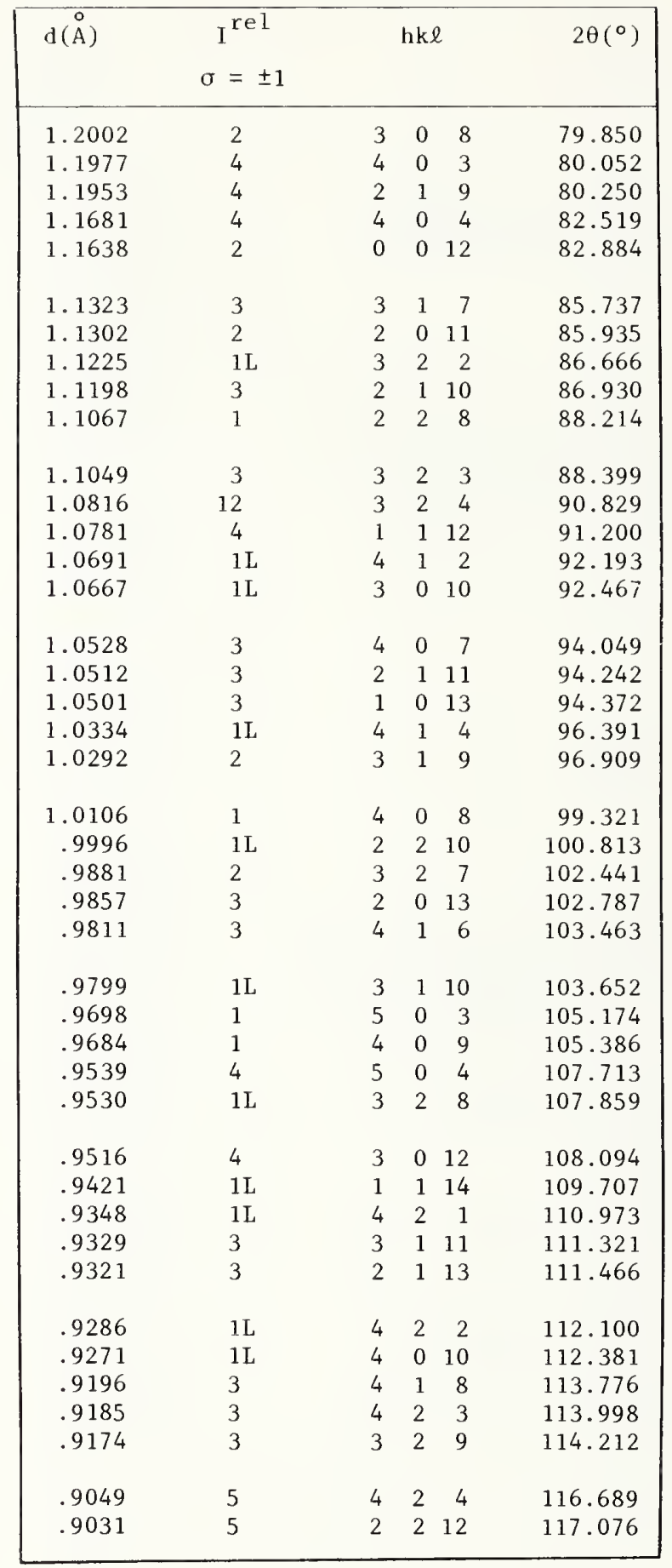


Barium Titanium 0xide, BaTi ${ }_{2} \mathrm{O}_{5}$

Synonyms

Barium titanate

Barium metadititanate

Sample

The sample was prepared by fusing an equimolar mixture of $\mathrm{BaTiO}_{3}$ and $\mathrm{TiO}_{2}$ at $1375{ }^{\circ} \mathrm{C}$ for one hour, then quenching in water.

Color

Light gray

Structure

Monoclinic, $\mathrm{C} 2 / \mathrm{m}$ (12). The structure of

$\mathrm{BaTi}_{2} \mathrm{O}_{5}$ was determined by Harrison (1956).

Crystallographic constants of this sample

$$
\begin{aligned}
& a=16.9140(12) \AA \\
& b=3.9345(3) \\
& c=9.4122(7) \\
& \beta=103.114(6)^{\circ}
\end{aligned}
$$

$\mathrm{a} / \mathrm{b}=4.2989$

$c / b=2.3922$

$Z=6$

$V=610.04 \mathrm{~A}^{3}$

Density $(\mathrm{calc})=5.112 \mathrm{~g} / \mathrm{cm}^{3}$

Figure of merit

$\mathrm{F}_{30}=90.6(0.0077,43)$

Additional pattern

PDF card 8-368 (Rase and Roy, 1955)

References

Harrison, F. W. (1956). Acta Cryst. 9, 495.

Rase, D. E. and Roy, R. (1955). J. Am. Ceram. Soc. 38 , No. 3, 102 .

CuKo $_{1} \lambda=1.540598 \AA$; mean $\mathrm{T}=26 \pm 2{ }^{\circ} \mathrm{C}$ Internal standards $W, a=4.0861 \AA$ F]uorophlogopite, SRM 675

$\begin{array}{cccc}\mathrm{d}(\mathrm{A}) & \mathrm{I}^{\mathrm{rel}} & \text { hkl } & 20\left(^{\circ}\right) \\ & \sigma= \pm 3 & \end{array}$

8.25

6.976

4.585

4.460

4.123

3.827

3.668

3.473

3.152

3. 105

3.056

3.016

2.904

2.864

2.802

2. 772

2.746

2.674

2.634

2.552

2.525

2.479

2. 451

2. 3957

2.3717

2.3333

2. 3292

2. 2910

2. 2237

2.1504

2. 1377

2. 1138

2. 0891

2.0758

2.0610

2.0200

2.0137

1.9960

1.9674

$1.9221 \mathrm{M}$

1. $9221 \mathrm{M}$

1.9175

1.8823

\begin{tabular}{|c|}
\hline 9 \\
\hline 7 \\
\hline 19 \\
\hline 4 \\
\hline 28 \\
\hline
\end{tabular}

1.8666

1.8600

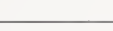

7
6
100
86
35

$\begin{array}{rrr}2 & 0 & 0 \\ -2 & 0 & 1 \\ 0 & 0 & 2 \\ -2 & 0 & 2 \\ -4 & 0 & 1\end{array}$

10.718

12.680

19.344

19.893

110

21.537

202

23.225

$\begin{array}{lll}4 & 0 & 1\end{array}$

24.246

$\begin{array}{lll}-3 & 1 & 1\end{array}$

25.632

28.287

28.727

\section{3}

$\begin{array}{ll}-2 & 0\end{array}$

$\begin{array}{lll}0 & 0 & 3\end{array}$

29.200

76

$\begin{array}{lll}-1 & 1 & 2\end{array}$

29.596

30.764

31.207

$\begin{array}{lll}1 & 1 & 2\end{array}$

$\begin{array}{lll}-3 & 1 & 2\end{array}$

31.909

28

$\begin{array}{lll}-4 & 0 & 3\end{array}$

32.266

69

$\begin{array}{lll}6 & 0 & 0\end{array}$

32.581

$\begin{array}{lll}2 & 0 & 3\end{array}$

$\begin{array}{lll}-6 & 0 & 2\end{array}$

33.480

34.008

35.141

$\begin{array}{lll}5 & 1 & 0\end{array}$

35.520

$\begin{array}{lll}6 & 0 & 1\end{array}$

36.201

$\begin{array}{lll}-1 & 1 & 3\end{array}$

36.627

$\begin{array}{lll}-5 & 1 & 2\end{array}$

37.511

$\begin{array}{lll}-3 & 1 & 3\end{array}$

37.906

51

$\begin{array}{lll}1 & 1 & 3\end{array}$

38.554

$\begin{array}{lll}0 & 0 & 4\end{array}$

$\begin{array}{lll}4 & 0 & 3\end{array}$

38.624

39.294

602

40.535

41.981

$\begin{array}{lll}-5 & 1 & 3\end{array}$

42.242

42.743

43.274

43.566

43.893

$\begin{array}{rrr}3 & 1 & 3 \\ -8 & 0 & 2\end{array}$

44.833

$\begin{array}{lll}7 & 1 & 0\end{array}$

44.982

$\begin{array}{lll}-1 & 1 & 4\end{array}$

$\begin{array}{lll}-6 & 0 & 4\end{array}$

45.401

020

46.100

47.252

$\begin{array}{lll}-8 & 0 & 3\end{array}$

47.252

$\begin{array}{lll}8 & 0 & 1\end{array}$

47.372

$\begin{array}{lll}-2 & 0 & 5\end{array}$

48.313

$\begin{array}{lll}-5 & 1 & 4\end{array}$

48.747 
Barium Titanium $0 x i d e, B a T i_{2} 0_{5}$ - (continued)

\begin{tabular}{|c|c|c|c|c|c|}
\hline$d(\stackrel{\circ}{A})$ & $\mathrm{I}^{\mathrm{rel}}$ & & hkl & & $2 \theta\left({ }^{\circ}\right)$ \\
\hline 1.8452 & $3 \mathrm{~L}$ & 6 & 0 & 3 & 49.349 \\
\hline $1.8329 \mathrm{M}$ & 7 & 4 & 0 & 4 & 49.701 \\
\hline $1.8329 M$ & & 0 & 0 & 5 & 49.701 \\
\hline 1.8081 & 10 & 0 & 2 & 2 & 50.430 \\
\hline 1.7992 & 30 & 5 & 1 & 3 & 50.700 \\
\hline $1.7751 \mathrm{M}$ & 8 & -4 & 2 & 1 & 51.437 \\
\hline $1.7751 \mathrm{M}$ & & 4 & 2 & 0 & 51.437 \\
\hline 1.7559 & 6 & 3 & 1 & 4 & 52.040 \\
\hline 1.7365 & 7 & 8 & 0 & 2 & 52.668 \\
\hline 1.7281 & 12 & 7 & 1 & 2 & 52.943 \\
\hline 1.7144 & 20 & -6 & 0 & 5 & 53.400 \\
\hline 1.7116 & $3 \mathrm{~L}$ & 4 & 2 & 1 & 53.494 \\
\hline $1.6896 \mathrm{M}$ & 28 & -3 & 1 & 5 & 54.248 \\
\hline $1.6896 \mathrm{M}$ & & -10 & 0 & 1 & 54.248 \\
\hline 1.6867 & 28 & -7 & 1 & 4 & 54.348 \\
\hline 1.6744 & 5 & -9 & 1 & 2 & 54.779 \\
\hline 1.6613 & 20 & -2 & 2 & 3 & 55.248 \\
\hline 1.6598 & 21 & 9 & 1 & 0 & 55.302 \\
\hline 1.6246 & 5 & -5 & 1 & 5 & 56.606 \\
\hline 1.6108 & 7 & -10 & 0 & 3 & 57.137 \\
\hline 1.6041 & 20 & -4 & 2 & 3 & 57.396 \\
\hline 1.5996 & 19 & 6 & 2 & 0 & 57.576 \\
\hline 1.5909 & $3 \mathrm{~L}$ & 6 & 0 & 4 & 57.918 \\
\hline 1.5667 & 3 & 5 & 1 & 4 & 58.903 \\
\hline 1.5610 & 10 & 10 & 0 & 1 & 59.138 \\
\hline $1.5520 \mathrm{M}$ & 5 & 8 & 0 & 3 & 59.514 \\
\hline $1.5520 \mathrm{M}$ & & -4 & 0 & 6 & 59.514 \\
\hline 1.5491 & 5 & 4 & 0 & 5 & 59.638 \\
\hline 1.5281 & 7 & 0 & 0 & 6 & 60.544 \\
\hline 1.5117 & $3 \mathrm{~L}$ & -7 & 1 & 5 & 61.270 \\
\hline 1.5070 & 7 & 3 & 1 & 5 & 61.480 \\
\hline 1.4984 & 7 & -9 & 1 & 4 & 61.871 \\
\hline 1.4928 & 7 & 0 & 2 & 4 & 62.131 \\
\hline 1.4856 & 12 & -6 & 0 & 6 & 62.463 \\
\hline 1.4733 & 5 & 4 & 2 & 3 & 63.045 \\
\hline 1.4667 & 8 & 9 & 1 & 2 & 63.362 \\
\hline 1.4553 & 3 & -3 & 1 & 6 & 63.918 \\
\hline 1.4492 & 10 & 10 & 0 & 2 & 64.219 \\
\hline 1.4323 & 23 & 2 & 2 & 4 & 65.068 \\
\hline 1.4293 & 25 & -11 & 1 & 1 & 65.224 \\
\hline 1.4230 & 35 & -8 & 2 & 2 & 65.546 \\
\hline 1.4049 & 5 & -12 & 0 & 1 & 66.500 \\
\hline 1.4013 & 7 & -6 & 2 & 4 & 66.693 \\
\hline 1.3938 & 19 & 1 & 1 & 6 & 67.098 \\
\hline 1.3890 & 25 & -11 & 1 & 3 & 67.362 \\
\hline 1.3861 & 20 & 6 & 0 & 5 & 67.523 \\
\hline 1.3835 & 20 & 8 & 0 & 4 & 67.665 \\
\hline $1.3746 \mathrm{M}$ & 12 & -8 & 2 & 3 & 68.164 \\
\hline $1.3746 \mathrm{M}$ & & 5 & 1 & 5 & 68.164 \\
\hline
\end{tabular}


Synonym

Barium tetratitanate

CAS registry no. 12009-31-3

Sample

The sample was prepared using $\mathrm{BaTiO}_{3}$ and $\mathrm{TiO}_{2}$ (rutile). The reactants in a $1: 3$ molar ratio were blended by grinding under acetone. The following heating schedule, with periodic grinding, yielded a nearly single phase product, containing less than $1 \%$ of an extra phase: $800{ }^{\circ} \mathrm{C}$ for 20 hours; $1175{ }^{\circ} \mathrm{C}$ for 25 hours; $1250{ }^{\circ} \mathrm{C}$ for 20 hours; $1325^{\circ} \mathrm{C}$ for 4 days.

Color

Colorless

Structure

Orthorhombic, Pnmm (59). The structure was determined by Lukaszewicz (1957). Temperature factors as well as atomic coordinates were given by Templeton and Dauben (1960).

Crystallographic constants of this sample

$$
\begin{aligned}
& a=6.2940(5) \AA \\
& b=14.5324(11) \\
& c=3.7972(3) \\
& a / b=0.4331 \\
& c / b=0.2613 \\
& Z=2 \\
& V=347.32 \AA^{3} \\
& \text { Density }(\mathrm{calc})=4.522 \mathrm{~g} / \mathrm{cm}^{3}
\end{aligned}
$$

Figure of merit

$\mathrm{F}_{30}=124.6(0.0065,37)$

Additional pattern

PDF card 8-367 (Rase and Roy, 1955)

References

Lukaszewicz, K. (1957). Rocz. Chem. 31, 1111.

Rase, D. E. and Roy, R. (1955). J. Am. Ceram. Soc. 38, 102.

Templeton, D. H. and Dauben, C. H. (1960).

J. Chem. Phys. 32, 1515.

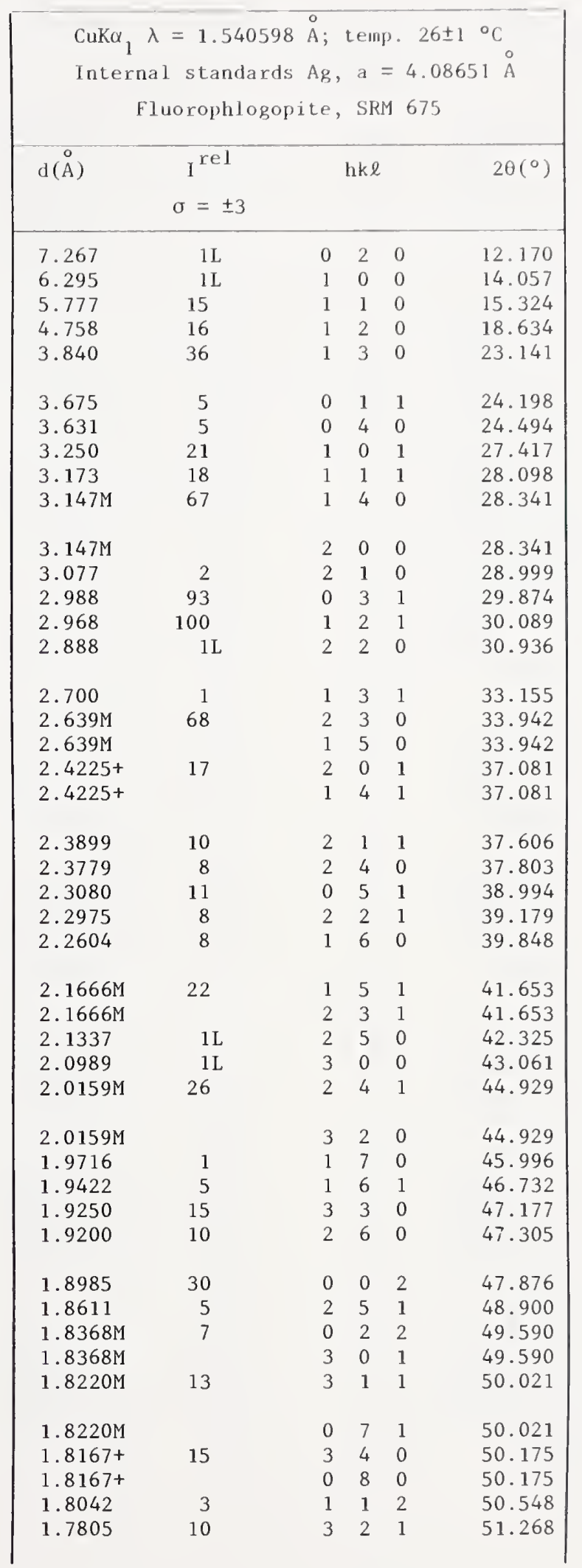


Barium Titanium 0xide, $\mathrm{BaTi}_{4} \mathrm{O}_{9}$ - (continued)

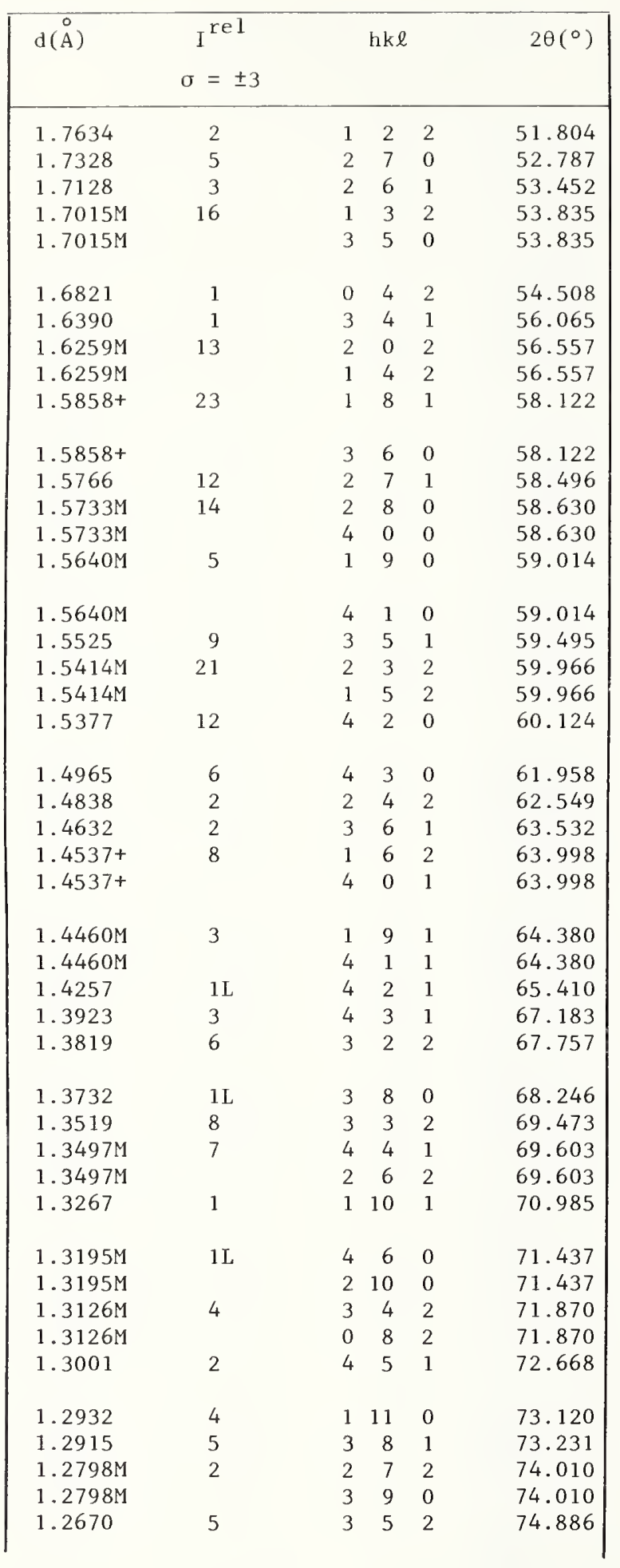

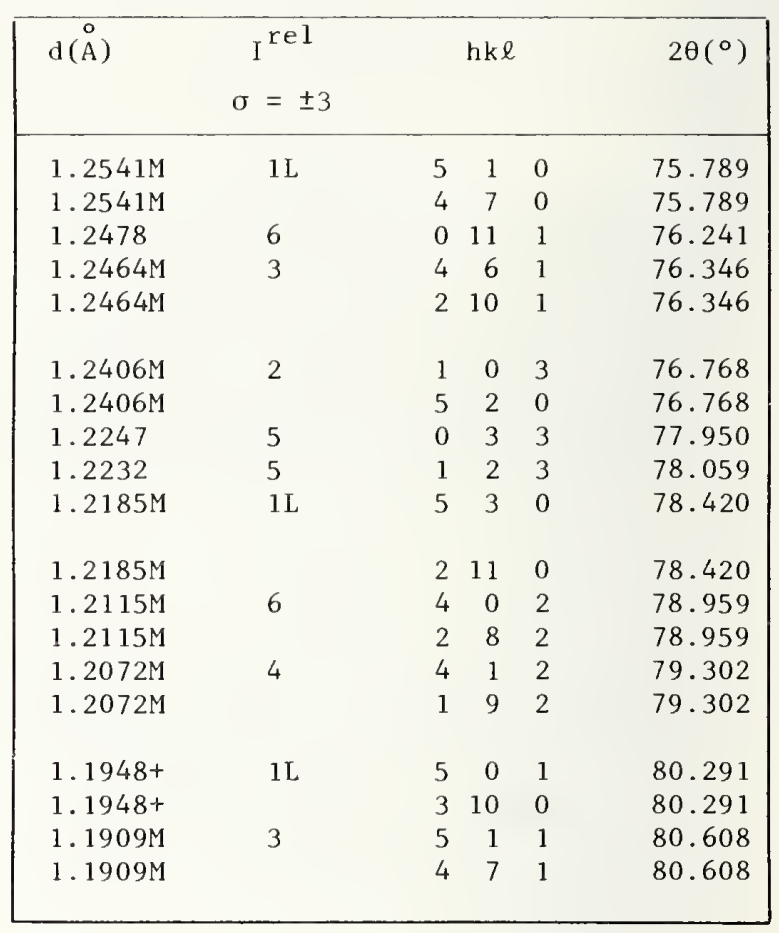


Sample

The sample was prepared by heating stoichiometric proportions of $\mathrm{BaCO}_{3}, \mathrm{TiO}_{2}$ (anatase), and $\left(\mathrm{NH}_{4}\right)_{2} \mathrm{HPO}_{4}$ at $500{ }^{\circ} \mathrm{C}$. The phase was then reground and heated at $1200{ }^{\circ} \mathrm{C}$ for 3 days.

Color

Bluish white

Structure

Rhombohedra1, R $\overline{3} \mathrm{c}$ (167). Similar to

$\mathrm{BaZr}_{4}\left(\mathrm{PO}_{4}\right)_{6}, \mathrm{NaZr}_{2}\left(\mathrm{PO}_{4}\right)_{3}$, and other phosphates of this type. The structure of $\mathrm{NaZr}_{2}\left(\mathrm{PO}_{4}\right)_{3}$ was determined by Hagman and Kierkegaard (1968).

Crystallographic constants of this sample (Hexagonal axes)

$a=8.3483(2) \AA$

$c=23.0193(9)$

$c / a=2.7574$

$Z=3$

$Z=3$
$V=1389.37 \AA^{3}$

Density $(\mathrm{calc})=3.222 \mathrm{~g} / \mathrm{cm}^{3}$

Figure of merit

$F_{30}=188.2(0.0048,33)$

Additional pattern

PDF card 12-685 (Harrison, 1960)

References

Hagman, L.-O. and Kierkegaard, P. (1968). Acta Chem. Scand. 22, 1822.

Harrison, D. E. (1960). J. Electrochem. Soc. $107,217$.

\begin{tabular}{|c|c|c|c|c|c|}
\hline \multicolumn{6}{|c|}{$\begin{array}{l}\text { CuKar } \lambda=1.540598 \AA \text {; mean } \mathrm{T}=26.0^{\circ} \mathrm{C} \\
\text { Internal standard Si, SRM } 640 \mathrm{a}\end{array}$} \\
\hline \multirow[t]{2}{*}{$\mathrm{d}(\AA)$} & \multirow{2}{*}{$\begin{array}{l}I^{r e 1} \\
\sigma= \pm 3\end{array}$} & \multirow{2}{*}{\multicolumn{3}{|c|}{ hke }} & \multirow[t]{2}{*}{$2 \theta\left(^{\circ}\right)$} \\
\hline & & & & & \\
\hline 6.124 & 5 & 0 & 1 & 2 & 14.453 \\
\hline 4.502 & 4 & 1 & 0 & 4 & 19.702 \\
\hline 4.176 & 59 & 1 & 1 & 0 & 21.261 \\
\hline 3.667 & 100 & 1 & 1 & 3 & 24.253 \\
\hline 3.449 & 12 & 2 & 0 & 2 & 25.812 \\
\hline 3.061 & 16 & 0 & 2 & 4 & 29.146 \\
\hline 2.8245 & 93 & 1 & 1 & 6 & 31.652 \\
\hline 2.7132 & 12 & 2 & 1 & 1 & 32.987 \\
\hline 2.6731 & 12 & 0 & 1 & 8 & 33.496 \\
\hline 2.6584 & 14 & 1 & 2 & 2 & 33.687 \\
\hline 2.4682 & 2 & 2 & 1 & 4 & 36.370 \\
\hline 2.4098 & 22 & 3 & 0 & 0 & 37.284 \\
\hline 2.3502 & 3 & 1 & 2 & 5 & 38.266 \\
\hline 2.2511 & 5 & 2 & 0 & 8 & 40.020 \\
\hline 2.1934 & 5 & 1 & 0 & 10 & 41.121 \\
\hline 2.1809 & 8 & 1 & 1 & 9 & 41.367 \\
\hline 2.1014 & 2 & 2 & 1 & 7 & 43.007 \\
\hline 2.0872 & 2 & 2 & 2 & 0 & 43.314 \\
\hline 2.0409 & 13 & 3 & 0 & 6 & 44.349 \\
\hline 2.0137 & 3 & 2 & 2 & 3 & 44.980 \\
\hline 1.9976 & 2 & 1 & 3 & 1 & 45.363 \\
\hline 1.9815 & 12 & 1 & 2 & 8 & 45.753 \\
\hline 1.9411 & 2 & 0 & 2 & 10 & 46.762 \\
\hline 1.9176 & 4 & 0 & 0 & 12 & 47.370 \\
\hline 1.8938 & $1 \mathrm{~L}$ & 1 & 3 & 4 & 48.002 \\
\hline 1.8331 & 30 & 2 & 2 & 6 & 49.697 \\
\hline 1.7854 & 1 & 0 & 4 & 2 & 51.118 \\
\hline 1.7602 & 6 & 2 & 1 & 10 & 51.906 \\
\hline 1.7430 & 3 & 1 & 1 & 12 & 52.455 \\
\hline 1.7240 & 3 & 4 & 0 & 4 & 53.078 \\
\hline 1.7119 & 6 & 1 & 3 & 7 & 53.484 \\
\hline 1.6613 & $1 \mathrm{~L}$ & 1 & 2 & 11 & 55.247 \\
\hline 1.6451 & 10 & 3 & 1 & 8 & 55.841 \\
\hline 1.6412 & 4 & 2 & 3 & 2 & 55.985 \\
\hline 1.6169 & 1 & 2 & 2 & 9 & 56.900 \\
\hline 1.6032 & 2 & 0 & 1 & 14 & 57.434 \\
\hline 1.5935 & 1 & 3 & 2 & 4 & 57.815 \\
\hline 1.5776 & 13 & 4 & 1 & 0 & 58.454 \\
\hline 1.5605 & 1 & 2 & 3 & 5 & 59.158 \\
\hline 1.5452 & 3 & 4 & 1 & 3 & 59.802 \\
\hline 1.5306 & 6 & 0 & 4 & 8 & 60.435 \\
\hline 1.5121 & 5 & 1 & 3 & 10 & 61.253 \\
\hline 1.5008 & 11 & 3 & 0 & 12 & 61.762 \\
\hline 1.4969 & 11 & 2 & 0 & 14 & 61.941 \\
\hline 1.4808 & 2 & 3 & 2 & 7 & 62.689 \\
\hline
\end{tabular}


Barium Titanium Phosphate, $\mathrm{BaTi}_{4}\left(\mathrm{PO}_{4}\right)_{6}-$ (continued)

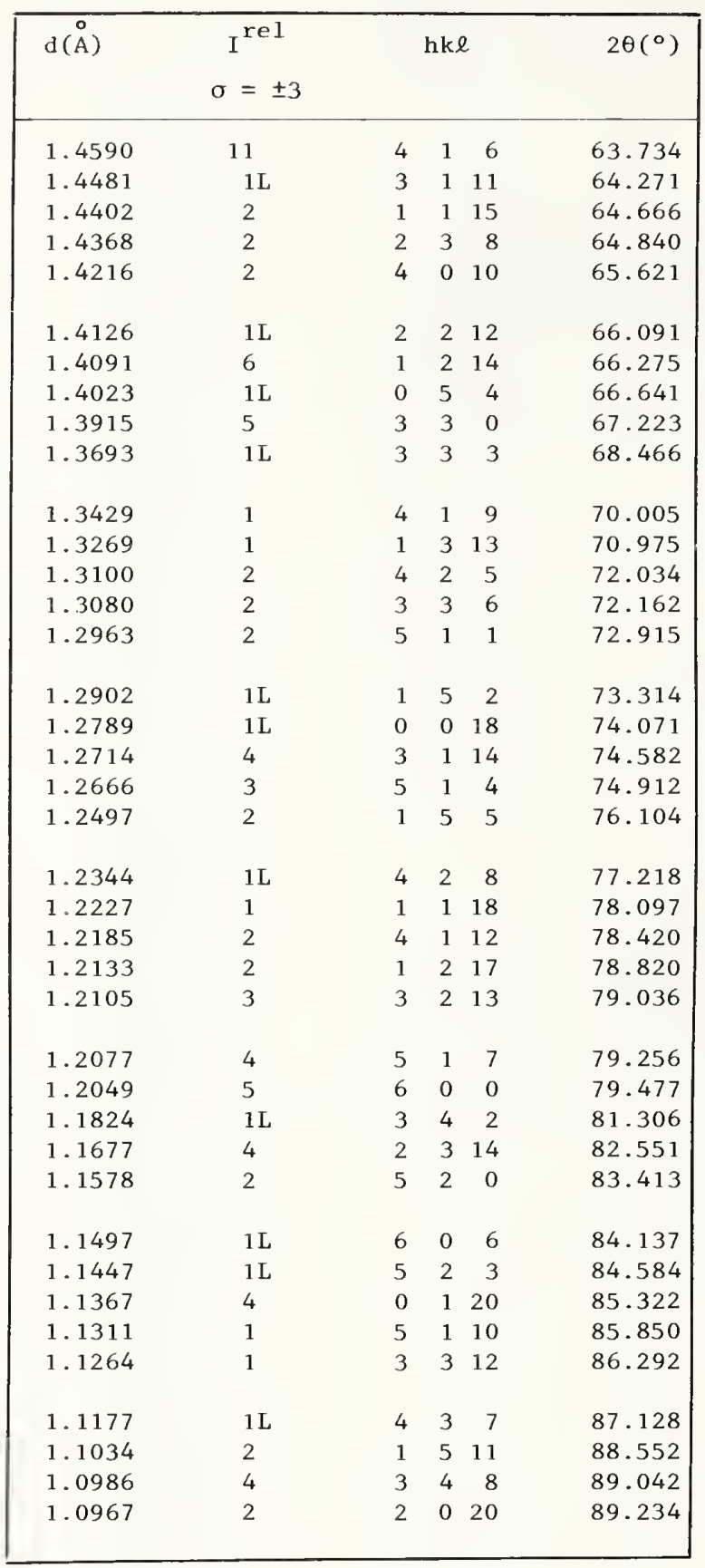


Synonym

Barium zirconium orthophosphate

CAS registry no.

6792-89-8

Sample

The sample was made by heating a $1: 4: 6$ molar mixture of $\mathrm{BaCO}_{3}, \mathrm{ZrO} \mathrm{rO}_{2}$, and $\left(\mathrm{NH}_{4}\right)_{2} \mathrm{HPO}_{4}$ at $500{ }^{\circ} \mathrm{C}$. This was followed by grinding and heating to $1600{ }^{\circ} \mathrm{C}$ for 1 day.

Color

Colorless

Structure

Rhombohedral, R*t (166). The structure was similar to that of $\mathrm{NaZr}_{2}\left(\mathrm{PO}_{4}\right)_{3}$ (Chernorukov et a1., 1978).

Crystallographic constants of this sample (Hexagonal axes)

$a=8.6468(5) \AA$

$c=23.984(2)$

$c / a=2.7737$

$\begin{array}{ll}\mathrm{Z} & =3 \\ \mathrm{~V} & =1552.97 \AA^{3}\end{array}$

Density $(\mathrm{calc})=3.439 \mathrm{~g} / \mathrm{cm}^{3}$

Figure of merit

$F_{30}=91.7(0.0091,36)$

Reference

Chernorukov, N. G., Korshunov, I. A., and Prokof'eva, T. V. (1978). Sov. Phys.

Crystallogr. (Engl. Trans1.) 23, 475.

\begin{tabular}{|c|c|c|c|c|c|}
\hline \multicolumn{6}{|c|}{$\begin{array}{c}\mathrm{CuK \alpha}_{1} \lambda=1.540598 \AA \text {; mean } \mathrm{T}=25.0{ }^{\circ} \mathrm{C} \\
\text { Internal standards Si, SRM } 640 \mathrm{a} \\
\text { Fluorophlogopite, SRM } 675\end{array}$} \\
\hline $\mathrm{d}(\stackrel{\circ}{\mathrm{A}})$ & $\begin{array}{l}I^{\text {rel }} \\
= \pm 4\end{array}$ & & $\mathrm{hk}$ & & $2 \theta\left(^{\circ}\right)$ \\
\hline 8.01 & $1 L$ & 0 & 0 & 3 & 11.039 \\
\hline 7.16 & 1 & 1 & 0 & 1 & 12.353 \\
\hline 6.359 & 1 & 0 & 1 & 2 & 13.915 \\
\hline 4.681 & 7 & 1 & 0 & 4 & 18.942 \\
\hline 4.324 & 36 & 1 & 1 & 0 & 20.522 \\
\hline 4.040 & 2 & 0 & 1 & 5 & 21.986 \\
\hline 3.999 & 1 & 0 & 0 & 6 & 22.213 \\
\hline 3.804 & 40 & 1 & 1 & 3 & 23.368 \\
\hline 3.695 & 3 & 0 & 2 & 1 & 24.063 \\
\hline 3.576 & 2 & 2 & 0 & 2 & 24.876 \\
\hline
\end{tabular}

\begin{tabular}{|c|c|c|c|c|c|}
\hline $\mathrm{d}(\stackrel{\mathrm{A}}{)})$ & $\begin{array}{l}\text { rel } \\
\sigma= \pm 4\end{array}$ & & $\mathrm{~h} k \mathrm{l}$ & & $2 \theta\left(^{\circ}\right)$ \\
\hline 3.176 & 20 & 0 & 2 & 4 & 28.071 \\
\hline 3.114 & 2 & 1 & 0 & 7 & 28.647 \\
\hline 2.936 & 100 & 1 & 1 & 6 & 30.422 \\
\hline 2.811 & 8 & 2 & 1 & 1 & 31.810 \\
\hline 2.784 & 7 & 0 & 1 & 8 & 32.126 \\
\hline 2.755 & 2 & 1 & 2 & 2 & 32.473 \\
\hline 2.559 & 2 & 2 & 1 & 4 & 35.032 \\
\hline 2.496 & 30 & 3 & 0 & 0 & 35.952 \\
\hline 2.437 & 2 & 1 & 2 & 5 & 36.850 \\
\hline 2.384 & 1 & 3 & 0 & 3 & 37.709 \\
\hline 2.340 & 6 & 2 & 0 & 8 & 38.435 \\
\hline 2. 2684 & 4 & 1 & 1 & 9 & 39.703 \\
\hline 2.1829 & 4 & 2 & 1 & 7 & 41.328 \\
\hline 2.1613 & 6 & 2 & 2 & 0 & 41.760 \\
\hline 2.1173 & 14 & 3 & 0 & 6 & 42.669 \\
\hline 2.0865 & 2 & 2 & 2 & 3 & 43.330 \\
\hline 2.0683 & 4 & 1 & 3 & 1 & 43.731 \\
\hline 2.0581 & 11 & 1 & 2 & 8 & 43.960 \\
\hline 2.0193 & 4 & 0 & 2 & 10 & 44.849 \\
\hline 1.9977 & 2 & 0 & 0 & 12 & 45.360 \\
\hline 1.9625 & 2 & 1 & 3 & 4 & 46.221 \\
\hline 1.9014 & 28 & 2 & 2 & 6 & 47.798 \\
\hline 1.8501 & $1 L$ & 0 & 4 & 2 & 49.209 \\
\hline 1.8295 & 12 & 2 & 1 & 10 & 49.801 \\
\hline 1.8140 & 2 & 1 & 1 & 12 & 50.255 \\
\hline 1.7760 & 2 & 1 & 3 & 7 & 51.409 \\
\hline 1.7072 & 6 & 3 & 1 & 8 & 53.642 \\
\hline 1.6788 & 2 & 2 & 2 & 9 & 54.623 \\
\hline 1.6702 & 4 & 0 & 1 & 14 & 54.929 \\
\hline 1.6513 & 4 & 3 & 2 & 4 & 55.613 \\
\hline 1.6339 & 14 & 4 & 1 & 0 & 56.257 \\
\hline 1.6167 & $1 \mathrm{~L}$ & 2 & 3 & 5 & 56.909 \\
\hline 1.6008 & $1 \mathrm{~L}$ & 4 & 1 & 3 & 57.526 \\
\hline 1.5877 & 2 & 0 & 4 & 8 & 58.048 \\
\hline 1.5700 & 6 & 1 & 31 & 10 & 58.766 \\
\hline 1.5602 & 9 & 3 & 0 & 12 & 59.172 \\
\hline 1.5573 & 9 & 2 & 0 & 14 & 59.292 \\
\hline 1.5356 & 2 & 3 & 2 & 7 & 60.214 \\
\hline 1.5126 & 15 & 4 & 1 & 6 & 61.230 \\
\hline 1.4995 & 1 & 1 & 1 & 15 & 61.823 \\
\hline 1.4905 & $1 \mathrm{~L}$ & 2 & 3 & 8 & 62.235 \\
\hline 1.4863 & 1 & 5 & 0 & 2 & 62.432 \\
\hline 1.4756 & 2 & 4 & 0 & 10 & 62.934 \\
\hline 1.4657 & 10 & 1 & 2 & 14 & 63.413 \\
\hline 1.4531 & 2 & 0 & 5 & 4 & 64.026 \\
\hline 1.4411 & 5 & 3 & 3 & 0 & 64.625 \\
\hline
\end{tabular}


Synonyms

Bismuth germanate, BGO

Bismuth orthogermanate

Germanium bismuthate

CAS registry no.

12233-56-6

Sample

The sample was donated by Harshaw Chemical Co.

Color

Colorless

Structure

Cubic, $1 \overline{4} 3 \mathrm{~d}(220)$, isostructural with

$\mathrm{Bi}_{4}\left(\mathrm{SiO}_{4}\right)_{3}$. The structure of $\mathrm{Bi}_{4}\left(\mathrm{SiO}_{4}\right)_{3}$ was determined by Durif (1957).

Crystallographic constants of this sample

$\mathrm{a}=10.5187(2) \AA$

$\mathrm{Z}=4$

$\mathrm{Z}=4$
$\mathrm{~V}=1163.8 \mathrm{~A}^{3}$

Density $(\mathrm{calc})=7.109 \mathrm{~g} / \mathrm{cm}^{3}$

Figure of merit

$\mathrm{F}_{30}=106.9(0.0091,31)$

Additional patterns

PDF card 11-320 (Durif, 1958)

PDF card 17-809 (Gattow and Fricke, 1963)

References

Durif, A. (1958). Anal. Chem. 30, 1161.

Durif, A. (1957). C. R. Hebd. Séances

Acad. Sci. 244, 2815, and ibid. 245,

1095 .

Gattow, G. and Fricke, H. (1963). Z.

Anorg. Allg. Chem. 324, 287.

\begin{tabular}{|c|c|c|c|c|c|}
\hline \multicolumn{6}{|c|}{$\begin{array}{l}\mathrm{CuK} \alpha_{1} \lambda=1.540598 \AA \text {; mean } \mathrm{T}=24.9{ }^{\circ} \mathrm{C} \\
\text { Internal standard } \mathrm{Si}, \mathrm{SRM} 640 \mathrm{a}\end{array}$} \\
\hline $\mathrm{d}(\stackrel{\circ}{\mathrm{A}})$ & $\begin{array}{l}I^{\text {rel }} \\
\sigma= \pm 3\end{array}$ & & hk $\ell$ & & $2 \theta\left(^{\circ}\right)$ \\
\hline 4.293 & 62 & 2 & 1 & 1 & 20.673 \\
\hline 3.719 & 1 & 2 & 2 & 0 & 23.911 \\
\hline 3.326 & 94 & 3 & 1 & 0 & 26.786 \\
\hline 2.811 & 100 & 3 & 2 & 1 & 31.803 \\
\hline 2.631 & 14 & 4 & 0 & 0 & 34.052 \\
\hline 2.352 & 2 & 4 & 2 & 0 & 38.242 \\
\hline 2.2423 & 2 & 3 & 3 & 2 & 40.185 \\
\hline 2.1470 & 44 & 4 & 2 & 2 & 42.051 \\
\hline 2.0622 & 34 & 5 & 1 & 0 & 43.867 \\
\hline 1.9200 & 6 & 5 & 2 & 1 & 47.306 \\
\hline
\end{tabular}

\begin{tabular}{|c|c|c|c|c|c|}
\hline $\mathrm{d}(\stackrel{\circ}{\mathrm{A}})$ & $\mathrm{I}^{\mathrm{rel}}$ & & hke & & $2 \theta\left(^{\circ}\right)$ \\
\hline 1.8594 & 2 & 4 & 4 & 0 & 48.946 \\
\hline 1.8038 & 18 & 5 & 3 & 0 & 50.560 \\
\hline 1.7059 & 38 & 6 & 1 & 1 & 53.685 \\
\hline 1.6626 & 14 & 6 & 2 & 0 & 55.203 \\
\hline 1.6226 & 22 & 5 & 4 & 1 & 56.685 \\
\hline 1.5505 & 15 & 6 & 3 & 1 & 59.577 \\
\hline 1.5183 & 6 & 4 & 4 & 4 & 60.975 \\
\hline 1.4874 & 21 & 7 & 1 & 0 & 62.382 \\
\hline 1.4585 & 2 & 6 & 4 & 0 & 63.759 \\
\hline 1.4313 & 8 & 7 & 2 & 1 & 65.120 \\
\hline 1.4053 & 7 & 6 & 4 & 2 & 66.478 \\
\hline 1. 3809 & 1 & 7 & 3 & 0 & 67.813 \\
\hline 1.3360 & 3 & 6 & 5 & 1 & 70.422 \\
\hline 1.2947 & 8 & 7 & 4 & 1 & 73.022 \\
\hline 1. 2758 & $1 \mathrm{~L}$ & 8 & 2 & 0 & 74.280 \\
\hline 1.2571 & 3 & 6 & 5 & 3 & 75.579 \\
\hline 1.2397 & 14 & 6 & 6 & 0 & 76.831 \\
\hline 1.2227 & 10 & 7 & 5 & 0 & 78.100 \\
\hline 1.1908 & 6 & 7 & 5 & 2 & 80.610 \\
\hline 1.1760 & 1 & 8 & 4 & 0 & 81.842 \\
\hline 1.1617 & 2 & 9 & 1 & 0 & 83.071 \\
\hline 1.1477 & $1 \mathrm{~L}$ & 8 & 4 & 2 & 84.319 \\
\hline 1.1343 & 9 & 7 & 6 & 1 & 85.551 \\
\hline 1.1213 & 2 & 6 & 6 & 4 & 86.779 \\
\hline 1.1088 & 9 & 9 & 3 & 0 & 88.007 \\
\hline 1.0850 & 3 & 7 & 6 & 3 & 90.461 \\
\hline 1.0736 & 4 & 8 & 4 & 4 & 91.698 \\
\hline 1.0626 & 10 & 9 & 4 & 1 & 92.923 \\
\hline 1.0415 & 6 & 10 & 1 & 1 & 95.393 \\
\hline 1.0314 & 1 & 10 & 2 & 0 & 96.632 \\
\hline 1.0216 & 2 & 9 & 5 & 0 & 97.873 \\
\hline 1.0029 & 9 & 10 & 3 & 1 & 100.355 \\
\hline .9852 & 2 & 8 & 7 & 1 & 102.869 \\
\hline .9766 & 1 & 10 & 4 & 0 & 104.136 \\
\hline .9683 & 1 & 9 & 6 & 1 & 105.402 \\
\hline .9602 & 4 & 10 & 4 & 2 & 106.681 \\
\hline .9523 & 6 & 11 & 1 & 0 & 107.966 \\
\hline .9371 & 1 & 10 & 5 & 1 & 110.568 \\
\hline .9298 & $1 \mathrm{~L}$ & 8 & 8 & 0 & 111.882 \\
\hline .9226 & 2 & 9 & 7 & 0 & 113.216 \\
\hline .9087 & 11 & 9 & 7 & 2 & 115.925 \\
\hline .9020 & 5 & 10 & 6 & 0 & 117.298 \\
\hline .8954 & 1 & 11 & 4 & 1 & 118.687 \\
\hline
\end{tabular}


CAS registry no. $12233-73-7$

\section{Sample}

$\mathrm{Bi}_{2} \mathrm{O}_{3}$ and $\mathrm{GeO}_{2}$ were melted together in a 6 to 1 molar ratio and a single crystal was pulled from the melt by the Czochralski technique (Levin and Roth, 1964).

Color

Single crystal: dark yellow

Ground: yellowish white

Structure

Cubic, I23 (197). The structure was

determined by Abrahams et al. (1967).

Crystallographic constants of this sample $a=10.14558(14) \AA$

$Z=2$

$V=144.31 \AA^{3}$

Density $(\mathrm{calc})=9.223 \mathrm{~g} / \mathrm{cm}^{3}$

Figure of merit $F_{30}=117.3(0.0085,30)$

Additional patterns PDF card 17-812 (Gattow and Fricke, 1963) PDF card 23-71 (Bernstein, 1967)

References

Abrahams, S. C., Jamieson, P. B., and Bernstein, J. L. (1967). J. Chem. Phys . 47,4034 .

Bernstein, J. L. (1967). J. Cryst. Growth 1,45 .

Gattow, G. and Fricke, H. (1963). Z. Anorg. Allg. Chem. 324, 287.

Levin, E. M. and Roth, R. S. (1964). J. Res. Nat1. Bur. Stand., Sect. A, 68, 197.
$\mathrm{CuK} \alpha_{1} \lambda=1.540598 \AA$; mean $\mathrm{T}=25.8{ }^{\circ} \mathrm{C}$ Internal standard $W, a=3.16524 \AA$

\begin{tabular}{|c|c|c|c|c|c|}
\hline \multirow[t]{2}{*}{$d(\stackrel{\circ}{A})$} & $I^{r e l}$ & \multicolumn{3}{|c|}{$h k \ell$} & \multirow[t]{2}{*}{$2 \theta\left(^{\circ}\right)$} \\
\hline & $\sigma= \pm 2$ & & & & \\
\hline 7.18 & 1 & 1 & 1 & 0 & 12.323 \\
\hline 5.072 & 2 & 2 & 0 & 0 & 17.470 \\
\hline 4.146 & 4 & 2 & 1 & 1 & 21.415 \\
\hline 3.587 & 22 & 2 & 2 & 0 & 24.800 \\
\hline 3.208 & 100 & 3 & 1 & 0 & 27.785 \\
\hline 2.930 & 26 & 2 & 2 & 2 & 30.484 \\
\hline 2.712 & 76 & 3 & 2 & 1 & 33.002 \\
\hline 2.537 & 4 & 4 & 0 & 0 & 35.344 \\
\hline 2.391 & 12 & 3 & 3 & 0 & 37.585 \\
\hline 2.2681 & 12 & 4 & 2 & 0 & 39.707 \\
\hline 2.1628 & 19 & 3 & 3 & 2 & 41.729 \\
\hline 2.0712 & 15 & 4 & 2 & 2 & 43.667 \\
\hline 1.9899 & 23 & 5 & 1 & 0 & 45.549 \\
\hline 1.8519 & 12 & 5 & 2 & 1 & 49.158 \\
\hline 1.7927 & 1 & 4 & 4 & 0 & 50.894 \\
\hline 1.7396 & 46 & 5 & 3 & 0 & 52.567 \\
\hline 1.6910 & 25 & 6 & 0 & 0 & 54.198 \\
\hline 1.6460 & 36 & 5 & 3 & 2 & 55.808 \\
\hline 1.6036 & 1 & 6 & 2 & 0 & 57.418 \\
\hline 1.5656 & 6 & 5 & 4 & 1 & 58.945 \\
\hline 1.5294 & 6 & 6 & 2 & 2 & 60.485 \\
\hline 1.4960 & 28 & 6 & 3 & 1 & 61.981 \\
\hline 1.4640 & 4 & 4 & 4 & 4 & 63.493 \\
\hline 1.4347 & 12 & 5 & 5 & 0 & 64.945 \\
\hline 1.4070 & 3 & 6 & 4 & 0 & 66.387 \\
\hline 1.3807 & 8 & 7 & 2 & 1 & 67.824 \\
\hline 1.3559 & 5 & 6 & 4 & 2 & 69.234 \\
\hline 1.3320 & 2 & 7 & 3 & 0 & 70.663 \\
\hline 1.2887 & 7 & 6 & 5 & 1 & 73.414 \\
\hline 1.2683 & 1 & 8 & 0 & 0 & 74.799 \\
\hline 1.2487 & 6 & 7 & 4 & 1 & 76.177 \\
\hline 1.2303 & 3 & 6 & 4 & 4 & 77.524 \\
\hline 1.2128 & 17 & 6 & 5 & 3 & 78.861 \\
\hline 1.1956 & 16 & 6 & 6 & 0 & 80.219 \\
\hline 1.1795 & 17 & 7 & 5 & 0 & 81.549 \\
\hline 1.1640 & 1 & 6 & 6 & 2 & 82.869 \\
\hline 1.1489 & 6 & 7 & 5 & 2 & 84.204 \\
\hline 1.1343 & 2 & 8 & 4 & 0 & 85.543 \\
\hline 1.1205 & 4 & 9 & 1 & 0 & 86.860 \\
\hline 1.1071 & 6 & 8 & 4 & 2 & 88.182 \\
\hline 1.0942 & 9 & 7 & 6 & 1 & 89.500 \\
\hline 1.0814 & 1 & 6 & 6 & 4 & 90.845 \\
\hline 1.0695 & 8 & 9 & 3 & 0 & 92.148 \\
\hline 1.0464 & 5 & 9 & 3 & 2 & 94.804 \\
\hline 1.0355 & $1 \mathrm{~L}$ & 8 & 4 & 4 & 96.133 \\
\hline
\end{tabular}


Bismuth Germanium Oxide, $\mathrm{Bi}_{12} \mathrm{GeO}_{20} \sim$ (continued)

\begin{tabular}{|c|c|c|c|c|c|}
\hline$d(\stackrel{\circ}{A})$ & $\mathrm{I}^{\text {rel }}$ & & hke & & $2 \theta\left({ }^{\circ}\right)$ \\
\hline 1.0248 & 16 & 7 & 7 & 0 & 97.468 \\
\hline 1.0145 & 3 & 8 & 6 & 0 & 98.802 \\
\hline 1.0047 & 1 & 10 & 1 & 1 & 100.122 \\
\hline .9948 & 3 & 10 & 2 & 0 & 101.482 \\
\hline .9854 & 9 & 9 & 5 & 0 & 102.835 \\
\hline .9763 & 3 & 10 & 2 & 2 & 104.187 \\
\hline .9673 & 9 & 10 & 3 & 1 & 105.571 \\
\hline .9501 & 4 & 8 & 7 & 1 & 108.333 \\
\hline .9419 & 3 & 10 & 4 & 0 & 109.729 \\
\hline .9340 & 6 & 10 & 3 & 3 & 111.130 \\
\hline .9261 & 2 & 10 & 4 & 2 & 112.569 \\
\hline .9185 & 7 & 11 & 1 & 0 & 113.999 \\
\hline .9038 & 7 & 10 & 5 & 1 & 116.923 \\
\hline .8897 & 8 & 9 & 7 & 0 & 119.940 \\
\hline .8831 & 10 & 10 & 4 & 4 & 121.448 \\
\hline .8764 & 12 & 9 & 7 & 2 & 123.021 \\
\hline .8699 & 3 & 10 & 6 & 0 & 124.630 \\
\hline .8637 & 5 & 11 & 4 & 1 & 126.225 \\
\hline .8514 & 6 & 9 & 6 & 5 & 129.591 \\
\hline .8454 & 4 & 12 & 0 & 0 & 131.320 \\
\hline .8397 & 17 & 11 & 5 & 0 & 133.089 \\
\hline .8284 & 6 & 10 & 7 & 1 & 136.826 \\
\hline .8230 & 2 & 10 & 6 & 4 & 138.783 \\
\hline .8176 & 4 & 12 & 3 & 1 & 140.829 \\
\hline .8072 & 5 & 11 & 6 & 1 & 145.237 \\
\hline
\end{tabular}


CAS registry no.

12441-73-5

Sample

$\mathrm{Bi}_{2} \mathrm{O}_{3}$ and $\mathrm{TiO}_{2}$ were melted together in the ratio of 6 to 1 moles and a single crystal was pulled by the Czochralski technique (Levin and Roth, 1964).

Color

Single crystal: dark red brown

Ground: grayish yellow

Structure

Cubic, I23 (197). Assumed to be isomorphous with $\mathrm{Bi}_{12} \mathrm{GeO}_{20}$ (Abrahams et al., 1967).

Crystallographic constants of this sample $\mathrm{a}=10.17391(13) \AA$

$Z=2$

$\mathrm{V}=1053.08 \AA^{3}$

Density $(\mathrm{calc})=9.069 \mathrm{~g} / \mathrm{cm}^{3}$

Figure of merit

$\mathrm{F}_{30}=111.0(0.0090,30)$

References

Abrahams, S. C., Jamieson, P. B., and Bernstein, J. L. (1967). J. Chem. Phys . 47,4034 .

Levin, E. M. and Roth, R. S. (1964). J. Res. Nat1. Bur. Stand., Sect. A, 68, 197.

\begin{tabular}{|c|c|c|c|c|c|}
\hline \multicolumn{6}{|c|}{$\begin{array}{l}\mathrm{CuK} \alpha_{1} \lambda=1.540598 \AA \text {; mean } \mathrm{T}=25.9{ }^{\circ} \mathrm{C} \\
\text { Internal standard } \mathrm{W}, \mathrm{a}=3.16524 \AA\end{array}$} \\
\hline $\mathrm{d}(\stackrel{\circ}{\mathrm{A}})$ & $\begin{array}{l}I^{\text {rel }} \\
\sigma= \pm 3\end{array}$ & & hke & & $2 \theta\left({ }^{\circ}\right)$ \\
\hline 7.19 & 1 & 1 & 1 & 0 & 12.296 \\
\hline 5.080 & 3 & 2 & 0 & 0 & 17.444 \\
\hline 4.152 & 4 & 2 & 1 & 1 & 21.381 \\
\hline 3.595 & 23 & 2 & 2 & 0 & 24.747 \\
\hline 3.218 & 100 & 3 & 1 & 0 & 27.701 \\
\hline 2.936 & 25 & 2 & 2 & 2 & 30.424 \\
\hline 2.720 & 77 & 3 & 2 & 1 & 32.901 \\
\hline 2.543 & 3 & 4 & 0 & 0 & 35.263 \\
\hline 2.398 & 11 & 3 & 3 & 0 & 37.482 \\
\hline 2.2750 & 12 & 4 & 2 & 0 & 39.583 \\
\hline 2.1691 & 20 & 3 & 3 & 2 & 41.603 \\
\hline 2.0763 & 13 & 4 & 2 & 2 & 43.554 \\
\hline 1.9959 & 20 & 5 & 1 & 0 & 45.403 \\
\hline 1.8578 & 11 & 5 & 2 & 1 & 48.993 \\
\hline 1.7982 & 1 & 4 & 4 & 0 & 50.729 \\
\hline 1.7447 & 46 & 5 & 3 & 0 & 52.401 \\
\hline 1.6959 & 22 & 6 & 0 & 0 & 54.028 \\
\hline 1.6506 & 35 & 6 & 1 & 1 & 55.637 \\
\hline 1.6090 & 1 & 6 & 2 & 0 & 57.207 \\
\hline 1.5700 & 4 & 5 & 4 & 1 & 58.766 \\
\hline
\end{tabular}

\begin{tabular}{|c|c|c|c|c|c|}
\hline $\mathrm{d}(\stackrel{\circ}{\AA})$ & $I^{r e l}$ & & hel & & $2 \theta(0)$ \\
\hline 1.5335 & 6 & 6 & 2 & 2 & 60.307 \\
\hline 1.4996 & 26 & 6 & 3 & 1 & 61.816 \\
\hline 1.4687 & 3 & 4 & 4 & 4 & 63.268 \\
\hline 1.4389 & 10 & 5 & 5 & 0 & 64.736 \\
\hline 1.4107 & 2 & 6 & 4 & 0 & 66.192 \\
\hline 1.3844 & 9 & 7 & 2 & 1 & 67.617 \\
\hline 1.3599 & 4 & 6 & 4 & 2 & 69.002 \\
\hline 1.3359 & 2 & 7 & 3 & 0 & 70.426 \\
\hline 1.2920 & 6 & 6 & 5 & 1 & 73.196 \\
\hline 1.2716 & 1 & 8 & 0 & 0 & 74.569 \\
\hline 1.2522 & 5 & 7 & 4 & 1 & 75.929 \\
\hline 1.2336 & 2 & 6 & 4 & 4 & 77.277 \\
\hline 1.2158 & 16 & 6 & 5 & 3 & 78.626 \\
\hline 1.1991 & 14 & 6 & 6 & 0 & 79.939 \\
\hline 1.1827 & 13 & 7 & 5 & 0 & 81.277 \\
\hline 1.1670 & 1 & 6 & 6 & 2 & 82.612 \\
\hline 1.1519 & 4 & 7 & 5 & 2 & 83.941 \\
\hline 1.1374 & 2 & 8 & 4 & 0 & 85.256 \\
\hline 1.1235 & 4 & 9 & 1 & 0 & 86.569 \\
\hline 1.1100 & 5 & 8 & 4 & 2 & 87.886 \\
\hline 1.0972 & 8 & 7 & 6 & 1 & 89.189 \\
\hline 1.0847 & 1 & 6 & 6 & 4 & 90.498 \\
\hline 1.0724 & 5 & 9 & 3 & 0 & 91.824 \\
\hline 1.0493 & 3 & 9 & 3 & 2 & 94.463 \\
\hline 1.0384 & 1 & 8 & 4 & 4 & 95.770 \\
\hline 1.0278 & 12 & 7 & 7 & 0 & 97.094 \\
\hline 1.0174 & 3 & 10 & 0 & 0 & 98.423 \\
\hline 1.0075 & 3 & 10 & 1 & 1 & 99.738 \\
\hline .9976 & 3 & 10 & 2 & 0 & 101.089 \\
\hline .9882 & 5 & 9 & 5 & 0 & 102.433 \\
\hline .9792 & 2 & 10 & 2 & 2 & 103.752 \\
\hline .9701 & 6 & 10 & 3 & 1 & 105.132 \\
\hline .9529 & 3 & 8 & 7 & 1 & 107.877 \\
\hline .9446 & 3 & 10 & 4 & 0 & 109.269 \\
\hline .9365 & 5 & 9 & 6 & 1 & 110.673 \\
\hline .9287 & 2 & 10 & 4 & 2 & 112.081 \\
\hline .9211 & 4 & 11 & 1 & 0 & 113.501 \\
\hline .9064 & 5 & 10 & 5 & 1 & 116.398 \\
\hline .8923 & 3 & 9 & 7 & 0 & 119.364 \\
\hline .8855 & 6 & 10 & 4 & 4 & 120.891 \\
\hline .8789 & 9 & 9 & 7 & 2 & 122.442 \\
\hline .8725 & 2 & 10 & 6 & 0 & 123.984 \\
\hline .8661 & 4 & 11 & 4 & 1 & 125.586 \\
\hline .8538 & 4 & 9 & 6 & 5 & 128.910 \\
\hline .8478 & 3 & 12 & 0 & 0 & 130.612 \\
\hline .8419 & 10 & 11 & 5 & 0 & 132.384 \\
\hline .8307 & 4 & 10 & 7 & 1 & 136.025 \\
\hline .8253 & 2 & 12 & 2 & 2 & 137.941 \\
\hline .8198 & 3 & 12 & 3 & 1 & 139.973 \\
\hline .8093 & 3 & 11 & 6 & 1 & 144.260 \\
\hline .7993 & 2 & 9 & 9 & 0 & 149.017 \\
\hline
\end{tabular}


CAS registry no. $10294-33-4$

Sample

The sample was obtained from the Carborundum Co., Niagara Falls, N.Y.

Color Colorless

Structure Hexagona $1, \mathrm{P}_{3} / \mathrm{mmc}(194)$. The structure was determined by Pease (1952).

Crystallographic constants of this sample

$\mathrm{a}=2.50441(7) \stackrel{\circ}{\mathrm{A}}$

$c=6.6562(4)$

$c / a=2.6578$

$Z=2$
$V=36.158 \AA^{3}$

Density $(\mathrm{calc})=2.280 \mathrm{~g} / \mathrm{cm}^{3}$

Figure of merit

$F_{20}=71.5(0.011,25)$

Polymorphism

A high pressure wurtzite-type form (borazon) was described by Soma et al. (1974) and another high pressure zinc blende type structure was described by Coleburn and Forbes (1968).

Additional pattern

PDF card 9-12 (Hatfield Ltd., Sheffield and A.E.R.E. Harwel1, Didcot, Berks, England).

\section{References}

Coleburn, N. L. and Forbes, J. W. (1968).

J. Chem. Phys. 48, 555.

Pease, R. S. (1952). Acta Crystallogr. 5, 356.

Soma, T., Sawaoka, A., and Saito, S. (1974).

Mater. Rés. Bull. 9, 755.

\begin{tabular}{|c|c|c|c|c|c|}
\hline \multicolumn{6}{|c|}{$\begin{array}{c}\mathrm{CuK}_{1} \lambda=1.540598 \mathrm{~A} \text {; mean } \mathrm{T}=26 \pm 2{ }^{\circ} \mathrm{C} \\
\text { Internal standard Si, SRM } 640 \mathrm{a}\end{array}$} \\
\hline \multirow[t]{2}{*}{$d(\stackrel{\circ}{A})$} & $\mathrm{I}^{\text {rel }}$ & \multirow{2}{*}{\multicolumn{2}{|c|}{ hke }} & & \multirow[t]{2}{*}{$2 \theta\left(^{\circ}\right)$} \\
\hline & $\sigma= \pm 1$ & & & & \\
\hline 3.328 & 100 & 0 & 0 & 2 & 26.765 \\
\hline 2.169 & 15 & 1 & 0 & 0 & 41.598 \\
\hline 2.0619 & 6 & 1 & 0 & 1 & 43.874 \\
\hline 1.8176 & 9 & 1 & 0 & 2 & 50.150 \\
\hline 1.6636 & 6 & 0 & 0 & 4 & 55.165 \\
\hline 1.5509 & $1 \mathrm{~L}$ & 1 & 0 & 3 & 59.561 \\
\hline 1.3198 & 2 & 1 & 0 & 4 & 71.412 \\
\hline 1.2521 & 5 & 1 & 1 & 0 & 75.934 \\
\hline 1.1720 & 5 & 1 & 1 & 2 & 82.177 \\
\hline 1.1346 & $1 \mathrm{~L}$ & 1 & 0 & 5 & 85.521 \\
\hline 1.1095 & $1 \mathrm{~L}$ & 0 & 0 & 6 & 87.943 \\
\hline 1.0843 & $1 \mathrm{~L}$ & 2 & 0 & 0 & 90.535 \\
\hline 1.0311 & 1 & 2 & 0 & 2 & 96.667 \\
\hline 1.0005 & 3 & 1 & 1 & 4 & 100.690 \\
\hline .9877 & $1 \mathrm{~L}$ & 1 & 0 & 6 & 102.501 \\
\hline .9086 & $1 \mathrm{~L}$ & 2 & 0 & 4 & 115.941 \\
\hline .8319 & $1 \mathrm{~L}$ & 0 & 0 & 8 & 135.641 \\
\hline .8304 & 1 & 1 & 1 & 6 & 136.150 \\
\hline .8197 & $1 \mathrm{~L}$ & 2 & 1 & 0 & 140.003 \\
\hline .7960 & $1 \mathrm{~L}$ & 2 & 1 & 2 & 150.810 \\
\hline
\end{tabular}


Synonym

Boron orthophosphate

Sample

The sample was obtained from City Chemical

Corp., New York, N.Y.

Color

Colorless

Structure

Tetragonal, $1 \overline{4}(82)$. The structure was

determined by Schulze (1934).

Crystallographic constants of this sample

$\mathrm{a}=4.34253(15) \AA$

$c=6.6415(4)$

$c / a=1.5294$

$\mathrm{Z}=2$

$V=125.24 \AA^{3}$

Density $(\mathrm{calc})=2.805 \mathrm{~g} / \mathrm{cm}^{3}$

Figure of merit

$\mathrm{F}_{28}=112.1(0.0063,36)$

Polymorphism

Three other forms of $\mathrm{BPO}_{4}$ are reported. A quartz-like hexagonal (Shafex et al., 1956) and two high pressure hexagonal forms

(Mackenzie et al., 1959, and Dachille and Dent-Glasser, 1959).

Additional pattern

PDF card 14-696 (deWolff, priv, comm.)

References

Dachille, F. and Dent-Glasser, L. S. (1959). Acta Crystallogr. 12, 280.

Mackenzie, J. D., Roth, W. L., and Wentorf, R. H. (1959). Acta Crystallogr. 12, 79.

Schulze, G. E. R. (1934). Z. Phys. Chem. B. 24,215 .

Shafer, E. C., Shafer, M. W., and Roy, R.

(1956). Z. Kristallogr. 108, 263.

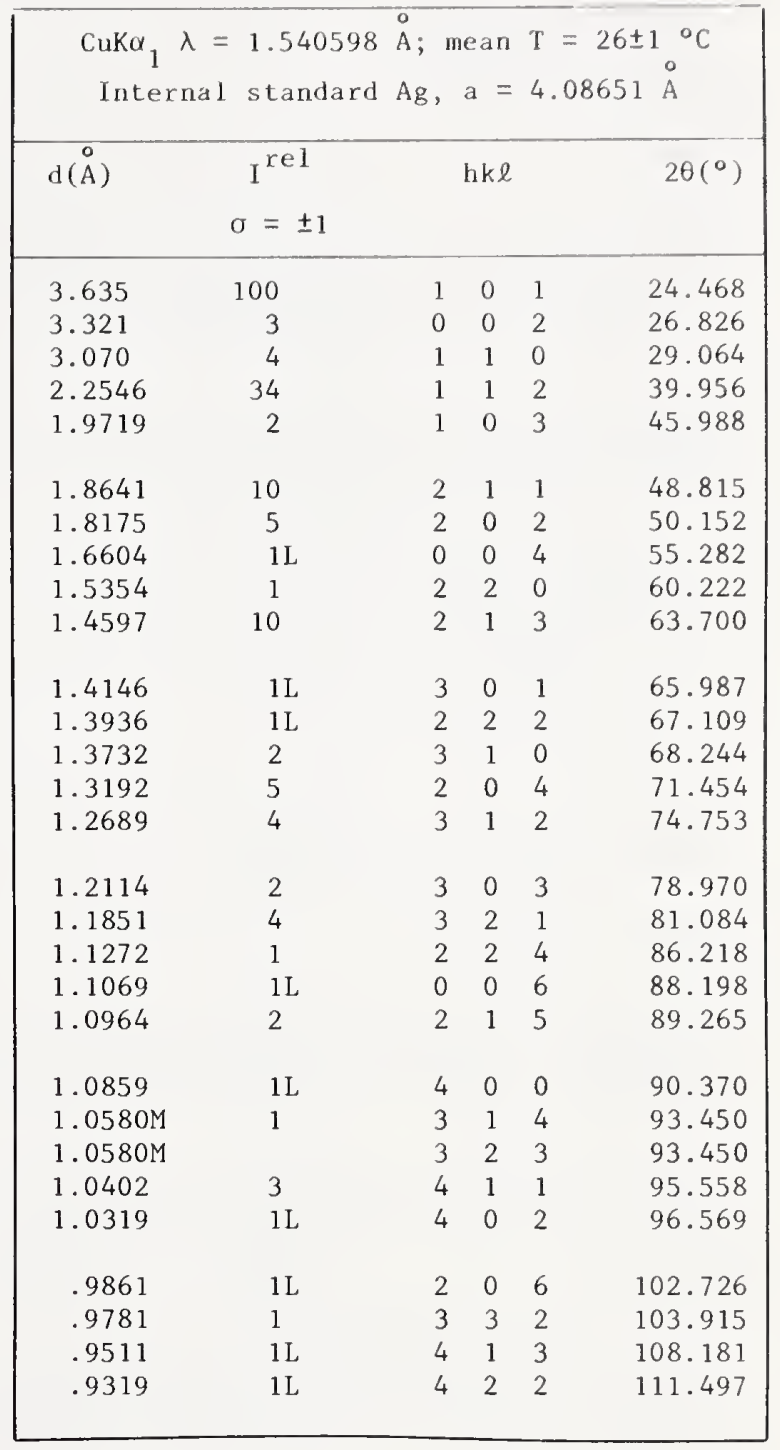


Cadmium Aluminum Oxide, $\mathrm{CdAl}_{2} \mathrm{O}_{4}$

Synonyms

Cadmium aluminate

Cadmium metaaluminate

CAS registry no.

$12252-16-3$

Sample

The sample was prepared by heating $\mathrm{CdO}$ and $\gamma-\mathrm{Al}_{2} \mathrm{O}_{3}$ in a sealed platinum tube for 18 hours at $975{ }^{\circ} \mathrm{C}$, followed by 20 hours at $1200{ }^{\circ} \mathrm{C}$.

Structure

Rhombohedral, R $\overline{3}$ (148). Phenakite type

(Colin and Théry, 1966).

Crystallographic constants of this sample (Hexagonal axes)

$a=14.2146(3) \AA$

$c=9.5698(3)$

$\mathrm{c} / \mathrm{a}=0.6732$

$Z=18$

$\mathrm{V}=1674.57 \AA^{3}$

Density $(\mathrm{calc})=4.112 \mathrm{~g} / \mathrm{cm}^{3}$

Figure of merit

$F_{30}=228.6(0.0031,43)$

Polmorphism

At higher temperatures a cubic (spinel) form has been reported (Hahn et al., 1955).

Additional pattern

PDF card 22-119 (Colin and Théry, 1966)

References

Colin, F. and Théry, J. (1966). C. R. Séances

Acad. Sci. Ser. C, 263, 1220.

Hahn, H., Günter, F., Klingler, W., Störger, D., and Störger, G. (1955). Z. Anorg. Allg. Chem. 279,241

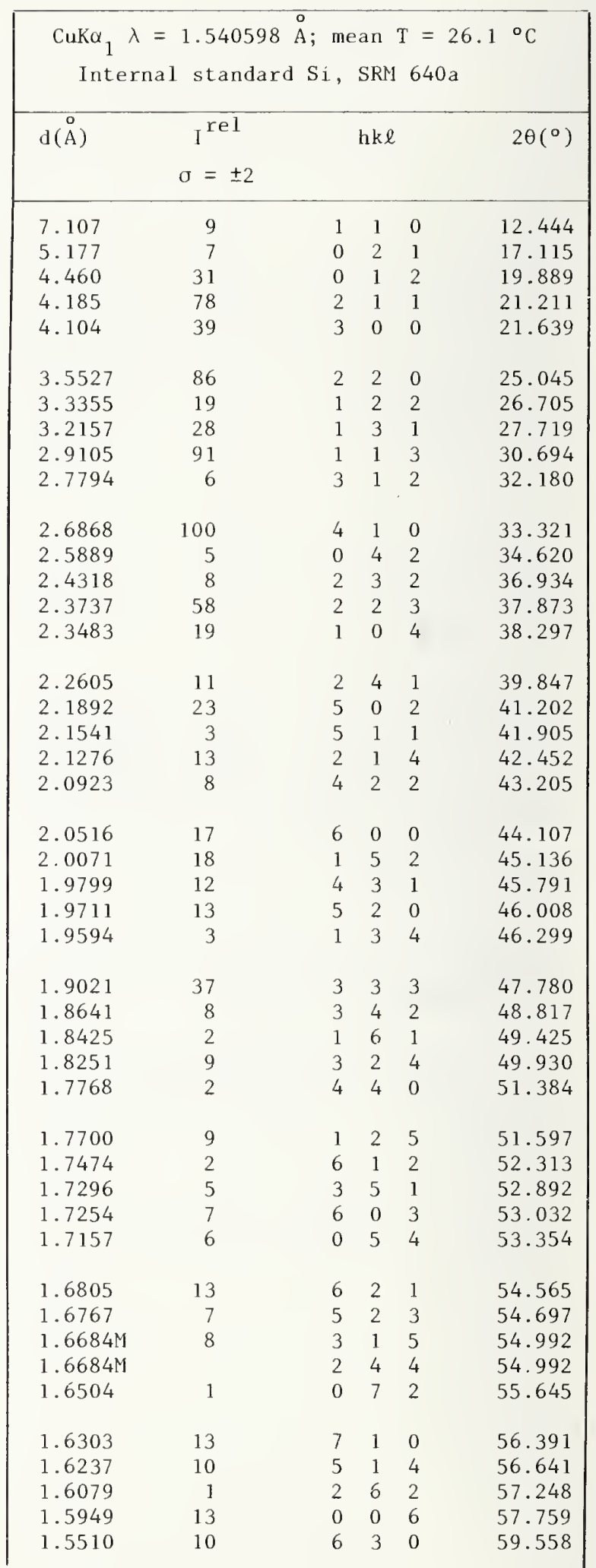


Cadmium Aluminum Oxide, $\mathrm{CdAl}_{2} \mathrm{O}_{4}$ - (continued)

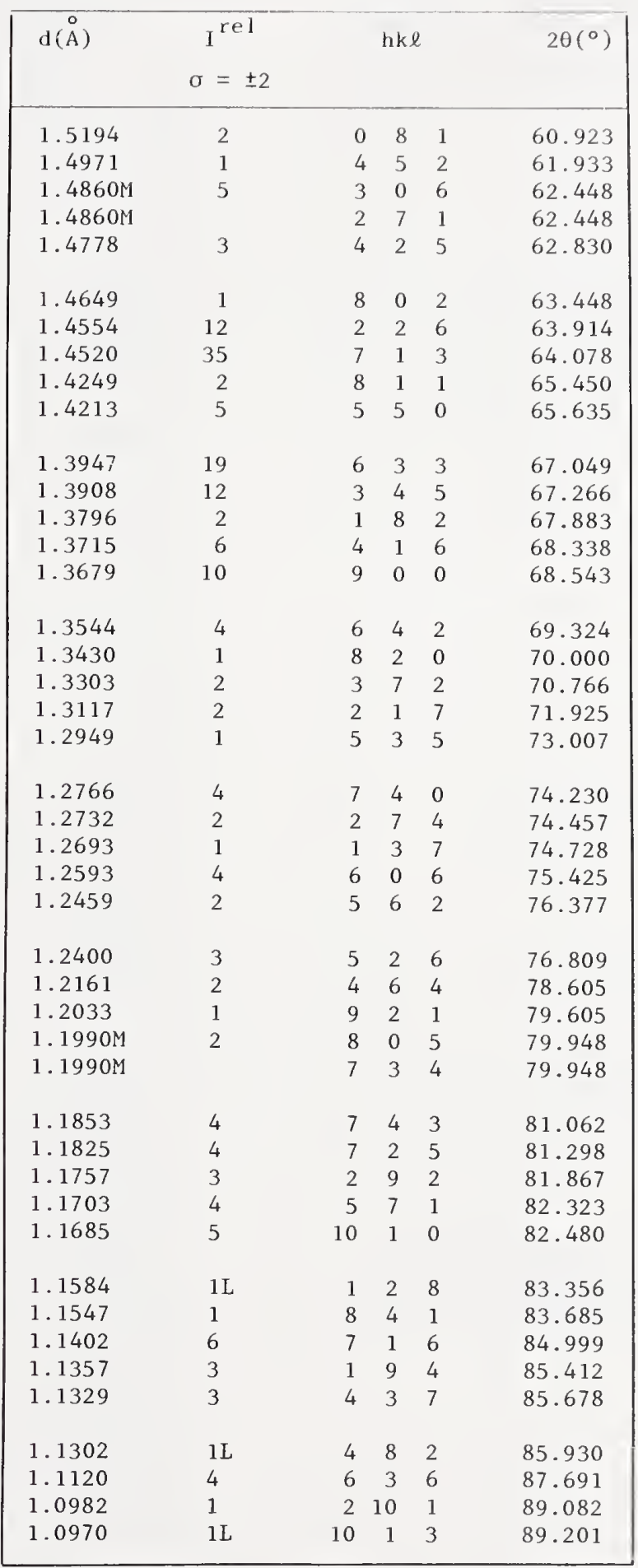


Sample

The sample was made by heating a mixture of $\mathrm{CdCO}_{3}, \mathrm{ZnO}$, and $\left(\mathrm{NH}_{4}\right)_{2} \mathrm{HPO}_{4}$ for 19 hours at $800{ }^{\circ} \mathrm{C}$, followed by regrinding and heating for 2 days at $800{ }^{\circ} \mathrm{C}$.

Color

Colorless

Structure

Monoclinic, P21/c (14). The structure was determined by Calvo and Stephens (1968).

The structure of this phase is related to graftonite $(\mathrm{Fe}, \mathrm{Mn}, \mathrm{Ca})_{3}\left(\mathrm{PO}_{4}\right)_{2}$ and $\mathrm{Cd}_{2} \mathrm{Zn}\left(\mathrm{PO}_{4}\right)_{2}$. This phase called "Bss" has a range of composition from $20 \%$ to $35 \% \mathrm{Cd}_{3}\left(\mathrm{PO}_{4}\right)_{2}$ in the system $\mathrm{Cd}_{3}\left(\mathrm{PO}_{4}\right)_{2}-\mathrm{Zn}_{3}\left(\mathrm{PO}_{4}\right)_{2}$ (Brown and Hummel, 1963).

Crystallographic constants of this sample

$\mathrm{a}=9.0594(14) \stackrel{\circ}{\mathrm{A}}$

$\mathrm{b}=11.5369(14)$

$c=5.9197(8)$

$\beta=98.556(14)^{\circ}$

$a / b=0.7853$

$c / b=0.5131$

$\mathrm{Z}=4$

$\mathrm{V}=611.83 \mathrm{\AA}^{3}$

Density $(\mathrm{calc})=4.651 \mathrm{~g} / \mathrm{cm}^{3}$

Figure of merit

$\mathrm{F}_{30}=61.5(0.012,41)$

Additional patterns

Brown and Hummel (1963)

Majling et al. (1979) Calculated pattern

References

Brown, J. J. and Hummel, F. A. (1963). J.

Electrochem. Soc. 110, 1218.

Calvo, C. and Stephens, J. S. (1968). Can.

J. Chem. 46, 903.

Majling. J., Raninec, Ц́., and Durovič, S. (1979). Calculated Powder Diffraction Patterns for Anhydrous Phosphates (VEDA, Bratislava, Czechoslovakia).
$\mathrm{CuK} \alpha_{1} \lambda=1.540598 \stackrel{\circ}{\mathrm{A}}$; mean $\mathrm{T}=25.1{ }^{\circ} \mathrm{C}$

Internal standards Si, SRM 640a

Fluorophlogopite, SRM 675

\begin{tabular}{|c|c|c|c|c|c|}
\hline$d(\stackrel{\circ}{A})$ & $I^{\text {rel }}$ & & hke & & $2 \theta\left(^{\circ}\right)$ \\
\hline 8.96 & 10 & 1 & 0 & 0 & 9.868 \\
\hline 7.06 & $1 \mathrm{~L}$ & 1 & 1 & 0 & 12.523 \\
\hline 5.760 & $1 \mathrm{~L}$ & 0 & 2 & 0 & 15.370 \\
\hline 5.212 & $1 \mathrm{~L}$ & 0 & 1 & 1 & 16.997 \\
\hline 4.800 & 2 & -1 & 1 & 1 & 18.471 \\
\hline 4.480 & 8 & 2 & 0 & 0 & 19.803 \\
\hline 4.274 & 4 & 1 & 1 & 1 & 20.765 \\
\hline 4.177 & 13 & 2 & 1 & 0 & 21.253 \\
\hline 4.112 & 6 & 0 & 2 & 1 & 21.594 \\
\hline 3.889 & 13 & -1 & 2 & 1 & 22.850 \\
\hline 3.648 & 33 & -2 & 1 & 1 & 24.383 \\
\hline 3.592 & 22 & 1 & 2 & 1 & 24.768 \\
\hline 3.532 & 53 & 1 & 3 & 0 & 25.196 \\
\hline 3.213 & 10 & 0 & 3 & 1 & 27.744 \\
\hline 3.107 & 9 & -1 & 3 & 1 & 28.713 \\
\hline 2.988 & 35 & 3 & 0 & 0 & 29.880 \\
\hline 2.950 & 47 & 1 & 3 & 1 & 30.277 \\
\hline 2.926 & 47 & 0 & 0 & 2 & 30.522 \\
\hline 2.916 & 49 & 2 & 3 & 0 & 30.633 \\
\hline $2.883 \mathrm{M}$ & 100 & 0 & 4 & 0 & 30.989 \\
\hline $2.883 \mathrm{M}$ & & 2 & 2 & 1 & 30.989 \\
\hline 2.838 & 32 & 0 & 1 & 2 & 31.496 \\
\hline 2.824 & 31 & -1 & 1 & 2 & 31.655 \\
\hline 2.756 & 67 & -3 & 1 & 1 & 32.462 \\
\hline 2.745 & 66 & 1 & 4 & 0 & 32.590 \\
\hline 2.668 & 8 & 1 & 0 & 2 & 33.558 \\
\hline 2.635 & 8 & -2 & 0 & 2 & 33.989 \\
\hline 2.611 & 13 & 0 & 2 & 2 & 34.320 \\
\hline $2.600 \mathrm{M}$ & 17 & -1 & 2 & 2 & 34.473 \\
\hline $2.600 \mathrm{M}$ & & 1 & 1 & 2 & 34.473 \\
\hline 2.569 & 8 & -2 & 1 & 2 & 34.892 \\
\hline 2.546 & 2 & -3 & 2 & 1 & 35.228 \\
\hline 2.455 & 19 & 3 & 1 & 1 & 36.574 \\
\hline 2.424 & 9 & 2 & 4 & 0 & 37.054 \\
\hline 2.398 & 2 & -2 & 2 & 2 & 37.467 \\
\hline 2.329 & 15 & 0 & 3 & 2 & 38.623 \\
\hline 2.308 & 5 & -2 & 4 & 1 & 39.002 \\
\hline 2.284 & 12 & -3 & 3 & 1 & 39.424 \\
\hline 2.266 & 4 & -3 & 0 & 2 & 39.744 \\
\hline 2.240 & 1 & 4 & 0 & 0 & 40.222 \\
\hline 2.198 & 12 & 4 & 1 & 0 & 41.039 \\
\hline 2.135 & 2 & 2 & 2 & 2 & 42.302 \\
\hline 2.114 & 5 & -1 & 5 & 1 & 42.732 \\
\hline 2.109 & 8 & -3 & 2 & 2 & 42.851 \\
\hline 2.103 & 2 & 3 & 3 & 1 & 42.971 \\
\hline 2.063 & 3 & 1 & 5 & 1 & 43.860 \\
\hline 2.051 & 10 & 2 & 5 & 0 & 44.109 \\
\hline 1.9786 & 8 & -2 & 5 & 1 & 45.825 \\
\hline 1.9734 & 1 & 2 & 3 & 2 & 45.951 \\
\hline 1.9517 & 25 & -3 & 3 & 2 & 46.492 \\
\hline
\end{tabular}


Cadmium Zinc Phosphate, $\mathrm{Cd}_{0.9} \mathrm{Zn}_{2.1}\left(\mathrm{PO}_{4}\right)_{2}-$ (continued)

\begin{tabular}{|c|c|c|c|c|c|}
\hline $\mathrm{d}(\stackrel{\circ}{\mathrm{A}})$ & $\begin{array}{c}l^{\text {re] }} \\
\sigma= \pm 4\end{array}$ & & hke & & $2 \theta\left({ }^{\circ}\right)$ \\
\hline 1.9346 & 10 & 4 & 3 & 0 & 46.927 \\
\hline $1.9234+$ & 10 & 3 & 1 & 2 & 47.216 \\
\hline $1.9234+$ & & 0 & 6 & 0 & 47.216 \\
\hline 1.9123 & 6 & -4 & 3 & 1 & 47.509 \\
\hline $1.8968 \mathrm{M}$ & 12 & 2 & 5 & 1 & 47.920 \\
\hline $1.8968 \mathrm{M}$ & & -4 & 1 & 2 & 47.920 \\
\hline 1.8699 & 13 & -2 & 1 & 3 & 48.654 \\
\hline 1.8494 & 2 & 0 & 2 & 3 & 49.230 \\
\hline $1.8274 M$ & 22 & 1 & 1 & 3 & 49.862 \\
\hline $1.8274 \mathrm{M}$ & & 0 & 6 & 1 & 49.862 \\
\hline 1.8100 & 6 & -1 & 5 & 2 & 50.374 \\
\hline 1.8061 & 7 & -1 & 6 & 1 & 50.492 \\
\hline 1.7976 & 7 & 2 & 4 & 2 & 50.746 \\
\hline 1.7903 & 4 & -3 & 5 & 1 & 50.968 \\
\hline 1.7719 & 22 & 4 & 3 & 1 & 51.535 \\
\hline 1.7671 & 12 & 2 & 6 & 0 & 51.686 \\
\hline $1.7519 \mathrm{M}$ & 4 & -1 & 3 & 3 & 52.169 \\
\hline $1.7519 \mathrm{M}$ & & -4 & 4 & 1 & 52.169 \\
\hline 1.7452 & 10 & 1 & 5 & 2 & 52.385 \\
\hline 1.7404 & 15 & 0 & 3 & 3 & 52.539 \\
\hline 1.7361 & 15 & -2 & 5 & 2 & 52.679 \\
\hline 1.7111 & 2 & 5 & 2 & 0 & 53.510 \\
\hline $1.7002 \mathrm{M}$ & 3 & -2 & 3 & 3 & 53.882 \\
\hline $1.7002 \mathrm{M}$ & & 3 & 5 & 1 & 53.882 \\
\hline $1.6813 \mathrm{M}$ & 9 & -3 & 2 & 3 & 54.536 \\
\hline $1.6813 \mathrm{M}$ & & 2 & 1 & 3 & 54.536 \\
\hline 1.6644 & 3 & 2 & 6 & 1 & 55.137 \\
\hline $1.6410 \mathrm{M}$ & 2 & 4 & 4 & 1 & 55.990 \\
\hline $1.6410 \mathrm{M}$ & & -5 & 0 & 2 & 55.990 \\
\hline $1.6288 \mathrm{M}$ & 8 & 2 & 2 & 3 & 56.447 \\
\hline $1.6288 \mathrm{M}$ & & 2 & 5 & 2 & 56.447 \\
\hline $1.6248 \mathrm{M}$ & 6 & -5 & 1 & 2 & 56.601 \\
\hline $1.6248 \mathrm{M}$ & & 5 & 3 & 0 & 56.601 \\
\hline 1.6211 & 6 & 1 & 7 & 0 & 56.742 \\
\hline $1.6162 \mathrm{M}$ & 7 & -3 & 5 & 2 & 56.930 \\
\hline $1.6162 \mathrm{M}$ & & 0 & 4 & 3 & 56.930 \\
\hline 1.5938 & 5 & -4 & 5 & 1 & 57.802 \\
\hline 1.5837 & 3 & -2 & 4 & 3 & 58.206 \\
\hline $1.5786 \mathrm{M}$ & 4 & -4 & 1 & 3 & 58.413 \\
\hline $1.5786 \mathrm{M}$ & & -5 & 2 & 2 & 58.413 \\
\hline 1.5739 & 1 & -1 & 7 & 1 & 58.603 \\
\hline 1.5352 & 1 & -4 & 2 & 3 & 60.231 \\
\hline $1.5271 \mathrm{M}$ & 14 & 3 & 6 & 1 & 60.586 \\
\hline $1.5271 \mathrm{M}$ & & 4 & 3 & 2 & 60.586 \\
\hline 1.5209 & 10 & -5 & 4 & 1 & 60.860 \\
\hline 1.5147 & 1 & -2 & 7 & 1 & 61.133 \\
\hline 1.4980 & 1 & -1 & 5 & 3 & 61.892 \\
\hline $1.4896 \mathrm{M}$ & 1 & 0 & 5 & 3 & 62.278 \\
\hline $1.4896 \mathrm{M}$ & & 3 & 5 & 2 & 62.278 \\
\hline 1.4805 & 2 & 6 & 1 & 0 & 62.705 \\
\hline $1.4768 \mathrm{M}$ & 1 & 2 & 7 & 1 & 62.881 \\
\hline $1.4768 \mathrm{M}$ & & -4 & 5 & 2 & 62.881 \\
\hline
\end{tabular}


Calcium Iron Silicate (Kirschsteinite), CaFeSiO 4

Sample

The sample was prepared from $\mathrm{CaCO}_{3}, \mathrm{Fe}_{2} \mathrm{O}_{3}$, and $\mathrm{SiO}_{2}$. The components, in stoichiometric proportions, were blended with a mortar and pestle in acetone. After drying, the sample was heated in an iron crucible in three eight hour stages; $1075{ }^{\circ} \mathrm{C}, 1110^{\circ} \mathrm{C}$, and $1140{ }^{\circ} \mathrm{C}$ in a controlled atmosphere with the partial pressure of oxygen less than or equal to $10^{-18} \mathrm{~atm}$.

Color

Yellowish gray

Structure

Orthorhombic, Pmnb (62). The structure was studied by Sahama and Hytönen (1958). Their results were derived by analogy of the fosterite-fayalite series as reported by Yoder and Sahama (1957).

Crystallographic constants of this sample

$$
\begin{aligned}
& a=6.4413(7) \AA \\
& b=11.1586(12) \\
& c=4.8753(3) \\
& a / b=0.5772 \\
& c / b=0.4369 \\
& Z=4 \\
& V=350.42 \AA^{3} \\
& \text { Density }(\mathrm{calc})=3.564 \mathrm{~g} / \mathrm{cm}^{3} \\
& \text { Figure of merit } \\
& \mathrm{F}_{30}=92.1(0.0072,45)
\end{aligned}
$$

\begin{tabular}{|c|c|c|c|c|c|}
\hline \multicolumn{6}{|c|}{$\begin{array}{c}\mathrm{CuK} \alpha_{1} \lambda=1.540598 \mathrm{~A} ; \text { mean } \mathrm{T}=26.0{ }^{\circ} \mathrm{C} \\
\text { Internal standard Si, SRM } 640 \mathrm{a}\end{array}$} \\
\hline $\mathrm{d}(\stackrel{\circ}{\mathrm{A}})$ & $I^{r e l}$ & & hal & & $2 \theta\left(^{\circ}\right)$ \\
\hline 5.579 & 31 & 0 & 2 & 0 & 15.872 \\
\hline 4.466 & 13 & 0 & 1 & 1 & 19.865 \\
\hline 4.217 & 16 & 1 & 2 & 0 & 21.049 \\
\hline 3.886 & 11 & 1 & 0 & 1 & 22.864 \\
\hline $3.672 \mathrm{M}$ & 39 & 0 & 2 & 1 & 24.221 \\
\hline $3.672 \mathrm{M}$ & & 1 & 1 & 1 & 24.221 \\
\hline 3.222 & 9 & 2 & 0 & 0 & 27.662 \\
\hline 3.190 & 8 & 1 & 2 & 1 & 27.946 \\
\hline 2.955 & 74 & 0 & 3 & 1 & 30.217 \\
\hline $2.789 \mathrm{M}$ & 34 & 0 & 4 & 0 & 32.062 \\
\hline $2.789 \mathrm{M}$ & & 2 & 2 & 0 & 32.062 \\
\hline 2.687 & 67 & 1 & 3 & 1 & 33.315 \\
\hline 2.612 & 100 & 2 & 1 & 1 & 34.298 \\
\hline 2.5604 & 16 & 1 & 4 & 0 & 35.018 \\
\hline 2.4375 & 12 & 0 & 0 & 2 & 36.845 \\
\hline $2.4208 \mathrm{M}$ & 27 & 0 & 4 & 1 & 37.108 \\
\hline $2.4208 \mathrm{M}$ & & 2 & 2 & 1 & 37.108 \\
\hline 2.3809 & 7 & 0 & 1 & 2 & 37.753 \\
\hline $2.2328 M$ & 8 & 0 & 2 & 2 & 40.362 \\
\hline $2.2328 \mathrm{M}$ & & 1 & 1 & 2 & 40.362 \\
\hline 2.1779 & 15 & 2 & 3 & 1 & 41.426 \\
\hline $2.1097 \mathrm{M}$ & 3 & 1 & 2 & 2 & 42.831 \\
\hline $2.1097 \mathrm{M}$ & & 2 & 4 & 0 & 42.831 \\
\hline 2.0381 & 5 & 0 & 3 & 2 & 44.414 \\
\hline 2.0294 & 9 & 0 & 5 & 1 & 44.613 \\
\hline 1.9644 & $1 \mathrm{~L}$ & 3 & 0 & 1 & 46.174 \\
\hline $1.9351+$ & 10 & 2 & 4 & 1 & 46.914 \\
\hline $1.9351+$ & & 3 & 1 & 1 & 46.914 \\
\hline 1.9150 & 2 & 2 & 1 & 2 & 47.437 \\
\hline 1.8534 & 5 & 3 & 2 & 1 & 49.116 \\
\hline $1.8353 \mathrm{M}$ & 85 & 0 & 4 & 2 & 49.634 \\
\hline $1.8353 \mathrm{M}$ & & 2 & 2 & 2 & 49.634 \\
\hline 1.7870 & 8 & 1 & 6 & 0 & 51.071 \\
\hline 1.7649 & 9 & 1 & 4 & 2 & 51.757 \\
\hline $1.7375 \mathrm{M}$ & 17 & 0 & 6 & 1 & 52.635 \\
\hline $1.7375 \mathrm{M}$ & & 3 & 3 & 1 & 52.635 \\
\hline 1.7167 & 18 & 2 & 5 & 1 & 53.322 \\
\hline 1.7015 & 10 & 3 & 4 & 0 & 53.835 \\
\hline $1.6105 \mathrm{M}$ & 51 & 2 & 6 & 0 & 57.150 \\
\hline $1.6105 \mathrm{M}$ & & 4 & 0 & 0 & 57.150 \\
\hline $1.5949 \mathrm{M}$ & 12 & 1 & 5 & 2 & 57.760 \\
\hline $1.5949 \mathrm{M}$ & & 2 & 4 & 2 & 57.760 \\
\hline $1.5603 \mathrm{M}$ & 7 & 0 & 2 & 3 & 59.167 \\
\hline $1.5603 \mathrm{M}$ & & 1 & 1 & 3 & 59.167 \\
\hline $1.5475 \mathrm{M}$ & 4 & 3 & 2 & 2 & 59.703 \\
\hline
\end{tabular}

Additional pattern

PDF card 11-477 (Sahama and Hytönen, 1958)

References

Sahama, Th. G. and Hytönen, K. (1958). Am.

Mineral. 43,862 .

Yoder, H. S., Jr. and Sahama, Th. G. (1957). Am. Mineral. 42, 475. 
Calcium Iron Silicate (Kirschsteinite), CaFeSiO $4^{-}$(continued)

\begin{tabular}{|c|c|c|c|c|c|}
\hline $\mathrm{d}(\stackrel{\circ}{\mathrm{A}})$ & $I^{\text {rel }}$ & & $\mathrm{h} \mathrm{k} \ell$ & & $2 \theta\left(^{\circ}\right)$ \\
\hline $1.5475 M$ & & 4 & 2 & 0 & 59.703 \\
\hline $1.5154 M$ & 17 & 0 & 7 & 1 & 61.103 \\
\hline $1.5154 M$ & & 4 & 1 & 1 & 61.103 \\
\hline 1.4893 & 6 & 0 & 3 & 3 & 62.294 \\
\hline $1.4743+$ & 1 & 3 & 5 & 1 & 62.997 \\
\hline $1.4743+$ & & 4 & 2 & 1 & 62.997 \\
\hline 1.4659 & $1 \mathrm{~L}$ & 2 & 5 & 2 & 63.403 \\
\hline 1.4508 & 4 & 1 & 3 & 3 & 64.139 \\
\hline 1.4387 & 9 & 2 & 1 & 3 & 64.745 \\
\hline 1.4143 & 8 & 4 & 3 & 1 & 66.004 \\
\hline $1.4040 \mathrm{M}$ & 9 & 0 & 4 & 3 & 66.546 \\
\hline $1.4040 \mathrm{M}$ & & 2 & 2 & 3 & 66.546 \\
\hline $1.3949+$ & 5 & 0 & 8 & 0 & 67.042 \\
\hline $1.3949+$ & & 4 & 4 & 0 & 67.042 \\
\hline 1.3723 & 2 & 1 & 4 & 3 & 68.295 \\
\hline 1.3518 & 2 & 2 & 3 & 3 & 69.478 \\
\hline $1.3439 \mathrm{M}$ & 9 & 2 & 6 & 2 & 69.948 \\
\hline $1.3439 \mathrm{M}$ & & 4 & 0 & 2 & 69.948 \\
\hline $1.3344 \mathrm{M}$ & 1 & 0 & 7 & 2 & 70.514 \\
\hline $1.3344 \mathrm{M}$ & & 4 & 1 & 2 & 70.514 \\
\hline 1.3137 & 1 & 0 & 5 & 3 & 71.796 \\
\hline $1.3061+$ & 1 & 3 & 5 & 2 & 72.281 \\
\hline $1.3061+$ & & 4 & 2 & 2 & 72.281 \\
\hline 1.2960 & 1 & 3 & 0 & 3 & 72.937 \\
\hline $1.2871+$ & 2 & 2 & 4 & 3 & $73 . j 20$ \\
\hline $1.2871+$ & & 3 & 1 & 3 & 73.520 \\
\hline 1.2800 & 3 & 2 & 8 & 0 & 73.999 \\
\hline 1.2614 & 2 & 4 & 5 & 1 & 75.279 \\
\hline $1.2380+$ & $1 \mathrm{~L}$ & 2 & 8 & 1 & 76.955 \\
\hline $1.2380+$ & & 3 & 7 & 1 & 76.955 \\
\hline $1.2235 \mathrm{M}$ & 1 & 0 & 6 & 3 & 78.040 \\
\hline $1.2235 \mathrm{M}$ & & 3 & 3 & 3 & 78.040 \\
\hline 1.2189 & 6 & 0 & 0 & 4 & 78.391 \\
\hline 1.2164 & 7 & 2 & 5 & 3 & 78.585 \\
\hline $1.2106 \mathrm{M}$ & 6 & 0 & 8 & 2 & 79.029 \\
\hline $1.2106 \mathrm{M}$ & & 4 & 4 & 2 & 79.029 \\
\hline
\end{tabular}




\section{Calcium Magnesium Silicate (Monticellite), CaMgSiO}

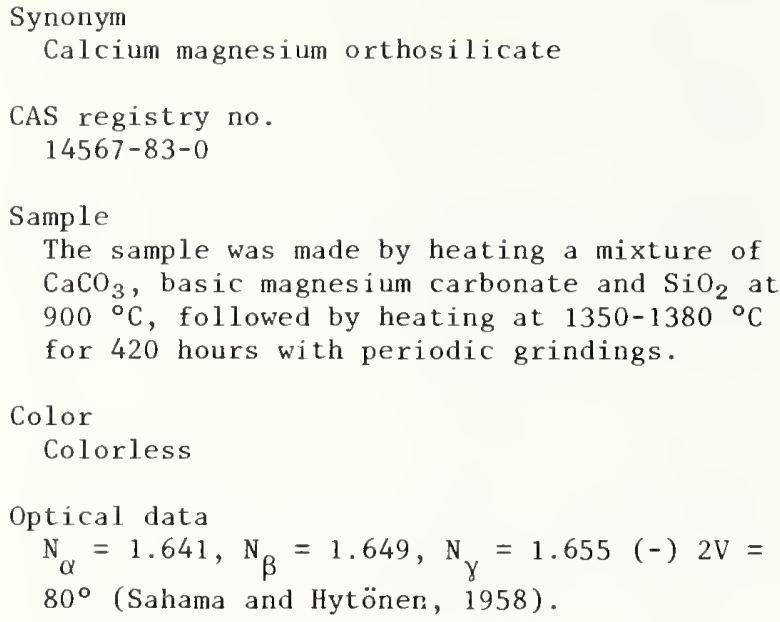

Structure

Orthorhombic, Pmnb (62). Olivine type (Brown and West, 1927). The structure was determined by Brown and West (1927) and refined by Onken (1964).

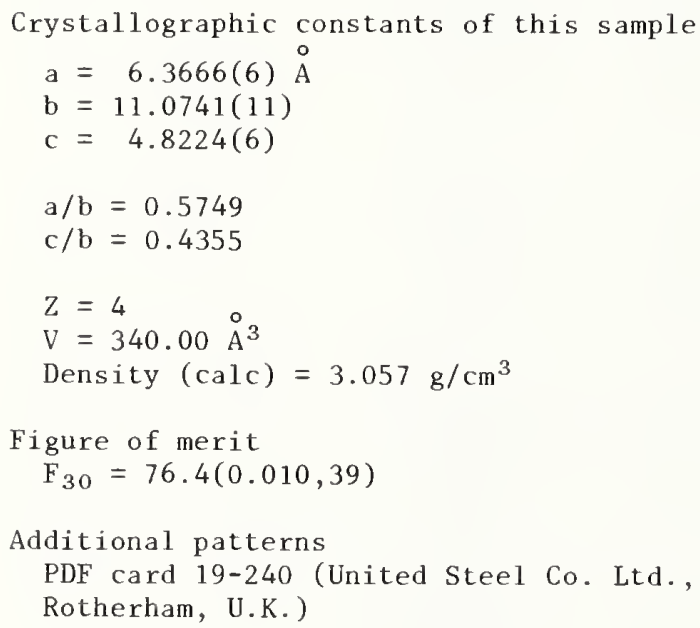

Sahama and Hytönen (1958)

References

Brown, G. B. and West, J. (1927). Z.

Kristallogr., 66, 154.

Onken, H. (1964). Naturwissenschaften 51, 334.

Sahama, Th. G. and Hytönen, K. (1958). Am. Mineral. 43, 862 .

\begin{tabular}{|c|c|c|c|c|c|}
\hline \multicolumn{6}{|c|}{$\begin{array}{c}\mathrm{CuK \alpha}_{1} \lambda=1.540598 \mathrm{~A} \text {; mean } \mathrm{T}=26 \pm 2{ }^{\circ} \mathrm{C} \\
\text { Internal standards Ag, } \mathrm{a}=4.08651 \mathrm{~A} \\
\text { Fluorophlogopite, SRM } 675\end{array}$} \\
\hline $\mathrm{d}(\stackrel{\circ}{\mathrm{A}})$ & $\begin{array}{c}\mathrm{I} \text { rel } \\
\sigma= \pm 4\end{array}$ & & hke & & $2 \theta\left(^{\circ}\right)$ \\
\hline 5.538 & 8 & 0 & 2 & 0 & 15.990 \\
\hline 4.425 & 3 & 0 & 1 & 1 & 20.052 \\
\hline 4.183 & 25 & 1 & 2 & 0 & 21.225 \\
\hline 3.845 & 13 & 1 & 0 & 1 & 23.113 \\
\hline $3.634 \mathrm{M}$ & 51 & 0 & 2 & 1 & 24.474 \\
\hline $3.634 \mathrm{M}$ & & 1 & 1 & 1 & 24.474 \\
\hline 3.185 & 26 & 2 & 0 & 0 & 27.995 \\
\hline 3.159 & 10 & 1 & 2 & 1 & 28.229 \\
\hline 2.932 & 44 & 0 & 3 & 1 & 30.465 \\
\hline 2.768 & 6 & 0 & 4 & 0 & 32.312 \\
\hline 2.761 & 6 & 2 & 2 & 0 & 32.402 \\
\hline 2.664 & 100 & 1 & 3 & 1 & 33.620 \\
\hline 2.584 & 59 & 2 & 1 & 1 & 34.684 \\
\hline 2.540 & 21 & 1 & 4 & 0 & 35.301 \\
\hline 2.396 & 29 & 2 & 2 & 1 & 37.499 \\
\hline 2.357 & 9 & 0 & 1 & 2 & 38.150 \\
\hline 2.247 & 1 & 1 & 4 & 1 & 40.095 \\
\hline $2.210 \mathrm{M}$ & 10 & 0 & 2 & 2 & 40.798 \\
\hline $2.210 \mathrm{M}$ & & 1 & 1 & 2 & 40.798 \\
\hline 2.1557 & 1 & 2 & 3 & 1 & 41.872 \\
\hline 2.0893 & 4 & 2 & 4 & 0 & 43.269 \\
\hline 2.0188 & 2 & 0 & 3 & 2 & 44.861 \\
\hline 2.0127 & 2 & 0 & 5 & 1 & 45.004 \\
\hline 1.9815 & $1 \mathrm{~L}$ & 3 & 2 & 0 & 45.754 \\
\hline 1.9424 & $1 \mathrm{~L}$ & 3 & 0 & 1 & 46.729 \\
\hline 1.9222 & 5 & 2 & 0 & 2 & 47.248 \\
\hline 1.9131 & 9 & 3 & 1 & 1 & 47.486 \\
\hline 1.8941 & 2 & 2 & 1 & 2 & 47.994 \\
\hline 1.8462 & 1 & 0 & 6 & 0 & 49.320 \\
\hline 1.8327 & 4 & 3 & 2 & 1 & 49.709 \\
\hline 1.8160 & 60 & 2 & 2 & 2 & 50.198 \\
\hline 1.7730 & 8 & 1 & 6 & 0 & 51.503 \\
\hline 1.7485 & 10 & 1 & 4 & 2 & 52.276 \\
\hline 1.7194 & 21 & 3 & 3 & 1 & 53.232 \\
\hline 1.7013 & 6 & 2 & 5 & 1 & 53.842 \\
\hline 1.6844 & 9 & 3 & 4 & 0 & 54.427 \\
\hline 1.6637 & $1 \mathrm{~L}$ & 1 & 6 & 1 & 55.161 \\
\hline 1.5965 & 25 & 2 & 6 & 0 & 57.697 \\
\hline 1.5919 & 27 & 4 & 0 & 0 & 57.877 \\
\hline $1.5796 \mathrm{M}$ & 8 & 1 & 5 & 2 & 58.372 \\
\hline
\end{tabular}


Calcium Magnesium Silicate (Monticellite), $\mathrm{CaMgSiO}_{4}$ - (continued)

\begin{tabular}{|lccccc|}
\hline $\begin{array}{l}\text { ( } \mathrm{A}) \\
\end{array}$ & $\mathrm{I}^{\mathrm{rel}}$ & \multicolumn{3}{c|}{$\mathrm{hk \ell}$} & $2 \theta\left(^{\circ}\right)$ \\
& $\sigma= \pm 4$ & & & & \\
\hline $1.5796 \mathrm{M}$ & & 2 & 4 & 2 & 58.372 \\
1.5433 & 9 & 1 & 1 & 3 & 59.883 \\
1.5311 & 3 & 3 & 2 & 2 & 60.409 \\
1.5032 & 12 & 0 & 7 & 1 & 61.653 \\
1.4736 & $1 \mathrm{~L}$ & 0 & 3 & 3 & 63.029 \\
& & & & & \\
1.4578 & 1 & 4 & 2 & 1 & 63.796 \\
1.4514 & 1 & 2 & 5 & 2 & 64.110 \\
1.4357 & 1 & 1 & 3 & 3 & 64.897 \\
1.4229 & 2 & 2 & 1 & 3 & 65.552 \\
1.3987 & 6 & 4 & 3 & 1 & 66.833 \\
1.3891 & 12 & 2 & 2 & 3 & 67.355 \\
1.3804 & 3 & 3 & 4 & 2 & 67.837 \\
1.3592 & 4 & 2 & 7 & 1 & 69.047 \\
1.3312 & 1 & 2 & 6 & 2 & 70.708 \\
1.3280 & 2 & 4 & 0 & 2 & 70.907 \\
1.3188 & $1 \mathrm{~L}$ & 4 & 1 & 2 & 71.477 \\
1.2932 & 1 & 3 & 5 & 2 & 73.118 \\
1.2731 & 4 & 3 & 1 & 3 & 74.466 \\
1.2692 & 4 & 2 & 8 & 0 & 74.733 \\
& & & & & \\
\hline
\end{tabular}


Sample

The sample was prepared from basic magnesium carbonate, $\mathrm{CaCO}_{3}$ and $\mathrm{SiO}_{2}$. The product was calcined at $850{ }^{\circ} \mathrm{C}$ then heated for 6 days between $1300-1380{ }^{\circ} \mathrm{C}$ with periodic grinding. To adjust the composition, $2 \% \mathrm{SiO}_{2}$ was added before the final heating at $1380{ }^{\circ} \mathrm{C}$ for 48 hours.

Color

Colorless

Structure

Tetragona 1, $\mathrm{P} \overline{4} 2{ }_{1} \mathrm{~m}$ (113), isostructural with $\mathrm{Ca}_{2} \mathrm{ZnSi}_{2} \mathrm{O}_{7}$ (Andrews, 1948). The structure of $\mathrm{Ca}_{2} \mathrm{ZnSi}_{2} \mathrm{O}_{7}$ was determined by Warren and Trautz (1930).

Crystallographic constants of this sample

$a=7.8332(2) \AA$

$c=5.0069(2)$

$c / a=0.6392$

$Z=2$

$\mathrm{V}=307.22 \AA^{3}$

Density $($ calc $)=2.947 \mathrm{~g} / \mathrm{cm}^{3}$

Figure of merit

$\mathrm{F}_{30}=93.3(0.011,30)$

Additional patterns

PDF card 10-391 (Ervin and Osborn, 1949)

Andrews (1948)

0'Daniel and Tscheischwili (1945-48)

References

Andrews, K. W. (1948). Mineral. Mag. 28, 374.

Ervin, G., Jr. and Osborn, E. F. (1949). Am. Mineral. 34, 717 .

O'Daniel, H. and Tscheischwili, L. (1945-48).

Neues Jahrb. Mineral., Monatsh. A65, 60 .

Warren, B. E. and Trautz, O. R. (1930). Z.

Kristallogr. 75, 525 .

\begin{tabular}{|c|c|c|c|c|c|}
\hline \multicolumn{6}{|c|}{$\begin{array}{c}\mathrm{CuK \alpha}_{1} \lambda=1.540598 \AA \text {; mean } \mathrm{T}=25 \pm 1{ }^{\circ} \mathrm{C} \\
\text { Internal standards } \mathrm{W}, \mathrm{a}=3.16524 \mathrm{~A} \\
\text { Fluorophlogopite, SRM } 675\end{array}$} \\
\hline $\mathrm{d}(\stackrel{\circ}{\mathrm{A}})$ & $\begin{array}{c}I^{\text {rel }} \\
\sigma= \pm 2\end{array}$ & & hke & & $2 \theta\left({ }^{\circ}\right)$ \\
\hline 5.538 & 9 & 1 & 1 & 0 & 15.991 \\
\hline 5.010 & 2 & 0 & 0 & 1 & 17.689 \\
\hline 4.222 & 7 & 1 & 0 & 1 & 21.027 \\
\hline 3.917 & 4 & 2 & 0 & 0 & 22.685 \\
\hline 3.716 & 12 & 1 & 1 & 1 & 23.928 \\
\hline 3.504 & 5 & 2 & 1 & 0 & 25.399 \\
\hline 3.087 & 23 & 2 & 0 & 1 & 28.903 \\
\hline 2.872 & 100 & 2 & 1 & 1 & 31.117 \\
\hline 2.771 & 4 & 2 & 2 & 0 & 32.277 \\
\hline 2.505 & 4 & 0 & 0 & 2 & 35.821 \\
\hline 2.478 & 15 & 3 & 1 & 0 & 36.224 \\
\hline 2.425 & 7 & 2 & 2 & 1 & 37.046 \\
\hline 2.386 & 8 & 1 & 0 & 2 & 37.673 \\
\hline 2.316 & 9 & 3 & 0 & 1 & 38.849 \\
\hline 2.282 & 3 & 1 & 1 & 2 & 39.452 \\
\hline 2.221 & 1 & 3 & 1 & 1 & 40.589 \\
\hline 2.172 & $1 \mathrm{~L}$ & 3 & 2 & 0 & 41.536 \\
\hline 2.110 & 1 & 2 & 0 & 2 & 42.828 \\
\hline 2.0372 & 13 & 2 & 1 & 2 & 44.434 \\
\hline 1.9928 & 2 & 3 & 2 & 1 & 45.480 \\
\hline 1.9581 & 6 & 4 & 0 & 0 & 46.331 \\
\hline 1.9005 & 6 & 4 & 1 & 0 & 47.822 \\
\hline 1.8572 & 5 & 2 & 2 & 2 & 49.009 \\
\hline 1.8462 & 9 & 3 & 3 & 0 & 49.321 \\
\hline 1.8237 & $1 \mathrm{~L}$ & 4 & 0 & 1 & 49.969 \\
\hline 1.8075 & 1 & 3 & 0 & 2 & 50.448 \\
\hline 1.7764 & 10 & 4 & 1 & 1 & 51.396 \\
\hline 1.7609 & 20 & 3 & 1 & 2 & 51.883 \\
\hline 1.7519 & 8 & 4 & 2 & 0 & 52.169 \\
\hline 1.7327 & 3 & 3 & 3 & 1 & 52.791 \\
\hline 1.6690 & 2 & 0 & 0 & 3 & 54.974 \\
\hline 1.6531 & 1 & 4 & 2 & 1 & 55.546 \\
\hline 1.6411 & 2 & 3 & 2 & 2 & 55.989 \\
\hline 1.6325 & 1 & 1 & 0 & 3 & 56.309 \\
\hline 1.5980 & 2 & 1 & 1 & 3 & 57.637 \\
\hline 1.5421 & $1 \mathrm{~L}$ & 4 & 0 & 2 & 59.935 \\
\hline 1.5355 & 2 & 2 & 0 & 3 & 60.221 \\
\hline 1.5133 & $1 \mathrm{~L}$ & 4 & 1 & 2 & 61.195 \\
\hline 1.5066 & 5 & 2 & 1 & 3 & 61.499 \\
\hline 1.4951 & $1 \mathrm{~L}$ & 5 & 0 & 1 & 62.025 \\
\hline 1.4861 & 2 & 3 & 3 & 2 & 62.442 \\
\hline 1.4683 & 3 & 5 & 1 & 1 & 63.286 \\
\hline 1.4544 & $1 \mathrm{~L}$ & 5 & 2 & 0 & 63.959 \\
\hline 1.4352 & 1 & 4 & 2 & 2 & 64.919 \\
\hline 1.4296 & 4 & 2 & 2 & 3 & 65.208 \\
\hline
\end{tabular}


Calcium Magnesium Silicate (Akermanite), $\mathrm{Ca}_{2} \mathrm{MgSi}_{2} \mathrm{O}_{7}$ - (continued)

\begin{tabular}{|c|c|c|c|c|c|}
\hline $\mathrm{d}(\stackrel{\circ}{\AA})$ & $I^{r e l}$ & & hke & & $2 \theta\left(^{\circ}\right)$ \\
\hline 1.4063 & 3 & 3 & 0 & 3 & 66.428 \\
\hline 1.3966 & 7 & 5 & 2 & 1 & 66.949 \\
\hline 1.3846 & 3 & 4 & 4 & 0 & 67.608 \\
\hline 1.3435 & 3 & 5 & 3 & 0 & 69.968 \\
\hline 1.3344 & 2 & 4 & 4 & 1 & 70.514 \\
\hline 1.3282 & 1L & 4 & 3 & 2 & 70.893 \\
\hline 1.3096 & 2 & 5 & 1 & 2 & 72.058 \\
\hline 1.3054 & 2 & 6 & 0 & 0 & 72.324 \\
\hline 1.2974 & 1 & 5 & 3 & 1 & 72.843 \\
\hline 1.2879 & 1 & 6 & 1 & 0 & 73.469 \\
\hline 1.2702 & 1 & 4 & 0 & 3 & 74.668 \\
\hline 1.2576 & 4 & 5 & 2 & 2 & 75.546 \\
\hline 1.2538 & 6 & 4 & 1 & 3 & 75.811 \\
\hline 1.2516 & 2 & 0 & 0 & 4 & 75.971 \\
\hline 1.2471 & 1 & 6 & 1 & 1 & 76.291 \\
\hline 1.2380 & 2 & 3 & 3 & 3 & 76.956 \\
\hline 1.2119 & 1 & 4 & 4 & 2 & 78.933 \\
\hline 1.2083 & 1 & 4 & 2 & 3 & 79.213 \\
\hline 1.2022 & 2 & 6 & 2 & 1 & 79.695 \\
\hline 1.1922 & $1 \mathrm{~L}$ & 2 & 0 & 4 & 80.495 \\
\hline 1.1884 & 3 & 5 & 4 & 1 & 80.809 \\
\hline 1.1838 & 1 & 5 & 3 & 2 & 81.193 \\
\hline 1.1787 & 1 & 2 & 1 & 4 & 81.615 \\
\hline 1.1677 & 1 & 6 & 3 & 0 & 82.551 \\
\hline 1.1577 & 1 & 6 & 0 & 2 & 83.422 \\
\hline 1.1450 & $1 \mathrm{~L}$ & 6 & 1 & 2 & 84.557 \\
\hline 1.1421 & 1 & 4 & 3 & 3 & 84.824 \\
\hline 1.1405 & 1 & 2 & 2 & 4 & 84.976 \\
\hline 1.1302 & 3 & 5 & 1 & 3 & 85.931 \\
\hline 1.1287 & 2 & 3 & 0 & 4 & 86.072 \\
\hline 1.1173 & 1 & 3 & 1 & 4 & 87.170 \\
\hline 1.1101 & 2 & 6 & 2 & 2 & 87.882 \\
\hline 1.1076 & $1 \mathrm{~L}$ & 5 & 5 & 0 & 88.125 \\
\hline 1.0990 & 1 & 5 & 4 & 2 & 88.997 \\
\hline 1.0966 & 1 & 5 & 2 & 3 & 89.247 \\
\hline 1.0864 & $1 \mathrm{~L}$ & 6 & 4 & 0 & 90.314 \\
\hline 1.0760 & $1 \mathrm{~L}$ & 7 & 2 & 0 & 91.427 \\
\hline 1.0584 & $1 \mathrm{~L}$ & 6 & 3 & 2 & 93.408 \\
\hline 1.0519 & $1 \mathrm{~L}$ & 7 & 2 & 1 & 94.159 \\
\hline 1.0452 & 1 & 4 & 1 & 4 & 94.948 \\
\hline
\end{tabular}


Synonym

Tricalcium magnesium orthosilicate

CAS registry no.

$13596-18-4$

Sample

The sample was made by heating $\mathrm{CaCO}_{3}$, basic magnesium carbonate and $\mathrm{SiO}_{2}$ overnight at $900{ }^{\circ} \mathrm{C}$, followed by heating at $1380{ }^{\circ} \mathrm{C}$ for 39 hours, and $1350{ }^{\circ} \mathrm{C}$ for 240 hours with intermediate grindings.

Color

Colorless

Structure

Monoclinic, P2/a (14) (Yamaguchi and

Suzuki, 1967). The structure was determined by Moore and Araki (1972).

Crystallographic constants of this sample

$\mathrm{a}=13.298(3) \AA$

$\mathrm{b}=5.3046(12)$

$\mathrm{c}=9.352(3)$

$\beta=92.09(2)^{\circ}$

$\mathrm{a} / \mathrm{b}=2.5069$

$\mathrm{c} / \mathrm{b}=1.7630$

$Z=4$

$\mathrm{V}=659.26 \AA^{3}$
Density $(\mathrm{calc})$

Density (calc) $3.312 \mathrm{~g} / \mathrm{cm}^{3}$

Figure of merit

$\mathrm{F}_{30}=33.6(0.012,73)$

Additional patterns

PDF card 4-728 (Phemister et al., 1942)

PDF card 25-161 (Moore and Araki, 1972)

Yamaguchi and Suzuki (1967)

References

Moore, P. B. and Araki, T. (1972). Am.

Mineral. 57, 1355.

Phemister, J., Nurse, R. W., and Bannister,

F. A. (1942). Mineral. Mag. 26, 225.

Yamaguchi, G. and Suzuki, K. (1967). Yogyo Kyokaishi (J. Ceram. Assoc. Jpn.) 75, 220.

\begin{tabular}{|c|c|c|c|c|c|}
\hline \multicolumn{6}{|c|}{$\begin{array}{c}\mathrm{CuK} \alpha_{1} \lambda=1.540598 \AA ; \text { mean } \mathrm{T}=26 \pm 2{ }^{\circ} \mathrm{C} \\
\text { Internal standards } \mathrm{Ag}, \mathrm{a}=4.08651 \AA \\
\text { Fluorophlogopite, SRM } 675\end{array}$} \\
\hline $\mathrm{d}(\stackrel{\circ}{\mathrm{A}})$ & $\mathrm{I}^{\text {rel }}$ & & hke & & $2 \theta\left(^{\circ}\right)$ \\
\hline 9.33 & 1 & 0 & 0 & 1 & 9.473 \\
\hline 6.64 & 3 & 2 & 0 & 0 & 13.317 \\
\hline 4.609 & 3 & 0 & 1 & 1 & 19.242 \\
\hline 4.379 & 2 & -1 & 1 & 1 & 20.264 \\
\hline 4.335 & 3 & 1 & 1 & 1 & 20.471 \\
\hline 3.890 & 6 & -2 & 0 & 2 & 22.843 \\
\hline $3.759 \mathrm{M}$ & 2 & 2 & 0 & 2 & 23.647 \\
\hline $3.759 \mathrm{M}$ & & 2 & 1 & 1 & 23.647 \\
\hline 3.508 & 1 & 0 & 1 & 2 & 25.371 \\
\hline 3.325 & 9 & 4 & 0 & 0 & 26.792 \\
\hline $3.169 \mathrm{M}$ & 7 & -4 & 0 & 1 & 28.137 \\
\hline $3.169 \mathrm{M}$ & & 3 & 1 & 1 & 28.137 \\
\hline 3.067 & 2 & 2 & 1 & 2 & 29.096 \\
\hline 2.816 & 2 & 4 & 1 & 0 & 31.746 \\
\hline 2.756 & 26 & -4 & 0 & 2 & 32.458 \\
\hline 2.719 & 12 & -4 & 1 & 1 & 32.920 \\
\hline 2.687 & 100 & 0 & 1 & 3 & 33.324 \\
\hline 2.671 & 65 & 4 & 1 & 1 & 33.521 \\
\hline 2.653 & 47 & 0 & 2 & 0 & 33.758 \\
\hline 2.509 & 1 & -1 & 2 & 1 & 35.765 \\
\hline $2.465 \mathrm{M}$ & 3 & 2 & 1 & 3 & 36.423 \\
\hline $2.465 \mathrm{M}$ & & 2 & 2 & 0 & 36.423 \\
\hline 2.330 & 5 & -3 & 1 & 3 & 38.614 \\
\hline 2.321 & 12 & -5 & 1 & 1 & 38.773 \\
\hline 2.307 & 10 & 0 & 2 & 2 & 39.015 \\
\hline 2.285 & 7 & 5 & 1 & 1 & 39.401 \\
\hline $2.267 \mathrm{M}$ & 5 & 1 & 2 & 2 & 39.735 \\
\hline $2.267 M$ & & 3 & 1 & 3 & 39.735 \\
\hline 2.230 & 9 & -2 & 0 & 4 & 40.413 \\
\hline 2.214 & 19 & 6 & 0 & 0 & 40.724 \\
\hline 2.192 & 2 & -2 & 2 & 2 & 41.155 \\
\hline 2.179 & 10 & 2 & 0 & 4 & 41.411 \\
\hline 2.168 & 11 & 2 & 2 & 2 & 41.626 \\
\hline $2.137 \mathrm{M}$ & 1 & 0 & 1 & 4 & 42.256 \\
\hline $2.137 \mathrm{M}$ & & 6 & 0 & 1 & 42.256 \\
\hline $2.121 M$ & 2 & -1 & 1 & 4 & 42.592 \\
\hline $2.121 \mathrm{M}$ & & -4 & 1 & 3 & 42.592 \\
\hline 2.100 & 1 & 1 & 1 & 4 & 43.037 \\
\hline 2.061 & 10 & -3 & 2 & 2 & 43.895 \\
\hline $2.031 \mathrm{M}$ & 14 & 3 & 2 & 2 & 44.574 \\
\hline $2.031 \mathrm{M}$ & & -6 & 0 & 2 & 44.574 \\
\hline 2.014 & 1 & 4 & 2 & 1 & 44.974 \\
\hline 1.9834 & 1 & 6 & 1 & 1 & 45.706 \\
\hline 1.9111 & 35 & -4 & 2 & 2 & 47.539 \\
\hline $1.8787 \mathrm{M}$ & 24 & 4 & 0 & 4 & 48.412 \\
\hline $1.8787 \mathrm{M}$ & & 4 & 2 & 2 & 48.412 \\
\hline 1.8258 & 1 & -4 & 1 & 4 & 49.909 \\
\hline
\end{tabular}


Calcium Manganese Phosphate Fluoride, $\mathrm{Ca}_{8} \mathrm{Mn}_{2}\left(\mathrm{PO}_{4}\right)_{6} \mathrm{~F}_{2}$

Synonym

Calcium manganese orthophosphate fluoride

Sample

The sample was obtained from the General

Electric Co., Chemical Products Plant,

$\mathrm{Clevel}$ and, $\mathrm{OH}$.

Color

Pink white

Structure

Hexagonal, $\mathrm{P}_{3} / \mathrm{m}$ (176) (Schroeder, 1982).

Crystallographic constants of this sample

$a=9.3286(4) \AA$

$c=6.7678(9)$

$c / a=0.7255$

$Z=1$

$\mathrm{V}=510.05 \AA^{3}$

Density $(\mathrm{calc})=3.380 \mathrm{~g} / \mathrm{cm}^{3}$

Figure of merit

$F_{30}=127.7(0.0073,32)$

Reference

Schroeder, L. W. (1982). Private communication.

\begin{tabular}{|c|c|c|c|c|c|}
\hline \multicolumn{6}{|c|}{$\begin{array}{l}\mathrm{CuK \alpha}{ }_{1} \lambda=1.540598 \AA \text {; temp. } 26 \pm 1{ }^{\circ} \mathrm{C} \\
\text { Internal standard Ag, } a=4.08651 \AA\end{array}$} \\
\hline $\mathrm{d}(\stackrel{0}{\mathrm{~A}})$ & $\begin{array}{c}\mathrm{rel} \\
\sigma= \pm 2\end{array}$ & & hke & & $2 \theta\left(^{\circ}\right)$ \\
\hline 8.08 & 7 & 1 & 0 & 0 & 10.947 \\
\hline 5.187 & 3 & 1 & 0 & 1 & 17.082 \\
\hline 4.040 & 10 & 2 & 0 & 0 & 21.985 \\
\hline 3.843 & 7 & 1 & 1 & 1 & 23.128 \\
\hline 3.470 & 5 & 2 & 0 & 1 & 25.655 \\
\hline 3.386 & 26 & 0 & 0 & 2 & 26.300 \\
\hline 3.122 & 14 & 1 & 0 & 2 & 28.570 \\
\hline 3.054 & 18 & 2 & 1 & 0 & 29.219 \\
\hline 2.784 & 100 & 2 & 1 & 1 & 32.123 \\
\hline 2.740 & 37 & 1 & 1 & 2 & 32.657 \\
\hline 2.694 & 64 & 3 & 0 & 0 & 33.233 \\
\hline 2.595 & 22 & 2 & 0 & 2 & 34.542 \\
\hline 2.5018 & 18 & 3 & 0 & 1 & 35.865 \\
\hline 2.3318 & 1 & 2 & 2 & 0 & 38.580 \\
\hline 2.2664 & 6 & 2 & 1 & 2 & 39.738 \\
\hline 2.2410 & 20 & 3 & 1 & 0 & 40.209 \\
\hline 2.2061 & 3 & 2 & 2 & 1 & 40.873 \\
\hline 2.1271 & 6 & 3 & 1 & 1 & 42.462 \\
\hline 2.1073 & 2 & 3 & 0 & 2 & 42.882 \\
\hline 2.0312 & 3 & 1 & 1 & 3 & 44.573 \\
\hline
\end{tabular}

\begin{tabular}{|c|c|c|c|c|c|}
\hline $\mathrm{d}(\AA)$ & I rel & & hke & & $2 \theta\left(^{\circ}\right)$ \\
\hline 2.0200 & 2 & 4 & 0 & 0 & 44.834 \\
\hline 1.9701 & 2 & 2 & 0 & 3 & 46.032 \\
\hline 1.9358 & 3 & 4 & 0 & 1 & 46.897 \\
\hline 1.9203 & 25 & 2 & 2 & 2 & 47.299 \\
\hline 1.8680 & 12 & 3 & 1 & 2 & 48.707 \\
\hline 1.8532 & 5 & 3 & 2 & 0 & 49.122 \\
\hline 1.8143 & 23 & 2 & 1 & 3 & 50.246 \\
\hline 1.7874 & 15 & 3 & 2 & 1 & 51.056 \\
\hline 1.7628 & 15 & 4 & 1 & 0 & 51.821 \\
\hline 1.7346 & 11 & 4 & 0 & 2 & 52.730 \\
\hline 1.7060 & 2 & 4 & 1 & 1 & 53.684 \\
\hline 1.6917 & 7 & 0 & 0 & 4 & 54.175 \\
\hline 1.6256 & 7 & 3 & 2 & 2 & 56.569 \\
\hline 1.6159 & 1 & 5 & 0 & 0 & 56.941 \\
\hline $1.5900 \mathrm{M}$ & 2 & 1 & 1 & 4 & 57.954 \\
\hline $1.5900 \mathrm{M}$ & & 3 & 1 & 3 & 57.954 \\
\hline 1.5715 & 2 & 5 & 0 & 1 & 58.705 \\
\hline 1.5637 & 1 & 4 & 1 & 2 & 59.026 \\
\hline 1.5550 & 1 & 3 & 3 & 0 & 59.388 \\
\hline 1.5266 & 5 & 4 & 2 & 0 & 60.606 \\
\hline 1.5157 & 4 & 3 & 3 & 1 & 61.090 \\
\hline 1.4893 & 2 & 4 & 2 & 1 & 62.292 \\
\hline 1.4800 & 2 & 2 & 1 & 4 & 62.727 \\
\hline 1.4580 & 9 & 5 & 0 & 2 & 63.785 \\
\hline 1.4512 & 3 & 5 & 1 & 0 & 64.118 \\
\hline $1.4324 \mathrm{M}$ & 9 & 3 & 0 & 4 & 65.065 \\
\hline $1.4324 \mathrm{M}$ & & 3 & 2 & 3 & 65.065 \\
\hline 1.4187 & 6 & 5 & 1 & 1 & 65.772 \\
\hline 1.4123 & 2 & 3 & 3 & 2 & 66.105 \\
\hline 1.3890 & 2 & 4 & 1 & 3 & 67.361 \\
\hline 1.3468 & 1 & 6 & 0 & 0 & 69.771 \\
\hline 1.3339 & 2 & 5 & 1 & 2 & 70.546 \\
\hline 1.3285 & 1 & 4 & 3 & 0 & 70.879 \\
\hline 1.3032 & 3 & 4 & 3 & 1 & 72.466 \\
\hline 1.2936 & 3 & 5 & 2 & 0 & 73.094 \\
\hline 1.2705 & 2 & 5 & 2 & 1 & 74.641 \\
\hline 1.2640 & 2 & 4 & 2 & 3 & 75.094 \\
\hline 1.2507 & 1 & 6 & 0 & 2 & 76.037 \\
\hline 1.2366 & 5 & 4 & 3 & 2 & 77.061 \\
\hline 1.2320 & 4 & 6 & 1 & 0 & 77.400 \\
\hline $1.2202 \mathrm{M}$ & 6 & 4 & 1 & 4 & 78.290 \\
\hline $1.2202 \mathrm{M}$ & & 5 & 1 & 3 & 78.290 \\
\hline 1.2119 & 1 & 6 & 1 & 1 & 78.931 \\
\hline 1.2084 & 4 & 5 & 2 & 2 & 79.204 \\
\hline 1.1658 & 3 & 4 & 4 & 0 & 82.712 \\
\hline 1.1542 & 1 & 5 & 3 & 0 & 83.729 \\
\hline 1.1493 & 1 & 4 & 4 & 1 & 84.167 \\
\hline $1.1446 \mathrm{M}$ & 2 & 3 & 3 & 4 & 84.593 \\
\hline $1.1446 \mathrm{M}$ & & 4 & 3 & 3 & 84.593 \\
\hline 1.1334 & 2 & 4 & 2 & 4 & 85.632 \\
\hline 1.1223 & $1 \mathrm{~L}$ & 5 & 2 & 3 & 86.685 \\
\hline 1.1015 & 1 & 5 & 1 & 4 & 88.745 \\
\hline 1.0921 & 5 & 5 & 3 & 2 & 89.713 \\
\hline
\end{tabular}


Calcium Zirconium Phosphate, $\mathrm{CaZr}_{4}\left(\mathrm{PO}_{4}\right)_{6}$

Synonym

Calcium zirconium orthophosphate

CAS registry no. 67972-87-6

Sample

The sample was made by heating a 1:4:6 molar mixture of $\mathrm{CaCO}_{3}, \mathrm{ZrO}_{2}$ and $\left(\mathrm{NH}_{4}\right)_{2} \mathrm{HPO}_{4}$ up to $500{ }^{\circ} \mathrm{C}$. It was then reground and heated at $1300{ }^{\circ} \mathrm{C}$ overnight.

Color

Colorless

Structure

Rhombohedral, R*t. The structure is similar to that of $\mathrm{NaZr}_{2}\left(\mathrm{PO}_{4}\right)_{3}$ (Chernorukov et al., 1978).

Crystallographic constants of this sample (Hexagonal axes)

$\mathrm{a}=8.7852(4) \AA$

$c=22.682(2)$

$\mathrm{c} / \mathrm{a}=2.5819$

$Z=3$

$\mathrm{V}=1516.06 \AA^{3}$

Density $(\mathrm{calc})=3.203 \mathrm{~g} / \mathrm{cm}^{3}$

Figure of merit

$\mathrm{F}_{30}=163.9(0.0045,41)$

Reference

Chernorukov, N. G., Korshunov, I. A., and

Prokof'eva, T. V. (1978). Sov. Phys.

Crystallogr. Eng1. Trans1. 23, 475.

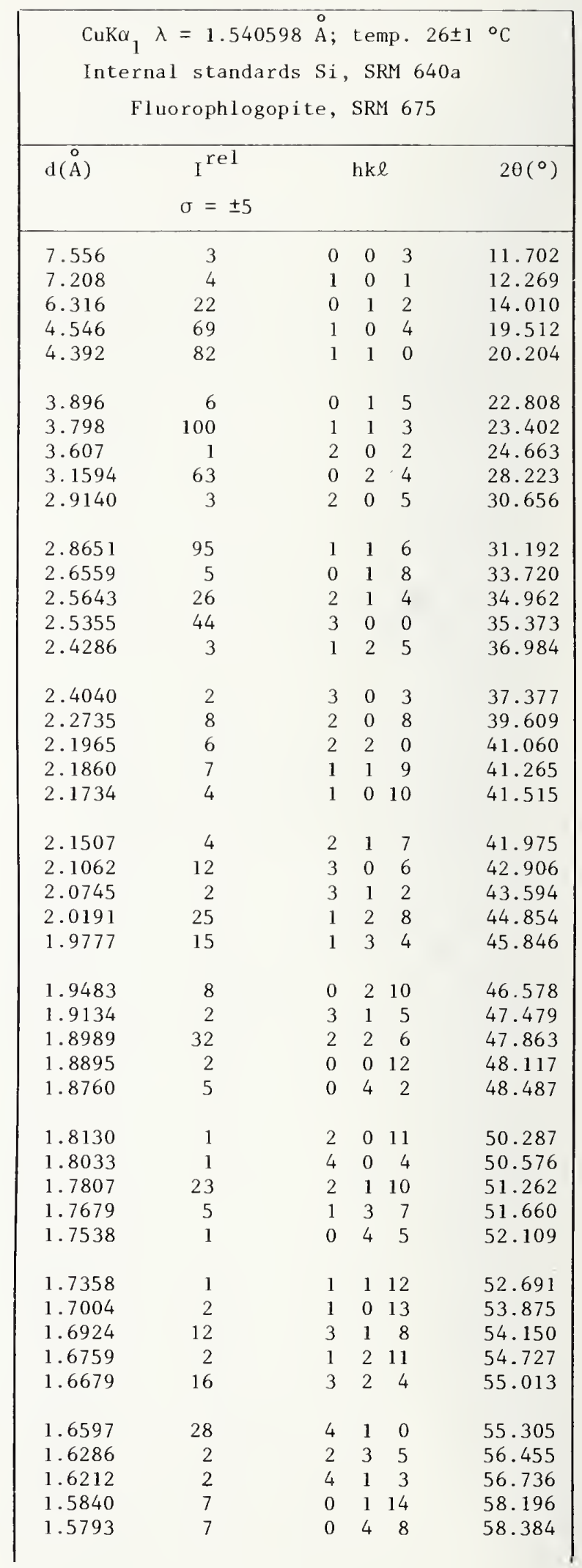


Calcium Zirconium Phosphate, $\mathrm{CaZr}_{4}\left(\mathrm{PO}_{4}\right)_{6}-$ (continued)

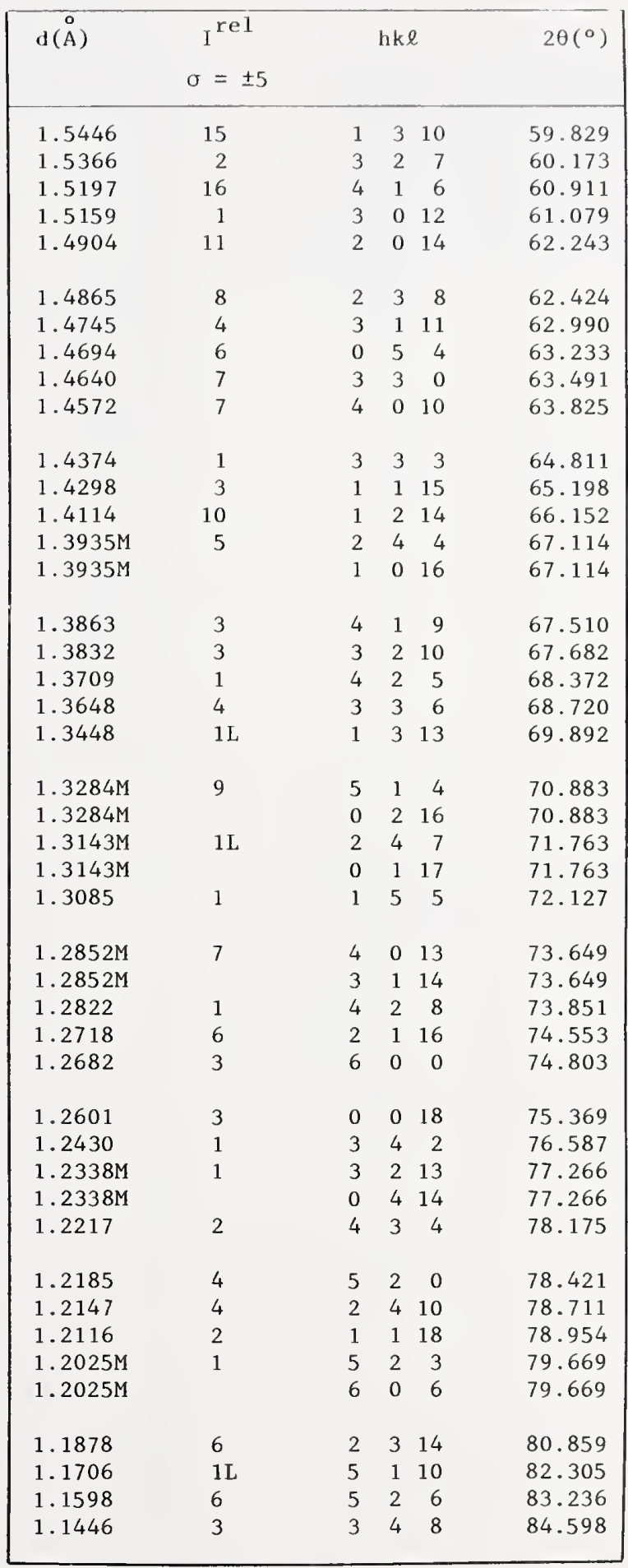


CAS registry no.

$1306-38-3$

Sample

This yttria-stabilized phase was prepared by Dragoo and Domingues (1982) from coprecipitation of the oxides. The powder was calcined at $620^{\circ} \mathrm{C}$ and then formed into a billet without binder, isostatically pressed, and then hot-pressed in an alumina die for 30 minutes at $1350{ }^{\circ} \mathrm{C}$ with an applied stress of $28 \mathrm{MPa}$.

Color

Light gray yellowish brown

Structure

Cubic, Fm3m (225). Isostructural with

fluorite, $\mathrm{CaF}_{2}$. The structure of fluorite was determined by Bragg (1914).

Crystallographic constants of this sample $a=5.41134(12) \AA$

$Z=4$

$V=158.46 \AA^{3}$

Density $($ calc $)=7.215 \mathrm{~g} / \mathrm{cm}^{3}$

Figure of merit

$F_{16}=134.1(0.0075,16)$

$*$ Comment

The true formula for this yttria-stabilized phase is $\mathrm{Ce}_{0.914} \mathrm{Y}_{0.086} \mathrm{O}_{1.957}$. This sample was prepared to produce a high density ceramic.

References

Bragg, W. L. (1914). Proc. R. Soc. London, A89, 468.

Dragoo, A. L. and Domingues, L. P. (1982).

J. Am. Ceram. Soc. 65, 非5, 253.

\begin{tabular}{|c|c|c|c|c|c|}
\hline \multicolumn{6}{|c|}{$\begin{array}{l}\mathrm{CuK \alpha}_{1} \lambda=1.540598 \AA \text {; mean } \mathrm{T}=26.1{ }^{\circ} \mathrm{C} \\
\text { Internal standard } \mathrm{Ag}, \mathrm{a}=4.08651 \AA\end{array}$} \\
\hline $\mathrm{d}(\stackrel{\circ}{\mathrm{A}})$ & $\begin{array}{l}\text { rel } \\
\sigma= \pm 1\end{array}$ & & hke & & $2 \theta\left(^{\circ}\right)$ \\
\hline 3.123 & 100 & 1 & 1 & 1 & 28.555 \\
\hline 2.706 & 30 & 2 & 0 & 0 & 33.082 \\
\hline 1.9134 & 52 & 2 & 2 & 0 & 47.479 \\
\hline 1.6318 & 42 & 3 & 1 & 1 & 56.335 \\
\hline 1.5622 & 8 & 2 & 2 & 2 & 59.087 \\
\hline 1.3531 & 8 & 4 & 0 & 0 & 69.402 \\
\hline 1.2415 & 14 & 3 & 3 & 1 & 76.700 \\
\hline 1.2101 & 8 & 4 & 2 & 0 & 79.070 \\
\hline 1.1048 & 14 & 4 & 2 & 2 & 88.412 \\
\hline 1.0415 & 11 & 5 & 1 & 1 & 95.397 \\
\hline .9566 & 4 & 4 & 4 & 0 & 107.265 \\
\hline .9147 & 13 & 5 & 3 & 1 & 114.730 \\
\hline .9019 & 6 & 6 & 0 & 0 & 117.317 \\
\hline .8556 & 9 & 6 & 2 & 0 & 128.393 \\
\hline .8252 & 6 & 5 & 3 & 3 & 137.969 \\
\hline .8158 & 5 & 6 & 2 & 2 & 141.566 \\
\hline
\end{tabular}


Cesium Hydrogen Phosphate, $\mathrm{CsH}_{5}\left(\mathrm{PO}_{4}\right)_{2}$

Synonym

Cesium pentahydrogen diphosphate

Sample

The sample was prepared by mixing an $85 \%$

solution of $\mathrm{H}_{3} \mathrm{PO}_{4}$ with $\mathrm{Cs}_{2} \mathrm{CO}_{3}$. The pre-

cipitate was washed with alcohol and dried in air one week.

Color

Colorless

Structure

Monoclinic, P2 1/c (14) (Ferrari, 1956), confirmed by Norbert and André (1970).

Crystallographic constants of this sample

$\mathrm{a}=10.8814(11) \AA$

$b=7.7717(8)$

$c=9.5320(9)$

$\beta=96.586(10)^{\circ}$

$\mathrm{a} / \mathrm{b}=1.4001$

$\mathrm{c} / \mathrm{b}=1.2265$

$Z=4$
$V=800.77 \AA^{3}$

Density $(\mathrm{calc})=2.720 \mathrm{~g} / \mathrm{cm}^{3}$

Figure of merit

$F_{30}=62.5(0.0098,49)$

Additional pattern

PDF card 24-252 (Norbert, Univ. of Montpelier,

Lab. de Chimie Min., C, 1971)

References

Ferrari, A., Nardelli, M., and Cingi, M.

(1956). Gazz Chim. Ita1. 86, 1174.

Norbert, A., and André, D. (1970). C. R.

Séances Acad. Sci. Ser. C. 270, 1718.

CuKa ${ }_{1} \lambda=1.540598 \AA$; mean $\mathrm{T}=25.6{ }^{\circ} \mathrm{C}$

Internal standards Si, SRM $640 \mathrm{a}$

Fluorophlogopite, SRM 675

\begin{tabular}{|c|c|c|c|c|c|}
\hline $\mathrm{d}(\stackrel{\circ}{\mathrm{A}})$ & $\begin{array}{c}I^{\text {rel }} \\
\sigma= \pm 3\end{array}$ & & hke & & $2 \theta\left({ }^{\circ}\right)$ \\
\hline 6.311 & 22 & 1 & 1 & 0 & 14.022 \\
\hline 6.005 & 7 & 0 & 1 & 1 & 14.741 \\
\hline 5.403 & 8 & 2 & 0 & 0 & 16.394 \\
\hline 4.531 & 13 & -1 & 0 & 2 & 19.577 \\
\hline $4.166 M$ & 39 & -2 & 1 & 1 & 21.311 \\
\hline 4. $166 M$ & & 1 & 0 & 2 & 21.311 \\
\hline 3.918 & 37 & -1 & 1 & 2 & 22.678 \\
\hline 3.879 & 40 & 2 & 1 & 1 & 22.909 \\
\hline 3.785 & 37 & -2 & 0 & 2 & 23.485 \\
\hline 3.672 & 52 & 1 & 1 & 2 & 24.217 \\
\hline
\end{tabular}

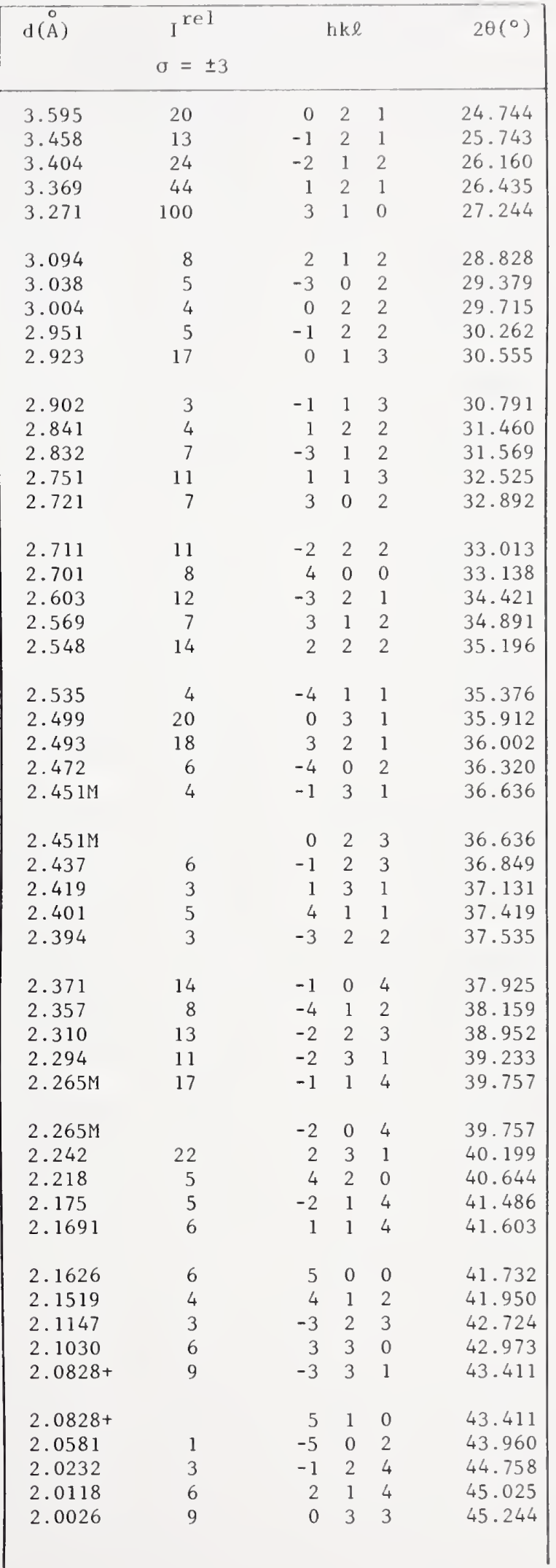




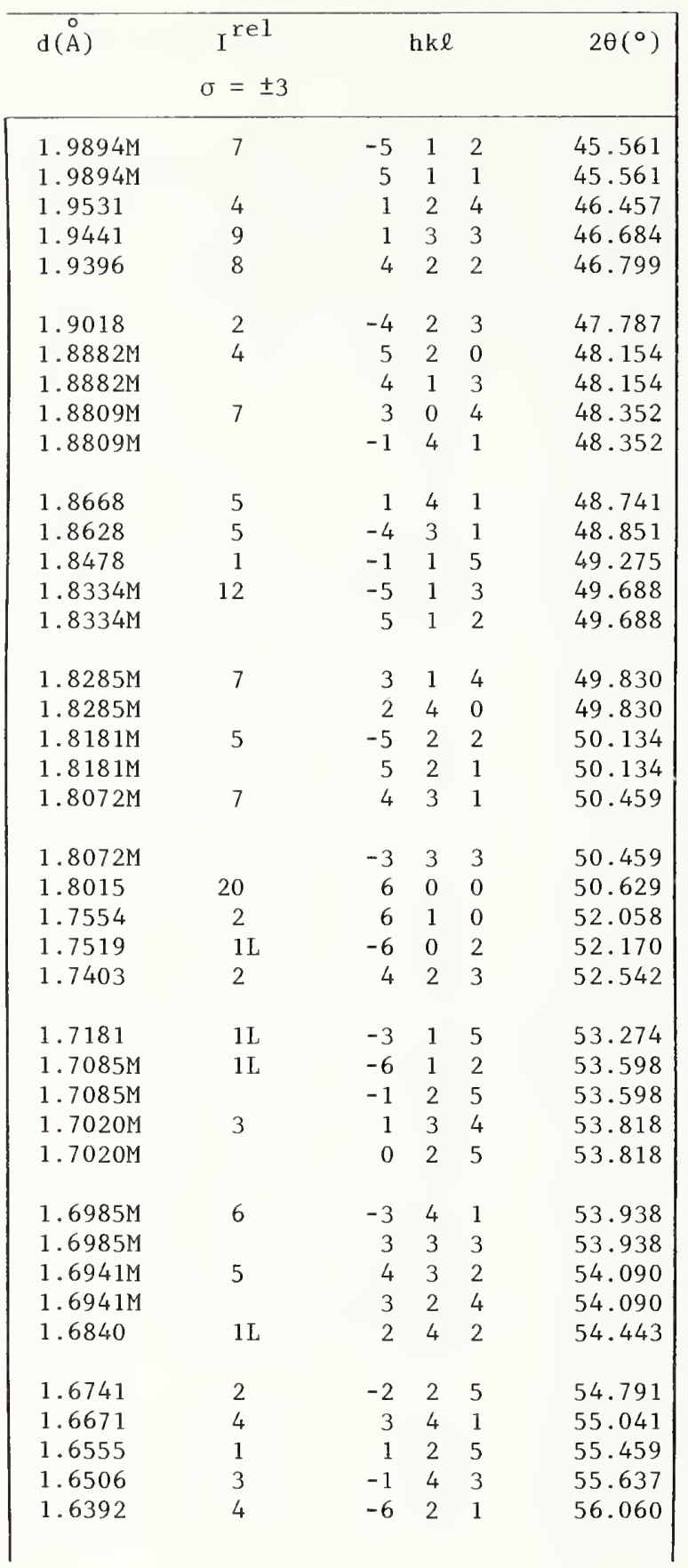

\begin{tabular}{|c|c|c|c|c|c|}
\hline $\mathrm{d}(\AA)$ & $\begin{array}{c}\text { rel } \\
\sigma= \pm 3\end{array}$ & & hkl & & $2 \theta\left(^{\circ}\right)$ \\
\hline 1.6344 & 9 & 6 & 2 & 0 & 56.238 \\
\hline 1.6089 & 2 & -2 & 4 & 3 & 57.212 \\
\hline $1.5777+$ & 2 & 2 & 2 & 5 & 58.450 \\
\hline $1.5777+$ & & 4 & 4 & 0 & 58.450 \\
\hline 1.5728 & 2 & -4 & 4 & 1 & 58.649 \\
\hline $1.5558+$ & 5 & -1 & 1 & 6 & 59.355 \\
\hline $1.5558+$ & & 4 & 3 & 3 & 59.355 \\
\hline 1.5465 & 2 & 0 & 1 & 6 & 59.746 \\
\hline $1.5389+$ & 5 & -3 & 4 & 3 & 60.073 \\
\hline $1.5389+$ & & 1 & 5 & 0 & 60.073 \\
\hline $1.5329 \mathrm{M}$ & 11 & -1 & 3 & 5 & 60.332 \\
\hline $1.5329 M$ & & -2 & 1 & 6 & 60.332 \\
\hline 1.5247 & 4 & 5 & 3 & 2 & 60.690 \\
\hline $1.5148 \mathrm{M}$ & 5 & 1 & 5 & 1 & 61.128 \\
\hline $1.5148 \mathrm{M}$ & & 7 & 1 & 0 & 61.128 \\
\hline $1.5121 \mathrm{M}$ & 3 & -4 & 2 & 5 & 61.249 \\
\hline $1.5121 \mathrm{M}$ & & 5 & 0 & 4 & 61.249 \\
\hline $1.4944 \mathrm{M}$ & 2 & 1 & 3 & 5 & 62.056 \\
\hline $1.4944 \mathrm{M}$ & & 2 & 5 & 0 & 62.056 \\
\hline $1.4823 \mathrm{M}$ & $1 \mathrm{~L}$ & -6 & 3 & 1 & 62.622 \\
\hline $1.4823 M$ & & -2 & 5 & 1 & 62.622 \\
\hline $1.4704+$ & 8 & 3 & 4 & 3 & 63.185 \\
\hline $1.4704+$ & & -1 & 5 & 2 & 63.185 \\
\hline 1.4558 & $1 \mathrm{~L}$ & 1 & 5 & 2 & 63.893 \\
\hline $1.4448+$ & 2 & 5 & 4 & 0 & 64.436 \\
\hline $1.4448+$ & & 2 & 1 & 6 & 64.436 \\
\hline 1.4408 & 3 & 6 & 3 & 1 & 64.639 \\
\hline 1.4290 & 1 & 1 & 2 & 6 & 65.235 \\
\hline $1.4121 \mathrm{M}$ & $1 \mathrm{~L}$ & 5 & 4 & 1 & 66.119 \\
\hline $1.4121 \mathrm{M}$ & & 2 & 5 & 2 & 66.119 \\
\hline $1.3973 M$ & $1 \mathrm{~L}$ & 7 & 1 & 2 & 66.909 \\
\hline $1.3973 M$ & & 7 & 2 & 1 & 66.909 \\
\hline $1.3939 M$ & $1 \mathrm{~L}$ & 0 & 5 & 3 & 67.095 \\
\hline $1.3939 \mathrm{M}$ & & 6 & 2 & 3 & 67.095 \\
\hline 1.3878 & 3 & 3 & 0 & 6 & 67.426 \\
\hline 1.3742 & 2 & 1 & 5 & 3 & 68.185 \\
\hline 1.3628 & 3 & 3 & 3 & 5 & 68.839 \\
\hline
\end{tabular}


Cesium Zirconium Phosphate, $\mathrm{CsZr}_{2}\left(\mathrm{PO}_{4}\right)_{3}$

Synonyms

Cesium zirconium orthophosphate

Cesium dizirconium tris (phosphate)

CAS registry no. 19527-88-9

Sample

The sample was prepared from a mixture of $\left(\mathrm{NH}_{4}\right)_{2} \mathrm{HPO}_{4}, \mathrm{Cs}_{2} \mathrm{CO}_{3}$ and $\mathrm{ZrO}_{2}$ in molar ratios of $6: 1: 4$. The mixture was heated for 2 hours at $500{ }^{\circ} \mathrm{C}$, reground, and heated for 2 hours at $1000{ }^{\circ} \mathrm{C}$. It was reground; a small amount of $\mathrm{Cs}_{2} \mathrm{CO}_{3}$ was added, and the mixture was heated at $900{ }^{\circ} \mathrm{C}$ for 16 hours.

Color

Colorless

Structure

Rhombohedral, R $\overline{3} \mathrm{c}$ (167), (Matković et al., 1968).

Crystallographic constants of this sample (Hexagonal axes)

$\mathrm{a}=8.5807(3) \stackrel{\circ}{\mathrm{A}}$

$c=24.9654(13)$

$c / a=2.9095$

$Z=6$

$Z=6$
$V=1591.90 \AA^{3}$

Density $($ calc $)=3.757 \mathrm{~g} / \mathrm{cm}^{3}$

Figure of merit

$\mathrm{F}_{30}=157.5(0.0054,35)$

Additional pattern

Roy et al. (1982)

References

Matković, B., Prodić, B., and Šljukić, M.

(1968). Bul1. Soc. Chim. Fr., 1777.

Roy, R., Vance, E. R., and Alamo, J. (1982). Mater. Res. Bul1. 17, 585.

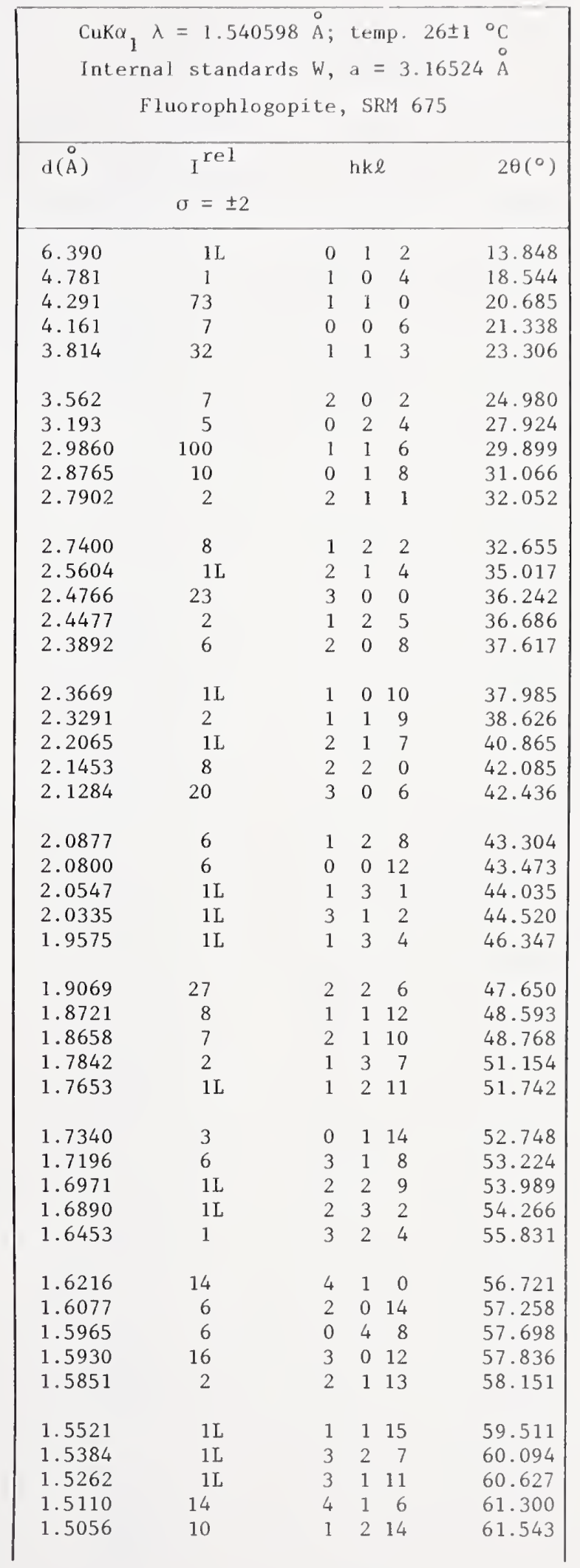


Cesium Zirconium Phosphate, $\mathrm{CsZr}_{2}\left(\mathrm{PO}_{4}\right)_{3}$ - (continued)

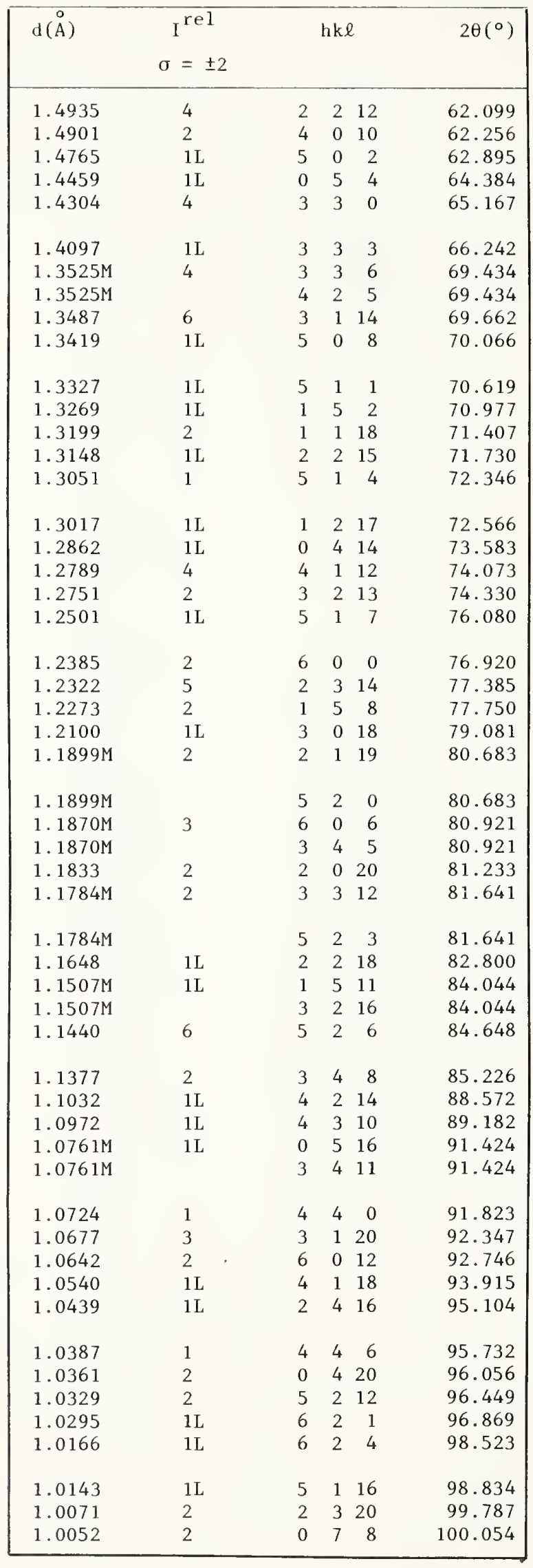


Synonym

Chromium tungstate

CAS registry no. 56729-81-8

Sample

The sample was prepared from $\mathrm{Cr}_{2} \mathrm{O}_{3}$ and

$\mathrm{WO}_{3}$. The constituent oxides in a $1: 2$

molar ratio were blended by grinding in an agate mortar under acetone. The powders were heated in a tungsten metal tray in a controlled atmosphere furnace. Temperatures and partial pressures of oxygen were chosen from the work of Trumm (1979). The following heat treatments were satisfactory:

$800{ }^{\circ} \mathrm{C}$ for 8 hours, at partial oxygen pressure $10^{-20} \mathrm{~atm}$;

$1000{ }^{\circ} \mathrm{C}$ for 8 hours, at partial oxygen pressure $10^{-20} \mathrm{~atm}$;

$1300{ }^{\circ} \mathrm{C}$ for 8 hours, at partial oxygen pressure $10^{-17} \mathrm{~atm}$.

Traces of $\mathrm{Cr}_{2} \mathrm{O}_{3}$ and $\mathrm{W}$ metal were present as extra phases.

Color

Very dark greenish blue

St ructure

Monoclinic, C2/m (12), distorted rutile type, isostructural with $\mathrm{AlWO}_{4}$. The structure was determined by Vlasse et al. (1976).

Crystallographic constants of this sample

$\mathrm{a}=9.2708(6) \AA$

$\mathrm{b}=5.8282(3)$

$\mathrm{c}=4.6445(3)$

$\beta=91.926(5)^{\circ}$

$\mathrm{a} / \mathrm{b}=1.5907$

$c / b=0.7969$

$Z=4$

$Z=4$
$V=250.81 \AA^{3}$

Density $(\mathrm{calc})=7.941 \mathrm{~g} / \mathrm{cm}^{3}$

Figure of merit

$F_{30}=78.9(0.0093,41)$

Additional patterns

PDF card 29-461 (Doumerc et al., 1975)

Trumm (1979)

References

Doumerc, J.-P., Pouchard, M., and Hagenmuller, P. (1975). C. R. Séances Acad. Sci. Ser. C 280, 1397 .

Trumm, A. (1979). Neues Jahrb. Mineral. Monatsh., 267.

Vlasse, M., Doumerc, J.-P., Peshev, P., Chaminade, J.-P., and Pouchard, M. (1976).

Rev. Chim. Miner. 13, 451.

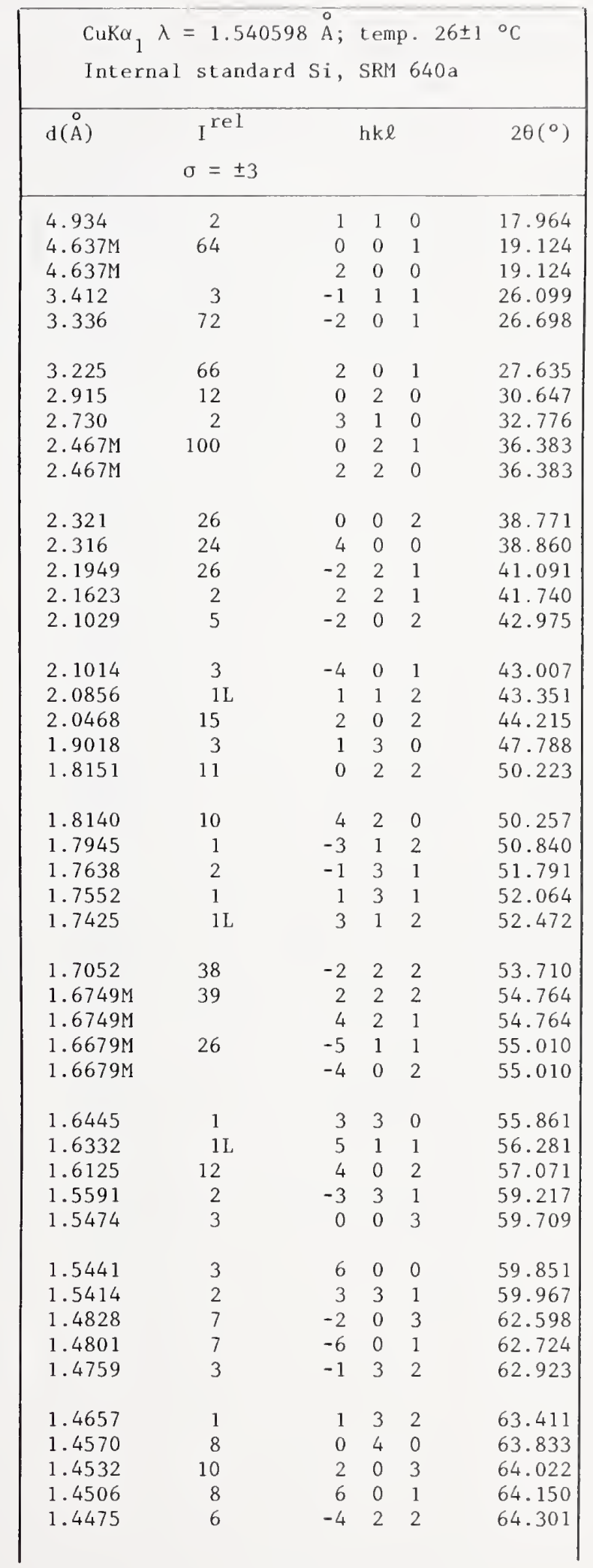


Chromium Tungsten $0 x i d e, \mathrm{CrWO}_{4}-$ (continued)

\begin{tabular}{|c|c|c|c|c|c|}
\hline$d(\stackrel{\circ}{A})$ & $I^{\text {rel }}$ & & hke & & $2 \theta\left(^{\circ}\right)$ \\
\hline 1.4109 & 2 & 4 & 2 & 2 & 66.179 \\
\hline $1.3902 \mathrm{M}$ & 4 & 0 & 4 & 1 & 67.298 \\
\hline 1. $3902 \mathrm{M}$ & & 2 & 4 & 0 & 67.298 \\
\hline 1.3666 & 15 & 0 & 2 & 3 & 68.620 \\
\hline 1.3644 & 15 & 6 & 2 & 0 & 68.748 \\
\hline 1.3532 & $1 \mathrm{~L}$ & -3 & 3 & 2 & 69.396 \\
\hline 1.3410 & 1 & 5 & 3 & 0 & 70.120 \\
\hline 1.3354 & 6 & -2 & 4 & 1 & 70.457 \\
\hline 1.3278 & 7 & 2 & 4 & 1 & 70.921 \\
\hline 1.3215 & 2 & -2 & 2 & 3 & 71.307 \\
\hline 1.3199 & 2 & -6 & 2 & 1 & 71.410 \\
\hline 1.3069 & 3 & -4 & 0 & 3 & 72.232 \\
\hline 1.3004 & 4 & 2 & 2 & 3 & 72.648 \\
\hline 1.2987 & 5 & 6 & 2 & 1 & 72.760 \\
\hline 1.2799 & $1 \mathrm{~L}$ & 5 & 3 & 1 & 74.006 \\
\hline 1.2665 & 1 & 6 & 0 & 2 & 74.918 \\
\hline 1.2338 & 6 & 0 & 4 & 2 & 77.267 \\
\hline 1.2045 & $1 \mathrm{~L}$ & -1 & 3 & 3 & 79.515 \\
\hline 1.1978 & 1 & -2 & 4 & 2 & 80.049 \\
\hline 1.1960 & 1 & 1 & 3 & 3 & 80.188 \\
\hline 1.1925 & 7 & -4 & 2 & 3 & 80.477 \\
\hline $1.1869 \mathrm{M}$ & 3 & 2 & 4 & 2 & 80.929 \\
\hline $1.1869 \mathrm{M}$ & & 4 & 4 & 1 & 80.929 \\
\hline 1.1737 & $1 \mathrm{~L}$ & -5 & 3 & 2 & 82.034 \\
\hline 1.1618 & 7 & 4 & 2 & 3 & 83.061 \\
\hline 1.1583 & 6 & 8 & 0 & 0 & 83.365 \\
\hline 1.1565 & 2 & 1 & 5 & 0 & 83.530 \\
\hline 1.1490 & $1 \mathrm{~L}$ & 5 & 3 & 2 & 84.194 \\
\hline 1.1460 & $1 \mathrm{~L}$ & 5 & 1 & 3 & 84.469 \\
\hline 1.1439 & $1 \mathrm{~L}$ & -7 & 1 & 2 & 84.659 \\
\hline 1.1372 & 1 & -3 & 3 & 3 & 85.278 \\
\hline 1.1347 & 1 & -2 & 0 & 4 & 85.505 \\
\hline 1.1326 & 1 & -8 & 0 & 1 & 85.703 \\
\hline 1.1233 & 1 & -1 & 5 & 1 & 86.589 \\
\hline 1.1210 & $1 \mathrm{~L}$ & 1 & 5 & 1 & 86.814 \\
\hline $1.1169 \mathrm{M}$ & 2 & 3 & 3 & 3 & 87.207 \\
\hline $1.1169 \mathrm{M}$ & & 2 & 0 & 4 & 87.207 \\
\hline 1.1119 & 4 & -6 & 0 & 3 & 87.696 \\
\hline 1.0974 & $1 \mathrm{~L}$ & -4 & 4 & 2 & 89.165 \\
\hline 1.0906 & 4 & 3 & 5 & 0 & 89.870 \\
\hline 1.0810 & 3 & 4 & 4 & 2 & 90.886 \\
\hline
\end{tabular}


CAS registry no.

$12017-13-9$

Sample

The sample was donated by CERAC/PURE, Inc., Butler, Wisc. The sample contained less than $5 \% \mathrm{CoTe}_{2}$ as a second phase.

Color

Very dark gray

Structure

Hexagona $1, \mathrm{P} 6_{3} / \mathrm{mmc}$ (194). Isostructural with NiAs (Oftedal, 1927).

Crystallographic constants of this sample

$\mathrm{a}=3.89276(15) \AA$

$c=5.3746(3)$

$Z=2$
$V=70.533 \AA^{3}$

Density $(\mathrm{calc})=8.783 \mathrm{~g} / \mathrm{cm}^{3}$

Figure of merit

$\mathrm{F}_{30}=75.5(0.011,36)$

Additional pattern

oftedal (1927)

Reference

Oftedal, I. (1927). Z. Phys. Chem. (Leipzig) 128,135 .

\begin{tabular}{|c|c|c|c|c|c|}
\hline \multirow{2}{*}{$\begin{array}{l}\text { CuK } \\
\text { In } \\
\mathrm{d}(\stackrel{\mathrm{A}}{)})\end{array}$} & $\begin{array}{l}=1.540 \\
1 \text { stand }\end{array}$ & \multicolumn{2}{|c|}{$\begin{array}{l}\text { A; mean } \\
\text { Si, SRM }\end{array}$} & \multicolumn{2}{|c|}{$\begin{array}{l}\mathrm{T}=26.4^{\circ} \mathrm{C} \\
640 \mathrm{a}\end{array}$} \\
\hline & $\begin{array}{c}\text { rel } \\
\sigma= \pm 3\end{array}$ & & $h k \ell$ & & $2 \theta\left(^{\circ}\right)$ \\
\hline 3.369 & 2 & 1 & 0 & 0 & 26.436 \\
\hline 2.856 & 100 & 1 & 0 & 1 & 31.295 \\
\hline 2.688 & 10 & 0 & 0 & 2 & 33.310 \\
\hline 2.101 & 45 & 1 & 0 & 2 & 43.007 \\
\hline 1.9470 & 37 & 1 & 1 & 0 & 46.612 \\
\hline 1.6082 & 16 & 2 & 0 & 1 & 57.239 \\
\hline 1.5819 & 20 & 1 & 0 & 3 & 58.282 \\
\hline 1.5768 & 18 & 1 & 1 & 2 & 58.486 \\
\hline 1.4280 & 11 & 2 & 0 & 2 & 65.290 \\
\hline 1.3433 & 4 & 0 & 0 & 4 & 69.981 \\
\hline 1.2744 & 11 & 2 & 1 & 0 & 74.376 \\
\hline 1.2484 & $1 \mathrm{~L}$ & 1 & 0 & 4 & 76.197 \\
\hline 1.2400 & 13 & 2 & 1 & 1 & 76.809 \\
\hline 1.2278 & 7 & 2 & 0 & 3 & 77.718 \\
\hline 1.1514 & 10 & 2 & 1 & 2 & 83.979 \\
\hline 1.1236 & 6 & 3 & 0 & 0 & 86.561 \\
\hline 1.1058 & 11 & 1 & 1 & 4 & 88.310 \\
\hline 1.0384 & 5 & 2 & 1 & 3 & 95.775 \\
\hline 1.0368 & 3 & 3 & 0 & 2 & 95.974 \\
\hline 1.0240 & 3 & 1 & 0 & 5 & 97.564 \\
\hline .9730 & 4 & 2 & 2 & 0 & 104.680 \\
\hline .9246 & 2 & 2 & 1 & 4 & 112.841 \\
\hline .9211 & 6 & 3 & 1 & 1 & 113.497 \\
\hline .9149 & 2 & 2 & 2 & 2 & 114.692 \\
\hline .9063 & 2 & 2 & 0 & 5 & 116.402 \\
\hline .8957 & 2 & 0 & 0 & 6 & 118.624 \\
\hline .8830 & 4 & 3 & 1 & 2 & 121.470 \\
\hline .8657 & 4 & 1 & 0 & 6 & 125.699 \\
\hline .8621 & 7 & 3 & 0 & 4 & 126.634 \\
\hline .8327 & 2 & 4 & 0 & 1 & 135.370 \\
\hline .8290 & 4 & 3 & 1 & 3 & 136.631 \\
\hline
\end{tabular}


Synonym

Copper chromite

CAS registry no. 12018-10-9

Sample

The sample was prepared by blending stoichiometric amounts of $\mathrm{CuO}$ and $\mathrm{Cr}_{2} \mathrm{O}_{3}$ and heating in a platinum boat in pure oxygen for extended periods at temperatures as high as $975{ }^{\circ} \mathrm{C}$, with periodic grinding.

Color

Black

Structure

Tetragonal, I $\overline{4} 2 \mathrm{~d}$ (122). Distorted normal spinel. The structure was determined by Prince (1957).

Crystallographic constants of this sample $\mathrm{a}=6.0336(3) \AA$

$c=7.7824(6)$

$c / a=1.2898$

$Z=4$

$V=283.31 \AA^{3}$

Density $(\mathrm{calc})=5.428 \mathrm{~g} / \mathrm{cm}^{3}$

Figure of merit

$\mathrm{F}_{30}=52.1(0.011,53)$

Polymorphism

A cubic form was found at $580{ }^{\circ} \mathrm{C}$ by

Ust'yantsev and Mar'evich (1973).

Additional pattern

PDF card 26-508 (Ust'yantsev and Mar'evich, 1973)

References

Prince, E. (1957). Acta Crystallogr. 10, 554.

Ust'yantsev, V. M. and Mar'evich, V. P. (1973). Inorg. Mater. (Engl. Trans.) 9, 306.

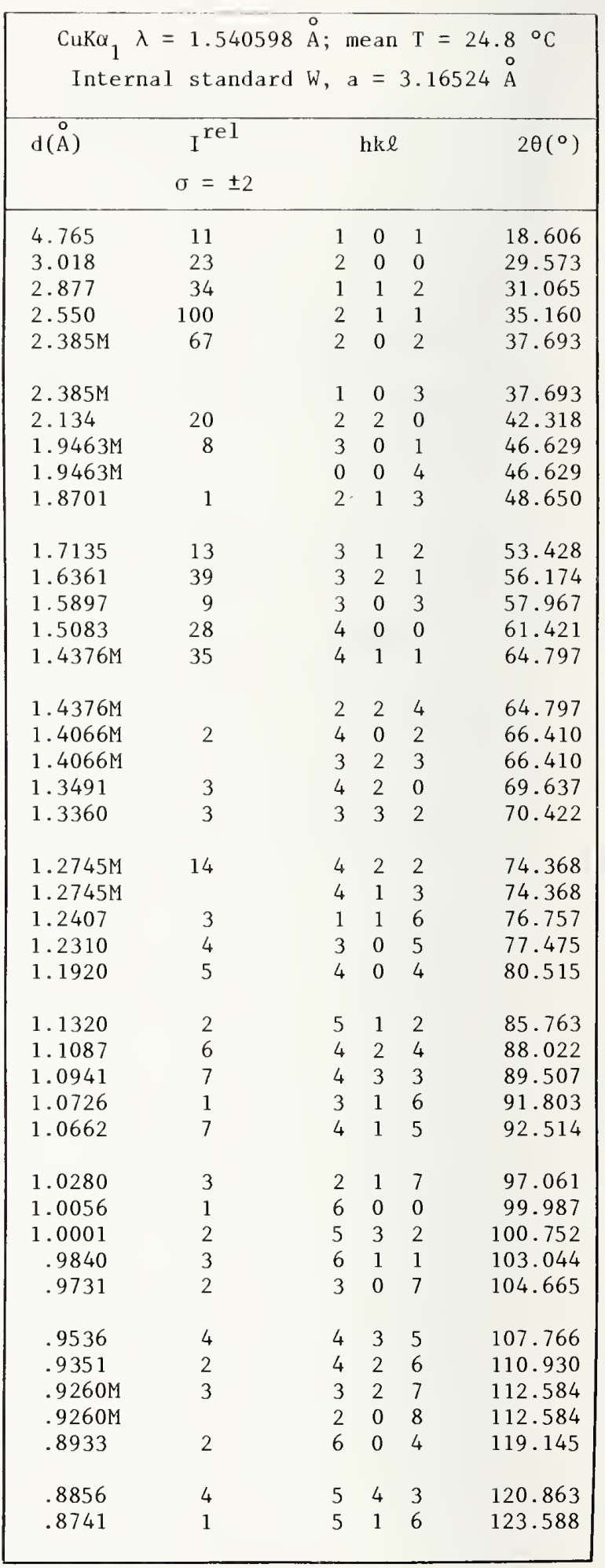


Copper Iron 0xide, $\mathrm{CuFe}_{2} \mathrm{O}_{4}$

Synonym

Copper ferrite

CAS registry no. 12018-79-0

Samp le

The sample was prepared by blending stoichiometric amounts of $\mathrm{CuO}$ and $\mathrm{Fe}_{2} \mathrm{O}_{3}$ and heating in a flowing atmosphere of pure oxygen for extended periods at temperatures as high as $975{ }^{\circ} \mathrm{C}$, with periodic grinding. The sample contained traces of $\mathrm{CuO}$ and an unidentified phase.

Color

Olive black

Structure

Tetragonal, I $4_{1} /$ and (141). It is a tetragonally distorted inverse spinel. The structure was determined by Prince and Treuting (1956). The "a" dimension given by Prince and Treuting was divided by $\sqrt{2}$ to make it conform to the body-centered space group.

Crystallographic constants of this sample $a=5.8444(4) \AA$

$c=8.6304(10)$

$c / a=1.4767$

$Z=4$

$\mathrm{V}=294.79 \AA^{3}$

Density $(\mathrm{calc})=5.390 \mathrm{~g} / \mathrm{cm}^{3}$

Figure of merit

$\mathrm{F}_{25}=86.2(0.0097,30)$

Polymorphism

A cubic phase has also been reported by Vervey and Heilman (1947).

Additional pattern

PDF card 6-545 (Weil et al., 1950)

References

Weil, L., Bertaut, F., and Bochirol, L,

(1950). J. Phys. Rad. 11, 208.

Prince, E. and Treuting, R. G. (1956).

Acta Crystallogr. 9, 1025.

Vervey, E. J. W. and Heilman, E. L. (1947).

J. Chem. Phys. 15, 174.

\begin{tabular}{|c|c|c|c|c|c|}
\hline \multicolumn{6}{|c|}{$\begin{array}{l}\mathrm{CuK} \alpha_{1} \lambda=1.540598 \AA ; \text { mean } \mathrm{T}=26.1{ }^{\circ} \mathrm{C} \\
\text { Internal standard Ag, } \mathrm{a}=4.08651 \AA\end{array}$} \\
\hline $\mathrm{d}(\stackrel{\circ}{\mathrm{A}})$ & $\begin{array}{l}\mathrm{Iel} \\
\sigma= \pm 2\end{array}$ & & hke & & $2 \theta\left(^{\circ}\right)$ \\
\hline 4.839 & 17 & 1 & 0 & 1 & 18.319 \\
\hline 2.985 & 32 & 1 & 1 & 2 & 29.908 \\
\hline 2.923 & 13 & 2 & 0 & 0 & 30.559 \\
\hline 2.582 & 53 & 1 & 0 & 3 & 34.719 \\
\hline 2.502 & 100 & 2 & 1 & 1 & 35.862 \\
\hline 2.420 & 14 & 2 & 0 & 2 & 37.122 \\
\hline 2.1580 & 11 & 0 & 0 & 4 & 41.826 \\
\hline 2.0667 & 22 & 2 & 2 & 0 & 43.768 \\
\hline 1.9345 & 1 & 2 & 1 & 3 & 46.930 \\
\hline 1.7360 & 5 & 2 & 0 & 4 & 52.683 \\
\hline 1.6990 & 10 & 3 & 1 & 2 & 53.923 \\
\hline 1.6550 & 12 & 1 & 0 & 5 & 55.477 \\
\hline 1.6134 & 11 & 3 & 0 & 3 & 57.035 \\
\hline 1.5932 & 24 & 3 & 2 & 1 & 57.829 \\
\hline 1.4922 & 40 & 2 & 2 & 4 & 62.158 \\
\hline 1.4611 & 16 & 4 & 0 & 0 & 63.632 \\
\hline 1.4119 & 1 & 3 & 2 & 3 & 66.127 \\
\hline 1.3583 & 2 & 1 & 1 & 6 & 69.099 \\
\hline 1.3118 & 2 & 3 & 3 & 2 & 71.920 \\
\hline 1.3067 & 2 & 4 & 2 & 0 & 72.241 \\
\hline 1.2922 & 5 & 3 & 0 & 5 & 73.184 \\
\hline 1.2900 & 4 & 2 & 0 & 6 & 73.331 \\
\hline 1.2713 & 9 & 4 & 1 & 3 & 74.586 \\
\hline 1.2507 & 4 & 4 & 2 & 2 & 76.037 \\
\hline 1.2098 & 5 & 4 & 0 & 4 & 79.094 \\
\hline
\end{tabular}


Copper Mercury Iodide, $\beta-\mathrm{Cu}_{2} \mathrm{HgI}_{4}$

Synonym

Cuprous mercuric tetraiodide

CAS registry no.

$55945-38-5$

Sample

The sample was obtained from City Chemical

Corp., New York, N.Y.

Color

Vivid red

Structure

Tetragonal, İ̄2m (121). Pseudocubic, isostructural with $\mathrm{Ag}_{2} \mathrm{HgI}_{4}$ (Ketelaar, 1931).

The structure of $\mathrm{Cu}_{2} \mathrm{HgI}_{4}$ was determined by Hahn et al. (1955).

Crystallographic constants of this sample

$\mathrm{a}=6.07802(15) \AA$

$c=12.2540(4)$

$\mathrm{c} / \mathrm{a}=2.0161$

$Z=2$

$V=452.69 \AA^{3}$

Density $(\mathrm{calc})=6.128 \mathrm{~g} / \mathrm{cm}^{3}$

Figure of merit

$\mathrm{F}_{30}=113.4(0.0070,38)$

Polymorphism

$\beta-\mathrm{Cu}_{2} \mathrm{HgI}_{4}$ transforms to a cubic form above $90{ }^{\circ} \mathrm{C}$ (Ketelaar, 1934).

Additional patterns

PDF card 18-450 (Hahn et al., 1955)

Ketelaar (1931)

\section{References}

Hahn, H., Frank, G., and Klinger, W. (1955).

Z. Anorg. Allg. Chem. 279, 271.

Ketelaar, J. A. A. (1931). Z. Kristallogr., 80,190 .

Ketelaar, J. A. A. (1934). Z. Kristallogr., 87,440 .

\begin{tabular}{|c|c|c|c|c|c|}
\hline \multicolumn{6}{|c|}{$\begin{array}{c}\mathrm{CuK} \alpha_{1} \lambda=1.540598 \AA \text {; mean } \mathrm{T}=26 \pm 2{ }^{\circ} \mathrm{C} \\
\text { Internal standard Si, SRM } 640 \mathrm{a}\end{array}$} \\
\hline $\mathrm{d}(\stackrel{\circ}{\mathrm{A}})$ & $I^{\text {rel }}$ & & $\mathrm{hke}$ & & $2 \theta\left(^{\circ}\right)$ \\
\hline & $\sigma= \pm 2$ & & & & \\
\hline 6.118 & $1 \mathrm{~L}$ & 0 & 0 & 2 & 14.466 \\
\hline 5.446 & 12 & 1 & 0 & 1 & 16.263 \\
\hline 4.299 & 2 & 1 & 1 & 0 & 20.645 \\
\hline 3.517 & 100 & 1 & 1 & 2 & 25.302 \\
\hline 3.389 & 14 & 1 & 0 & 3 & 26.275 \\
\hline 3.063 & 3 & 0 & 0 & 4 & 29.133 \\
\hline 3.038 & 4 & 2 & 0 & 0 & 29.375 \\
\hline 2.722 & $1 \mathrm{~L}$ & 2 & 0 & 2 & 32.876 \\
\hline 2.654 & 17 & 2 & 1 & 1 & 33.751 \\
\hline 2.4945 & 1 & 1 & 1 & 4 & 35.974 \\
\hline 2.2725 & 7 & 1 & 0 & 5 & 39.628 \\
\hline 2.2623 & 7 & 2 & 1 & 3 & 39.813 \\
\hline 2.1572 & 62 & 2 & 0 & 4 & 41.842 \\
\hline 2.0418 & $1 \mathrm{~L}$ & 0 & 0 & 6 & 44.328 \\
\hline 2.0274 & $1 \mathrm{~L}$ & 2 & 2 & 2 & 44.661 \\
\hline 1.9985 & 6 & 3 & 0 & 1 & 45.341 \\
\hline 1.8445 & 18 & 1 & 1 & 6 & 49.369 \\
\hline 1.8338 & 30 & 3 & 1 & 2 & 49.675 \\
\hline 1.8200 & 4 & 2 & 1 & 5 & 50.079 \\
\hline 1.7591 & 2 & 2 & 2 & 4 & 51.939 \\
\hline 1.6820 & $1 \mathrm{~L}$ & 1 & 0 & 7 & 54.513 \\
\hline 1.6698 & $1 \mathrm{~L}$ & 3 & 2 & 1 & 54.945 \\
\hline 1.6280 & $1 \mathrm{~L}$ & 3 & 1 & 4 & 56.478 \\
\hline 1.5580 & 5 & 3 & 2 & 3 & 59.261 \\
\hline 1.5317 & 3 & 0 & 0 & 8 & 60.387 \\
\hline 1.5194 & 5 & 4 & 0 & 0 & 60.924 \\
\hline 1.4801 & 2 & 2 & 2 & 6 & 62.725 \\
\hline 1.4716 & 2 & 2 & 1 & 7 & 63.125 \\
\hline 1.4635 & $1 \mathrm{~L}$ & 4 & 1 & 1 & 63.516 \\
\hline 1.3996 & 10 & 3 & 1 & 6 & 66.783 \\
\hline 1.3889 & 8 & 3 & 2 & 5 & 67.370 \\
\hline 1.3863 & 3 & 4 & 1 & 3 & 67.512 \\
\hline 1.3681 & 1 & 2 & 0 & 8 & 68.530 \\
\hline 1.3610 & $1 \mathrm{~L}$ & 4 & 0 & 4 & 68.942 \\
\hline 1.3286 & $1 \mathrm{~L}$ & 1 & 0 & 9 & 70.868 \\
\hline 1.3245 & 2 & 3 & 0 & 7 & 71.121 \\
\hline 1.2630 & 2 & 4 & 1 & 5 & 75.165 \\
\hline 1.2472 & 6 & 2 & 2 & 8 & 76.287 \\
\hline 1.2422 & 9 & 4 & 2 & 4 & 76.650 \\
\hline 1.2173 & 1 & 2 & 1 & 9 & 78.513 \\
\hline 1.2141 & 1 & 3 & 2 & 7 & 78.758 \\
\hline 1.2096 & 1 & 4 & 3 & 1 & 79.107 \\
\hline 1.1783 & 2 & 1 & $\begin{array}{ll}1 & 1\end{array}$ & 10 & 81.646 \\
\hline 1.1728 & 3 & 3 & 3 & 6 & 82.112 \\
\hline 1.1700 & 5 & 5 & 1 & 2 & 82.352 \\
\hline
\end{tabular}


Copper Mercury Iodide, $\beta-\mathrm{Cu}_{2} \mathrm{HgI}_{4}-$ (continued)

\begin{tabular}{|c|c|c|c|c|}
\hline $\mathrm{d}(\AA)$ & $\begin{array}{c}I^{\text {rel }} \\
\sigma= \pm 2\end{array}$ & & hke & $2 \theta\left({ }^{\circ}\right)$ \\
\hline 1.1650 & 2 & 4 & 33 & 82.784 \\
\hline 1.1300 & 1 & 3 & $\begin{array}{ll}0 & 9\end{array}$ & 85.951 \\
\hline 1.1275 & 1 & 4 & 17 & 86.190 \\
\hline 1.1239 & 2 & 5 & $\begin{array}{ll}2 & 1\end{array}$ & 86.530 \\
\hline 1.0960 & $1 \mathrm{~L}$ & 1 & $\begin{array}{ll}0 & 11\end{array}$ & 89.313 \\
\hline 1.0889 & 1 & 4 & 35 & 90.046 \\
\hline 1.0788 & 2 & 4 & $\begin{array}{ll}0 & 8\end{array}$ & 91.130 \\
\hline 1.0746 & 1 & 4 & 40 & 91.589 \\
\hline 1.0463 & $1 \mathrm{~L}$ & 3 & 38 & 94.816 \\
\hline 1.0333 & 3 & 3 & 110 & 96.402 \\
\hline 1.0308 & 2 & 2 & $\begin{array}{ll}1 & 11\end{array}$ & 96.714 \\
\hline 1.0296 & 2 & 5 & 16 & 96.865 \\
\hline 1.0275 & 2 & 5 & 32 & 97.119 \\
\hline 1.0167 & $1 \mathrm{~L}$ & 4 & 28 & 98.521 \\
\hline 1.0141 & $1 \mathrm{~L}$ & 4 & 44 & 98.857 \\
\hline 1.0001 & $1 \mathrm{~L}$ & 4 & 19 & 100.748 \\
\hline .9985 & $1 \mathrm{~L}$ & 4 & $\begin{array}{ll}3 & 7\end{array}$ & 100.972 \\
\hline .9959 & $1 \mathrm{~L}$ & 6 & $\begin{array}{ll}1 & 1\end{array}$ & 101.328 \\
\hline .9706 & 1 & 6 & 13 & 105.059 \\
\hline .9681 & 1 & 2 & $\begin{array}{ll}0 & 12\end{array}$ & 105.447 \\
\hline .9619 & 1 & 6 & 04 & 106.418 \\
\hline .9610 & $1 \mathrm{~L}$ & 6 & 20 & 106.565 \\
\hline .9486 & $1 \mathrm{~L}$ & 5 & 27 & 108.595 \\
\hline .9313 & 1 & 3 & 310 & 111.617 \\
\hline .9294 & 1 & 3 & 211 & 111.946 \\
\hline .9285 & 1 & 5 & 36 & 112.118 \\
\hline .9254 & 1 & 6 & 15 & 112.696 \\
\hline .9246 & $1 \mathrm{~L}$ & 5 & 43 & 112.836 \\
\hline .9102 & 1 & 4 & 210 & 115.631 \\
\hline .9068 & $1 \mathrm{~L}$ & 4 & 39 & 116.302 \\
\hline .9036 & $1 \mathrm{~L}$ & 6 & $\begin{array}{ll}3 & 1\end{array}$ & 116.966 \\
\hline .9018 & $1 \mathrm{~L}$ & 3 & 112 & 117.343 \\
\hline
\end{tabular}


Synonym

Europium sesquioxide

CAS registry no. 1308-96-9

Sample

The sample was obtained from American Potash and Chemical Corp., Research Division, West Chicago, IL. It was heated at $1000{ }^{\circ} \mathrm{C}$ for 5 days.

Color Colorless

Structure Cubic, Ia3 (206). Its structure was determined by Zachariasen (1928).

Crystallographic constants of this sample $\mathrm{a}=10.8683(2) \AA$

$Z=16$

$V=1283.76 \stackrel{\circ}{\mathrm{A}^{3}}$

Density $(\mathrm{calc})=7.283 \mathrm{~g} / \mathrm{cm}^{3}$

Figure of merit

$\mathrm{F}_{30}=125.6(0.0075,32)$

Polymorphism

There is a monoclinic modification formed above $1075{ }^{\circ} \mathrm{C}$ (Roth and Schneider, 1960) and a hexagonal form was found at $2330{ }^{\circ} \mathrm{C}$ (Foëx and Traverse, 1966).

Additional patterns

PDF card 12-393 (General Electric, ANP Dept., Cincinnati, OH)

PDF card 32-380 (Ferguson, I. and Hughes, UK Atomic Energy Authority. See UKAEA Report ND-R-150 (S). Gives a as $1 / 2$ of value used here.)

\section{References}

Foëx, M. and Traverse, J. P. (1966). Bull.

Soc. Fr. Minéral. Cristallogr. 89, 184.

Roth, R. S. and Schneider, S. J. (1960). J. Res. Nat. Bur. Stand. 64A, 309.

Zachariasen, W. H. (1928). Skr. Nor. Vidensk. Akad., Kl. 1: Mat-Naturvidensk. Kl. 1, 4.

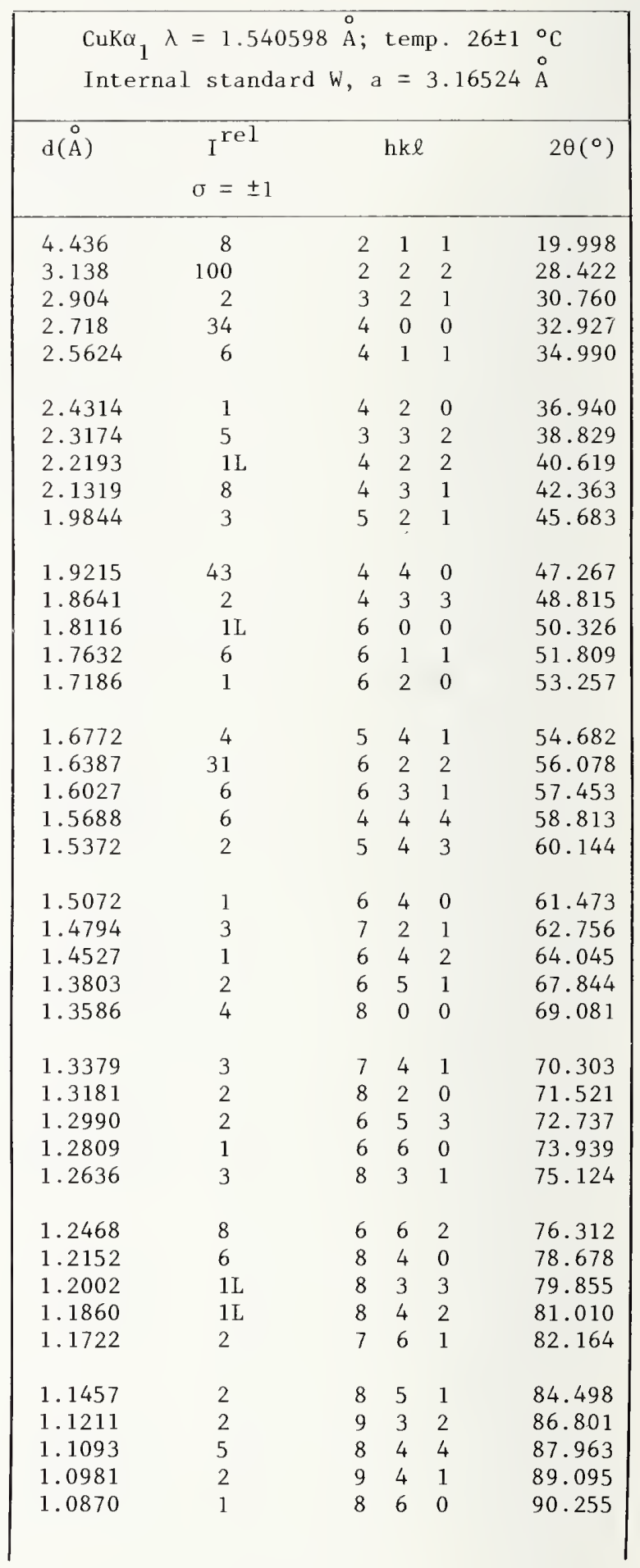


Europium 0xide, $\mathrm{Eu}_{2} \mathrm{O}_{3}$ - (continued)

\begin{tabular}{|c|c|c|c|c|c|}
\hline$d(\stackrel{\circ}{)}$ & $\mathrm{I}^{\text {rel }}$ & & hke & & $2 \theta\left({ }^{\circ}\right)$ \\
\hline 1.0763 & $1 \mathrm{~L}$ & 10 & 1 & 1 & 91.403 \\
\hline 1.0658 & 3 & 10 & 2 & 0 & 92.559 \\
\hline 1.0558 & $1 \mathrm{~L}$ & 9 & 4 & 3 & 93.708 \\
\hline 1.0458 & 5 & 10 & 2 & 2 & 94.877 \\
\hline 1.0363 & 2 & 10 & 3 & 1 & 96.025 \\
\hline 1.0179 & 2 & 8 & 7 & 1 & 98.355 \\
\hline 1.0091 & 2 & 10 & 4 & 0 & 99.519 \\
\hline 1.0006 & 2 & 9 & 6 & 1 & 100.679 \\
\hline .9922 & 2 & 10 & 4 & 2 & 101.863 \\
\hline .9840 & 2 & 8 & 7 & 3 & 103.035 \\
\hline .9683 & 2 & 10 & 5 & 1 & 105.413 \\
\hline .9605 & 2 & 8 & 8 & 0 & 106.631 \\
\hline .9460 & $1 \mathrm{~L}$ & 8 & 8 & 2 & 109.028 \\
\hline .9389 & ] & 11 & 3 & 2 & 110.258 \\
\hline .9319 & $1 \mathrm{~L}$ & 10 & 6 & 0 & 111.508 \\
\hline .9251 & 1 & 11 & 4 & 1 & 112.744 \\
\hline .9184 & 3 & 10 & 6 & 2 & 114.006 \\
\hline .9120 & $1 \mathrm{~L}$ & 9 & 6 & 5 & 115.271 \\
\hline .9056 & 2 & 12 & 0 & 0 & 116.560 \\
\hline .8995 & 2 & 9 & 8 & 1 & 117.817 \\
\hline .8933 & 1 & 12 & 2 & 0 & 119.143 \\
\hline .8873 & 1 & 10 & 7 & 1 & 120.487 \\
\hline .8815 & 2 & 12 & 2 & 2 & 121.824 \\
\hline .8757 & 1 & 12 & 3 & 1 & 123.201 \\
\hline .8646 & 2 & 11 & 6 & 1 & 125.990 \\
\hline .8592 & 2 & 12 & 4 & 0 & 127.408 \\
\hline .8539 & 1 & 12 & 3 & 3 & 128.860 \\
\hline .8486 & 3 & 10 & 8 & 0 & 130.381 \\
\hline .8435 & 2 & 11 & 6 & 3 & 131.901 \\
\hline .8384 & 2 & 10 & 8 & 2 & 133.482 \\
\hline .8336 & 1 & 12 & 5 & 1 & 135.053 \\
\hline
\end{tabular}


Europium $0 x i d e, \mathrm{Eu}_{2} \mathrm{O}_{3}$

Synonym

Europium sesquioxide

CAS registry no. 1308-96-9

Sample

The sample was obtained from American Potash and Chemical Corp., Research Division, West Chicago, IL. It was heated to $1250{ }^{\circ} \mathrm{C}$ for 3 hours to convert it to the monoclinic form.

Color

Colorless

Structure

Monoclinic, Roth and Schneider (1960). Its aspect $\mathrm{C} \% / \%$ was assumed to be the same as that of $\mathrm{Sm}_{2} \mathrm{O}_{3}$ determined by Douglass and Staritzky (1956).

Crystallographic constants of this sample $\mathrm{a}=14.1126(12) \AA$

$\mathrm{b}=3.6025(3)$

$c^{\prime}=8.8089(4)$

$\beta=100.050(6)^{\circ}$

$\mathrm{a} / \mathrm{b}=3.9174$

$c / b=2.4452$

$Z=6$
$V=440.98 \AA^{3}$

Density $(\mathrm{calc})=7.951 \mathrm{~g} / \mathrm{cm}^{3}$

Figure of merit

$F_{30}=62.1(0.0070,69)$

Polymorphism

There is a cubic form existing below $1050{ }^{\circ} \mathrm{C}$, Roth and Schneider (1960). A hexagonal form was found at $2330^{\circ} \mathrm{C}$, Foëx and Traverse (1966).

Additional pattern

PDF card 12-384 (General Electric ANP Dept., Cincinnati, $\mathrm{OH}$ )

References

Douglass, R. M. and Staritzky, E. (1956).

Anal. Chem. 28, 552.

Foëx, M. and Traverse, J. P. (1966). Bull. Soc. Fr. Mineral Crystallogr. 89, 184.

Roth, R. S. and Schneider, S. J. (1960). J. Res. Nat1. Bur. Stand. 64A, 309.

\begin{tabular}{|c|c|c|c|c|c|}
\hline \multicolumn{6}{|c|}{$\begin{array}{c}\mathrm{CuK} \alpha_{1} \lambda=1.540598 \AA \text {; mean } \mathrm{T}=25.3{ }^{\circ} \mathrm{C} \\
\text { Internal standard } \mathrm{W}, \mathrm{a}=3.16524 \AA\end{array}$} \\
\hline $\mathrm{d}(\stackrel{\circ}{\mathrm{A}})$ & $\begin{array}{l}\mathrm{rel} \\
\sigma= \pm 3\end{array}$ & & $\mathrm{hk}$ & & $2 \theta\left({ }^{\circ}\right)$ \\
\hline 5.953 & 4 & -2 & 0 & 1 & 14.869 \\
\hline 5.014 & $1 \mathrm{~L}$ & 2 & 0 & 1 & 17.676 \\
\hline 4.003 & 8 & -2 & 0 & 2 & 22.192 \\
\hline 3.419 & 18 & 2 & 0 & 2 & 26.042 \\
\hline 3.187 & 83 & 1 & 1 & 1 & 27.971 \\
\hline 3.046 & 85 & 4 & 0 & 1 & 29.297 \\
\hline 2.977 & 87 & -4 & 0 & 2 & 29.996 \\
\hline 2.891 & 58 & 0 & 0 & 3 & 30.906 \\
\hline 2.844 & 89 & 3 & 1 & 0 & 31.432 \\
\hline 2.778 & 100 & -1 & 1 & 2 & 32.196 \\
\hline 2.662 & 4 & 1 & 1 & 2 & 33.642 \\
\hline 2.5179 & 2 & 2 & 0 & 3 & 35.628 \\
\hline 2.3162 & 16 & 6 & 0 & 0 & 38.850 \\
\hline 2.2756 & 6 & -1 & 1 & 3 & 39.571 \\
\hline 2.2072 & 26 & -5 & 1 & 1 & 40.852 \\
\hline $2.1800 \mathrm{M}$ & 9 & -2 & 0 & 4 & 41.385 \\
\hline $2.1800 \mathrm{M}$ & & 1 & 1 & 3 & 41.385 \\
\hline $2.1459 \mathrm{M}$ & 56 & 6 & 0 & 1 & 42.072 \\
\hline $2.1459 \mathrm{M}$ & & -3 & 1 & 3 & 42.072 \\
\hline 2.0662 & 1 & 5 & 1 & 1 & 43.778 \\
\hline 2.0534 & $1 \mathrm{~L}$ & 4 & 0 & 3 & 44.064 \\
\hline 1.9739 & 2 & 2 & 0 & 4 & 45.940 \\
\hline 1.9267 & 56 & 3 & 1 & 3 & 47.132 \\
\hline 1.8804 & 4 & -5 & 1 & 3 & 48.366 \\
\hline 1.8614 & 4 & 5 & 1 & 2 & 48.892 \\
\hline 1.8213 & 3 & -3 & 1 & 4 & 50.042 \\
\hline 1.8014 & 32 & 0 & 2 & 0 & 50.632 \\
\hline $1.7635 \mathrm{M}$ & 17 & 0 & 2 & 1 & 51.799 \\
\hline $1.7635 \mathrm{M}$ & & -8 & 0 & 1 & 51.799 \\
\hline 1.7425 & $1 \mathrm{~L}$ & -6 & 0 & 4 & 52.472 \\
\hline 1.7061 & 39 & -7 & 1 & 2 & 53.679 \\
\hline 1.6729 & 19 & -4 & 0 & 5 & 54.834 \\
\hline $1.6635 \mathrm{M}$ & 17 & -5 & 1 & 4 & 55.168 \\
\hline $1.6635 \mathrm{M}$ & & 0 & 2 & 2 & 55.168 \\
\hline 1.6563 & 34 & 7 & 1 & 1 & 55.429 \\
\hline 1.6443 & 7 & 5 & 1 & 3 & 55.868 \\
\hline 1.6410 & 7 & 3 & 1 & 4 & 55.993 \\
\hline 1.6178 & 4 & 2 & 0 & 5 & 56.866 \\
\hline 1.5939 & 4 & 2 & 2 & 2 & 57.801 \\
\hline 1.5809 & 18 & -1 & 1 & 5 & 58.322 \\
\hline 1.5569 & 6 & -3 & 1 & 5 & 59.308 \\
\hline 1.5504 & 23 & 4 & 2 & 1 & 59.580 \\
\hline 1.5412 & 24 & -4 & 2 & 2 & 59.975 \\
\hline 1.5289 & 18 & 0 & 2 & 3 & 60.507 \\
\hline $1.5232 \mathrm{M}$ & 14 & 8 & 0 & 2 & 60.758 \\
\hline
\end{tabular}


Europium 0xide, $\mathrm{Eu}_{2} \mathrm{O}_{3}$ - (continued)

\begin{tabular}{|c|c|c|c|c|c|}
\hline$d(\AA)$ & $\begin{array}{c}\mathrm{I}^{r \mathrm{el}} \\
\sigma= \pm 3\end{array}$ & & $h \mathrm{ke}$ & & $2 \theta\left(^{\circ}\right)$ \\
\hline $1.5232 \mathrm{M}$ & & -2 & 2 & 3 & 60.758 \\
\hline 1.4882 & 9 & -8 & 0 & 4 & 62.345 \\
\hline 1.4673 & 3 & -2 & 0 & 6 & 63.332 \\
\hline $1.4648 \mathrm{M}$ & 4 & 2 & 2 & 3 & 63.456 \\
\hline $1.4648 \mathrm{M}$ & & -5 & 1 & 5 & 63.456 \\
\hline 1.4459 & 6 & 0 & 0 & 6 & 64.384 \\
\hline 1.4218 & 5 & 6 & 2 & 0 & 65.610 \\
\hline $1.3961 \mathrm{M}$ & 3 & -6 & 2 & 2 & 66.975 \\
\hline $1.3961 \mathrm{M}$ & & -10 & 0 & 2 & 66.975 \\
\hline 1.3796 & 3 & 6 & 2 & 1 & 67.881 \\
\hline 1.3683 & 3 & 2 & 0 & 6 & 68.519 \\
\hline 1.3635 & 4 & -9 & 1 & 3 & 68.797 \\
\hline 1.3488 & 3 & -3 & 1 & 6 & 69.654 \\
\hline 1.3362 & 8 & 10 & 0 & 1 & 70.406 \\
\hline $1.3341 \mathrm{M}$ & 7 & -7 & 1 & 5 & 70.535 \\
\hline $1.3341 \mathrm{M}$ & & -6 & 2 & 3 & 70.535 \\
\hline 1.3308 & 4 & 2 & 2 & 4 & 70.739 \\
\hline 1.3156 & 2 & 1 & 1 & 6 & 71.681 \\
\hline 1.2652 & 8 & 7 & 1 & 4 & 75.008 \\
\hline 1.2600 & 10 & -8 & 2 & 1 & 75.373 \\
\hline 1.2574 & 8 & -2 & 2 & 5 & 75.558 \\
\hline 1.2405 & 6 & 4 & 2 & 4 & 76.770 \\
\hline 1.2363 & 7 & 3 & 1 & 6 & 77.081 \\
\hline 1.2258 & 10 & -4 & 2 & 5 & 77.862 \\
\hline 1.2083 & 8 & -11 & 1 & 1 & 79.215 \\
\hline 1.2044 & 4 & -8 & 2 & 3 & 79.521 \\
\hline $1.1967 \mathrm{M}$ & 1 & -9 & 1 & 5 & 80.136 \\
\hline $1.1967 \mathrm{M}$ & & 1 & 3 & 0 & 80.136 \\
\hline 1.1829 & 5 & 1 & 3 & 1 & 81.266 \\
\hline $1.1628+$ & 9 & 8 & 2 & 2 & 82.977 \\
\hline $1.1628+$ & & -6 & 2 & 5 & 82.977 \\
\hline $1.1579 \mathrm{M}$ & 7 & 12 & 0 & 0 & 83.402 \\
\hline 1. $1579 \mathrm{M}$ & & -1 & 3 & 2 & 83.402 \\
\hline 1.1474 & 8 & -8 & 2 & 4 & 84.345 \\
\hline 1.1401 & 3 & 6 & 0 & 6 & 85.005 \\
\hline 1.1378 & 3 & -2 & 2 & 6 & 85.223 \\
\hline 1.1351 & 1 & -3 & 3 & 2 & 85.471 \\
\hline 1.1327 & 1 & 8 & 0 & 5 & 85.694 \\
\hline 1.1274 & 6 & 0 & 2 & 6 & 86.196 \\
\hline 1.1258 & 7 & -11 & 1 & 4 & 86.351 \\
\hline 1.1091 & 3 & 9 & 1 & 4 & 87.980 \\
\hline $1.1040 \mathrm{M}$ & 10 & 11 & 1 & 2 & 88.492 \\
\hline $1.1040 \mathrm{M}$ & & -8 & 0 & 7 & 88.492 \\
\hline 1.1010 & 6 & -2 & 0 & 8 & 88.798 \\
\hline 1.0951 & 4 & -3 & 3 & 3 & 89.398 \\
\hline 1.0903 & 3 & -4 & 0 & 8 & 89.902 \\
\hline 1.0871 & 3 & 10 & 0 & 4 & 90.243 \\
\hline
\end{tabular}


Hafnium Oxide, $\mathrm{HfO}_{2}$

CAS registry no. $12055-23-1$

Sample

A commercial sample was heated at $1450{ }^{\circ} \mathrm{C}$ for 96 hours, then at $1550{ }^{\circ} \mathrm{C}$ for 48 hours.

Color

Colorless

Structure

Monoclinic, P2/a (14), isostructural with $\mathrm{ZrO}_{2}$ (Geller and Corenzwit, 1953). The structure of $\mathrm{ZrO}_{2}$ was redetermined by McCullough and Trueblood (1959) and Adams and Rogers (1959).

Crystallographic constants of this sample

$\mathrm{a}=5.2851(2) \AA$

$\mathrm{b}=5.1819(2)$

$c=5.1157(2)$

$\beta=99.259(3)^{\circ}$

$\mathrm{a} / \mathrm{b}=1.0199$

$c / b=0.9872$

$\mathrm{Z}=4$

$V=138.28 \AA^{3}$

Density $(\mathrm{calc})=10.111 \mathrm{~g} / \mathrm{cm}^{3}$

Figure of merit

$\mathrm{F}_{30}=129.1(0.0061,38)$

Polymorphism

Hafnium oxide has been reported to exist in 3 other polymorphic phases. Curtis, Doney, and Johnson (1954) report a tetragonal phase. Ruh et al. (1968) and Ruh and Corfield (1970) report inversion temperatures of the tetragonal to the cubic phase. Bocquillon et al. (1968) report an orthorhombic phase.

Additional pattern PDF card 6-318 (Geller and Corenzwit, 1953)

References

Adams, J. and Rogers, M. D. (1959). Acta Crystallogr. 12, 951 .

Bocquillon, G., Susse, C., and Vodar, B. (1968). Rev. Int. Hautes Temp. Refract. 5,247 .

Curtis, C. E., Doney, L. M., and Johnson, J. R. (1954). J. Am. Ceram. Soc. 37, 458.

Geller, S. and Corenzwit, E. (1953). Anal. Chem. 25, 1774.

McCullough, J. D. and Trueblood, K. N. (1959). Acta Crystallogr. 12, 507.

Ruh, R. and Corfield, P. W. R. (1970). J. Am. Ceram. Soc. 53, No. 3, 126.

Ruh, R., Garrett, H. J., Domagala, R. F., and Tallan, N. M. (1968). J. Am. Ceram. Soc. 51, No. $1,23$.

\begin{tabular}{|c|c|c|c|c|c|}
\hline \multicolumn{6}{|c|}{$\begin{array}{c}\mathrm{CuK} \alpha_{1} \lambda=1.540598 \AA \text {; mean } \mathrm{T}=25.4{ }^{\circ} \mathrm{C} \\
\text { Internal standards Ag, } \mathrm{a}=4.08651 \mathrm{~A} \\
\text { Fluo rophlogopite, SRM } 675\end{array}$} \\
\hline $\mathrm{d}(\stackrel{\circ}{\AA})$ & $\begin{array}{l}\mathrm{Iel} \\
\sigma= \pm 1\end{array}$ & & $h \mathrm{k} \ell$ & & $2 \theta\left(^{\circ}\right)$ \\
\hline 5.051 & 6 & 0 & 0 & 1 & 17.544 \\
\hline 3.677 & 16 & 1 & 1 & 0 & 24.185 \\
\hline 3.616 & 12 & 0 & 1 & 1 & 24.597 \\
\hline 3.147 & 100 & -1 & 1 & 1 & 28.336 \\
\hline 2.823 & 78 & 1 & 1 & 1 & 31.665 \\
\hline 2.6080 & 25 & 2 & 0 & 0 & 34.358 \\
\hline 2.5907 & 20 & 0 & 2 & 0 & 34.595 \\
\hline 2.5244 & 23 & 0 & 0 & 2 & 35.534 \\
\hline 2.4870 & 7 & -2 & 0 & 1 & 36.086 \\
\hline 2.3204 & 9 & 1 & 2 & 0 & 38.776 \\
\hline 2.3050 & 1 & 0 & 2 & 1 & 39.046 \\
\hline 2.2690 & $1 \mathrm{~L}$ & 0 & 1 & 2 & 39.691 \\
\hline 2.2423 & 2 & -2 & 1 & 1 & 40.185 \\
\hline 2.2010 & 15 & -1 & 1 & 2 & 40.971 \\
\hline 2.1792 & 5 & 2 & 0 & 1 & 41.401 \\
\hline 2.1678 & 5 & -1 & 2 & 1 & 41.628 \\
\hline 2.0088 & 5 & 2 & 1 & 1 & 45.096 \\
\hline 1.9803 & 7 & -2 & 0 & 2 & 45.783 \\
\hline 1.8494 & 3 & -2 & 1 & 2 & 49.230 \\
\hline 1.8379 & 18 & 2 & 2 & 0 & 49.559 \\
\hline 1.8079 & 23 & 0 & 2 & 2 & 50.436 \\
\hline 1.7936 & 12 & -2 & 2 & 1 & 50.868 \\
\hline 1.7727 & 6 & -1 & 2 & 2 & 51.513 \\
\hline $1.6834 \mathrm{M}$ & 12 & 2 & 0 & 2 & 54.461 \\
\hline $1.6834 \mathrm{M}$ & & 0 & 0 & 3 & 54.461 \\
\hline 1.6673 & 1 & 2 & 2 & 1 & 55.032 \\
\hline 1.6483 & 13 & 3 & 1 & 0 & 55.722 \\
\hline 1.6431 & 10 & -3 & 1 & 1 & 55.914 \\
\hline 1.6342 & 9 & 0 & 3 & 1 & 56.246 \\
\hline $1.6006+$ & 9 & 0 & 1 & 3 & 57.535 \\
\hline $1.6006+$ & & -1 & 1 & 3 & 57.535 \\
\hline 1.5833 & 7 & -1 & 3 & 1 & 58.225 \\
\hline 1.5732 & 5 & -2 & 2 & 2 & 58.635 \\
\hline 1.5370 & 9 & 1 & 3 & 1 & 60.155 \\
\hline 1.5306 & 9 & -2 & 0 & 3 & 60.433 \\
\hline 1.5009 & 6 & 3 & 1 & 1 & 61.759 \\
\hline 1.4881 & 8 & -3 & 1 & 2 & 62.350 \\
\hline 1.4687 & 11 & 1 & 1 & 3 & 63.265 \\
\hline 1.4439 & 2 & 3 & 2 & 0 & 64.484 \\
\hline $1.4401 \mathrm{M}$ & 3 & 2 & 3 & 0 & 64.676 \\
\hline $1.4401 \mathrm{M}$ & & -3 & 2 & 1 & 64.676 \\
\hline 1.4252 & 1 & 0 & 3 & 2 & 65.433 \\
\hline 1.4184 & 3 & -2 & 3 & 1 & 65.784 \\
\hline $1.4118 \mathrm{M}$ & 9 & 2 & 2 & 2 & 66.133 \\
\hline $1.4118 \mathrm{M}$ & & 0 & 2 & 3 & 66.133 \\
\hline 1.4083 & 4 & -1 & 3 & 2 & 66.318 \\
\hline 1.3537 & 3 & 2 & 3 & 1 & 69.367 \\
\hline 1.3414 & 1 & 3 & 2 & 1 & 70.094 \\
\hline 1.3325 & 1 & -3 & 2 & 2 & 70.634 \\
\hline $1.3181 \mathrm{M}$ & 4 & 1 & 2 & 3 & 71.520 \\
\hline
\end{tabular}


Hafnium Oxide, $\mathrm{HfO}_{2}-$ (continued)

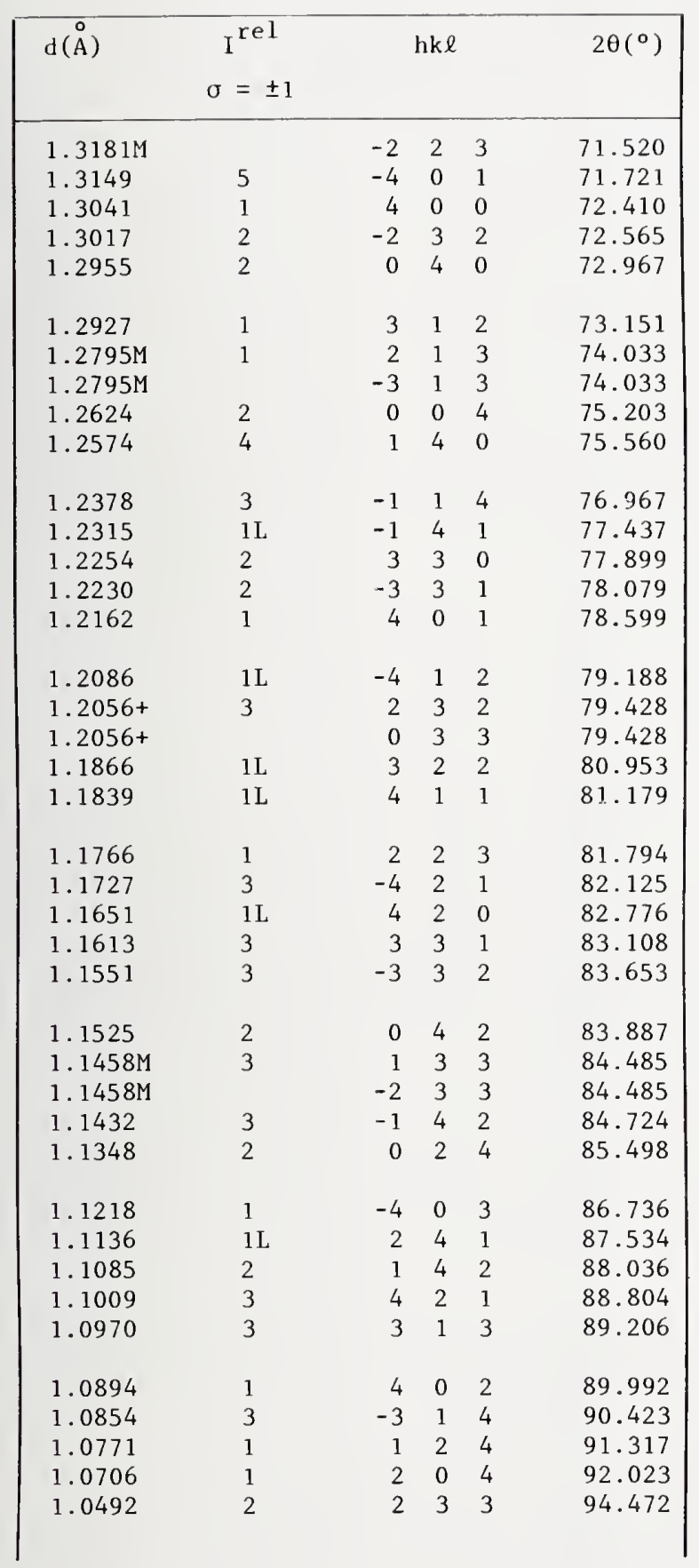

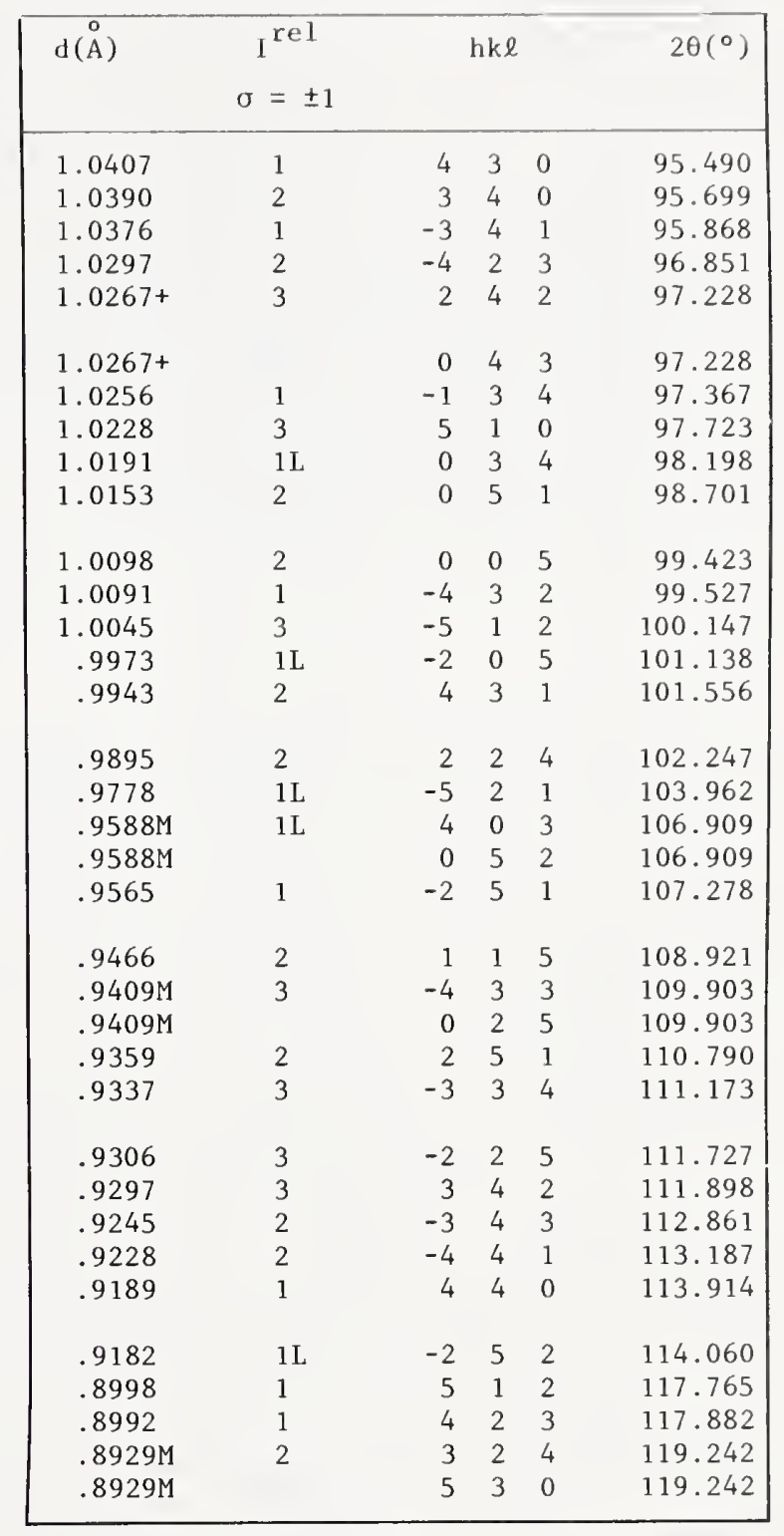


Synonyms

Iron niobate

Ferrous niobate

CAS registry no. 12022-59-2

Sample

The sample was prepared according to the paper by Turnock (1966). Stoichiometric amounts of $\mathrm{Fe}_{2} \mathrm{O}_{3}$ and $\mathrm{Nb}_{2} \mathrm{O}_{5}$ were mixed and heated in a platinum boat (presaturated with $\mathrm{Fe}$ by heating a sample of $\mathrm{FeNb}_{2} \mathrm{O}_{6}$ under the same conditions as in the synthesis). Two heatings in the $1130-1160{ }^{\circ} \mathrm{C}$ range in a controlled atmosphere with the partial pressure of oxygen less than or equal to $10^{-14} \mathrm{~atm}$. The sample contains a trace of $\mathrm{NbO}_{2}$.

Color

Black

\section{Structure}

Orthorhombic, Pcan (60). It has the columbite structure, solved by Sturdivant (1930). The structure was qualitatively done by Brandt (1943).

Crystallographic constants of this sample

$\mathrm{a}=5.7350(3) \AA$

$\mathrm{b}=14.2688(7)$

$\mathrm{c}=5.0504(3)$

$\mathrm{a} / \mathrm{b}=0.4019$

$\mathrm{c} / \mathrm{b}=0.3539$

$\begin{array}{ll}Z=4 & \circ \\ V & =413.29 \AA^{3}\end{array}$

Density $(\mathrm{calc})=5.427 \mathrm{~g} / \mathrm{cm}^{3}$

Figure of merit

$\mathrm{F}_{30}=49.3(0.0086,71)$

Additional pattern

PDF card 31-644 (Husson, et al., 1977)

References

Brandt, K. (1943). Ark. Kemi Mineral. Geol.

17 a, No. 15.

Husson, E., Repelin, Y., Nguyen, Q. D., and Brusset, H. (1977). Mater. Res. Bull. 12, 1199.

Sturdivant, J. H. (1930). Z. Kristallogr. $68,239$.

Turnock, A. C. (1966). J. Am. Ceram. Soc. 49,177 .

\begin{tabular}{|c|c|c|c|c|c|}
\hline \multicolumn{6}{|c|}{$\begin{array}{c}\mathrm{CuK} \alpha_{1} \lambda=1.540598 \AA \text { 皮 } \text { mean } \mathrm{T}=26.8^{\circ} \mathrm{C} \\
\text { Internal standard Si, SRM } 640 \mathrm{a}\end{array}$} \\
\hline $\mathrm{d}(\stackrel{\circ}{\mathrm{A}})$ & $\mathrm{I}^{\text {rel }}$ & & $\mathrm{hk \ell}$ & & $2 \theta\left(^{\circ}\right)$ \\
\hline & $\sigma= \pm 2$ & & & & \\
\hline 7.14 & 4 & 0 & 2 & 0 & 12.394 \\
\hline 5.319 & 1 & 1 & 1 & 0 & 16.655 \\
\hline $3.661 \mathrm{M}$ & 38 & 1 & 1 & 1 & 24.290 \\
\hline $3.661 \mathrm{M}$ & & 1 & 3 & 0 & 24.290 \\
\hline 3.567 & 5 & 0 & 4 & 0 & 24.944 \\
\hline 2.965 & 100 & 1 & 3 & 1 & 30.118 \\
\hline 2.868 & 11 & 2 & 0 & 0 & 31.164 \\
\hline 2.662 & 1 & 2 & 2 & 0 & 33.642 \\
\hline 2.526 & 14 & 0 & 0 & 2 & 35.510 \\
\hline 2.495 & 14 & 2 & 0 & 1 & 35.972 \\
\hline $2.379 \mathrm{M}$ & 7 & 0 & 2 & 2 & 37.790 \\
\hline $2.379 \mathrm{M}$ & & 0 & 6 & 0 & 37.790 \\
\hline 2.354 & $1 \mathrm{~L}$ & 2 & 2 & 1 & 38.196 \\
\hline 2.2302 & 4 & 0 & 3 & 2 & 40.411 \\
\hline 2.2088 & 8 & 2 & 3 & 1 & 40.820 \\
\hline 2.0790 & 10 & 1 & 3 & 2 & 43.495 \\
\hline 2.0613 & 2 & 0 & 4 & 2 & 43.887 \\
\hline 2.0438 & 2 & 2 & 4 & 1 & 44.283 \\
\hline 1.9212 & $1 \mathrm{~L}$ & 1 & 7 & 0 & 47.274 \\
\hline $1.8952 \mathrm{M}$ & 8 & 2 & 0 & 2 & 47.964 \\
\hline $1.8952 \mathrm{M}$ & & 3 & 1 & 0 & 47.964 \\
\hline $1.8307 \mathrm{M}$ & 8 & 2 & 2 & 2 & 49.765 \\
\hline $1.8307 \mathrm{M}$ & & 2 & 6 & 0 & 49.765 \\
\hline $1.7957 \mathrm{M}$ & 2 & 1 & 5 & 2 & 50.805 \\
\hline $1.7957 \mathrm{M}$ & & 1 & 7 & 1 & 50.805 \\
\hline $1.7740 \mathrm{M}$ & 14 & 3 & 1 & 1 & 51.471 \\
\hline $1.7740 \mathrm{M}$ & & 3 & 3 & 0 & 51.471 \\
\hline 1.7315 & 17 & 0 & 6 & 2 & 52.829 \\
\hline 1.7211 & 22 & 2 & 6 & 1 & 53.175 \\
\hline $1.6738 \mathrm{M}$ & 2 & 2 & 4 & 2 & 54.801 \\
\hline $1.6738 \mathrm{M}$ & & 3 & 3 & 1 & 54.801 \\
\hline 1.6052 & $1 \mathrm{~L}$ & 1 & 1 & 3 & 57.355 \\
\hline $1.5294 M$ & 14 & 1 & 3 & 3 & 60.485 \\
\hline $1.5294 \mathrm{M}$ & & 1 & 7 & 2 & 60.485 \\
\hline $1.5155 \mathrm{M}$ & $1 \mathrm{~L}$ & 3 & 1 & 2 & 61.101 \\
\hline $1.5155 \mathrm{M}$ & & 3 & 5 & 1 & 61.101 \\
\hline 1.4821 & 5 & 2 & 6 & 2 & 62.631 \\
\hline 1.4625 & 12 & 1 & 9 & 1 & 63.563 \\
\hline $1.4517 \mathrm{M}$ & 18 & 2 & 0 & 3 & 64.094 \\
\hline $1.4517 \mathrm{M}$ & & 3 & 3 & 2 & 64.094 \\
\hline 1.4338 & 2 & 4 & 0 & 0 & 64,995 \\
\hline 1.4271 & $1 \mathrm{~L}$ & 0 & 10 & 0 & 65.337 \\
\hline 1.4231 & $1 \mathrm{~L}$ & 2 & 2 & 3 & 65.544 \\
\hline 1.3946 & 1 & 3 & 7 & 0 & 67.056 \\
\hline 1.3792 & 5 & 4 & 0 & 1 & 67.905 \\
\hline
\end{tabular}


Iron Niobium Oxide, $\mathrm{Fe}\left(\mathrm{NbO}_{3}\right)_{2}$ - (continued)

\begin{tabular}{|c|c|c|c|c|c|}
\hline $\mathrm{d}(\stackrel{\circ}{\mathrm{A}})$ & $\begin{array}{c}I \text { rel } \\
\sigma= \pm 2\end{array}$ & & hke & & $2 \theta\left({ }^{\circ}\right)$ \\
\hline $1.3445+$ & $1 \mathrm{~L}$ & 2 & 4 & 3 & 69.907 \\
\hline $1.3445+$ & & 3 & 5 & 2 & 69.907 \\
\hline 1.3380 & 1L & 2 & 9 & 1 & 70.299 \\
\hline 1.3072 & 1 & 1 & 9 & 2 & 72.210 \\
\hline 1.2864 & $1 \mathrm{~L}$ & 4 & 4 & 1 & 73.571 \\
\hline 1.2776 & 1L & 2 & 10 & 0 & 74.161 \\
\hline 1.2659 & $1 \mathrm{~L}$ & 1 & 7 & 3 & 74.960 \\
\hline $1.2626 \mathrm{M}$ & 1 & 3 & 8 & 1 & 75.191 \\
\hline $1.2626 \mathrm{M}$ & & 0 & 0 & 4 & 75.191 \\
\hline $1.2422 \mathrm{M}$ & $1 \mathrm{~L}$ & 0 & 10 & 2 & 76.647 \\
\hline $1.2422 \mathrm{M}$ & & 4 & 1 & 2 & 76.647 \\
\hline 1.2390 & 2 & 2 & 6 & 3 & 76.883 \\
\hline $1.2204+$ & 2 & 3 & 9 & 0 & 78.275 \\
\hline $1.2204+$ & & 0 & 3 & 4 & 78.275 \\
\hline 1.2060 & $1 \mathrm{~L}$ & 4 & 3 & 2 & 79.391 \\
\hline 1.1932 & 6 & 4 & 6 & 1 & 80.416 \\
\hline 1.1901 & 3 & 0 & 4 & 4 & 80.671 \\
\hline 1.1862 & 1 & 3 & 9 & 1 & 80.992 \\
\hline $1.1554 \mathrm{M}$ & 1 & 2 & 0 & 4 & 83.625 \\
\hline $1.1554 \mathrm{M}$ & & 3 & 5 & 3 & 83.625 \\
\hline 1.1400 & $1 \mathrm{~L}$ & 2 & 10 & 2 & 85.014 \\
\hline $1.1314 \mathrm{M}$ & 3 & 1 & 9 & 3 & 85.821 \\
\hline $1.1314 \mathrm{M}$ & & 1 & 11 & 2 & 85.821 \\
\hline $1.1152+$ & 3 & 3 & 10 & 1 & 87.377 \\
\hline $1.1152+$ & & 0 & 6 & 4 & 87.377 \\
\hline 1.1042 & $1 \mathrm{~L}$ & 4 & 6 & 2 & 88.466 \\
\hline 1.0987 & 4 & 3 & 9 & 2 & 89.028 \\
\hline 1.0915 & 1 & 4 & 0 & 3 & 89.782 \\
\hline 1.0887 & 2 & 5 & 3 & 1 & 90.066 \\
\hline 1.0789 & $1 \mathrm{~L}$ & 4 & 2 & 3 & 91.122 \\
\hline 1.0779 & $1 \mathrm{~L}$ & 1 & 13 & 0 & 91.230 \\
\hline 1.0758 & 1 & 0 & 12 & 2 & 91.453 \\
\hline $1.0733+$ & 1 & 0 & 7 & 4 & 91.729 \\
\hline $1.0733+$ & & 2 & 12 & 1 & 91.729 \\
\hline 1.0542 & $1 \mathrm{~L}$ & 1 & 13 & 1 & 93.892 \\
\hline 1.0507 & $1 \mathrm{~L}$ & 3 & 1 & 4 & 94.302 \\
\hline 1.0438 & $1 \mathrm{~L}$ & 4 & 4 & 3 & 95.117 \\
\hline $1.0414+$ & $1 \mathrm{~L}$ & 5 & 1 & 2 & 95.408 \\
\hline $1.0414+$ & & 5 & 5 & 1 & 95.408 \\
\hline 1.0394 & 1 & 2 & 6 & 4 & 95.656 \\
\hline 1.0287 & 2 & 3 & 3 & 4 & 96.980 \\
\hline 1.0201 & 2 & 5 & 3 & 2 & 98.076 \\
\hline 1.0176 & 1 & 2 & 10 & 3 & 98.402 \\
\hline 1.0073 & 1 & 2 & 12 & 2 & 99.758 \\
\hline .9996 & $1 \mathrm{~L}$ & 5 & 7 & 0 & 100.816 \\
\hline .9920 & 3 & 4 & 6 & 3 & 101.886 \\
\hline $.9879 \mathrm{M}$ & 1 & 3 & 9 & 3 & 102.473 \\
\hline $.9879 M$ & & 3 & 11 & 2 & 102.473 \\
\hline
\end{tabular}


Synonym

Iron niobate

Sample

The sample was prepared by blending $\mathrm{Fe}^{2} \mathrm{Nb}_{2} \mathrm{O}_{6}$ with $\mathrm{Fe}_{2} \mathrm{O}_{3}$ in the appropriate molar ratio. The mixture was heated in an iron boat at $1160{ }^{\circ} \mathrm{C}$ for 8 hours, in a controlled atmosphere with the partial pressure of oxygen less than or equal to $10^{-16} \mathrm{~atm}$. The product was essentially single phase and contained only a trace of $\mathrm{Fe}^{2} \mathrm{Nb}_{2} \mathrm{O}_{6}$ as a second phase.

Color

Colorless

Structure

Hexagona 1, $\mathrm{P} 6 /$ \%st, $\mathrm{Z}=2$, isostructural

with $\mathrm{Co}_{4} \mathrm{Nb}_{2} \mathrm{O}_{9}$ (Bertaut et al., 1960).

Crystallographic constants of this sample

$\mathrm{a}=5.2253(2) \AA$

$c=14.2070(8)$

$c / a=2.7189$

$Z=2$

$\mathrm{V}=335.94 \AA^{3}$

Density $(\mathrm{calc})=5.469 \mathrm{~g} / \mathrm{cm}^{3}$

Figure of merit

$\mathrm{F}_{30}=46.6(0.0097,66)$

Reference

Bertaut, F., Corliss, L., and Forrat, F.

(1960). C. R. Hebd. Séances Acad. Sci.

$251,1733$.

\begin{tabular}{|c|c|c|c|c|c|}
\hline \multicolumn{6}{|c|}{$\begin{array}{r}\mathrm{CuKa}_{1} \lambda=1.540598 \mathrm{~A} ; \text { mean } \mathrm{T}=27 \\
\text { Internal standards } \mathrm{W}, \mathrm{a}=3.165 \\
\text { Fluorophlogopite, SRM } 675\end{array}$} \\
\hline$d(\stackrel{\circ}{A})$ & $\begin{array}{c}I^{\text {rel }} \\
\sigma= \pm 2\end{array}$ & & $\mathrm{hkl}$ & & $2 \theta\left({ }^{\circ}\right)$ \\
\hline 7.12 & 1 & 0 & 0 & 2 & 12.428 \\
\hline 4.524 & 20 & 1 & 0 & 0 & 19.605 \\
\hline 3.818 & 23 & 1 & 0 & 2 & 23.282 \\
\hline 3.553 & 2 & 0 & 0 & 4 & 25.040 \\
\hline 2.793 & 100 & 1 & 0 & 4 & 32.019 \\
\hline 2.611 & 72 & 1 & 1 & 0 & 34.314 \\
\hline 2.368 & 3 & 0 & 0 & 6 & 37.961 \\
\hline 2.287 & 12 & 1 & 1 & 3 & 39.369 \\
\hline 2.263 & 2 & 2 & 0 & 0 & 39.801 \\
\hline 2.1557 & 2 & 2 & 0 & 2 & 41.873 \\
\hline 2.1045 & 2 & 1 & 1 & 4 & 42.941 \\
\hline 2.0979 & 2 & 1 & 0 & 6 & 43.083 \\
\hline 1.9084 & 29 & 2 & 0 & 4 & 47.612 \\
\hline 1.7753 & 4 & 0 & 0 & 8 & 51.432 \\
\hline 1.7540 & 31 & 1 & 1 & 6 & 52.101 \\
\hline
\end{tabular}

\begin{tabular}{|c|c|c|c|c|c|}
\hline $\mathrm{d}(\stackrel{\circ}{\mathrm{A}})$ & $\begin{array}{c}I^{\text {rel }} \\
\sigma= \pm 2\end{array}$ & & hk $\ell$ & & $2 \theta\left(^{\circ}\right)$ \\
\hline 1.7108 & 2 & 2 & 1 & 0 & 53.520 \\
\hline 1.6630 & 3 & 2 & 1 & 2 & 55.187 \\
\hline 1.6530 & 7 & 1 & 0 & 8 & 55.552 \\
\hline 1.6362 & 1 & 2 & 0 & 6 & 56.172 \\
\hline 1.5413 & 23 & 2 & 1 & 4 & 59.972 \\
\hline 1.5087 & 21 & 3 & 0 & 0 & 61.405 \\
\hline 1.4684 & 2 & 1 & 1 & 8 & 63.279 \\
\hline 1.3973 & 3 & 2 & 0 & 8 & 66.911 \\
\hline 1.3886 & 3 & 3 & 0 & 4 & 67.385 \\
\hline 1.3555 & 8 & 1 & $\begin{array}{ll}0 & 1\end{array}$ & 10 & 69.261 \\
\hline 1.3065 & 4 & 2 & 2 & 0 & 72.255 \\
\hline 1.2721 & 2 & 3 & 0 & 6 & 74.531 \\
\hline 1.2594 & 1 & 2 & 2 & 3 & 75.419 \\
\hline 1.2553 & $1 \mathrm{~L}$ & 3 & 1 & 0 & 75.704 \\
\hline 1.2360 & 2 & 3 & 1 & 2 & 77.103 \\
\hline 1.2322 & 3 & 2 & 1 & 8 & 77.388 \\
\hline 1.2032 & 4 & 2 & 01 & 10 & 79.617 \\
\hline 1.1835 & 6 & 3 & 1 & 4 & 81.216 \\
\hline 1.1496 & 1 & 3 & 0 & 8 & 84.143 \\
\hline 1.1440 & 4 & 2 & 2 & 6 & 84.655 \\
\hline 1.1316 & $1 \mathrm{~L}$ & 4 & 0 & 0 & 85.800 \\
\hline $1.0929 M$ & 4 & 2 & 11 & 10 & 89.626 \\
\hline $1.0929 \mathrm{M}$ & & 0 & $\begin{array}{ll}0 & 1\end{array}$ & 13 & 89.626 \\
\hline 1.0781 & 2 & 4 & 0 & 4 & 91.206 \\
\hline 1.0522 & $1 \mathrm{~L}$ & 2 & 2 & 8 & 94.120 \\
\hline 1.0270 & $1 \mathrm{~L}$ & 3 & 2 & 2 & 97.185 \\
\hline 1.0251 & 1 & 3 & 1 & 8 & 97.426 \\
\hline .9965 & 3 & 3 & 2 & 4 & 101.247 \\
\hline .9903 & 2 & 1 & $\begin{array}{ll}0 & 1\end{array}$ & 14 & 102.129 \\
\hline .9875 & 4 & 4 & 1 & 0 & 102.524 \\
\hline .9541 & 1 & 4 & 0 & 8 & 107.679 \\
\hline .9508 & 1 & 3 & 2 & 6 & 108.215 \\
\hline .9460 & 1 & 1 & 11 & 14 & 109.033 \\
\hline .9407 & 3 & 3 & 11 & 10 & 109.944 \\
\hline .9260 & 2 & 2 & $\begin{array}{ll}0 & 1\end{array}$ & 14 & 112.582 \\
\hline .9114 & 3 & 4 & 1 & 6 & 115.386 \\
\hline .8962 & $1 \mathrm{~L}$ & 3 & 2 & 8 & 118.532 \\
\hline $.8849 M$ & $1 \mathrm{~L}$ & 4 & $\begin{array}{ll}0 & 1\end{array}$ & 10 & 121.021 \\
\hline $.8849 M$ & & 3 & 01 & 13 & 121.021 \\
\hline .8770 & $1 \mathrm{~L}$ & 5 & 0 & 4 & 122.887 \\
\hline .8727 & 2 & 2 & 11 & 14 & 123.940 \\
\hline .8709 & 3 & 3 & 3 & 0 & 124.382 \\
\hline .8629 & $1 \mathrm{~L}$ & 4 & 1 & 8 & 126.414 \\
\hline
\end{tabular}


Synonym

Ferrous orthosilicate

Sample

The sample was prepared by grinding together equimolar parts of $\mathrm{Fe}_{2} \mathrm{O}_{3}$ and $\mathrm{SiO}_{2}$ under acetone. After drying, the specimen was transferred to a high purity iron boat. It was heated twice for 8 hours each time in a controlled atmosphere with the partial pressure of oxygen less than or equal to $10^{-20} \mathrm{~atm}$, first at $1110^{\circ} \mathrm{C}$ and then at $1150{ }^{\circ} \mathrm{C}$.

Color

Grayish olive

Structure

Orthorhombic, Pmnb (62). The structure was determined by Rinne et al. (1924) and an indexing error was corrected by Bragg and Brown (1926).

Crystallographic constants of this sample

$$
\begin{aligned}
& a=6.0902(5) \AA \\
& b=10.4805(8) \\
& c=4.8215(5) \\
& a / b=0.5811 \\
& c / b=0.4600 \\
& Z=4 \\
& V=307.75 \AA^{3} \\
& \text { Density (calc) }=4.398 \mathrm{~g} / \mathrm{cm}^{3}
\end{aligned}
$$

Figure of merit

$$
F_{30}=70.4(0.010,44)
$$

\section{Polymorphism}

Fayalite is converted to a cubic form with spinel structure at high pressure and temperature (Ringwood, 1958).

\section{Additional patterns}

PDF card 20-1139 (Feier, British Museum (Natural History), London)

PDF card 31-649 (Smith et al., Penn State U.,

\begin{tabular}{|c|c|c|c|c|c|}
\hline \multicolumn{6}{|c|}{$\begin{array}{l}\mathrm{CuK} \alpha_{1} \lambda=1.540598 \AA \text {; temp. } 26 \pm 1{ }^{\circ} \mathrm{C} \\
\text { Internal standard } \mathrm{W}, \mathrm{a}=3.16524 \AA\end{array}$} \\
\hline \multirow[t]{2}{*}{$\mathrm{d}(\stackrel{\circ}{\mathrm{A}})$} & \multirow{2}{*}{$\begin{array}{c}\text { rel } \\
\sigma= \pm 3\end{array}$} & \multirow{2}{*}{\multicolumn{3}{|c|}{ hk $\ell$}} & \multirow[t]{2}{*}{$2 \theta\left({ }^{\circ}\right)$} \\
\hline & & & & & \\
\hline 5.242 & 6 & 0 & 2 & 0 & 16.901 \\
\hline 4.380 & 9 & 0 & 1 & 1 & 20.257 \\
\hline 3.974 & 11 & 1 & 2 & 0 & 22.353 \\
\hline 3.783 & 7 & 1 & 0 & 1 & 23.496 \\
\hline 3.556 & 55 & 1 & 1 & 1 & 25.019 \\
\hline 3.064 & 6 & 1 & 2 & 1 & 29.123 \\
\hline 3.046 & 7 & 2 & 0 & 0 & 29.295 \\
\hline 2.829 & 86 & 0 & 3 & 1 & 31.599 \\
\hline 2.633 & 32 & 2 & 2 & 0 & 34.020 \\
\hline 2.619 & 23 & 0 & 4 & 0 & 34.205 \\
\hline 2.565 & 45 & 1 & 3 & 1 & 34.952 \\
\hline 2.500 & 100 & 2 & 1 & 1 & 35.893 \\
\hline 2.407 & 25 & 1 & 4 & 0 & 37.331 \\
\hline 2.351 & 10 & 0 & 1 & 2 & 38.259 \\
\hline 2.311 & 23 & 2 & 2 & 1 & 38.949 \\
\hline 2.303 & 21 & 0 & 4 & 1 & 39.073 \\
\hline 2.193 & 9 & 1 & 1 & 2 & 41.134 \\
\hline 2.1532 & 7 & 1 & 4 & 1 & 41.923 \\
\hline 2.0723 & 8 & 2 & 3 & 1 & 43.643 \\
\hline $1.9855 \mathrm{M}$ & 5 & 2 & 4 & 0 & 45.655 \\
\hline $1.9855 \mathrm{M}$ & & 0 & 3 & 2 & 45.655 \\
\hline 1.9220 & 5 & 0 & 5 & 1 & 47.253 \\
\hline 1.8418 & 12 & 3 & 1 & 1 & 49.446 \\
\hline 1.8327 & 10 & 1 & 5 & 1 & 49.709 \\
\hline 1.7781 & 79 & 2 & 2 & 2 & 51.343 \\
\hline 1.7735 & 65 & 0 & 4 & 2 & 51.486 \\
\hline 1.7624 & 6 & 3 & 2 & 1 & 51.834 \\
\hline 1.7032 & 13 & 1 & 4 & 2 & 53.779 \\
\hline 1.6789 & 15 & 1 & 6 & 0 & 54.621 \\
\hline 1.6496 & 17 & 3 & 3 & 1 & 55.674 \\
\hline 1.6253 & 17 & 2 & 5 & 1 & 56.581 \\
\hline 1.6044 & 9 & 3 & 4 & 0 & 57.386 \\
\hline 1.5885 & 6 & 0 & 1 & 3 & 58.014 \\
\hline $1.5367 \mathrm{M}$ & 11 & 0 & 2 & 3 & 60.166 \\
\hline $1.5367 \mathrm{M}$ & & 1 & 1 & 3 & 60.166 \\
\hline 1.5331 & 10 & 2 & 4 & 2 & 60.326 \\
\hline $1.5225 \mathrm{M}$ & 26 & 4 & 0 & 0 & 60.789 \\
\hline $1.5225 \mathrm{M}$ & & 3 & 4 & 1 & 60.789 \\
\hline 1.5151 & 31 & 2 & 6 & 0 & 61.115 \\
\hline $1.4896 \mathrm{M}$ & 3 & 1 & 2 & 3 & 62.276 \\
\hline
\end{tabular}
University Park, PA) calculated pattern

\section{References}

Bragg, W. L. and Brown, G. B. (1926).

Z. Kristallogr. 63, 538 .

Ringwood, A. E. (1958). Geochim. Cosmochim. Acta, 15, 18 .

Rinne, F., Leonhardt, J., and Hentschel, H. (1924). Z. Kristallogr. 59, 548. 
Iron Silicate (Fayalite), $\mathrm{Fe}_{2} \mathrm{SiO}_{4}-$ (continued)

\begin{tabular}{|c|c|c|c|c|c|}
\hline $\mathrm{d}(\stackrel{\circ}{\mathrm{A}})$ & $I^{r e l}$ & & $\mathrm{hk} \ell$ & & $2 \theta\left(^{\circ}\right)$ \\
\hline $1.4896 \mathrm{M}$ & & 3 & 2 & 2 & 62.276 \\
\hline 1.4602 & 9 & 0 & 3 & 3 & 63.679 \\
\hline 1.4383 & 2 & 4 & 1 & 1 & 64.765 \\
\hline 1.4300 & 15 & 0 & 7 & 1 & 65.186 \\
\hline 1.4201 & 8 & 1 & 3 & 3 & 65.698 \\
\hline 1.4085 & 10 & 2 & 1 & 3 & 66.311 \\
\hline 1.3962 & 3 & 3 & 5 & 1 & 66.971 \\
\hline 1.3778 & 6 & 1 & 6 & 2 & 67.986 \\
\hline 1.3719 & 9 & 2 & 2 & 3 & 68.315 \\
\hline 1.3408 & 13 & 4 & 3 & 1 & 70.130 \\
\hline 1.3365 & 12 & 1 & 4 & 3 & 70.386 \\
\hline 1.3240 & 5 & 3 & 6 & 0 & 71.156 \\
\hline $1.3165 \mathrm{M}$ & 3 & 4 & 4 & 0 & 71.624 \\
\hline $1.3165 \mathrm{M}$ & & 2 & 3 & 3 & 71.624 \\
\hline 1.2868 & 6 & 4 & 0 & 2 & 73.542 \\
\hline 1.2832 & 6 & 2 & 6 & 2 & 73.780 \\
\hline 1.2699 & $1 \mathrm{~L}$ & 4 & 4 & 1 & 74.686 \\
\hline 1.2507 & 2 & 3 & 1 & 3 & 76.032 \\
\hline $1.2478 \mathrm{M}$ & 3 & 3 & 5 & 2 & 76.241 \\
\hline $1.2478 M$ & & 1 & 5 & 3 & 76.241 \\
\hline 1.2053 & 5 & 0 & 0 & 4 & 79.446 \\
\hline 1.2034 & 3 & 2 & 8 & 0 & 79.601 \\
\hline 1.1935 & 1 & 4 & 5 & 1 & 80.395 \\
\hline 1.1855 & 4 & 3 & 3 & 3 & 81.045 \\
\hline 1.1830 & 3 & 0 & 6 & 3 & 81.258 \\
\hline 1.1763 & 8 & 2 & 5 & 3 & 81.818 \\
\hline $1.1737 \mathrm{M}$ & 7 & 2 & 7 & 2 & 82.037 \\
\hline $1.1737 \mathrm{M}$ & & 5 & 1 & 1 & 82.037 \\
\hline 1.1673 & 1 & 2 & 8 & 1 & 82.581 \\
\hline $1.1606 \mathrm{M}$ & 4 & 1 & 6 & 3 & 83.168 \\
\hline $1.1606 \mathrm{M}$ & & 3 & 6 & 2 & 83.168 \\
\hline 1.1556 & 6 & 4 & 4 & 2 & 83.611 \\
\hline 1.1512 & 4 & 0 & 8 & 2 & 84.004 \\
\hline 1.1355 & 5 & 3 & 4 & 3 & 85.430 \\
\hline 1.1311 & 1 & 1 & 8 & 2 & 85.848 \\
\hline 1.1188 & 4 & 5 & 3 & 1 & 87.020 \\
\hline 1.1143 & 3 & 2 & 1 & 4 & 87.467 \\
\hline 1.0992 & 2 & 4 & 1 & 3 & 88.981 \\
\hline 1.0959 & 13 & 2 & 2 & 4 & 89.319 \\
\hline $1.0811 \mathrm{M}$ & 2 & 5 & 1 & 2 & 90.875 \\
\hline $1.0811 \mathrm{M}$ & & 4 & 2 & 3 & 90.875 \\
\hline $1.0767 \mathrm{M}$ & 2 & 2 & 8 & 2 & 91.355 \\
\hline $1.0767 \mathrm{M}$ & & 5 & 4 & 1 & 91.355 \\
\hline
\end{tabular}


Iron Titanium Oxide (UTvöspinel), $\mathrm{Fe}_{2} \mathrm{TiO}_{4}$

Synonym

Iron titanate

CAS registry no. 12434-86-5

Sample

The sample was prepared from stoichiometric amounts of $\mathrm{Fe}_{2} \mathrm{O}_{3}$ and $\mathrm{TiO}_{2}$, blended in an agate mortar. The mixture was dried and transferred to a high-purity iron boat for heat treatments. All heatings were done in a controlled atmosphere at an oxygen partial pressure of $10^{-18}$ atmosphere or lower. Three heatings, $1140{ }^{\circ} \mathrm{C}$ for 8 hours, $1150{ }^{\circ} \mathrm{C}$ for 8 hours, and $1200{ }^{\circ} \mathrm{C}$ for 8 hours were sufficient to yield a single phase, well crystallized product.

Color

Dark gray

Structure

Cubic, Fd3m (227), isostructural with spinel, $\mathrm{MgAl}_{2} \mathrm{O}_{4}$ (Barth and Posnjak, 1932). The structure was refined by Forster and Hall (1965) by neutron diffraction methods.

Crystallographic constants of this sample

$a=8.53520(12) \AA$

$Z=8$

$V=621.786 \AA^{3}$

Density $(\mathrm{calc})=4.777 \mathrm{~g} / \mathrm{cm}^{3}$

Figure of merit

$\mathrm{F}_{30}=124.2(0.0069,35)$

Additional patterns

PDF card 24-537 (Ashworth, Dept. of Geology, Univ. of Durham, U.K.)

Forster and Hall (1965)

Pouillard (1950)

\section{References}

Barth, T. F. W. and Posnjak, E. (1932). Z.

Kristallogr. 82, 325.

Forster, R. H. and Hall, E. O. (1965). Acta Crystallogr. 18, 857.

Pouillard, E. (1950). Ann. Chim. Paris 5, 164.

\begin{tabular}{|c|c|c|c|c|c|}
\hline \multicolumn{6}{|c|}{$\begin{array}{c}\mathrm{CuK \alpha}_{1} \lambda=1.540598 \AA \text {; temp. } 26 \pm 1{ }^{\circ} \mathrm{C} \\
\text { Internal standards } \mathrm{W}, \mathrm{a}=3.16524 \AA \\
\text { Fluorophlogopite, SRM } 675\end{array}$} \\
\hline $\mathrm{d}(\AA)$ & $\begin{array}{c}I^{\text {rel }} \\
\sigma= \pm 2\end{array}$ & & hke & & $2 \theta\left(^{\circ}\right)$ \\
\hline 4.924 & 8 & 1 & 1 & 1 & 18.002 \\
\hline 3.018 & 33 & 2 & 2 & 0 & 29.573 \\
\hline 2.573 & 100 & 3 & 1 & 1 & 34.837 \\
\hline 2.4640 & 6 & 2 & 2 & 2 & 36.434 \\
\hline 2.1339 & 19 & 4 & 0 & 0 & 42.321 \\
\hline 1.7419 & 10 & 4 & 2 & 2 & 52.490 \\
\hline 1.6426 & 33 & 5 & 1 & 1 & 55.932 \\
\hline 1.5089 & 39 & 4 & 4 & 0 & 61.395 \\
\hline 1.4427 & 1 & 5 & 3 & 1 & 64.545 \\
\hline 1.3495 & 3 & 6 & 2 & 0 & 69.615 \\
\hline 1.3016 & 7 & 5 & 3 & 3 & 72.572 \\
\hline I. 2867 & 3 & 6 & 2 & 2 & 73.551 \\
\hline 1. 2318 & 2 & 4 & 4 & 4 & 77.413 \\
\hline 1.1952 & 1L & 7 & 1 & 1 & 80.255 \\
\hline 1. 1406 & 3 & 6 & 4 & 2 & 84.960 \\
\hline 1.1111 & 8 & 7 & 3 & 1 & 87.782 \\
\hline 1.0669 & 2 & 8 & 0 & 0 & 92.435 \\
\hline 1.0059 & 1 & 6 & 6 & 0 & 99.948 \\
\hline .9856 & 5 & 7 & 5 & 1 & 102.805 \\
\hline .9792 & 2 & 6 & 6 & 2 & 103.754 \\
\hline .9543 & 2 & 8 & 4 & 0 & 107.649 \\
\hline .9369 & $1 \mathrm{~L}$ & 7 & 5 & 3 & 110.606 \\
\hline .9099 & $1 \mathrm{~L}$ & 6 & 6 & 4 & 115.689 \\
\hline .8947 & 4 & 9 & 3 & 1 & 118.843 \\
\hline .8711 & 10 & 8 & 4 & 4 & 124.332 \\
\hline .8578 & $1 \mathrm{~L}$ & 7 & 7 & 1 & 127.778 \\
\hline .8370 & 2 & 10 & 2 & 0 & 133.953 \\
\hline .8251 & 4 & 7 & 7 & 3 & 138.003 \\
\hline .8212 & 2 & 10 & 2 & 2 & 139.428 \\
\hline .7959 & $1 \mathrm{~L}$ & 9 & 5 & 3 & 150.838 \\
\hline
\end{tabular}


Lanthanum Boride, $\mathrm{LaB}_{6}$

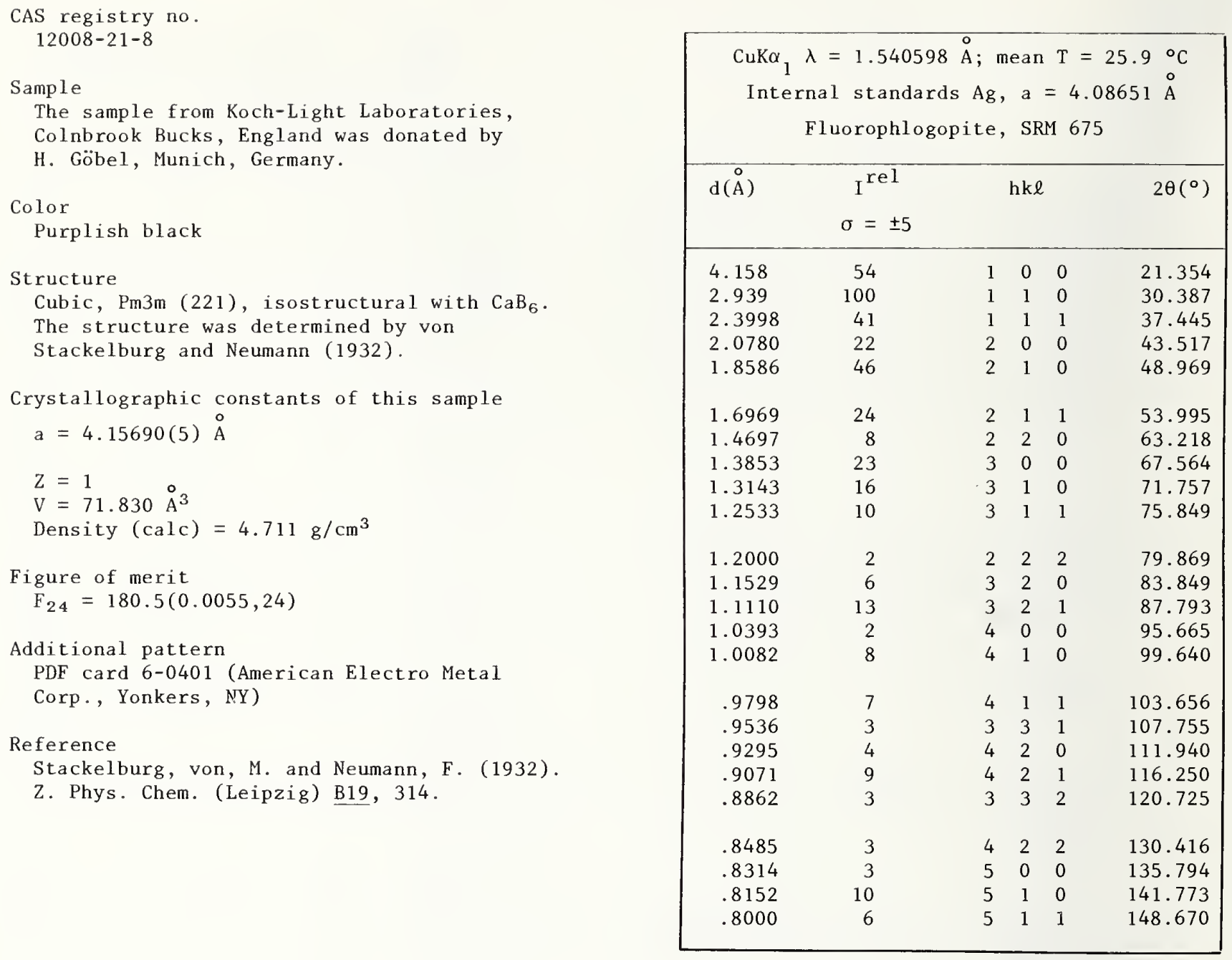


Lithium Thoriumi Molybdenum Oxide, $\mathrm{Li}_{4} \mathrm{Th}_{7}\left(\mathrm{MoO}_{4}\right)_{16}$

Synonym

Lithium thorium molybdate

Sample

One mole of $\mathrm{Li}_{2} \mathrm{MoO}_{4}, 3.5$ moles of $\mathrm{ThO}_{2}$ and 7 moles of $\mathrm{MoO}_{3}$ were heated at $700{ }^{\circ} \mathrm{C}$ for 21 hours.

Color

Colorless

Structure

Cubic, $1 * 3 *$ Martel and Roth (1981).

Apparently isostructural with cubic phase in the system lithium thorium tungsten oxide, Finch and Clark (1965).

Crystallographic constants of this sample $a=14.4504(3) \AA$

$Z=2$

$V=3017.5 \AA^{3}$

Density $(\mathrm{calc})=4.635 \mathrm{~g} / \mathrm{cm}^{3}$

Figure of merit

$F_{30}=108.8(0.0073,38)$

References

Finch, C. B. and Clark, G. W. (1965). J. Appl. Phys. 36, 2143.

Martel, L. C. and Roth, R. C. (1981). Am. Ceram. Soc. Bull. 60, 376.

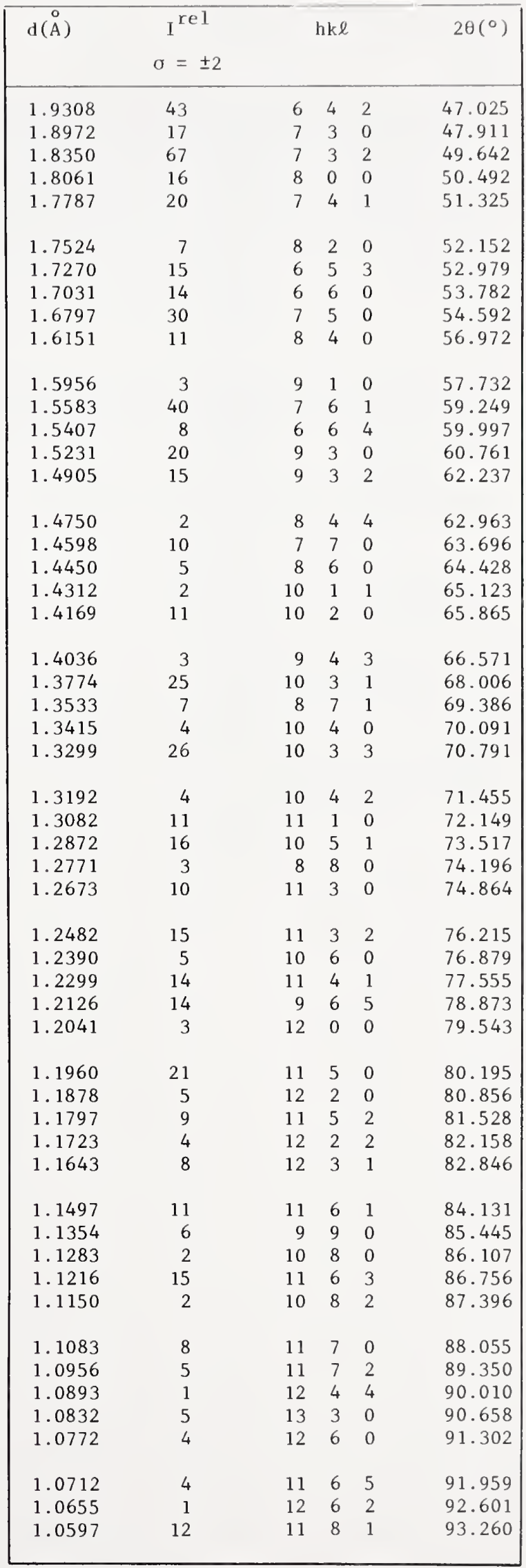


Synonym

Lithium thorium tungstate

Sample

$\mathrm{Li}_{2} \mathrm{WO}_{4}$ was prepared by reacting $\mathrm{Li}_{2} \mathrm{CO}_{3}$ and $\mathrm{WO}_{3}$ in equimolar proportions at $700{ }^{\circ} \mathrm{C}$ for 24 hours to form $\mathrm{Li}_{2} \mathrm{WO}_{4}$. Then $\mathrm{Li}_{2} \mathrm{WO}_{4}$, $\mathrm{ThO}_{2}$, and $\mathrm{WO}_{3}$ in the molar ratios of $1: 3.5: 7$ were ground together under acetone, heated in gold to $700{ }^{\circ} \mathrm{C}$ for 48 hours with regrinding every 24 hours, then heated a further 16 hours at $700{ }^{\circ} \mathrm{C}$, reground and heaced at $750{ }^{\circ} \mathrm{C}$ for 21 hours.

Color

Colorless

Structure

Cubic, I*3*. Finch and Clark (1965) referred to a body-centered cubic phase of this approximate composition.

Crystallographic constants of this sample $\mathrm{a}=14.4969(4) \AA$

$Z=2$

$\mathrm{V}=3046.7 \AA^{3}$

Density $(\mathrm{calc})=6.124 \mathrm{~g} / \mathrm{cm}^{3}$

Figure of merit

$\mathrm{F}_{30}=159.6(0.0049,38)$

\section{Reference}

Finch, C. B. and Clark, G. W. (1965). J. Appl. Phys. 36, 2143.

\begin{tabular}{|c|c|c|c|c|c|}
\hline \multicolumn{6}{|c|}{$\begin{array}{c}\mathrm{CuK \alpha}{ }_{1} \lambda=1.540598 \AA \text {; mean } \mathrm{T}=26.0{ }^{\circ} \mathrm{C} \\
\text { Internal standards } \mathrm{W}, \mathrm{a}=3.16524 \AA \\
\text { Fluorophlogopite, SRM } 675\end{array}$} \\
\hline \multirow[t]{2}{*}{$d(\stackrel{0}{A})$} & \multirow{2}{*}{$\begin{array}{c}I^{\text {rel }} \\
\sigma= \pm 4\end{array}$} & \multirow{2}{*}{\multicolumn{3}{|c|}{ hke }} & \multirow[t]{2}{*}{$2 \theta\left({ }^{\circ}\right)$} \\
\hline & & & & & \\
\hline 5.920 & 8 & 2 & 1 & 1 & 14.952 \\
\hline 5.127 & 10 & 2 & 2 & 0 & 17.281 \\
\hline 4.586 & 7 & 3 & 1 & 0 & 19.340 \\
\hline 3.875 & 68 & 3 & 2 & 1 & 22.931 \\
\hline 3.625 & 80 & 4 & 0 & 0 & 24.540 \\
\hline 3.242 & 50 & 4 & 2 & 0 & 27.489 \\
\hline 3.091 & 100 & 3 & 3 & 2 & 28.860 \\
\hline 2.9593 & 44 & 4 & 2 & 2 & 30.175 \\
\hline 2.8430 & 15 & 5 & 1 & 0 & 31.441 \\
\hline 2.6465 & 19 & 5 & 2 & 1 & 33.843 \\
\hline 2.5625 & 24 & 4 & 4 & 0 & 34.988 \\
\hline 2.3518 & 14 & 6 & 1 & 1 & 38.239 \\
\hline 2.2922 & 4 & 6 & 2 & 0 & 39.273 \\
\hline 2.2376 & 10 & 5 & 4 & 1 & 40.272 \\
\hline 2.1378 & 30 & 6 & 3 & 1 & 42.240 \\
\hline 2.0931 & 1 & 4 & 4 & 4 & 43.187 \\
\hline 2.0500 & 13 & 5 & 5 & 0 & 44.142 \\
\hline 2.0103 & 12 & 6 & 4 & 0 & 45.062 \\
\hline 1.9725 & 53 & 7 & 2 & 1 & 45.973 \\
\hline 1.9369 & 28 & 6 & 4 & 2 & 46.868 \\
\hline 1.9031 & 18 & 7 & 3 & 0 & 47.751 \\
\hline 1.8412 & 61 & 6 & 5 & 1 & 49.463 \\
\hline 1.8122 & 16 & 8 & 0 & 0 & 50.310 \\
\hline 1.7844 & 13 & 7 & 4 & 1 & 51.148 \\
\hline 1.7582 & 12 & 8 & 2 & 0 & 51.969 \\
\hline 1.7327 & 20 & 6 & 5 & 3 & 52.790 \\
\hline 1.7088 & 9 & 8 & 2 & 2 & 53.588 \\
\hline 1.6853 & 35 & 7 & 5 & 0 & 54.395 \\
\hline 1.6417 & $1 \mathrm{~L}$ & 7 & 5 & 2 & 55.967 \\
\hline 1.6210 & 9 & 8 & 4 & 0 & 56.746 \\
\hline 1.5634 & 40 & 7 & 6 & 1 & 59.038 \\
\hline 1.5455 & 5 & 6 & 6 & 4 & 59.792 \\
\hline 1.5280 & 11 & 9 & 3 & 0 & 60.548 \\
\hline 1.4954 & 13 & 9 & 3 & 2 & 62.011 \\
\hline 1.4794 & 1 & 8 & 4 & 4 & 62.756 \\
\hline 1.4645 & 6 & 7 & 7 & 0 & 63.471 \\
\hline 1.4499 & 7 & 8 & 6 & 0 & 64.184 \\
\hline 1.4354 & 1 & 10 & 1 & 1 & 64.910 \\
\hline 1.4215 & 9 & 10 & 2 & 0 & 65.624 \\
\hline 1.4082 & 4 & 9 & 5 & 0 & 66.324 \\
\hline 1.3823 & 29 & 10 & 3 & 1 & 67.733 \\
\hline 1.3579 & 5 & 8 & 7 & 1 & 69.123 \\
\hline 1.3460 & 5 & 10 & 4 & 0 & 69.819 \\
\hline 1.3347 & 28 & 9 & 6 & 1 & 70.498 \\
\hline 1.3235 & 3 & 10 & 4 & 2 & 71.183 \\
\hline
\end{tabular}


Lithium Thorium Tungsten Oxide, $\mathrm{Li}_{4} \mathrm{Th}_{7}\left(\mathrm{WO}_{4}\right)_{16}$ - (continued)

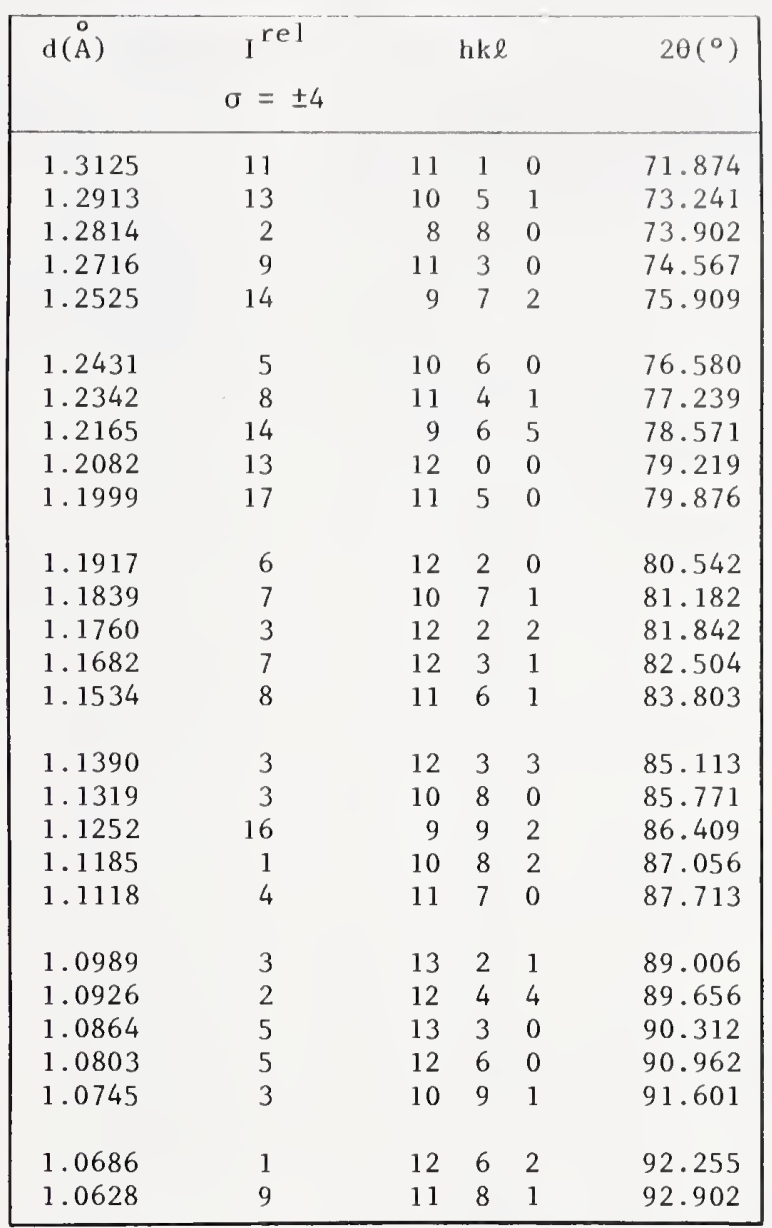


Synonym

Lithium titanate

Sample

The sample was obtained from J. C. Mikkelson of Xerox Palo Alto Research Center, Palo Alto, CA.

\section{Color \\ Colorless}

Structure

Orthorhombic, Pbnm (62). Its ramsdellitelike structure was qualitatively determined by Lundberg and Andersson (1964).

Crystallographic constants of this sample

$\mathrm{a}=5.0182(4) \AA$

$\mathrm{b}=9.5523(8)$

c $=2.9455(3)$

$\mathrm{a} / \mathrm{b}=0.5253$

$\mathrm{c} / \mathrm{b}=0.3084$

$Z=1$

$V=141.19 \AA^{3}$

Density $(\mathrm{calc})=3.170 \mathrm{~g} / \mathrm{cm}^{3}$

Figure of merit

$\mathrm{F}_{30}=62.0(0.012,42)$

Reference

Lundberg, M. and Andersson, S. (1964). Acta Chem. Scand. 18, 817.

\begin{tabular}{|c|c|c|c|c|c|}
\hline \multicolumn{6}{|c|}{$\begin{array}{l}\mathrm{CuK} \alpha_{1} \lambda=1.540598 \mathrm{~A} ; \text { temp. } 26 \pm 1{ }^{\circ} \mathrm{C} \\
\text { Internal standard } \mathrm{W}, \mathrm{a}=3.16524 \AA\end{array}$} \\
\hline $\mathrm{d}(\stackrel{\circ}{\mathrm{A}})$ & $\begin{array}{l}\mathrm{rel} \\
\sigma= \pm 4\end{array}$ & & hke & & $2 \theta\left(^{\circ}\right)$ \\
\hline 4.777 & 3 & 0 & 2 & 0 & 18.561 \\
\hline 4.440 & 100 & 1 & 1 & 0 & 19.982 \\
\hline 3.461 & 9 & 1 & 2 & 0 & 25.721 \\
\hline 2.688 & 46 & 1 & 3 & 0 & 33.303 \\
\hline 2.541 & 3 & 1 & 0 & 1 & 35.291 \\
\hline $2.507 \mathrm{M}$ & 24 & 2 & 0 & 0 & 35.790 \\
\hline $2.507 \mathrm{M}$ & & 0 & 2 & 1 & 35.790 \\
\hline 2.457 & 23 & 1 & 1 & 1 & 36.543 \\
\hline 2.388 & 2 & 0 & 4 & 0 & 37.637 \\
\hline 2.243 & 32 & 1 & 2 & 1 & 40.179 \\
\hline 2.222 & 3 & 2 & 2 & 0 & 40.569 \\
\hline 2.156 & 6 & 1 & 4 & 0 & 41.861 \\
\hline 1.9857 & 13 & 1 & 3 & 1 & 45.651 \\
\hline 1.9707 & 12 & 2 & 3 & 0 & 46.017 \\
\hline 1.8554 & 3 & 0 & 4 & 1 & 49.060 \\
\hline 1.7733 & 32 & 2 & 2 & 1 & 51.494 \\
\hline 1.7301 & 21 & 2 & 4 & 0 & 52.878 \\
\hline 1.6382 & 9 & 2 & 3 & 1 & 56.095 \\
\hline 1.5923 & $1 \mathrm{~L}$ & 0 & 6 & 0 & 57.863 \\
\hline 1.5792 & 3 & 3 & 2 & 0 & 58.389 \\
\hline
\end{tabular}

\begin{tabular}{|c|c|c|c|c|c|}
\hline$d(\stackrel{\circ}{)})$ & $\begin{array}{c}I^{\text {rel }} \\
\sigma= \pm 4\end{array}$ & & hke & & $2 \theta\left(^{\circ}\right)$ \\
\hline 1.5265 & 17 & 1 & 5 & 1 & 60.612 \\
\hline 1.4917 & 2 & 2 & 4 & 1 & 62.179 \\
\hline 1.4814 & 7 & 3 & 3 & 0 & 62.663 \\
\hline 1.4729 & 7 & 0 & 0 & 2 & 63.067 \\
\hline 1.4540 & 5 & 3 & 0 & 1 & 63.981 \\
\hline 1.4380 & 14 & 3 & 1 & 1 & 64.782 \\
\hline 1.4001 & 10 & 0 & 6 & 1 & 66.760 \\
\hline 1.3976 & 9 & 1 & 1 & 2 & 66.895 \\
\hline 1.3913 & 2 & 3 & 2 & 1 & 67.236 \\
\hline 1.3697 & 2 & 3 & 4 & 0 & 68.444 \\
\hline 1.3444 & 2 & 2 & 6 & 0 & 69.916 \\
\hline 1.3233 & 1 & 3 & 3 & 1 & 71.198 \\
\hline 1.3168 & 4 & 1. & 7 & 0 & 71.605 \\
\hline 1.2914 & 6 & 1 & 3 & 2 & 73.235 \\
\hline 1.2703 & 1 & 2 & 0 & 2 & 74.658 \\
\hline $1.2589 \mathrm{M}$ & 3 & 2 & 1 & 2 & 75.451 \\
\hline $1.2589 \mathrm{M}$ & & 3 & 5 & 0 & 75.451 \\
\hline 1.2440 & 3 & 4 & 1 & 0 & 76.519 \\
\hline 1.2418 & 3 & 3 & 4 & 1 & 76.678 \\
\hline 1.2230 & 1 & 2 & 6 & 1 & 78.078 \\
\hline 1.2162 & 1L & 1 & 4 & 2 & 78.601 \\
\hline 1.1940 & 1 & 0 & 8 & 0 & 80.354 \\
\hline 1.1798 & 2 & 2 & 3 & 2 & 81.523 \\
\hline 1.1617 & 1 & 1 & 8 & 0 & 83.074 \\
\hline 1.1573 & 1 & 3 & 5 & 1 & 83.457 \\
\hline 1.1530 & 2 & 3 & 6 & 0 & 83.837 \\
\hline 1.1458 & 2 & 4 & 1 & 1 & 84.485 \\
\hline $1.1216 \mathrm{M}$ & 6 & 4 & 2 & 1 & 86.750 \\
\hline $1.1216 \mathrm{M}$ & & 2 & 4 & 2 & 86.750 \\
\hline $1.1103 \mathrm{M}$ & 1 & 4 & 4 & 0 & 87.861 \\
\hline $1.1103 \mathrm{M}$ & & 2 & 7 & 1 & 87.861 \\
\hline 1.1069 & 2 & 0 & 8 & 1 & 88.200 \\
\hline 1.0979 & $1 \mathrm{~L}$ & 3 & 1 & 2 & 89.108 \\
\hline 1.0849 & 1 & 4 & 3 & 1 & 90.469 \\
\hline 1.0781 & 2 & 2 & 8 & 0 & 91.201 \\
\hline 1.0769 & 2 & 3 & 2 & 2 & 91.332 \\
\hline 1.0740 & 2 & 3 & 6 & 1 & 91.648 \\
\hline $1.0575 \mathrm{M}$ & 3 & 2 & 5 & 2 & 93.511 \\
\hline $1.0575 \mathrm{M}$ & & 3 & 7 & 0 & 93.511 \\
\hline 1.0488 & 1 & 4 & 5 & 0 & 94.522 \\
\hline 1.0444 & 2 & 3 & 3 & 2 & 95.051 \\
\hline 1.0392 & $1 \mathrm{~L}$ & 4 & 4 & 1 & 95.673 \\
\hline 1.0124 & 2 & 2 & 8 & 1 & 99.082 \\
\hline .9982 & 1 & 5 & 1 & 0 & 101.013 \\
\hline
\end{tabular}


Magnesium Manganese Zinc Iron Sulfate Hydroxide Hydrate, Zincobotryogen, $(\mathrm{Zn}, \mathrm{Mg}, \mathrm{Mn}) \mathrm{Fe}\left(\mathrm{SO}_{4}\right)_{2}(\mathrm{OH}) \cdot 7 \mathrm{H}_{2} \mathrm{O}$

Sample

The specimen from the northern border of the Tsadam Basin, China was obtained by E. C. T. Chao of the U.S. Geological Survey.

Chemical analysis

$\mathrm{SO}_{3}-36.03, \mathrm{Fe}_{2} \mathrm{O}_{3}-18.34, \mathrm{MgO}-2.50, \mathrm{FeO}-0.85$, $\mathrm{MnO}-1.75, \mathrm{ZnO}-11.77, \mathrm{H}_{2} \mathrm{O}+29.13$, minor $\mathrm{H}_{2} \mathrm{O}-\mathrm{Na}_{2} \mathrm{O}$; this corresponds to the formula $\left(\mathrm{Zn}_{0} .59 \mathrm{Mg}_{0.26} \mathrm{Mn}_{0} .10 \mathrm{Fe}_{0.05}\right) \mathrm{Fe}^{+}\left(\mathrm{SO}_{4}\right)_{2}(\mathrm{OH}) \cdot$ $7 \mathrm{H}_{2} \mathrm{O}$ (Kwanchih et al., 1964).

Color

Deep orange

Optical data

Biaxial (+). $\quad \mathrm{N}_{\alpha}=1.542, \mathrm{~N}_{\beta}=1.551$,

$\mathrm{N}_{\gamma}=1.587 .2 \mathrm{~V}=54^{\circ}$ (calc.) Ibid.

Structure

Monoclinic, $\mathrm{P} 2{ }_{1} / \mathrm{n}$ (14). The structure was determined by Süsse (1968).

Crystallographic constants of this sample

$a=10.523(2) \AA$

$\mathrm{b}=17.841(3)$

$c=7.137(2)$

$\beta=100.11(2)^{\circ}$

$a / b=0.5898$

$c / b=0.4000$

$Z=4$

$Z=4$
$V=1310.10 \AA^{3}$

Density $(\mathrm{calc})=2.237 \mathrm{~g} / \mathrm{cm}^{3}$

Figure of merit

$\mathrm{F}_{30}=44.6(0.011,61)$

Additional pattern

Kwanchih et al. (1964)

\section{References}

Kwanchih, T., et al. (1964). Ti Chih K'o

Hsueh (Geol. Sci.), No. 11, 313.

Süsse, von P. (1968). Acta Crystallogr., Sect. B, 24, 760 .
$\mathrm{CuKa}_{1} \lambda=1.540598 \AA$ А mean $\mathrm{T}=25.7^{\circ} \mathrm{C}$

Internal standards Si, SRM 640a

Fluorophlogopite, SRM 675

\begin{tabular}{|c|c|c|c|c|c|}
\hline$d(\AA)$ & ${ }_{\mathrm{I}}^{\mathrm{rel}}$ & & hke & & $2 \theta\left(^{\circ}\right)$ \\
\hline 8.96 & 100 & 1 & 1 & 0 & 9.860 \\
\hline 6.76 & 8 & 1 & 2 & 0 & 13.090 \\
\hline 6.354 & 58 & -1 & 0 & 1 & 13.926 \\
\hline 5.990 & 8 & -1 & 1 & 1 & 14.778 \\
\hline 5.525 & 36 & 0 & 2 & 1 & 16.030 \\
\hline 5.395 & 28 & 1 & 0 & 1 & 16.419 \\
\hline $5.163 \mathrm{M}$ & 75 & 1 & 1 & 1 & 17.162 \\
\hline $5.163 \mathrm{M}$ & & 1 & 3 & 0 & 17.162 \\
\hline 4.613 & 17 & 1 & 2 & 1 & 19.226 \\
\hline 4.538 & 29 & 0 & 3 & 1 & 19.54 \\
\hline
\end{tabular}

$4.480 \quad 23$

$4.456 \quad 26$

$4.425 \quad 29$

4.340

40

4.095

53

3.905

27

3.766

3.655

3.541

3.451

41

9

41

25

3.389

3. 343

3.267

3.219

3. $181 \mathrm{M}$

32

19

26

51

3. $181 \mathrm{M}$

3. $112 \mathrm{M}$

3. $112 \mathrm{M}$

$3.027 \mathrm{M}$

$3.027 \mathrm{M}$

$\begin{array}{lll}2 & 2 & 0 \\ 0 & 4 & 0\end{array}$

19.800

19.910

20.049

$\begin{array}{lll}-2 & 1 & 1\end{array}$

$\begin{array}{lll}-1 & 3 & 1\end{array}$

20.447

21.684

2.987

2.905

2.867

2.858

2.813

17

$\begin{array}{lll}2 & 3 & 0\end{array}$

22.753

$\begin{array}{lll}0 & 4 & 1\end{array}$

23.603

24.333

25.128

$\begin{array}{ll}-1 & 4 \\ 2 & 2\end{array}$

$\begin{array}{lll}-1 & 1 & 2\end{array}$

25.792

$\begin{array}{lll}3 & 1 & 0\end{array}$

26.278

26.640

27.277

$\begin{array}{lll}0 & 2 & 2\end{array}$

27.680

28.030

$\begin{array}{lll}3 & 2 & 0 \\ 0 & 5 & 1\end{array}$

$\begin{array}{rrrr}-2 & 0 & 2 & 28.030 \\ 1 & 1 & 2 & 28.664\end{array}$

14

$\begin{array}{lll}-1 & 5 & 1\end{array}$

61

$\begin{array}{lll}-1 & 3 & 2\end{array}$

28.664

29.485

29.485

2.803

$2.762 \mathrm{M}$

$2.762 \mathrm{M}$

2.732

2. $695 \mathrm{M}$

\section{6}

032

$2.695 \mathrm{M}$

2.673

2.609

2.594

$2.580 \mathrm{M}$

$2.580 \mathrm{M}$

2.563

2.555

2.487

$2.480 \mathrm{M}$
29.894

30.750

31.172

31.269

31.787

330

$\begin{array}{ll}3 & 0 \\ 3 & 1\end{array}$

$\begin{array}{lll}1 & 6 & 0\end{array}$

$\begin{array}{lll}-2 & 5 & 1\end{array}$

31.907

32.384

32.384

32.752

33.210

$\begin{array}{llll}-1 & 6 & 1 & 33.210\end{array}$

$\begin{array}{lll}-3 & 4 & 1\end{array}$

33.502

34.349

34.554

34.747

$\begin{array}{lll}2 & 2 & 2\end{array}$

34.747

142

34.987

35.090

36.080

36.190 
Magnesium Manganese Zinc Iron Sulfate Hydroxide Hydrate, Zincobotryogen, $(\mathrm{Zn}, \mathrm{Mg}, \mathrm{Mn}) \mathrm{Fe}\left(\mathrm{SO}_{4}\right)_{2}(\mathrm{OH}) \cdot 7 \mathrm{H}_{2} \mathrm{O}$ - (continued)

\begin{tabular}{|c|c|c|c|c|c|}
\hline $\mathrm{d}(\AA)$ & $\begin{array}{c}I^{\text {rel }} \\
\sigma= \pm 4\end{array}$ & & hke & & $2 \theta\left(^{\circ}\right)$ \\
\hline $2.480 \mathrm{M}$ & & -4 & 2 & 1 & 36.190 \\
\hline 2.439 & 10 & -3 & 5 & 1 & 36.815 \\
\hline 2.432 & 8 & 3 & 4 & 1 & 36.926 \\
\hline 2.396 & 10 & 0 & 7 & 1 & 37.512 \\
\hline $2.368 \mathrm{M}$ & 23 & -4 & 3 & 1 & 37.963 \\
\hline $2.368 \mathrm{M}$ & & 1 & 5 & 2 & 37.963 \\
\hline 2.294 & 5 & -1 & 2 & 3 & 39.237 \\
\hline $2.284 \mathrm{M}$ & 10 & -4 & 0 & 2 & 39.414 \\
\hline $2.284 \mathrm{M}$ & & 4 & 1 & 1 & 39.414 \\
\hline $2.271+$ & 7 & -2 & 1 & 3 & 39.659 \\
\hline $2.271+$ & & -1 & 6 & 2 & 39.659 \\
\hline 2.255 & 9 & 3 & 6 & 0 & 39.947 \\
\hline $2.229 M$ & 12 & 0 & 8 & 0 & 40.440 \\
\hline $2.229 \mathrm{M}$ & & 4 & 2 & 1 & 40.440 \\
\hline $2.180 \mathrm{M}$ & 7 & 1 & 8 & 0 & 41.392 \\
\hline $2.180 \mathrm{M}$ & & 0 & 3 & 3 & 41.392 \\
\hline 2.172 & 20 & -2 & 6 & 2 & 41.537 \\
\hline 2.134 & 9 & -4 & 3 & 2 & 42.322 \\
\hline $2.097 \mathrm{M}$ & 14 & -1 & 4 & 3 & 43.106 \\
\hline $2.097 \mathrm{M}$ & & 4 & 5 & 0 & 43.106 \\
\hline 2.092 & 14 & -4 & 5 & 1 & 43.217 \\
\hline 2.0776 & 18 & 3 & 6 & 1 & 43.526 \\
\hline $2.0611 \mathrm{M}$ & 22 & -3 & 2 & 3 & 43.891 \\
\hline $2.0611 \mathrm{M}$ & & 1 & 8 & 1 & 43.891 \\
\hline 2.0241 & 8 & 3 & 4 & 2 & 44.738 \\
\hline $2.0032 \mathrm{M}$ & 7 & -2 & 8 & 1 & 45.229 \\
\hline $2.0032 \mathrm{M}$ & & -3 & 6 & 2 & 45.229 \\
\hline 1.9857 & 11 & 1 & 7 & 2 & 45.651 \\
\hline $1.9580 \mathrm{M}$ & 6 & 0 & 5 & 3 & 46.333 \\
\hline $1.9580 \mathrm{M}$ & & 2 & 2 & 3 & 46.333 \\
\hline 1.9521 & 7 & 4 & 6 & 0 & 46.481 \\
\hline 1.9473 & 7 & 1 & 9 & 0 & 46.604 \\
\hline $1.9275 \mathrm{M}$ & 10 & -5 & 1 & 2 & 47.110 \\
\hline $1.9275 \mathrm{M}$ & & -2 & 5 & 3 & 47.110 \\
\hline 1.9241 & 11 & -4 & 5 & 2 & 47.200 \\
\hline 1.9179 & 11 & 4 & 1 & 2 & 47.360 \\
\hline $1.8999 M$ & 9 & 2 & 3 & 3 & 47.837 \\
\hline $1.8999 \mathrm{M}$ & & 5 & 0 & 1 & 47.837 \\
\hline 1.8841 & 10 & -1 & 8 & 2 & 48.264 \\
\hline 1.8598 & 11 & 1 & 9 & 1 & 48.935 \\
\hline $1.8557 \mathrm{M}$ & 12 & -1 & 6 & 3 & 49.050 \\
\hline $1.8557 \mathrm{M}$ & & -3 & 8 & 1 & 49.050 \\
\hline 1.8296 & 7 & 2 & 4 & 3 & 49.799 \\
\hline 1.8242 & 9 & -2 & 8 & 2 & 49.955 \\
\hline $1.8195+$ & 13 & -4 & 3 & 3 & 50.093 \\
\hline $1.8195+$ & & -2 & 9 & 1 & 50.093 \\
\hline $1.8144 \mathrm{M}$ & 16 & -2 & 6 & 3 & 50.243 \\
\hline $1.8144 \mathrm{M}$ & & -4 & 7 & 1 & 50.243 \\
\hline
\end{tabular}


Synonym

Magnesium metasilicate

CAS registry no.

$13776-74-4$

\section{Sample}

The sample was prepared by solid state reaction of basic magnesium carbonate and $\mathrm{SiO}_{2}$. After an overnight calcine at $850{ }^{\circ} \mathrm{C}$, the sample was heated at temperatures in the $1450{ }^{\circ} \mathrm{C}$ to $1500{ }^{\circ} \mathrm{C}$ range for about 4 days with daily regrindings.

Color

Colorless

Structure Monoclinic, $\mathrm{P} 2{ }_{1} / \mathrm{c}(14)$. The structure was determined by Morimoto et al. (1960).

Crystallographic constants of this sample

$$
\begin{aligned}
& \mathrm{a}=9.6061(10) \AA \\
& \mathrm{b}=8.8185(8) \\
& \mathrm{c}=5.1710(5) \\
& \beta=108.289(10)^{\circ}
\end{aligned}
$$

$\mathrm{a} / \mathrm{b}=1.0893$

$c / b=0.5864$

$\mathrm{Z}=8$

$\mathrm{Z}=8$
$\mathrm{~V}=415.92 \AA^{3}$

Density $(\mathrm{calc})=3.206 \mathrm{~g} / \mathrm{cm}^{3}$

Figure of merit

$\mathrm{F}_{30}=56.7(0.010,52)$

Polymorphism

There has been and still is considerable controversy. The present view is that there is a stable high temperature form, orthorhombic protoenstatite and a stable low temperature form, orthorhombic enstatite. Protoenstatite on cooling reversibly transforms into metastable monoclinic clinoenstatite.

Additional patterns

PDF card 19-769 Stephenson et al. (1966) Smyth (1974)

References

Morimoto, N., Appleman, D. E., and Evans, H. T. (1960). Z. Kristallogr. 114, 120.

\begin{tabular}{|c|c|c|c|c|c|}
\hline \multicolumn{6}{|c|}{$\begin{array}{l}\mathrm{CuK} \alpha_{1} \lambda=1.540598 \AA ; \text { mean } \mathrm{T}=26 \pm 2{ }^{\circ} \mathrm{C} \\
\text { Internal standard Ag, } \mathrm{a}=4.08651 \AA\end{array}$} \\
\hline \multirow[t]{2}{*}{$d(\AA)$} & \multirow{2}{*}{$\begin{array}{l}r \text { rel } \\
\sigma= \pm 4\end{array}$} & \multirow{2}{*}{\multicolumn{2}{|c|}{ hke }} & & \multirow[t]{2}{*}{$2 \theta\left(^{\circ}\right)$} \\
\hline & & & & & \\
\hline 6.341 & I & I & I & 0 & 13.955 \\
\hline 4.410 & 9 & 0 & 2 & 0 & 20.119 \\
\hline 4.372 & 3 & -1 & 1 & 1 & 20.296 \\
\hline 4.293 & 8 & 0 & 1 & I & 20.673 \\
\hline 4.048 & $1 \mathrm{~L}$ & 2 & 1 & 0 & 21.937 \\
\hline 3.530 & 4 & 1 & 1 & 1 & 25.206 \\
\hline 3.316 & 8 & -1 & 2 & 1 & 26.863 \\
\hline 3.280 & 30 & 0 & 2 & 1 & 27.162 \\
\hline 3.170 & 47 & 2 & 2 & 0 & 28.125 \\
\hline 3.041 & 4 & 3 & 0 & 0 & 29.350 \\
\hline 2.976 & 69 & -2 & 2 & 1 & 30.000 \\
\hline 2.874 & 100 & 3 & 1 & 0 & 31.094 \\
\hline 2.798 & 3 & 1 & 3 & 0 & 31.956 \\
\hline 2.769 & 2 & 2 & 1 & 1 & 32.308 \\
\hline 2.582 & 2 & -1 & 0 & 2 & 34.716 \\
\hline 2.539 & 23 & -1 & 3 & 1 & 35.323 \\
\hline 2.523 & 19 & 0 & 3 & 1 & 35.555 \\
\hline 2.516 & 17 & -2 & 0 & 2 & 35.659 \\
\hline 2.472 & 13 & 2 & 3 & 0 & 36.319 \\
\hline 2.455 & 35 & 0 & 0 & 2 & 36.570 \\
\hline 2.433 & 14 & 2 & 2 & 1 & 36.916 \\
\hline 2.375 & 11 & -2 & 3 & 1 & 37.847 \\
\hline 2.280 & 4 & 4 & 0 & 0 & 39.483 \\
\hline 2.209 & 9 & 4 & 1 & 0 & 40.815 \\
\hline $2.204 \mathrm{M}$ & 9 & 0 & 4 & 0 & 40.921 \\
\hline $2.204 M$ & & 1 & 0 & 2 & 40.921 \\
\hline $2.1443 \mathrm{M}$ & 6 & 0 & 2 & 2 & 42.107 \\
\hline $2.1443 \mathrm{M}$ & & 1 & 4 & 0 & 42.107 \\
\hline 2.1380 & 5 & 1 & 1 & 2 & 42.237 \\
\hline 2.1166 & 26 & -3 & 3 & 1 & 42.684 \\
\hline 2.0901 & 5 & -4 & 2 & 1 & 43.253 \\
\hline 2.0359 & 3 & -3 & 2 & 2 & 44.465 \\
\hline 2.0247 & 6 & 4 & 2 & 0 & 44.722 \\
\hline 2.0193 & 10 & $-I$ & 4 & 1 & 44.849 \\
\hline 2.0114 & 9 & 0 & 4 & 1 & 45.035 \\
\hline 1.9862 & 6 & 2 & 4 & 0 & 45.639 \\
\hline 1.9655 & 4 & -4 & 1 & 2 & 46.148 \\
\hline 1.9349 & 8 & -2 & 4 & 1 & 46.921 \\
\hline 1.9238 & 5 & 2 & 0 & 2 & 47.206 \\
\hline 1.9135 & 2 & 1 & 4 & 1 & 47.476 \\
\hline 1.8847 & 2 & 0 & 3 & 2 & 48.249 \\
\hline 1.8737 & 2 & -5 & 1 & 1 & 48.549 \\
\hline 1.8455 & 3 & -4 & 3 & 1 & 49.339 \\
\hline 1.8085 & 2 & -3 & 3 & 2 & 50.420 \\
\hline 1.8038 & 1 & 3 & 3 & 1 & 50.560 \\
\hline $1.7862 \mathrm{M}$ & 9 & -3 & 4 & 1 & 51.095 \\
\hline $1.7862 \mathrm{M}$ & & 5 & 1 & 0 & 51.095 \\
\hline $1.7640 \mathrm{M}$ & 7 & 2 & 2 & 2 & 51.784 \\
\hline $1.7640 \mathrm{M}$ & & 1 & 3 & 2 & 51.784 \\
\hline $1.7586 \mathrm{M}$ & 8 & 2 & 4 & 1 & 51.955 \\
\hline
\end{tabular}

Smyth, J. R. (1974). Am. Mineral. 59, 345.

Stephenson, D. A., Sclar, C. B., and Smith, J. V. (1966). Mineral. Mag. 35, 838. 
Magnesium Silicate ( $\mathrm{Cl}$ inoenstatite), $\mathrm{MgSiO}_{3}-$ (continued)

\begin{tabular}{|c|c|c|c|c|c|}
\hline $\mathrm{d}(\AA)$ & $\mathrm{I}^{\text {rel }}$ & & hke & & $2 \theta\left({ }^{\circ}\right)$ \\
\hline $1.7586 \mathrm{M}$ & & -5 & 2 & 1 & 51.955 \\
\hline 1.7311 & 7 & 1 & 5 & 0 & 52.844 \\
\hline 1.7112 & 2 & 4 & 2 & 1 & 53.506 \\
\hline $1.6763 \mathrm{M}$ & 1 & -1 & 4 & 2 & 54.713 \\
\hline $1.6763 \mathrm{M}$ & & -1 & 1 & 3 & 54.713 \\
\hline 1.6713 & 2 & 3 & 0 & 2 & 54.890 \\
\hline 1.6595 & 5 & 0 & 5 & 1 & 55.315 \\
\hline 1.6480 & 5 & -3 & 1 & 3 & 55.733 \\
\hline 1.6412 & 7 & 3 & 1 & 2 & 55.983 \\
\hline 1.6273 & 2 & -5 & 2 & 2 & 56.504 \\
\hline 1.6054 & 30 & -5 & 3 & 1 & 57.347 \\
\hline 1.5920 & 4 & -1 & 2 & 3 & 57.875 \\
\hline 1.5852 & 5 & 4 & 4 & 0 & 58.147 \\
\hline 1.5702 & 1 & 4 & 3 & 1 & 58.755 \\
\hline 1.5608 & 1 & -4 & 1 & 3 & 59.146 \\
\hline $1.5340 \mathrm{M}$ & $1 \mathrm{~L}$ & 0 & 2 & 3 & 60.284 \\
\hline $1.5340 \mathrm{M}$ & & 5 & 1 & 1 & 60.284 \\
\hline 1.5257 & 9 & 3 & 5 & 0 & 60.648 \\
\hline 1.5201 & 7 & 6 & 0 & 0 & 60.896 \\
\hline 1.4857 & 10 & -2 & 3 & 3 & 62.459 \\
\hline 1.4757 & 10 & -1 & 3 & 3 & 62.933 \\
\hline 1.4695 & 11 & 0 & 6 & 0 & 63.229 \\
\hline $1.4571 \mathrm{M}$ & 2 & -3 & 3 & 3 & 63.829 \\
\hline $1.4571 \mathrm{M}$ & & -1 & 5 & 2 & 63.829 \\
\hline 1.4507 & 1 & 1 & 6 & 0 & 64.145 \\
\hline $1.4448 \mathrm{M}$ & 1 & 1 & 2 & 3 & 64.436 \\
\hline $1.4448 \mathrm{M}$ & & -2 & 5 & 2 & 64.436 \\
\hline 1.4401 & 1 & -6 & 2 & 2 & 64.675 \\
\hline 1.4320 & 3 & 0 & 5 & 2 & 65.085 \\
\hline 1.4084 & 1 & 0 & 6 & 1 & 66.316 \\
\hline 1.4049 & 1 & 5 & 4 & 0 & 66.503 \\
\hline 1.3981 & 2 & -3 & 5 & 2 & 66.865 \\
\hline 1. 3952 & 2 & 4 & 5 & 0 & 67.021 \\
\hline 1.3762 & 11 & 5 & 3 & 1 & 68.074 \\
\hline $1.3568 \mathrm{M}$ & 6 & -2 & 4 & 3 & 69.182 \\
\hline 1. $3568 \mathrm{M}$ & & 1 & 3 & 3 & 69.182 \\
\hline 1.3381 & 2 & -7 & 0 & 2 & 70.294 \\
\hline $1.3351 \mathrm{C}$ & 2 & -3 & 4 & 3 & 70.474 \\
\hline 1.3233 & 3 & 3 & 6 & 0 & 71.195 \\
\hline 1.3233 & 3 & 6 & 1 & 1 & 71.195 \\
\hline 1. $3139 M$ & 1 & 0 & 4 & 3 & 71.787 \\
\hline $1.3139 M$ & & -5 & 3 & 3 & 71.787 \\
\hline 1. 2888 & 2 & 7 & 1 & 0 & 73.411 \\
\hline 1.2867 & 2 & -3 & 0 & 4 & 73.548 \\
\hline $1.2776 \mathrm{M}$ & 2 & -2 & 1 & 4 & 74.161 \\
\hline $1.2776 \mathrm{M}$ & & -1 & 6 & 2 & 74.161 \\
\hline 1.2696 & 3 & 3 & 1 & 3 & 74.706 \\
\hline 1. 2680 & 2 & 5 & 5 & 0 & 74.819 \\
\hline 1. 2609 & 5 & 0 & 6 & 2 & 75.309 \\
\hline 1.2579 & 3 & -4 & 0 & 4 & 75.519 \\
\hline
\end{tabular}


Synonym

Magnesium orthosilicate

CAS registry no. 10034-94-3

Sample

$\mathrm{MgCO}_{3}$ and $\mathrm{SiO}_{2}$ were mixed in a 2:1 molar ratio and heated at $800{ }^{\circ} \mathrm{C}$ overnight, $1300{ }^{\circ} \mathrm{C}$ for 21 hours, $1500{ }^{\circ} \mathrm{C}$ for 25 hours, and $1525{ }^{\circ} \mathrm{C}$ for 24 hours with intermittent grinding. There was a trace of $\mathrm{MgO}$ in the sample.

Color

Colorless

Structure

Orthorhombic, Pmnb (62). The structure was solved by Rinne et al. (1924).

Crystallographic constants of this sample

$a=5.9817(5) \AA$

$\mathrm{b}=10.1978(8)$

$c=4.7553(3)$

$a / b=0.5866$

$c / b=0.4663$

$Z=4$

$Z=4$
$V=290.07 \AA^{3}$

Density $(\mathrm{calc})=3.222 \mathrm{~g} / \mathrm{cm}^{3}$

Figure of merit

$F_{30}=113.8(0.0068,39)$

Additional patterns

PDF card 7-74 (Gillery, Coll. of Min. Ind.,

Penn. State University, University Park, PA)

Swanson and Tatge (1951)

References

Rinne, F. (1924). Z. Kristallogr. 59, 230.

Rinne, F., Leonhardt, J., and Hentschel, H.

(1924) ibid 59, 548.

Swanson, H. E. and Tatge, E. (1953). Natl.

Bur. Stand. (U.S.) Circ. 539, Vol. 1, p. 83.

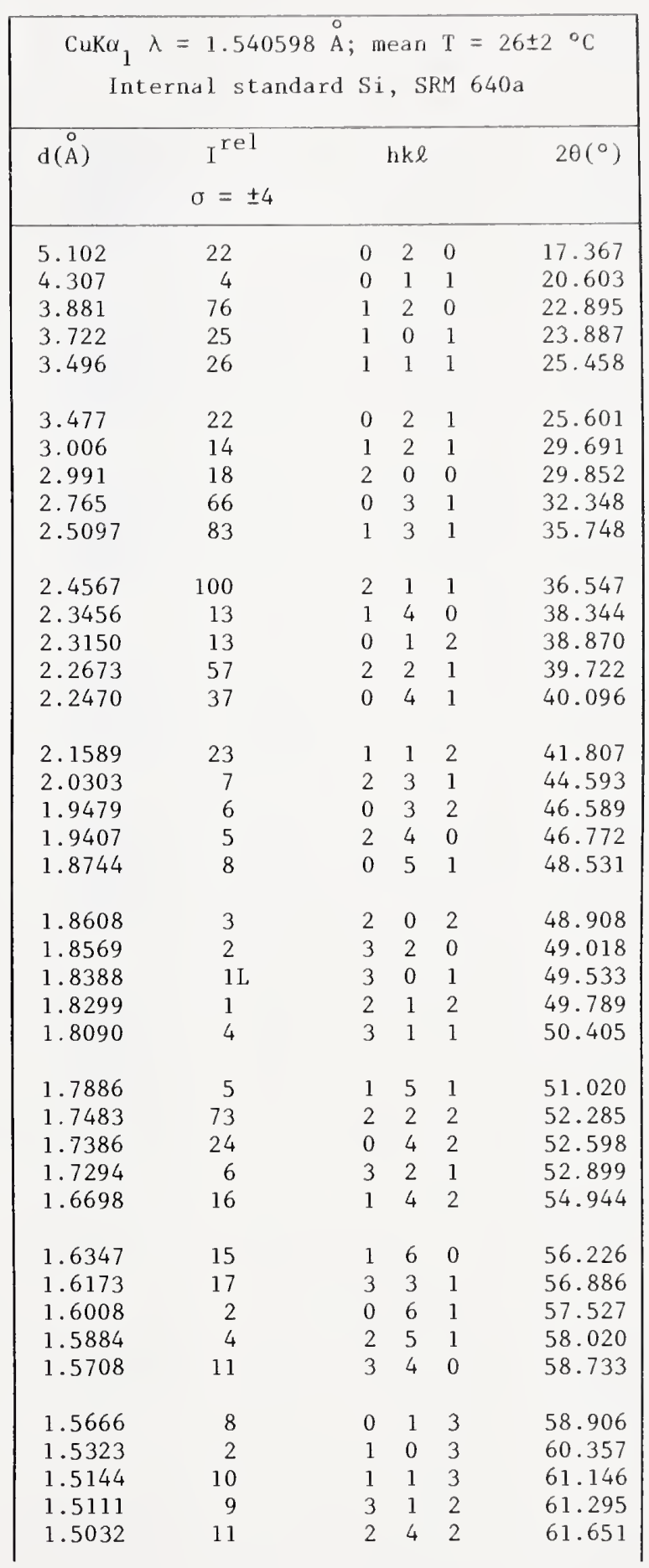


Magnesium Silicate (Forsterite), $\mathrm{Mg}_{2} \mathrm{SiO}_{4}-$ (continued)

\begin{tabular}{|c|c|c|c|c|c|}
\hline$d(\stackrel{\bar{A}}{)})$ & $\begin{array}{c}I^{\text {rel }} \\
\sigma= \pm 4\end{array}$ & \multicolumn{3}{|c|}{ hkl } & $2 \theta\left({ }^{\circ}\right)$ \\
\hline 1.4990 & 20 & 1 & 5 & 2 & 61.845 \\
\hline 1.4954 & 30 & 4 & 0 & 0 & 62.008 \\
\hline 1.4780 & 33 & 2 & 6 & 0 & 62.825 \\
\hline 1.4674 & 3 & 1 & 2 & 3 & 63.328 \\
\hline 1.4638 & 3 & 3 & 2 & 2 & 63.505 \\
\hline 1.4365 & 4 & 0 & 3 & 3 & 64.853 \\
\hline 1.4111 & 2 & 2 & 6 & 1 & 66.172 \\
\hline 1.3968 & 13 & 1 & 3 & 3 & 66.934 \\
\hline $1.3930 \mathrm{M}$ & 14 & 3 & 3 & 2 & 67.143 \\
\hline $1.3930 \mathrm{M}$ & & 0 & 7 & 1 & 67.143 \\
\hline 1.3874 & 9 & 2 & 1 & 3 & 67.448 \\
\hline 1.3748 & 2 & 2 & 5 & 2 & 68.155 \\
\hline 1.3657 & $1 \mathrm{~L}$ & 3 & 5 & 1 & 68.668 \\
\hline 1.3505 & 22 & 2 & 2 & 3 & 69.553 \\
\hline 1.3465 & 15 & 0 & 4 & 3 & 69.793 \\
\hline 1.3155 & 11 & 4 & 3 & 1 & 71.685 \\
\hline 1.3131 & 9 & 1 & 4 & 3 & 71.838 \\
\hline 1.2937 & 5 & 3 & 6 & 0 & 73.085 \\
\hline 1.2661 & 2 & 4 & 0 & 2 & 74.950 \\
\hline 1.2627 & 1 & 2 & 7 & 1 & 75.185 \\
\hline 1.2561 & 3 & 4 & 1 & 2 & 75.651 \\
\hline 1.2515 & 1 & 0 & 5 & 3 & 75.975 \\
\hline 1.2477 & 3 & 3 & 6 & 1 & 76.246 \\
\hline 1.2448 & 3 & 4 & 4 & 1 & 76.456 \\
\hline 1.2411 & 3 & 3 & 0 & 3 & 76.726 \\
\hline 1.2317 & 1 & 3 & 1 & 3 & 77.420 \\
\hline 1.2276 & 2 & 2 & 4 & 3 & 77.732 \\
\hline 1.2228 & 2 & 3 & 5 & 2 & 78.089 \\
\hline 1.2160 & $1 \mathrm{~L}$ & 1 & 7 & 2 & 78.615 \\
\hline 1.2060 & 1L & 1 & 8 & 1 & 79.391 \\
\hline
\end{tabular}


Potassium Barium Niobium Oxide, $\mathrm{KBa}_{2}\left(\mathrm{NbO}_{3}\right)_{5}$

Synonym

Potassium barium niobate

CAS registry no. 12400-06-5

Sample

The sample was made by heating a mixture of $\mathrm{KNbO}_{3}, \mathrm{BaCO}_{3}$, and $\mathrm{Nb}_{2} \mathrm{O}_{5}$ at temperatures up to $1340{ }^{\circ} \mathrm{C}$ for 56 hours with intermediate grindings.

Color Colorless

Structure Tetragonal, P4bm (100). Tungsten bronze structure (Giess et al., 1969).

Crystallographic constants of this sample $\mathrm{a}=12.5407(5) \AA$

$c=4.0210(3)$

$c / a=0.3206$

$Z=2$

$V=632.38 \AA^{3}$

Density $(\mathrm{calc})=5.348 \mathrm{~g} / \mathrm{cm}^{3}$

Figure of merit

$\mathrm{F}_{30}=104.2(0.0082,35)$

Additional pattern

PDF card 28-375 (Giess et al., 1969)

Reference

Giess, E. A., Scott, B. A., Burns, G., O'Kane, D. F., and Segmüller, A. (1969). J. Am. Ceram. Soc. 52,276 .

\begin{tabular}{|c|c|c|c|c|c|}
\hline \multicolumn{6}{|c|}{$\begin{array}{c}\mathrm{CuK}_{1} \lambda=1.540598 \AA \text {; mean } \mathrm{T}=26 \pm 2{ }^{\circ} \mathrm{C} \\
\text { Internal standards } \mathrm{W}, \mathrm{a}=3.16524 \AA \\
\text { Fluorophlogopite, SRM } 675\end{array}$} \\
\hline $\mathrm{d}(\stackrel{\circ}{\AA})$ & $\begin{array}{c}I^{\mathrm{rel}} \\
\sigma= \pm 4\end{array}$ & & hk & & $2 \theta\left(^{\circ}\right)$ \\
\hline 8.87 & 1 & 1 & 1 & 0 & 9.968 \\
\hline 6.279 & $1 \mathrm{~L}$ & 2 & 0 & 0 & 14.093 \\
\hline 5.609 & 1 & 2 & 1 & 0 & 15.787 \\
\hline 4.433 & $1 \mathrm{~L}$ & 2 & 2 & 0 & 20.014 \\
\hline 4.021 & 13 & 0 & 0 & 1 & 22.086 \\
\hline 3.967 & 4 & 3 & 1 & 0 & 22.394 \\
\hline 3.664 & 1 & 1 & 1 & 1 & 24.274 \\
\hline 3.479 & 29 & 3 & 2 & 0 & 25.585 \\
\hline 3.386 & 3 & 2 & 0 & 1 & 26.303 \\
\hline 3.269 & 59 & 2 & 1 & 1 & 27.255 \\
\hline 3.136 & 9 & 4 & 0 & 0 & 28.440 \\
\hline 3.042 & 68 & 4 & 1 & 0 & 29.332 \\
\hline 2.981 & 23 & 2 & 2 & 1 & 29.955 \\
\hline 2.957 & 15 & 3 & 3 & 0 & 30.204 \\
\hline 2.824 & 100 & 3 & 1 & 1 & 31.661 \\
\hline
\end{tabular}

\begin{tabular}{|c|c|c|c|c|c|}
\hline $\mathrm{d}(\stackrel{\circ}{\mathrm{A}})$ & $\mathrm{I}^{\text {rel }}$ & & hke & & $2 \theta\left(^{\circ}\right)$ \\
\hline 2.804 & 36 & 4 & 2 & 0 & 31.889 \\
\hline 2.631 & 16 & 3 & 2 & 1 & 34.052 \\
\hline 2.474 & 4 & 4 & 0 & 1 & 36.280 \\
\hline 2.427 & 1 & 4 & 1 & 1 & 37.013 \\
\hline 2.3811 & 1 & 3 & 3 & 1 & 37.750 \\
\hline 2.3291 & 9 & 5 & 2 & 0 & 38.626 \\
\hline 2.3015 & 1 & 4 & 2 & 1 & 39.108 \\
\hline 2.2167 & 2 & 4 & 4 & 0 & 40.669 \\
\hline 2.1506 & 17 & 5 & 3 & 0 & 41.976 \\
\hline 2.0981 & 2 & 5 & 1 & 1 & 43.079 \\
\hline 2.0901 & 3 & 6 & 0 & 0 & 43.252 \\
\hline 2.0616 & 1 & 6 & 1 & 0 & 43.880 \\
\hline 2.0100 & 25 & 0 & 0 & 2 & 45.069 \\
\hline 1.9823 & 15 & 6 & 2 & 0 & 45.734 \\
\hline 1.9581 & 1 & 5 & 4 & 0 & 46.332 \\
\hline 1.9416 & 1 & 4 & 4 & 1 & 46.748 \\
\hline 1.8963 & 4 & 5 & 3 & 1 & 47.934 \\
\hline 1.8690 & 8 & 6 & 3 & 0 & 48.678 \\
\hline 1.8544 & 14 & 6 & 0 & 1 & 49.088 \\
\hline 1.7785 & 21 & 6 & 2 & 1 & 51.332 \\
\hline 1.7733 & 27 & 5 & 5 & 0 & 51.492 \\
\hline 1.7606 & 18 & 5 & 4 & 1 & 51.892 \\
\hline 1.7404 & 7 & 3 & 2 & 2 & 52.540 \\
\hline 1.7225 & 2 & 7 & 2 & 0 & 53.127 \\
\hline 1.6951 & 9 & 6 & 3 & 1 & 54.056 \\
\hline 1.6770 & 20 & 4 & 1 & 2 & 54.687 \\
\hline 1.6622 & 4 & 3 & 3 & 2 & 55.217 \\
\hline 1.6464 & 2 & 7 & 3 & 0 & 55.792 \\
\hline 1.6339 & 11 & 4 & 2 & 2 & 56.256 \\
\hline 1.6226 & 26 & 5 & 5 & 1 & 56.684 \\
\hline 1.6058 & $1 \mathrm{~L}$ & 6 & 5 & 0 & 57.332 \\
\hline 1.5837 & 4 & 7 & 2 & 1 & 58.209 \\
\hline 1.5677 & 1 & 8 & 0 & 0 & 58.858 \\
\hline 1.5553 & 3 & 7 & 4 & 0 & 59.375 \\
\hline 1.5206 & 12 & 8 & 2 & 0 & 60.871 \\
\hline 1.4912 & 1 & 6 & 5 & 1 & 62.202 \\
\hline 1.4780 & 3 & 6 & 6 & 0 & 62.825 \\
\hline 1.4680 & 12 & 8 & 3 & 0 & 63.300 \\
\hline 1.4578 & 2 & 7 & 5 & 0 & 63.793 \\
\hline 1.4509 & 3 & 7 & 4 & 1 & 64.134 \\
\hline 1.4117 & 5 & 6 & 2 & 2 & 66.139 \\
\hline 1.4018 & 1 & 8 & 4 & 0 & 66.665 \\
\hline 1.3847 & 2 & 9 & 1 & 0 & 67.597 \\
\hline 1.3787 & 3 & 8 & 3 & 1 & 67.931 \\
\hline 1.3690 & 6 & 6 & 3 & 2 & 68.480 \\
\hline 1.3298 & 7 & 5 & 5 & 2 & 70.795 \\
\hline 1.3239 & 2 & 8 & 4 & 1 & 71.162 \\
\hline 1.3095 & 1 & 9 & 1 & 1 & 72.067 \\
\hline 1.3034 & 2 & 2 & 1 & 3 & 72.453 \\
\hline 1.2886 & 1 & 7 & 6 & 1 & 73.421 \\
\hline 1.2830 & 1 & 2 & 2 & 3 & 73.796 \\
\hline 1.2735 & 2 & 9 & 4 & 0 & 74.442 \\
\hline 1.2700 & 3 & 3 & 1 & 3 & 74.680 \\
\hline 1.2668 & 2 & 7 & 7 & 0 & 74.900 \\
\hline
\end{tabular}


Potassium Strontium Niobium Oxide, $\mathrm{KSr}_{2}\left(\mathrm{NbO}_{3}\right)_{5}$

Synonym

Potassium strontium niobate

CAS registry no.

$12358-94-0$

Sample

The sample was made by heating $\mathrm{KNbO}_{3}$, $\mathrm{SrCO}_{3}$, and $\mathrm{Nb}_{2} \mathrm{O}_{5}$ at $850{ }^{\circ} \mathrm{C}$ for 18 hours, at $1300{ }^{\circ} \mathrm{C}$ for 18 hours, and at $1340{ }^{\circ} \mathrm{C}$ for 20 hours.

Color

Colorless

\section{Structure}

Tetragonal, P4bm (100). Tungsten bronze structure (Giess et al., 1969).

Crystallographic constants of this sample $\mathrm{a}=12.461(1) \AA$

$c=3.9431(8)$

$\mathrm{c} / \mathrm{a}=0.3164$

$Z=2$

$V=612.27 \AA^{3}$

Density $(\mathrm{calc})=4.984 \mathrm{~g} / \mathrm{cm}^{3}$

Figure of merit

$\mathrm{F}_{30}=49.1(0.012,50)$

\section{Reference}

Giess, E. A., Scott, B. A., Burns, G., O'Kane, D. F., and Segmüller, A. (1969).

J. Am. Ceram. Soc. 52, 276.

\begin{tabular}{|c|c|c|c|c|c|}
\hline \multicolumn{6}{|c|}{$\begin{array}{c}\text { CuK } \alpha_{1} \lambda=1.540598 \AA \text {; mean } \mathrm{T}=2 \\
\text { Internal standards Si, SRM } 640 \mathrm{a} \\
\text { Fluorophlogopite, SRM } 675\end{array}$} \\
\hline$d(\stackrel{\circ}{A})$ & $\begin{array}{c}\mathrm{Iel} \\
\sigma= \pm 1\end{array}$ & & hke & & $2 \theta\left(^{\circ}\right)$ \\
\hline 8.82 & 2 & 1 & 1 & 0 & 10.025 \\
\hline 6.24 & 5 & 2 & 0 & 0 & 14.189 \\
\hline 5.578 & 2 & 2 & 1 & 0 & 15.876 \\
\hline $3.944 \mathrm{M}$ & 34 & 0 & 0 & 1 & 22.527 \\
\hline $3.944 \mathrm{M}$ & & 3 & 1 & 0 & 22.527 \\
\hline 3.602 & 4 & 1 & 1 & 1 & 24.700 \\
\hline 3.458 & 37 & 3 & 2 & 0 & 25.745 \\
\hline 3.219 & 43 & 2 & 1 & 1 & 27.687 \\
\hline 3.116 & 12 & 4 & 0 & 0 & 28.627 \\
\hline 3.023 & 52 & 4 & 1 & 0 & 29.521 \\
\hline $2.939 \mathrm{M}$ & 25 & 2 & 2 & 1 & 30.392 \\
\hline $2.939 \mathrm{M}$ & & 3 & 3 & 0 & 30.392 \\
\hline $2.788 \mathrm{M}$ & 100 & 3 & 1 & 1 & 32.077 \\
\hline $2.788 \mathrm{M}$ & & 4 & 2 & 0 & 32.077 \\
\hline 2.600 & 20 & 3 & 2 & 1 & 34.472 \\
\hline $2.446 \mathrm{M}$ & 2 & 4 & 0 & 1 & 36.711 \\
\hline $2.446 \mathrm{M}$ & & 5 & 1 & 0 & 36.711 \\
\hline 2.399 & 1 & 4 & 1 & 1 & 37.454 \\
\hline 2.356 & 2 & 3 & 3 & 1 & 38.176 \\
\hline 2.315 & 3 & 5 & 2 & 0 & 38.875 \\
\hline 2.202 & 1 & 4 & 4 & 0 & 40.960 \\
\hline 2.137 & 8 & 5 & 3 & 0 & 42.252 \\
\hline $2.077 \mathrm{M}$ & 3 & 5 & 1 & 1 & 43.539 \\
\hline $2.077 \mathrm{M}$ & & 6 & 0 & 0 & 43.539 \\
\hline 2.048 & 3 & 6 & 1 & 0 & 44.179 \\
\hline $1.9709 \mathrm{M}$ & 36 & 0 & 0 & 2 & 46.014 \\
\hline $1.9709 \mathrm{M}$ & & 6 & 2 & 0 & 46.014 \\
\hline 1.9468 & 6 & 5 & 4 & 0 & 46.616 \\
\hline $1.9229 \mathrm{M}$ & 1 & 1 & 1 & 2 & 47.231 \\
\hline $1.9229 \mathrm{M}$ & & 4 & 4 & 1 & 47.231 \\
\hline $1.8786 \mathrm{M}$ & 1 & 2 & 0 & 2 & 48.414 \\
\hline $1.8786 \mathrm{M}$ & & 5 & 3 & 1 & 48.414 \\
\hline $1.8576 \mathrm{M}$ & 12 & 2 & 1 & 2 & 48.998 \\
\hline $1.8576 \mathrm{M}$ & & 6 & 3 & 0 & 48.998 \\
\hline 1.8375 & 8 & 6 & 0 & 1 & 49.568 \\
\hline $1.7625+$ & 20 & 6 & 2 & 1 & 51.831 \\
\hline $1.7625+$ & & 5 & 5 & 0 & 51.831 \\
\hline 1.7452 & 10 & 5 & 4 & 1 & 52.385 \\
\hline 1.7288 & $1 \mathrm{~L}$ & 6 & 4 & 0 & 52.918 \\
\hline 1.7127 & 6 & 3 & 2 & 2 & 53.456 \\
\hline 1.6805 & 7 & 6 & 3 & 1 & 54.563 \\
\hline 1.6656 & 1 & 4 & 0 & 2 & 55.093 \\
\hline 1.6513 & 12 & 4 & 1 & 2 & 55.613 \\
\hline 1.6377 & 2 & 3 & 3 & 2 & 56.116 \\
\hline $1.6090 \mathrm{M}$ & 31 & 4 & 2 & 2 & 57.206 \\
\hline
\end{tabular}


Potassium Strontium Niobium Oxide, $\mathrm{KSr}_{2}\left(\mathrm{NbO}_{3}\right)_{5}$ - (continued)

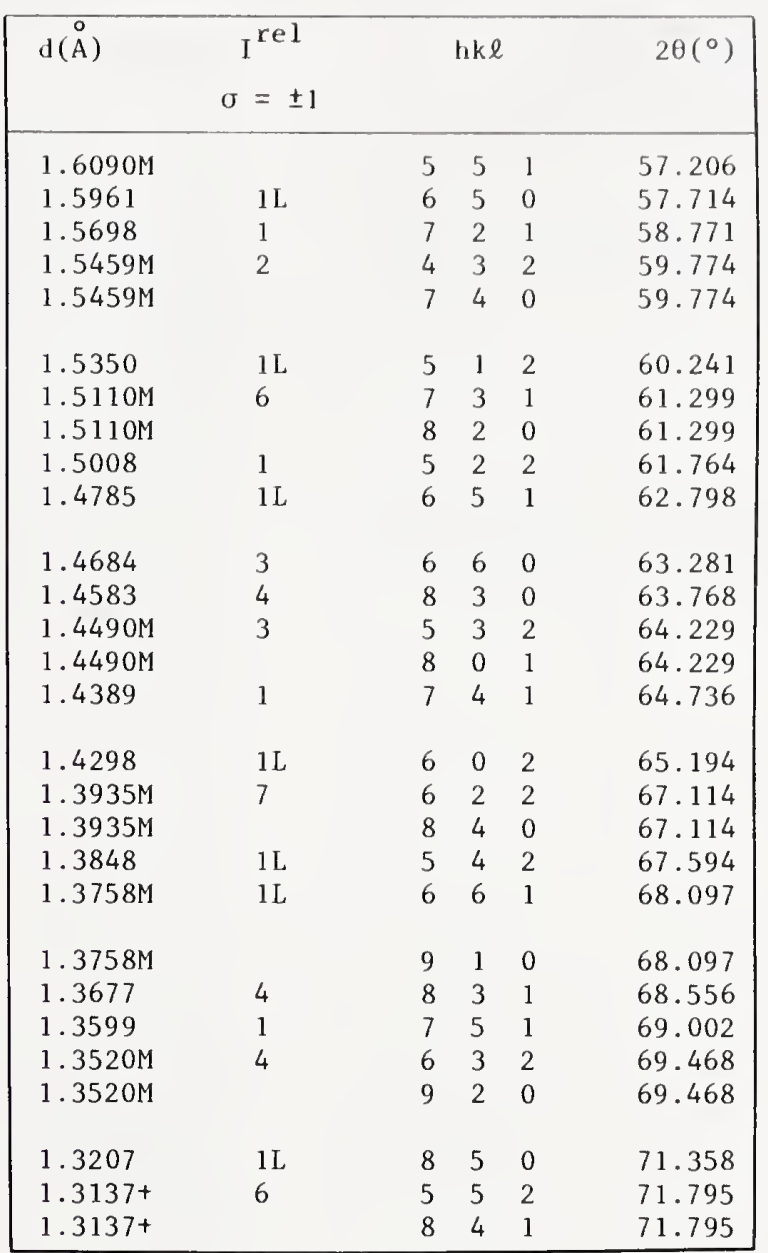


Potassium Titanium Phosphate, $\mathrm{KTi}_{2}\left(\mathrm{PO}_{4}\right)_{3}$

Synonym

Potassium titanium orthophosphate

CAS registry no. 30622-41-4

Sample

The sample was made by heating a mixture of $\mathrm{KH}_{2} \mathrm{PO}_{4}, \mathrm{TiO}_{2}$ (anatase) and $\left(\mathrm{NH}_{4}\right)_{2} \mathrm{HPO}_{4}$ up to $1000{ }^{\circ} \mathrm{C}$ for 2 days.

Color

Colorless

Structure

Rhombohedral, $\mathrm{R}^{* *}$. Isostructural with many other similar double phosphates (Masse, 1970).

Crystallographic constants of this sample (Hexagonal axes)

$\mathrm{a}=8.3582(5) \AA$

$c=23.086(2)$

$c / a=2.7621$

$Z=6$

$\mathrm{V}=1396.71 \AA^{3}$

Density $(\mathrm{calc})=2.995 \mathrm{~g} / \mathrm{cm}^{3}$

Figure of merit

$\mathrm{F}_{30}=83.0(0.0070,52)$

Polymorphism

Masse et al. (1972) reported a cubic form of $\mathrm{KTi}_{2}\left(\mathrm{PO}_{4}\right)_{3}$.

Additional pattern

PDF card 25-691 (Masse, 1970)

References

Masse, R. (1970). Bull. Soc. Fr. Mineral.

Crystallogr. 93, 500 .

Masse, R., Durif, A., Guitel, J.-C., and

Tordjman, I. (1972). Bull. Soc. Fr. Mineral.

Crystallogr. 95, 47.

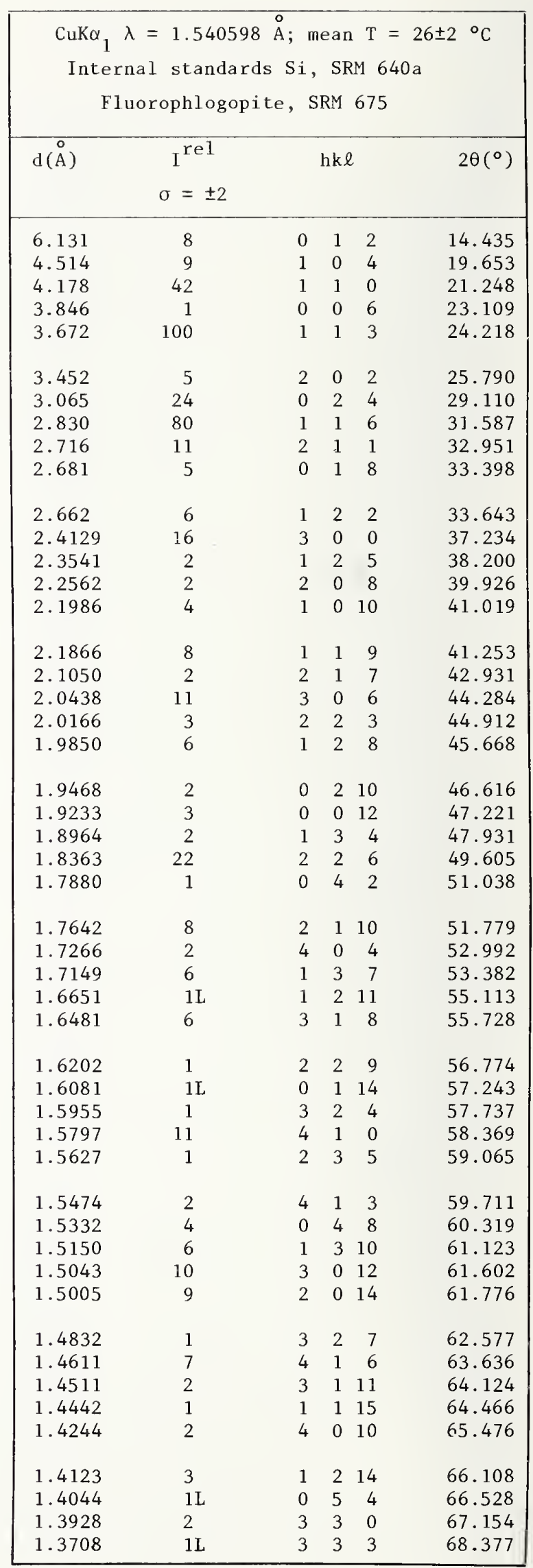


Potassium Zinc Phosphate, $\mathrm{KZnPO}_{4}$

Synonym

Potassium zinc orthophosphate

CAS registry no.

$13826-55-6$

Sample

The sample was prepared by heating $\mathrm{KH}_{2} \mathrm{PO}_{4}$ and $\mathrm{ZnO}$ at $800{ }^{\circ} \mathrm{C}$ for 2 days. After grinding and sieving, it was held at $800{ }^{\circ} \mathrm{C}$ overnight.

Color Colorless

Structure Hexagona1, $\mathrm{P} 6_{3} / *$ (Averbuch-Pouchot and Durif, 1973).

Crystallographic constants of this sample $\mathrm{a}=18.1626(12) \AA$

$c=8.5066(11)$

$c / a=0.4684$

$Z=24$

$V=2430.20 \AA^{3}$

Density $($ calc $)=3.2702 \mathrm{~g} / \mathrm{cm}^{3}$

Figure of merit

$\mathrm{F}_{30}=57.4(0.0093,56)$

Additional patterns

PDF card 20-1447 (Salutsky and Steiger, 1964)

PDF card 20-1448 (Frazier et al., 1966)

PDF card 25-1342 (Averbuch-Pouchot and Durif, 1973)

Barbou des Courières and Simonot-Grange (1979) Grins and Nygren (1982)

References

Averbuch-Pouchot, M. T. and Durif, A. (1973). Mat. Res. Bul1. 8, 353.

Barbou des Courieres, T. and Simonot-Grange, M.-H. (1979). Mat. Res. Bull, 14, 1419.

Grins, J. and Nygren, M. (1982). Mat. Res. Bull. 17,895 .

Frazier, A. W., Smith, J. P., and Lehr, J. R. (1966). J. Agric. Food Chem. 14, 522.

Salutsky, M. L. and Steiger, R. P. (1964). J. Agric. Food Chem. 12, 6.

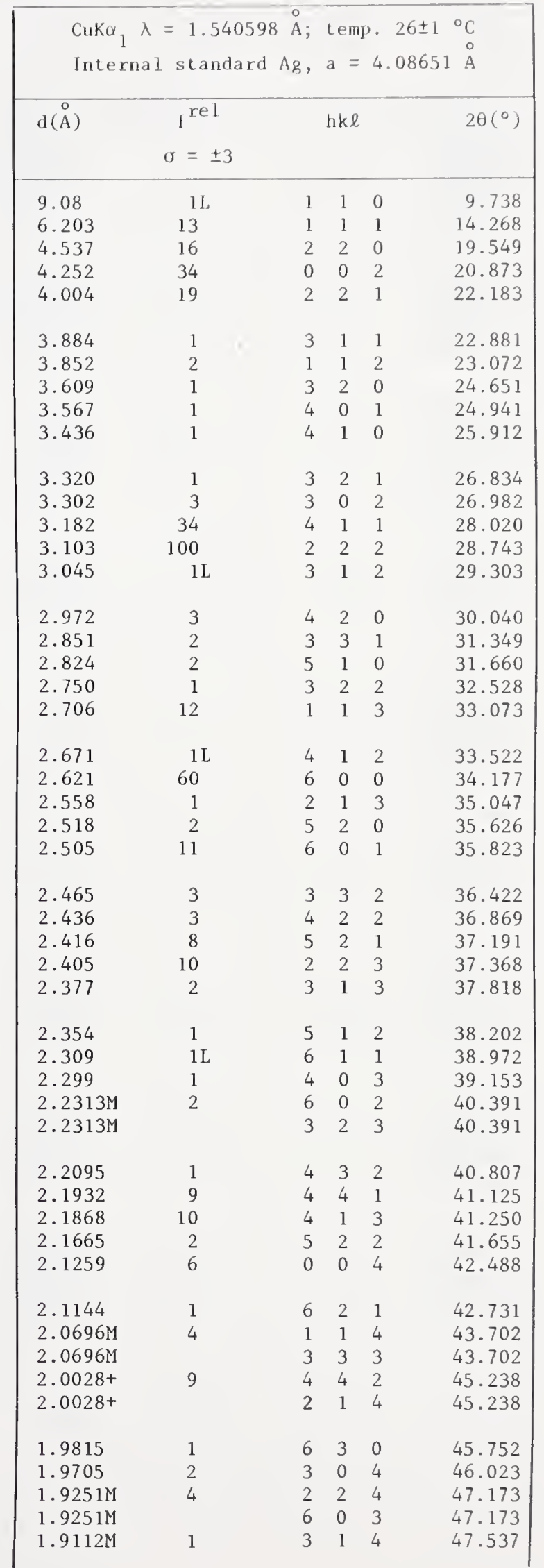


Potassium Zinc Phosphate, $\mathrm{KZnPO}_{4}$ - (continued)

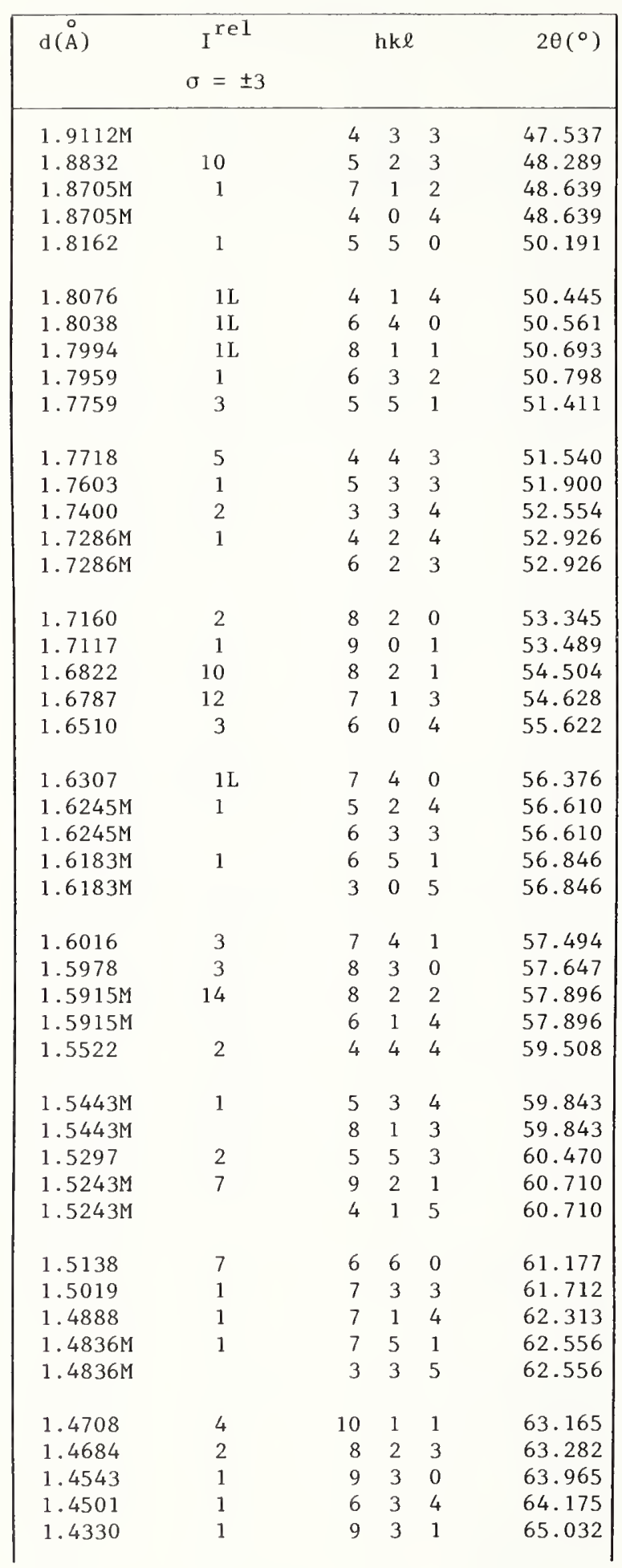

\begin{tabular}{|lcrrrr|}
\hline $\mathrm{d}(\AA)$ & $\mathrm{I}^{\mathrm{rel}}$ & \multicolumn{3}{c}{ hk $\ell$} & $2 \theta\left(^{\circ}\right)$ \\
& $\sigma= \pm 3$ & & & & \\
\hline $1.4261 \mathrm{M}$ & 3 & 6 & 6 & 2 & 65.386 \\
$1.4261 \mathrm{M}$ & & 7 & 2 & 4 & 65.386 \\
1.4183 & 1 & 0 & 0 & 6 & 65.794 \\
1.4141 & 2 & 7 & 4 & 3 & 66.010 \\
$1.4101 \mathrm{M}$ & 4 & 11 & 0 & 1 & 66.222 \\
& & & & & \\
$1.4101 \mathrm{M}$ & & 5 & 2 & 5 & 66.222 \\
$1.3757+$ & 1 & 6 & 4 & 4 & 68.102 \\
$1.3757+$ & & 10 & 0 & 3 & 68.102 \\
$1.3610 \mathrm{M}$ & 2 & 4 & 4 & 5 & 68.941 \\
$1.3610 \mathrm{M}$ & & 7 & 3 & 4 & 68.941 \\
& & & & & \\
1.3504 & 1 & 9 & 0 & 4 & 69.558 \\
& & & & & \\
\hline
\end{tabular}


Rubidium Hydrogen Phosphate, $\mathrm{RbH}_{2} \mathrm{PO}_{4}$

Synonym

Rubidium dihydrogen orthophosphate

CAS registry no. 13774-16-8

Sample

The sample was made by adding $\mathrm{Rb}_{2} \mathrm{CO}_{3}$ to an $85 \%$ solution of $\mathrm{H}_{3} \mathrm{PO}_{4}$. The precipitate was washed with alcohol.

Color

Colorless

Structure

Tetragonal, I $\overline{4} 2 \mathrm{~d}(122)$. The structure was determined by Al-Karaghouli et al. (1978). Isostructural with $\mathrm{KH}_{2} \mathrm{PO}_{4}$.

Crystallographic constants of this sample

$$
\begin{aligned}
& a=7.6080(3) \AA \\
& c=7.2979(6) \\
& c / a=0.9592 \\
& Z=4 \\
& V=422.42 \AA^{3} \\
& \text { Density }(\mathrm{calc})=2.869 \mathrm{~g} / \mathrm{cm}^{3}
\end{aligned}
$$

Figure of merit

$\mathrm{F}_{30}=90.5(0.011,31)$

Additional patterns PDF card 30-1064 (Smith et al., 1979) (calculated pattern)

Nirsha et al. (1981)

\section{References}

Al-Karaghouli, A. R., Abdul-Wahab, B., Ajaj, E., and Sequeira, A. (1978). Acta Crystallogr. B34, 1040.

Nirsha, B. M., Budinitsa, E. N., Efremov, V. A., Zhadanov, B. V., Olikova, V. A., and Fakeer, A. A. (1981). Russ. J. Inorg. Chem. 26,1560 .

\begin{tabular}{|c|c|c|c|c|c|}
\hline$d(\AA)$ & $\begin{array}{c}I^{\text {rel }} \\
\sigma= \pm 1\end{array}$ & & $\mathrm{hk} \ell$ & & $2 \theta\left(^{\circ}\right)$ \\
\hline 5.262 & 2 & 1 & 0 & 1 & 16.837 \\
\hline 3.803 & 100 & 2 & 0 & 0 & 23.375 \\
\hline 3.083 & 15 & 2 & 1 & 1 & 28.934 \\
\hline 3.020 & 67 & 1 & 1 & 2 & 29.555 \\
\hline 2.689 & 24 & 2 & 2 & 0 & 33.292 \\
\hline
\end{tabular}

Smith, D. E. (1979). Ann. Report to Joint Committee on Powder Diffraction Standards.

$\mathrm{CuK} \alpha_{1} \lambda=1.540598 \AA$ A; mean $\mathrm{T}=25.7{ }^{\circ} \mathrm{C}$ Internal standards $\mathrm{Ag}, \mathrm{a}=4.08651 \AA$ Fluorophlogopite, SRM 675

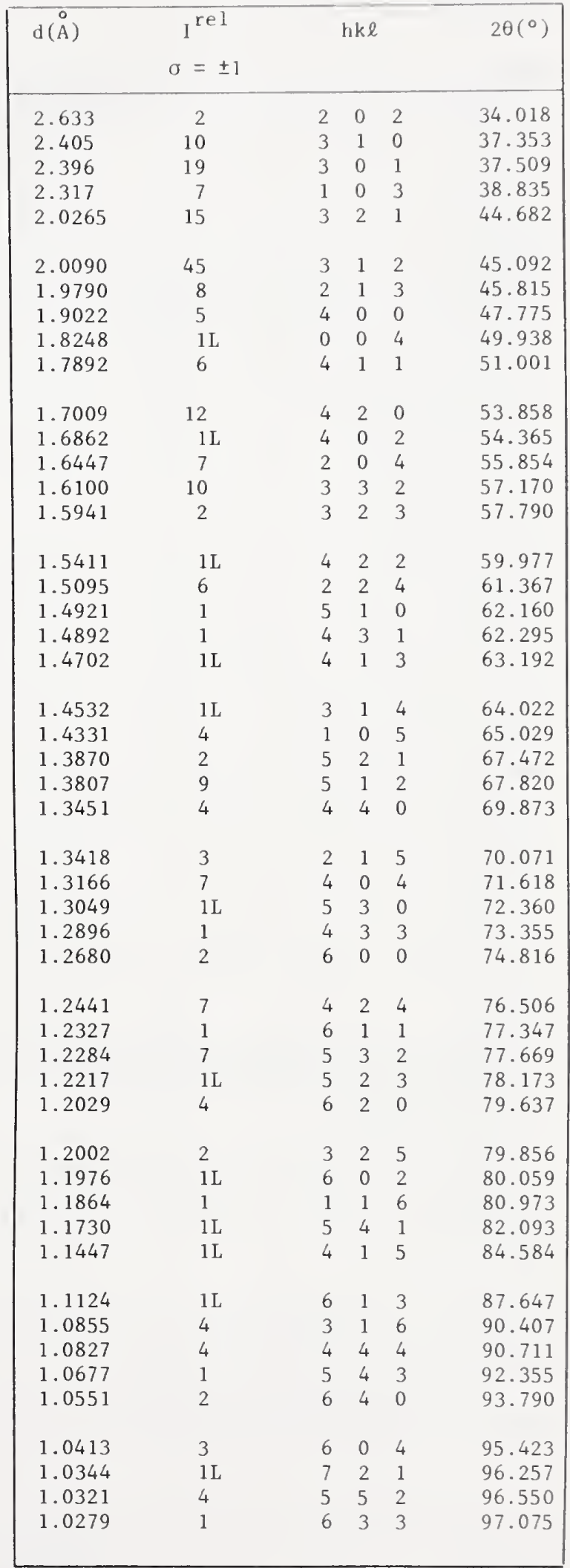


Rubidium Phosphate, $\mathrm{RbPO}_{3}$

Synonym

Rubidium metaphosphate

Sample

The sample was prepared by heating $\mathrm{RbH}_{2} \mathrm{PO}_{4}$ at $250{ }^{\circ} \mathrm{C}$ for 17 hours

Color

Colorless

Structure

Monoclinic, $\mathrm{P} 2{ }_{1} / \mathrm{n}$ (14). The structure of

$\mathrm{RbPO}_{3}$ was determined by Corbridge (1956)

and refined by Cruickshank (1964).

Crystallographic constants of this sample

$\mathrm{a}=12.1221(14) \AA$

$\mathrm{b}=4.2307(5)$

$c=6.4773(9)$

$\beta=95.980(11)^{\circ}$

$\mathrm{a} / \mathrm{b}=2.8652$

$\mathrm{c} / \mathrm{b}=1.5310$

$\begin{array}{ll}Z=4 & \circ \\ V & =330.38 \AA^{3}\end{array}$

Density $(\mathrm{calc})=3.306 \mathrm{~g} / \mathrm{cm}^{3}$

Figure of merit

$\mathrm{F}_{30}=123.3(0.0055,44)$

Additional patterns

Majling et al. (1979) (calculated pattern)

Nirsha et al. (1981)

References

Corbridge, D. E. C. (1956). Acta Crystallogr.

9, 308 .

Cruickshank, D. W. J. (1964). Acta Crystallogr. 17,681 .

Majling, J., Raninec, ¿̌s., and Durovič, S. (1979).

Calculated Powder Diffraction Patterns for

Anhydrous Phosphates (VEDA, Bratislava,

Czechoslovakia).

Nirsha, B. M., Gudinitsa, E. N., Efremov, V. A., Zhadanov, B. V., Olikova, V. A., and Fakeev, A. A. (1981). Russ. J. Inorg. Chem. 26,1560

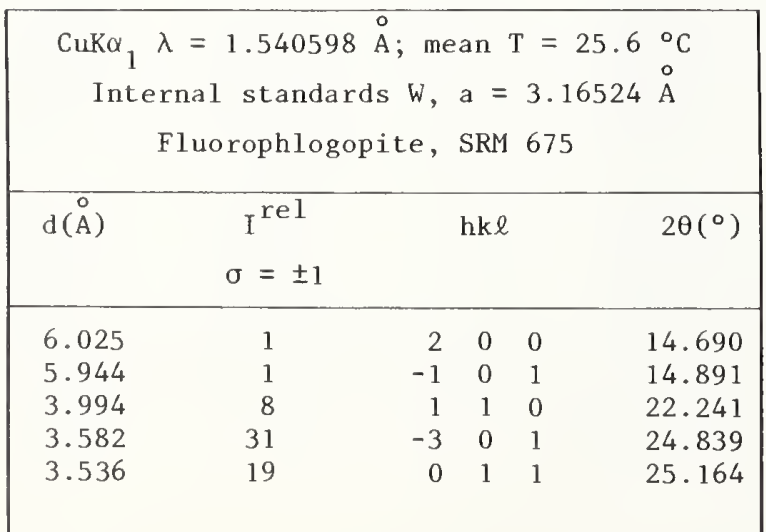

\begin{tabular}{|c|c|c|c|c|c|}
\hline $\mathrm{d}(\stackrel{\circ}{\AA})$ & $\begin{array}{c}\mathrm{r}^{\text {rel }} \\
\sigma= \pm 1\end{array}$ & & hke & & $2 \theta\left({ }^{\circ}\right)$ \\
\hline 3.463 & 68 & 2 & 1 & 0 & 25.704 \\
\hline 3.447 & 50 & -1 & 1 & 1 & 25.822 \\
\hline 3.342 & 13 & 1 & 1 & 1 & 26.651 \\
\hline 3.260 & 72 & 3 & 0 & 1 & 27.338 \\
\hline 3.220 & 72 & 0 & 0 & 2 & 27.681 \\
\hline 3.129 & 100 & -2 & 1 & 1 & 28.501 \\
\hline 3.014 & 24 & 4 & 0 & 0 & 29.617 \\
\hline 2.976 & 55 & 2 & 1 & 1 & 30.003 \\
\hline 2.914 & 10 & 3 & 1 & 0 & 30.657 \\
\hline 2.5832 & 7 & 3 & 1 & 1 & 34.698 \\
\hline 2.5623 & 8 & 0 & 1 & 2 & 34.991 \\
\hline 2.4550 & 40 & 4 & 1 & 0 & 36.572 \\
\hline 2.4326 & 37 & -2 & 1 & 2 & 36.922 \\
\hline 2.3613 & 6 & -4 & 1 & 1 & 38.079 \\
\hline 2.3398 & 6 & -5 & 0 & 1 & 38.443 \\
\hline 2.3253 & 6 & -4 & 0 & 2 & 38.692 \\
\hline 2.2918 & 13 & 2 & 1 & 2 & 39.281 \\
\hline 2.2325 & 3 & 4 & 1 & 1 & 40.369 \\
\hline $2.0946 \mathrm{M}$ & 26 & 5 & 1 & 0 & 43.155 \\
\hline $2.0946 \mathrm{M}$ & & 4 & 0 & 2 & 43.155 \\
\hline 2.0829 & 12 & 1 & 2 & 0 & 43.409 \\
\hline 2.0773 & 11 & 1 & 0 & 3 & 43.533 \\
\hline 2.0474 & 8 & -5 & 1 & 1 & 44.202 \\
\hline 2.0369 & 8 & -4 & 1 & 2 & 44.442 \\
\hline $2.0094 M$ & 28 & 0 & 2 & 1 & 45.083 \\
\hline $2.0094 M$ & & 6 & 0 & 0 & 45.083 \\
\hline 1.9958 & 1 & 2 & 2 & 0 & 45.406 \\
\hline 1.9815 & 3 & -3 & 0 & 3 & 45.753 \\
\hline 1.9408 & 1 & 5 & 1 & 1 & 46.769 \\
\hline 1.9253 & 3 & -2 & 2 & 1 & 47.167 \\
\hline 1.9153 & 4 & 0 & 1 & 3 & 47.430 \\
\hline 1.8892 & 3 & 2 & 2 & 1 & 48.125 \\
\hline 1.8716 & 15 & 3 & 2 & 0 & 48.607 \\
\hline 1.8340 & 1 & -5 & 1 & 2 & 49.670 \\
\hline 1.8157 & 5 & 6 & 1 & 0 & 50.206 \\
\hline $1.7909 \mathrm{M}$ & 9 & -6 & 1 & 1 & 50.951 \\
\hline $1.7909 \mathrm{M}$ & & -6 & 0 & 2 & 50.951 \\
\hline $1.7779 M$ & 2 & 2 & 1 & 3 & 51.350 \\
\hline $1.7744 \mathrm{M}$ & 1 & 3 & 2 & 1 & 51.460 \\
\hline 1.7637 & $1 \mathrm{~L}$ & -1 & 2 & 2 & 51.793 \\
\hline 1.7351 & 2 & 1 & 2 & 2 & 52.712 \\
\hline 1.7086 & 3 & -7 & 0 & 1 & 53.595 \\
\hline 1.6976 & 4 & -4 & 2 & 1 & 53.971 \\
\hline 1.6937 & 4 & -5 & 0 & 3 & 54.103 \\
\hline 1.6874 & 6 & 5 & 1 & 2 & 54.323 \\
\hline 1.6706 & 4 & 2 & 2 & 2 & 54.915 \\
\hline
\end{tabular}


Rubidium Strontium Niobium Oxide, $\mathrm{RbSr}_{2}\left(\mathrm{NbO}_{3}\right)_{5}$

Synonym

Rubidium strontium niobate

Sample

The sample was prepared by heating $\mathrm{Rb}_{2} \mathrm{CO}_{3}$,

$\mathrm{SrCO}_{3}$, and $\mathrm{Nb}_{2} \mathrm{O}_{5}$ at $900{ }^{\circ} \mathrm{C}$ overnight,

followed by heating at $1275{ }^{\circ} \mathrm{C}$ overnight with intermediate grindings.

Color

Colorless

Structure

Tetragonal, P4bm (100). Tungsten bronze

type (Giess et a1., 1969).

Crystallographic constants of this sample

$\mathrm{a}=12.5127(7) \AA$

$c=3.9600(4)$

$c / a=0.3165$

$Z=2$

$\mathrm{V}=620.01 \AA^{3}$

Density $(\mathrm{calc})=5.172 \mathrm{~g} / \mathrm{cm}^{3}$

Figure of merit

$\mathrm{F}_{30}=75.7(0.0076,52)$

Reference

Giess, E. A., Scott, B. A., Burns, G.,

O'Kane, D. F., and Segmüller, A. (1969).

J. Am. Ceram. Soc. 52, 276.

$\mathrm{CuK \alpha}{ }_{1} \lambda=1.540598 \stackrel{\circ}{\mathrm{A}}$; mean $\mathrm{T}=26 \pm 2{ }^{\circ} \mathrm{C}$

Internal standards Ag, $a=4.08651 \AA$

Fluorophlogopite, SRM 675

\begin{tabular}{|c|c|c|c|c|c|}
\hline $\mathrm{d}(\stackrel{\circ}{\AA})$ & $\mathrm{I}^{\text {rel }}$ & & hkl & & $2 \theta\left(^{\circ}\right)$ \\
\hline 8.86 & 1 & 1 & 1 & 0 & 9.971 \\
\hline 6.263 & 3 & 2 & 0 & 0 & 14.129 \\
\hline $3.961 M$ & 22 & 0 & 0 & 1 & 22.428 \\
\hline $3.961 \mathrm{M}$ & & 3 & 1 & 0 & 22.428 \\
\hline 3.616 & 3 & 1 & 1 & 1 & 24.601 \\
\hline 3.472 & 29 & 3 & 2 & 0 & 25.638 \\
\hline 3.346 & 1 & 2 & 0 & 1 & 26.620 \\
\hline 3.233 & 51 & 2 & 1 & 1 & 27.564 \\
\hline 3.129 & 8 & 4 & 0 & 0 & 28.506 \\
\hline 3.036 & 54 & 4 & 1 & 0 & 29.400 \\
\hline 2.951 & 26 & 2 & 2 & 1 & 30.260 \\
\hline $2.799 \mathrm{M}$ & 100 & 3 & 1 & 1 & 31.952 \\
\hline $2.799 M$ & & 4 & 2 & 0 & 31.952 \\
\hline 2.610 & 17 & 3 & 2 & 1 & 34.327 \\
\hline 2.455 & 2 & 4 & 0 & 1 & 36.574 \\
\hline
\end{tabular}

\begin{tabular}{|c|c|c|c|c|c|}
\hline $\mathrm{d}(\stackrel{\circ}{\AA})$ & $I^{\text {rel }}$ & & hke & & $2 \theta\left(^{\circ}\right)$ \\
\hline 2.4097 & 1 & 4 & 1 & 1 & 37.285 \\
\hline 2.3233 & 5 & 5 & 2 & 0 & 38.727 \\
\hline 2.2846 & 1 & 4 & 2 & 1 & 39.409 \\
\hline 2.2110 & 1 & 4 & 4 & 0 & 40.779 \\
\hline 2.1461 & 10 & 5 & 3 & 0 & 42.069 \\
\hline 2.0851 & 3 & 6 & 0 & 0 & 43.361 \\
\hline 1.9796 & 33 & 0 & 0 & 2 & 45.799 \\
\hline 1.9538 & 2 & 5 & 4 & 0 & 46.440 \\
\hline 1.9314 & 2 & 4 & 4 & 1 & 47.009 \\
\hline 1.8867 & 3 & 5 & 3 & 1 & 48.193 \\
\hline 1.8653 & 10 & 6 & 3 & 0 & 48.781 \\
\hline 1.8455 & 8 & 6 & 0 & 1 & 49.340 \\
\hline $1.7696 \mathrm{M}$ & 20 & 6 & 2 & 1 & 51.609 \\
\hline $1.7696 \mathrm{M}$ & & 5 & 5 & 0 & 51.609 \\
\hline 1.7524 & 11 & 5 & 4 & 1 & 52.154 \\
\hline 1.7197 & 6 & 3 & 2 & 2 & 53.223 \\
\hline 1.6874 & 7 & 6 & 3 & 1 & 54.323 \\
\hline 1.6729 & 2 & 4 & 0 & 2 & 54.834 \\
\hline 1.6583 & 14 & 4 & 1 & 2 & 55.356 \\
\hline 1.6433 & 3 & 7 & 3 & 0 & 55.906 \\
\hline 1.6158 & 29 & 5 & 5 & 1 & 56.943 \\
\hline 1.5766 & 2 & 7 & 2 & 1 & 58.495 \\
\hline 1.5520 & 2 & 7 & 4 & 0 & 59.516 \\
\hline 1.5415 & $1 \mathrm{~L}$ & 5 & 1 & 2 & 59.961 \\
\hline 1.5173 & 6 & 8 & 2 & 0 & 61.018 \\
\hline 1.5069 & 1 & 5 & 2 & 2 & 61.486 \\
\hline 1.4849 & 1 & 6 & 5 & 1 & 62.500 \\
\hline $1.4750 \mathrm{M}$ & 3 & 4 & 4 & 2 & 62.967 \\
\hline $1.4750 \mathrm{M}$ & & 6 & 6 & 0 & 62.967 \\
\hline 1.4646 & 5 & 8 & 3 & 0 & 63.465 \\
\hline 1.4551 & 5 & 5 & 3 & 2 & 63.925 \\
\hline 1.4452 & 1 & 7 & 4 & 1 & 64.420 \\
\hline 1.4359 & $1 \mathrm{~L}$ & 6 & 0 & 2 & 64.883 \\
\hline $1.3994 \mathrm{M}$ & 5 & 6 & 2 & 2 & 66.797 \\
\hline $1.3994 M$ & & 8 & 4 & 0 & 66.797 \\
\hline 1.3907 & 1 & 5 & 4 & 2 & 67.272 \\
\hline 1.3736 & 4 & 8 & 3 & 1 & 68.219 \\
\hline 1.3654 & 2 & 7 & 5 & 1 & 68.685 \\
\hline 1.3577 & 5 & 6 & 3 & 2 & 69.133 \\
\hline 1.3263 & 1 & 8 & 5 & 0 & 71.015 \\
\hline $1.3194 \mathrm{M}$ & 7 & 7 & 1 & 2 & 71.441 \\
\hline 1. $3194 \mathrm{M}$ & & 8 & 4 & 1 & 71.441 \\
\hline 1.2846 & 2 & 2 & 1 & 3 & 73.689 \\
\hline 1.2705 & 2 & 9 & 4 & 0 & 74.645 \\
\hline 1.2646 & 3 & 7 & 3 & 2 & 75.051 \\
\hline
\end{tabular}


Sodium Antimony Fluoride, $\mathrm{NaSbF}_{4}$

Synonyms

Sodium fluoantimonite

Sodium antimony tetrafluoride

Sample

The sample was from Harshaw Chemical Co., Cleveland, Ohio. There were a number of impurity lines, the strongest being at $\mathrm{d}=$ $3.20,4.91$, and 4.20 with relative intensities of 5,6 , and 12 , respectively.

Because of the impurity, the intensities may be somewhat in error.

Color

Colorless

Optical data

Biaxial (-) $\mathrm{N}_{\alpha}=1.475, \mathrm{~N}_{\beta}=1.521, \mathrm{~N}_{\gamma}=$

1.540. $2 \mathrm{~V}$ is about $75^{\circ}$ (Byström et al., 1953)

Structure

Monoclinic, $\mathrm{P} 21$ (4). The structure of

$\mathrm{NaSbF}_{4}$ was determined by Byström et al.

(1953). The authors gave the space group as $\mathrm{P} 2{ }_{1}$ or $\mathrm{P} 2{ }_{1} / \mathrm{a} . \mathrm{P} 21$ indexed our data more completely than $\mathrm{P} 2{ }_{1} / \mathrm{a}$.

Crystallographic constants of this sample

$$
\begin{aligned}
& \mathrm{a}=8.5749(12) \AA \\
& \mathrm{b}=5.5299(10) \\
& \mathrm{c}=8.0703(9) \\
& \beta=94.089(14)^{\circ} \\
& Z=4 \\
& V=381.71 \AA^{3} \\
& \text { Density }(\mathrm{calc})=3.841 \mathrm{~g} / \mathrm{cm}^{3}
\end{aligned}
$$

\begin{tabular}{|c|c|c|c|c|c|}
\hline \multicolumn{6}{|c|}{$\begin{array}{c}\mathrm{CuK} \alpha_{1} \lambda=1.540598 \AA \text {; mean } \mathrm{T}=26.5{ }^{\circ} \mathrm{C} \\
\text { Internal standard Si, SRM } 640 \mathrm{a}\end{array}$} \\
\hline $\mathrm{d}(\stackrel{\circ}{\mathrm{A}})$ & $\begin{array}{c}\mathrm{I} \text { rel } \\
\sigma= \pm 3\end{array}$ & & hkl & & $2 \theta\left(^{\circ}\right)$ \\
\hline 8.05 & 22 & 0 & 0 & 1 & 10.983 \\
\hline 4.643 & 21 & 1 & 1 & 0 & 19.100 \\
\hline 4.554 & 93 & 0 & 1 & 1 & 19.475 \\
\hline 4.273 & 19 & 2 & 0 & 0 & 20.771 \\
\hline 4.092 & 8 & -1 & 1 & 1 & 21.702 \\
\hline 4.022 & 59 & 0 & 0 & 2 & 22.083 \\
\hline 3.959 & 2 & 1 & 1 & 1 & 22.440 \\
\hline 3.890 & 23 & -2 & 0 & 1 & 22.840 \\
\hline 3.669 & 47 & 2 & 0 & 1 & 24.239 \\
\hline 3.381 & 100 & 2 & 1 & 0 & 26.338 \\
\hline 3.254 & 94 & 0 & 1 & 2 & 27.383 \\
\hline 3.184 & 16 & -2 & 1 & 1 & 28.004 \\
\hline 3.041 & 21 & -2 & 0 & 2 & 29.345 \\
\hline 2.985 & 3 & 1 & 1 & 2 & 29.907 \\
\hline 2.832 & 9 & 2 & 0 & 2 & 31.571 \\
\hline 2.765 & 10 & 0 & 2 & 0 & 32.349 \\
\hline 2.747 & 3 & -3 & 0 & 1 & 32.573 \\
\hline 2.684 & 9 & 0 & 0 & 3 & 33.361 \\
\hline 2.666 & 3 & -2 & 1 & 2 & 33.590 \\
\hline $2.631 \mathrm{M}$ & 6 & 1 & 2 & 0 & 34.049 \\
\hline $2.631 \mathrm{M}$ & & 3 & 0 & 1 & 34.049 \\
\hline 2.520 & 18 & 2 & 1 & 2 & 35.595 \\
\hline 2.415 & 2 & 0 & 1 & 3 & 37.201 \\
\hline 2.375 & 10 & 3 & 1 & 1 & 37.856 \\
\hline 2.363 & 8 & -1 & 1 & 3 & 38.052 \\
\hline 2.349 & 5 & -2 & 0 & 3 & 38.280 \\
\hline 2.285 & 5 & 1 & 1 & 3 & 39.398 \\
\hline 2.2790 & 6 & 0 & 2 & 2 & 39.510 \\
\hline 2.2545 & 17 & -2 & 2 & 1 & 39.957 \\
\hline 2.2241 & 15 & -1 & 2 & 2 & 40.528 \\
\hline $2.2095 \mathrm{M}$ & 10 & -3 & 1 & 2 & 40.808 \\
\hline $2.2095 \mathrm{M}$ & & 2 & 2 & 1 & 40.808 \\
\hline 2.1629 & 20 & -2 & 1 & 3 & 41.726 \\
\hline 2.1388 & 20 & 4 & 0 & 0 & 42.219 \\
\hline $2.0463 \mathrm{M}$ & 44 & 2 & 1 & 3 & 44.227 \\
\hline $2.0463 \mathrm{M}$ & & -2 & 2 & 2 & 44.227 \\
\hline 2.0123 & 13 & 0 & 0 & 4 & 45.015 \\
\hline 1.9903 & 6 & -1 & 0 & 4 & 45.538 \\
\hline 1.9779 & 15 & 2 & 2 & 2 & 45.841 \\
\hline 1.9669 & 10 & -4 & 1 & 1 & 46.111 \\
\hline 1.9256 & 9 & 0 & 2 & 3 & 47.161 \\
\hline 1.9067 & 9 & 4 & 1 & 1 & 47.655 \\
\hline 1.8916 & 22 & 0 & 1 & 4 & 48.062 \\
\hline $1.8731 \mathrm{M}$ & 5 & -1 & 1 & 4 & 48.565 \\
\hline $1.8731 \mathrm{M}$ & & -2 & 0 & 4 & 48.565 \\
\hline 1.8585 & 3 & 1 & 2 & 3 & 48.973 \\
\hline 1.8360 & 21 & -4 & 1 & 2 & 49.614 \\
\hline 1.8164 & 3 & -3 & 2 & 2 & 50.184 \\
\hline 1.7955 & 3 & 0 & 3 & 1 & 50.809 \\
\hline 1.7741 & 17 & -2 & 1 & 4 & 51.466 \\
\hline
\end{tabular}

Figure of merit

$$
\mathrm{F}_{30}=68.2(0.0088,50)
$$

\section{Reference}

Byström, A., Bäcklund, S., and Wilhelmi, K.-A. (1953). Ark. Kemi 6, 77. 
Sodium Barium Niobium 0xide, $\mathrm{NaBa}_{2}\left(\mathrm{NbO}_{3}\right)_{5}$

Synonym

$B-S-N$

Sample

The sample was obtained from Johnson Matthey

Chemicals Limited, Royston, Hertfordshire, UK.

Chemical analysis

Analysis given by the manufacturer: parts per million $\mathrm{Mg}-10, \mathrm{Ca}-2, \mathrm{Al}, \mathrm{Fe}, \mathrm{Si}$, and $\mathrm{Ag}$ - less than 1 .

\section{Structure}

Tetragonal. Bobb et al. (1969) reported

this phase as orthorhombic ( Ccc2), with a and $b$ very nearly equal. Our sample showed no definitive departure from tetragonal. It is a distorted tungsten bronze structure with the $c$ axis doubled. Barns (1968) reported the phase as orthorhombic, with a and b equal.

Crystallographic constants of this sample

$$
\begin{aligned}
& a=17.609(2) \AA \\
& c=7.987(2) \\
& c / a=0.4536 \\
& Z=8 \\
& V=2476.58 \AA^{3} \\
& \text { Density (calc) }=5.376 \mathrm{~g} / \mathrm{cm}^{3}
\end{aligned}
$$

Figure of merit

$\mathrm{F}_{30}=20.0(0.0094,160)$

\section{Polymorphism}

Above $\sim 290{ }^{\circ} \mathrm{C}$ this composition is truly

tetragonal (Abell et al., 1973).

Additional patterns

PDF card 27-1408 (Abell et al., 1973)

PDF card 27-1409 (Cook, W., Priv. Com.)

PDF card 23-654 (Barns, 1968)

\section{References}

Abell, J. S., Harris, I. R., and Cockayne, B. (1973). J. Mater. Sci. 8, 667.

Barns, R. L. (1968). J. Appl. Crystallogr. 1,290 .

Bobb, L. C., Lefkowitz, I., and Muldauer, L. (1969). J. Appl. Crystallogr. 2, 189.

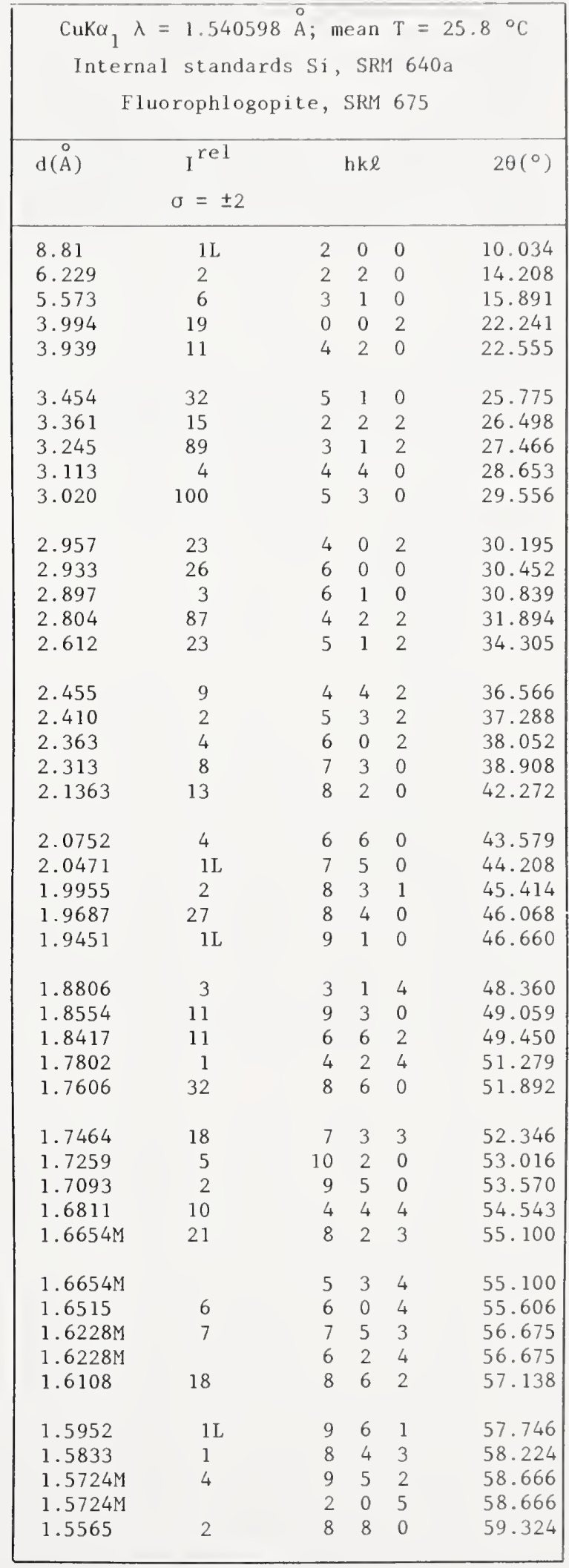


Sodium Germanium Phosphate, $\mathrm{NaGe}_{2}\left(\mathrm{PO}_{4}\right)_{3}$

Synonym

Sodium germanium orthophosphate

CAS registry no. 22239-25 -

Sample

The sample was made by heating a $1: 4: 6$ molar mixture of $\mathrm{Na}_{2} \mathrm{CO}_{3}, \mathrm{GeO}_{2}$, and $\left(\mathrm{NH}_{4}\right)_{2} \mathrm{HPO}_{4}$ up to $500{ }^{\circ} \mathrm{C}$. Then it was reground and heated at $1050{ }^{\circ} \mathrm{C}$ overnight.

Color

Colorless

Structure

Rhombohedral, R*ir. This phase was reported to be isostructural with $\mathrm{NaZr}_{2}\left(\mathrm{PO}_{4}\right)_{3}$ in space group R $\tilde{3} \mathrm{C}$ (Hagman and Kierkegaard, 1968). However several lines could not be indexed on that space group.

Crystallographic constants of this sample (Hexagonal axes)

$a=8.0894(6) \AA$

$c=21.522(3)$

$c / a=2.6605$

$Z=6$

$\mathrm{V}=1219.68 \AA^{3}$

Density $(\mathrm{calc})=3.701 \mathrm{~g} / \mathrm{cm}^{3}$

Figure of merit

$\mathrm{F}_{30}=62.9(0.010,46)$

Additional pattern

PDF card 22-1219 (Hagman and Kierkegaard, 1968)

Reference

Hagman, L.-O. and Kierkegaard, P. (1968).

Acta Chem. Scand. 22, 1822.

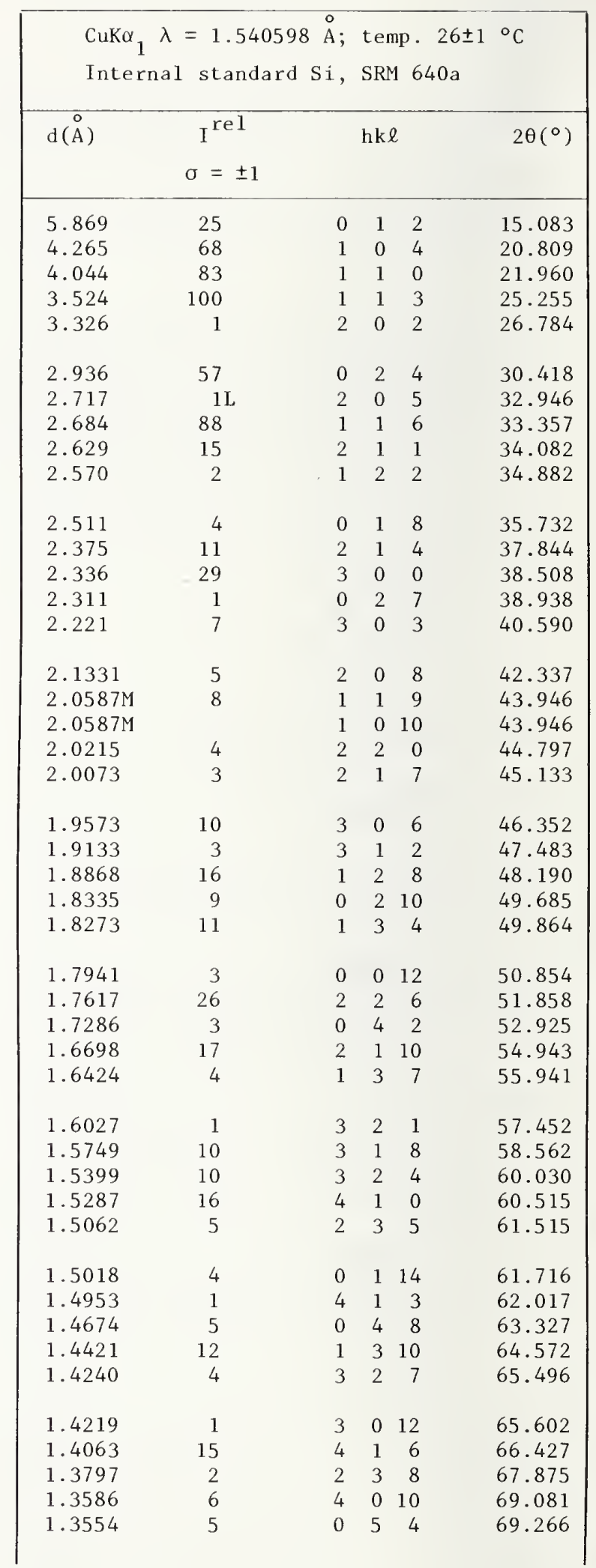


Sodium Germanium Phosphate, $\mathrm{NaGe}_{2}\left(\mathrm{PO}_{4}\right)_{3}$ - (continued)

\begin{tabular}{|llllrl|}
\hline$d(\AA)$ & $I^{\text {rel }}$ & \multicolumn{3}{l}{ hke } & $2 \theta\left(^{\circ}\right)$ \\
& $\sigma= \pm 1$ & & & \\
\hline 1.3525 & 5 & 1 & 1 & 15 & 69.436 \\
1.3480 & 8 & 3 & 3 & 0 & 69.699 \\
1.3293 & 6 & 1 & 2 & 14 & 70.826 \\
1.3213 & $1 \mathrm{~L}$ & 2 & 4 & 1 & 71.319 \\
1.2880 & 4 & 4 & 1 & 9 & 73.463 \\
1.2657 & $1 \mathrm{~L}$ & 4 & 2 & 5 & 74.975 \\
\hline
\end{tabular}




\section{Sodium Iron Silicate (Acmite), $\operatorname{NaFe}\left(\mathrm{SiO}_{3}\right)_{2}$}

Synonyms

Sodium iron metasilicate

Aegirine

Aegirite

CAS registry no.

14483-20-6

Sample

The sample was made by heating $\mathrm{Na}_{2} \mathrm{CO}_{3}, \mathrm{Fe}_{2} \mathrm{O}_{3}$, and $\mathrm{SiO}_{2}$ for about 10 days at temperatures up to $950{ }^{\circ} \mathrm{C}$. The sample contained a small amount of $\mathrm{Fe}_{2} \mathrm{O}_{3}$.

Color

Deep brown

Structure

Monoclinic, I2/a (15). Clinopyroxene type

(Nolan and Edgar, 1963) (Frondel and Klein,

1965). The structure of acmite was

determined by Clark et al. (1969).

Crystallographic constants of this sample

$\mathrm{a}=9.5277(14) \AA$

$\mathrm{b}=8.8067(14)$

$c=5.287(1)$

$\beta=104.686(13)^{\circ}$

$\mathrm{a} / \mathrm{b}=1.0819$

$c / b=0.6003$

$Z=4$

$\begin{array}{ll}Z & =4 \\ V & =429.13 \AA^{3}\end{array}$

Density $(\mathrm{calc})=3.576 \mathrm{~g} / \mathrm{cm}^{3}$

Figure of merit

$\mathrm{F}_{30}=53.3(0.011,51)$

Additional patterns

PDF card 18-1222 (Frondel and Klein, 1965)

Nat. Min.

PDF card 31-1309 (Nickel and Mark, 1965)

Nat. Min.

Borg and Smith (1969)

Brown (1982)

\section{References}

Borg, I. Y. and Smith, D. K. (1969).

Geological Society of America Inc., Memoir 122, Boulder, CO.

Brown, I. W. (1982). J. Non Cryst. Solids 50, 233 .

Clark, J., Appleman, D. E., and Papike, J. J.

(1969). Contrib. Mineral. Petrol., 20, 81.

Frondel, C. and Klein, C. (1965). Science

(Washington, D.C.), 149, 742 .

Nickel, E. H. and Mark, E. (1965). Can. Mineral. 8,185 .

Nolan, J. and Edgar, A. D. (1963). Mineral. Mag. J. Minera1. Soc. 33, 634.

\begin{tabular}{|c|c|c|c|c|c|}
\hline \multicolumn{6}{|c|}{$\begin{array}{c}\mathrm{CuK}_{1} \lambda=1.540598 \AA \text {; mean } \mathrm{T}=2 \\
\text { Internal standards Si, SRM } 640 a \\
\text { Fluorophlogopite, SRM } 675\end{array}$} \\
\hline $\mathrm{d}(\AA)$ & $\begin{array}{c}I^{\text {rel }} \\
\sigma= \pm 2\end{array}$ & & thke & & $2 \theta\left(^{\circ}\right)$ \\
\hline 6.37 & 21 & 1 & 1 & 0 & 13.887 \\
\hline 4.609 & 1 & 2 & 0 & 0 & 19.242 \\
\hline 4.423 & 29 & 0 & 1 & 1 & 20.058 \\
\hline 3.613 & 9 & -2 & 1 & 1 & 24.620 \\
\hline 3.185 & 6 & 2 & 2 & 0 & 27.994 \\
\hline 2.985 & 100 & 1 & 2 & 1 & 29.914 \\
\hline 2.901 & 50 & 3 & 1 & 0 & 30.799 \\
\hline 2.798 & 1 & 1 & 3 & 0 & 31.965 \\
\hline 2.546 & 28 & 0 & 3 & 1 & 35.228 \\
\hline 2.523 & 45 & -2 & 0 & 2 & 35.550 \\
\hline 2.473 & 37 & -3 & 2 & 1 & 36.303 \\
\hline 2.256 & 3 & -4 & 1 & 1 & 39.926 \\
\hline 2.212 & 8 & 0 & 2 & 2 & 40.767 \\
\hline 2.196 & 20 & -3 & 1 & 2 & 41.061 \\
\hline 2.190 & 7 & -2 & 2 & 2 & 41.185 \\
\hline 2.118 & 20 & 2 & 3 & 1 & 42.650 \\
\hline 2.094 & 12 & 3 & 2 & 1 & 43.160 \\
\hline 2.0294 & 10 & 2 & 0 & 2 & 44.613 \\
\hline 2.0186 & 10 & -1 & 4 & 1 & 44.865 \\
\hline 1.9796 & 7 & -4 & 0 & 2 & 45.800 \\
\hline 1.9341 & 5 & 1 & 4 & 1 & 46.940 \\
\hline 1.8807 & 2 & 4 & 1 & 1 & 48.357 \\
\hline 1.8262 & 2 & -4 & 3 & 1 & 49.897 \\
\hline $1.8045 \mathrm{M}$ & 1 & -4 & 2 & 2 & 50.538 \\
\hline $1.8045 \mathrm{M}$ & & 5 & 1 & 0 & 50.538 \\
\hline 1.7732 & 1 & -3 & 4 & 1 & 51.497 \\
\hline 1.7403 & 2 & -5 & 2 & 1 & 52.542 \\
\hline 1.7303 & 9 & 1 & 5 & 0 & 52.870 \\
\hline 1.7244 & 7 & 3 & 1 & 2 & 53.066 \\
\hline 1.6829 & 2 & -5 & 1 & 2 & 54.481 \\
\hline 1.6588 & 4 & -2 & 4 & 2 & 55.339 \\
\hline 1.6327 & 11 & -1 & 2 & 3 & 56.301 \\
\hline 1.6104 & 12 & 4 & 3 & 1 & 57.151 \\
\hline 1.5915 & 6 & 4 & 4 & 0 & 57.893 \\
\hline 1.5362 & 7 & 6 & 0 & 0 & 60.191 \\
\hline 1.5295 & 9 & 4 & 0 & 2 & 60.479 \\
\hline
\end{tabular}


Synonym

Sodium molybdate hydrate

CAS registry no. $10102-40-6$

Sample

The sample was obtained from the General Chemical Division of Allied Chemical, Morristown, NJ.

Color

Colorless

Structure

Orthorhombic, Pcab (61) (Pistorius, 1960).

The structure of $\mathrm{Na}_{2} \mathrm{MoO}_{4} \cdot 2 \mathrm{H}_{2} \mathrm{O}$ was determined by Matsumato et al. (1975).

Crystallographic constants of this sample

$$
\begin{aligned}
& \mathrm{a}=10.566(2) \AA \\
& \mathrm{b}=13.842(2) \\
& \mathrm{c}=8.4823(15) \\
& \mathrm{a} / \mathrm{b}=0.7633 \\
& \mathrm{c} / \mathrm{b}=0.6128 \\
& \mathrm{Z}=8 \\
& \mathrm{~V}=1240.65 \AA^{3} \\
& \text { Density (calc) } 2.591 \mathrm{~g} / \mathrm{cm}^{3}
\end{aligned}
$$

Figure of merit

$$
\mathrm{F}_{30}=44.4(0.012,59)
$$

Additional pattern

PDF card 13-236 (Pistorius, 1960)

\section{References}

Matsumato, K., Kobayashi, A., and Sasaki, Y. (1975). Bull. Chem. Soc. Jpn. 48, 1009.

Pistorius, C. W. F. T. (1960). Z. Kristallogr. $114,154$.

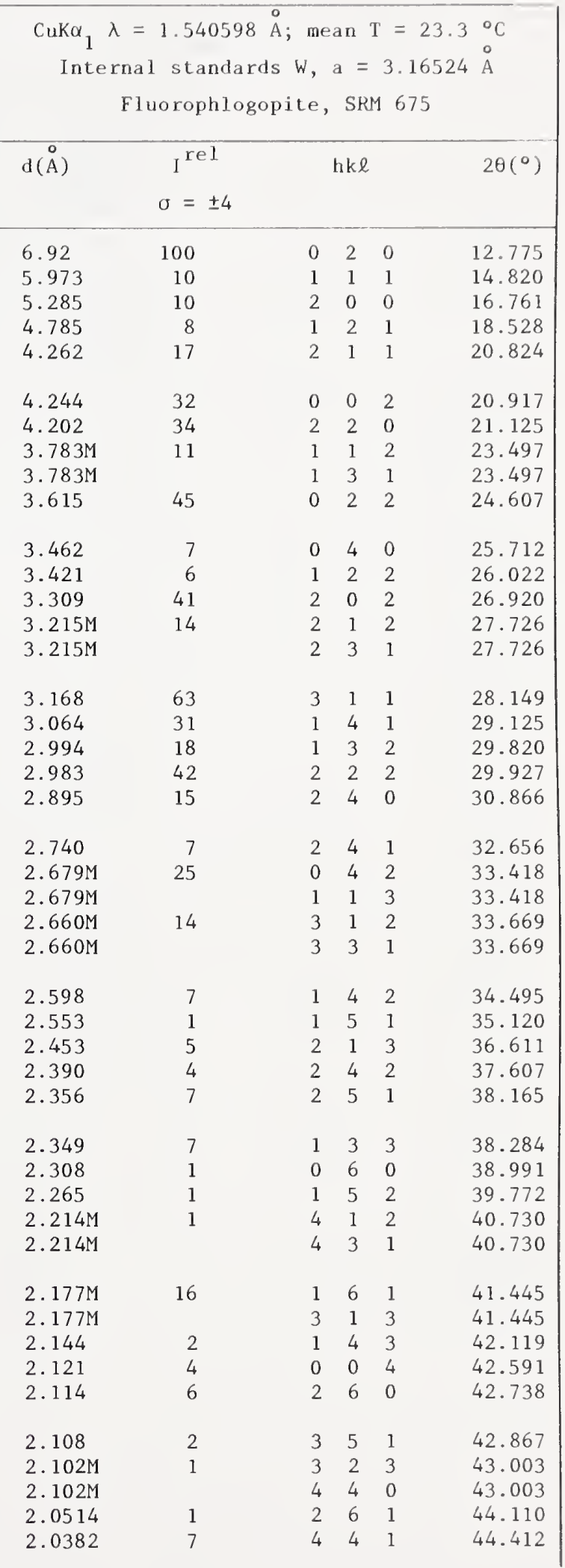


Sodium Molybdenum Oxide Hydrate, $\mathrm{Na}_{2} \mathrm{MoO}_{4} \cdot 2 \mathrm{H}_{2} \mathrm{O}$ - (continued)

\begin{tabular}{|c|c|c|c|c|c|}
\hline$d(\stackrel{\circ}{A})$ & $I^{\text {rel }}$ & & hke & & $2 \theta\left({ }^{\circ}\right)$ \\
\hline 2.0294 & 17 & 5 & 1 & 1 & 44.613 \\
\hline $1.9908 \mathrm{M}$ & 11 & 1 & 2 & 4 & 45.528 \\
\hline $1.9908 \mathrm{M}$ & & 1 & 6 & 2 & 45.528 \\
\hline 1.9687 & 3 & 2 & 0 & 4 & 46.067 \\
\hline $1.8927 \mathrm{M}$ & 12 & 2 & 2 & 4 & 48.032 \\
\hline $1.8927 \mathrm{M}$ & & 2 & 6 & 2 & 48.032 \\
\hline $1.8810 \mathrm{M}$ & 7 & 3 & 6 & 1 & 48.348 \\
\hline $1.8810 \mathrm{M}$ & & 4 & 4 & 2 & 48.348 \\
\hline $1.8747 \mathrm{M}$ & 9 & 5 & 1 & 2 & 48.523 \\
\hline $1.8747 \mathrm{M}$ & & 5 & 3 & 1 & 48.523 \\
\hline $1.8597 \mathrm{M}$ & 1 & 3 & 4 & 3 & 48.940 \\
\hline $1.8597 \mathrm{M}$ & & 4 & 2 & 3 & 48.940 \\
\hline 1.8525 & 1 & 2 & 5 & 3 & 49.140 \\
\hline 1.8081 & 4 & 0 & 4 & 4 & 50.431 \\
\hline 1.8033 & 2 & 5 & 4 & 0 & 50.574 \\
\hline 1.7809 & 5 & 4 & 3 & 3 & 51.257 \\
\hline $1.7619 \mathrm{M}$ & 6 & 1 & 6 & 3 & 51.851 \\
\hline $1.7619 M$ & & 6 & 0 & 0 & 51.851 \\
\hline 1.7426 & 5 & 4 & 5 & 2 & 52.470 \\
\hline $1.7240 \mathrm{M}$ & 3 & 3 & 5 & 3 & 53.077 \\
\hline $1.7240 \mathrm{M}$ & & 6 & 0 & 1 & 53.077 \\
\hline $1.7104 \mathrm{M}$ & 3 & 6 & 1 & 1 & 53.533 \\
\hline $1.7104 \mathrm{M}$ & & 2 & 4 & 4 & 53.533 \\
\hline 1.6942 & 2 & 2 & 6 & 3 & 54.088 \\
\hline 1.6798 & 13 & 5 & 1 & 3 & 54.588 \\
\hline 1.6728 & 12 & 6 & 2 & 1 & 54.835 \\
\hline $1.6634 \mathrm{M}$ & 1 & 1 & 1 & 5 & 55.172 \\
\hline $1.6634 \mathrm{M}$ & & 1 & 5 & 4 & 55.172 \\
\hline 1.6479 & 2 & 5 & 5 & 1 & 55.738 \\
\hline $1.6436 \mathrm{M}$ & 1 & 2 & 8 & 0 & 55.896 \\
\hline $1.6436 \mathrm{M}$ & & 5 & 2 & 3 & 55.896 \\
\hline
\end{tabular}


Synonym

Sodium strontium niobate

Sample

The sample was made by heating $\mathrm{NaNbO}_{3}, \mathrm{SrCO}_{3}$, and $\mathrm{Nb}_{2} \mathrm{O}_{5}$ up to $1300{ }^{\circ} \mathrm{C}$ for about 48 hours, with intermediate grindings.

Color

Colorless

Structure

Tetragonal. Tungsten bronze structure (Morin, 1973). This phase has been reported as orthorhombic (Giess et al., 1969) but this work found no evidence for symmetry lower than tetragonal. This phase occurs in the region of the $\mathrm{NaNbO}_{3}-\mathrm{SrNb}_{2} \mathrm{O}_{6}$ system from 65 to $79 \%$ $\mathrm{SrNb}_{2} \mathrm{O}_{6}$ and changes cell size little over the region (Morin, 1973).

Crystallographic constants of this sample $\mathrm{a}=12.3562(9) \AA$

$c=3.8979(6)$

$c / a=0.3155$

$Z=2$

$\begin{array}{ll}\mathrm{V} & =595.11 \mathrm{~A}^{3}\end{array}$

Density $(\mathrm{calc})=5.038 \mathrm{~g} / \mathrm{cm}^{3}$

Figure of merit

$\mathrm{F}_{30}=57.3(0.011,50)$

Polymorphism

Van Uitert (1968) reports that $\mathrm{NaSr}_{2} \mathrm{Nb}_{5} \mathrm{O}_{15}$

is stable as tetragonal only above $184^{\circ} \mathrm{C}$.

Additional pattern

PDF card 27-787 (Morin, Priv. Comm., 1974).

The composition given was $\mathrm{Na}_{0}{ }_{1}{ }_{8} \mathrm{Sr}_{0},{ }_{41} \mathrm{NbO}_{3}$.

\section{References}

Giess, E. A., Scott, B. A., Burns, G., O'Kane, D. F., and Segmüller, A. (1969). J. Am.

Ceram. Soc. 52, 276.

Morin, D., Colin, J.-P., LeRoux, G. , Pateau, L., and Toledano, J.-C. (1973).

Mater. Res. Bull. 8, 1189.

Van Uitert, L. G., Levinstein, H. J., Rubin, J. J., Capio, C. D., Dearborn, E. F., and Bonner, W. A. (1968). Mater. Res. Bul1. 3, 47.

\begin{tabular}{|c|c|c|c|c|c|}
\hline \multicolumn{6}{|c|}{$\begin{array}{l}\mathrm{CuKa}_{1} \lambda=1.540598 \AA \text {; mean } \mathrm{T}=26.1{ }^{\circ} \mathrm{C} \\
\text { Internal standard Si, SRM } 640 \mathrm{a}\end{array}$} \\
\hline$d(\AA)$ & $\mathrm{I}^{\mathrm{rel}}$ & & hk 2 & & $2 \theta\left(^{\circ}\right)$ \\
\hline 6.184 & 1 & 2 & 0 & 0 & 14.312 \\
\hline 5.526 & 1 & 2 & 1 & 0 & 16.025 \\
\hline 4.369 & 1 & 2 & 2 & 0 & 20.312 \\
\hline 3.899 & 45 & 0 & 0 & 1 & 22.789 \\
\hline 3.556 & 2 & 1 & 1 & 1 & 25.022 \\
\hline 3.426 & 32 & 3 & 2 & 0 & 25.989 \\
\hline 3.297 & 2 & 2 & 0 & 1 & 27.025 \\
\hline 3.185 & 47 & 2 & 1 & 1 & 27.992 \\
\hline 3.088 & 8 & 4 & 0 & 0 & 28.887 \\
\hline 2.996 & 62 & 4 & 1 & 0 & 29.795 \\
\hline 2.909 & 23 & 2 & 2 & 1 & 30.711 \\
\hline 2.760 & 100 & 3 & 1 & 1 & 32.414 \\
\hline 2.574 & 25 & 3 & 2 & 1 & 34.831 \\
\hline 2.421 & 5 & 4 & 0 & 1 & 37.101 \\
\hline 2.376 & 2 & 4 & 1 & 1 & 37.841 \\
\hline 2.295 & 4 & 5 & 2 & 0 & 39.232 \\
\hline 2.255 & 2 & 4 & 2 & 1 & 39.952 \\
\hline 2.185 & $1 \mathrm{~L}$ & 4 & 4 & 0 & 41.287 \\
\hline 2.1186 & 7 & 5 & 3 & 0 & 42.641 \\
\hline $2.0591 \mathrm{M}$ & 1 & 6 & 0 & 0 & 43.936 \\
\hline $2.0591 \mathrm{M}$ & & 5 & 1 & 1 & 43.936 \\
\hline 2.0312 & 2 & 6 & 1 & 0 & 44.572 \\
\hline 1.9774 & 1 & 5 & 2 & 1 & 45.854 \\
\hline 1.9546 & 9 & 6 & 2 & 0 & 46.419 \\
\hline 1.9491 & 23 & 0 & 0 & 2 & 46.558 \\
\hline 1.9314 & 1 & 5 & 4 & 0 & 47.011 \\
\hline 1.9064 & 1 & 4 & 4 & 1 & 47.664 \\
\hline 1.8608 & 2 & 5 & 3 & 1 & 48.908 \\
\hline 1.8421 & 12 & 6 & 3 & 0 & 49.437 \\
\hline 1.8206 & 9 & 6 & 0 & 1 & 50.060 \\
\hline 1.7606 & 4 & 3 & 0 & 2 & 51.890 \\
\hline $1.7470 \mathrm{M}$ & 33 & 5 & 5 & 0 & 52.326 \\
\hline $1.7470 \mathrm{M}$ & & 6 & 2 & 1 & 52.326 \\
\hline 1.7295 & 12 & 5 & 4 & 1 & 52.896 \\
\hline 1.6943 & 8 & 3 & 2 & 2 & 54.085 \\
\hline 1.6656 & 9 & 6 & 3 & 1 & 55.095 \\
\hline 1.6479 & 2 & 4 & 0 & 2 & 55.736 \\
\hline 1.6336 & 17 & 4 & 1 & 2 & 56.268 \\
\hline 1.6197 & 3 & 3 & 3 & 2 & 56.794 \\
\hline 1.6080 & 4 & 7 & 0 & 1 & 57.246 \\
\hline
\end{tabular}


Sodium Strontium Niobium Oxide, $\mathrm{NaSr}_{2}\left(\mathrm{NbO}_{3}\right)_{5}-$ (continued)

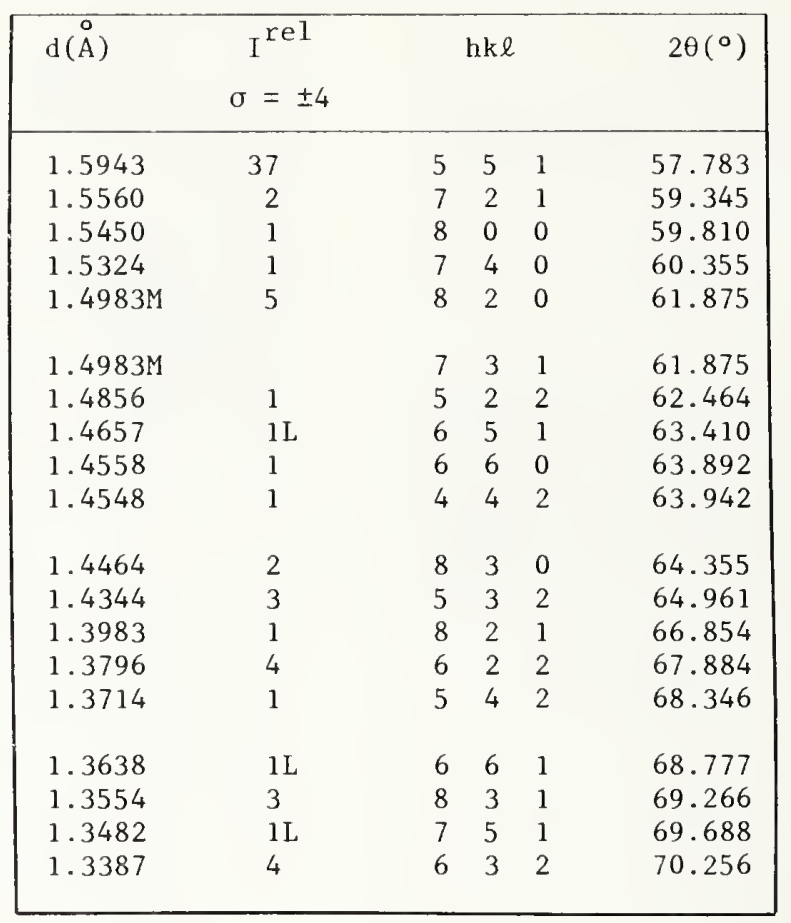


Strontium Aluminum Oxide, $\mathrm{SrAl}_{2} \mathrm{O}_{4}$

Synonym

Strontium aluminate

CAS registry no. 12004-37-4

Sample

One mole of $\mathrm{SrCO}_{3}$ was reacted with two moles of $\mathrm{Al}(\mathrm{OH})_{3}$ by heating from $500^{\circ}$ to $1000{ }^{\circ} \mathrm{C}$ where it was held for 3 hours, then at $1500{ }^{\circ} \mathrm{C}$ for 40 hours. The sample was ground and heated at $550{ }^{\circ} \mathrm{C}$ for 7 hours.

\section{Color}

Colorless

\section{Structure}

Monoclinic, $\mathrm{P} * / *$. The cell was found by use of the Visser program. A unit cell given by Ito et al. (1977) was that of a supercell with four times the volume of the Visser cell.

Crystallographic constants of this sample

$$
\begin{aligned}
& a=8.4424(8) \AA \\
& b=8.8221(10) \\
& c=5.1607(6) \\
& \beta=93.415(6)^{\circ} \\
& a / b=0.9570 \\
& c / b=0.5850 \\
& Z=4 \\
& V=383.68 \AA^{3} \\
& \text { Density (calc) }=3.559 \mathrm{~g} / \mathrm{cm}^{3}
\end{aligned}
$$

Figures of merit

$$
\mathrm{F}_{30}=83.1(0.0071,51)
$$

$M_{20}=47.6$

\section{Polymorphism}

There is a hexagonal phase formed above $650{ }^{\circ} \mathrm{C}$ (Ito et al., 1977)

Additional pattern PDF card 9-39 (Carlson, 1955)

\section{References}

Carlson, E. (1955). J. Res. Nat1.

Bur. Stand. 54, 332 .

Ito, S., Banno, S., Suzuki, K., and Inagaki, M. (1977). Z. Phys. Chem. Wiesbaden 105, 173.

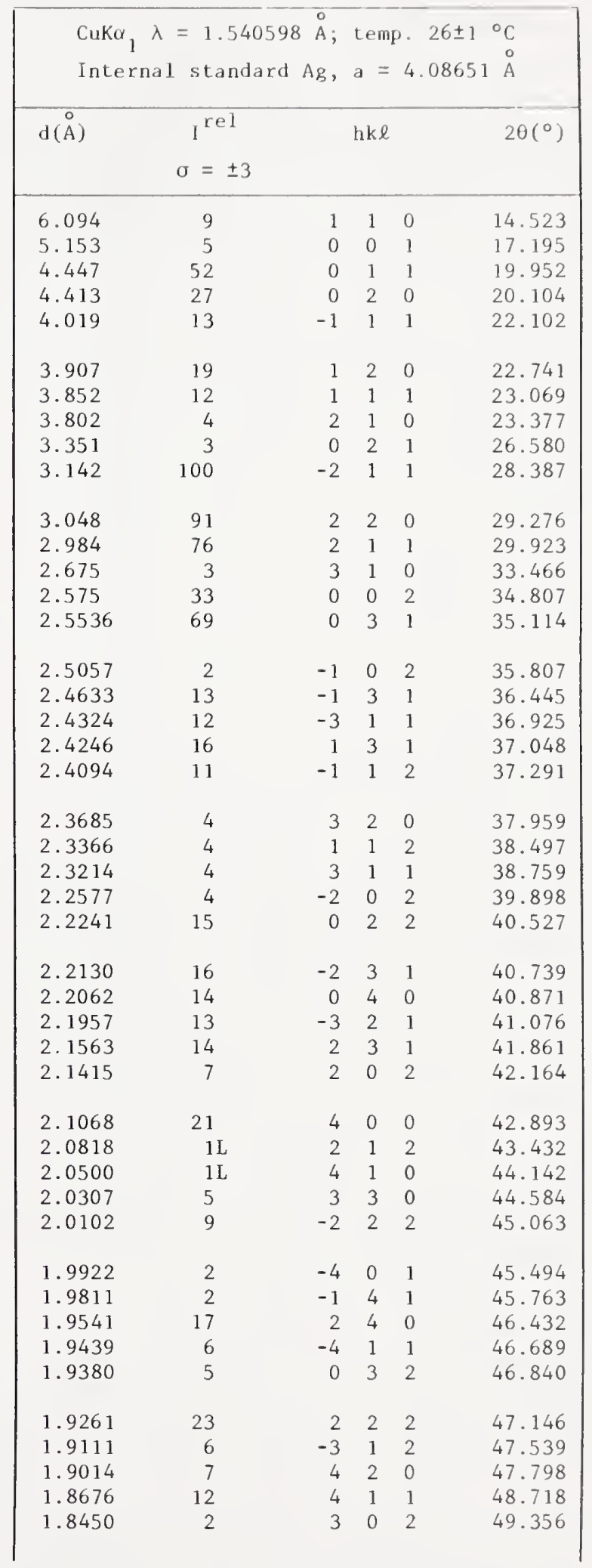


Strontium Aluminum Oxide, $\mathrm{SrAl}_{2} \mathrm{O}_{4}$ - (continued)

\begin{tabular}{|c|c|c|c|c|c|}
\hline $\mathrm{d}(\AA)$ & $I^{\text {rel }}$ & & hke & & $2 \theta\left({ }^{\circ}\right)$ \\
\hline 1.8155 & 3 & -4 & 2 & 1 & 50.211 \\
\hline 1.7891 & 3 & -3 & 2 & 2 & 51.004 \\
\hline 1.7344 & 4 & 3 & 4 & 0 & 52.736 \\
\hline 1.7013 & 3 & 3 & 2 & 2 & 53.845 \\
\hline 1.6804 & 5 & -4 & 0 & 2 & 54.567 \\
\hline 1.6757 & 5 & 0 & 4 & 2 & 54.734 \\
\hline 1.6688 & 6 & 0 & 5 & 1 & 54.978 \\
\hline 1.6557 & 7 & 5 & 1 & 0 & 55.450 \\
\hline 1.6505 & 6 & -4 & 1 & 2 & 55.640 \\
\hline 1.6293 & 8 & -3 & 3 & 2 & 56.428 \\
\hline 1.6021 & 11 & 4 & 3 & 1 & 57.477 \\
\hline 1.5975 & 10 & -2 & 1 & 3 & 57.657 \\
\hline 1.5884 & 4 & -1 & 2 & 3 & 58.018 \\
\hline 1.5851 & 6 & 4 & 0 & 2 & 58.152 \\
\hline 1.5780 & 6 & -2 & 4 & 2 & 58.439 \\
\hline 1.5741 & 6 & 5 & 0 & 1 & 58.595 \\
\hline 1.5704 & 6 & -4 & 2 & 2 & 58.747 \\
\hline 1.5623 & 8 & -2 & 5 & 1 & 59.082 \\
\hline 1.5496 & 2 & 5 & 1 & 1 & 59.615 \\
\hline 1.5417 & 7 & 2 & 5 & 1 & 59.954 \\
\hline 1.5343 & 12 & 2 & 1 & 3 & 60.270 \\
\hline 1.5234 & 5 & 4 & 4 & 0 & 60.750 \\
\hline 1.5055 & 2 & -3 & 0 & 3 & 61.550 \\
\hline 1.4913 & 4 & 4 & 2 & 2 & 62.197 \\
\hline 1.4825 & 13 & 5 & 2 & 1 & 62.610 \\
\hline 1.4791 & 8 & -4 & 4 & 1 & 62.772 \\
\hline 1.4706 & 9 & 0 & 6 & 0 & 63.177 \\
\hline 1.4589 & 3 & -4 & 3 & 2 & 63.742 \\
\hline 1.4315 & 3 & -5 & 1 & 2 & 65.110 \\
\hline 1.4143 & 3 & 0 & 6 & 1 & 66.000 \\
\hline 1.4100 & 3 & 3 & 1 & 3 & 66.230 \\
\hline 1.4044 & 5 & 6 & 0 & 0 & 66.525 \\
\hline
\end{tabular}


Synonym

Strontium metasilicate

CAS registry no.

127 12-63-9

Sample

The sample was made by heating a mixture of $\mathrm{SrCO}_{3}$ and amorphous $\mathrm{SiO}_{2}$ for 21 hours at $1400{ }^{\circ} \mathrm{C}$, followed by grinding and heating about 5 days at $1300{ }^{\circ} \mathrm{C}$.

Color

Colorless

Structure

Monoclinic, C2 (5). The structure of $\alpha-\mathrm{SrSiO}_{3}$ was determined by Machida et al. (1982). This phase was earlier indexed as hexagona 1 .

Crystallographic constants of this sample

$$
\begin{aligned}
& a=12.340(3) \AA \\
& b=7.136(2) \\
& c=10.880(3) \\
& \beta=111.51(3)^{\circ} \\
& a / b=1.7293 \\
& c / b=1.5247 \\
& Z=12 \\
& V=891.35 \AA^{3} \\
& \text { Density (calc) }=3.660 \mathrm{~g} / \mathrm{cm}^{3}
\end{aligned}
$$

Figure of merit

$$
\mathrm{F}_{30}=16.7(0.015,117)
$$

\section{Polymorphism}

There are 2 high pressure forms reported by Shimizu et al. (1970).

\section{Additional patterns}

PDF card 30-1302 (Shimizu et al., 1970)

PDF card 32-1258 (Moir et al., 1975).

\begin{tabular}{|c|c|c|c|c|c|}
\hline \multicolumn{6}{|c|}{$\begin{array}{c}\mathrm{CuK}_{1} \lambda=1.540598 \AA \text {; mean } \mathrm{T}=25.2{ }^{\circ} \mathrm{C} \\
\text { Internal standards Si, SRM } 640 \mathrm{a} \\
\text { Fluorophlogopite, SRM } 675\end{array}$} \\
\hline $\mathrm{d}(\stackrel{\circ}{\mathrm{A}})$ & $\begin{array}{c}\text { rel } \\
\sigma= \pm 2\end{array}$ & & hke & & $2 \theta\left({ }^{\circ}\right)$ \\
\hline 6.05 & $1 \mathrm{~L}$ & 1 & 1 & 0 & 14.624 \\
\hline 5.73 & $1 \mathrm{~L}$ & 2 & 0 & 0 & 15.457 \\
\hline 5.056 & 17 & 0 & 0 & 2 & 17.527 \\
\hline 4.803 & $1 \mathrm{~L}$ & 1 & 1 & 1 & 18.456 \\
\hline 4.755 & 1 & -2 & 0 & 2 & 18.647 \\
\hline 4. 320 & 1 & -1 & 1 & 2 & 20.541 \\
\hline $3.566 \mathrm{M}$ & 66 & 0 & 2 & 0 & 24.951 \\
\hline $3.566 \mathrm{M}$ & & -3 & 1 & 1 & 24.951 \\
\hline 3.365 & 30 & 0 & 2 & 1 & 26.466 \\
\hline 3.254 & 1 & 2 & 0 & 2 & 27.389 \\
\hline 3.225 & $1 \mathrm{~L}$ & -1 & 1 & 3 & 27.639 \\
\hline 2.928 & 50 & 3 & 1 & 1 & 30.510 \\
\hline 2.917 & 100 & 0 & 2 & 2 & 30.627 \\
\hline 2.760 & 1 & 2 & 2 & 1 & 32.409 \\
\hline $2.531+$ & 21 & 2 & 0 & 3 & 35.437 \\
\hline $2.531+$ & & 0 & 0 & 4 & 35.437 \\
\hline 2.514 & 9 & -1 & 1 & 4 & 35.692 \\
\hline 2.462 & 1 & 3 & 1 & 2 & 36.463 \\
\hline 2.452 & 1 & 0 & 2 & 3 & 36.621 \\
\hline 2.324 & 1 & -5 & 1 & 2 & 38.715 \\
\hline 2.185 & $1 L$ & 5 & 1 & 0 & 41.287 \\
\hline 2.073 & 27 & 3 & 1 & 3 & 43.629 \\
\hline 2.060 & 75 & -3 & 3 & 1 & 43.906 \\
\hline 1.914 & 18 & 6 & 0 & 0 & 47.453 \\
\hline 1.909 & 22 & 3 & 3 & 1 & 47.593 \\
\hline 1.897 & 3 & -6 & 0 & 4 & 47.913 \\
\hline 1.854 & $1 \mathrm{~L}$ & 1 & 3 & 3 & 49.092 \\
\hline 1.808 & $1 \mathrm{~L}$ & -2 & 0 & 6 & 50.430 \\
\hline 1.7837 & 10 & 0 & 4 & 0 & 51.169 \\
\hline 1.7579 & 2 & -6 & 2 & 1 & 51.976 \\
\hline $1.7525+$ & 2 & -3 & 3 & 4 & 52.151 \\
\hline $1.7525+$ & & -3 & 1 & 6 & 52.151 \\
\hline $1.6858 \mathrm{M}$ & 9 & 0 & 0 & 6 & 54.378 \\
\hline $1.6858 \mathrm{M}$ & & 6 & 2 & 0 & 54.378 \\
\hline 1.6821 & 11 & 0 & 4 & 2 & 54.507 \\
\hline 1.6767 & 7 & -6 & 2 & 4 & 54.699 \\
\hline 1.6724 & 2 & -7 & 1 & 1 & 54.851 \\
\hline 1.6023 & 4 & 3 & 3 & 3 & 57.470 \\
\hline 1.5983 & 5 & 7 & 1 & 0 & 57.626 \\
\hline 1.5967 & 4 & 5 & 1 & 3 & 57.689 \\
\hline
\end{tabular}

\section{References}

Machida, K., Adachi, G., Shiokawa, J., Shimada, M., and Koizumi, M. (1982). Acta Crystallogr. B38, 386.

Moir, G. K., Gand, J. A., and Glasser, F. P. (1975). Z. Kristallogr., 141, 437.

Shimizu, Y., Syono, Y., and Akimoto, S.

(1970). High Temp. High Pressures 2, 113. 
Synonym

Strontium zirconium orthophosphate

CAS registry no. 67972-88-7

Sample

The sample was made by heating a 1:4:6 molar mixture of $\mathrm{SrCO}_{3}, \mathrm{ZrO}_{2}$, and $\left(\mathrm{NH}_{4}\right)_{2} \mathrm{HPO}_{4}$ up to $500{ }^{\circ} \mathrm{C}$. After regrinding it was heated to $1300{ }^{\circ} \mathrm{C}$ for 70 hours.

Color

Colorless

Structure

Rhombohedral, $\mathrm{R} * *$ The structure is similar to that of $\mathrm{NaZr}_{2}\left(\mathrm{PO}_{4}\right)_{3}$ (Chernorukov et al.,
1978.)

Crystallographic constants of this sample (Hexagonal axes)

$\mathrm{a}=8.6935(4) \AA$

$c=23.389(2)$

$c / a=2.6904$

$Z=3$

$V=1530.85 \AA^{3}$

Density $(\mathrm{calc})=3.327 \mathrm{~g} / \mathrm{cm}^{3}$

Figure of merit

$\mathrm{F}_{30}=119.9(0.0068,37)$

Reference

Chernorukov, N. G., Korshunov, I. A., and Prokof'eva, T. V. (1978). Sov. Phys.

Crystallogr. Engl. Transl. 23, 475.

\begin{tabular}{|c|c|c|c|c|c|}
\hline \multicolumn{6}{|c|}{$\begin{array}{l}\text { CuK } \alpha_{1} \lambda=1.540598 \AA \text {; temp. } \\
\text { Internal standards } \mathrm{Si}, \text { SRM } 6 \\
\text { Fluorophlogopite, SRM } 675\end{array}$} \\
\hline $\mathrm{d}(\stackrel{\circ}{\mathrm{A}})$ & $\begin{array}{c}\text { rel } \\
\sigma= \pm 3\end{array}$ & & hke & & $2 \theta\left(^{\circ}\right)$ \\
\hline 7.796 & 4 & 0 & 0 & 3 & 11.341 \\
\hline 7.170 & 9 & 1 & 0 & 1 & 12.334 \\
\hline 6.333 & 10 & 0 & 1 & 2 & 13.972 \\
\hline 4.619 & 35 & 1 & 0 & 4 & 19.198 \\
\hline 4.348 & 75 & 1 & 1 & 0 & 20.407 \\
\hline 3.972 & 5 & 0 & 1 & 5 & 22.366 \\
\hline 3.795 & 74 & 1 & 1 & 3 & 23.420 \\
\hline 3.716 & 2 & 0 & 2 & 1 & 23.926 \\
\hline 3.583 & 2 & .2 & 0 & 2 & 24.829 \\
\hline 3.165 & 36 & 0 & 2 & 4 & 28.172 \\
\hline 3.053 & 1 & 1 & 0 & 7 & 29.232 \\
\hline 2.933 & 6 & 2 & 0 & 5 & 30.451 \\
\hline 2.900 & 100 & 1 & 1 & 6 & 30.804 \\
\hline 2.825 & 12 & 2 & 1 & 1 & 31.646 \\
\hline 2.766 & 1 & 1 & 2 & 2 & 32.341 \\
\hline 2.726 & 5 & 0 & 1 & 8 & 32.830 \\
\hline 2.5579 & 10 & 2 & 1 & 4 & 35.053 \\
\hline 2.5084 & 34 & 3 & 0 & 0 & 35.768 \\
\hline 2.4307 & 4 & 1 & 2 & 5 & 36.951 \\
\hline 2.3884 & 3 & 3 & 0 & 3 & 37.630 \\
\hline 2.3089 & 5 & 2 & 0 & 8 & 38.977 \\
\hline 2.2305 & 5 & 1 & 1 & 9 & 40.406 \\
\hline 2.1736 & 8 & 2 & 2 & 0 & 41.512 \\
\hline 2.1665 & 8 & 2 & 1 & 7 & 41.655 \\
\hline 2.1098 & 13 & 3 & 0 & 6 & 42.827 \\
\hline 2.0933 & 4 & 2 & 2 & 3 & 43.182 \\
\hline 2.0395 & 16 & 1 & 2 & 8 & 44.381 \\
\hline 1.9862 & 7 & 0 & & 10 & 45.639 \\
\hline 1.9664 & 7 & 1 & 3 & 4 & 46.125 \\
\hline 1.9486 & 2 & 0 & 0 & 12 & 46.570 \\
\hline 1.9067 & 4 & 3 & 1 & 5 & 47.655 \\
\hline 1.8980 & 29 & 2 & 2 & 6 & 47.888 \\
\hline 1.8762 & 1 & 4 & 0 & 1 & 48.480 \\
\hline 1.8582 & 2 & 0 & 4 & 2 & 48.981 \\
\hline 1.8526 & 1 & 2 & 0 & 11 & 49.138 \\
\hline $1.8064 \mathrm{M}$ & 16 & 2 & 1 & 10 & 50.482 \\
\hline $1.8064 \mathrm{M}$ & & 3 & 0 & 9 & 50.482 \\
\hline 1.7702 & 3 & 1 & 3 & 7 & 51.588 \\
\hline 1.7495 & 1 & 1 & 0 & 13 & 52.245 \\
\hline 1.7456 & 1 & 0 & 4 & 5 & 52.372 \\
\hline 1.6989 & 8 & 3 & 1 & 8 & 53.926 \\
\hline 1.6665 & 1 & 2 & 2 & 9 & 55.061 \\
\hline 1.6561 & 6 & 3 & 2 & 4 & 55.438 \\
\hline 1.6426 & 20 & 4 & 1 & 0 & 55.931 \\
\hline 1.6308 & 5 & 0 & 1 & 14 & 56.374 \\
\hline
\end{tabular}


Strontium Zirconium Phosphate, $\mathrm{SrZr}_{4}\left(\mathrm{PO}_{4}\right)_{6}-$ (continued)

\begin{tabular}{|c|c|c|c|c|}
\hline $\mathrm{d}(\stackrel{\circ}{\mathrm{A}})$ & $\begin{array}{c}\mathrm{Iel} \\
\sigma= \pm 3\end{array}$ & & hke & $2 \theta\left({ }^{\circ}\right)$ \\
\hline 1.6200 & 2 & 2 & 35 & 56.781 \\
\hline 1.6075 & 2 & 4 & 13 & 57.263 \\
\hline 1.5824 & 4 & 0 & 48 & 58.262 \\
\hline 1.5573 & 10 & 1 & 310 & 59.290 \\
\hline 1.5391 & 5 & 3 & $\begin{array}{ll}0 & 12\end{array}$ & 60.063 \\
\hline 1.5350 & 4 & 3 & 27 & 60.242 \\
\hline 1.5268 & 7 & 2 & $0 \quad 14$ & 60.600 \\
\hline 1.5139 & 15 & 4 & 16 & 61.172 \\
\hline 1.4898 & 2 & 3 & 111 & 62.270 \\
\hline 1.4868 & 2 & 2 & 38 & 62.411 \\
\hline 1.4667 & 5 & 4 & $0 \quad 10$ & 63.360 \\
\hline 1.4582 & 2 & 0 & 54 & 63.776 \\
\hline 1.4489 & 4 & 3 & 30 & 64.232 \\
\hline 1.4406 & 8 & 1 & 214 & 64.649 \\
\hline 1.4244 & 1 & 3 & 33 & 65.472 \\
\hline 1. $3893 \mathrm{M}$ & 3 & 3 & 210 & 67.346 \\
\hline $1.3893 \mathrm{M}$ & & 4 & 19 & 67.346 \\
\hline 1.3824 & 2 & 2 & 44 & 67.728 \\
\hline $1.3625 \mathrm{M}$ & 1 & 1 & 313 & 68.854 \\
\hline $1.3625 M$ & & 0 & 216 & 68.854 \\
\hline 1.3582 & 3 & 3 & 36 & 69.102 \\
\hline 1.3499 & 1 & 5 & 11 & 69.588 \\
\hline 1.3175 & 2 & 5 & 14 & 71.558 \\
\hline 1.3088 & 2 & 2 & 47 & 72.112 \\
\hline 1.3044 & 2 & 3 & 114 & 72.394 \\
\hline $1.2991 \mathrm{M}$ & 1 & 0 & $\begin{array}{ll}0 & 18\end{array}$ & 72.736 \\
\hline $1.2991 \mathrm{M}$ & & 1 & $5 \quad 5$ & 72.736 \\
\hline 1.2791 & 1 & 4 & 28 & 74.056 \\
\hline 1.2548 & 2 & 6 & 00 & 75.739 \\
\hline 1.2465 & $1 \mathrm{~L}$ & 3 & 213 & 76.339 \\
\hline $1.2385 \mathrm{M}$ & $1 \mathrm{~L}$ & 6 & 03 & 76.917 \\
\hline $1.2385 \mathrm{M}$ & & 1 & 217 & 76.917 \\
\hline 1.2309 & $1 \mathrm{~L}$ & 3 & 42 & 77.480 \\
\hline 1.2156 & 1 & 2 & 410 & 78.645 \\
\hline 1.2057 & 2 & 5 & 20 & 79.419 \\
\hline 1.2008 & 2 & 2 & 314 & 79.804 \\
\hline 1.1944 & $1 \mathrm{~L}$ & 6 & 06 & 80.323 \\
\hline 1.1913 & $1 \mathrm{~L}$ & 5 & 23 & 80.571 \\
\hline 1.1707 & $1 \mathrm{~L}$ & 5 & 110 & 82.289 \\
\hline 1.1629 & $1 \mathrm{~L}$ & 3 & 312 & 82.968 \\
\hline 1.1557 & 1 & 0 & 120 & 83.599 \\
\hline 1.1518 & 2 & 5 & 26 & 83.945 \\
\hline 1.1411 & 1 & 1 & $\begin{array}{ll}5 & 11\end{array}$ & 84.912 \\
\hline 1.1399 & $1 \mathrm{~L}$ & 3 & 48 & 85.027 \\
\hline $1.1299 \mathrm{M}$ & $1 \mathrm{~L}$ & 2 & 119 & 85.960 \\
\hline $1.1299 \mathrm{M}$ & & 6 & 09 & 85.960 \\
\hline 1.1269 & $1 \mathrm{~L}$ & 1 & 64 & 86.244 \\
\hline 1.1187 & $1 \mathrm{~L}$ & 5 & $0 \quad 14$ & 87.036 \\
\hline $1.1160 \mathrm{M}$ & 1 & 2 & 413 & 87.293 \\
\hline $1.1160 \mathrm{M}$ & & 3 & 216 & 87.293 \\
\hline 1.0942 & 2 & 4 & 310 & 89.492 \\
\hline
\end{tabular}


Tantalum Tungsten Oxide, $\mathrm{Ta}_{2} \mathrm{WO}_{8}$

Synonym

Tantalum tungstate

Sample

The single phase composition was prepared by heating 52.4 mole $\%$ of $\mathrm{Ta}_{2} \mathrm{O}_{5}$ with 47.6 mole \% of $\mathrm{WO}_{3}$ for 30 hours at $1350^{\circ} \mathrm{C}$. (A mixture of 50 mole \% each yielded 2 phases.) The composition reported here may be regarded either as oxygen deficient $(\mathrm{Ta}, \mathrm{W})_{3} 0_{7.986}$ or as containing excess metal ( $\mathrm{Ta}, \mathrm{W})_{3.012} \mathrm{O}_{8}$ (Santoro et a1., 1979).

Color

Pale yellow gray

\section{Structure}

Orthorhombic, Pmab (57). The structure was determined by Santoro et al. (1979).

Crystallographic constants of this sample

$$
\begin{aligned}
& a=16.6997(15) \AA \\
& b=17.7296(15) \\
& c=3.8798(4) \\
& a / b=0.9419 \\
& c / b=0.2188 \\
& Z=8 \\
& V=1148.7 A^{3} \\
& \text { Density }\left(\mathrm{calc}^{3}=7.791 \mathrm{~g} / \mathrm{cm}^{3}\right.
\end{aligned}
$$

Figure of merit

$$
F_{30}=73.6(0.0074,55)
$$

\section{Additional patterns}

PDF card 19-1375 (Kovba and Trunov, 1965)

PDF card 29-1322 (Holcombe, 1976)

References

Holcombe, C., Jr., Oak Ridge Nat. Lab.

Rep. No. Y2031 (1976).

Kovba, L. M. and Trunov, V. K. (1965).

J. Struct. Chem. Engl. Trans 1., 6, 224.

Santoro, A., Roth, R. S., and Minor, D.

(1979). Acta Crystallogr., B35, 1202.
$\mathrm{CuK \alpha}_{1} \lambda=1.540598 \AA$ A; temp. $26 \pm 1{ }^{\circ} \mathrm{C}$ Internal standard Ag, $a=4.08651 \AA$

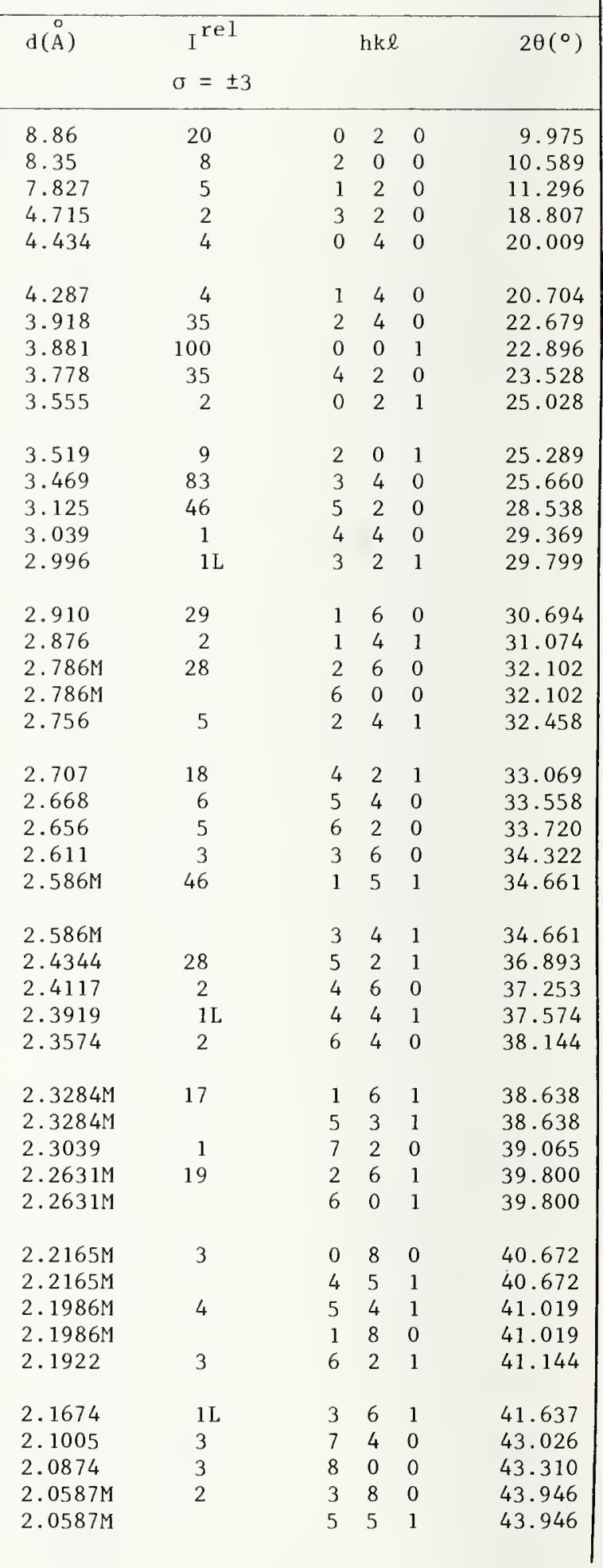


Tantalum Tungsten Oxide, $\mathrm{Ta}_{2} \mathrm{WO}_{8}-$ (continued)

\begin{tabular}{|c|c|c|c|c|c|}
\hline$d(\AA)$ & $I^{r e l}$ & & $\mathrm{hk \ell}$ & & $2 \theta\left({ }^{\circ}\right)$ \\
\hline 2.0488 & 2 & 4 & 6 & 1 & 44.169 \\
\hline 2.0316 & 6 & 8 & 2 & 0 & 44.564 \\
\hline 2.0142 & 1 & 6 & 4 & 1 & 44.969 \\
\hline $1.9812 \mathrm{M}$ & 1 & 7 & 2 & 1 & 45.760 \\
\hline $1.9812 \mathrm{M}$ & & 3 & 7 & 1 & 45.760 \\
\hline 1.9574 & 6 & 4 & 8 & 0 & 46.350 \\
\hline 1.9398 & 21 & 0 & 0 & 2 & 46.794 \\
\hline 1.9240 & 3 & 0 & 8 & 1 & 47.202 \\
\hline $1.8883 \mathrm{M}$ & 6 & 8 & 4 & 0 & 48.149 \\
\hline $1.8883 M$ & & 2 & 0 & 2 & 48.149 \\
\hline 1.8561 & 6 & 7 & 6 & 0 & 49.039 \\
\hline $1.8466+$ & 13 & 5 & 8 & 0 & 49.310 \\
\hline $1.8466+$ & & 7 & 4 & 1 & 49.310 \\
\hline 1.8381 & 4 & 8 & 0 & 1 & 49.553 \\
\hline 1.8185 & 4 & 3 & 8 & 1 & 50.123 \\
\hline 1.7999 & 6 & 8 & 2 & 1 & 50.677 \\
\hline 1.7768 & 2 & 0 & 4 & 2 & 51.384 \\
\hline 1.7727 & 4 & 0 & 10 & 0 & 51.513 \\
\hline $1.7628 \mathrm{M}$ & 4 & 1 & 10 & 0 & 51.822 \\
\hline $1.7628 \mathrm{M}$ & & 7 & 5 & 1 & 51.822 \\
\hline $1.7474 \mathrm{M}$ & 6 & 4 & 8 & 1 & 52.313 \\
\hline $1.7474 \mathrm{M}$ & & 1 & 9 & 1 & 52.313 \\
\hline 1.7381 & 3 & 2 & 4 & 2 & 52.615 \\
\hline $1.7341 \mathrm{M}$ & 6 & 2 & 10 & 0 & 52.746 \\
\hline $1.7341 \mathrm{M}$ & & 6 & 8 & 0 & 52.746 \\
\hline 1.7255 & 5 & 4 & 2 & 2 & 53.029 \\
\hline 1.7047 & 15 & 8 & 6 & 0 & 53.727 \\
\hline 1.6983 & 8 & 8 & 4 & 1 & 53.946 \\
\hline $1.6930 \mathrm{M}$ & 16 & 1 & 5 & 2 & 54.128 \\
\hline $1.6930 \mathrm{M}$ & & 3 & 4 & 2 & 54.128 \\
\hline $1.6744 \mathrm{M}$ & 6 & 7 & 6 & 1 & 54.779 \\
\hline $1.6744 \mathrm{M}$ & & 3 & 9 & 1 & 54.779 \\
\hline $1.6701 \mathrm{M}$ & 12 & 5 & 1 & 2 & 54.933 \\
\hline $1.6701 \mathrm{M}$ & & 10 & 0 & 0 & 54.933 \\
\hline $1.6670 \mathrm{M}$ & 12 & 5 & 8 & 1 & 55.045 \\
\hline $1.6670 \mathrm{M}$ & & 9 & 1 & 1 & 55.045 \\
\hline 1.6482 & 12 & 5 & 2 & 2 & 55.727 \\
\hline $1.6317 \mathrm{M}$ & 6 & 4 & 10 & 0 & 56.340 \\
\hline $1.6317 \mathrm{M}$ & & 8 & 5 & 1 & 56.340 \\
\hline 1.6235 & 2 & 7 & 8 & 0 & 56.650 \\
\hline $1.6138 \mathrm{M}$ & 9 & 5 & 3 & 2 & 57.019 \\
\hline $1.6138 \mathrm{M}$ & & 1 & 6 & 2 & 57.019 \\
\hline 1.6050 & 3 & 1 & 10 & 1 & 57.364 \\
\hline 1.5917 & 7 & 2 & 6 & 2 & 57.885 \\
\hline $1.5917 \mathrm{M}$ & & 6 & 0 & 2 & 57.885 \\
\hline $1.5831 \mathrm{M}$ & 5 & 2 & 10 & 1 & 58.230 \\
\hline $1.5831 \mathrm{M}$ & & 6 & 8 & 1 & 58.230 \\
\hline 1.5713 & 5 & 9 & 6 & 0 & 58.711 \\
\hline $1.5663+$ & 7 & 6 & 2 & 2 & 58.919 \\
\hline $1.5663+$ & & 5 & 10 & 0 & 58.919 \\
\hline
\end{tabular}

\begin{tabular}{|c|c|c|c|c|c|}
\hline \multirow{2}{*}{$\begin{array}{c}\mathrm{d}(\stackrel{\mathrm{A}}{)}) \\
1.5608\end{array}$} & $\begin{array}{c}I^{\text {rel }} \\
\sigma= \pm 3\end{array}$ & \multicolumn{3}{|c|}{ hke } & \multirow{2}{*}{$20\left(^{\circ}\right)$} \\
\hline & 15 & 8 & 6 & 1 & \\
\hline 1.5490 & $1 \mathrm{~L}$ & 3 & 10 & 1 & 59.640 \\
\hline $1.5336 \mathrm{M}$ & 7 & 1 & 7 & 2 & 60.300 \\
\hline $1.5336 \mathrm{M}$ & & 10 & 0 & 1 & 60.300 \\
\hline 1.5195 & $1 \mathrm{~L}$ & 8 & 8 & 0 & 60.921 \\
\hline $1.5112 \mathrm{M}$ & 1 & 10 & 2 & 1 & 61.291 \\
\hline $1.5112 \mathrm{M}$ & & 4 & 6 & 2 & 61.291 \\
\hline 1.5041 & 7 & 4 & 10 & 1 & 61.611 \\
\hline $1.4974 \mathrm{M}$ & 5 & 7 & 8 & 1 & 61.918 \\
\hline $1.4974 \mathrm{M}$ & & 6 & 4 & 2 & 61.918 \\
\hline $1.4840+$ & $1 \mathrm{~L}$ & 7 & 2 & 2 & 62.542 \\
\hline $1.4840+$ & & 3 & 7 & 2 & 62.542 \\
\hline 1.4717 & 2 & 1 & 12 & 0 & 63.122 \\
\hline $1.4587 \mathrm{M}$ & 4 & 5 & 6 & 2 & 63.752 \\
\hline $1.4587 \mathrm{M}$ & & 7 & 3 & 2 & 63.752 \\
\hline 1.4563 & 5 & 9 & 6 & 1 & 63.867 \\
\hline $1.4525 \mathrm{M}$ & 6 & 5 & 10 & 1 & 64.056 \\
\hline $1.4525 \mathrm{M}$ & & 6 & 5 & 2 & 64.056 \\
\hline
\end{tabular}


Thorium Molybdenum 0xide, $\alpha-\operatorname{Th}\left(\mathrm{MoO}_{4}\right)_{2}$

Synonym

Thorium molybdate

Sample

$\mathrm{ThO}_{2}$ and $\mathrm{MoO}_{3}$ in a molar ratio of 1 to 2

were ground together and heated to $700{ }^{\circ} \mathrm{C}$

for 70 hours in a Pt crucible.

Color

Colorless

Structure

Orthorhombic, Pcab (61) (Thoret, 1974).

Crystallographic constants of this sample

$a=10.3174(7) \AA$

$b=14.4835(10)$

$c=9.7491(7)$

$\mathrm{a} / \mathrm{b}=0.7124$

$c / b=0.6731$

$Z=8$
$V=1456.8 \AA^{3}$

Density $(\mathrm{calc})=5.032 \mathrm{~g} / \mathrm{cm}^{3}$

Figure of merit

$\mathrm{F}_{30}=105.5(0.0071,40)$

Polymorphism

There is a hexagonal $\beta$-form existing above $950{ }^{\circ} \mathrm{C}$ (Thoret, 1974).

Additional pattern

PDF card 27-1468 (Thoret, 1974)

Reference

Thoret, J. (1974). Rev. Chim. Minér. 11, 237.

\begin{tabular}{|c|c|c|c|c|c|}
\hline \multicolumn{6}{|c|}{$\begin{array}{l}\mathrm{CuK \alpha}_{1} \lambda=1.540598 \AA ; \text { mean } \mathrm{T}=25.9{ }^{\circ} \mathrm{C} \\
\text { Internal standard Ag, } \mathrm{a}=4.08651 \AA\end{array}$} \\
\hline $\mathrm{d}(\stackrel{\circ}{\mathrm{A}})$ & $\begin{array}{l}I^{\text {rel }} \\
\sigma= \pm 3\end{array}$ & & hke & & $2 \theta\left({ }^{\circ}\right)$ \\
\hline 7.241 & 15 & 0 & 2 & 0 & 12.213 \\
\hline 6.371 & 8 & 1 & 1 & 1 & 13.890 \\
\hline 5.929 & 5 & 1 & 2 & 0 & 14.929 \\
\hline 5.156 & 29 & 2 & 0 & 0 & 17.185 \\
\hline 5.065 & 77 & 1 & 2 & 1 & 17.496 \\
\hline 4.874 & 27 & 0 & 0 & 2 & 18.185 \\
\hline 4.348 & 16 & 2 & 1 & 1 & 20.408 \\
\hline 4.202 & 6 & 2 & 2 & 0 & 21.125 \\
\hline 4.045 & 10 & 0 & 2 & 2 & 21.955 \\
\hline 3.991 & 15 & 1 & 3 & 1 & 22.256 \\
\hline 3.859 & 10 & 2 & 2 & 1 & 23.031 \\
\hline 3.766 & 63 & 1 & 2 & 2 & 23.604 \\
\hline 3.621 & 47 & 0 & 4 & 0 & 24.563 \\
\hline 3.544 & 100 & 2 & 0 & 2 & 25.105 \\
\hline 3.417 & 21 & 1 & 4 & 0 & 26.059 \\
\hline 3.315 & 7 & 2 & 3 & 1 & 26.877 \\
\hline 3.257 & 11 & 1 & 3 & 2 & 27.364 \\
\hline 3.225 & 33 & 1 & 4 & 1 & 27.635 \\
\hline 3.184 & 23 & 2 & 2 & 2 & 28.003 \\
\hline 3.163 & 7 & 3 & 1 & 1 & 28.194 \\
\hline 3.107 & 62 & 3 & 2 & 0 & 28.713 \\
\hline 3.032 & 25 & 1 & 1 & 3 & 29.437 \\
\hline $2.961 \mathrm{M}$ & 71 & 2 & 4 & 0 & 30.154 \\
\hline $2.961 \mathrm{M}$ & & 3 & 2 & 1 & 30.154 \\
\hline 2.908 & 31 & 0 & 4 & 2 & 30.722 \\
\hline 2.850 & 22 & 1 & 2 & 3 & 31.361 \\
\hline 2.799 & 11 & 1 & 4 & 2 & 31.947 \\
\hline 2.750 & 2 & 2 & 0 & 3 & 32.531 \\
\hline 2.701 & 7 & 2 & 1 & 3 & 33.135 \\
\hline 2.682 & 2 & 1 & 5 & 1 & 33.382 \\
\hline 2.620 & 17 & 3 & 2 & 2 & 34.197 \\
\hline 2.609 & 11 & 1 & 3 & 3 & 34.347 \\
\hline 2.579 & 4 & 4 & 0 & 0 & 34.755 \\
\hline 2.574 & 3 & 2 & 2 & 3 & 34.833 \\
\hline 2.5326 & 25 & 2 & 4 & 2 & 35.414 \\
\hline $2.4936 \mathrm{M}$ & 10 & 3 & 4 & 0 & 35.987 \\
\hline $2.4936 \mathrm{M}$ & & 4 & 0 & 1 & 35.987 \\
\hline 2.4582 & 6 & 4 & 1 & 1 & 36.524 \\
\hline 2.4374 & 15 & 0 & 0 & 4 & 36.846 \\
\hline $2.4297 \mathrm{M}$ & 9 & 4 & 2 & 0 & 36.968 \\
\hline $2.4297 \mathrm{M}$ & & 3 & 3 & 2 & 36.968 \\
\hline $2.4151 \mathrm{M}$ & 5 & 3 & 4 & 1 & 37.199 \\
\hline $2.4151 \mathrm{M}$ & & 0 & 6 & 0 & 37.199 \\
\hline 2.3538 & 8 & 1 & 4 & 3 & 38.205 \\
\hline 2.2849 & 24 & 1 & 6 & 1 & 39.404 \\
\hline
\end{tabular}


Thorium Molybdenum Oxide, $\alpha$-Th $\left(\mathrm{MoO}_{4}\right)_{2}$ - (continued)

\begin{tabular}{|c|c|c|c|c|}
\hline $\mathrm{d}(\stackrel{\circ}{\mathrm{A}})$ & $I^{\text {rel }}$ & & $\mathrm{hk} \ell$ & $2 \theta\left({ }^{\circ}\right)$ \\
\hline 2.2786 & 15 & 4 & $\begin{array}{ll}0 & 2\end{array}$ & 39.517 \\
\hline 2.2460 & 26 & 3 & 23 & 40.115 \\
\hline 2.2195 & 3 & 3 & 42 & 40.616 \\
\hline 2.2039 & 5 & 2 & 04 & 40.916 \\
\hline 2.1901 & 12 & 2 & 43 & 41.185 \\
\hline $2.1747 \mathrm{M}$ & 6 & 0 & 34 & 41.491 \\
\hline $2.1747 \mathrm{M}$ & & 4 & 22 & 41.491 \\
\hline 2.1599 & 2 & 3 & $\begin{array}{ll}5 & 1\end{array}$ & 41.787 \\
\hline 2.1334 & 11 & 2 & $\begin{array}{ll}6 & 1\end{array}$ & 42.331 \\
\hline 2.1279 & 8 & 1 & 34 & 42.447 \\
\hline $2.1161 \mathrm{M}$ & 7 & 1 & 62 & 42.694 \\
\hline $2.1161 \mathrm{M}$ & & 1 & 53 & 42.694 \\
\hline 2.0617 & 5 & 4 & 32 & 43.878 \\
\hline 2.0535 & 12 & 4 & 41 & 44.062 \\
\hline $2.0212 \mathrm{M}$ & 10 & 0 & 44 & 44.805 \\
\hline $2.0212 \mathrm{M}$ & & 4 & $\begin{array}{ll}0 & 3\end{array}$ & 44.805 \\
\hline 2.0164 & 8 & 3 & 52 & 44.918 \\
\hline 1.9995 & 21 & 5 & $\begin{array}{ll}1 & 1\end{array}$ & 45.319 \\
\hline $1.9950 M$ & 31 & 2 & 62 & 45.427 \\
\hline $1.9950 \mathrm{M}$ & & 2 & 53 & 45.427 \\
\hline $1.9844+$ & 15 & 5 & 20 & 45.683 \\
\hline $1.9844+$ & & 1 & 44 & 45.683 \\
\hline 1.9793 & 11 & 3 & 43 & 45.806 \\
\hline 1.9446 & 18 & 5 & 21 & 46.672 \\
\hline 1.9362 & 19 & 3 & $\begin{array}{ll}6 & 1\end{array}$ & 46.886 \\
\hline 1.9179 & 12 & 3 & 24 & 47.361 \\
\hline $1.9046 \mathrm{M}$ & 18 & 0 & 72 & 47.712 \\
\hline $1.9046 \mathrm{M}$ & & 1 & 63 & 47.712 \\
\hline 1.8994 & 15 & 1 & 15 & 47.850 \\
\hline 1.8897 & 7 & 4 & $5 \quad 1$ & 48.113 \\
\hline $1.8834+$ & 10 & 2 & $\begin{array}{ll}7 & 1\end{array}$ & 48.284 \\
\hline $1.8834+$ & & 5 & 12 & 48.284 \\
\hline 1.8639 & 11 & 4 & 33 & 48.822 \\
\hline 1.8519 & 10 & 1 & 25 & 49.158 \\
\hline $1.8380 \mathrm{M}$ & 24 & 3 & 34 & 49.554 \\
\hline $1.8380 \mathrm{M}$ & & 5 & 22 & 49.554 \\
\hline $1.8312 \mathrm{M}$ & 25 & 3 & 62 & 49.752 \\
\hline $1.8312 \mathrm{M}$ & & 3 & 53 & 49.752 \\
\hline 1.8139 & 6 & 2 & 63 & 50.258 \\
\hline $1.8097 \mathrm{M}$ & 7 & 0 & 80 & 50.384 \\
\hline $1.8097 \mathrm{M}$ & & 2 & 15 & 50.384 \\
\hline 1.7917 & 4 & 4 & 52 & 50.925 \\
\hline 1.7870 & 5 & 2 & 72 & 51.071 \\
\hline 1.7804 & 7 & 1 & 35 & 51.271 \\
\hline 1.7717 & 11 & 4 & 04 & 51.544 \\
\hline $1.7627 \mathrm{M}$ & 14 & 5 & 41 & 51.824 \\
\hline $1.7627 \mathrm{M}$ & & 4 & $\begin{array}{ll}6 & 0\end{array}$ & 51.824 \\
\hline 1.7585 & 14 & 4 & 14 & 51.957 \\
\hline $1.7541 \mathrm{M}$ & 20 & 1 & 81 & 52.098 \\
\hline $1.7541 \mathrm{M}$ & & 2 & 54 & 52.098 \\
\hline
\end{tabular}

\begin{tabular}{|c|c|c|c|c|c|}
\hline $\mathrm{d}(\stackrel{\circ}{\mathrm{A}})$ & $I^{\text {rel }}$ & & hke & & $2 \theta\left(^{\circ}\right)$ \\
\hline $1.7434 \mathrm{M}$ & 8 & 3 & 7 & 1 & 52.442 \\
\hline $1.7434 \mathrm{M}$ & & 3 & 4 & 4 & 52.442 \\
\hline 1.7344 & 7 & 4 & 6 & $i$ & 52.735 \\
\hline 1.7299 & 6 & 5 & 1 & 3 & 52.883 \\
\hline $1.7211 \mathrm{M}$ & 4 & 1 & 7 & 3 & 53.174 \\
\hline $1.7211 \mathrm{M}$ & & 4 & 2 & 4 & 53.174 \\
\hline 1.7156 & 7 & 0 & 6 & 4 & 53.360 \\
\hline 1.7083 & 14 & 2 & 8 & 0 & 53.605 \\
\hline 1.6975 & 16 & 0 & 8 & 2 & 53.975 \\
\hline $1.6934 t$ & 24 & 1 & 4 & 5 & 54.115 \\
\hline $1.6934 t$ & & 6 & 0 & 1 & 54.115 \\
\hline 1.6888 & 19 & 3 & 6 & 3 & 54.274 \\
\hline $1.6830+$ & 16 & 2 & 8 & 1 & 54.476 \\
\hline $1.6830+$ & & 5 & 4 & 2 & 54.476 \\
\hline 1.6658 & 3 & 3 & 7 & 2 & 55.085 \\
\hline 1.6625 & 2 & 4 & 3 & 4 & 55.205 \\
\hline $1.6573+$ & 2 & 4 & 6 & 2 & 55.395 \\
\hline $1.6573+$ & & 4 & 5 & 3 & 55.395 \\
\hline 1.6531 & 2 & 2 & 7 & 3 & 55.545 \\
\hline 1.6509 & 2 & 3 & 2 & 5 & 55.625 \\
\hline 1.6386 & 3 & 5 & 3 & 3 & 56.082 \\
\hline 1.6289 & 3 & 2 & 4 & 5 & 56.444 \\
\hline $1.6117 \mathrm{M}$ & 3 & 2 & 8 & 2 & 57.104 \\
\hline $1.6117 \mathrm{M}$ & & 6 & 1 & 2 & 57.104 \\
\hline $1.5976 \mathrm{M}$ & 5 & 1 & 5 & 5 & 57.654 \\
\hline $1.5976 \mathrm{M}$ & & 6 & 3 & 1 & 57.654 \\
\hline $1.5920 \mathrm{M}$ & 4 & 4 & 7 & 1 & 57.876 \\
\hline $1.5920 \mathrm{M}$ & & 4 & 4 & 4 & 57.876 \\
\hline 1.5827 & 3 & 6 & 2 & 2 & 58.246 \\
\hline 1.5807 & 4 & 3 & 8 & 1 & 58.327 \\
\hline 1.5770 & 4 & 0 & 7 & 4 & 58.479 \\
\hline 1.5681 & 7 & 5 & 6 & 0 & 58.842 \\
\hline 1.5636 & 10 & 1 & 8 & 3 & 59.030 \\
\hline 1.5592 & 8 & 1 & 7 & 4 & 59.213 \\
\hline $1.5485 \mathrm{M}$ & 6 & 4 & 6 & 3 & 59.662 \\
\hline $1.5485 \mathrm{M}$ & & 5 & 6 & 1 & 59.662 \\
\hline $1.5393 \mathrm{M}$ & 6 & 0 & 3 & 6 & 60.055 \\
\hline $1.5393 M$ & & 5 & 2 & 4 & 60.055 \\
\hline 1.5339 & 12 & 6 & 4 & 1 & 60.289 \\
\hline
\end{tabular}


Thorium Molybdenum 0xide, $\beta-\operatorname{Th}\left(\mathrm{MoO}_{4}\right)_{2}$

Synonym

Thorium molybdate

Sample

The sample was prepared by heating $\mathrm{ThO}_{2}$ and $\mathrm{MoO}_{3}$ in a 1 to 2 molar ratio in a $\mathrm{Pt}$ crucible at $700{ }^{\circ} \mathrm{C}$ for $203 / 4$ hours, then reheated at $1000{ }^{\circ} \mathrm{C}$ for $21 / 4$ hours.

Color Colorless

Structure

Hexagonal, $\mathrm{P} \overline{6}$ (174). The structure was determined by Thoret et al. (1968).

Crystallographic constants of this sample $\mathrm{a}=17.5764(6) \AA$

$c=6.2265(2)$

$c / a=0.3543$

$\mathrm{Z}=9$

$\mathrm{V}=1665.84 \AA^{3}$

Density $(\mathrm{calc})=4.951 \mathrm{~g} / \mathrm{cm}^{3}$

Figure of merit $\mathrm{F}_{30}=42.7(0.011,64)$

Polymorphism

There is an orthorhombic $\alpha$-form existing below $950{ }^{\circ} \mathrm{C}$ (Thoret, 1974).

Additional pattern PDF card 27-1469 (Thoret, 1974)

\section{References}

Thoret, J. (1974). Rev. Chim. Minér. 11, 237.

Thoret, J., Rimsky, A., and Freundlich, W. (1968). C. R. Séances Acad. Sci., Ser. C 267, 1682 .

\begin{tabular}{|c|c|c|c|c|c|}
\hline \multicolumn{6}{|c|}{$\begin{array}{l}\mathrm{CuK \alpha}_{1} \lambda=1.540598 \AA \text {; mean } \mathrm{T}=25.6{ }^{\circ} \mathrm{C} \\
\text { Internal standard } \mathrm{W}, \mathrm{a}=3.16524 \AA\end{array}$} \\
\hline $\mathrm{d}(\stackrel{\circ}{\mathrm{A}})$ & $\begin{array}{l}I^{\mathrm{rel}} \\
\sigma= \pm 3\end{array}$ & & $\mathrm{hk}$ & & $2 \theta\left({ }^{\circ}\right)$ \\
\hline 8.79 & 2 & 1 & 1 & 0 & 10.051 \\
\hline $5.764 M$ & 8 & 1 & 0 & 1 & 15.360 \\
\hline $5.764 M$ & & 2 & 1 & 0 & 15.360 \\
\hline $5.075 \mathrm{M}$ & 48 & 1 & 1 & 1 & 17.461 \\
\hline $5.075 \mathrm{M}$ & & 3 & 0 & 0 & 17.461 \\
\hline 4.821 & 7 & 2 & 0 & 1 & 18.389 \\
\hline $4.227 M$ & 17 & 2 & 1 & 1 & 21.002 \\
\hline $4.227 \mathrm{M}$ & & 3 & 1 & 0 & 21.002 \\
\hline 3.935 & 51 & 3 & 0 & 1 & 22.580 \\
\hline 3.592 & 79 & 2 & 2 & 1 & 24.768 \\
\hline
\end{tabular}

\begin{tabular}{|c|c|c|c|c|c|}
\hline $\mathrm{d}(\stackrel{\circ}{\mathrm{A}})$ & $\begin{array}{c}1^{\text {rel }} \\
\sigma= \pm 3\end{array}$ & & hke & & $2 \theta\left({ }^{\circ}\right)$ \\
\hline $3.495 \mathrm{M}$ & 10 & 3 & 1 & 1 & 25.465 \\
\hline $3.495 \mathrm{M}$ & & 3 & 2 & 0 & 25.465 \\
\hline 3.321 & 34 & 4 & 1 & 0 & 26.820 \\
\hline 3.247 & 5 & 4 & 0 & 1 & 27.443 \\
\hline 3.115 & 14 & 0 & 0 & 2 & 28.635 \\
\hline $3.047 \mathrm{M}$ & 6 & 3 & 2 & 1 & 29.283 \\
\hline $3.047 \mathrm{M}$ & & 5 & 0 & 0 & 29.283 \\
\hline $2.932 \mathrm{M}$ & 100 & 1 & 4 & 1 & 30.461 \\
\hline $2.932 \mathrm{M}$ & & 3 & 3 & 0 & 30.461 \\
\hline $2.735 \mathrm{M}$ & 2 & 5 & 0 & 1 & 32.714 \\
\hline $2.735 \mathrm{M}$ & & 5 & 1 & 0 & 32.714 \\
\hline 2.650 & 3 & 3 & 3 & 1 & 33.793 \\
\hline 2.612 & 4 & 4 & 2 & 1 & 34.309 \\
\hline 2.541 & 23 & 2 & 2 & 2 & 35.298 \\
\hline $2.503 \mathrm{M}$ & 3 & 5 & 1 & 1 & 35.852 \\
\hline $2.503 \mathrm{M}$ & & 4 & 3 & 0 & 35.852 \\
\hline 2.439 & 5 & 5 & 2 & 0 & 36.826 \\
\hline 2.348 & 1 & 6 & 0 & 1 & 38.299 \\
\hline $2.322 \mathrm{M}$ & 7 & 4 & 3 & 1 & 38.755 \\
\hline $2.322 \mathrm{M}$ & & 6 & 1 & 0 & 38.755 \\
\hline 2.271 & 46 & 4 & 1 & 2 & 39.646 \\
\hline 2.198 & 1 & 4 & 4 & 0 & 41.027 \\
\hline $2.175 \mathrm{M}$ & 4 & 6 & 1 & 1 & 41.481 \\
\hline $2.175 \mathrm{M}$ & & 5 & 3 & 0 & 41.481 \\
\hline 2.133 & 10 & 3 & 3 & 2 & 42.337 \\
\hline 2.0715 & 1 & 4 & 4 & 1 & 43.660 \\
\hline 2.0528 & 7 & 5 & 3 & 1 & 44.079 \\
\hline 2.0196 & 4 & 1 & 1 & 3 & 44.843 \\
\hline 1.9985 & 4 & 6 & 2 & 1 & 45.341 \\
\hline 1.9671 & 2 & 6 & 0 & 2 & 46.108 \\
\hline 1.9523 & 2 & 2 & 1 & 3 & 46.478 \\
\hline $1.9181 \mathrm{M}$ & 94 & 7 & 1 & 1 & 47.357 \\
\hline $1.9181 \mathrm{M}$ & & 6 & 3 & 0 & 47.357 \\
\hline 1.8769 & 16 & 2 & 2 & 3 & 48.461 \\
\hline 1.8628 & 3 & 3 & 1 & 3 & 48.853 \\
\hline $1.8592 \mathrm{M}$ & 2 & 5 & 4 & 1 & 48.954 \\
\hline $1.8592 \mathrm{M}$ & & 7 & 2 & 0 & 48.954 \\
\hline 1.8326 & 3 & 6 & 3 & 1 & 49.711 \\
\hline 1.8196 & 2 & 8 & 0 & 1 & 50.089 \\
\hline 1.7954 & 21 & 4 & 4 & 2 & 50.815 \\
\hline 1.7842 & 3 & 3 & 2 & 3 & 51.154 \\
\hline $1.7807 \mathrm{M}$ & 2 & 7 & 2 & 1 & 51.263 \\
\hline $1.7807 \mathrm{M}$ & & 8 & 1 & 0 & 51.263 \\
\hline 1.7602 & 14 & 4 & 1 & 3 & 51.906 \\
\hline $1.7129 \mathrm{M}$ & 2 & 8 & 1 & 1 & 53.450 \\
\hline $1.7129 \mathrm{M}$ & & 7 & 3 & 0 & 53.450 \\
\hline $1.6920 \mathrm{M}$ & 32 & 7 & 1 & 2 & 54.164 \\
\hline $1.6920 \mathrm{M}$ & & 5 & 5 & 1 & 54.164 \\
\hline 1.6811 & 4 & 6 & 4 & 1 & 54.545 \\
\hline 1.6608 & 3 & 8 & 2 & 0 & 55.266 \\
\hline
\end{tabular}


Thorium Molybdenum 0xide, $\beta$ - Th $\left(\mathrm{MoO}_{4}\right)_{2}-$ (continued)

\begin{tabular}{|c|c|c|c|c|c|c|c|c|c|c|c|}
\hline$d(\AA)$ & $\begin{array}{c}\text { I rel } \\
\sigma= \pm 3\end{array}$ & \multicolumn{2}{|c|}{ hk $\ell$} & & $2 \theta\left({ }^{\circ}\right)$ & $\begin{array}{c}0 \\
d(\AA)\end{array}$ & \multicolumn{2}{|c|}{$\begin{array}{c}\text { rel } \\
\sigma= \pm 3\end{array}$} & \multicolumn{2}{|c|}{$\mathrm{hk} \ell$} & $2 \theta\left(^{\circ}\right)$ \\
\hline $1.6510 \mathrm{M}$ & 3 & 5 & 4 & 2 & 55.623 & $1.2308 \mathrm{M}$ & & 7 & 7 & 1 & 77.486 \\
\hline $1.6510 \mathrm{M}$ & & 7 & 3 & 1 & 55.623 & 1.2169 & 2 & 2 & 1 & 5 & 78.542 \\
\hline $1.6328 \mathrm{M}$ & 11 & 6 & 3 & 2 & 56.297 & $1.2145 \mathrm{M}$ & 1 & 9 & 5 & 1 & 78.726 \\
\hline $1.6328 \mathrm{M}$ & & 9 & 0 & 1 & 56.297 & $1.2145 \mathrm{M}$ & & 12 & 1 & 0 & 78.726 \\
\hline 1.6048 & 8 & 8 & 2 & 1 & 57.371 & 1.2085 & 12 & 6 & 3 & 4 & 79.199 \\
\hline 1.5972 & 2 & 4 & 3 & 3 & 57.667 & 1.1982 & 5 & 2 & 2 & 5 & 80.015 \\
\hline 1.5787 & 5 & 7 & 4 & 0 & 58.408 & 1.1962 & 6 & 10 & 4 & 1 & 80.173 \\
\hline 1.5569 & 5 & 0 & 0 & 4 & 59.308 & 1.1858 & 4 & 10 & 1 & 3 & 81.019 \\
\hline $1.5458 \mathrm{M}$ & 3 & 6 & 5 & 1 & 59.776 & 1.1745 & 3 & 12 & 0 & 2 & 81.968 \\
\hline $1.5458 \mathrm{M}$ & & 8 & 3 & 0 & 59.776 & 1.1660 & 7 & 4 & 1 & 5 & 82.699 \\
\hline $1.5303 \mathrm{M}$ & 19 & 5 & 5 & 2 & 60.444 & $1.1641 \mathrm{M}$ & 17 & 7 & 7 & 2 & 82.860 \\
\hline $1.5303 \mathrm{M}$ & & 7 & 4 & 1 & 60.444 & $1.1641 \mathrm{M}$ & & 9 & 6 & 0 & 82.860 \\
\hline $1.4995 M$ & 2 & 8 & 3 & 1 & 61.822 & $1.1508 \mathrm{M}$ & 1 & 8 & 7 & 1 & 84.039 \\
\hline $1.4995 M$ & & 9 & 2 & 0 & 61.822 & $1.1508 \mathrm{M}$ & & 10 & 5 & 0 & 84.039 \\
\hline 1.4881 & 4 & 3 & 0 & 4 & 62.347 & 1.1453 & 3 & 9 & 0 & 4 & 84.531 \\
\hline 1.4797 & 2 & 6 & 2 & 3 & 62.744 & 1.1441 & 1 & 9 & 6 & 1 & 84.646 \\
\hline $1.4650 \mathrm{M}$ & 3 & 8 & 2 & 2 & 63.444 & 1.1426 & 2 & 4 & 2 & 5 & 84.780 \\
\hline $1.4650 \mathrm{M}$ & & 6 & 6 & 0 & 63.444 & 1.1411 & 1 & 12 & 2 & 1 & 84.913 \\
\hline $1.4580 \mathrm{M}$ & 2 & 9 & 2 & 1 & 63.786 & 1.1348 & 7 & 10 & 4 & 2 & 85.495 \\
\hline $1.4580 \mathrm{M}$ & & 7 & 5 & 0 & 63.786 & 1.1258 & 7 & 8 & 5 & 3 & 86.348 \\
\hline 1.4461 & 16 & 7 & 1 & 3 & 64.371 & $1.1134 \mathrm{M}$ & 1 & 11 & 3 & 2 & 87.557 \\
\hline 1.4259 & 4 & 6 & 6 & 1 & 65.398 & $1.1134 \mathrm{M}$ & & 11 & 4 & 1 & 87.557 \\
\hline $1.4196 \mathrm{M}$ & 2 & 6 & 5 & 2 & 65.724 & 1.1089 & 2 & 5 & 2 & 5 & 88.002 \\
\hline $1.4196 \mathrm{M}$ & & 7 & 5 & 1 & 65.724 & $1.1072 \mathrm{M}$ & 6 & 13 & 1 & 1 & 88.167 \\
\hline 1.4096 & 10 & 4 & 1 & 4 & 66.248 & $1.1072 \mathrm{M}$ & & 12 & 3 & 0 & 88.167 \\
\hline $1.4075 M$ & 21 & 7 & 4 & 2 & 66.362 & $1.0902 \mathrm{M}$ & 6 & 9 & 6 & 2 & 89.913 \\
\hline $1.4075 \mathrm{M}$ & & 10 & 1 & 1 & 66.362 & $1.0902 \mathrm{M}$ & & 12 & 3 & 1 & 89.913 \\
\hline 1.4011 & 2 & 8 & 4 & 1 & 66.703 & 1.0818 & 1 & 8 & 8 & 1 & 90.808 \\
\hline $1.3847 M$ & 2 & 7 & 2 & 3 & 67.597 & 1.0806 & $1 \mathrm{~L}$ & 5 & 3 & 5 & 90.934 \\
\hline $1.3847 \mathrm{M}$ & & 8 & 3 & 2 & 67.597 & $1.0792 \mathrm{M}$ & 1 & 10 & 5 & 2 & 91.085 \\
\hline 1.3750 & 8 & 3 & 3 & 4 & 68.139 & $1.0792 \mathrm{M}$ & & 9 & 7 & 1 & 91.085 \\
\hline 1.3723 & 5 & 9 & 3 & 1 & 68.295 & 1.0742 & 3 & 7 & 7 & 3 & 91.624 \\
\hline 1.3533 & 1 & 5 & 1 & 4 & 69.389 & 1.0715 & 2 & 8 & 6 & 3 & 91.932 \\
\hline $1.3513 M$ & 2 & 9 & 2 & 2 & 69.508 & 1.0668 & 2 & 6 & 6 & 4 & 92.447 \\
\hline $1.3513 M$ & & 11 & 0 & 1 & 69.508 & $1.0631 \mathrm{M}$ & 1 & 11 & 4 & 2 & 92.864 \\
\hline 1.3412 & 3 & 5 & 5 & 3 & 70.106 & $1.0631 \mathrm{M}$ & & 13 & 2 & 1 & 92.864 \\
\hline 1.3353 & 1 & 10 & 2 & 1 & 70.464 & 1.0596 & 3 & 7 & 1 & 5 & 93.269 \\
\hline 1.3268 & 5 & 6 & 0 & 4 & 70.981 & $1.0581 \mathrm{M}$ & 4 & 13 & 1 & 2 & 93.443 \\
\hline $1.3206 \mathrm{M}$ & 2 & 7 & 3 & 3 & 71.362 & $1.0581 \mathrm{M}$ & & 11 & 5 & 1 & 93.443 \\
\hline $1.3206 \mathrm{M}$ & & 7 & 5 & 2 & 71.362 & 1.0509 & 2 & 10 & 4 & 3 & 94.272 \\
\hline $1.3102 \mathrm{M}$ & 20 & 10 & 1 & 2 & 72.018 & 1.0484 & 2 & 12 & 1 & 3 & 94.567 \\
\hline $1.3102 \mathrm{M}$ & & 8 & 5 & 1 & 72.018 & 1.0440 & 4 & 9 & 3 & 4 & 95.099 \\
\hline 1.2967 & 4 & 8 & 2 & 3 & 72.891 & 1.0379 & 3 & 0 & 0 & 6 & 95.839 \\
\hline 1.2824 & 4 & 9 & 3 & 2 & 73.838 & 1.0360 & 1 & 8 & 8 & 2 & 96.072 \\
\hline 1.2685 & 5 & 12 & 0 & 0 & 74.785 & $1.0334 \mathrm{M}$ & 1 & 14 & 1 & 1 & 96.385 \\
\hline 1.2652 & 4 & 6 & 5 & 3 & 75.010 & $1.0334 \mathrm{M}$ & & 9 & 8 & 0 & 96.385 \\
\hline 1.2563 & 6 & 7 & 4 & 3 & 75.635 & 1.0307 & 1 & 1 & 1 & 6 & 96.724 \\
\hline 1.2412 & 1 & 1 & 0 & 5 & 76.718 & 1.0281 & 1 & 2 & 0 & 6 & 97.056 \\
\hline 1.2396 & 1 & 8 & 3 & 3 & 76.838 & $1.0196 \mathrm{M}$ & 1 & 13 & 2 & 2 & 98.141 \\
\hline $1.2308 \mathrm{M}$ & 10 & 8 & 5 & 2 & 77.486 & $1.0196 \mathrm{M}$ & & 9 & 8 & 1 & 98.141 \\
\hline
\end{tabular}


Sample

The sample was prepared by heating an equimolar mixture of $\mathrm{ThO}_{2}$ and $\mathrm{SiO}_{2}$ for a total of 60 hours at $1550{ }^{\circ} \mathrm{C}$, with periodic grinding. The sample contains a small amount of $\mathrm{ThO}_{2}$ as a second phase.

Color

Colorless

Structure

Monoclinic, $\mathrm{P} 21 / \mathrm{n}$ (14). Isostructural with monazite (Pabst and Hutton, 1951). The structure of monazite, $\mathrm{CePO}_{4}$, was determined by Mooney (1948).

Crystallographic constants of this sample

$$
\begin{aligned}
& a=6.7759(4) \AA \\
& b=6.9648(4) \\
& c=6.4982(4) \\
& \beta=104.99(1)^{\circ} \\
& Z=4 \\
& V=296.23 \AA^{3}
\end{aligned}
$$$$
\text { Density }(\mathrm{calc})=7.267 \mathrm{~g} / \mathrm{cm}^{3}
$$

Figure of merit

$$
F_{30}=142.8(0.0045,47)
$$

\section{Polymorphism}

A tetragonal modification, thorite, also exists (Pabst and Hutton, 1951). This tetragonal modification has the zircon structure.

Additional pattern

PDF card 4-613 (Pabst and Hutton, 1951).

\section{References}

Mooney, R. C. L. (1948). J. Chem. Phys. 16,1003 .

Pabst, A. and Hutton, C. O. (1951). Am. Mineral. 36, 60 .

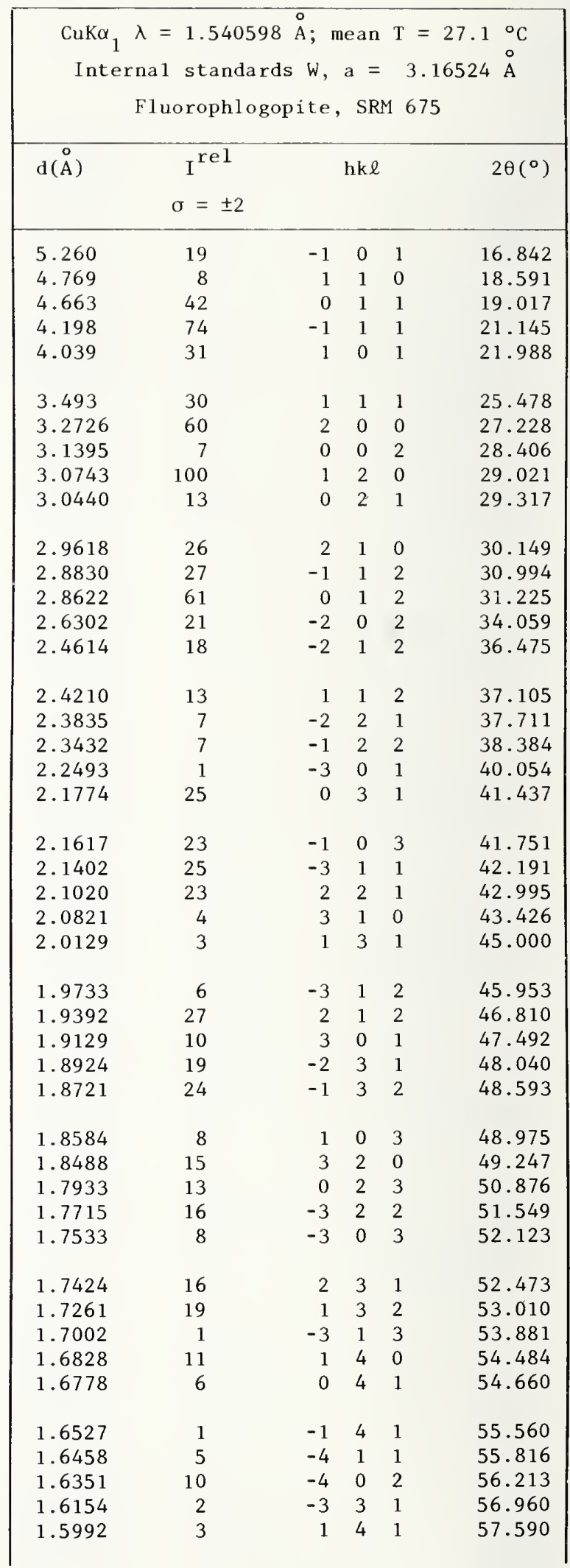


Thorium Silicate (Huttonite), $\beta$ - $\mathrm{ThSiO}_{4}$ - (continued)

\begin{tabular}{|c|c|c|c|c|c|}
\hline $\mathrm{d}(\stackrel{\circ}{\mathrm{A}})$ & $\begin{array}{c}I^{r e l} \\
\sigma= \pm 2\end{array}$ & & hke & & $2 \theta\left(^{\circ}\right)$ \\
\hline 1.5929 & 8 & 4 & 1 & 0 & 57.840 \\
\hline 1.5895 & 8 & 3 & 3 & 0 & 57.973 \\
\hline $1.5819 \mathrm{M}$ & 4 & -1 & 3 & 3 & 58.279 \\
\hline $1.5819 \mathrm{M}$ & & -1 & 1 & 4 & 58.279 \\
\hline 1.5690 & 5 & 0 & 0 & 4 & 58.806 \\
\hline 1.5656 & 7 & 3 & 1 & 2 & 58.947 \\
\hline 1.5441 & 8 & -2 & 1 & 4 & 59.849 \\
\hline 1.5405 & 9 & -3 & 3 & 2 & 60.005 \\
\hline 1.5367 & 8 & -2 & 4 & 1 & 60.167 \\
\hline 1.5315 & 4 & 0 & 1 & 4 & 60.394 \\
\hline 1.5259 & 3 & -1 & 4 & 2 & 60.640 \\
\hline 1.5227 & 3 & 0 & 4 & 2 & 60.780 \\
\hline 1.4799 & 2 & -4 & 2 & 2 & 62.731 \\
\hline 1.4720 & 4 & -1 & 2 & 4 & 63.110 \\
\hline 1.4593 & 2 & 3 & 2 & 2 & 63.720 \\
\hline 1.4527 & 5 & 2 & 4 & 1 & 64.044 \\
\hline $1.4439 \mathrm{M}$ & 3 & 2 & 2 & 3 & 64.481 \\
\hline $1.4439 \mathrm{M}$ & & 1 & 4 & 2 & 64.481 \\
\hline 1.4348 & 2 & -3 & 1 & 4 & 64.940 \\
\hline 1.3993 & 2 & -3 & 3 & 3 & 66.801 \\
\hline 1.3716 & 5 & 4 & 2 & 1 & 68.332 \\
\hline 1.3684 & 9 & -4 & 3 & 1 & 68.514 \\
\hline 1.3609 & 2 & 3 & 4 & 0 & 68.947 \\
\hline 1.3559 & 3 & -1 & 4 & 3 & 69.234 \\
\hline 1.3467 & 5 & -1 & 5 & 1 & 69.778 \\
\hline 1.3385 & 3 & 0 & 4 & 3 & 70.270 \\
\hline 1.3337 & 7 & 1 & 2 & 4 & 70.557 \\
\hline 1.3307 & 8 & -1 & 3 & 4 & 70.740 \\
\hline 1.3293 & 7 & -3 & 4 & 2 & 70.830 \\
\hline $1.3215 \mathrm{M}$ & 7 & 3 & 1 & 3 & 71.311 \\
\hline $1.3215 \mathrm{M}$ & & 3 & 3 & 2 & 71.311 \\
\hline $1.3183 \mathrm{M}$ & 6 & 2 & 4 & 2 & 71.510 \\
\hline $1.3183 \mathrm{M}$ & & 4 & 0 & 2 & 71.510 \\
\hline 1.3097 & 3 & 2 & 3 & 3 & 72.055 \\
\hline 1.2949 & 3 & 4 & 1 & 2 & 73.004 \\
\hline 1.2926 & 7 & -4 & 1 & 4 & 73.160 \\
\hline 1.2879 & 7 & 3 & 4 & 1 & 73.470 \\
\hline 1.2814 & 4 & -2 & 5 & 1 & 73.902 \\
\hline 1.2733 & 8 & 0 & 5 & 2 & 74.450 \\
\hline 1.2674 & 3 & -2 & 1 & 5 & 74.857 \\
\hline
\end{tabular}


Synonym

Thorium tantalate

Sample

The sample was prepared from stoichiometric proportions of $\mathrm{ThO}_{2}$ and $\mathrm{Ta}_{2} \mathrm{O}_{5}$. The following heating schedule, alternating with further grinding, yielded a nearly single phase product: $1400{ }^{\circ} \mathrm{C}$ for $4 \frac{1}{2}$ days, at $1625{ }^{\circ} \mathrm{C}$ for 2 hours, at $1625{ }^{\circ} \mathrm{C}$ again for 2 hours, and finally at $1625{ }^{\circ} \mathrm{C}$ for $2 \frac{1}{2}$ hours.

Traces of unreacted $\mathrm{ThO}_{2}$ remained.

\section{Comment}

Due to the minor radiation hazard, only one intensity chart was processed. Because of the $\mathrm{ThO}_{2}$ impurity and lack of repetitive measurements, the intensities may be in error by a few percent.

Color

Yellowish white

Structure

Monoclinic. The cell was found by use of the Visser program, followed by calculation of the supercell given below. The data may also be indexed on a $\mathrm{C}$-centered orthorhomic cell with twice the volume of the given monoclinic cell. The phase is apparently not isostructural with $\mathrm{Th}_{2} \mathrm{Nb}_{2} \mathrm{O}_{9}$, for which single crystal work has been done by Cava et al. (1981).

Crystallographic constants of this sample

$$
\begin{aligned}
& a=9.681(2) \AA \\
& b=7.4006(11) \\
& c=9.6766(15) \\
& \beta=106.398(10)^{\circ} \\
& a / b=1.3081 \\
& c / b=1.3075 \\
& Z=4 \text { (assumed) } \\
& V=665.08 \AA^{3} \\
& \text { Density (calc) }=9.687 \mathrm{~g} / \mathrm{cm}^{3}
\end{aligned}
$$

\begin{tabular}{|c|c|c|c|c|c|}
\hline \multicolumn{6}{|c|}{$\begin{array}{c}\mathrm{CuK \alpha}_{1} \lambda=1.540598 \AA \text {; mean } \mathrm{T}=25.8{ }^{\circ} \mathrm{C} \\
\text { Internal standards } \mathrm{W}, \mathrm{a}=3.16524 \AA \\
\text { Fluorophlogopite, SRM } 675\end{array}$} \\
\hline $\mathrm{d}(\stackrel{\circ}{\mathrm{A}})$ & $I^{\text {rel }}$ & & hke & & $2 \theta\left(^{\circ}\right)$ \\
\hline $4.722 \mathrm{M}$ & 2 & -2 & 0 & 1 & 18.777 \\
\hline $4.722 \mathrm{M}$ & & -1 & 0 & 2 & 18.777 \\
\hline $3.976 \mathrm{M}$ & 4 & -2 & 1 & 1 & 22.344 \\
\hline $3.976 \mathrm{M}$ & & -1 & 1 & 2 & 22.344 \\
\hline $3.932 \mathrm{M}$ & 3 & 2 & 1 & 0 & 22.596 \\
\hline $3.932 \mathrm{M}$ & & 0 & 1 & 2 & 22.596 \\
\hline $3.749 \mathrm{M}$ & 4 & 2 & 0 & 1 & 23.711 \\
\hline $3.749 \mathrm{M}$ & & 1 & 0 & 2 & 23.711 \\
\hline 3.701 & 6 & 0 & 2 & 0 & 24.024 \\
\hline $3.437 \mathrm{M}$ & 8 & 1 & 2 & 0 & 25.900 \\
\hline $3.437 \mathrm{M}$ & & 0 & 2 & 1 & 25.900 \\
\hline 3.336 & 7 & -1 & 2 & 1 & 26.700 \\
\hline $3.223 \mathrm{M}$ & 100 & -3 & 0 & 1 & 27.659 \\
\hline $3.223 M$ & & -1 & 0 & 3 & 27.659 \\
\hline 3.119 & 64 & 1 & 2 & 1 & 28.594 \\
\hline $3.094 \mathrm{M}$ & 7 & 3 & 0 & 0 & 28.837 \\
\hline $3.094 \mathrm{M}$ & & 0 & 0 & 3 & 28.837 \\
\hline $2.995 \mathrm{M}$ & 5 & -3 & 0 & 2 & 29.810 \\
\hline $2.995 \mathrm{M}$ & & -2 & 0 & 3 & 29.810 \\
\hline $2.955 \mathrm{M}$ & 3 & -3 & 1 & 1 & 30.221 \\
\hline $2.955 \mathrm{M}$ & & -1 & 1 & 3 & 30.221 \\
\hline $2.912 \mathrm{M}$ & 48 & -2 & 2 & 1 & 30.679 \\
\hline $2.912 \mathrm{M}$ & & -1 & 2 & 2 & 30.679 \\
\hline $2.894 M$ & 46 & 2 & 2 & 0 & 30.869 \\
\hline $2.894 M$ & & 0 & 2 & 2 & 30.869 \\
\hline $2.856 \mathrm{M}$ & 1 & 3 & 1 & 0 & 31.291 \\
\hline $2.856 \mathrm{M}$ & & 0 & 1 & 3 & 31.291 \\
\hline 2.676 & 39 & -2 & 2. & 2 & 33.462 \\
\hline $2.634 M$ & 11 & 2 & 2 & 1 & 34.004 \\
\hline $2.634 M$ & & 1 & 2 & 2 & 34.004 \\
\hline $2.430 \mathrm{M}$ & 5 & -3 & 2 & 1 & 36.959 \\
\hline $2.430 \mathrm{M}$ & & -1 & 2 & 3 & 36.959 \\
\hline $2.375 \mathrm{M}$ & 3 & 3 & 2 & 0 & 37.859 \\
\hline $2.375 \mathrm{M}$ & & 0 & 2 & 3 & 37.859 \\
\hline 2.350 & 1 & -1 & 3 & 1 & 38.271 \\
\hline $2.299 M$ & 1 & -4 & 1 & 1 & 39.154 \\
\hline $2.299 \mathrm{M}$ & & -1 & 1 & 4 & 39.154 \\
\hline 2.2821 & 1 & 2 & 2 & 2 & 39.454 \\
\hline $2.1895+$ & 7 & 3 & 2 & 1 & 41.196 \\
\hline $2.1895+$ & & 1 & 2 & 3 & 41.196 \\
\hline $2.1742 \mathrm{M}$ & 1 & -4 & 0 & 3 & 41.500 \\
\hline $2.1742 \mathrm{M}$ & & -3 & 0 & 4 & 41.500 \\
\hline 2.1180 & 1 & -3 & 2 & 3 & 42.654 \\
\hline $2.0875 \mathrm{M}$ & 2 & -4 & 1 & 3 & 43.309 \\
\hline $2.0875 \mathrm{M}$ & & -3 & 1 & 4 & 43.309 \\
\hline 2.0811 & 2 & -2 & 3 & 2 & 43.449 \\
\hline $2.0342 \mathrm{M}$ & 1 & 4 & 1 & 1 & 44.503 \\
\hline $2.0342 \mathrm{M}$ & & 1 & 1 & 4 & 44.503 \\
\hline $1.9887 \mathrm{M}$ & 2 & -4 & 2 & 2 & 45.579 \\
\hline $1.9887 \mathrm{M}$ & & -2 & 2 & 4 & 45.579 \\
\hline
\end{tabular}

Figures of merit

$\mathrm{F}_{30}=30.4(0.0092,107)$

$\mathrm{M}_{20}=24.0$

\section{Reference}

Cava, R. J., Roth, R. S., and Minor, D. B.

(1981). J. Am. Ceram. Soc. 64, 非, C-64. 
Thorium Tantalum 0xide, $\mathrm{Th}_{2} \mathrm{Ta}_{2} \mathrm{O}_{9}$ - (continued)

\begin{tabular}{|c|c|c|c|c|c|}
\hline$d(\AA)$ & $\mathrm{I}^{\mathrm{rel}}$ & & $\mathrm{hk \ell}$ & & $2 \theta\left(^{\circ}\right)$ \\
\hline $1.9660 \mathrm{M}$ & 33 & 4 & 2 & 0 & 46.135 \\
\hline $1.9660 \mathrm{M}$ & & 0 & 2 & 4 & 46.135 \\
\hline 1.9374 & 9 & -4 & 0 & 4 & 46.855 \\
\hline $1.9211 \mathrm{M}$ & 2 & -5 & 0 & 2 & 47.278 \\
\hline $1.9211 \mathrm{M}$ & & -2 & 0 & 5 & 47.278 \\
\hline $1.9036 \mathrm{M}$ & 1 & -3 & 3 & 2 & 47.739 \\
\hline $1.9036 \mathrm{M}$ & & -2 & 3 & 3 & 47.739 \\
\hline $1.8751+$ & 8 & 2 & 0 & 4 & 48.511 \\
\hline $1.8751+$ & & -3 & 2 & 4 & 48.511 \\
\hline $1.8657 \mathrm{M}$ & 4 & -5 & 1 & 1 & 48.771 \\
\hline $1.8657 \mathrm{M}$ & & -1 & 1 & 5 & 48.771 \\
\hline $1.8564 \mathrm{M}$ & 7 & 5 & 0 & 0 & 49.031 \\
\hline $1.8564 \mathrm{M}$ & & 0 & 0 & 5 & 49.031 \\
\hline 1.8505 & 15 & 0 & 4 & 0 & 49.198 \\
\hline $1.8368+$ & 14 & 4 & 2 & 1 & 49.590 \\
\hline $1.8368+$ & & 1 & 2 & 4 & 49.590 \\
\hline $1.8258 \mathrm{M}$ & 2 & 3 & 3 & 1 & 49.910 \\
\hline $1.8258 \mathrm{M}$ & & 1 & 3 & 3 & 49.910 \\
\hline $1.7291 \mathrm{M}$ & 13 & 5 & 0 & 1 & 52.908 \\
\hline $1.7291 \mathrm{M}$ & & 1 & 0 & 5 & 52.908 \\
\hline 1.7161 & 13 & -4 & 2 & 4 & 53.341 \\
\hline 1.7128 & 17 & 3 & 2 & 3 & 53.453 \\
\hline $1.7052+$ & 15 & -4 & 3 & 2 & 53.711 \\
\hline $1.7052+$ & & -2 & 3 & 4 & 53.711 \\
\hline $1.6903 \mathrm{M}$ & 3 & 4 & 3 & 0 & 54.221 \\
\hline $1.6903 \mathrm{M}$ & & 0 & 3 & 4 & 54.221 \\
\hline $1.6798 \mathrm{M}$ & 1L & 3 & 3 & 2 & 54.588 \\
\hline $1.6798 \mathrm{M}$ & & 2 & 3 & 3 & 54.588 \\
\hline $1.6725 \mathrm{M}$ & 3 & 4 & 2 & 2 & 54.848 \\
\hline $1.6725 \mathrm{M}$ & & 2 & 2 & 4 & 54.848 \\
\hline $1.6596+$ & 4 & -4 & 1 & 5 & 55.309 \\
\hline $1.6596+$ & & 0 & 2 & 5 & 55.309 \\
\hline $1.6458 \mathrm{M}$ & 10 & -5 & 2 & 3 & 55.816 \\
\hline $1.6458 \mathrm{M}$ & & -3 & 2 & 5 & 55.816 \\
\hline $1.6310 \mathrm{M}$ & 1 & -4 & 3 & 3 & 56.366 \\
\hline $1.6310 \mathrm{M}$ & & -3 & 3 & 4 & 56.366 \\
\hline $1.6108 \mathrm{M}$ & 13 & -6 & 0 & 2 & 57.137 \\
\hline $1.6108 \mathrm{M}$ & & -2 & 0 & 6 & 57.137 \\
\hline $1.6046 \mathrm{M}$ & 29 & -3 & 4 & 1 & 57.379 \\
\hline $1.6046 \mathrm{M}$ & & -1 & 4 & 3 & 57.379 \\
\hline $1.5885 \mathrm{M}$ & 2 & 3 & 4 & 0 & 58.013 \\
\hline $1.5885 \mathrm{M}$ & & 0 & 4 & 3 & 58.013 \\
\hline $1.5774 \mathrm{M}$ & 4 & 5 & 0 & 2 & 58.464 \\
\hline $1.5774 \mathrm{M}$ & & 2 & 0 & 5 & 58.464 \\
\hline $1.5662+$ & 3 & 1 & 2 & 5 & 58.923 \\
\hline $1.5662+$ & & -6 & 1 & 1 & 58.923 \\
\hline 1.5599 & 11 & 2 & 4 & 2 & 59.183 \\
\hline $1.5389 \mathrm{M}$ & 1 & -6 & 1 & 3 & 60.074 \\
\hline $1.5389 \mathrm{M}$ & & -3 & 1 & 6 & 60.074 \\
\hline 1.5172 & $1 \mathrm{~L}$ & -5 & 1 & 5 & 61.021 \\
\hline $1.5154+$ & $1 \mathrm{~L}$ & -2 & 3 & 5 & 61.102 \\
\hline $1.5154+$ & & 6 & 1 & 0 & 61.102 \\
\hline $1.5049+$ & 4 & 4 & 2 & 3 & 61.575 \\
\hline $1.5049+$ & & 3 & 2 & 4 & 61.575 \\
\hline $1.4972 \mathrm{M}$ & 1 & -6 & 0 & 4 & 61.927 \\
\hline
\end{tabular}

\begin{tabular}{|c|c|c|c|c|c|}
\hline $\mathrm{d}(\stackrel{\circ}{\AA})$ & $I^{r e l}$ & & $h k \ell$ & & $2 \theta\left(^{\circ}\right)$ \\
\hline $1.4972 \mathrm{M}$ & & -4 & 0 & 6 & 61.927 \\
\hline $1.4769 \mathrm{M}$ & 3 & -6 & 2 & 2 & 62.876 \\
\hline $1.4769 \mathrm{M}$ & & -2 & 2 & 6 & 62.876 \\
\hline $1.4736 \mathrm{M}$ & 1 & -5 & 3 & 3 & 63.034 \\
\hline $1.4736 \mathrm{M}$ & & -3 & 3 & 5 & 63.034 \\
\hline $1.4695+$ & 2 & -4 & 4 & 1 & 63.228 \\
\hline $1.4695+$ & & -1 & 2 & 6 & 63.228 \\
\hline 1.4496 & 4 & 4 & 0 & 4 & 64.201 \\
\hline $1.4162 \mathrm{M}$ & $1 \mathrm{~L}$ & 5 & 3 & 1 & 65.904 \\
\hline $1.4162 \mathrm{M}$ & & 1 & 3 & 5 & 65.904 \\
\hline $1.4106 \mathrm{M}$ & $1 \mathrm{~L}$ & 2 & 5 & 0 & 66.197 \\
\hline $1.4106 \mathrm{M}$ & & 0 & 5 & 2 & 66.197 \\
\hline $1.4020 M$ & 1 & -5 & 3 & 4 & 66.655 \\
\hline $1.4020 \mathrm{M}$ & & -4 & 3 & 5 & 66.655 \\
\hline $1.3585+$ & 1 & -2 & 1 & 7 & 69.086 \\
\hline $1.3585+$ & & 1 & 2 & 6 & 69.086 \\
\hline 1.3381 & 5 & -4 & 4 & 4 & 70.293 \\
\hline $1.3295+$ & $1 \mathrm{~L}$ & 5 & 2 & 3 & 70.817 \\
\hline 1. $3295^{+}$ & & 3 & 2 & 5 & 70.817 \\
\hline $1.3265+$ & 1 & 7 & 0 & 0 & 70.998 \\
\hline $1.3265^{+}$ & & -6 & 3 & 3 & 70.998 \\
\hline $1.3107+$ & 4 & 5 & 4 & 0 & 71.987 \\
\hline $1.3107+$ & & 0 & 3 & 6 & 71.987 \\
\hline $1.3081 \mathrm{M}$ & 3 & -6 & 2 & 5 & 72.154 \\
\hline $1.3081 \mathrm{M}$ & & -5 & 2 & 6 & 72.154 \\
\hline $1.2833 \mathrm{M}$ & 3 & -7 & 2 & 1 & 73.773 \\
\hline $1.2833 \mathrm{M}$ & & -1 & 2 & 7 & 73.773 \\
\hline $1.2746 \mathrm{M}$ & 4 & 6 & 2 & 2 & 74.364 \\
\hline $1.2746 \mathrm{M}$ & & 2 & 2 & 6 & 74.364 \\
\hline $1.2636+$ & 7 & 5 & 4 & 1 & 75.121 \\
\hline $1.2636+$ & & 1 & 4 & 5 & 75.121 \\
\hline $1.2604 M$ & 7 & -7 & 0 & 5 & 75.346 \\
\hline $1.2604 \mathrm{M}$ & & -5 & 0 & 7 & 75.346 \\
\hline $1.2573 \mathrm{M}$ & 1 & 6 & 3 & 1 & 75.567 \\
\hline $1.2573 M$ & & 1 & 3 & 6 & 75.567 \\
\hline $1.2485 \mathrm{M}$ & 4 & 7 & 2 & 0 & 76.189 \\
\hline $1.2485 \mathrm{M}$ & & 0 & 2 & 7 & 76.189 \\
\hline $1.2306 \mathrm{M}$ & $1 \mathrm{~L}$ & 4 & 4 & 3 & 77.504 \\
\hline $1.2306 \mathrm{M}$ & & 3 & 4 & 4 & 77.504 \\
\hline 1.2196 & 2 & -6 & 2 & 6 & 78.338 \\
\hline $1.2150 \mathrm{M}$ & 5 & -6 & 4 & 2 & 78.691 \\
\hline $1.2150 \mathrm{M}$ & & -2 & 4 & 6 & 78.691 \\
\hline $1.2066 \mathrm{M}$ & 3 & 1 & 6 & 1 & 79.348 \\
\hline $1.2066 \mathrm{M}$ & & -7 & 3 & 2 & 79.348 \\
\hline 1.2007 & 1 & 5 & 4 & 2 & 79.810 \\
\hline $1.1983 \mathrm{M}$ & 2 & -6 & 4 & 3 & 80.008 \\
\hline $1.1983 \mathrm{M}$ & & -3 & 4 & 6 & 80.008 \\
\hline $1.1934+$ & 3 & -7 & 2 & 5 & 80.398 \\
\hline $1.1934+$ & & -2 & 6 & 1 & 80.398 \\
\hline $1.1846 \mathrm{M}$ & 3 & 6 & 2 & 3 & 81.119 \\
\hline 1. $1846 \mathrm{M}$ & & 3 & 2 & 6 & 81.119 \\
\hline 1.1784 & $1 \mathrm{~L}$ & -1 & 1 & 8 & 81.640 \\
\hline 1.1755 & 2 & -2 & 6 & 2 & 81.886 \\
\hline
\end{tabular}


Thorium Tungsten 0xide, $\alpha-\operatorname{Th}\left(\mathrm{WO}_{4}\right)_{2}$

Synonym

Thorium tungstate

Sample

The sample was prepared by grinding

together stoichiometric amounts of $\mathrm{ThO}_{2}$ and $\mathrm{WO}_{3}$, heating with daily intermittent grinding for extended periods at temperatures up to $875{ }^{\circ} \mathrm{C}$. The sample contained a small amount of $\mathrm{ThO}_{2}$ as impurity. Hence, the intensity of lines $2 \theta=27.370,31.927$, 45.765, and 54.293 may be somewhat in error.

Color

Colorless

Structure

Orthorhombic, Pcab (61). The unit cell and space group were determined by Freundich and Pagès (1969).

Crystallographic constants of this sample

$\mathrm{a}=10.3709(14) \AA$

$\mathrm{b}=14.518(2)$

$c=9.7133(13)$

$\mathrm{a} / \mathrm{b}=0.7143$

$c / b=0.6601$

$\mathrm{Z}=8$
$\mathrm{~V}=1462.5 \AA^{3}$

Density $(\mathrm{calc})=6.610 \mathrm{~g} / \mathrm{cm}^{3}$

Figure of merit

$F_{30}=81.5(0.0092,40)$

Polymorphism

A hexagonal high temperature form was

described by Spitsyn et al. (1969).

Additional pattern

PDF card 28-1373 (Thoret, 1974)

\section{References}

Freundlich, W. and Pagès, M. (1969). C. R.

Séances Acad. Sci., Ser. C, 269, 392.

Spitsyn, V. I., Pokrovskii, A. N., Afonskii, N. S., and Trunov, V. K. (1969). Dok1. Chem.

(Eng1. Trans1.), 188, 825 .

Thoret, J. (1974). Rev. Chim. Minér. 11, 237.

\begin{tabular}{|c|c|c|c|c|c|}
\hline \multicolumn{6}{|c|}{$\begin{array}{l}\mathrm{CuK}_{1} \lambda=1.540598 \AA \text {; mean } \mathrm{T}=25.6{ }^{\circ} \mathrm{C} \\
\text { Internal standard Ag, } \mathrm{a}=4.08651 \AA\end{array}$} \\
\hline $\mathrm{d}(\stackrel{\circ}{\mathrm{A}})$ & $\begin{array}{l}\mathrm{I} \text { rel } \\
\sigma= \pm 3\end{array}$ & & hkl & & $2 \theta\left(^{\circ}\right)$ \\
\hline 7.26 & 13 & 0 & 2 & 0 & 12.180 \\
\hline 6.374 & 6 & 1 & 1 & 1 & 13.883 \\
\hline 5.939 & 1 & 1 & 2 & 0 & 14.904 \\
\hline 5.183 & 8 & 2 & 0 & 0 & 17.094 \\
\hline 5.072 & 19 & 1 & 2 & 1 & 17.472 \\
\hline 4.854 & 13 & 0 & 0 & 2 & 18.263 \\
\hline 4.608 & 7 & 0 & 1 & 2 & 19.246 \\
\hline 4.573 & 8 & 2 & 0 & 1 & 19.393 \\
\hline 4.362 & 19 & 2 & 1 & 1 & 20.342 \\
\hline 4.219 & 13 & 2 & 2 & 0 & 21.042 \\
\hline 4.038 & 16 & 0 & 2 & 2 & 21.997 \\
\hline 3.994 & 16 & 1 & 3 & 1 & 22.239 \\
\hline 3.872 & 11 & 2 & 2 & 1 & 22.951 \\
\hline 3.762 & 75 & 1 & 2 & 2 & 23.630 \\
\hline 3.628 & 36 & 0 & 4 & 0 & 24.514 \\
\hline 3.546 & 100 & 2 & 0 & 2 & 25.091 \\
\hline $3.425 \mathrm{M}$ & 29 & 0 & 3 & 2 & 25.993 \\
\hline $3.425 \mathrm{M}$ & & 1 & 4 & 0 & 25.993 \\
\hline 3.325 & 6 & 2 & 3 & 1 & 26.792 \\
\hline 3.256 & 13 & 1 & 3 & 2 & 27.370 \\
\hline 3.232 & 27 & 1 & 4 & 1 & 27.580 \\
\hline 3.184 & 30 & 2 & 2 & 2 & 28.003 \\
\hline 3.121 & 75 & 3 & 2 & 0 & 28.581 \\
\hline 3.022 & 18 & 1 & 1 & 3 & 29.537 \\
\hline $2.972 \mathrm{M}$ & 55 & 2 & 4 & 0 & 30.044 \\
\hline $2.972 M$ & & 3 & 2 & 1 & 30.044 \\
\hline 2.908 & 21 & 0 & 4 & 2 & 30.722 \\
\hline $2.844 M$ & 10 & 1 & 2 & 3 & 31.427 \\
\hline $2.844 M$ & & 2 & 4 & 1 & 31.427 \\
\hline 2.801 & 13 & 1 & 4 & 2 & 31.927 \\
\hline 2.767 & 1 & 3 & 1 & 2 & 32.330 \\
\hline 2.699 & 11 & 2 & 1 & 3 & 33.160 \\
\hline 2.626 & 14 & 3 & 2 & 2 & 34.115 \\
\hline 2.606 & 10 & 1 & 3 & 3 & 34.390 \\
\hline 2.537 & 14 & 2 & 4 & 2 & 35.350 \\
\hline $2.503 \mathrm{M}$ & 9 & 4 & 0 & 1 & 35.848 \\
\hline $2.503 \mathrm{M}$ & & 3 & 4 & 0 & 35.848 \\
\hline 2.434 & 17 & 3 & 3 & 2 & 36.892 \\
\hline 2.355 & 11 & 1 & 6 & 0 & 38.181 \\
\hline $2.335 \mathrm{M}$ & 3 & 1 & 1 & 4 & 38.531 \\
\hline $2.335 \mathrm{M}$ & & 3 & 1 & 3 & 38.531 \\
\hline $2.288 \mathrm{M}$ & 9 & 1 & 6 & 1 & 39.342 \\
\hline $2.288 \mathrm{M}$ & & 4 & 0 & 2 & 39.342 \\
\hline 2.2596 & 15 & 4 & 1 & 2 & 39.863 \\
\hline $2.2467 \mathrm{M}$ & 18 & 3 & 2 & 3 & 40.103 \\
\hline
\end{tabular}


Thorium Tungsten $0 x i d e, \alpha-\operatorname{Th}\left(\mathrm{WO}_{4}\right)_{2}-$ (continued)

\begin{tabular}{|c|c|c|c|c|c|}
\hline$d(\stackrel{\circ}{A})$ & $I^{\text {rel }}$ & & $\mathrm{hk \ell}$ & & $2 \theta\left(^{\circ}\right)$ \\
\hline $2.2467 \mathrm{M}$ & & 2 & 5 & 2 & 40.103 \\
\hline $2.2234 \mathrm{M}$ & 10 & 3 & 4 & 2 & 40.540 \\
\hline $2.2234 \mathrm{M}$ & & 4 & 3 & 1 & 40.540 \\
\hline 2.1984 & 4 & 2 & 0 & 4 & 41.023 \\
\hline 2.1934 & 4 & 2 & 6 & 0 & 41.120 \\
\hline 2.1807 & 6 & 4 & 2 & 2 & 41.370 \\
\hline 2.1712 & 4 & 0 & 3 & 4 & 41.560 \\
\hline 2.1396 & 12 & 2 & 6 & 1 & 42.203 \\
\hline 2.1180 & 6 & 1 & 5 & 3 & 42.655 \\
\hline 2.0614 & 6 & 4 & 4 & 1 & 43.886 \\
\hline 2.0188 & 7 & 0 & 4 & 4 & 44.862 \\
\hline 2.0035 & 29 & 4 & 1 & 3 & 45.223 \\
\hline 1.9992 & 30 & 2 & 6 & 2 & 45.325 \\
\hline $1.9810+$ & 10 & 1 & 4 & 4 & 45.765 \\
\hline $1.9810+$ & & 3 & 4 & 3 & 45.765 \\
\hline 1.9543 & 19 & 5 & 2 & 1 & 46.427 \\
\hline 1.9424 & 14 & 3 & 6 & 1 & 46.728 \\
\hline 1.9175 & 13 & 3 & 2 & 4 & 47.372 \\
\hline 1.9073 & 11 & 0 & 7 & 2 & 47.641 \\
\hline 1.8968 & 13 & 4 & 5 & 1 & 47.922 \\
\hline 1.8914 & 18 & 5 & 1 & 2 & 48.066 \\
\hline 1.8807 & 8 & 2 & 4 & 4 & 48.356 \\
\hline 1.8671 & 12 & 4 & 3 & 3 & 48.732 \\
\hline 1.8625 & 9 & 0 & 5 & 4 & 48.861 \\
\hline 1.8447 & 27 & 5 & 2 & 2 & 49.362 \\
\hline 1.8381 & 27 & 3 & 3 & 4 & 49.552 \\
\hline 1.8352 & 25 & 3 & 6 & 2 & 49.637 \\
\hline 1.8191 & 5 & 2 & 0 & 5 & 50.106 \\
\hline 1.8167 & 5 & 2 & 6 & 3 & 50.177 \\
\hline 1.8049 & 6 & 2 & 1 & 5 & 50.526 \\
\hline 1.7963 & 6 & 4 & 5 & 2 & 50.786 \\
\hline 1.7759 & 16 & 1 & 3 & 5 & 51.413 \\
\hline 1.7723 & 20 & 4 & 0 & 4 & 51.525 \\
\hline 1.7576 & 20 & 1 & 8 & 1 & 51.986 \\
\hline 1.7409 & 11 & 4 & 6 & 1 & 52.524 \\
\hline $1.7228 \mathrm{M}$ & 7 & 1 & 7 & 3 & 53.119 \\
\hline $1.7228 \mathrm{M}$ & & 4 & 2 & 4 & 53.119 \\
\hline 1.7128 & 13 & 2 & 8 & 0 & 53.454 \\
\hline 1.6977 & 25 & 5 & 2 & 3 & 53.966 \\
\hline 1.6883 & 20 & 5 & 4 & 2 & 54.293 \\
\hline $1.6634 \mathrm{M}$ & 2 & 4 & 3 & 4 & 55.174 \\
\hline $1.6634 \mathrm{M}$ & & 5 & 5 & 1 & 55.174 \\
\hline 1.6431 & 6 & 5 & 3 & 3 & 55.913 \\
\hline 1.6395 & 3 & 3 & 5 & 4 & 56.049 \\
\hline
\end{tabular}


Vanadium $0 x i d e$ (Karelianite), $\mathrm{V}_{2} \mathrm{O}_{3}$

Synonyms

Vanadium sesquioxide

Vanadium trioxide

Vanadic oxide

Sample

The sample was prepared by reducing $\mathrm{V}_{2} \mathrm{O}_{5}$ in a controlled atmosphere with the partial pressure of oxygen less than or equal to $10^{-20} \mathrm{~atm}$. It was then heated at $600{ }^{\circ} \mathrm{C}$ for 8 hours, at $925{ }^{\circ} \mathrm{C}$ for 18 hours, and at $900{ }^{\circ} \mathrm{C}$ for 30 hours with intermittent grinding.

Color

Black

Structure

Rhombohedral, $R \overline{3} c$ (167). The structure was

solved by Zachariasen (1928).

Crystallographic constants of this sample (Hexagonal axes)

$a=4.9540(2) \AA$

$c=14.0083(10)$

$c / a=2.8277$

$Z=6$

$\mathrm{V}=297.7 \mathrm{~A}^{3}$

Density $(\mathrm{calc})=5.016 \mathrm{~g} / \mathrm{cm}^{3}$

Figure of merit

$\mathrm{F}_{30}=64.6(0.0097,48)$

Additional pattern

PDF card 26-278 (Hanawalt et al., 1938)

References

Hanawalt, J. D., Rinn, H. W., and Frevel,

L. K. (1938). Ind. Eng. Chem. Anal. Ed.

$10,457$.

Zachariasen, F. W. H. (1928). Skr. Nor.

Vidensk.-Akad., Kl. 1: Mat.-Naturvidensk.

$\mathrm{Kl}$. 4.

\begin{tabular}{|c|c|c|c|c|c|}
\hline \multicolumn{6}{|c|}{$\begin{array}{l}\mathrm{CuK}_{1} \lambda=1.540598 \AA ; \text { mean } \mathrm{T}=27.4{ }^{\circ} \mathrm{C} \\
\text { Internal standard } \mathrm{W}, \mathrm{a}=3.16524 \AA\end{array}$} \\
\hline $\mathrm{d}(\stackrel{\circ}{\mathrm{A}})$ & $\begin{array}{l}I^{\mathrm{rel}} \\
\sigma= \pm 2\end{array}$ & & $\mathrm{hk} \ell$ & & $2 \theta\left({ }^{\circ}\right)$ \\
\hline 3.658 & 71 & 0 & 1 & 2 & 24.314 \\
\hline 2.713 & 100 & 1 & 0 & 4 & 32.987 \\
\hline 2.477 & 80 & 1 & 1 & 0 & 36.231 \\
\hline 2.336 & 6 & 0 & 0 & 6 & 38.514 \\
\hline 2.189 & 36 & 1 & 1 & 3 & 41.216 \\
\hline 2.0519 & 7 & 2 & 0 & 2 & 44.099 \\
\hline 1.8292 & 34 & 0 & 2 & 4 & 49.811 \\
\hline 1.6990 & 85 & 1 & 1 & 6 & 53.923 \\
\hline 1.6210 & 1 & 0 & 1 & 8 & 56.745 \\
\hline 1.5798 & 7 & 1 & 2 & 2 & 58.366 \\
\hline 1.4718 & 24 & 2 & 1 & 4 & 63.115 \\
\hline 1.4298 & 31 & 3 & 0 & 0 & 65.195 \\
\hline 1.3316 & 14 & 1 & 0 & 10 & 70.688 \\
\hline 1.3180 & 3 & 1 & 1 & 9 & 71.527 \\
\hline 1.2385 & 6 & 2 & 2 & 0 & 76.916 \\
\hline 1.2193 & 4 & 3 & 0 & 6 & 78.357 \\
\hline 1.1972 & 1 & 2 & 2 & 3 & 80.092 \\
\hline $1.1728 \mathrm{M}$ & 9 & 3 & 1 & 2 & 82.114 \\
\hline $1.1728 \mathrm{M}$ & & 0 & 2 & 10 & 82.114 \\
\hline 1.1674 & 2 & 0 & 0 & 12 & 82.575 \\
\hline 1.1267 & 4 & 1 & 3 & 4 & 86.267 \\
\hline 1.0940 & 12 & 2 & 2 & 6 & 89.515 \\
\hline $1.0601 \mathrm{M}$ & 9 & 0 & 4 & 2 & 93.207 \\
\hline $1.0601 \mathrm{M}$ & & 2 & 1 & 10 & 93.207 \\
\hline 1.0559 & 3 & 1 & 1 & 12 & 93.693 \\
\hline 1.0255 & 1 & 4 & 0 & 4 & 97.381 \\
\hline $.9745 \mathrm{M}$ & 1 & 2 & 3 & 2 & 104.456 \\
\hline $.9745 \mathrm{M}$ & & 0 & 1 & 14 & 104.456 \\
\hline .9690 & $1 \mathrm{~L}$ & 2 & 2 & 9 & 105.295 \\
\hline .9476 & 8 & 3 & 2 & 4 & 108.760 \\
\hline .9363 & 4 & 4 & 1 & 0 & 110.711 \\
\hline $.9068 \mathrm{M}$ & 4 & 1 & 3 & 10 & 116.309 \\
\hline $.9068 \mathrm{M}$ & & 2 & 0 & 14 & 116.309 \\
\hline .9044 & 5 & 3 & 0 & 12 & 116.799 \\
\hline .8689 & 5 & 4 & 1 & 6 & 124.874 \\
\hline
\end{tabular}


Ytterbium Fluoride, $\mathrm{YbF}_{3}$

CAS registry no.

$13760-80-0$

Sample

The sample was obtained from Research Chemicals, Nuclear Corp. of America, Phoenix, AR.

Structure

Orthorhombic, Pnma (62); isostructural with

$\mathrm{YF}_{3}$. The structure of $\mathrm{YF}_{3}$ was determined by Zalkin and Templeton (1953).

Crystallographic constants of this sample

$$
\begin{aligned}
& a=6.2164(2) \AA \\
& b=6.7857(2) \\
& c=4.4316(2) \\
& a / b=0.9161 \\
& c / b=0.6531 \\
& Z=4 \\
& V=186.94 \AA^{3} \\
& \text { Density }(\mathrm{calc})=8.173 \mathrm{~g} / \mathrm{cm}^{3}
\end{aligned}
$$

Figure of merit

$\mathrm{F}_{30}=130.2(0.0062,37)$

\section{Polymorphism}

Thoma and Brunton (1966) report that a hexagonal form exists. The transition temperature is reported to be $985^{\circ} \mathrm{C}$.

Additional patterns

PDF card 5-551 (Zalkin, Thesis, U. Calif, Berkeley, 1951)

PDF card 32-1418 (Greis, Thesis, U. Freiburg i. Br., Germany, 1976)

\section{References}

Thoma, R. E. and Brunton, G. D. (1966).

Inorg. Chem. 11, 1937.

Zalkin, A. and Templeton, D. H. (1953).

J. Am. Chem. Soc. 75, 2453.

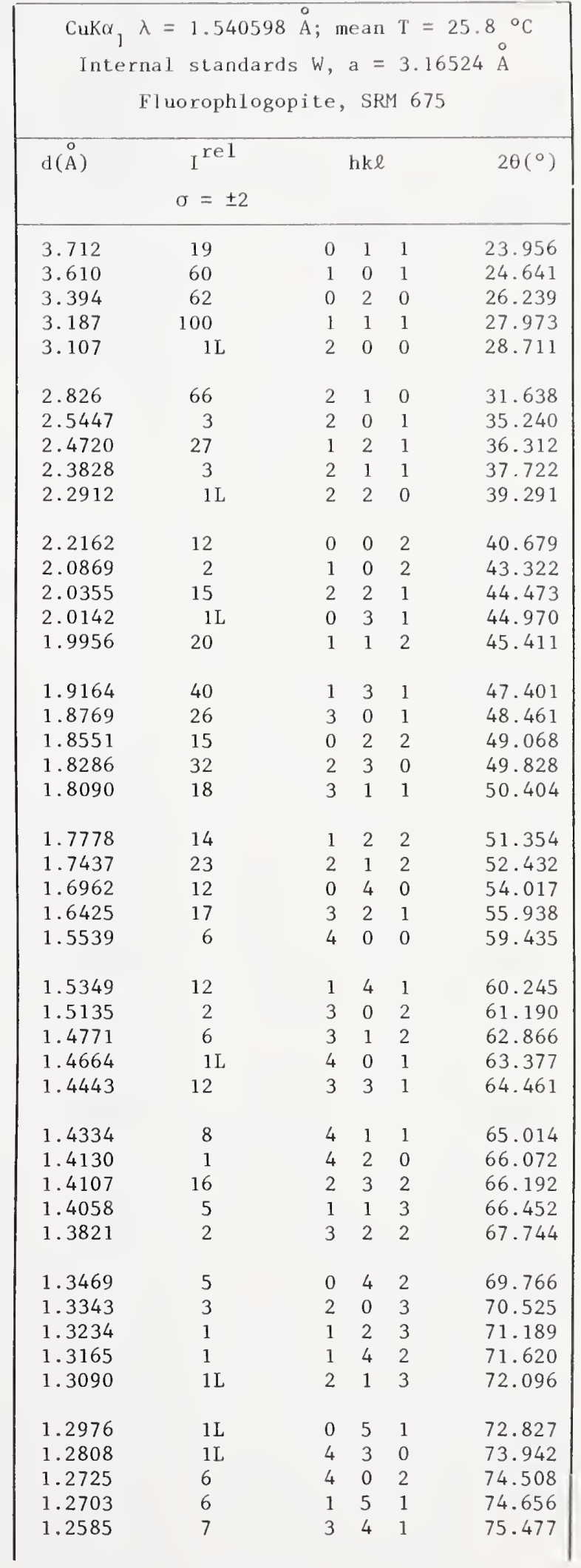


Ytterbium Fluoride, $\mathrm{YbF}_{3}$ - (continued)

\begin{tabular}{|c|c|c|c|c|c|}
\hline$d(\AA)$ & $I^{\text {rel }}$ & & hke & & $2 \theta\left(^{\circ}\right)$ \\
\hline 1.2507 & $1 \mathrm{~L}$ & 4 & 1 & 2 & 76.035 \\
\hline 1.2438 & 9 & 2 & 5 & 0 & 76.535 \\
\hline 1.2418 & 9 & 2 & 2 & 3 & 76.678 \\
\hline 1.2369 & $1 \mathrm{~L}$ & 0 & 3 & 3 & 77.040 \\
\hline 1.2306 & $1 \mathrm{~L}$ & 4 & 3 & 1 & 77.508 \\
\hline 1.2130 & $1 \mathrm{~L}$ & 1 & 3 & 3 & 78.849 \\
\hline 1.2033 & $1 \mathrm{~L}$ & 3 & 0 & 3 & 79.611 \\
\hline $1.1974 \mathrm{M}$ & $1 \mathrm{~L}$ & 2 & 5 & 1 & 80.078 \\
\hline $1.1974 \mathrm{M}$ & & 5 & 0 & 1 & 80.078 \\
\hline 1.1914 & 5 & 4 & 2 & 2 & 80.567 \\
\hline 1.1847 & 1 & 3 & 1 & 3 & 81.117 \\
\hline 1. 1790 & 4 & 5 & 1 & 1 & 81.593 \\
\hline 1.1460 & 4 & 4 & 4 & 0 & 84.470 \\
\hline 1.1379 & 3 & 1 & 5 & 2 & 85.211 \\
\hline 1.1337 & 5 & 3 & 2 & 3 & 85.603 \\
\hline 1.1310 & 5 & 0 & 6 & 0 & 85.861 \\
\hline 1.1290 & 5 & 5 & 2 & 1 & 86.047 \\
\hline 1.1087 & $1 \mathrm{~L}$ & 4 & 3 & 2 & 88.019 \\
\hline 1.0998 & 2 & 3 & 5 & 1 & 88.919 \\
\hline 1.0968 & 2 & 1 & 4 & 3 & 89.231 \\
\hline 1.0906 & $1 \mathrm{~L}$ & 1 & 0 & 4 & 89.870 \\
\hline 1.0846 & 5 & 2 & 5 & 2 & 90.503 \\
\hline 1.0792 & 3 & 1 & 6 & 1 & 91.080 \\
\hline 1.0767 & 3 & 1 & 1 & 4 & 91.351 \\
\hline $1.0707 \mathrm{M}$ & $1 \mathrm{~L}$ & 4 & 0 & 3 & 92.016 \\
\hline $1.0707 \mathrm{M}$ & & 5 & 1 & 2 & 92.016 \\
\hline 1.0620 & $1 \mathrm{~L}$ & 3 & 3 & 3 & 92.991 \\
\hline $1.0578 \mathrm{M}$ & 9 & 5 & 3 & 1 & 93.468 \\
\hline $1.0578 \mathrm{M}$ & & 4 & 1 & 3 & 93.468 \\
\hline 1.0488 & 4 & 2 & 4 & 3 & 94.528 \\
\hline 1.0384 & $1 \mathrm{~L}$ & 1 & 2 & 4 & 95.769 \\
\hline 1.0329 & 3 & 5 & 2 & 2 & 96.454 \\
\hline 1.0243 & 2 & 6 & 1 & 0 & 97.532 \\
\hline 1.0179 & 3 & 4 & 4 & 2 & 98.358 \\
\hline 1.0104 & 4 & 3 & 5 & 2 & 99.349 \\
\hline 1.0074 & 2 & 0 & 6 & 2 & 99.746 \\
\hline .9994 & $1 \mathrm{~L}$ & 0 & 5 & 3 & 100.850 \\
\hline .9961 & $1 \mathrm{~L}$ & 4 & 5 & 1 & 101.307 \\
\hline .9944 & $1 \mathrm{~L}$ & 1 & 6 & 2 & 101.542 \\
\hline .9867 & $1 \mathrm{~L}$ & 1 & 5 & 3 & 102.652 \\
\hline .9825 & 1 & 1 & 3 & 4 & 103.267 \\
\hline .9812 & $1 \mathrm{~L}$ & 3 & 4 & 3 & 103.451 \\
\hline .9780 & 1 & 5 & 4 & 1 & 103.925 \\
\hline .9687 & 8 & 3 & 6 & 1 & 105.350 \\
\hline .9677 & 7 & 4 & 3 & 3 & 105.495 \\
\hline $.9669 \mathrm{M}$ & 6 & 3 & 1 & 4 & 105.620 \\
\hline $.9669 M$ & & 6 & 2 & 1 & 105.620 \\
\hline $.9513 \mathrm{M}$ & $1 \mathrm{~L}$ & 2 & 5 & 3 & 108.140 \\
\hline $.9513 \mathrm{M}$ & & 5 & 0 & 3 & 108.140 \\
\hline $.9420 \mathrm{M}$ & 4 & 5 & 1 & 3 & 109.716 \\
\hline $.9420 \mathrm{M}$ & & 6 & 3 & 0 & 109.716 \\
\hline
\end{tabular}


Synonym

Zinc vanadate

Sample

The sample was made by heating a $3: 1$ molar mixture of $\mathrm{ZnO}$ and $\mathrm{V}_{2} \mathrm{O}_{5}$ at $780{ }^{\circ} \mathrm{C}$ for 3 days, with several regrindings.

Color

Yellowish white

Structure

Orthorhombic, Abam (64) (Brisi, 1960).

Isostructural with $\mathrm{Co}_{3}\left(\mathrm{VO}_{4}\right)_{2}$.

Crystallographic constants of this sample

$\mathrm{a}=8.299(1) \AA$

$\mathrm{b}=11.5284(14)$

$\mathrm{c}=6.1116(7)$

$a / b=0.7199$

$c / b=0.5301$

$Z=4$

$\mathrm{V}=584.72 \AA^{3}$

Density $(\mathrm{calc})=4.839 \mathrm{~g} / \mathrm{cm}^{3}$

Figure of merit

$\mathrm{F}_{30}=66.6(0.010,44)$

Polymorphism

Brown and Hummel (1965) report two polymorphic changes in $\mathrm{Zn}_{3}\left(\mathrm{VO}_{4}\right)_{2}$ at $795^{\circ}$ and $815{ }^{\circ} \mathrm{C}$. This is disputed by Makarov et al. (1971).

Additional patterns

PDF card 16-830 (Brisi, 1960)

PDF card 19-1469 (Brown and Hummel, 1965)

Makarov et al. (1971)

References

Brisi, C. (1960). Ric. Sci. 30, 1339.

Brown, J. J. and Hummel, F. A. (1965).

Trans. Br. Ceram. Soc. 64, 419.

Makarov, V. A., Fotiev, A. A., and

Serebryakova, L. N. (1971). Russ.

J. Inorg. Chem. Eng. Trans1. 16, 1515 .

\begin{tabular}{|c|c|c|c|c|c|}
\hline \multicolumn{6}{|c|}{$\begin{array}{l}{\text { CuK } \alpha_{1}}_{1}=1.540598 \AA \\
\text { Internal standard Si, SRM } 640 \mathrm{a}\end{array}$} \\
\hline \multirow[t]{2}{*}{$\mathrm{d}(\stackrel{\circ}{\mathrm{A}})$} & \multirow{2}{*}{\multicolumn{2}{|c|}{$\begin{array}{c}\text { rel } \\
\sigma= \pm 5\end{array}$}} & \multirow{2}{*}{\multicolumn{2}{|c|}{ hke }} & \multirow[t]{2}{*}{$2 \theta\left({ }^{\circ}\right)$} \\
\hline & & & & & \\
\hline 5.762 & 13 & 0 & 2 & 0 & 15.364 \\
\hline 4.731 & 18 & 1 & 2 & 0 & 18.742 \\
\hline 4.147 & 7 & 2 & 0 & 0 & 21.410 \\
\hline 3.367 & 20 & 2 & 2 & 0 & 26.452 \\
\hline 3.290 & 27 & 2 & 1 & 1 & 27.080 \\
\hline 3.059 & 1 & 0 & 0 & 2 & 29.165 \\
\hline 3.030 & 57 & 1 & 3 & 1 & 29.460 \\
\hline 2.882 & 30 & 0 & 4 & 0 & 31.004 \\
\hline 2.723 & 4 & 1 & 4 & 0 & 32.866 \\
\hline 2.700 & 9 & 0 & 2 & 2 & 33.156 \\
\hline 2.568 & 100 & 1 & 2 & 2 & 34.907 \\
\hline 2.494 & 47 & 3 & 2 & 0 & 35.980 \\
\hline $2.462 \mathrm{M}$ & 25 & 3 & 1 & 1 & 36.466 \\
\hline $2.462 \mathrm{M}$ & & 2 & 0 & 2 & 36.466 \\
\hline 2.367 & 3 & 2 & 4 & 0 & 37.984 \\
\hline 2.263 & 3 & 2 & 2 & 2 & 39.797 \\
\hline 2.0964 & 37 & 0 & 4 & 2 & 43.116 \\
\hline 2.0878 & 36 & 1 & 5 & 1 & 43.302 \\
\hline 2.0747 & 1 & 4 & 0 & 0 & 43.589 \\
\hline 2.0323 & 1 & 1 & 4 & 2 & 44.546 \\
\hline 1.9533 & 3 & 4 & 2 & 0 & 46.453 \\
\hline 1.9319 & 4 & 3 & 2 & 2 & 46.998 \\
\hline 1.9203 & 4 & 0 & 6 & 0 & 47.299 \\
\hline 1.9154 & 4 & 2 & 5 & 1 & 47.426 \\
\hline $1.8716 \mathrm{M}$ & 5 & 1 & 6 & 0 & 48.608 \\
\hline $1.8716 \mathrm{M}$ & & 2 & 4 & 2 & 48.608 \\
\hline 1.8061 & 4 & 2 & 1 & 3 & 50.491 \\
\hline 1.7588 & 6 & 1 & 3 & 3 & 51.950 \\
\hline 1.7167 & 8 & 4 & 0 & 2 & 53.323 \\
\hline 1.6509 & 3 & 2 & 3 & 3 & 55.627 \\
\hline 1.6457 & 4 & 4 & 2 & 2 & 55.817 \\
\hline 1.6237 & 7 & 3 & 1 & 3 & 56.641 \\
\hline 1.5963 & 23 & 1 & 6 & 2 & 57.704 \\
\hline 1.5863 & 8 & 5 & 1 & 1 & 58.103 \\
\hline 1.5778 & 12 & 3 & 6 & 0 & 58.444 \\
\hline 1.5276 & 13 & 0 & 0 & 4 & 60.562 \\
\hline 1.5145 & 6 & 2 & 6 & 2 & 61.145 \\
\hline 1.5014 & 3 & 1 & 5 & 3 & 61.734 \\
\hline 1.4848 & 4 & 2 & 7 & 1 & 62.503 \\
\hline 1.4748 & 38 & 4 & 4 & 2 & 62.975 \\
\hline 1.4542 & 2 & 1 & 2 & 4 & 63.972 \\
\hline 1.4414 & 13 & 0 & 8 & 0 & 64.609 \\
\hline 1.4377 & 2 & 5 & 4 & 0 & 64.793 \\
\hline $1.4331 \mathrm{M}$ & 1 & 2 & 0 & 4 & 65.028 \\
\hline $1.4331 \mathrm{M}$ & & 2 & 5 & 3 & 65.028 \\
\hline
\end{tabular}


Zinc Vanadium 0xide, $\mathrm{Zn}_{3}\left(\mathrm{VO}_{4}\right)_{2}-$ (continued)

\begin{tabular}{|llllll|}
\hline$d(\AA)$ & $I^{\text {rel }}$ & \multicolumn{3}{c|}{ hke } & $2 \theta\left(^{\circ}\right)$ \\
& $\sigma= \pm 5$ & & & & \\
\hline 1.4095 & 1 & 4 & 6 & 0 & 66.254 \\
1.3918 & 1 & 2 & 2 & 4 & 67.211 \\
1.3786 & 2 & 3 & 7 & 1 & 67.937 \\
1.3495 & 3 & 0 & 4 & 4 & 69.610 \\
1.3400 & $1 \mathrm{~L}$ & 6 & 1 & 1 & 70.180 \\
& & & & & \\
1.3028 & 9 & 3 & 2 & 4 & 72.493 \\
1.2838 & 2 & 2 & 4 & 4 & 73.744 \\
1.2789 & 4 & 5 & 1 & 3 & 74.070 \\
1.2563 & 1 & 5 & 6 & 0 & 75.638 \\
1.2471 & 3 & 6 & 4 & 0 & 76.290 \\
1.2436 & 2 & 2 & 8 & 2 & 76.543 \\
1.2302 & 3 & 4 & 0 & 4 & 77.534 \\
\hline
\end{tabular}


Synonym

Zirconium diboride

CAS registry no. 12045-64-6

Sample

The sample was prepared at NBS by S. Lang.

Color

Very dark gray

Structure

Hexagona 1, P6/mmm (191). Kiessling (1949)

and Norton et al. (1949) studied the structure

of $\mathrm{ZrB}_{2}$. This phase is isomorphous with $\mathrm{AlB}_{2}$.

Crystallographic constants of this sample

$\mathrm{a}=3.16870(8) \AA$

$c=3.53002(10)$

$c / a=1.1140$

$Z=1$

$V=30.70 \AA^{3}$

Density $(\mathrm{calc})=6.104 \mathrm{~g} / \mathrm{cm}^{3}$

Figure of merit

$\mathrm{F}_{23}=175.3(0.0057,23)$

Additional pattern

PDF card 6-610 (Am. Electro Metal Co.)

References

Kiessling, R. (1949). Acta Chem. Scand. 3 , 90.

Norton, J. T., Blumenthal, H., and Sindeband,

J. (1949). Metal1. Trans. 185, 749.

\begin{tabular}{|c|c|c|c|c|c|}
\hline \multicolumn{6}{|c|}{$\begin{array}{c}\mathrm{CuK \alpha}{ }_{1} \lambda=1.540598 \AA \text {; mean } \mathrm{T}=24.9{ }^{\circ} \mathrm{C} \\
\text { Internal standard Si, SRM } 640 \mathrm{a}\end{array}$} \\
\hline$d(\AA)$ & $\begin{array}{c}\text { rel } \\
\sigma= \pm 1\end{array}$ & & hke & & $2 \theta\left({ }^{\circ}\right)$ \\
\hline 3.530 & 30 & 0 & 0 & 1 & 25.205 \\
\hline 2.7445 & 67 & 1 & 0 & 0 & 32.601 \\
\hline 2.1663 & 100 & 1 & 0 & 1 & 41.658 \\
\hline 1.7652 & 9 & 0 & 0 & 2 & 51.746 \\
\hline 1.5843 & 19 & 1 & 1 & 0 & 58.184 \\
\hline 1.4845 & 21 & 1 & 0 & 2 & 62.515 \\
\hline 1.4455 & 16 & 1 & 1 & 1 & 64.404 \\
\hline 1.3722 & 8 & 2 & 0 & 0 & 68.298 \\
\hline 1.2789 & 16 & 2 & 0 & 1 & 74.071 \\
\hline 1.1790 & 13 & 1 & 1 & 2 & 81.585 \\
\hline 1.1769 & 1 & 0 & 0 & 3 & 81.770 \\
\hline 1.0832 & 8 & 2 & 0 & 2 & 90.654 \\
\hline 1.0813 & 10 & 1 & 0 & 3 & 90.853 \\
\hline 1.0372 & 7 & 2 & 1 & 0 & 95.918 \\
\hline .9951 & 14 & 2 & 1 & 1 & 101.442 \\
\hline .9446 & 4 & 1 & 1 & 3 & 109.266 \\
\hline .9147 & 3 & 3 & 0 & 0 & 114.733 \\
\hline .8942 & 10 & 2 & 1 & 2 & 118.958 \\
\hline .8932 & 6 & 2 & 0 & 3 & 119.185 \\
\hline .8855 & 4 & 3 & 0 & 1 & 120.899 \\
\hline .8825 & 2 & 0 & 0 & 4 & 121.593 \\
\hline .8402 & 4 & 1 & 0 & 4 & 132.938 \\
\hline .8122 & 7 & 3 & 0 & 2 & 143.047 \\
\hline
\end{tabular}


Synonym

Zirconium hydrogen orthophosphate hydrate

CAS registry no.

13933-56-7

\section{Sample}

The sample was prepared by the method of Alberti and Torracca (1968). $\mathrm{ZrOCl} \cdot 8 \mathrm{H}_{2} \mathrm{O}$ was dissolved in $\mathrm{H}_{2} \mathrm{O}$. $\mathrm{HF}$ and $\mathrm{H}_{3} \mathrm{PO}_{4}$ were added and the solution allowed to evaporate slowly at room temperature. The crystals formed were very fine needles.

Color

Colorless

\section{Structure}

Monoclinic, $\mathrm{P} 21 / \mathrm{n}$ (14) (Ahrland and

Albertsson, 1969). The structure was

determined by Clearfield and Smith (1969).

Crystallographic constants of this sample

$$
\begin{aligned}
& a=15.463(4) \AA \\
& b=5.294(2) \\
& c=9.0674(15) \\
& \beta=101.70(2)^{\circ} \\
& a / b=2.9209 \\
& c / b=1.7128 \\
& Z=4 \\
& V=726.83 \AA^{3} \\
& \text { Density (calc) }=2.753 \mathrm{~g} / \mathrm{cm}^{3}
\end{aligned}
$$

Figure of merit

$$
\mathrm{F}_{30}=21.0(0.013,108)
$$

Additional patterns

PDF card 19-1489 (Sedlakova and Pekarks, 1966)

PDF card 21-396 (Michel and Weiss, 1967)

PDF card 21-397 (Priv. Comm., Winkler, 1968)

PDF card 22-1022 (Ahrland and Albertsson, 1969)

References

Ahrland, S. and Albertsson, J. (1969). Acta

Chem. Scand. 23, 1446.

Alberti, G. and Torracca, E. (1968). J.

Inorg. Nucl. Chem. 30, 317.

\begin{tabular}{|c|c|c|c|c|c|}
\hline \multirow{2}{*}{$\begin{array}{c}\mathrm{d}(\mathrm{A}) \\
7.59\end{array}$} & \multirow{2}{*}{$\begin{array}{c}\mathrm{I}^{\mathrm{rel}} \\
\sigma= \pm 1 \\
100\end{array}$} & \multicolumn{3}{|c|}{ hke } & \multirow{2}{*}{$\frac{2 \theta\left(^{\circ}\right)}{11.653}$} \\
\hline & & 2 & 0 & 0 & \\
\hline 4.489 & 14 & -1 & 1 & 1 & 19.761 \\
\hline 4.441 & 8 & 0 & 0 & 2 & 19.978 \\
\hline 4.052 & $1 \mathrm{~L}$ & 3 & 0 & 1 & 21.917 \\
\hline 3.785 & 1 & 4 & 0 & 0 & 23.483 \\
\hline 3.568 & 51 & -3 & 1 & 1 & 24.934 \\
\hline 3.531 & 31 & 2 & 0 & 2 & 25.198 \\
\hline 3.220 & 3 & -4 & 0 & 2 & 2.7 .683 \\
\hline 3.082 & 1 & 4 & 1 & 0 & 28.948 \\
\hline 3.045 & 2 & -3 & 1 & 2 & 29.309 \\
\hline 3.022 & $1 \mathrm{~L}$ & -1 & 0 & 3 & 29.539 \\
\hline $2.649 \mathrm{M}$ & 14 & -5 & 1 & 1 & 33.811 \\
\hline $2.649 \mathrm{M}$ & & 0 & 2 & 0 & 33.811 \\
\hline 2.626 & 14 & -1 & 1 & 3 & 34.112 \\
\hline $2.524 \mathrm{M}$ & 4 & -1 & 2 & 1 & 35.539 \\
\hline $2.524 \mathrm{M}$ & & 6 & 0 & 0 & 35.539 \\
\hline 2.475 & $1 \mathrm{~L}$ & 1 & 1 & 3 & 36.263 \\
\hline 2.408 & 6 & 5 & 1 & 1 & 37.305 \\
\hline 2.353 & 2 & 3 & 0 & 3 & 38.219 \\
\hline 2.277 & $1 \mathrm{~L}$ & 6 & 1 & 0 & 39.544 \\
\hline 2.219 & $1 \mathrm{~L}$ & 0 & 0 & 4 & 40.631 \\
\hline 2.197 & 1 & -6 & 1 & 2 & 41.059 \\
\hline 2.169 & 3 & 4 & 2 & 0 & 41.600 \\
\hline 2.164 & 2 & -5 & 1 & 3 & 41.705 \\
\hline 2.118 & 3 & 2 & 2 & 2 & 42.663 \\
\hline 2.111 & 2 & -4 & 0 & 4 & 42.806 \\
\hline 2.045 & 5 & -4 & 2 & 2 & 44.258 \\
\hline 2.038 & 4 & -7 & 1 & 1 & 44.425 \\
\hline 2.023 & 3 & 2 & 0 & 4 & 44.769 \\
\hline 1.996 & 1 & 3 & 2 & 2 & 45.407 \\
\hline 1.968 & $1 \mathrm{~L}$ & -7 & 1 & 2 & 46.077 \\
\hline 1.930 & $1 \mathrm{I}$ & 5 & 0 & 3 & 47.055 \\
\hline $1.8922 \mathrm{M}$ & 5 & 8 & 0 & 0 & 48.044 \\
\hline $1.8922 \mathrm{M}$ & & 5 & 2 & 1 & 48.044 \\
\hline 1.8791 & 5 & 7 & 1 & 1 & 48.400 \\
\hline $1.8647 \mathrm{M}$ & 5 & 4 & 2 & 2 & 48.800 \\
\hline $1.8647 \mathrm{M}$ & & -6 & 0 & 4 & 48.800 \\
\hline
\end{tabular}

Clearfield, A. and Smith, G. D. (1969).

Inorg. Chem. 8, 431 .

Michel, E. and Weiss, A. (1967). Z.

Naturforsch. 22B, 1100 .

Sedlakova, L, and Pekarks, V. (1966). J .

Less-Common Met. 10, 130.

CuK ${ }_{1} \lambda=1.540598 \stackrel{\circ}{\AA}$; mean $\mathrm{T}=26 \pm 2{ }^{\circ} \mathrm{C}$

Internal standards Si, SRM 640a

Fluorophlogopite, SRM 675 
Zirconium Titanium Oxide, $\mathrm{ZrTiO}_{4}$

Synonym

Zirconium titanate

CAS registry no. $12036-70-3$

Sample

The sample was prepared by heating equimolar amounts of $\mathrm{ZrO}_{2}$ and $\mathrm{TiO}_{2}$ at $1500{ }^{\circ} \mathrm{C}$ for 66 hours. It was then heat-treated at $1500{ }^{\circ} \mathrm{C}$ for 17 hours and quenched in distilled water.

Color

Pale pink

Structure Orthorhombic, Pnab (60). The unit cell was determined by Coughanour et al. (1954) and the space group by Newnham (1967).

Crystallographic constants of this sample

$$
\begin{aligned}
& a=5.0358(5) \AA \\
& b=5.4874(6) \\
& c=4.8018(6) \\
& a / b=0.9177 \\
& c / b=0.8751 \\
& Z=2 \\
& V=132.69 \AA^{3} \\
& \text { Density (calc) }=5.084 \mathrm{~g} / \mathrm{cm}^{3}
\end{aligned}
$$

Figure of merit

$\mathrm{F}_{30}=68.0(0.011,41)$

Additional pattern

\begin{tabular}{|c|c|c|c|c|c|}
\hline \multicolumn{6}{|c|}{$\begin{array}{l}\mathrm{CuK}_{1} \lambda=1.540598 \AA \\
\text { Internal mean } \mathrm{T}=25.5{ }^{\circ} \mathrm{C} \\
\end{array}$} \\
\hline$d(\stackrel{\circ}{A})$ & $\begin{array}{l}\text { rel } \\
\sigma= \pm 1\end{array}$ & & hkl & & $2 \theta\left(^{\circ}\right)$ \\
\hline 3.614 & 16 & 0 & 1 & 1 & 24.614 \\
\hline 2.934 & 100 & 1 & 1 & 1 & 30.443 \\
\hline 2.744 & 13 & 0 & 2 & 0 & 32.612 \\
\hline 2.517 & 10 & 2 & 0 & 0 & 35.642 \\
\hline 2.408 & 9 & 1 & 2 & 0 & 37.312 \\
\hline 2.229 & 3 & 2 & 0 & 1 & 40.431 \\
\hline 2.154 & 10 & 1 & 2 & 1 & 41.911 \\
\hline 2.0661 & 4 & 2 & 1 & 1 & 43.781 \\
\hline 2.0158 & $1 \mathrm{~L}$ & 1 & 1 & 2 & 44.931 \\
\hline 1.8542 & 13 & 2 & 2 & 0 & 49.092 \\
\hline 1.8067 & 11 & 0 & 2 & 2 & 50.472 \\
\hline 1.7376 & 13 & 2 & 0 & 2 & 52.632 \\
\hline 1.7096 & 9 & 0 & 3 & 1 & 53.562 \\
\hline 1.7013 & 12 & 1 & 2 & 2 & 53.843 \\
\hline 1.6192 & 6 & 1 & 3 & 1 & 56.813 \\
\hline 1.5366 & $1 \mathrm{~L}$ & 0 & 1 & 3 & 60.174 \\
\hline 1.5224 & 10 & 3 & 1 & 1 & 60.794 \\
\hline 1.4679 & 11 & 2 & 2 & 2 & 63.305 \\
\hline 1.4318 & 4 & 3 & 2 & 0 & 65.096 \\
\hline 1.4144 & 6 & 2 & 3 & 1 & 65.996 \\
\hline 1.3506 & $1 \mathrm{~L}$ & 2 & 0 & 3 & 69.548 \\
\hline 1.3345 & $1 \mathrm{~L}$ & 3 & 1 & 2 & 70.508 \\
\hline 1.3233 & 4 & 1 & 4 & 0 & 71.199 \\
\hline 1.3121 & $1 \mathrm{~L}$ & 2 & 1 & 3 & 71.899 \\
\hline 1.2763 & 1 & 1 & 4 & 1 & 74.250 \\
\hline 1.2590 & 1 & 4 & 0 & 0 & 75.441 \\
\hline 1.2298 & $1 \mathrm{~L}$ & 3 & 2 & 2 & 77.562 \\
\hline 1.2174 & $1 \mathrm{~L}$ & 4 & 0 & 1 & 78.503 \\
\hline $1.2046 \mathrm{M}$ & 2 & 2 & 4 & 0 & 79.504 \\
\hline $1.2046 \mathrm{M}$ & & 0 & 3 & 3 & 79.504 \\
\hline 1.2003 & 1 & 0 & 0 & 4 & 79.844 \\
\hline 1.1978 & 1 & 3 & 3 & 1 & 80.044 \\
\hline 1.1910 & 1 & 0 & 4 & 2 & 80.594 \\
\hline 1.1890 & 1 & 4 & 1 & 1 & 80.764 \\
\hline 1.1716 & $1 \mathrm{~L}$ & 1 & 3 & 3 & 82.215 \\
\hline 1.1591 & 3 & 1 & 4 & 2 & 83.296 \\
\hline 1.1443 & 1 & 4 & 2 & 0 & 84.627 \\
\hline 1.1336 & 1 & 3 & 1 & 3 & 85.608 \\
\hline 1.1152 & 1 & 4 & 0 & 2 & 87.379 \\
\hline $1.0997 \mathrm{M}$ & $1 \mathrm{~L}$ & 0 & 2 & 4 & 88.930 \\
\hline $1.0997 \mathrm{M}$ & & 3 & 3 & 2 & 88.930 \\
\hline 1.0864 & 2 & 2 & 3 & 3 & 90.312 \\
\hline
\end{tabular}

PDF card 7-290 (Coughanour et al., 1954)

\section{References}

Coughanour, L. W., Roth, R. S., and DeProsse, V. A. (1954). J. Res. Nat1. Bur. Stand. (U.S.) , 52, 37 .

Newnham, R. E. (1967). J. Am. Ceram. Soc. 50, 216. 
Zirconium Titanium 0xide, $\mathrm{Zr}_{5} \mathrm{Ti}_{7} \mathrm{O}_{24}$

Synonym

Zirconium titanate

Sample

The sample was prepared by blending $\mathrm{ZrO}_{2}$ and $\mathrm{TiO}_{2}$ in the molar ratio of $5: 7$, doped with $1 / 2$ mole $\%$ of $\mathrm{Y}_{2} \mathrm{O}_{3}$ to promote crystallinity, and calcined at $1000{ }^{\circ} \mathrm{C}$ for 24 hours, twice reground and fired at $1500{ }^{\circ} \mathrm{C}$ for 20 hours, heat-treated at $1300{ }^{\circ} \mathrm{C}$ for several hours and cooled at $1{ }^{\circ} \mathrm{C}$ /hour to $650{ }^{\circ} \mathrm{C}$ (McHale et al., 1983).

\section{Color}

Pale yellowish pink

\section{Structure}

Orthorhombic, Pcan (60). The material has the columbite structure (McHale et al., 1983). The structure was confirmed by neutron powder diffraction (Bourdot, 1983).

Crystallographic constants of this sample $\mathrm{a}=5.3255(5) \AA$

$\mathrm{b}=14.374(2)$

$c=5.0236(5)$

$a / b=0.3705$

$c / b=0.3495$

$\begin{array}{ll}Z=1 & \\ V & =384.55 \AA^{3}\end{array}$

Density $(\mathrm{calc})=5.075 \mathrm{~g} / \mathrm{cm}^{3}$

Figure of merit

$\mathrm{F}_{30}=66.8(0.011,43)$

References

Bourdot, P. (1983). Private communication.

McHale, A. E., Roth, R. S., Santoro, A., and Simmons, J. (1983). Am. Ceram. Soc. Bu11. 62, 387 .

\begin{tabular}{|c|c|c|c|c|c|}
\hline \multirow{2}{*}{$\begin{array}{c}\begin{array}{c}\text { CuK } \\
\text { In }\end{array} \\
\mathrm{d}(\dot{\mathrm{A}})\end{array}$} & $\begin{array}{l}=1.5405 \\
1 \text { stand }\end{array}$ & \multicolumn{2}{|c|}{$\begin{array}{l}\text { A; mean } \\
\text { Si, SRM }\end{array}$} & \multicolumn{2}{|c|}{$\begin{array}{l}\mathrm{T}=26.0{ }^{\circ} \mathrm{C} \\
640 \mathrm{a}\end{array}$} \\
\hline & $\begin{array}{c}\text { rel } \\
\sigma= \pm 1\end{array}$ & & hke & & $2 \theta\left({ }^{\circ}\right)$ \\
\hline 7.19 & 4 & 0 & 2 & 0 & 12.305 \\
\hline 3.592 & 6 & 0 & 4 & 0 & 24.765 \\
\hline 3.561 & 14 & 1 & 3 & 0 & 24.986 \\
\hline 3.543 & 11 & 1 & 1 & 1 & 25.116 \\
\hline 3.257 & 8 & 1 & 2 & 1 & 27.358 \\
\hline 2.904 & 100 & 1 & 3 & 1 & 30.760 \\
\hline 2.663 & 15 & 2 & 0 & 0 & 33.628 \\
\hline 2.561 & 3 & 1 & 4 & 1 & 35.013 \\
\hline 2.513 & 9 & 0 & 0 & 2 & 35.704 \\
\hline 2.498 & 4 & 2. & 2 & 0 & 35.921 \\
\hline 2.475 & 2 & 0 & 1 & 2 & 36.264 \\
\hline 2.396 & 4 & 0 & 6 & 0 & 37.506 \\
\hline 2.353 & 4 & 2 & 0 & 1 & 38.217 \\
\hline 2.321 & $1 \mathrm{~L}$ & 2 & 1 & 1 & 38.758 \\
\hline 2.260 & 4 & 1 & 5 & 1 & 39.863 \\
\hline 2.225 & 1 & 0 & 3 & 2 & 40.513 \\
\hline 2.1389 & 4 & 2 & 4 & 0 & 42.218 \\
\hline 2.1123 & 10 & 2 & 3 & 1 & 42.776 \\
\hline 2.0594 & 5 & 0 & 4 & 2 & 43.931 \\
\hline 2.0531 & 4 & 1 & 3 & 2 & 44.071 \\
\hline 1.9680 & 1 & 2 & 4 & 1 & 46.084 \\
\hline 1.9190 & 2 & 1 & 4 & 2 & 47.332 \\
\hline 1.8904 & 2 & 0 & 5 & 2 & 48.092 \\
\hline 1.8267 & 14 & 2 & 0 & 2 & 49.883 \\
\hline 1.8127 & 2 & 2 & 1 & 2 & 50.293 \\
\hline 1.7905 & 3 & 1 & 7 & 1 & 50.964 \\
\hline 1.7814 & 15 & 2 & 6 & 0 & 51.241 \\
\hline 1.7705 & 6 & 2 & 2 & 2 & 51.580 \\
\hline 1.7333 & 10 & 0 & 6 & 2 & 52.772 \\
\hline 1.6788 & 7 & 2 & 6 & 1 & 54.626 \\
\hline 1.6651 & 11 & 3 & 3 & 0 & 55.112 \\
\hline 1.6620 & 8 & 3 & 1 & 1 & 55.222 \\
\hline 1.6285 & 2 & 2 & 4 & 2 & 56.461 \\
\hline 1.5880 & 5 & 1 & 1 & 3 & 58.035 \\
\hline 1.5804 & 11 & 3 & 3 & 1 & 58.342 \\
\hline 1.5592 & 3 & 1 & 2 & 3 & 59.211 \\
\hline 1.5425 & 1 & 2 & 5 & 2 & 59.920 \\
\hline 1.5293 & $1 \mathrm{~L}$ & 1 & 9 & 0 & 60.489 \\
\hline 1.5153 & 5 & 1 & 3 & 3 & 61.108 \\
\hline 1.4895 & 1 & 2 & 8 & 0 & 62.283 \\
\hline 1.4634 & 6 & 1 & 9 & 1 & 63.520 \\
\hline 1.4612 & 5 & 0 & 8 & 2 & 63.627 \\
\hline 1.4592 & 4 & 1 & 4 & 3 & 63.724 \\
\hline 1.4534 & 4 & 2 & 6 & 2 & 64.012 \\
\hline 1.4459 & 2 & 3 & 5 & 1 & 64.381 \\
\hline
\end{tabular}


Zirconium Titanium 0xide, $Z \mathrm{Zr}_{5} \mathrm{Ti}_{7} \mathrm{O}_{24}$ - (continued)

\begin{tabular}{|c|c|c|c|c|c|}
\hline$d(\AA)$ & $\begin{array}{c}I^{r e]} \\
\sigma= \pm 1\end{array}$ & & $\mathrm{k} \ell$ & & $2 \theta\left({ }^{\circ}\right)$ \\
\hline 1.4209 & $1 \mathrm{~L}$ & 3 & 2 & 2 & 65.655 \\
\hline 1.4176 & 1 & 2 & 0 & 3 & 65.827 \\
\hline 1.4109 & 3 & 2 & 1 & 3 & 66.179 \\
\hline 1.3877 & 2 & 3 & 3 & 2 & 67.434 \\
\hline 1.3649 & 1 & 2 & 7 & 2 & 68.714 \\
\hline 1.3448 & $1 \mathrm{~L}$ & 3 & 4 & 2 & 69.889 \\
\hline 1.3093 & 2 & 4 & 2 & 0 & 72.076 \\
\hline 1.2971 & $1 \mathrm{~L}$ & 3 & 7 & 1 & 72.862 \\
\hline 1.2872 & 2 & 4 & 0 & 1 & 73.517 \\
\hline 1.2817 & 1 & 4 & 1 & 1 & 73.886 \\
\hline 1.2612 & $1 \mathrm{~L}$ & 1 & 7 & 3 & 75.292 \\
\hline 1.2556 & $1 \mathrm{~L}$ & 0 & 0 & 4 & 75.681 \\
\hline 1.2511 & 1 & 0 & 1 & 4 & 76.007 \\
\hline 1.2371 & 1 & 0 & 2 & 4 & 77.022 \\
\hline 1.2135 & 1 & 3 & 1 & 3 & 78.804 \\
\hline 1.2050 & $1 \mathrm{~L}$ & 1 & 2 & 4 & 79.470 \\
\hline 1.1979 & $1 \mathrm{~L}$ & $\begin{array}{lll}0 & 1\end{array}$ & 2 & 0 & 80.041 \\
\hline 1.1874 & 1 & 3 & 9 & 0 & 80.892 \\
\hline $1.1846 \mathrm{M}$ & $1 \mathrm{~L}$ & 1 & 3 & 4 & 81.121 \\
\hline $1.1846 \mathrm{M}$ & & 3 & 7 & 2 & 81.121 \\
\hline 1.1807 & 1 & 3 & 3 & 3 & 81.443 \\
\hline 1.1762 & 1 & 4 & 0 & 2 & 81.827 \\
\hline 1.1636 & 2 & 4 & 6 & 0 & 82.901 \\
\hline 1.1607 & 2 & 4 & 2 & 2 & 83.156 \\
\hline 1.1553 & 2 & 3 & 9 & 1 & 83.632 \\
\hline 1.1336 & 2 & 4 & 6 & 1 & 85.613 \\
\hline $1.1297 \mathrm{M}$ & $1 \mathrm{~L}$ & 21 & 0 & 2 & 85.981 \\
\hline $1.1297 \mathrm{M}$ & & 1 & 9 & 3 & 85.981 \\
\hline
\end{tabular}


This pattern is calculated from published crystal structure data. The calculation procedure follows the method described in sections 15 and 16 of NBS Monograph 25.

Synonym

Molybdenum trioxide

CAS registry no.

$$
\text { 1313-27-5 }
$$

Structure

Orthorhombic, Pbnm (62). The structure was refined from single crystal data (Kihlborg, 1963).

Atom positions

All atoms were in special positions $4(\mathrm{c})$.

Intensities

The peak intensities here differ from those on PDF card 5-508 (Swanson et al., 1954), especially at hkl's 040 and 060 . From a spray-dried sample, recent experimental intensity measurements at NBS support the intensity values given in this calculated pattern.

Crystallographic constants of this sample

$$
\begin{aligned}
& a=3.9630 \AA \\
& b=13.856 \\
& c=3.6966 \\
& a / b=0.2860 \\
& c / b=0.2668
\end{aligned}
$$

(published values: $3.9628,13.855,3.6964 \AA$ A; Kihlborg, 1963)

$Z=4$

$\mathrm{V}=202.99 \AA^{3}$

Density $(\mathrm{calc})=4.710 \mathrm{~g} / \mathrm{cm}^{3}$

Thermal parameters

Isotropic (Kihlborg, 1963)

Scattering factors

$\mathrm{O}^{-2}$ (Suzuki, 1960)

$\mathrm{Mo}^{\circ}$ (Thomas and Umeda, 1957), corrected for the real part of the anomalous dispersion (Cromer and Liberman, 1970).

Scale factors

$\gamma=0.686 \times 10^{-3}$

$I / I$ corundum (calculated) $=4.80$, for the scale reflection hk $=021$.

Polymorphism

A hexagonal form has been reported (PDF card 21-569, Spangenberg, Westinghouse Electric Corp., Horseheads, NY).

Additional pattern

PDF card 5-508 (Swanson et al., 1954)

References

Cromer, D. T. and Liberman, D. (1970).

J. Chem. Phys. 53, 1891 .
Kihlborg, L. (1963). Ark. Kemi 21, 357.

Suzuki, T. (1960). Acta Crystallogr. 13, 279 .

\begin{tabular}{|c|c|c|c|c|c|}
\hline \multicolumn{6}{|c|}{$\begin{array}{c}\text { Calculated Pattern (Peak heights) } \\
\lambda=1.540598 \AA\end{array}$} \\
\hline$d(\AA)$ & $\mathrm{r}^{\mathrm{rel}}$ & & hke & & $2 \theta\left(^{\circ}\right)$ \\
\hline 6.921 & 36 & 0 & 2 & 0 & 12.78 \\
\hline 3.808 & 77 & 1 & 1 & 0 & 23.34 \\
\hline 3.464 & 38 & 0 & 4 & 0 & 25.70 \\
\hline 3.440 & 31 & 1 & 2 & 0 & 25.88 \\
\hline 3.259 & 100 & 0 & 2 & 1 & 27.34 \\
\hline 3.008 & 7 & 1 & 3 & 0 & 29.68 \\
\hline 2.703 & 15 & 1 & 0 & 1 & 33.12 \\
\hline 2.653 & 26 & 1 & 1 & 1 & 33.76 \\
\hline 2.608 & 3 & 1 & 4 & 0 & 34.36 \\
\hline 2.5267 & 7 & 0 & 4 & 1 & 35.50 \\
\hline 2.3329 & 8 & 1 & 3 & 1 & 38.56 \\
\hline 2.3088 & 21 & 0 & 6 & 0 & 38.98 \\
\hline 2.2707 & 13 & 1 & 5 & 0 & 39.66 \\
\hline 2.1311 & 7 & 1 & 4 & 1 & 42.38 \\
\hline 1.9953 & 2 & 1 & 6 & 0 & 45.42 \\
\hline 1.9812 & 8 & 2 & 0 & 0 & 45.76 \\
\hline $1.9586+$ & 12 & 0 & 6 & 1 & 46.32 \\
\hline 1.9349 & 1 & 1 & 5 & 1 & 46.92 \\
\hline 1.9051 & 1 & 2 & 2 & 0 & 47.70 \\
\hline 1.8483 & 15 & 0 & 0 & 2 & 49.26 \\
\hline 1.8206 & 9 & 2 & 3 & 0 & 50.06 \\
\hline 1.7860 & 2 & 0 & 2 & 2 & 51.10 \\
\hline 1.7705 & 2 & 1 & 7 & 0 & 51.58 \\
\hline 1.7559 & 4 & 1 & 6 & 1 & 52.04 \\
\hline 1.7324 & 13 & 2 & 1 & 1 & 52.80 \\
\hline 1.7203 & 2 & 2 & 4 & 0 & 53.20 \\
\hline 1.6933 & 5 & 2 & 2 & 1 & 54.12 \\
\hline 1.6627 & 10 & 1 & 1 & 2 & 55.20 \\
\hline 1.6301 & 9 & 0 & 4 & 2 & 56.40 \\
\hline 1.6285 & 9 & 1 & 2 & 2 & 56.46 \\
\hline 1.5969 & 12 & 1 & 7 & 1 & 57.68 \\
\hline 1.5869 & 3 & 1 & 8 & 0 & 58.08 \\
\hline 1.5745 & 2 & 1 & 3 & 2 & 58.58 \\
\hline 1.5682 & 11 & 0 & 8 & 1 & 58.84 \\
\hline 1.5079 & 1 & 1 & 4 & 2 & 61.44 \\
\hline 1.5039 & 3 & 2 & 6 & 0 & 61.62 \\
\hline 1.4776 & 10 & 2 & 5 & 1 & 62.84 \\
\hline 1.4585 & 1 & 1 & 8 & 1 & 63.76 \\
\hline 1.4431 & 7 & 0 & 6 & 2 & 64.52 \\
\hline $1.4337+$ & 8 & 1 & 5 & 2 & 65.00 \\
\hline
\end{tabular}

Swanson, H. E., Fuyat, R. K., and Ugrinic, G. M. (1954). Nat1. Bur. Stand. (U.S.)

Circ. 539, 3, 30 .

Thomas, L. H. and Umeda, K. (1957). J. Chem. Phys. 26, 293. 
Molybdenum Oxide (Molybdite), $\mathrm{MoO}_{3}$ - (continued)

\begin{tabular}{|c|c|c|c|c|c|}
\hline$d(\stackrel{\circ}{A})$ & $\mathrm{I}^{\mathrm{rel}}$ & hke & & & $2 \theta\left(^{\circ}\right)$ \\
\hline 1.4004 & 4 & 2 & 7 & 0 & 66.74 \\
\hline 1.3931 & 1 & 2 & 6 & 1 & 67.14 \\
\hline 1.3854 & 3 & 0 & 10 & 0 & 67.56 \\
\hline 1.3558 & 1 & 1 & 6 & 2 & 69.24 \\
\hline 1.3518 & 3 & 2 & 0 & 2 & 69.48 \\
\hline 1.3453 & 3 & 2 & 1 & 2 & 69.86 \\
\hline 1.3380 & 1 & 1 & 9 & 1 & 70.30 \\
\hline 1.3096 & 1 & 2 & 7 & 1 & 72.06 \\
\hline $1.2975+$ & 8 & 2 & 3 & 2 & 72.84 \\
\hline 1.2788 & 1 & 1 & 7 & 2 & 74.08 \\
\hline 1.2591 & 1 & 2 & 4 & 2 & 75.44 \\
\hline 1.2440 & 4 & 3 & 0 & 1 & 76.52 \\
\hline $1.2331+$ & 2 & 1 & 10 & 1 & 77.32 \\
\hline 1.2299 & 3 & 2 & 8 & 1 & 77.56 \\
\hline 1.2157 & 1 & 2 & 9 & 0 & 78.64 \\
\hline 1.2131 & 4 & 0 & 2 & 3 & 78.84 \\
\hline 1. 2041 & 2 & 1 & 8 & 2 & 79.54 \\
\hline $1.2011+$ & 2 & 3 & 3 & 1 & 79.78 \\
\hline
\end{tabular}

\begin{tabular}{|c|c|c|c|c|c|}
\hline \multicolumn{6}{|c|}{$\begin{array}{c}\text { Calculated Pattern (Integrated) } \\
\lambda=1.540598 \AA\end{array}$} \\
\hline$d(\AA)$ & $\mathrm{I}^{\mathrm{rel}}$ & & hke & & $2 \theta\left(^{\circ}\right)$ \\
\hline 6.927 & 31 & 0 & 2 & 0 & 12.77 \\
\hline 3.8098 & 73 & 1 & 1 & 0 & 23.33 \\
\hline 3.4636 & 34 & 0 & 4 & 0 & 25.70 \\
\hline 3.4399 & 25 & 1 & 2 & 0 & 25.88 \\
\hline 3.2618 & 100 & 0 & 2 & 1 & 27.32 \\
\hline 3.0076 & 7 & 1 & 3 & 0 & 29.68 \\
\hline 2.7034 & 15 & 1 & 0 & 1 & 33.11 \\
\hline 2.6528 & 27 & 1 & 1 & 1 & 33.76 \\
\hline 2.6079 & 3 & 1 & 4 & 0 & 34.36 \\
\hline 2.5274 & 7 & 0 & 4 & 1 & 35.49 \\
\hline 2.5185 & 1 & 1 & 2 & 1 & 35.62 \\
\hline 2.3329 & 8 & 1 & 3 & 1 & 38.56 \\
\hline 2.3093 & 22 & 0 & 6 & 0 & 38.97 \\
\hline 2.2713 & 14 & 1 & 5 & 0 & 39.65 \\
\hline 2.1311 & 8 & 1 & 4 & 1 & 42.38 \\
\hline 1.9953 & 2 & 1 & 6 & 0 & 45.42 \\
\hline 1.9816 & 8 & 2 & 0 & 0 & 45.75 \\
\hline 1.9613 & 8 & 2 & 1 & 0 & 46.25 \\
\hline 1.9586 & 8 & 0 & 6 & 1 & 46.32 \\
\hline 1.9349 & 1 & 1 & 5 & 1 & 46.92 \\
\hline 1.9051 & 1 & 2 & 2 & 0 & 47.70 \\
\hline 1.8483 & 17 & 0 & 0 & 2 & 49.26 \\
\hline 1.8210 & 11 & 2 & 3 & 0 & 50.05 \\
\hline 1.7860 & 2 & 0 & 2 & 2 & 51.10 \\
\hline 1.7708 & 2 & 1 & 7 & 0 & 51.57 \\
\hline
\end{tabular}

\begin{tabular}{|c|c|c|c|c|c|}
\hline $\mathrm{d}(\stackrel{\circ}{\mathrm{A}})$ & $\mathrm{I}^{\mathrm{rel}}$ & & hke & & $2 \theta\left({ }^{\circ}\right)$ \\
\hline 1.7559 & 4 & 1 & 6 & 1 & 52.04 \\
\hline 1.7327 & 15 & 2 & 1 & 1 & 52.79 \\
\hline 1.7200 & 2 & 2 & 4 & 0 & 53.21 \\
\hline 1.6935 & 6 & 2 & 2 & 1 & 54.11 \\
\hline 1.6629 & 12 & 1 & 1 & 2 & 55.19 \\
\hline 1.6335 & 2 & 2 & 3 & 1 & 56.27 \\
\hline 1.6306 & 7 & 0 & 4 & 2 & 56.38 \\
\hline 1.6282 & 5 & 1 & 2 & 2 & 56.47 \\
\hline 1.5969 & 14 & 1 & 7 & 1 & 57.68 \\
\hline 1.5871 & 2 & 1 & 8 & 0 & 58.07 \\
\hline 1.5748 & 2 & 1 & 3 & 2 & 58.57 \\
\hline 1.5684 & 13 & 0 & 8 & 1 & 58.83 \\
\hline 1.5081 & 1 & 1 & 4 & 2 & 61.43 \\
\hline 1.5037 & 3 & 2 & 6 & 0 & 61.63 \\
\hline 1.4774 & 12 & 2 & 5 & 1 & 62.85 \\
\hline 1.4583 & 1 & 1 & 8 & 1 & 63.77 \\
\hline 1.4429 & 8 & 0 & 6 & 2 & 64.53 \\
\hline 1.4350 & 4 & 1 & 9 & 0 & 64.93 \\
\hline 1.4334 & 7 & 1 & 5 & 2 & 65.01 \\
\hline 1.4004 & 5 & 2 & 7 & 0 & 66.74 \\
\hline 1.3929 & 1 & 2 & 6 & 1 & 67.15 \\
\hline 1.3856 & 3 & 0 & 10 & 0 & 67.55 \\
\hline 1.3558 & 1 & 1 & 6 & 2 & 69.24 \\
\hline 1.3516 & 4 & 2 & 0 & 2 & 69.49 \\
\hline 1.3452 & 4 & 2 & 1 & 2 & 69.87 \\
\hline 1.3378 & 1 & 1 & 9 & 1 & 70.31 \\
\hline 1.3096 & 1 & 2 & 7 & 1 & 72.06 \\
\hline 1.2976 & 4 & 3 & 2 & 0 & 72.83 \\
\hline 1.2972 & 7 & 2 & 3 & 2 & 72.86 \\
\hline 1.2786 & 1 & 1 & 7 & 2 & 74.09 \\
\hline 1.2591 & 1 & 2 & 4 & 2 & 75.44 \\
\hline 1.2440 & 4 & 3 & 0 & 1 & 76.52 \\
\hline 1.2343 & 1 & 3 & 4 & 0 & 77.23 \\
\hline 1.2331 & 2 & 1 & 10 & 1 & 77.32 \\
\hline 1.2297 & 3 & 2 & 8 & 1 & 77.57 \\
\hline 1.2158 & 1 & 2 & 9 & 0 & 78.63 \\
\hline 1.2132 & 4 & 0 & 2 & 3 & 78.83 \\
\hline 1.2040 & 2 & 1 & 8 & 2 & 79.55 \\
\hline 1.2011 & 1 & 3 & 3 & 1 & 79.78 \\
\hline 1.2005 & 1 & 1 & 11 & 0 & 79.83 \\
\hline
\end{tabular}


CUMULATIVE INDEX TO CIRCULAR 539, VOLUMES 1-10 and MONOGRAPH 25, SECTIONS 1-20, inclusive.

\section{INORGANIC NAMES}

Vol. or

Sec. Page

Aluminum, Al ................ 111

Aluminum antimony, Alsb ......... $4 \quad 72$

Aluminum bismuth oxide, $\mathrm{Al}_{4} \mathrm{Bi}_{2} \mathrm{O}_{9} \ldots 1 \mathrm{~m} \quad 5$

Aluminum borate, $\mathrm{Al}_{18} \mathrm{~B}_{4} \mathrm{O}_{33} \ldots \ldots \ldots \ldots .17 \mathrm{~m}$

Aluminum chloride, $\mathrm{AlCl}_{3} \ldots \ldots \ldots .9 \mathrm{~m}$

Aluminum chloride hydrate

(chloraluminite), $\mathrm{AlCl}_{3} \cdot 6 \mathrm{H}_{2} \mathrm{O} \ldots \ldots$.

Aluminum fluoride hydroxide silicate,

topaz, $\mathrm{Al}_{2}(\mathrm{~F}, \mathrm{OH})_{2} \mathrm{SiO}_{4} \ldots \ldots \ldots \ldots \ldots$ 1m

Aluminum iron, AlFe ........... 18m

Aluminum iron antimony oxide, bahianite,

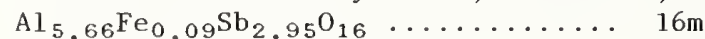

Aluminum iron oxide, $\mathrm{AlFeO}_{3} \ldots \ldots \ldots .15 \mathrm{~m}$

Aluminum lithium, $\mathrm{Al}_{4} \mathrm{Li}_{9} \ldots \ldots \ldots \ldots$ 10m

Aluminum nickel, AlNi ......... $6 \mathrm{~m}$

Aluminum nitride, AlN .......... 12m

Aluminum nitrate hydrate,

$\mathrm{Al}\left(\mathrm{NO}_{3}\right)_{3} \cdot 9 \mathrm{H}_{2} \mathrm{O} \ldots \ldots \ldots \ldots \ldots \ldots . \ldots . \ldots 1 \mathrm{~m}$

Aluminum oxide (corundum),$\alpha-\mathrm{Al}_{2} \mathrm{O}_{3} \ldots$

Aluminum oxide hydrate (boehmite),

$\alpha-\mathrm{Al}_{2} \mathrm{O}_{3} \cdot \mathrm{H}_{2} \mathrm{O} \ldots \ldots \ldots \ldots \ldots \ldots \ldots$

Aluminum oxide hydrate, diaspore,

$\beta-\mathrm{Al}_{2} \mathrm{O}_{3} \cdot \mathrm{H}_{2} \mathrm{O} \ldots \ldots \ldots \ldots \ldots \ldots \ldots$

Aluminum phosphate, $\mathrm{Al}\left(\mathrm{PO}_{3}\right)_{3} \ldots \ldots$.

Aluminum phosphate (berlinite),

$\mathrm{AlPO}_{4}$ (trigonal) .............. 10

Aluminum phosphate, $\mathrm{AlPO}_{4}$

(orthorhombic) .............. 10

Aluminum plutonium, $\mathrm{Al}_{3} \mathrm{Pu} \ldots \ldots \ldots \ldots 15 \mathrm{~m}$

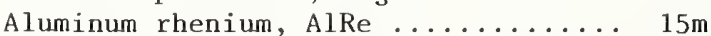

Aluminum rhenium, $\mathrm{Al}_{12} \operatorname{Re} \ldots \ldots \ldots \ldots$ 15m

Aluminum rhodium, AlRh ......... 15m

Aluminum ruthenium, AlRu ......... 15m

Aluminum ruthenium, $\mathrm{Al}_{6} \mathrm{Ru} \ldots \ldots \ldots \ldots 15 \mathrm{~m}$

Aluminum samarium, $\mathrm{AlSm}_{2} \ldots \ldots \ldots \ldots \ldots$ 15m

Aluminum samarium, $\mathrm{AlSm}_{3} \ldots \ldots \ldots \ldots 15 \mathrm{~m}$

Aluminum samarium, $\mathrm{Al}_{2} \mathrm{Sm} \ldots \ldots \ldots \ldots 15 \mathrm{~m}$

Aluminum samarium, $\mathrm{Al}_{3} \mathrm{Sm} \ldots \ldots \ldots \ldots 15 \mathrm{~m}$

Aluminum silicate (mullite),

$\mathrm{Al}_{6} \mathrm{Si}_{2} \mathrm{O}_{13} \ldots \ldots \ldots \ldots \ldots \ldots \ldots \ldots \ldots \ldots \ldots$

Aluminum sulfate, $\mathrm{Al}_{2}\left(\mathrm{SO}_{4}\right)_{3} \ldots \ldots \ldots 15 \mathrm{~m}$

Aluminum technetium, $\mathrm{Al}_{6} \mathrm{Tc} \ldots \ldots \ldots .15 \mathrm{~m}$

Aluminum terbium, $\mathrm{Al}_{2} \mathrm{~Tb} \ldots \ldots \ldots \ldots . . \ldots 15 \mathrm{~m}$

Aluminum terbium, $\mathrm{Al}_{2} \mathrm{~Tb}_{3} \ldots \ldots \ldots \ldots$ 15m

Aluminum thorium uranium, $\mathrm{Al}_{6} \mathrm{ThU} \ldots$ 15m

Aluminum tungsten, $\mathrm{Al}_{5} \mathrm{~W}, \delta$-phase ... $15 \mathrm{~m}$

Aluminum tungsten oxide, $\mathrm{Al}_{2}\left(\mathrm{WO}_{4}\right)_{3} \ldots 11 \mathrm{~m}$

Aluminum vanadium, $\mathrm{Al}_{10} \mathrm{~V} \ldots \ldots \ldots \ldots 15 \mathrm{~m}$

Aluminum vanadium, $A 1_{10.25} \mathrm{~V} \ldots \ldots \ldots 15 \mathrm{~m}$

Aluminum vanadium, $\mathrm{Al}_{23} \mathrm{~V}_{4} \ldots \ldots \ldots 15 \mathrm{~m}$

Aluminum vanadium, $\mathrm{Al}_{45} \mathrm{~V}_{7}, \alpha^{\prime}$-phase. $15 \mathrm{~m}$

Aluminum ytterbium, $\mathrm{Al}_{2} \mathrm{Yb} \ldots \ldots \ldots$ 15m

Aluminum yttrium, $\mathrm{Al}_{3} \mathrm{Y} \ldots \ldots \ldots \ldots .15 \mathrm{~m}$

Aluminium yttrium oxide, $\mathrm{AlYO}_{3} \ldots \ldots$ 19m

Aluminium yttrium oxide, $\mathrm{A}_{2} \mathrm{Y}_{4} \mathrm{O}_{9} \ldots .19 \mathrm{~m}$

Aluminium yttrium oxide, $\mathrm{A}_{1}{ }_{5} \mathrm{Y}_{3} \mathrm{O}_{12} \ldots 1.19 \mathrm{~m}$

Further work on this program is in progress, and it is anticipated that additional sections will be issued. Therefore, the cumulative index here is not necessarily the concluding index for the project.

m - Monograph 25.

A mineral name in ( ) indicates a synthetic sample.
Ammonium aluminum fluoride,

$\left(\mathrm{NH}_{4}\right)_{3} \mathrm{AlF}_{6} \ldots \ldots \ldots \ldots \ldots \ldots \ldots \ldots \ldots$

Ammonium aluminum selenate hydrate,

$\mathrm{NH}_{4} \mathrm{Al}\left(\mathrm{SeO}_{4}\right)_{2} \cdot 12 \mathrm{H}_{2} \mathrm{O} \ldots \ldots \ldots \ldots$

Ammonium aluminum sulfate,

$\mathrm{NH}_{4} \mathrm{All}\left(\mathrm{SO}_{4}\right)_{2} \ldots \ldots \ldots \ldots \ldots \ldots \ldots$

Ammonium aluminum sulfate hydrate

(tschermigite), $\mathrm{NH}_{4} \mathrm{Al}\left(\mathrm{SO}_{4}\right)_{2} \cdot 12 \mathrm{H}_{2} \mathrm{O}$

Ammonium azide, $\mathrm{NH}_{4} \mathrm{~N}_{3} \ldots \ldots \ldots \ldots$

Vol. or

Sec. Page

Ammonium beryllium fluoride,

$\left(\mathrm{NH}_{4}\right)_{2} \mathrm{BeF}_{4} \ldots \ldots \ldots \ldots \ldots \ldots \ldots \ldots$

Ammonium borate hydrate,

$\mathrm{NH}_{4} \mathrm{~B}_{5} \mathrm{O}_{8} \cdot 4 \mathrm{H}_{2} \mathrm{O} \ldots \ldots \ldots \ldots \ldots \ldots \ldots$

Ammonium boron fluoride, $\mathrm{NH}_{4} \mathrm{BF}_{4} \ldots$

Ammonium bromide, $\mathrm{NH}_{4} \mathrm{Br} \ldots \ldots \ldots$

Ammonium cadmium bromide, $\left(\mathrm{NH}_{4}\right)_{4} \mathrm{CdBr}_{6}$

Ammonium cadmium chloride, $\mathrm{NH}_{4} \mathrm{CdCl}_{3}$

Ammonium cadmium phosphate hydrate,

$\mathrm{NH}_{4} \mathrm{CdPO}_{4} \cdot \mathrm{H}_{2} \mathrm{O} \ldots \ldots \ldots \ldots \ldots$

Ammonium cadmium sulfate,

$\left(\mathrm{NH}_{4}\right)_{2} \mathrm{Cd}_{2}\left(\mathrm{SO}_{4}\right)_{3} \ldots \ldots \ldots \ldots \ldots \ldots$

Ammonium cadmium sulfate hydrate,

$\left(\mathrm{NH}_{4}\right)_{2} \mathrm{Cd}\left(\mathrm{SO}_{4}\right)_{2} \cdot 6 \mathrm{H}_{2} \mathrm{O} \ldots \ldots \ldots \ldots$

Ammonium calcium sulfate,

$\left(\mathrm{NH}_{4}\right)_{2} \mathrm{Ca}_{2}\left(\mathrm{SO}_{4}\right)_{3} \ldots \ldots \ldots \ldots \ldots$

Ammonium cerium nitrate,

$\left(\mathrm{NH}_{4}\right)_{2} \mathrm{Ce}\left(\mathrm{NO}_{3}\right)_{6} \ldots \ldots \ldots \ldots \ldots \ldots \ldots$

Ammonium chlorate, $\mathrm{NH}_{4} \mathrm{ClO}_{4}$

(orthorhombic) ............ 7

Ammonium chloride (salammoniac),

$\mathrm{NH}_{4} \mathrm{Cl} \ldots \ldots \ldots \ldots \ldots \ldots \ldots \ldots \ldots \ldots \ldots \ldots$

Ammonium chromium sulfate hydrate,

$\mathrm{NH}_{4} \mathrm{Cr}\left(\mathrm{SO}_{4}\right)_{2} \cdot 12 \mathrm{H}_{2} \mathrm{O} \ldots \ldots \ldots \ldots$.

Ammonium cobalt (II) chloride,

$\mathrm{NH}_{4} \mathrm{CoCl}_{3} \ldots \ldots \ldots \ldots \ldots \ldots$

Ammonium cobalt fluoride, $\mathrm{NH}_{4} \mathrm{CoF}_{3}$

Ammonium copper bromide hydrate,

$\left(\mathrm{NH}_{4}\right)_{2} \mathrm{CuBr}_{4} \cdot 2 \mathrm{H}_{2} \mathrm{O} \ldots \ldots \ldots \ldots \ldots \ldots$

Ammonium copper chloride, $\mathrm{NH}_{4} \mathrm{CuCl}_{3} \quad 7 \mathrm{~m}$

Ammonium copper chloride hydrate;

$\left(\mathrm{NH}_{4}\right)_{2} \mathrm{CuCl}_{4} \cdot 2 \mathrm{H}_{2} \mathrm{O} \ldots \ldots \ldots \ldots \ldots \ldots$

Ammonium copper fluoride, $\mathrm{NH}_{4} \mathrm{CuF}_{3}$

Ammonium gallium sulfate hydrate,

$\mathrm{NH}_{4} \mathrm{Ga}\left(\mathrm{SO}_{4}\right)_{2} \cdot 12 \mathrm{H}_{2} \mathrm{O} \ldots \ldots \ldots \ldots \ldots$

Ammonium germanium fluoride,

$\left(\mathrm{NH}_{4}\right)_{2} \mathrm{GeF}_{6} \ldots \ldots \ldots \ldots \ldots \ldots \ldots$

Ammonium hydrogen arsenate,

$\mathrm{NH}_{4} \mathrm{H}_{2} \mathrm{AsO}_{4} \ldots \ldots \ldots \ldots \ldots \ldots \ldots \ldots$

Ammonium hydrogen carbonate

(teschemacherite), $\left(\mathrm{NH}_{4}\right) \mathrm{HCO}_{3} \ldots \ldots$

Ammonium hydrogen phosphate,

$\mathrm{NH}_{4} \mathrm{H}_{2} \mathrm{PO}_{4} \ldots \ldots \ldots \ldots \ldots \ldots \ldots \ldots$

Ammonium iodate, $\mathrm{NH}_{4} \mathrm{IO}_{3} \ldots \ldots \ldots \ldots$

Ammonium iodide, $\mathrm{NH}_{4} \mathrm{I} \ldots \ldots \ldots \ldots$

Ammonium iridium chloride,

$\left(\mathrm{NH}_{4}\right)_{2} \mathrm{IrCl} 1_{6} \ldots \ldots \ldots \ldots \ldots \ldots$

Ammonium iron chloride hydrate,

$\left(\mathrm{NH}_{4}\right)_{2} \mathrm{FeCl}_{5} \cdot \mathrm{H}_{2} \mathrm{O} \ldots \ldots \ldots \ldots \ldots$

Ammonium iron fluoride, $\left(\mathrm{NH}_{4}\right)_{3} \mathrm{FeF}_{6}$

Ammonium iron sulfate, $\mathrm{NH}_{4} \mathrm{Fe}\left(\mathrm{SO}_{4}\right)_{2}$

Ammonium iron sulfate hydrate,

$\mathrm{NH}_{4} \mathrm{Fe}\left(\mathrm{SO}_{4}\right)_{2} \cdot 12 \mathrm{H}_{2} \mathrm{O} \ldots \ldots \ldots \ldots \ldots$

Ammonium lead chloride, $\left(\mathrm{NH}_{4}\right)_{2} \mathrm{PbCl}_{6} \quad 11 \mathrm{~m}$

Ammonium magnesium aluminum fluoride,

$\mathrm{NH}_{4} \mathrm{MgAlF}_{6} \ldots \ldots \ldots \ldots \ldots \ldots, 10 \mathrm{~m}$ 
Ammonium magnesium chromium oxide hydrate, $\left(\mathrm{NH}_{4}\right)_{2} \mathrm{Mg}\left(\mathrm{CrO}_{4}\right)_{2} \cdot 6 \mathrm{H}_{2} \mathrm{O} \ldots$ Ammonium magnesium phosphate hydrate (struvite), $\mathrm{NH}_{4} \mathrm{MgPO}_{4} \cdot 6 \mathrm{H}_{2} \mathrm{O} \ldots \ldots$. Ammonium manganese chloride hydrate,

$\left(\mathrm{NH}_{4}\right)_{2} \mathrm{MnCl}_{4} \cdot 2 \mathrm{H}_{2} \mathrm{O} \ldots \ldots \ldots \ldots \ldots$ Ammonium manganese(II) fluoride,

$\mathrm{NH}_{4} \mathrm{MnF}_{3} \ldots \ldots \ldots \ldots \ldots \ldots \ldots$

Ammonium manganese sulfate,

$\left(\mathrm{NH}_{4}\right)_{2} \mathrm{Mn}_{2}\left(\mathrm{SO}_{4}\right)_{3} \ldots \ldots \ldots \ldots$

Ammonium manganese sulfate hydrate,

$\left(\mathrm{NH}_{4}\right)_{2} \mathrm{Mn}\left(\mathrm{SO}_{4}\right)_{2} \cdot 6 \mathrm{H}_{2} \mathrm{O} \ldots \ldots \ldots \ldots$

Ammonium mercury chloride, $\mathrm{NH}_{4} \mathrm{HgCl}_{3}$

Ammonium molybdenum oxide phosphate

hydrate, $\left(\mathrm{NH}_{4}\right)_{3}\left(\mathrm{MoO}_{3}\right)_{12} \mathrm{PO}_{4} \cdot 4 \mathrm{H}_{2} \mathrm{O} \ldots$

Ammonium nickel(II) chloride,

$\mathrm{NH}_{4} \mathrm{NiCl}_{3} \ldots \ldots \ldots \ldots \ldots \ldots$

Ammonium nickel chromium oxide

hydrate, $\left(\mathrm{NH}_{4}\right)_{2} \mathrm{Ni}\left(\mathrm{CrO}_{4}\right)_{2} \cdot 6 \mathrm{H}_{2} \mathrm{O} \ldots$.

Ammonium nickel sulfate hydrate,

$\left(\mathrm{NH}_{4}\right)_{2} \mathrm{Ni}\left(\mathrm{SO}_{4}\right)_{2} \cdot 6 \mathrm{H}_{2} \mathrm{O} \ldots \ldots \ldots \ldots$

Ammonium nitrate (nitrammite),

$\mathrm{NH}_{4} \mathrm{NO}_{3} \ldots \ldots \ldots \ldots \ldots \ldots$

Ammonium osmium bromide, $\left(\mathrm{NH}_{4}\right)_{2} \mathrm{OsBr}_{6}$ Ammonium osmium chloride,

$\left(\mathrm{NH}_{4}\right)_{2} \mathrm{OsCl}_{6} \ldots \ldots \ldots \ldots \ldots \ldots$

Ammonium palladium chloride,

$\left(\mathrm{NH}_{4}\right)_{2} \mathrm{PdCl}_{4} \ldots \ldots \ldots \ldots \ldots \ldots \ldots$

Ammonium palladium chloride,

$\left(\mathrm{NH}_{4}\right)_{2} \mathrm{PdCl}_{6} \ldots \ldots \ldots \ldots \ldots \ldots \ldots$

Ammonium platinum bromide,

$\left(\mathrm{NH}_{4}\right)_{2} \mathrm{PtBr}_{6} \ldots \ldots \ldots \ldots \ldots$

Ammonium platinum chloride,

$\left(\mathrm{NH}_{4}\right)_{2} \mathrm{PtCl}_{6} \ldots \ldots \ldots \ldots \ldots \ldots \ldots$

Ammonium potassium iron chloride

hydrate (kremersite),

$\left(\mathrm{NH}_{4}, \mathrm{~K}\right)_{2} \mathrm{FeCl}_{5} \cdot \mathrm{H}_{2} \mathrm{O} \ldots \ldots \ldots \ldots \ldots$

Ammonium rhenium oxide, $\mathrm{NH}_{4} \mathrm{ReO}_{4} \ldots$

Ammonium selenium bromide,

$\left(\mathrm{NH}_{4}\right)_{2} \mathrm{SeBr}_{6} \ldots \ldots \ldots \ldots \ldots \ldots$

Ammonium silicon fluoride

(cryptohalite), $\left(\mathrm{NH}_{4}\right)_{2} \mathrm{SiF}_{6} \ldots \ldots$

Ammonium strontium chromium oxide,

$\left(\mathrm{NH}_{4}\right)_{2} \mathrm{Sr}\left(\mathrm{CrO}_{4}\right)_{2} \ldots \ldots \ldots \ldots \ldots$

Ammonium strontium sulfate,

$\left(\mathrm{NH}_{4}\right)_{2} \mathrm{Sr}\left(\mathrm{SO}_{4}\right)_{2} \ldots \ldots \ldots \ldots \ldots$

Ammonium sulfate (mascagnite),

$\left(\mathrm{NH}_{4}\right)_{2} \mathrm{SO}_{4} \ldots \ldots \ldots \ldots \ldots \ldots \ldots \ldots \ldots \ldots \ldots \ldots$

Ammonium sulfate, $\left(\mathrm{NH}_{4}\right)_{2} \mathrm{~S}_{2} \mathrm{O}_{3} \ldots \ldots$.

Ammonium sulfate, $\left(\mathrm{NH}_{4}\right)_{2} \mathrm{~S}_{2} \mathrm{O}_{8} \ldots \ldots$

Ammonium tellurium bromide,

$\left(\mathrm{NH}_{4}\right)_{2} \mathrm{TeBr}_{6} \ldots \ldots \ldots \ldots \ldots \ldots$

Ammonium tellurium chloride,

$\left(\mathrm{NH}_{4}\right)_{2} \mathrm{TeCl}_{6} \ldots \ldots \ldots \ldots \ldots \ldots \ldots$

Ammonium tin chloride, $\left(\mathrm{NH}_{4}\right)_{2} \mathrm{SnCl}_{6}$

Ammonium tin fluoride, $\mathrm{NH}_{4} \mathrm{SnF}_{3} \ldots$.

Ammonium titanium fluoride,

$\left(\mathrm{NH}_{4}\right)_{2} \mathrm{TiF}_{6} \ldots \ldots \ldots \ldots \ldots \ldots$

Ammonium vanadium oxide, $\mathrm{NH}_{4} \mathrm{VO}_{3} \ldots$ Ammonium zinc chloride, $\left(\mathrm{NH}_{4}\right)_{3} \mathrm{ZnCl}_{5}$

Ammonium zinc fluoride, $\mathrm{NH}_{4} \mathrm{ZnF}_{3} \ldots$ Ammonium zirconium fluoride,

$\left(\mathrm{NH}_{4}\right)_{3} \mathrm{ZrF}_{7} \ldots \ldots \ldots \ldots \ldots \ldots \ldots$ Antiomonic acid, $\mathrm{H}_{14} \mathrm{Sb}_{14} \mathrm{O}_{21}(\mathrm{OH})_{42}$. Antimony, $\mathrm{Sb} \ldots \ldots \ldots \ldots \ldots \ldots \ldots$ Antimony bromide, $\alpha-\operatorname{SbBr}_{3} \ldots \ldots \ldots$

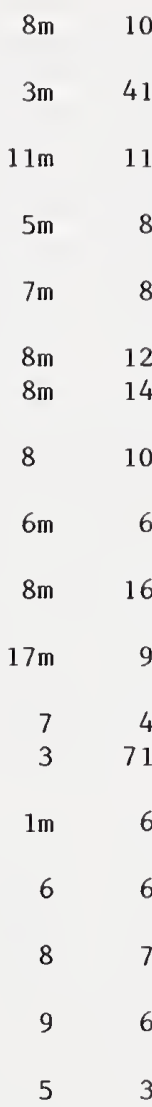

Antimony ceriun, CeSb ...........

Antimony cobalt, CoSb ...........

Antimony cobalt, $\mathrm{CoSb}_{2} \ldots \ldots \ldots \ldots$

Antimony cobalt titanium, CoSbTi ..

Antimony cobalt vanadium, CoSbV ...

Antimony dysprosium, DySb ........

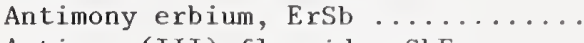

Antimony(III) fluoride, $\mathrm{SbF}_{3} \ldots \ldots$

Antimony gadolinium, GdSb ........

Antimony gallium, GaSb ..........

Antimony gold (aurostibite), $\mathrm{AuSb}_{2}$

Antimony indium, InSb ...........

Antimony(III) iodide, $\mathrm{SbI}_{3} \ldots . .$.

Antimony iron titanium oxide

hydroxide, derbylite,

$\mathrm{SbFe}_{4} \mathrm{Ti}_{3} \mathrm{O}_{13}(\mathrm{OH})$

Antimony lanthanum, LaSb ......... Antimony neodymium, NdSb .......... Antimony(III) oxide (senarmontite),

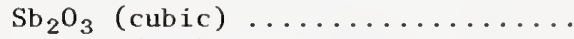

Antimony(III) oxide, valentinite,

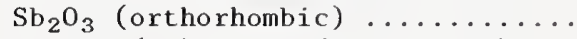

Antimony(IV) oxide (cervantite),

$\mathrm{Sb}_{2} \mathrm{O}_{4} \ldots \ldots \ldots \ldots \ldots \ldots \ldots$

Antimony oxide, $\mathrm{Sb}_{6} \mathrm{O}_{13} \ldots \ldots \ldots \ldots$

Antimony praseodymium, PrSb ......

Antimony scandium, $\operatorname{SbSc} \ldots \ldots \ldots \ldots$

Antimony selenide, $\mathrm{Sb}_{2} \mathrm{Se}_{3} \ldots \ldots \ldots$

Antimony silver sulfide, $\mathrm{AgSbS}_{2}$

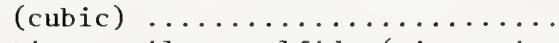

Antimony silver sulfide (miargyrite), $\mathrm{AgSbS}_{2}$ (monoclinic) .......... $5 \mathrm{~m}$

Antimony silver sulfide (pyrargyrite),

$\mathrm{Ag}_{3} \mathrm{SbS}_{3}$ (trigonal) .......... $5 \mathrm{~m}$

Antimony silver telluride, $\mathrm{AgSbTe}_{2} \quad 3 \mathrm{~m}$

Antimony(III) sulfide (stibnite),

$\mathrm{Sb}_{2} \mathrm{~S}_{3} \ldots \ldots \ldots \ldots \ldots \ldots \ldots \ldots \ldots \ldots \ldots \ldots$

Antimony telluride, $\mathrm{Sb}_{2} \mathrm{Te}_{3} \ldots \ldots \ldots$

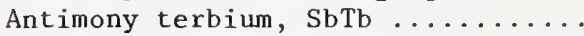

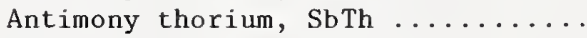

Antimony thulium, $\operatorname{SbTm} \ldots \ldots \ldots \ldots$

Antimony $t i n, \operatorname{SbSn} \ldots \ldots \ldots \ldots$

Antimony ytterbium, $\mathrm{SbYb} \ldots . . \ldots \ldots$

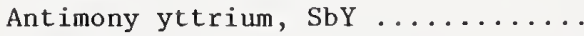

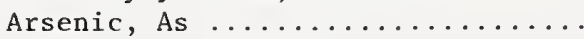

Arsenic bromide, $\mathrm{AsBr}_{3} \ldots \ldots \ldots \ldots$

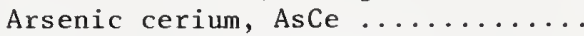

Arsenic(III) iodide, $\mathrm{AsI}_{3} \ldots \ldots \ldots$

Arsenic oxide (arsenolite),

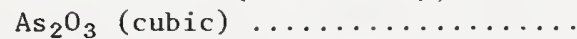

Arsenic oxide, claudetite, $\mathrm{As}_{2} \mathrm{O}_{3}$

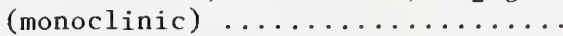

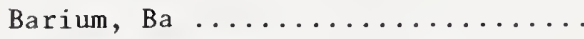

Barium aluminum oxide, $\mathrm{BaAl}_{2} \mathrm{O}_{4} \ldots$

Barium aluminum oxide, $\mathrm{Ba}_{3} \mathrm{Al}_{2} \mathrm{O}_{6} \ldots$

Barium aluminum titanium oxide,

$\mathrm{BaAl}_{6} \mathrm{TiO}_{12} \ldots \ldots \ldots \ldots \ldots \ldots \ldots$

Barium aluminum titanium oxide,

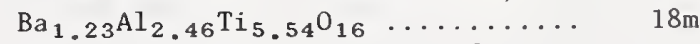

Barium aluminum titanium oxide,

$\mathrm{Ba}_{3} \mathrm{Al}_{10} \mathrm{TiO}_{20} \ldots \ldots \ldots \ldots \ldots \ldots$

Barium arsenate, $\mathrm{Ba}_{3}\left(\mathrm{AsO}_{4}\right)_{2} \ldots \ldots$

Barium borate, $\mathrm{BaB}_{4} \mathrm{O}_{7} \ldots \ldots \ldots \ldots$

Barium borate, high form, $\mathrm{BaB}_{2} \mathrm{O}_{4} \ldots$

Barium borate, $\mathrm{BaB}_{8} \mathrm{O}_{13} \ldots \ldots \ldots \ldots$

Barium boride, $\mathrm{BaB}_{6} \ldots \ldots \ldots \ldots$
$18 \mathrm{~m}$

$\begin{array}{rr}4 m & 40 \\ 15 m & 121 \\ 15 m & 122 \\ 15 m & 124 \\ 15 m & 125 \\ 4 m & 41 \\ 4 m & 41 \\ 2 m & 4 \\ 4 m & 42 \\ 6 & 30 \\ 7 & 18 \\ 4 & 73 \\ 6 & 16\end{array}$

$16 \mathrm{~m}$

$4 \mathrm{~m}$

89

42

3

31

10

10

$16 \mathrm{~m}$

$4 m$

$4 \mathrm{~m}$

$5 \mathrm{~m}$

51

5

$3 \mathrm{~m}$

$4 \mathrm{~m}$

$4 \mathrm{~m}$

$16 \mathrm{~m}$

$4 \mathrm{~m}$

$4 m$

3
$18 \mathrm{~m}$

$4 \mathrm{~m}$

$13 m$

$$
1
$$

51

9

7

11
7

$12 \mathrm{~m}$

$19 m$

$2 \mathrm{~m}$

$4 m$

$4 m$

$7 \mathrm{~m}$

$19 \mathrm{~m}$ 
Barium bromate hydrate,

$\mathrm{Ba}\left(\mathrm{BrO}_{3}\right)_{2} \cdot \mathrm{H}_{2} \mathrm{O} \ldots \ldots \ldots \ldots \ldots \ldots$

Barium bromide, $\mathrm{BaBr}_{2} \ldots \ldots \ldots \ldots$ Barium bromide fluoride, BaBrF ....

Barium bromide hydrate, $\mathrm{BaBr}_{2} \cdot \mathrm{H}_{2} \mathrm{O}$

Barium bromide hydrate, $\mathrm{BaBr}_{2} \cdot 2 \mathrm{H}_{2} \mathrm{O}$

Barium cadmium chloride hydrate,

$\mathrm{BaCdCl}_{4} \cdot 4 \mathrm{H}_{2} \mathrm{O} \ldots \ldots \ldots \ldots \ldots \ldots$

Barium cadmium phosphate,

$\operatorname{BaCd}\left(\mathrm{PO}_{3}\right)_{4} \ldots \ldots \ldots \ldots \ldots \ldots$

Barium calcium nitrate,

$\mathrm{Ba} .2{ }_{2} \mathrm{Ca} .75\left(\mathrm{NO}_{3}\right)_{2} \ldots \ldots \ldots \ldots \ldots$

Barium calcium nitrate,

$\mathrm{Ba} .{ }_{50} \mathrm{Ca} .{ }_{50}\left(\mathrm{NO}_{3}\right)_{2} \ldots \ldots \ldots \ldots \ldots$

Barium calcium nitrate,

$\mathrm{Ba} .{ }_{75} \mathrm{Ca} .25\left(\mathrm{NO}_{3}\right)_{2} \ldots \ldots \ldots \ldots$

Barium calcium tungsten oxide,

$\mathrm{Ba}_{2} \mathrm{CaWO}_{6} \ldots \ldots \ldots \ldots \ldots \ldots \ldots$

Barium carbonate (witherite), $\mathrm{BaCO}_{3}$

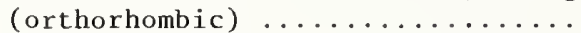

Barium carbonate, $\mathrm{BaCO}_{3}$ (cubic)

at $1075{ }^{\circ} \mathrm{C} \ldots \ldots \ldots \ldots \ldots \ldots \ldots$

Barium chlorate, $\mathrm{Ba}\left(\mathrm{ClO}_{3}\right)_{2} \ldots \ldots \ldots$

Barium chlorate hydrate,

$\mathrm{Ba}\left(\mathrm{ClO}_{3}\right)_{2} \cdot \mathrm{H}_{2} \mathrm{O} \ldots \ldots \ldots \ldots \ldots \ldots$

Barium chlorate hydrate,

$\mathrm{Ba}\left(\mathrm{ClO}_{4}\right)_{2} \cdot 3 \mathrm{H}_{2} \mathrm{O} \ldots \ldots \ldots \ldots \ldots$

Barium chloride, $\mathrm{BaCl}_{2}$, (cubic) ...

Barium chloride, $\mathrm{BaCl}_{2}$,

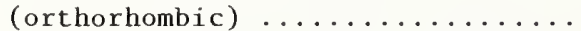

Barium chloride fluoride, BaClF ...

Barium chloride hydrate, $\mathrm{BaCl}_{2} \cdot 2 \mathrm{H}_{2} \mathrm{O}$

Barium chromium oxide,

$\mathrm{Ba}_{3}\left(\mathrm{CrO}_{4}\right)_{2} \ldots \ldots \ldots \ldots \ldots \ldots$

Barium fluoride, $\mathrm{BaF}_{2} \ldots \ldots \ldots \ldots$

Barium hydroxide phosphate,

$\mathrm{Ba}_{5}(\mathrm{OH})\left(\mathrm{PO}_{4}\right)_{3} \ldots \ldots \ldots \ldots \ldots \ldots$

Barium iodide, $\mathrm{BaI}_{2} \ldots \ldots \ldots \ldots$

Barium iodide hydrate, $\mathrm{BaI}_{2} \cdot 2 \mathrm{H}_{2} \mathrm{O}$.

Barium lead chloride, $\mathrm{BaPbCl}_{4} \ldots$.

Barium lead nitrate,

$\mathrm{Ba}{ }_{33} \mathrm{~Pb}{ }_{.67}\left(\mathrm{NO}_{3}\right)_{2} \ldots \ldots \ldots \ldots$

Barium lead nitrate,

$\mathrm{Ba}{ }_{67} \mathrm{~Pb}{ }_{33}\left(\mathrm{NO}_{3}\right)_{2} \ldots \ldots \ldots \ldots$

Barium manganese oxide, $\mathrm{BaMnO}_{4} \ldots$

Barium manganese oxide,

$\mathrm{Ba}\left(\mathrm{MnO}_{4}\right)_{2} \ldots \ldots \ldots \ldots \ldots$

Barium molybdenum oxide, $\mathrm{BaMoO}_{4} \ldots$

Barium molybdenum oxide, $\mathrm{Ba}_{2} \mathrm{MoO}_{5} \ldots$

Barium neodymium titanium oxide,

$\mathrm{BaNd}_{2} \mathrm{Ti}_{3} \mathrm{O}_{10} \ldots \ldots \ldots \ldots \ldots \ldots \ldots$

Barium neodymium titanium oxide,

$\mathrm{BaNd}_{2} \mathrm{Ti}_{5} \mathrm{O}_{14} \ldots \ldots \ldots \ldots \ldots$

Barium nitrate (nitrobarite),

$\mathrm{Ba}\left(\mathrm{NO}_{3}\right)_{2} \ldots \ldots \ldots \ldots \ldots \ldots$

Barium nitrite hydrate,

$\mathrm{Ba}\left(\mathrm{NO}_{2}\right)_{2} \cdot \mathrm{H}_{2} \mathrm{O} \ldots \ldots \ldots \ldots \ldots \ldots$

Barium oxide, $\mathrm{BaO} \ldots \ldots \ldots \ldots \ldots \ldots$

Barium oxide, $\mathrm{BaO}_{2} \ldots \ldots \ldots \ldots$

Barium phosphate, $\mathrm{Ba}_{2} \mathrm{P}_{2} \mathrm{O}_{7}$,

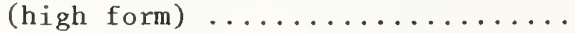

Barium phosphate, $\mathrm{Ba}_{3}\left(\mathrm{PO}_{4}\right)_{2} \ldots \ldots$.

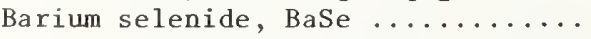

Barium silicate, $\mathrm{\beta}-\mathrm{BaSiO}_{3} \ldots \ldots \ldots$

Barium silicate (sanbornite),

$\beta-\mathrm{BaSi}_{2} \mathrm{O}_{5} \ldots \ldots \ldots \ldots \ldots \ldots \ldots$

Barium silicate, $\mathrm{Ba}_{2} \mathrm{SiO}_{4} \ldots \ldots \ldots$.
$8 \mathrm{~m} \quad 19$

$10 \mathrm{~m} \quad 63$

$10 \mathrm{~m} \quad 10$

$3 \mathrm{~m} \quad 10$

$16 \mathrm{~m} \quad 16$

$15 \mathrm{~m} \quad 14$

$20 \mathrm{~m} \quad 7$

$12 \mathrm{~m} \quad 38$

$12 \mathrm{~m}$

38

$12 m$

38

$9 m$

10

$16 \mathrm{~m}$

$8 m$

$2 m$

$9 \mathrm{~m}$

7

$9 \mathrm{~m}$

$10 \mathrm{~m}$

$12 \mathrm{~m}$

$15 \mathrm{~m}$

1

$11 \mathrm{~m}$

$10 \mathrm{~m}$

$16 \mathrm{~m}$

$11 \mathrm{~m}$

$12 \mathrm{~m}$

$12 \mathrm{~m}$

$18 \mathrm{~m}$

$15 \mathrm{~m}$

7

$12 \mathrm{~m}$

$18 \mathrm{~m}$

$19 m$

$11 \mathrm{~m}$

14

$15 \mathrm{~m}$

18.

$9 \mathrm{~m}$

6

$16 \mathrm{~m}$

$12 \mathrm{~m}$

$5 \mathrm{~m}$

$13 \mathrm{~m}$

$13 m$

$13 \mathrm{~m}$
Barium silicate, $\mathrm{Ba}_{2} \mathrm{Si}_{3} \mathrm{O}_{8} \ldots \ldots \ldots$

Barium silicate, $\mathrm{Ba}_{3} \mathrm{SiO}_{5} \ldots \ldots \ldots$

Barium silicate, $\mathrm{Ba}_{3} \mathrm{Si}_{5} \mathrm{O}_{13} \ldots \ldots \ldots$

Barium silicon fluoride, $\mathrm{BaSiF}_{6} \ldots$

Barium strontium nitrate,

$\mathrm{Ba} .{ }_{25} \mathrm{Sr} .75\left(\mathrm{NO}_{3}\right)_{2} \ldots \ldots \ldots \ldots \ldots \ldots, 12 \mathrm{~m}$

Barium strontium nitrate,

Ba ${ }_{50} \mathrm{Sr}{ }_{50}\left(\mathrm{NO}_{3}\right)_{2} \ldots \ldots \ldots \ldots \ldots \ldots . \ldots \ldots$

Barium strontium nitrate,

Ba ${ }_{75} \mathrm{Sr}{ }_{25}\left(\mathrm{NO}_{3}\right)_{2} \ldots \ldots \ldots \ldots \ldots . \ldots \ldots$ 12m

Barium sulfate (baryte), $\mathrm{BaSO}_{4} \ldots .10 \mathrm{~m}$

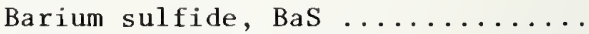

Barium thiosulfate hydrate,

$\mathrm{BaS}_{2} \mathrm{O}_{3} \cdot \mathrm{H}_{2} \mathrm{O} \ldots \ldots \ldots \ldots \ldots \ldots \ldots \ldots \ldots \ldots \ldots \ldots$

Barium tin oxide, $\mathrm{BaSnO}_{3} \ldots \ldots \ldots . . .3 \mathrm{~m}$

Barium titanium oxide, $\mathrm{BaTiO}_{3}$

(hexagonal) ............... 20m

Barium titanium oxide, $\mathrm{BaTiO}_{3}$

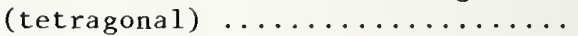

Barium titanium oxide, $\mathrm{BaTi}_{2} \mathrm{O}_{5} \ldots 2$ 20m

Barium titanium oxide, $\mathrm{BaTi}_{4} \mathrm{O}_{9} \ldots .20 \mathrm{~m}$

Barium titanium phosphate,

$\mathrm{BaTi}_{4}\left(\mathrm{PO}_{4}\right)_{6} \ldots \ldots \ldots \ldots \ldots \ldots \ldots$ 20m

Barium titanium silicate (fresnoite),

$\mathrm{Ba}_{2} \mathrm{TiSi}_{2} \mathrm{O}_{8} \ldots \ldots \ldots \ldots \ldots \ldots$

Barium tungsten oxide, $\mathrm{BaWO}_{4} \ldots \ldots$.

Barium tungsten oxide, $\mathrm{Ba}_{2} \mathrm{WO}_{5} \ldots \ldots$

Barium tungsten oxide, $\mathrm{Ba}_{3} \mathrm{WO}_{6} \ldots \ldots$

Barium vanadium oxide, $\mathrm{Ba}_{3}\left(\mathrm{VO}_{4}\right)_{2} \ldots$

Barium zirconium oxide, $\mathrm{BaZrO}_{3} \ldots$

Barium zirconium phosphate,

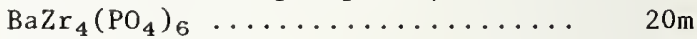

Beryllium, alpha, Be ......... 9m

Beryllium aluminum oxide

(chrysoberyl), $\mathrm{BeAl}_{2} \mathrm{O}_{4} \ldots \ldots \ldots \ldots, 9$

Beryllium aluminum silicate, beryl,

$\mathrm{Be}_{3} \mathrm{Al}_{2}\left(\mathrm{SiO}_{3}\right)_{6} \ldots \ldots \ldots \ldots \ldots \ldots$

Beryllium calcium iron magnesium

aluminum phosphate hydroxide

hydrate, roscherite (monoclinic),

$\mathrm{Be}_{2} \mathrm{Ca}\left(\mathrm{Fe} .{ }_{3} \mathrm{Mg} .7\right)_{2} \mathrm{Al} .67\left(\mathrm{PO}_{4}\right)_{3}(\mathrm{OH})_{3} \cdot 2 \mathrm{H}_{2} \mathrm{O} \quad 16 \mathrm{~m}$

Beryllium calcium manganese

aluminum iron phosphate hydroxide

hydrate, roscherite (triclinic),

$\mathrm{Be}_{4} \mathrm{Ca}_{2}\left(\mathrm{Mn}_{3} .9{ }_{1} \mathrm{Mg} .04{ }_{4} \mathrm{Ca} .05\right)\left(\mathrm{Al} .{ }_{13} \mathrm{Fe} .42\right.$

$\left.\mathrm{Mn}{ }_{12}\right)\left(\mathrm{PO}_{4}\right)_{6}(\mathrm{OH})_{4} \cdot 6 \mathrm{H}_{2} \mathrm{O} \ldots \ldots \ldots \ldots$

$16 \mathrm{~m}$

Beryllium calcium oxide,

$\mathrm{Be}_{17} \mathrm{Ca}_{12} \mathrm{O}_{29} \ldots \ldots \ldots \ldots \ldots \ldots \ldots$

Beryllium carbide, $\mathrm{Be}_{2} \mathrm{C} \ldots \ldots \ldots \ldots$

Beryllium chromium oxide, $\mathrm{BeCr}_{2} \mathrm{O}_{4}$

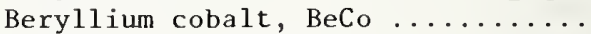

Beryllium germanium oxide, $\mathrm{Be}_{2} \mathrm{GeO}_{4}$

Beryllium lanthanum oxide, $\mathrm{Be}_{2} \mathrm{La}_{2} \mathrm{O}_{5}$

Beryllium niobium, $\mathrm{Be}_{2} \mathrm{Nb} \ldots \ldots \ldots$

Beryllium nitride, $\mathrm{Be}_{3} \mathrm{~N}_{2} \ldots \ldots \ldots$

Beryllium oxide (bromellite), BeO

Beryllium palladium, BePd .......

Beryllium silicate, phenakite,

$\mathrm{Be}_{2} \mathrm{SiO}_{4} \ldots \ldots \ldots \ldots \ldots \ldots \ldots$

Beryllium sulfate, $\mathrm{BeSO}_{4} \ldots \ldots \ldots \ldots$

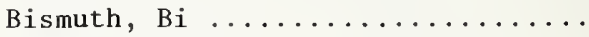

Bismuth bromide oxide, $\mathrm{BiOBr} . .$. .

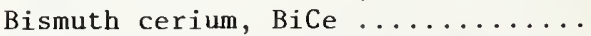

Bismuth chloride oxide (bismoclite),

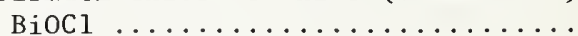

Bismuth dysprosium, BiDy ........

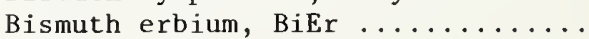

$7 \mathrm{~m}$

$19 \mathrm{~m}$

10

$5 \mathrm{~m}$

10

$7 \mathrm{~m}$

$18 \mathrm{~m}$
$9 \mathrm{~m}$

1

13

15

42

12

8

20

11

9

45

11

13

15

14

9

14

21

10

8

17

64

10

100 
Bismuth fluoride, $\mathrm{BiF}_{3} \ldots \ldots \ldots$

Bismuth germanium oxide,

$\mathrm{Bi}_{4}\left(\mathrm{GeO}_{4}\right)_{3} \ldots \ldots \ldots \ldots \ldots \ldots \ldots$

Bismuth germanium oxide,

$\mathrm{Bi}_{12} \mathrm{GeO}_{20} \ldots \ldots \ldots \ldots \ldots \ldots \ldots \ldots \ldots \ldots \ldots$

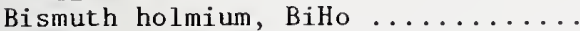

Bismuth(IIl) iodide, $\mathrm{Bil}_{3} \ldots \ldots \ldots$

Bismuth iodide oxide, BiOl .......

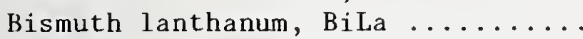

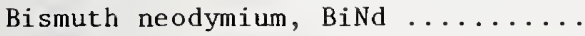

Bismuth oxide (bismite), $\alpha-\mathrm{Bi}_{2} \mathrm{O}_{3} \ldots$

Bismuth phosphate, $\mathrm{BiPO}_{4}$

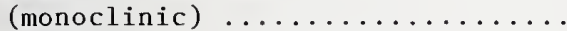

Bismuth phosphate, $\mathrm{BiPO}_{4}$ (trigonal)

Bismuth praseodymium, BiPr .......

Bismuth selenide (paraguanajuatite),

$\mathrm{Bi}_{2} \mathrm{Se}_{3} \ldots \ldots \ldots \ldots \ldots \ldots \ldots$

Bismuth sulfide (bismuthinite),

$\mathrm{Bi}_{2} \mathrm{~S}_{3} \ldots \ldots \ldots \ldots \ldots \ldots \ldots \ldots \ldots$

Bismuth telluride, BiTe .........

Bismuth telluride (tellurobis-

muthite), $\mathrm{Bi}_{2} \mathrm{Te}_{3} \ldots \ldots \ldots \ldots \ldots$

Bismuth titanium oxide, $\mathrm{Bi}_{12} \mathrm{TiO}_{20}$.

Bismuth vanadium oxide, high form,

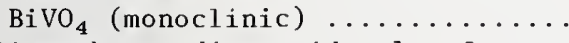

Bismuth vanadium oxide, low form,

$\mathrm{BiVO}_{4}$ (tetragonal) $\ldots \ldots \ldots \ldots \ldots$

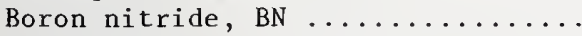

Boron oxide, $\mathrm{B}_{2} \mathrm{O}_{3}$, phase $1 \ldots \ldots$.

Boron phosphate, $\mathrm{BPO}_{4} \ldots \ldots \ldots \ldots$

Cadmium, $\mathrm{Cd} \ldots \ldots \ldots \ldots \ldots \ldots$

Cadmium aluminum oxide, $\mathrm{CdAl}_{2} \mathrm{O}_{4} \ldots$

Cadmium ammine chloride,

$\mathrm{Cd}\left(\mathrm{NH}_{3}\right)_{2} \mathrm{Cl}_{2} \ldots \ldots \ldots \ldots \ldots$

Cadmium borate, $\mathrm{CdB}_{4} \mathrm{O}_{7} \ldots \ldots \ldots$.

Cadmium bromate hydrate,

$\mathrm{Cd}\left(\mathrm{BrO}_{3}\right) \cdot 2 \mathrm{H}_{2} \mathrm{O} \ldots \ldots \ldots \ldots \ldots \ldots$

Cadmium bromide, $\mathrm{CdBr}_{2} \ldots \ldots \ldots \ldots$

Cadmium bromide chloride, $\mathrm{CdBrCl}$. .

Cadmium carbonate (otavite), $\mathrm{CdCO}_{3}$

Cadmium cerium, CdCe ............

Cadmium chlorate hydrate,

$\mathrm{Cd}\left(\mathrm{ClO}_{4}\right)_{2} \cdot 6 \mathrm{H}_{2} \mathrm{O} \ldots \ldots \ldots \ldots \ldots \ldots$

Cadmium chloride, $\mathrm{CdCl}_{2} \ldots \ldots \ldots$

Cadmium chromium oxide, $\mathrm{CdCr}_{2} \mathrm{O}_{4} \ldots$

Cadmium copper, $\mathrm{Cd}_{8} \mathrm{Cu}_{5} \ldots \ldots \ldots \ldots$

Cadmium cyanide, $\mathrm{Cd}(\mathrm{CN})_{2} \ldots \ldots \ldots$

Cadmium fluoride, $\mathrm{CdF}_{2} \ldots \ldots \ldots \ldots$

Cadmium iodide, $\alpha-\mathrm{CdI}_{2} \ldots \ldots \ldots \ldots$

Cadmium iron oxide, $\mathrm{CdFe}_{2} \mathrm{O}_{4} \ldots \ldots$.

Cadmium lanthanum, CdLa ..........

Cadmium manganese oxide, $\mathrm{CdMn}_{2} \mathrm{O}_{4} \ldots$

Cadmium molybdenum oxide, $\mathrm{CdMoO}_{4}$..

Cadmium nitrate hydrate,

$\mathrm{Cd}\left(\mathrm{NO}_{3}\right)_{2} \cdot 4 \mathrm{H}_{2} \mathrm{O} \ldots \ldots \ldots \ldots \ldots \ldots$

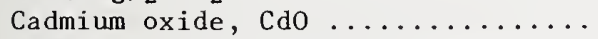

Cadmium oxide, CdO (ref. standard)

Cadmium phosphate, $\mathrm{Cd}_{2} \mathrm{P}_{2} \mathrm{O}_{7} \ldots \ldots \ldots$

Cadmium phosphate, $\mathrm{Cd}_{3}\left(\mathrm{PO}_{4}\right)_{2} \ldots \ldots$

Cadmium praseodymium, $\mathrm{CdPr} \ldots \ldots \ldots$

Cadmium selenide (cadmoselite),

CdSe (hexagonal) ...............

Cadmium silicate, $\mathrm{Cd}_{2} \mathrm{SiO}_{4} \ldots \ldots \ldots$.

Cadmium silicate, $\mathrm{Cd}_{3} \mathrm{SiO}_{5} \ldots \ldots \ldots$

Cadmium sulfate, $\mathrm{CdSO}_{4} \ldots \ldots \ldots \ldots$

Cadmium sulfate hydrate, $\mathrm{CdSO}_{4} \cdot \mathrm{H}_{2} \mathrm{O}$

Cadmium sulfate hydrate,

$3 \mathrm{CdSO}_{4} \cdot 8 \mathrm{H}_{2} \mathrm{O}$
$1 \mathrm{~m} \quad 7$

$20 \mathrm{~m}$

18

$20 \mathrm{~m}$

19

$4 \mathrm{~m} \quad 48$

620

916

$4 \mathrm{~m} \quad 48$

$4 \mathrm{~m} \quad 49$

$3 \mathrm{~m} \quad 17$

$3 \mathrm{~m} \quad 11$

$3 m \quad 13$

$4 \mathrm{~m} \quad 49$

$18 \mathrm{~m} \quad 16$

$5 m$

13

$4 \mathrm{~m} \quad 50$

$3 \mathrm{~m} \quad 16$

$20 \mathrm{~m} \quad 21$

$3 \mathrm{~m} \quad 14$

$3 m$

$20 \mathrm{~m}$

$10 \mathrm{~m}$

$20 \mathrm{~m}$

3
$20 \mathrm{~m}$

$10 \mathrm{~m}$

$16 \mathrm{~m}$

$17 \mathrm{~m}$

9

$11 \mathrm{~m}$

7

$5 m$

$3 m$

9

$5 \mathrm{~m}$

$11 \mathrm{~m}$

$2 \mathrm{~m}$

$10 \mathrm{~m}$

$19 \mathrm{~m}$

$9 \mathrm{~m}$

$5 \mathrm{~m}$

$10 \mathrm{~m}$

6

$7 \mathrm{~m}$

2

$8 \mathrm{~m}$

$16 \mathrm{~m}$

$16 \mathrm{~m}$

$5 m$

7

$13 \mathrm{~m}$

$13 \mathrm{~m}$

$3 m$

$6 m$

$6 m$
Cadmium sulfide (greenockite), CdS

Cadmium telluride, CdTe ..........

Cadmium tilanium oxide, $\mathrm{CdTiO}_{3} \ldots$

Cadmium tungsten oxide, $\mathrm{CdWO}_{4} \ldots$.

Cadmium zinc phosphate,

$\mathrm{Cd}_{\mathrm{O}} ._{9} \mathrm{Zn}_{2.1}\left(\mathrm{PO}_{4}\right)_{2} \ldots \ldots \ldots \ldots \ldots$

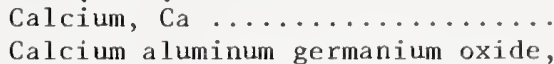

$\mathrm{Ca}_{3} \mathrm{Al}_{2}\left(\mathrm{GeO}_{4}\right)_{3} \ldots \ldots \ldots \ldots \ldots$

Calcium aluminum hydroxide,

$\mathrm{Ca}_{3} \mathrm{Al}_{2}(\mathrm{OH})_{12} \ldots \ldots \ldots \ldots \ldots \ldots$

(brownmillerite), $\mathrm{Ca}_{4} \mathrm{Al}_{2} \mathrm{Fe}_{2} \mathrm{O}_{10} \ldots$

Calcium aluminum oxide, $\mathrm{Ca}_{3} \mathrm{Al}_{2} \mathrm{O}_{6} \ldots$

Calcium aluminum oxide (mayenite),

$\mathrm{Ca}_{12} \mathrm{Al}_{14} \mathrm{O}_{33} \ldots \ldots \ldots \ldots \ldots \ldots \ldots$

Calcium aluminum oxide hydrate,

$\mathrm{Ca}_{4} \mathrm{Al}_{6} \mathrm{O}_{13} \cdot 3 \mathrm{H}_{2} \mathrm{O} \ldots \ldots \ldots \ldots \ldots \ldots$

Calcium aluminum silicate hydrate,

chabazite, $\mathrm{Ca}_{2} \mathrm{Al}_{4} \mathrm{Si}_{8} \mathrm{O}_{24} \cdot 12 \mathrm{H}_{2} \mathrm{O} \ldots$

Calcium aluminum sulfate hydrate

(ettringite), $\mathrm{Ca}_{6} \mathrm{Al}_{2} \mathrm{~S}_{3} \mathrm{O}_{18} \cdot 31 \mathrm{H}_{2} \mathrm{O} \ldots$

Calcium borate, $\mathrm{CaB}_{2} \mathrm{O}_{4} \ldots \ldots \ldots \ldots$

Calcium borate, $\mathrm{CaB}_{2} \mathrm{O}_{4}$

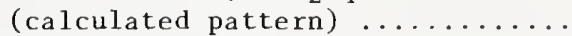

Calcium borate hydrate,

hexahydroborite, $\mathrm{Ca}\left[\mathrm{B}(\mathrm{OH})_{4}\right]_{2} \cdot 2 \mathrm{H}_{2} \mathrm{O}$

Calcium boride, $\mathrm{CaB}_{6} \ldots \ldots \ldots \ldots \ldots$

Calcium bromide, $\mathrm{CaBr}_{2} \ldots \ldots \ldots \ldots$

Calcium bromide hydrate, $\mathrm{CaBr}_{2} \cdot 6 \mathrm{H}_{2} \mathrm{O}$

Calcium carbonate (aragonite),

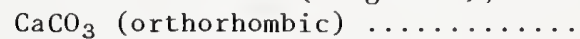

Calcium carbonate (aragonite),

$\mathrm{CaCO}_{3}$ (orthorhombic, calculated

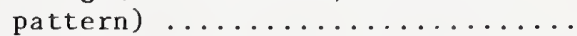

Calcium carbonate (calcite),

$\mathrm{CaCO}_{3}$ (hexagonal) $\ldots \ldots \ldots \ldots \ldots \ldots$

Calcium chloride (hydrophilite),

$\mathrm{CaCl}_{2} \ldots \ldots \ldots \ldots \ldots \ldots \ldots$

Calcium chloride fluoride, CaClF . .

Calcium chloride hydrate,

$\mathrm{CaCl}_{2} \cdot 4 \mathrm{H}_{2} \mathrm{O} \ldots \ldots \ldots \ldots$

Calcium chloride hydrate

(antarcticite), $\mathrm{CaCl}_{2} \cdot 6 \mathrm{H}_{2} \mathrm{O} \ldots \ldots$

Calcium chromium germanium oxide,

$\mathrm{Ca}_{3} \mathrm{Cr}_{2}\left(\mathrm{GeO}_{4}\right)_{3} \ldots \ldots \ldots \ldots \ldots \ldots$

Calcium chromium iron titanium

oxide, loveringite, $\mathrm{Ca} .{ }_{72} \mathrm{RE} .{ }_{33}(\mathrm{Y}$,

$\mathrm{Th}, \mathrm{U}, \mathrm{Pb}) .05 \mathrm{Ti}_{12.48} \mathrm{Fe}_{3.38} \mathrm{Cr}_{2.24}$

$\mathrm{Mg} .92 \mathrm{Zr} .{ }_{58} \mathrm{Al} .{ }_{39} \dot{\mathrm{V}}_{.21} \mathrm{Mn} .{ }_{04} \mathrm{O}_{38} \ldots \ldots$

Calcium chromium oxide (chromatite),

$\mathrm{CaCrO}_{4} \ldots \ldots \ldots \ldots \ldots \ldots \ldots$

Calcium chromium oxide, $\mathrm{Ca}_{3}\left(\mathrm{CrO}_{4}\right)_{2}$

Calcium chromium silicate

(uva rovite), $\mathrm{Ca}_{3} \mathrm{Cr}_{2}\left(\mathrm{SiO}_{4}\right)_{3} \ldots \ldots$

Calcium cyanamide, $\mathrm{CaCN}_{2} \ldots \ldots \ldots$

Calcium fluoride (fluorite), $\mathrm{CaF}_{2}$.

Calcium fluoride phosphate

(fluorapatite), $\mathrm{Ca}_{5} \mathrm{~F}\left(\mathrm{PO}_{4}\right)_{3} \ldots \ldots$

Calcium fluoride phosphate hydrate,

$\mathrm{CaFPO}_{3} \cdot 2 \mathrm{H}_{2} \mathrm{O} \ldots \ldots \ldots \ldots \ldots \ldots$

Calcium gallium germanium oxide,

$\mathrm{Ca}_{3} \mathrm{Ga}_{2}\left(\mathrm{GeO}_{4}\right)_{3} \ldots \ldots \ldots \ldots \ldots \ldots$

Calcium hydrogen phosphate hydrate, $\mathrm{Ca}_{8} \mathrm{H}_{2}\left(\mathrm{PO}_{4}\right)_{6} \cdot 5 \mathrm{H}_{2} \mathrm{O} \ldots \ldots \ldots \ldots \ldots$

Calcium hydrogen phosphate sulfate hydrate, $\mathrm{Ca}_{2} \mathrm{HPO}_{4} \mathrm{SO}_{4} \cdot 4 \mathrm{H}_{2} \mathrm{O} \ldots \ldots \ldots$

\begin{tabular}{rr}
4 & 15 \\
$3 \mathrm{~m}$ & 21 \\
$15 \mathrm{~m}$ & 21 \\
$2 \mathrm{~m}$ & 8 \\
& \\
$20 \mathrm{~m}$ & 26 \\
$9 \mathrm{~m}$ & 68 \\
& \\
10 & 15 \\
& \\
$11 \mathrm{~m}$ & 16 \\
& \\
$16 \mathrm{~m}$ & 28 \\
5 & 10 \\
& \\
9 & 20 \\
& \\
$19 \mathrm{~m}$ & 25 \\
& \\
$19 \mathrm{~m}$ & 27 \\
& \\
8 & 3 \\
$18 \mathrm{~m}$ & 17 \\
& \\
$15 \mathrm{~m}$ & 136 \\
& \\
$16 \mathrm{~m}$ & 104 \\
$16 \mathrm{~m}$ & 29 \\
$11 \mathrm{~m}$ & 70 \\
8 & 15 \\
3 & 53 \\
& \\
\hline
\end{tabular}

$14 \mathrm{~m}$

44

$11 \mathrm{~m}$

18

$10 \mathrm{~m}$

$11 \mathrm{~m}$

$12 m$

16

10

16

$16 \mathrm{~m}$

106

7

13

$15 \mathrm{~m}$

10

17

$18 \mathrm{~m}$

19

69

$3 m$

22

$15 m$

24

10

$13 m$

$16 \mathrm{~m}$ 
Calcium hydroxide (portlandite),

$\mathrm{Ca}(\mathrm{OH})_{2} \ldots \ldots \ldots \ldots \ldots \ldots \ldots \ldots \ldots$

Calcium iodate (lautarite),

$\mathrm{Ca}\left(\mathrm{IO}_{3}\right)_{2} \ldots \ldots \ldots \ldots \ldots \ldots \ldots \ldots \ldots$ 14m 12

Calcium iodate hydrate,

$\mathrm{Ca}\left(\mathrm{IO}_{3}\right)_{2} \cdot 6 \mathrm{H}_{2} \mathrm{O} \ldots \ldots \ldots \ldots \ldots \ldots \ldots \ldots \ldots$
$\mathrm{Calcium}$ iron germanium oxide,

$\mathrm{Ca}_{3} \mathrm{Fe}_{2}\left(\mathrm{GeO}_{4}\right)_{3} \ldots \ldots \ldots \ldots \ldots \ldots \ldots \ldots 10 \ldots \ldots$

Calcium iron oxide, $\mathrm{CaFe}_{2} \mathrm{O}_{4} \ldots \ldots, 18 \mathrm{~m} \quad 20$

Calcium iron silicate (kirschsteinite),

$\mathrm{CaFeSiO}_{4} \ldots \ldots \ldots \ldots \ldots \ldots \ldots \ldots$ 20m

Calcium iron silicate (andradite),

$\mathrm{Ca}_{3} \mathrm{Fe}_{2} \mathrm{Si}_{3} \mathrm{O}_{12} \ldots \ldots \ldots \ldots \ldots \ldots \ldots$

Calcium iron silicate

hydroxide, julgoldite,

$\mathrm{Ca}_{2} \mathrm{Fe}_{3} \mathrm{Si}_{3} \mathrm{O}_{10}(\mathrm{OH}, \mathrm{O})_{2}(\mathrm{OH})_{2} \ldots \ldots \ldots \quad 10 \mathrm{~m}$

Calcium lead nitrate,

$\mathrm{Ca} .{ }_{33} \mathrm{~Pb}{ }_{67}\left(\mathrm{NO}_{3}\right)_{2} \ldots \ldots \ldots \ldots \ldots \ldots \quad 12 \mathrm{~m}$

Calcium lead nitrate,

$\mathrm{Ca} .{ }_{67} \mathrm{~Pb}{ }_{.33}\left(\mathrm{NO}_{3}\right)_{2} \ldots \ldots \ldots \ldots \ldots, 12 \mathrm{~m}$

Calcium magnesium silicate (monticel-

lite), $\mathrm{CaMgSiO}_{4} \ldots \ldots \ldots \ldots \ldots \ldots \ldots \quad 20 \mathrm{~m}$
Calcium magnesium silicate

(diopside), $\mathrm{CaMg}\left(\mathrm{SiO}_{3}\right)_{2} \ldots \ldots \ldots \ldots{ }^{2}, 5 \mathrm{~m}$
Calcium magnesium silicate (akermanite),

$\mathrm{Ca}_{2} \mathrm{MgSi}_{2} \mathrm{O}_{7} \ldots \ldots \ldots \ldots \ldots \ldots \ldots \ldots \ldots \ldots \ldots \ldots$

Calcium magnesium silicate (merwinite),

$\mathrm{Ca}_{3} \mathrm{Mg}\left(\mathrm{SiO}_{4}\right)_{2} \ldots \ldots \ldots \ldots \ldots \ldots$ 20m

Calcium manganese phosphate fluoride,

$\mathrm{Ca}{ }_{8} \mathrm{Mn}_{2}\left(\mathrm{PO}_{4}\right)_{6} \mathrm{~F}_{2} \ldots \ldots \ldots \ldots \ldots \ldots \ldots, 20 \mathrm{~m}$

Calcium molybdenum oxide

(powellite), $\mathrm{CaMoO}_{4} \ldots \ldots \ldots \ldots \ldots$

Calcium nitrate, $\mathrm{Ca}\left(\mathrm{NO}_{3}\right)_{2} \ldots \ldots \ldots$

Calcium oxide (lime), $\mathrm{CaO} \ldots \ldots \ldots$

Calcium oxide (lime), $\mathrm{CaO}$

(calculated pattern) ...........

Calcium oxide phosphate, $\mathrm{Ca}_{4} \mathrm{O}\left(\mathrm{PO}_{4}\right)_{2}$

Calcium phosphate, $\beta-\mathrm{Ca}_{2} \mathrm{P}_{2} \mathrm{O}_{7} \ldots \ldots$.

Calcium platinum oxide, $\mathrm{Ca}_{4} \mathrm{PtO}_{6} \ldots$

Calcium selenide, CaSe ..........

Calcium silicate (larnite),

$\beta-\mathrm{Ca}_{2} \mathrm{SiO}_{4} \ldots \ldots \ldots \ldots \ldots \ldots \ldots$

Calcium silicon fluoride hydrate,

$\mathrm{CaSiF}_{6} \cdot 2 \mathrm{H}_{2} \mathrm{O} \ldots \ldots \ldots \ldots \ldots \ldots$

Calcium strontium nitrate,

$\left.\mathrm{Ca} .{ }_{33} \mathrm{Sr} .67 \mathrm{NO}_{3}\right)_{2} \ldots \ldots \ldots \ldots$

Calcium strontium nitrate,

$\mathrm{Ca}{ }_{67} \mathrm{Sr} .{ }_{33}\left(\mathrm{NO}_{3}\right)_{2} \ldots \ldots \ldots \ldots \ldots$

Calcium sulfate (anhydrite), $\mathrm{CaSO}_{4}$

Calcium sulfate hydrate (bassanite),

$\mathrm{CaSO}_{4} \cdot 0.5 \mathrm{H}_{2} \mathrm{O} \ldots \ldots \ldots \ldots \ldots \ldots$

Calcium sulfate hydrate (gypsum),

$\mathrm{CaSO}_{4} \cdot 2 \mathrm{H}_{2} \mathrm{O} \ldots \ldots \ldots \ldots \ldots \ldots \ldots$

Calcium sulfide (oldhamite), CaS ..

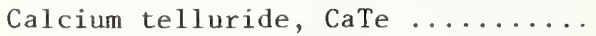

Calcium tin oxide, $\mathrm{CaSnO}_{3} \ldots \ldots \ldots$

Calcium titanium oxide

(perovskite), $\mathrm{CaTiO}_{3} \ldots \ldots \ldots \ldots$

Calcium tungsten oxide, $\mathrm{Ca}_{3} \mathrm{WO}_{6} \ldots$.

Calcium tungsten oxide, scheelite,

$\mathrm{CaWO}_{4} \ldots \ldots \ldots \ldots \ldots \ldots \ldots \ldots$

Calcium zirconium phosphate,

$\mathrm{CaZr}_{4}\left(\mathrm{PO}_{4}\right)_{6} \ldots \ldots \ldots \ldots \ldots \ldots$

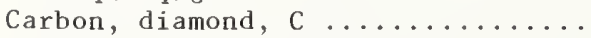

Cerium arsenate, $\mathrm{CeAsO}_{4} \ldots \ldots \ldots \ldots$

Cerium(III) chloride, $\mathrm{CeCl}_{3} \ldots \ldots \ldots$

Cerium cobalt, $\mathrm{CeCo}_{2} \ldots \ldots \ldots \ldots \ldots$

Cerium cobalt, $\mathrm{Ce}_{24} \mathrm{Co}_{11} \ldots \ldots \ldots \ldots$
Cerium copper, $\mathrm{CeCu}_{6} \ldots \ldots \ldots \ldots$.

Cerium(III) fluoride, $\mathrm{CeF}_{3} \ldots \ldots \ldots$

Cerium gallium, $\mathrm{CeGa}_{2} \ldots \ldots \ldots \ldots$.

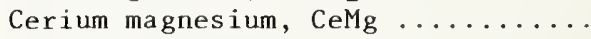

Cerium magnesium, $\mathrm{CeMg}_{3} \ldots \ldots \ldots \ldots$

Cerium nickel, $\mathrm{CeNi}_{2} \ldots \ldots \ldots \ldots \ldots$

Cerium niobium oxide, $\mathrm{CeNbO}_{4} \ldots \ldots$.

Cerium niobium titanium oxide

(aeschynite), $\mathrm{CeNbTiO}_{6} \ldots \ldots \ldots$

Cerium nitrate hydrate,

$\mathrm{Ce}\left(\mathrm{NO}_{3}\right)_{3} \cdot 6 \mathrm{H}_{2} \mathrm{O} \ldots \ldots \ldots \ldots \ldots \ldots$

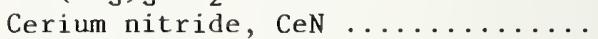

Cerium oxide, $\mathrm{CeO}_{2} \ldots \ldots \ldots \ldots \ldots$ Cerium(IV) oxide (cerianite), $\mathrm{CeO}_{2}$

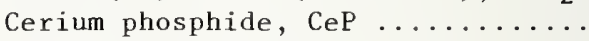

Cerium tantalum oxide, $\mathrm{CeTaO}_{4} \ldots$.

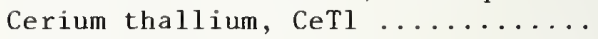

Cerium thallium, $\mathrm{CeTl}_{3} \ldots \ldots \ldots \ldots$ Cerium thallium, $\mathrm{Ce}_{3} \mathrm{Tl} \ldots \ldots \ldots \ldots$ Cerium(III) vanadium oxide, $\mathrm{CeVO}_{4}$

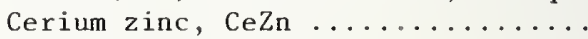

Cerium zinc, $\operatorname{Ce} \mathrm{n}_{3} \ldots \ldots \ldots \ldots \ldots$

Cerium zinc, $\mathrm{CeZn}_{5} \ldots \ldots \ldots \ldots \ldots$

Cerium zinc, $\mathrm{Ce}_{2} \mathrm{Zn}_{17} \ldots \ldots \ldots \ldots \ldots$

Cesium aluminum sulfate hydrate,

$\mathrm{CsAl}\left(\mathrm{SO}_{4}\right)_{2} \cdot 12 \mathrm{H}_{2} \mathrm{O} \ldots \ldots \ldots \ldots \ldots$

Cesium antimony fluoride, $\mathrm{CsSbF}_{6} \ldots$

Cesium beryllium fluoride, $\mathrm{CsBeF}_{3}$

Cesium boron fluoride, $\mathrm{CsBF}_{4} \ldots \ldots$.

Cesium bromate, $\mathrm{CsBrO}_{3} \ldots \ldots \ldots \ldots$

Cesium bromide, $\mathrm{CsBr}$..........

Cesium cadmium bromide, $\mathrm{CsCdBr}_{3}$

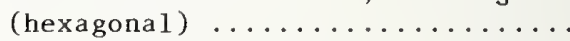

Cesium cadmium chloride, $\mathrm{CsCdCl}_{3}$

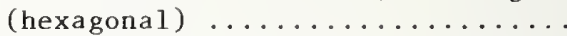

Cesium calcium chloride, $\mathrm{CsCaCl}_{3} \ldots$

Cesium calcium fluoride, $\mathrm{CsCaF}_{3} \ldots$

Cesium calcium sulfate,

$\mathrm{Cs}_{2} \mathrm{Ca}_{2}\left(\mathrm{SO}_{4}\right)_{3} \ldots \ldots \ldots \ldots \ldots \ldots$

Cesium cerium chloride, $\mathrm{Cs}_{2} \mathrm{CeCl}_{6} \ldots$

Cesium chlorate, $\mathrm{CsClO}_{3} \ldots \ldots \ldots$

Cesium chlorate, $\mathrm{CsClO}_{4}$,

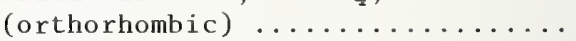

Cesium chloride, $\mathrm{CsCl} \ldots . . \ldots \ldots \ldots$

Cesium chromium oxide, $\mathrm{Cs}_{2} \mathrm{CrO}_{4} \ldots$

Cesium chromium sulfate hydrate,

$\mathrm{CsCr}\left(\mathrm{SO}_{4}\right)_{2} \cdot 12 \mathrm{H}_{2} \mathrm{O} \ldots \ldots \ldots \ldots \ldots \ldots$ Cesium cobalt(II) chloride, $\mathrm{CsCoCl}_{3}$ Cesium cobalt chloride, $\mathrm{Cs}_{2} \mathrm{CoCl}_{4} \ldots$ Cesium copper(II) chloride, $\mathrm{CsCuCl}_{3}$ Cesium copper chloride, $\mathrm{Cs}_{2} \mathrm{CuCl}_{4} \ldots$ Cesium copper sulfate hydrate,

$\mathrm{Cs}_{2} \mathrm{Cu}\left(\mathrm{SO}_{4}\right)_{2} \cdot 6 \mathrm{H}_{2} \mathrm{O} \ldots \ldots \ldots \ldots \ldots$

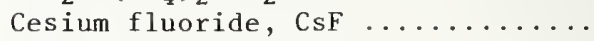

Cesium gallium sulfate hydrate,

$\mathrm{CsGa}\left(\mathrm{SO}_{4}\right)_{2} \cdot 12 \mathrm{H}_{2} \mathrm{O} \ldots \ldots \ldots \ldots . . . .$. Cesium germanium fluoride, $\mathrm{Cs}_{2} \mathrm{GeF}_{6}$ Cesium hydrogen phosphate,

$\mathrm{CsH}_{5}\left(\mathrm{PO}_{4}\right)_{2} \ldots \ldots \ldots \ldots \ldots \ldots$

Cesium iodate, $\mathrm{CsIO}_{3} \ldots \ldots \ldots \ldots \ldots$

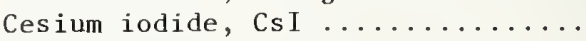

Cesium iodide, $\mathrm{CsI}_{3} \ldots \ldots \ldots \ldots \ldots$

Cesium iodine bromide, $\mathrm{CsI}_{2} \mathrm{Br} \ldots .$.

Cesium iodine chloride, $\mathrm{CsICl}_{2} \ldots$

Cesium iron chloride hydrate,

$\mathrm{Cs}_{2} \mathrm{FeCl}_{5} \cdot \mathrm{H}_{2} \mathrm{O} \ldots \ldots \ldots \ldots \ldots \ldots$

Cesium iron sulfate hydrate,

$\mathrm{Cs}_{2} \mathrm{Fe}\left(\mathrm{SO}_{4}\right)_{2} \cdot 6 \mathrm{H}_{2} \mathrm{O}$
Sec. Page

$\begin{array}{rr}7 \mathrm{~m} & 99 \\ 8 & 17 \\ 13 \mathrm{~m} & 54 \\ 5 \mathrm{~m} & 65 \\ 13 \mathrm{~m} & 56 \\ 13 \mathrm{~m} & 58 \\ 18 \mathrm{~m} & 25 \\ & \\ 3 \mathrm{~m} & 24 \\ & \\ 17 \mathrm{~m} & 20 \\ 4 \mathrm{~m} & 51 \\ 20 \mathrm{~m} & 38 \\ 1 & 56 \\ 4 \mathrm{~m} & 52 \\ 18 \mathrm{~m} & 27 \\ 13 \mathrm{~m} & 59 \\ 13 \mathrm{~m} & 60 \\ 13 \mathrm{~m} & 61 \\ 1 \mathrm{~m} & 9 \\ 5 \mathrm{~m} & 65 \\ 14 \mathrm{~m} & 50 \\ 14 \mathrm{~m} & 53 \\ 14 \mathrm{~m} & 55 \\ & \\ 6 & 25 \\ 4 \mathrm{~m} & 9 \\ 9 \mathrm{~m} & 69 \\ 8 & 22 \\ 8 & 18 \\ 3 & 49 \\ & \\ & \\ & \\ & \\ & \end{array}$

$10 \mathrm{~m}$

20

$5 \mathrm{~m} \quad 19$

$5 \mathrm{~m} \quad 21$

$8 \mathrm{~m} \quad 25$

$7 \mathrm{~m} \quad 12$

$14 \mathrm{~m} \quad 58$

$8 \quad 20$

$1 \mathrm{~m}$

2

$3 \mathrm{~m}$

8

$6 \mathrm{~m}$

$11 \mathrm{~m}$

$5 m$

$11 \mathrm{~m}$

$7 \mathrm{~m}$

$3 \mathrm{~m}$

$20 \mathrm{~m}$

$15 \mathrm{~m}$

4
$19 m$

$19 \mathrm{~m}$

$7 \mathrm{~m}$
3

$14 \mathrm{~m}$

$7 \mathrm{~m}$
9 54 5 .4. 20 51 60 61 65 53 55 25 9 . 


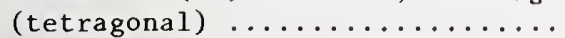

Cesium lead fluoride, $\mathrm{CsPbF}_{3} \ldots \ldots$

$5 m$

Cesium lithium cobalt cyanide,

$\mathrm{CsLiCo}(\mathrm{CN})_{6} \ldots \ldots \ldots \ldots \ldots \ldots$

Cesium lithium fluoride, $\mathrm{CsLiF}_{2} \ldots$

Cesium magnesium chromium oxide,

$\mathrm{Cs}_{2} \mathrm{Mg}_{2}\left(\mathrm{CrO}_{4}\right)_{3} \ldots \ldots \ldots \ldots \ldots \ldots \ldots$

Cesium magnesium chromium oxide

hydrate, $\mathrm{Cs}_{2} \mathrm{Mg}\left(\mathrm{CrO}_{4}\right)_{2} \cdot 6 \mathrm{H}_{2} \mathrm{O} \ldots \ldots$

Cesium magnesium sulfate hydrate,

$\mathrm{Cs}_{2} \mathrm{Mg}\left(\mathrm{SO}_{4}\right)_{2} \cdot 6 \mathrm{H}_{2} \mathrm{O} \ldots \ldots \ldots \ldots \ldots$

Cesium magnesium titanium oxide,

$\mathrm{Cs}_{1,45} \mathrm{Mgo} .724^{\mathrm{Ti}_{7}, 2{ }_{7} \mathrm{O}_{16} \ldots \ldots \ldots \ldots}$

Cesium manganese fluoride, $\mathrm{CsMnF}_{3}$

Cesium manganese sulfate hydrate,

$\mathrm{Cs}_{2} \mathrm{Mn}\left(\mathrm{SO}_{4}\right)_{2} \cdot 6 \mathrm{H}_{2} \mathrm{O} \ldots \ldots \ldots \ldots$

Cesium mercury chloride, $\mathrm{CsHgCl}_{3} \ldots$

Cesium molybdenum oxide,

$\mathrm{Cs}_{2} \mathrm{Mo}_{3} \mathrm{O}_{10} \ldots \ldots \ldots \ldots \ldots \ldots \ldots \ldots \ldots$

Cesium nickel(II) chloride, $\mathrm{CsNiCl}_{3}$

Cesium nickel sulfate hydrate,

$\mathrm{Cs}_{2} \mathrm{Ni}\left(\mathrm{SO}_{4}\right)_{2} \cdot 6 \mathrm{H}_{2} \mathrm{O} \ldots \ldots \ldots \ldots \ldots \ldots$

Cesium nitrate, $\mathrm{CsNO}_{3} \ldots \ldots \ldots \ldots$

Cesium osmium(IV) bromide, $\mathrm{Cs}_{2} \mathrm{OsBr}_{6}$

Cesium osmium chloride, $\mathrm{Cs}_{2} \mathrm{OsCl}_{6} \ldots$

Cesium platinum bromide, $\mathrm{Cs}_{2} \mathrm{PtBr}_{6}$.

Cesium platinum chloride, $\mathrm{Cs}_{2} \mathrm{PtCl}_{6}$

Cesium platinum fluoride, $\mathrm{Cs}_{2} \mathrm{PtF}_{6}$.

Cesium selenium bromide, $\mathrm{Cs}_{2} \mathrm{SeBr}_{6}$.

Cesium silicon fluoride, $\mathrm{Cs}_{2} \mathrm{SiF}_{6} \ldots$

Cesium strontium chloride, $\mathrm{CsSrCl}_{3}$

Cesium sulfate, $\mathrm{Cs}_{2} \mathrm{SO}_{4} \ldots \ldots \ldots \ldots$.

Cesium tellurium bromide, $\mathrm{Cs}_{2} \mathrm{TeBr}_{6}$

Cesium tin chloride, $\mathrm{Cs}_{2} \mathrm{SnCl}_{6} \ldots .$.

Cesium vanadium sulfate hydrate,

$\mathrm{CsV}\left(\mathrm{SO}_{4}\right)_{2} \cdot 12 \mathrm{H}_{2} \mathrm{O} \ldots \ldots \ldots \ldots \ldots \ldots$

Cesium zinc sulfate hydrate,

$\mathrm{Cs}_{2} \mathrm{Zn}\left(\mathrm{SO}_{4}\right)_{2} \cdot 6 \mathrm{H}_{2} \mathrm{O} \ldots \ldots \ldots \ldots \ldots$

Cesium zirconium phosphate

$\mathrm{CsZr}_{2}\left(\mathrm{PO}_{4}\right)_{3} \ldots \ldots \ldots \ldots \ldots \ldots \ldots$

Chromium, $\mathrm{Cr} \ldots \ldots \ldots \ldots \ldots \ldots$

Chromium boride, $\zeta-\mathrm{C}$ B $\ldots \ldots \ldots \ldots$

Chromium boride, $\mathrm{CrB}_{2} \ldots \ldots \ldots \ldots$

Chromium boride, $\mathrm{Cr}_{5} \mathrm{~B}_{3} \ldots \ldots \ldots \ldots$

Chromium chloride, $\mathrm{CrCl}_{2} \ldots \ldots \ldots \ldots$
Chromium chloride, $\mathrm{CrCl}_{3} \ldots \ldots \ldots$.

Chromium chloride, $\mathrm{CrCl}_{3} \ldots \ldots \ldots .6$

Chromium cobalt niobium, $\mathrm{CoC} r \mathrm{Nb}$...

Chromium cobalt silicide,

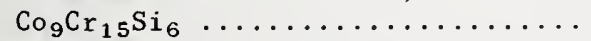

Chromium cobalt tantalum, CoCrTa ..

Chromium fluoride, $\mathrm{CrF}_{2} \ldots \ldots \ldots \ldots$

Chromium fluoride, $\mathrm{Cr}_{2} \mathrm{~F}_{5} \ldots \ldots \ldots$

Chromium(III) fluoride hydrate,

$\mathrm{CrF}_{3} \cdot 3 \mathrm{H}_{2} \mathrm{O} \ldots \ldots \ldots \ldots \ldots \ldots \ldots$

Chromium iridium, $\mathrm{Cr}_{3} \mathrm{Ir} \ldots \ldots \ldots$

Chromium iron oxide,

$\mathrm{Cr}_{1.3} \mathrm{Fe}_{0.7} \mathrm{O}_{3} \ldots \ldots \ldots \ldots \ldots \ldots$

Chromium niobium oxide, $\mathrm{CrNbO}_{4} \ldots$

Chromium oxide, $\mathrm{Cr}_{3} \ldots \ldots \ldots \ldots$

Chromium (III) oxide, $\mathrm{Cr}_{2} \mathrm{O}_{3} \ldots \ldots \ldots$

Chromium phosphate, $\alpha-\mathrm{CrPO}_{4} \ldots \ldots$.

Chromium phosphate, $\beta-\mathrm{CrPO}_{4} \ldots \ldots$

Chromium phosphate hydrate,

$\mathrm{CrPO}_{4} \cdot 6 \mathrm{H}_{2} \mathrm{O}$

$18 \mathrm{~m} \quad 29$

$10 \mathrm{~m} \quad 21$

$7 \mathrm{~m} \quad 20$

$7 \mathrm{~m} \quad 22$

$19 \mathrm{~m} \quad 35$

$6 \mathrm{~m} \quad 12$

$7 \mathrm{~m} \quad 23$

925

$2 \mathrm{~m} \quad 10$

$2 \mathrm{~m} \quad 11$

$8 \quad 19$

$5 \quad 14$

$6 \quad 27$

820

519

$6 \mathrm{~m} \quad 13$

$7 \quad 17$

924

516

$1 \mathrm{~m}$

11

$7 m$

25

$20 \mathrm{~m}$

41

520

$17 \mathrm{~m} \quad 22$

$19 \mathrm{~m} \quad 37$

$18 \mathrm{~m} \quad 30$

$11 \mathrm{~m} \quad 77$

$17 \mathrm{~m} \quad 23$

$16 \mathrm{~m} \quad 31$

$15 \mathrm{~m} \quad 140$

$14 \mathrm{~m} \quad 62$

$15 \mathrm{~m} \quad 142$

$10 \mathrm{~m} \quad 81$

$7 \mathrm{~m} \quad 108$

$5 \mathrm{~m} \quad 25$

$6 \mathrm{~m} \quad 14$

$17 \mathrm{~m} \quad 24$

$19 \mathrm{~m} \quad 38$

$17 \mathrm{~m} \quad 25$

$5 \quad 22$

$2 \mathrm{~m} \quad 12$

$9 \quad 26$

$15 \mathrm{~m}$
Chromium silicide, $\mathrm{Cr}_{3} \mathrm{Si} \ldots \ldots \ldots$.
Chromium rhodium, $\mathrm{Cr}_{3} \mathrm{Rh}_{2} \ldots \ldots \ldots \ldots$

Chromium sulfate, $\mathrm{Cr}_{2}\left(\mathrm{SO}_{4}\right)_{3} \ldots \ldots$

Chromium tungsten oxide, $\mathrm{CrWO}_{4} \ldots$

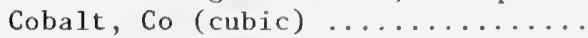

Cobalt aluminum oxide, $\mathrm{CoAl}_{2} \mathrm{O}_{4} \ldots$

Cobalt ammine iodide, $\mathrm{Co}\left(\mathrm{NH}_{3}\right)_{6} \mathrm{I}_{3} \ldots$

Cobalt antimony oxide, $\mathrm{CoSb}_{2} \mathrm{O}_{6} \ldots$

Cobalt arsenate hydrate (erythrite),

$\mathrm{Co}_{3}\left(\mathrm{AsO}_{4}\right)_{2} \cdot 8 \mathrm{H}_{2} \mathrm{O} \ldots \ldots \ldots \ldots \ldots$

Cobalt arsenide, $\mathrm{CoAs}_{2} \ldots \ldots \ldots \ldots$

Cobalt arsenide (skutterudite),

$\mathrm{CoAs}_{3} \ldots \ldots \ldots \ldots \ldots \ldots \ldots \ldots$

Cobalt borate, $\mathrm{Co}_{3}\left(\mathrm{BO}_{3}\right)_{2} \ldots \ldots \ldots$

Cobalt bromide hydrate, $\mathrm{CoBr}_{2} \cdot 6 \mathrm{H}_{2} \mathrm{O}$
Cobalt(II) carbonate (sphaero-

Sec. Page

$6 \mathrm{~m} \quad 15$

629

$16 \mathrm{~m} \quad 33$

$20 \mathrm{~m} \quad 43$

$4 \mathrm{~m} \quad 10$

$\begin{array}{rr}9 & 27 \\ 10 \mathrm{~m} & 83\end{array}$

$5 \mathrm{~m} \quad 26$

$19 \mathrm{~m} \quad 39$

$4 \mathrm{~m} \quad 10$

10

21

$12 \mathrm{~m}$

$12 \mathrm{~m}$

cobaltite), $\mathrm{CoCO}_{3} \ldots \ldots \ldots \ldots \ldots \ldots$

$10 \quad 24$

Cobalt chlorate hydrate,

$\mathrm{Co}\left(\mathrm{ClO}_{4}\right)_{2} \cdot 6 \mathrm{H}_{2} \mathrm{O} \ldots \ldots \ldots \ldots \ldots . . . . .3 \mathrm{~m}$

Cobalt chloride hydrate, $\mathrm{CoCl}_{2} \cdot 2 \mathrm{H}_{2} \mathrm{O} \quad 11 \mathrm{~m}$

Cobalt chloride hydrate, $\mathrm{CoCl}_{2} \cdot 6 \mathrm{H}_{2} \mathrm{O} \quad 1 \mathrm{Im}$

Cobalt chromium oxide, $\mathrm{CoCr}_{2} \mathrm{O}_{4} \ldots .99 \mathrm{~m}$

Cobalt copper tin, $\mathrm{CoCu}_{2} \mathrm{Sn} \ldots \ldots \ldots \quad 14 \mathrm{~m}$

Cobalt dysprosium, $\mathrm{Co}_{2} \mathrm{Dy} \ldots \ldots \ldots \ldots \quad 13 \mathrm{~m}$

Cobalt erbium, $\mathrm{Co}_{2} \mathrm{Er} \ldots \ldots \ldots \ldots \ldots \quad 13 \mathrm{~m}$

Cobalt erbium, $\mathrm{Co}_{7} \mathrm{Er}_{2} \ldots \ldots \ldots \ldots . . \ldots 13 \mathrm{~m}$

Cobalt fluoride, $\mathrm{CoF}_{2} \ldots \ldots \ldots \ldots$ 18m

Cobalt fluoride, $\mathrm{CoF}_{2}$ (calculated

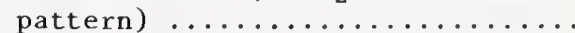

Cobalt fluoride hydrate, $\mathrm{CoF}_{2} \cdot 4 \mathrm{H}_{2} \mathrm{O}$

Cobalt gadolinium, $\mathrm{CoGd}_{3} \ldots . . . .$.

Cobalt gadolinium, $\mathrm{Co}_{2} \mathrm{Gd} \ldots \ldots \ldots$.

Cobalt gadolinium, $\mathrm{Co}_{7} \mathrm{Gd}_{2} \ldots \ldots \ldots$

Cobalt gallium hafnium, $\mathrm{Co}_{2} \mathrm{GaHf}$...

Cobalt gallium manganese, $\mathrm{Co}_{2} \mathrm{GaMn}$

Cobalt gallium niobium,

$\mathrm{Co}_{1,5} \mathrm{Ga}_{0,5} \mathrm{Nb} \ldots \ldots \ldots \ldots \ldots \ldots$

Cobalt gailium niobium, $\mathrm{CO}_{2} \mathrm{GaNb} \ldots$

Cobalt gallium oxide, $\mathrm{CoGa}_{2} \mathrm{O}_{4} \ldots \ldots$

Cobalt gallium tantalum,

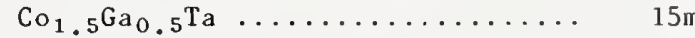

Cobait gailium tantalum, $\mathrm{Co}_{2} \mathrm{GaTa}$

Cobalt gallium titanium, $\mathrm{Co}_{2} \mathrm{GaTi}$.. 13m

Cobalt gallium vanadium, $\mathrm{Co}_{2} \mathrm{GaV} \ldots 13 \mathrm{~m}$

Cobalt germanium, $\mathrm{Co}_{3} \mathrm{Ge}_{2} \ldots \ldots \ldots .14 \mathrm{~m}$

Cobalt germanium, $\mathrm{Co}_{5} \mathrm{Ge}_{7} \ldots \ldots \ldots .15 \mathrm{~m}$

Cobalt germanium hafnium,

$\mathrm{Co}_{16} \mathrm{Ge}_{7} \mathrm{Hf}_{6} \ldots \ldots \ldots \ldots \ldots \ldots \ldots \ldots \ldots$

Cobalt germanium manganese,

$\mathrm{Co}_{2} \mathrm{GeMn} \ldots \ldots \ldots \ldots \ldots \ldots \ldots \ldots \ldots \ldots \ldots$

Cobalt germanium niobium,

$\mathrm{Co}_{1}{ }_{5} \mathrm{Ge}_{0.5} \mathrm{Nb} \ldots \ldots \ldots \ldots$. . .

$\mathrm{Co}_{16} \mathrm{Ge}_{7} \mathrm{Nb}_{6} \ldots \ldots \ldots \ldots \ldots \ldots \ldots$

$15 \mathrm{~m}$

Cobalt germanium oxide, $\mathrm{Co}_{2} \mathrm{GeO}_{4} \ldots$

$14 \mathrm{~m}$

Cobalt germanium tantalum,

$\mathrm{Co}_{1.5} \mathrm{Ge}_{0.5} \mathrm{Ta} \ldots \ldots \ldots \ldots \ldots \ldots \ldots \ldots \ldots$ 15m

Cobalt germanium tantalum,

$\mathrm{Co}_{16} \mathrm{Ge}_{7} \mathrm{Ta}_{6} \ldots \ldots \ldots \ldots \ldots \ldots \ldots \ldots \ldots$

Cobalt germanium titanium, $\mathrm{Co}_{2} \mathrm{GeTi} 13 \mathrm{~m}$

Cobalt hafnium tin, $\mathrm{Co}_{2} \mathrm{HfSn} \ldots . . .14 \mathrm{~m}$

Cobalt holmium, $\mathrm{Co}_{2} \mathrm{Ho} \ldots \ldots \ldots \ldots .14 \mathrm{~m}$

Cobalt holmium, $\mathrm{Co}_{9}{ }_{2} \mathrm{Ho}_{12} \ldots \ldots \ldots \ldots$. $15 \mathrm{~m}$

Cobalt hydroxide, $\beta-\mathrm{Co}(\mathrm{OH})_{2} \ldots \ldots . \quad 15 \mathrm{~m}$

Cobalt indium, $\mathrm{CoIn}_{3} \ldots \ldots \ldots \ldots \ldots$ 13m

Cobalt iodide, $\mathrm{CoI}_{2} \ldots \ldots \ldots \ldots .4 \mathrm{~m}$

Cobalt iron arsenide

(safflorite), $\mathrm{CoFeAs}_{4} \ldots \ldots \ldots \ldots$

10
150

152

28

23

21

64

63

64

65

85

24

68

71

72

65

45

144

27

146

76

77

67

148

69

71

27

73

80

75

76

154

29

81

52

28 
Cobalt iron oxide, $\mathrm{CoFe}_{2} \mathrm{O}_{4} \ldots \ldots \ldots$

Cobalt iron sulfide, $\mathrm{Co}_{8} \mathrm{FeS}_{8} \ldots \ldots$.

$9 \mathrm{~m} \quad 22$

Cobalt iron vanadium,

$\mathrm{Co}_{4.35} \mathrm{Fe}_{13.4{ }_{7} \mathrm{~V}_{12.18} \ldots \ldots \ldots \ldots \ldots}$

Cobalt lanthanum, $\mathrm{CoLa}_{3} \ldots \ldots \ldots \ldots$

Cobalt lutetium, $\mathrm{Co}_{2} \mathrm{Lu} \ldots \ldots \ldots \ldots$.

Cobalt magnesium, $\mathrm{Co}_{2} \mathrm{Mg} \ldots \ldots \ldots \ldots$

Cobalt manganese silicide, $\mathrm{Co}_{2} \mathrm{MnSi}$

Cobalt mercury thiocyanate,

$\mathrm{Co}\left[\mathrm{Hg}(\mathrm{CNS})_{4}\right] \ldots \ldots \ldots \ldots \ldots \ldots$

Cobalt molybdenum, $\mathrm{Co}_{2} \mathrm{Mo} \ldots \ldots \ldots$.

Cobalt molybdenum, $\mathrm{Co}_{2} \mathrm{Mo}_{3} \ldots \ldots \ldots$

Cobalt molybdenum, $\mathrm{Co}_{7} \mathrm{Mo}_{6} \ldots \ldots \ldots$

Cobalt molybdenum silicide,

$\mathrm{Co}_{3} \mathrm{Mo}_{2} \mathrm{Si} \ldots \ldots \ldots \ldots \ldots \ldots \ldots$

Cobalt neodymium, $\mathrm{Co}_{2} \mathrm{Nd} \ldots \ldots \ldots$

Cobalt nickel tin,

Co ${ }_{75} \mathrm{Ni},{ }_{75} \mathrm{Sn} .75 \ldots \ldots \ldots \ldots$

Cobalt niobium silicide, $\mathrm{Co}_{3} \mathrm{Nb}_{4} \mathrm{Si}_{7}$

Cobalt niobium tin, $\mathrm{Co}_{2} \mathrm{NbSn} \ldots . .$.

Cobalt nitrate hydrate,

$\alpha-\mathrm{Co}\left(\mathrm{NO}_{3}\right)_{2} \cdot 6 \mathrm{H}_{2} \mathrm{O} \ldots \ldots \ldots \ldots \ldots$

Cobalt(II) oxide, $\mathrm{CoO} \ldots \ldots \ldots \ldots$

Cobalt(II, III) oxide, $\mathrm{Co}_{3} \mathrm{O}_{4} \ldots \ldots$.

Cobalt phosphate, $\mathrm{Co}\left(\mathrm{PO}_{3}\right)_{2} \ldots \ldots$.

Cobalt phosphate hydrate,

$\mathrm{Co}_{3}\left(\mathrm{PO}_{4}\right)_{2} \cdot 8 \mathrm{H}_{2} \mathrm{O} \ldots \ldots \ldots \ldots \ldots$

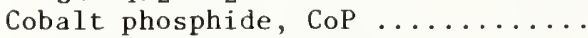

Cobalt phosphide, $\mathrm{CoP}_{3} \ldots \ldots \ldots \ldots$

Cobalt phosphide, $\mathrm{Co}_{2} \mathrm{P} \ldots \ldots \ldots \ldots$

Cobalt platinum, CoPt (disordered)

Cobalt platinum, CoPt (ordered)...

Cobalt platinum, $\mathrm{CoPt}_{3}$

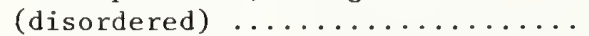

Cobalt platinum, CoPt $_{3}$ (ordered)..

Cobalt plutonium, $\mathrm{CoPu}_{2} \ldots \ldots \ldots \ldots$

Cobalt plutonium, $\mathrm{CoPu}_{3} \ldots \ldots \ldots \ldots$

Cobalt plutonium, $\mathrm{CoPu}_{6} \ldots \ldots \ldots \ldots$

Cobalt plutonium, $\mathrm{Co}_{2} \mathrm{Pu} \ldots \ldots \ldots \ldots$

Cobalt plutonium, $\mathrm{Co}_{3} \mathrm{Pu} \ldots \ldots \ldots \ldots$

Cobalt plutonium, $\mathrm{Co}_{17} \mathrm{Pu}_{2} \ldots \ldots \ldots$

Cobalt praseodymium, $\mathrm{Co}_{2} \mathrm{Pr} \ldots \ldots \ldots$

Cobalt rhodium sulfide, $\mathrm{Co}_{8} \mathrm{RhS}_{8} \ldots$

Cobalt ruthenium sulfide, $\mathrm{Cc}_{8} \mathrm{RuS}_{8}$.

Cobalt samarium, $\mathrm{Co}_{2} \mathrm{Sm} \ldots \ldots \ldots \ldots$

Cobalt samarium, $\mathrm{Co}_{5} \mathrm{Sm} \ldots \ldots \ldots \ldots$

Cobalt silicate, $\mathrm{Co}_{2} \mathrm{SiO}_{4}$

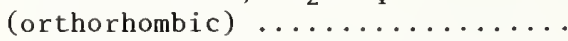

Cobalt silicon fluoride hydrate,

$\mathrm{CoSiF}_{6} \cdot 6 \mathrm{H}_{2} \mathrm{O} \ldots \ldots \ldots \ldots \ldots \ldots \ldots$

Cobalt sulfate, $\beta-\mathrm{CoSO}_{4} \ldots \ldots \ldots \ldots$

Cobalt tantalum silicide,

$\mathrm{Co}_{16} \mathrm{Ta}_{6} \mathrm{Si}_{7} \ldots \ldots \ldots \ldots \ldots \ldots \ldots$

Cobalt telluride, CoTe ..........

Cobalt thorium, $\mathrm{Co}_{17} \mathrm{Th}_{2} \ldots \ldots \ldots \ldots$

Cobalt tin, $\mathrm{Co}_{3} \mathrm{Sn}_{2} \ldots \ldots \ldots \ldots \ldots$

Cobalt tin oxide, $\mathrm{Co}_{2} \mathrm{SnO}_{4} \ldots \ldots \ldots$

Cobalt tin vanadium, $\mathrm{Co}_{2} \mathrm{SnV} \ldots \ldots$.

Cobalt tin zirconium, $\mathrm{Co}_{2} \mathrm{SnZr} \ldots \ldots$

Cobalt titanium oxide, $\mathrm{CoTiO}_{3} \ldots$.

Cobalt titanium silicide,

$\mathrm{Co}_{16} \mathrm{Ti}_{6} \mathrm{Si}_{7} \ldots \ldots \ldots \ldots \ldots \ldots \ldots$

Cobalt tungsten oxide, $\mathrm{CoWO}_{4} \ldots .$.

Cobalt vanadium silicide, $\mathrm{Co}_{2}$ VSi ..

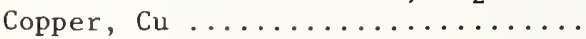

Copper ammine selenate,

$\mathrm{Cu}\left(\mathrm{NH}_{3}\right)_{4} \mathrm{SeO}$

\section{$14 \mathrm{~m}$}

$14 \mathrm{~m}$

$13 \mathrm{~m}$

$13 \mathrm{~m}$

$15 \mathrm{~m}$

79

83

86

$14 \mathrm{~m} \quad 81$

$2 \mathrm{~m}$

$14 \mathrm{~m}$

13

$15 \mathrm{~m}$

$15 \mathrm{~m}$

82

158

160

$15 \mathrm{~m}$

162

$13 \mathrm{~m}$

87

$13 \mathrm{~m}$

$15 \mathrm{~m}$

88

$15 \mathrm{~m}$

164

$12 \mathrm{~m}$

166

22

28

9

$13 \mathrm{~m}$

29

$19 \mathrm{~m}$

$14 \mathrm{~m}$

$14 \mathrm{~m}$

$18 \mathrm{~m}$

$15 \mathrm{~m}$

$15 \mathrm{~m}$

$15 \mathrm{~m}$

$15 \mathrm{~m}$

$14 \mathrm{~m}$

$15 \mathrm{~m}$

$14 \mathrm{~m}$

$14 \mathrm{~m}$

$14 \mathrm{~m}$

$14 \mathrm{~m}$

$14 \mathrm{~m}$

$14 \mathrm{~m}$

$14 \mathrm{~m}$

$15 \mathrm{~m}$

$13 \mathrm{~m}$

$4 \mathrm{~m}$
Copper ammine sulfate hydrate,

$\mathrm{Cu}\left(\mathrm{NH}_{3}\right)_{4} \mathrm{SO}_{4} \cdot \mathrm{H}_{2} \mathrm{O} \ldots \ldots \ldots \ldots \ldots$

$10 m$
$5 m$

90

Copper antimony oxide, $\mathrm{CuSb}_{2} \mathrm{O}_{6} \ldots$

Copper arsenate (trippkeite),

$\mathrm{CuAs}_{2} \mathrm{O}_{4} \ldots \ldots \ldots \ldots \ldots \ldots \ldots \ldots$

$16 \mathrm{~m}$

120

Copper(I) bromide, $\mathrm{CuBr} \ldots . . .$.
Copper(I) chloride (nantokite),

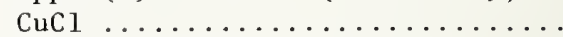

Copper chloride hydrate

(eriochalcite), $\mathrm{CuCl}_{2} \cdot 2 \mathrm{H}_{2} \mathrm{O}$

Copper chromium oxide, $\mathrm{CuCr}_{2} \mathrm{O}_{4} \ldots$.

Copper fluoride hydrate, $\mathrm{CuF}_{2} \cdot 2 \mathrm{H}_{2} \mathrm{O}$

Copper hydrogen phosphite hydrate,

$\mathrm{CuHPO}_{3} \cdot 2 \mathrm{H}_{2} \mathrm{O} \ldots \ldots \ldots \ldots \ldots \ldots$

Copper hydroxide carbonate,

azurite, $\mathrm{Cu}_{3}(\mathrm{OH})_{2}\left(\mathrm{CO}_{3}\right)_{2} \ldots \ldots \ldots$

Copper hydroxide carbonate

(malachite), $\mathrm{Cu}_{2}(\mathrm{OH})_{2} \mathrm{CO}_{3}$

Copper hydroxide phosphate

(libethenite), $\mathrm{Cu}_{2}(\mathrm{OH}) \mathrm{PO}_{4} \ldots \ldots \ldots$

Copper(I) iodide (marshite), CuI ..

Copper iron oxide, $\mathrm{CuFe}_{2} \mathrm{O}_{4} \ldots \ldots \ldots$

Copper lead hydroxide sulfate,

linarite, $\mathrm{CuPb}(\mathrm{OH})_{2}\left(\mathrm{SO}_{4}\right) \ldots \ldots \ldots$

Copper mercury iodide, $\beta-\mathrm{Cu}_{2} \mathrm{HgI}_{4} \ldots$

Copper(I) oxide (cuprite), $\mathrm{Cu}_{2} \mathrm{O} \ldots$

Copper(II) oxide (tenorite), CuO ..

Copper phosphate, $\mathrm{Cu}\left(\mathrm{PO}_{3}\right)_{2} \ldots \ldots \ldots$

Copper phosphate, $\alpha-\mathrm{Cu}_{2} \mathrm{P}_{2} \mathrm{O}_{7} \ldots \ldots$.

Copper sulfate (chalcocyanite),

$\mathrm{CuSO}_{4} \ldots \ldots \ldots \ldots \ldots \ldots \ldots \ldots$

Copper(II) sulfide (covellite), CuS

Copper uranium oxide, $\mathrm{CuUO}_{4} \ldots \ldots$.

Dichlorotetraaquochromium (III)

chloride dihydrate,

$\left[\mathrm{Cr}\left(\mathrm{H}_{2} \mathrm{O}\right)_{4} \mathrm{Cl}_{2}\right] \mathrm{Cl} \cdot 2 \mathrm{H}_{2} \mathrm{O} \ldots \ldots \ldots$

Dysprosium arsenate, DyAsO $_{4} \ldots \ldots \ldots$

Dysprosium arsenide, DyAs .......

Dysprosium gallium oxide,

$\mathrm{Dy}_{3} \mathrm{Ga}_{5} \mathrm{O}_{12} \ldots \ldots \ldots \ldots \ldots \ldots \ldots$

Dysprosium gold, DyAu ..........

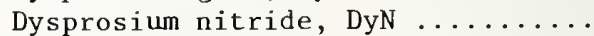

Dysprosium oxide, $\mathrm{Dy}_{2} \mathrm{O}_{3} \ldots \ldots \ldots \ldots$

Dysprosium silver, DyAg .........

Dysprosium telluride, DyTe ......

Dysprosium vanadium oxide, $\mathrm{DyVO}_{4} \ldots$

Erbium arsenate, $\mathrm{ErAsO}_{4} \ldots \ldots \ldots \ldots$

Erbium arsenide, ErAs ..........

Erbium gallium oxide, $\mathrm{Er}_{3} \mathrm{Ga}_{5} \mathrm{O}_{12} \ldots$

Erbium iron, $\mathrm{ErFe}_{2} \ldots \ldots \ldots \ldots \ldots$

Erbium manganese oxide, $\mathrm{ErMnO}_{3} \ldots$

Erbium nitride, $\operatorname{ErN} \ldots \ldots \ldots \ldots \ldots$

Erbium oxide, $\operatorname{Er}_{2} \mathrm{O}_{3} \ldots \ldots \ldots \ldots \ldots$

Erbium phosphate, $\mathrm{ErPO}_{4} \ldots \ldots \ldots \ldots$

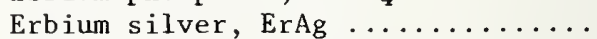

Erbium telluride, ErTe ..........

Erbium vanadium oxide, $\mathrm{ErVO}_{4} \ldots \ldots$.

Europium arsenate, $\mathrm{EuAs}_{4} \ldots \ldots \ldots$

Europium(III) chloride, $\mathrm{EuCl}_{3} \ldots \ldots$

Europium chloride oxide, EuCl0 ....

Europium gallium oxide,

$\mathrm{Eu}_{3} \mathrm{Ga}_{5} \mathrm{O}_{12} \ldots \ldots \ldots \ldots \ldots \ldots \ldots \ldots$

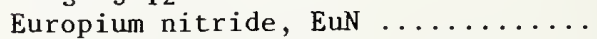

Europium oxide, EuO ............

Europium oxide, $\mathrm{Eu}_{2} \mathrm{O}_{3}$ (cubic) .....

Europium oxide, $\mathrm{Eu}_{2} \mathrm{O}_{3}$ (monoclinic).

Europium phosphate, $\mathrm{EuPO}_{4} \ldots \ldots \ldots$.

$4 \quad 36$

$\begin{array}{rr}3 m & 29 \\ 4 & 13 \\ 10 m & 93\end{array}$

$16 \mathrm{~m}$ 
VoI . or

Sec. Page

Europium(III) vanadium oxide, EuVO Gadolinium arsenate, $\mathrm{GdAsO}_{4} \ldots \ldots$. Gadolinium arsenide, GdAs ......... Gadolinium chloride hydrate,

$\mathrm{GdCl}_{3} \cdot 6 \mathrm{H}_{2} \mathrm{O} \ldots \ldots \ldots \ldots \ldots \ldots \ldots$ Gadolinium chloride oxide, GdClo . .

Gadolinium fluoride, $\mathrm{GdF}_{3} \ldots \ldots \ldots$ Gadolinium gallium oxide,

$\mathrm{Gd}_{3} \mathrm{Ga}_{5} \mathrm{O}_{12} \ldots \ldots \ldots \ldots \ldots \ldots \ldots$

Gadolinium indium, GdIn ..........

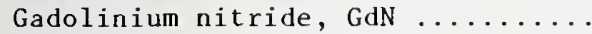

Gadolinium oxide, $\mathrm{Gd}_{2} \mathrm{O}_{3} \ldots \ldots \ldots \ldots$

Gadolinium silver, $\mathrm{GdAg} \ldots \ldots \ldots \ldots$

Gadolinium titanium oxide, $\mathrm{Gd}_{2} \mathrm{TiO}_{5}$

Gadolinium vanadium_oxide, $\mathrm{GdVO}_{4} \ldots$

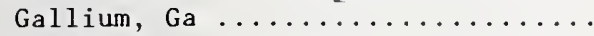

Gallium arsenide, GaAs ..........

Gallium lutetium oxide, $\mathrm{Ga}_{5} \mathrm{Lu}_{3} \mathrm{O}_{12}$

Gallium magnesium, $\mathrm{Ga}_{2} \mathrm{Mg} \ldots \ldots \ldots$

Gallium magnesium, $\mathrm{Ga}_{5} \mathrm{Mg}_{2} \ldots \ldots \ldots$

Gallium neodymium oxide, $\mathrm{Ga}_{5} \mathrm{Nd}_{3} \mathrm{O}_{12}$

Gallium oxide, $\alpha-\mathrm{Ga}_{2} \mathrm{O}_{3} \ldots \ldots \ldots \ldots$

Gallium phosphate ( $\alpha$-quartz type),

$\mathrm{GaPO}_{4} \ldots \ldots \ldots \ldots \ldots \ldots \ldots \ldots$

Gallium phosphate hydrate,

$\mathrm{GaPO}_{4} \cdot 2 \mathrm{H}_{2} \mathrm{O} \ldots \ldots \ldots \ldots \ldots \ldots \ldots$

Gallium samarium oxide, $\mathrm{Ga}_{5} \mathrm{Sm}_{3} \mathrm{O}_{12}$

Gallium ytterbium oxide, $\mathrm{Ga}_{5} \mathrm{Yb}_{3} \mathrm{O}_{12}$

Gallium yttrium oxide, $\mathrm{Ga}_{5} \mathrm{Y}_{3} \mathrm{O}_{12} \ldots$

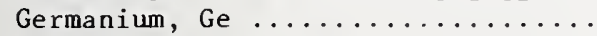

Germanium iodide, $\mathrm{GeI}_{2} \ldots \ldots \ldots \ldots$

Germanium(IV) iodide, $\mathrm{GeI}_{4} \ldots \ldots \ldots$

Germanium oxide, $\mathrm{GeO}_{2}$ (hexagonal)

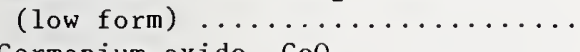

Germanium oxide, $\mathrm{GeO}_{2}$

(tetragonal) (high form) ........

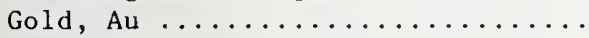

Gold chloride, AuCl ............

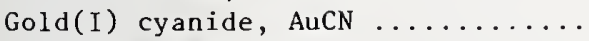

Gold holmium, AuHo .............

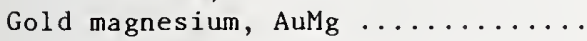

Gold niobium, $\mathrm{AuNb}_{3} \ldots \ldots \ldots \ldots \ldots$

Gold potassium cyanide, $\operatorname{AuK}(\mathrm{CN})_{2} \ldots$

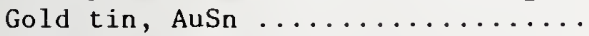

Gold titanium, $\operatorname{AuTi}_{3} \ldots \ldots \ldots \ldots \ldots$

Gold vanadium, $\mathrm{AuV}_{3} \ldots \ldots \ldots \ldots \ldots$

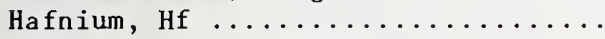

Hafnium nitride, $\mathrm{HfN} \ldots \ldots \ldots \ldots \ldots$

Hafnium oxide, $\mathrm{HfO}_{2} \ldots \ldots \ldots \ldots \ldots$

Holmium arsenate, $\mathrm{HoAsO}_{4} \ldots \ldots \ldots \ldots$

Holmium fluoride, $\mathrm{HoF}_{3} \ldots \ldots \ldots \ldots$

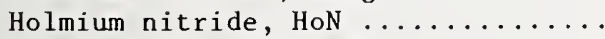

Holmium oxide, $\mathrm{Ho}_{2} \mathrm{O}_{3} \ldots \ldots \ldots \ldots \ldots$

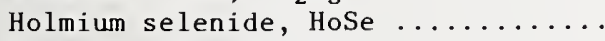

Holmium silver, HoAg ............

Holmium vanadium oxide, $\mathrm{HoVO}_{4} \ldots \ldots$.

Hydrazinium sulfate, $\left(\mathrm{NH}_{3}\right)_{2} \mathrm{SO}_{4} \ldots \ldots$

Hydrogen amidosulfate, $\mathrm{H}_{2} \mathrm{NSO}_{3} \mathrm{H} \ldots \ldots$

Hydrogen a rsenate, $\mathrm{H}_{5} \mathrm{As}_{3} \mathrm{O}_{10} \ldots \ldots \ldots$

Hydrogen borate, $\beta-\mathrm{HBO}_{2}$ (monoclinic)

Hydrogen borate (metaborite),

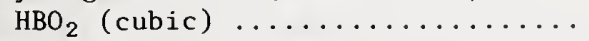

Hydrogen iodate, $\mathrm{HIO}_{3} \ldots \ldots \ldots \ldots$

Hydrogen iodate, $\mathrm{HI}_{3} \mathrm{O}_{8} \ldots \ldots \ldots \ldots$

Hydrogen phosphate hydrate,

$\mathrm{H}_{3} \mathrm{PO}_{4} \cdot 0.5 \mathrm{H}_{2} \mathrm{O} \ldots \ldots \ldots \ldots \ldots \ldots \ldots$

Hydrogen tellurate, $\mathrm{H}_{6} \mathrm{TeO}_{6} \ldots \ldots \ldots$

$\begin{array}{rr}4 \mathrm{~m} & 16 \\ 4 \mathrm{~m} & 17 \\ 4 \mathrm{~m} & 57 \\ & \\ 7 \mathrm{~m} & 118 \\ 1 \mathrm{~m} & 17 \\ 1 \mathrm{~m} & 14 \\ & \\ 2 \mathrm{~m} & 18 \\ 5 \mathrm{~m} & 67 \\ 4 \mathrm{~m} & 57 \\ 1 \mathrm{~m} & 16 \\ 6 \mathrm{~m} & 87 \\ 8 \mathrm{~m} & 32 \\ 5 \mathrm{~m} & 30 \\ 2 & 9 \\ 3 \mathrm{~m} & 33 \\ 2 \mathrm{~m} & 22 \\ 12 \mathrm{~m} & 48 \\ 12 \mathrm{~m} & 51 \\ 1 \mathrm{~m} & 34 \\ 4 & 25\end{array}$

Indium, In $\ldots \ldots \ldots \ldots \ldots \ldots \ldots \ldots$

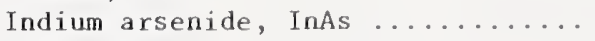

Indium oxide, $\operatorname{In}_{2} \mathrm{O}_{3} \ldots \ldots \ldots \ldots \ldots$

Indium sulfide, $\operatorname{In}_{2} \mathrm{~S}_{3}$

Iodine, $\mathrm{I}_{2} \ldots \ldots \ldots \ldots \ldots \ldots \ldots$

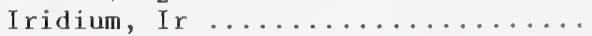

Iridium niobium, $\operatorname{IrNb}_{3} \ldots \ldots \ldots \ldots$

Iridium oxide, $\operatorname{IrO}_{2} \ldots \ldots \ldots \ldots$

Iridium titanium, $\operatorname{IrTi}_{3} \ldots \ldots \ldots \ldots$

Iridium vanadium, $\operatorname{Irv}_{3} \ldots \ldots \ldots \ldots$

Iron aluminum oxide (hercynite),

$\mathrm{FeAl}_{2} \mathrm{O}_{4} \ldots \ldots \ldots \ldots \ldots \ldots$

Iron antimony oxide, $\mathrm{FeSbO}_{4} \ldots \ldots$.

Iron arsenide, FeAs ............

Iron arsenide (loellingite), $\mathrm{FeAs}_{2}$

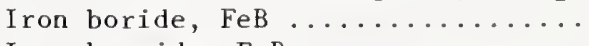

Iron bromide, $\mathrm{FeBr}_{2} \ldots \ldots \ldots \ldots \ldots$

Iron carbonate, siderite, $\mathrm{FeCO}_{3} \ldots$

Iron chloride hydrate (rokuhnite),

$\mathrm{FeCl}_{2} \cdot 2 \mathrm{H}_{2} \mathrm{O} \ldots \ldots \ldots \ldots \ldots \ldots \ldots \ldots . \ldots \ldots, 1 \mathrm{~m}$

Iron chloride hydrate (hydromolysite),

$\mathrm{FeCl}_{3} \cdot 6 \mathrm{H}_{2} \mathrm{O} \ldots \ldots \ldots \ldots \ldots \ldots \ldots \ldots, 17 \mathrm{~m}$

Iron chromium oxide (chromite),

$\mathrm{FeCr}_{2} \mathrm{O}_{4} \ldots \ldots \ldots \ldots \ldots \ldots \ldots \ldots \ldots \ldots$ 19m

Iron fluoride hydrate, $\mathrm{FeF}_{2} \cdot 4 \mathrm{H}_{2} \mathrm{O}$

Iron fluoride, $\mathrm{FeF}_{3} \ldots \ldots \ldots \ldots \ldots$

Iron fluoride hydrate, $\beta-\mathrm{FeF}_{3} \cdot 3 \mathrm{H}_{2} \mathrm{O}$

Iron hydroxide sulfate hydrate,

butlerite, $\mathrm{Fe}(\mathrm{OH}) \mathrm{SO}_{4} \cdot 2 \mathrm{H}_{2} \mathrm{O} \ldots \ldots \ldots$

Iron iodide, $\mathrm{FeI}_{2} \ldots \ldots \ldots \ldots \ldots$

Iron niobium oxide, $\mathrm{Fe}\left(\mathrm{NbO}_{3}\right)_{2} \ldots \ldots$

Iron niobium oxide, $\mathrm{Fe}_{4} \mathrm{Nb}_{2} \mathrm{O}_{9} \ldots \ldots$

Iron oxide (hematite), $\alpha-\mathrm{Fe}_{2} \mathrm{O}_{3} \ldots$

Iron(II, III) oxide (magnetite),

$\mathrm{Fe}_{3} \mathrm{O}_{4} \ldots \ldots \ldots \ldots \ldots \ldots \ldots \ldots \ldots$

Iron phosphate, $\mathrm{FePO}_{4} \ldots \ldots \ldots \ldots$

Iron phosphate hydrate (vivianite),

$\mathrm{Fe}_{3}\left(\mathrm{PO}_{4}\right)_{2} \cdot 8 \mathrm{H}_{2} \mathrm{O} \ldots \ldots \ldots \ldots \ldots$

Iron silicate (fayalite), $\mathrm{Fe}_{2} \mathrm{SiO}_{4}$.

Iron sulfate, $\mathrm{Fe}_{2}\left(\mathrm{SO}_{4}\right)_{3} \ldots \ldots \ldots \ldots$

Iron sulfate hydrate (melanterite),

$\mathrm{FeSO}_{4} \cdot 7 \mathrm{H}_{2} \mathrm{O} \ldots \ldots \ldots \ldots \ldots \ldots$

Iron sulfide (pyrite), $\mathrm{FeS}_{2} \ldots \ldots$

Iron thorium, $\mathrm{Fe}_{17} \mathrm{Th}_{2} \ldots \ldots \ldots \ldots$

Iron titanium oxide (ilmenite),

$\mathrm{FeTiO}_{3} \ldots \ldots \ldots \ldots \ldots \ldots \ldots \ldots \ldots \ldots$

Iron titanium oxide (ulvöspinel),

$\mathrm{Fe}_{2} \mathrm{TiO}_{4} \ldots \ldots \ldots \ldots \ldots \ldots \ldots \ldots$

Iron yttrium oxide, $\mathrm{Fe}_{5} \mathrm{Y}_{3} \mathrm{O}_{12} \ldots \ldots$

Lanthanum arsenate, $\mathrm{LaAsO}_{4} \ldots \ldots \ldots$

Lanthanum arsenide, LaAs ..........

Lanthanum borate, $\mathrm{LaBO}_{3} \ldots \ldots \ldots$

Lanthanum boride, $\mathrm{LaB}_{6} \ldots \ldots \ldots \ldots$

Lanthanum chloride, $\mathrm{LaCl}_{3} \ldots \ldots \ldots$

Lanthanum chloride oxide, LaClo ...

Lanthanum fluoride, $\mathrm{LaF}_{3} \ldots \ldots \ldots$

Lanthanum magnesium, $\mathrm{LaMg} \ldots \ldots \ldots$

Lanthanum nickel platinum,

$\mathrm{LaNi}_{0.25} \mathrm{Pt}_{4.75} \ldots \ldots \ldots \ldots \ldots \ldots$

Lanthanum niobium titanium oxide,

$\mathrm{LaNbTiO}_{6} \ldots \ldots \ldots \ldots \ldots \ldots . \ldots . . . .$.

Lanthanum nitrate hydrate,

$\mathrm{La}\left(\mathrm{NO}_{3}\right)_{3} \cdot 6 \mathrm{H}_{2} \mathrm{O} \ldots \ldots \ldots \ldots \ldots \ldots$

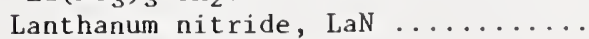

Lanthanum oxide, $\mathrm{La}_{2} \mathrm{O}_{3} \ldots \ldots \ldots \ldots$
Sec. Page

$\begin{array}{rr}3 & 12 \\ 3 \mathrm{~m} & 35 \\ 5 & 26 \\ 8 & 29 \\ 11 \mathrm{~m} & 30 \\ 3 & 16 \\ 4 & \\ 6 \mathrm{~m} & 19 \\ 4 \mathrm{~m} & 19 \\ 6 \mathrm{~m} & 2 \\ 6 \mathrm{~m} & 2 \\ 4 & \end{array}$

$19 \mathrm{~m} \quad 48$

$19 \mathrm{~m} \quad 49$

$1 \mathrm{~m} \quad 19$

$10 \quad 34$

$18 \mathrm{~m} \quad 35$

$4 \mathrm{~m} \quad 59$

$15 \mathrm{~m} \quad 32$

40

50

$11 \mathrm{~m} \quad 90$

$18 \mathrm{~m} \quad 36$

$17 \mathrm{~m} \quad 41$

$10 \mathrm{~m}$

$4 m$

$20 m$

$20 \mathrm{~m}$

$18 \mathrm{~m}$

$5 \mathrm{~m}$

$15 \mathrm{~m}$

$16 \mathrm{~m}$

$20 \mathrm{~m}$

$16 \mathrm{~m}$

$8 m$

$12 \mathrm{~m}$

38

29

$15 \mathrm{~m}$

34

$20 m$

$18 \mathrm{~m}$

$3 m$

$4 m$

$1 \mathrm{~m}$

$20 m$

$1 \mathrm{~m}$

7

7
$5 m$

$17 \mathrm{~m}$ 
Lanthanum phosphide, LaP .........

Lanthanum selenide, LaSe ..........

Lanthanum titanium oxide, $\mathrm{La}_{2} \mathrm{Ti}_{2} \mathrm{O}_{7}$

Lanthanum zinc, LaZn ...........

Lead, $\mathrm{Pb} \ldots \ldots \ldots \ldots \ldots \ldots$

Lead borate, $\mathrm{PbB}_{4} \mathrm{O}_{7} \ldots \ldots \ldots \ldots \ldots$

Lead bromide, $\mathrm{PbBr}_{2} \ldots \ldots \ldots \ldots \ldots$

Lead bromide chloride, $\mathrm{PbBrCl} \ldots .$.

Lead bromide fluoride, $\mathrm{PbBrF} \ldots .$.

Lead bromide hydroxide, $\operatorname{PbBr}(\mathrm{OH}) \ldots$

Lead bromide oxide, $\mathrm{Pb}_{3} \mathrm{O}_{2} \mathrm{Br}_{2} \ldots \ldots$

Lead carbonate (cerussite), $\mathrm{PbCO}_{3}$

Lead chloride (cotunnite), $\mathrm{PbCl}_{2}$..

Lead chloride fluoride (matlockite),

$\mathrm{PbClF} \ldots \ldots \ldots \ldots \ldots \ldots \ldots$

Lead chromium oxide, $\mathrm{Pb}_{2} \mathrm{CrO}_{5} \ldots \ldots$

Lead fluoride, $\alpha-\mathrm{PbF}_{2}$

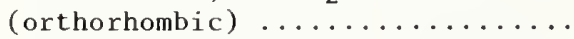

Lead fluoride, $\beta-\mathrm{PbF}_{2}$ (cubic) .....

Lead fluoride iodide, PbFI .......

Lead hydrogen arsenate (schultenite),

$\mathrm{PbHAsO}_{4} \ldots \ldots \ldots \ldots \ldots \ldots \ldots \ldots$

Lead hydrogen phosphate, $\mathrm{PbHPO}_{4} \ldots$

Lead hydroxide phosphate,

$\mathrm{Pb}_{5} \mathrm{OH}\left(\mathrm{PO}_{4}\right)_{3} \ldots \ldots \ldots \ldots \ldots \ldots \ldots$

Lead iodate, $\mathrm{Pb}\left(\mathrm{IO}_{3}\right)_{2} \ldots \ldots \ldots \ldots$

Lead(II) iodide, $\mathrm{PbI}_{2} \ldots \ldots \ldots \ldots \ldots$

Lead molybdenum oxide (wulfenite),

$\mathrm{PbMoO}_{4} \ldots \ldots \ldots \ldots \ldots \ldots \ldots \ldots$

Lead nitrate, $\mathrm{Pb}\left(\mathrm{NO}_{3}\right)_{2} \ldots \ldots \ldots \ldots$

Lead oxide (litharge), Pbo (red,

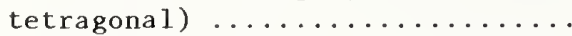

Lead oxide (massicot), Pbo (yellow,

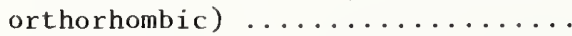

Lead(II, III) oxide (minium), $\mathrm{Pb}_{3} \mathrm{O}_{4}$

Lead oxide sulfate, $\mathrm{Pb}_{5} \mathrm{O}_{5} \mathrm{SO}_{4} \ldots \ldots$.

Lead selenide (clausthalite), PbSe

Lead strontium nitrate,

$\mathrm{Pb}{ }_{33} \mathrm{Sr}{ }_{67}\left(\mathrm{NO}_{3}\right)_{2} \ldots \ldots \ldots \ldots$

Lead strontium nitrate,

$\mathrm{Pb}{ }_{.67} \mathrm{Sr}_{.33}\left(\mathrm{NO}_{3}\right)_{2} \ldots \ldots \ldots \ldots \ldots$

Lead sulfate (anglesite), $\mathrm{PbSO}_{4} \ldots$

Lead sulfide (galena), $\mathrm{PbS} \ldots . . .$.

Lead tin oxide, $\mathrm{Pb}_{2} \mathrm{SnO}_{4} \ldots \ldots \ldots \ldots$

Lead titanium oxide (macedonite),

$\mathrm{PbTiO}_{3} \ldots \ldots \ldots \ldots \ldots \ldots \ldots$

Lead tungsten oxide (stolzite),

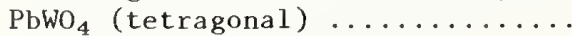

Lead uranium oxide, $\mathrm{Pb}_{3} \mathrm{UO}_{6} \ldots \ldots$.

Lithium aluminum fluoride,

$\alpha-\mathrm{Li}_{3} \mathrm{AlF}_{6} \ldots \ldots \ldots \ldots \ldots \ldots \ldots$

Lithium arsenate, $\mathrm{Li}_{3} \mathrm{AsO}_{4} \ldots \ldots \ldots$

Lithium azide, $\operatorname{LiN}_{3} \ldots \ldots \ldots \ldots$

Lithium barium fluoride, $\mathrm{LiBaF}_{3} \ldots$

Lithium beryllium fluoride, $\mathrm{Li}_{2} \mathrm{BeF}_{4}$

Lithium borate, $\mathrm{Li}_{2} \mathrm{~B}_{4} \mathrm{O}_{7} \ldots \ldots \ldots$

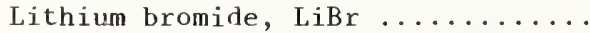

Lithium calcium aluminum boron

hydroxy silicate, liddicoatite,

$\mathrm{Ca}(\mathrm{Li}, \mathrm{Al})_{3} \mathrm{Al}_{6} \mathrm{~B}_{3} \mathrm{Si}_{6} \mathrm{O}_{27}(\mathrm{O}, \mathrm{OH})_{3}(\mathrm{OH}, \mathrm{F})$

Lithium carbonate, $\mathrm{Li}_{2} \mathrm{CO}_{3} \ldots \ldots \ldots$

Lithium chlorate hydrate,

$\mathrm{LiClO}_{4} \cdot 3 \mathrm{H}_{2} \mathrm{O} \ldots \ldots \ldots \ldots \ldots \ldots$

Lithium chloride, LiCl .........

Lithium chromium oxide hydrate,

$\mathrm{Li}_{2} \mathrm{CrO}_{4} \cdot 2 \mathrm{H}_{2} \mathrm{O} \ldots \ldots \ldots \ldots \ldots \ldots \ldots$

Lithium fluoride, LiF ...........

Lithium gallium oxide, $\mathrm{LiGaO}_{2} \ldots \ldots$

$\begin{array}{rl}5 \mathrm{~m} & 69 \\ 4 \mathrm{~m} & 61 \\ 15 \mathrm{~m} & 35 \\ 5 \mathrm{~m} & 70 \\ 1 & 34 \\ 4 \mathrm{~m} & 19 \\ 17 \mathrm{~m} & 43 \\ 11 \mathrm{~m} & 33 \\ 10 \mathrm{~m} & 25 \\ 16 \mathrm{~m} & 40 \\ 5 \mathrm{~m} & 32 \\ 2 & 56 \\ 12 \mathrm{~m} & 23\end{array}$

$13 \mathrm{~m} \quad 25$

$14 \mathrm{~m} \quad 16$

$\begin{array}{rr}5 & 31 \\ 5 & 33 \\ 10 \mathrm{~m} & 26\end{array}$

$14 \mathrm{~m} \quad 18$

$15 \mathrm{~m}$

37

$8 \quad 33$

$17 \mathrm{~m}$

5

45

7

5

2

2

8

$10 \mathrm{~m}$

5

$12 \mathrm{~m}$

$12 m$

3

2

$10 \mathrm{~m}$

5

$5 m$

$8 \mathrm{~m}$

$8 \mathrm{~m}$

$2 m$

$8 m$

$5 m$

$7 \mathrm{~m}$

126

$8 \mathrm{~m} \quad 114$

430

$16 \mathrm{~m} \quad 42$

$8 \mathrm{~m} \quad 42$

$8 \quad 34$

162

$16 \mathrm{~m}$

44

$10 \mathrm{~m}$

61
Lithium hydroxide, $\mathrm{LiOH} \ldots \ldots \ldots \ldots$

Lithium hydroxide hydrate, $\mathrm{LiOH} \cdot \mathrm{H}_{2} \mathrm{O}$

Lithium iodate, $\mathrm{LiIO}_{3}$ (hexagonal)

Lithium iodate, $\mathrm{LiIO}_{3}$ (tetragonal)

Lithium iodide hydrate, $\mathrm{LiI} \cdot 3 \mathrm{H}_{2} \mathrm{O}$.

Lithium molybdenum oxide, $\mathrm{Li}_{2} \mathrm{MoO}_{4}$

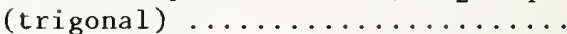

Lithium niobium oxide, $\mathrm{LiNbO}_{3} \ldots \ldots$

Lithium nitrate, $\mathrm{LiNO}_{3} \ldots \ldots \ldots \ldots$

Lithium oxide, $\mathrm{Li}_{2} \mathrm{O} \ldots \ldots \ldots \ldots \ldots$

Lithium phosphate, high form, $\mathrm{Li}_{3} \mathrm{PO}_{4}$

Lithium phosphate, low form

(1ithiophosphate), $\mathrm{Li}_{3} \mathrm{PO}_{4}$

Lithium phosphate hydrate,

$\mathrm{Li}_{3} \mathrm{P}_{3} \mathrm{O}_{9} \cdot 3 \mathrm{H}_{2} \mathrm{O} \ldots \ldots \ldots \ldots \ldots$

Lithium potassium sulfate, $\mathrm{KLiSO}_{4}$

Lithium rubidium fluoride, $\mathrm{LiRbF}_{2}$

Lithium selenide, $\mathrm{Li}_{2} \mathrm{Se} \ldots \ldots \ldots \ldots$

Lithium silicate, $\mathrm{Li}_{2} \mathrm{SiO}_{3} \ldots \ldots \ldots$

Lithium silver bromide,

$\mathrm{Li},{ }_{2} \mathrm{Ag},{ }_{8} \mathrm{Br} \ldots \ldots \ldots \ldots \ldots \ldots$

Lithium silver bromide, $\mathrm{Li} .{ }_{4} \mathrm{Ag} .{ }_{6} \mathrm{Br}$

Lithium silver bromide,

$\mathrm{Li},{ }_{6} \mathrm{Ag},{ }_{4} \mathrm{Br} \ldots \ldots \ldots \ldots$

Lithium silver bromide,

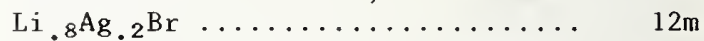

Lithium sodium aluminum fluoride,

cryolithionite, $\mathrm{Li}_{3} \mathrm{Na}_{3} \mathrm{Al}_{2} \mathrm{~F}_{12} \ldots \ldots$

Lithium sodium sulfate, $\mathrm{LiNaSO}_{4} \ldots$

Lithium sulfate, $\mathrm{Ii}_{2} \mathrm{SO}_{4} \ldots \ldots \ldots \ldots$

Lithium sulfate hydrate,

$\mathrm{Li}_{2} \mathrm{SO}_{4} \cdot \mathrm{H}_{2} \mathrm{O} \ldots \ldots \ldots \ldots \ldots \ldots$

Lithium sulfide, $\mathrm{Li}_{2} \mathrm{~S} \ldots \ldots \ldots \ldots$

Lithium tantalum oxide, $\mathrm{LiTaO}_{3} \ldots$

Lithium telluride, $\mathrm{Li}_{2} \mathrm{Te} \ldots \ldots \ldots \ldots$

Lithium thorium molybdenum oxide,

$\mathrm{Li}_{4} \mathrm{Th}_{7}\left(\mathrm{MoO}_{4}\right)_{16} \ldots \ldots \ldots \ldots$

Lithium thorium tungsten oxide,

$\mathrm{Li}_{4} \mathrm{Th}_{7}\left(\mathrm{WO}_{4}\right)_{16} \ldots \ldots \ldots \ldots \ldots$

Lithium tin oxide, $\mathrm{Li}_{2} \mathrm{SnO}_{3} \ldots \ldots \ldots$

Lithium titanium oxide, $\mathrm{Li}_{2} \mathrm{Ti}_{3} \mathrm{O}_{7} \ldots$

Lithium tungsten oxide, $\mathrm{Li}_{2} \mathrm{WO}_{4}$

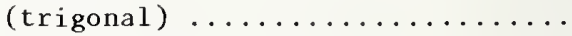

Lithium tungsten oxide hydrate,

$\mathrm{Li}_{2} \mathrm{WO}_{4} \cdot 0.5 \mathrm{H}_{2} \mathrm{O} \ldots \ldots \ldots \ldots \ldots$

Lithium uranium fluoride, $\mathrm{LiUF}_{5} \ldots$

Lithium zirconium oxide, $\mathrm{Li}_{2} \mathrm{ZrO}_{3} \ldots$

Lutetium arsenate, $\mathrm{LuAsO}_{4} \ldots \ldots \ldots$.

Lutetium manganese oxide, $\mathrm{LuMnO}_{3} \ldots$

Lutetium nitride, LuN ...........

Lutetium oxide, $\mathrm{Lu}_{2} \mathrm{O}_{3} \ldots \ldots \ldots \ldots$

Lutetium vanadium oxide, $\mathrm{LuVO}_{4} \ldots$.

Magnesium, $\mathrm{Mg} \ldots \ldots \ldots \ldots \ldots \ldots$

Magnesium aluminum oxide (spinel),

$\mathrm{MgAl}_{2} \mathrm{O}_{4} \ldots \ldots \ldots \ldots \ldots \ldots \ldots \ldots \ldots$

Magnesium aluminum silicate (low

cordierite), $\mathrm{Mg}_{2} \mathrm{Al}_{4} \mathrm{Si}_{5} \mathrm{O}_{18}$

(orthorhombic) ........................

Magnesium aluminum silicate

(indialite) $\mathrm{Mg}_{2} \mathrm{Al}_{4} \mathrm{Si}_{5} \mathrm{O}_{18}$

(hexagonal) .............. $1 \mathrm{~m}$

Magnesium aluminum silicate

(pyrope), $\mathrm{Mg}_{3} \mathrm{Al}_{2}\left(\mathrm{SiO}_{4}\right)_{3} \ldots \ldots \ldots \quad 4 \mathrm{~m}$

Magnesium arsenate hydrate

(hoernesite), $\mathrm{Mg}_{3}\left(\mathrm{AsO}_{4}\right)_{2} \cdot 8 \mathrm{H}_{2} \mathrm{O} \ldots$

Magnesium borate, $\mathrm{MgB}_{4} \mathrm{O}_{7} \ldots \ldots \ldots$

Magnesium borate, $\mathrm{Mg}_{2} \mathrm{~B}_{2} \mathrm{O}_{5}$

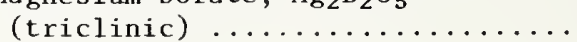

$17 \mathrm{~m}$

$11 \mathrm{~m}$

7

$10 \mathrm{~m}$

$18 \mathrm{~m}$

$1 \mathrm{~m}$

$6 \mathrm{~m}$

7

$1 \mathrm{~m}$

$3 m$

$4 m$

21

46

92

26

33

40

23

22

27

25

39

$2 m \quad 20$

$3 \mathrm{~m} \quad 43$

$7 \mathrm{~m} \quad 128$

$10 \mathrm{~m} \quad 100$

$14 \mathrm{~m} \quad 19$

$12 \mathrm{~m} \quad 55$

$12 \mathrm{~m} \quad 55$

$12 \mathrm{~m} \quad 55$

$9 \mathrm{~m} \quad 23$

$6 \mathrm{~m} \quad 26$

$4 \mathrm{~m} \quad 22$

$10 \mathrm{~m} \quad 101$

$14 \mathrm{~m} \quad 20$

$10 \mathrm{~m} \quad 102$

$20 \mathrm{~m}$

63

$20 \mathrm{~m}$

$16 \mathrm{~m}$

$20 \mathrm{~m}$

$1 \mathrm{~m}$

25

$2 \mathrm{~m}$

20

$7 \mathrm{~m} \quad 131$

$19 \mathrm{~m} \quad 51$

$5 \mathrm{~m} \quad 36$

$2 \mathrm{~m} \quad 23$

$4 \mathrm{~m} \quad 62$

$1 \mathrm{~m} \quad 27$

$5 \mathrm{~m} \quad 37$

$1 \quad 10$

$6 \mathrm{~m} \quad 24$ 
Magnesium bromide, $\mathrm{MgBr}_{2} \ldots \ldots \ldots$

$4 \mathrm{~m} \quad 62$

Magnesium bromide hydrate,

$\mathrm{MgBr}_{2} \cdot 6 \mathrm{H}_{2} \mathrm{O} \ldots \ldots \ldots \ldots \ldots \ldots$

Magnesium carbonate (magnesite),

$\mathrm{MgCO}_{3}$

Magnesium cerium nitrate hydrate,

$\mathrm{Mg}_{3} \mathrm{Ce}_{2}\left(\mathrm{NO}_{3}\right)_{12} \cdot 24 \mathrm{H}_{2} \mathrm{O} \ldots \ldots \ldots \ldots \ldots$

Magnesium chlorate hydrate,

$\mathrm{Mg}\left(\mathrm{ClO}_{4}\right)_{2} \cdot 6 \mathrm{H}_{2} \mathrm{O} \ldots \ldots \ldots \ldots$

Magnesium chloride, $\mathrm{MgCl}_{2} \ldots \ldots \ldots$

Magnesium chloride hydrate,

$\mathrm{MgCl}_{2} \cdot 12 \mathrm{H}_{2} \mathrm{O} \ldots \ldots \ldots \ldots \ldots \ldots$

Magnesium chloride hydrate

(bischofite), $\mathrm{MgCl}_{2} \cdot 6 \mathrm{H}_{2} \mathrm{O} \ldots \ldots \ldots$

Magnesium chromium oxide

(magnesiochromite), $\mathrm{MgCr}_{2} \mathrm{O}_{4} \ldots \ldots$

Magnesium chromium oxide hydrate,

$\mathrm{MgCrO}_{4} \cdot 5 \mathrm{H}_{2} \mathrm{O} \ldots \ldots \ldots \ldots \ldots \ldots$

Magnesium fluoride (sellaite), $\mathrm{MgF}_{2}$

Magnesium fluoride silicate

(humite), $\mathrm{Mg}_{7} \mathrm{~F}_{2} \mathrm{Si}_{3} \mathrm{O}_{12} \ldots \ldots \ldots$

Magnesium fluoride silicate

(norbergite), $\mathrm{Mg}_{3} \mathrm{~F}_{2} \mathrm{SiO}_{4} \ldots \ldots \ldots$

Magnesium gallium oxide, $\mathrm{MgGa}_{2} \mathrm{O}_{4} \ldots$

Magnesium germanium oxide,

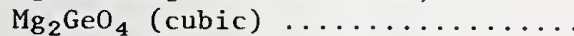

Magnesium germanium oxide,

$\mathrm{Mg}_{2} \mathrm{GeO}_{4}$ (orthorhombic) .........

Magnesium hydrogen phosphate

hydrate, newberyite, $\mathrm{MgHPO}_{4} \cdot 3 \mathrm{H}_{2} \mathrm{O}$

Magnesium hydroxide (brucite),

$\mathrm{Mg}(\mathrm{OH})_{2} \ldots \ldots \ldots \ldots \ldots \ldots \ldots \ldots$

Magnesium iodate hydrate,

$\mathrm{Mg}\left(\mathrm{IO}_{3}\right)_{2} \cdot 4 \mathrm{H}_{2} \mathrm{O} \ldots \ldots \ldots \ldots \ldots \ldots$

Magnesium iron hydroxide carbonate

hydrate, pyroaurite,

$\mathrm{Mg}_{6} \mathrm{Fe}_{2}(\mathrm{OH})_{16} \mathrm{CO}_{3} \cdot 4 \mathrm{H}_{2} \mathrm{O}$ (rhomb.) ...

Magnesium iron hydroxide carbonate

hydrate, sjögrenite,

$\mathrm{Mg}_{6} \mathrm{Fe}_{2}(\mathrm{OH})_{16} \mathrm{CO}_{3} \cdot 4 \mathrm{H}_{2} \mathrm{O}$, (hexag.) ...

Magnesium lanthanum nitrate

hydrate, $\mathrm{Mg}_{3} \mathrm{La}_{2}\left(\mathrm{NO}_{3}\right)_{12} \cdot 24 \mathrm{H}_{2} \mathrm{O} \ldots$.

Magnesium manganese oxide, $\mathrm{MgMn}_{2} \mathrm{O}_{4}$

Magnesium manganese zinc iron sulfate hydroxide hydrate, zincobotryogen,

$(\mathrm{Zn}, \mathrm{Mg}, \mathrm{Mn}) \mathrm{Fe}\left(\mathrm{SO}_{4}\right)_{2}(\mathrm{OH}) \cdot 7 \mathrm{H}_{2} \mathrm{O} \ldots \ldots$

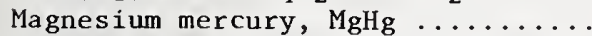

Magnesium molybdenum oxide, $\mathrm{MgMoO}_{4}$

Magnesium nickel oxide, $\mathrm{MgNiO}_{2} \ldots$.

Magnesium oxide (periclase), MgO ..

Magnesium phosphate, $\mathrm{Mg}\left(\mathrm{PO}_{3}\right)_{2} \ldots$.

Magnesium phosphate, $\alpha-\mathrm{Mg}_{2} \mathrm{P}_{2} \mathrm{O}_{7} \ldots$

Magnesium phosphate (farringtonite),

$\mathrm{Mg}_{3}\left(\mathrm{PO}_{4}\right)_{2} \ldots \ldots \ldots \ldots \ldots \ldots \ldots \ldots \ldots \ldots \ldots$

Magnesium selenide, MgSe .........

Magnesium selenite hydrate,

$\mathrm{MgSeO}_{3} \cdot 6 \mathrm{H}_{2} \mathrm{O} \ldots \ldots \ldots \ldots \ldots \ldots \ldots$

Magnesium silicate (clinoenstatite),

$\mathrm{MgSiO}_{3} \ldots \ldots \ldots \ldots \ldots \ldots$

Magnesium silicate, enstatite,

$\mathrm{MgSiO}_{3} \ldots \ldots \ldots \ldots \ldots \ldots$

Magnesium silicate (forsterite),

$\mathrm{Mg}_{2} \mathrm{SiO}_{4} \ldots \ldots \ldots \ldots \ldots \ldots \ldots$

Magnesium sulfate hydrate

(kieserite), $\mathrm{MgSO}_{4} \cdot \mathrm{H}_{2} \mathrm{O} \ldots \ldots \ldots \ldots \quad 16$

Magnesium sulfate hydrate

(epsomite), $\mathrm{MgSO}_{4} \cdot 7 \mathrm{H}_{2} \mathrm{O}$
$11 \mathrm{~m}$

35

$7 \quad 28$

$10 \quad 20$

$7 \mathrm{~m} \quad 30$

$11 \mathrm{~m} \quad 94$

$7 \mathrm{~m} \quad 135$

$11 \mathrm{~m}$

$15 \mathrm{~m}$

39

$1 \mathrm{~m}$

30

10

39

10

$7 m$

139

$17 m$

$10 \mathrm{~m}$

104

$10 \mathrm{~m}$

103

$1 \mathrm{~m}$

22

$10 \mathrm{~m}$

$20 \mathrm{~m}$

$6 \mathrm{~m}$

$7 \mathrm{~m}$

$10 \mathrm{~m}$

1

$13 \mathrm{~m}$

$18 \mathrm{~m}$

$19 \mathrm{~m}$

$5 \mathrm{~m}$

$8 \mathrm{~m}$

$20 m$

69

6

32

$20 m$

$16 \mathrm{~m}$

46

7

30

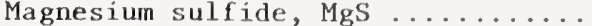

Magnesium sulfite hydrate,

$\mathrm{MgSO}_{3} \cdot 6 \mathrm{H}_{2} \mathrm{O} \ldots \ldots \ldots \ldots \ldots \ldots$

Magnesium $\mathrm{tin}, \mathrm{Mg}_{2} \mathrm{Sn} \ldots \ldots \ldots \ldots$

Magnesium tin oxide, $\mathrm{Mg}_{2} \mathrm{SnO}_{4} \ldots \ldots$

Magnesium titanium oxide

(geikielite), $\operatorname{MgTiO}_{3} \ldots \ldots \ldots \ldots$

Magnesium titanium oxide, $\mathrm{Mg}_{2} \mathrm{TiO}_{4}$

Magnesium tungsten oxide, $\mathrm{MgWO}_{4}$

Manganese, $\alpha-M n$ (calculated pattern)

Manganese, $\alpha-M n \ldots \ldots \ldots \ldots$.

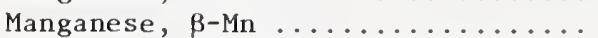

Manganese aluminum oxide (galaxite), $\mathrm{MnAl}_{2} \mathrm{O}_{4} \ldots \ldots \ldots \ldots \ldots \ldots \ldots$

Manganese bromide, $\mathrm{MnBr}_{2} \ldots \ldots \ldots$

Manganese(II) carbonate

(rhodochrosite), $\mathrm{MnCO}_{3}$

Manganese chloride (scacchite),

$\mathrm{MnCl}_{2} \ldots \ldots \ldots \ldots \ldots \ldots$

Manganese chloride hydrate,

$\mathrm{MnCl}_{2} \cdot 2 \mathrm{H}_{2} \mathrm{O} \ldots \ldots \ldots \ldots \ldots$

Manganese chloride hydrate,

$\mathrm{MnCl}_{2} \cdot 4 \mathrm{H}_{2} \mathrm{O} \ldots \ldots \ldots \ldots \ldots$

Manganese cobalt oxide, $\mathrm{MnCo}_{2} \mathrm{O}_{4} \ldots$

Manganese fluoride, $\mathrm{MnF}_{2} \ldots \ldots \ldots$

Manganese iodide, $\mathrm{MnI}_{2} \ldots \ldots \ldots \ldots$

Manganese iron oxide (jacobsite),

$\mathrm{MnFe}_{2} \mathrm{O}_{4} \ldots \ldots \ldots \ldots \ldots \ldots \ldots \ldots \ldots \ldots \ldots$

Manganese(II) oxide (manganosite),

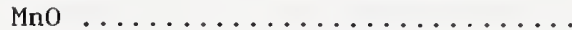

Manganese oxide (pyrolusite), $\mathrm{\beta}-\mathrm{MnO}_{2}$

Manganese oxide (bixbyite), $\alpha-\mathrm{Mn}_{2} \mathrm{O}_{3}$

Manganese oxide (hausmannite),

$\mathrm{Mn}_{3} \mathrm{O}_{4} \ldots \ldots \ldots \ldots \ldots \ldots \ldots \ldots \ldots$

Manganese oxide hydroxide, groutite,

$\alpha-\mathrm{MnOOH} \ldots \ldots \ldots \ldots \ldots \ldots \ldots$

Manganese phosphate, $\mathrm{Mn}\left(\mathrm{PO}_{3}\right)_{2} \ldots \ldots$

Manganese phosphate, $\mathrm{Mn}_{2} \mathrm{P}_{2} \mathrm{O}_{7} \ldots \ldots$

Manganese phosphate, $\mathrm{Mn}_{3}\left(\mathrm{PO}_{4}\right)_{2} \ldots$

Manganese selenide, MnSe .........

Manganese sulfate hydrate

(szmikite), $\mathrm{MnSO}_{4} \cdot \mathrm{H}_{2} \mathrm{O} \ldots \ldots \ldots \ldots \quad 16 \mathrm{~m}$

Manganese sulfide (alabandite),

$\alpha-\operatorname{MnS} \ldots \ldots \ldots \ldots \ldots \ldots \ldots$

Manganese titanium oxide

(pyrophanite), $\mathrm{MnTiO}_{3} \ldots \ldots \ldots \ldots \quad 15 \mathrm{~m}$

Manganese(II) tungsten oxide

(huebnerite), $\mathrm{MnWO}_{4} \ldots \ldots \ldots \ldots .2 \mathrm{~m}$

Manganese vanadium oxide, $\mathrm{Mn}_{2} \mathrm{~V}_{2} \mathrm{O}_{7} \quad 9 \mathrm{~m}$

Mercury amide chloride, $\mathrm{HgNH}_{2} \mathrm{Cl}$... 10m

Mercury ammine chloride,

$\mathrm{Hg}\left(\mathrm{NH}_{3}\right)_{2} \mathrm{Cl}_{2} \ldots \ldots \ldots \ldots \ldots \ldots \ldots \ldots . \ldots \ldots$

Mercury bromate, $\mathrm{Hg}\left(\mathrm{BrO}_{3}\right)_{2} \ldots \ldots \ldots \quad 10 \mathrm{~m}$

Mercury bromide, $\mathrm{HgBr}_{2} \ldots \ldots \ldots \ldots .10 \mathrm{~m}$

Mercury bromide, $\mathrm{Hg}_{2} \mathrm{Br}_{2} \ldots \ldots \ldots \ldots$

Mercury chloride, $\mathrm{HgCl}_{2} \ldots \ldots \ldots \ldots$ 13m

Mercury chloride (calomel),

$\mathrm{Hg}_{2} \mathrm{Cl}_{2} \ldots \ldots \ldots \ldots \ldots \ldots \ldots \ldots \ldots \ldots$ 13m

Mercury chloride sulfide,

$\alpha-\mathrm{Hg}_{3} \mathrm{Cl}_{2} \mathrm{~S}_{2} \ldots \ldots \ldots \ldots \ldots \ldots \ldots$

Mercury(II) cyanide, $\mathrm{Hg}(\mathrm{CN})_{2} \ldots \ldots$

Mercury(II) fluoride, $\mathrm{HgF}_{2} \ldots \ldots \ldots$

Mercury hydroxide nitrate,

$\mathrm{Hg}(\mathrm{OH}) \mathrm{NO}_{3} \ldots \ldots \ldots \ldots \ldots \ldots \ldots$

Mercury(I) iodide, $\mathrm{HgI} \ldots \ldots \ldots \ldots$

Mercury(II) iodide, $\mathrm{HgI}_{2}$ (tetragonal)

Mercury(II) oxide (montroydite),

$\mathrm{HgO}$

7

31

26

41

37

43

25

27

142

50

43

35

63

38

28

30

105

63

36

45

39

95

38

97

21

41

47

41

49

11

24

75

40

39

107

110

33

29

30

$8 \mathrm{~m}$

118

35

$2 \mathrm{~m} \quad 25$

$17 \mathrm{~m}$

52

49

7 
Mercury(I I) selenide (tiemannite),

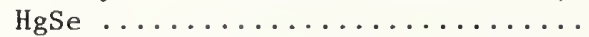
Mercury sulfate, $\mathrm{HgSO}_{4} \ldots \ldots \ldots \ldots$ Mercury sulfate, $\mathrm{Hg}_{2} \mathrm{SO}_{4} \ldots \ldots \ldots \ldots$ Mercury(II) sulfide (cinnabar),

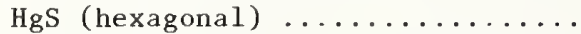
Mercury(II) sulfide (metacinnabar),

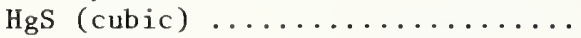

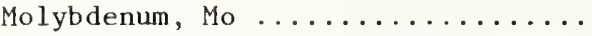
Molybdenum arsenide, $\mathrm{Mo}_{2} \mathrm{As}_{3} \ldots \ldots$ Molybdenum osmium, $\mathrm{Mo}_{3} \mathrm{Os} \ldots \ldots \ldots$ Molybdenum oxide, $\mathrm{MoO}_{2} \ldots \ldots \ldots \ldots$ Molybdenum oxide (molybdite), $\mathrm{MoO}_{3}$. Molybdenum oxide (molybdite), $\mathrm{MoO}_{3}$ Molybdenum silicide, $\mathrm{Mo}_{5} \mathrm{Si}_{3} \ldots . .$. Molybdenum sulfide (molybdenite),

$\mathrm{MoS}_{2} \ldots \ldots \ldots \ldots \ldots \ldots \ldots$ Neodymi um arsenate, $\mathrm{NdAsO}_{4} \ldots \ldots \ldots$ Neodymium arsenide, NdAs ......... Neodymi um borate, $\mathrm{NdBO}_{3} \ldots \ldots \ldots \ldots$ Neodymium chloride, $\mathrm{NdCl}_{3} \ldots \ldots \ldots$ Neodymium chloride oxide, NdOCl ... Neodymium fluoride, $\mathrm{NdF}_{3} \ldots \ldots \ldots \ldots$ Neodymium oxide, $\mathrm{Nd}_{2} \mathrm{O}_{3} \ldots \ldots \ldots \ldots$ Neodymium phosphate, $\mathrm{NdPO}_{4} \ldots \ldots \ldots$ Neodymium selenide, NdSe ......... Neodymi um silver, $\mathrm{NdAg}$........... Neodymium tantalum oxide, $\mathrm{NdTaO}_{4} \ldots$ Neodymium titanium oxide, $\mathrm{Nd}_{2} \mathrm{TiO}_{5}$ Neodymium titanium oxide, $\mathrm{Nd}_{2} \mathrm{Ti}_{2} \mathrm{O}_{7}$ Neodymium titanium oxide, $\mathrm{Nd}_{4} \mathrm{Ti}_{9} \mathrm{O}_{24}$ Neodymium vanadium oxide, $\mathrm{NdVO}_{4} \ldots$ Neptunium nitride, $\mathrm{NpN} \ldots \ldots \ldots \ldots$

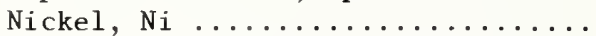
$\mathrm{Nickel}$ aluminum oxide, $\mathrm{NiAl}_{2} \mathrm{O}_{4} \ldots$. Nickel arsenate hydrate (annabergite),

$\mathrm{Ni}_{3}\left(\mathrm{AsO}_{4}\right)_{2} \cdot 8 \mathrm{H}_{2} \mathrm{O} \ldots \ldots \ldots \ldots . . . .$. Nickel arsenide (rammelsbergite),

$\mathrm{NiAs}_{2} \ldots \ldots \ldots \ldots \ldots \ldots \ldots \ldots$

Nickel arsenic sulfide

(gersdorffite), NiAsS .........

Nickel bromide, $\mathrm{NiBr}_{2} \ldots \ldots \ldots \ldots$.

Nickel(II) carbonate, $\mathrm{NiCO}_{3}$

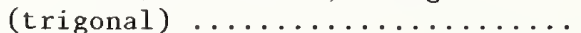

Nickel chloride, $\mathrm{NiCl}_{2} \ldots \ldots \ldots \ldots$

Nickel chloride hydrate,

$\mathrm{NiCl}_{2} \cdot 6 \mathrm{H}_{2} \mathrm{O} \ldots \ldots \ldots \ldots \ldots \ldots$

Nickel fluoride, $\mathrm{NiF}_{2} \ldots \ldots \ldots \ldots$

Nickel fluoride hydrate, $\mathrm{NiF}_{2} \cdot 4 \mathrm{H}_{2} \mathrm{O}$

$\mathrm{Nickel}$ gallium oxide, $\mathrm{NiGa}_{2} \mathrm{O}_{4} \ldots$. .

$\mathrm{Nickel}$ germanium oxide, $\mathrm{Ni}_{2} \mathrm{GeO}_{4} \ldots$

Nickel iron oxide (trevorite),

$\mathrm{NiFe}_{2} \mathrm{O}_{4} \ldots \ldots \ldots \ldots \ldots \ldots \ldots \ldots$

Nickel molybdenum oxide, $\mathrm{NiMoO}_{4} \ldots$

Nickel nitrate hydrate,

$\mathrm{Ni}\left(\mathrm{NO}_{3}\right)_{2} \cdot 6 \mathrm{H}_{2} \mathrm{O} \ldots \ldots \ldots \ldots \ldots \ldots$

Nickel(II) oxide (bunsenite), $\mathrm{NiO}$

Nickel phosphate, $\mathrm{Ni}\left(\mathrm{PO}_{3}\right)_{2} \ldots \ldots \ldots$

Nickel phosphate hydrate,

$\mathrm{Ni}_{3}\left(\mathrm{PO}_{4}\right)_{2} \cdot 8 \mathrm{H}_{2} \mathrm{O} \ldots \ldots \ldots \ldots \ldots$

Nickel phosphide, $\mathrm{Ni}_{12} \mathrm{P}_{5} \ldots \ldots \ldots$.

Nickel silicon fluoride hydrate,

$\mathrm{NiSiF}_{6} \cdot 6 \mathrm{H}_{2} \mathrm{O} \ldots \ldots \ldots \ldots \ldots \ldots \ldots$

Nickel sulfate, $\mathrm{NiSO}_{4} \ldots \ldots \ldots \ldots \ldots$

Nickel sulfate hydrate (retgersite),

$\mathrm{NiSO}_{4} \cdot 6 \mathrm{H}_{2} \mathrm{O}$ (tetragonal) .........

Nickel sulfate hydrate (nickel-

hexahydrite), $\beta-\mathrm{NiSO}_{4} \cdot 6 \mathrm{H}_{2} \mathrm{O}$

(monoclinic)

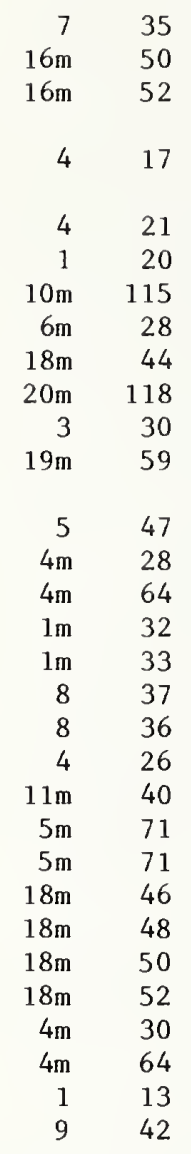

$19 \mathrm{~m} \quad 60$

10

42

$1 \mathrm{~m} \quad 35$

$10 \mathrm{~m} \quad 119$

$1 \mathrm{~m} \quad 36$

$9 \mathrm{~m} \quad 81$

$11 \mathrm{~m} \quad 42$

$10 \mathrm{~m} \quad 121$

$11 \mathrm{~m} \quad 43$

$10 \quad 45$

943

$10 \quad 44$

$19 \mathrm{~m} \quad 62$

$12 \mathrm{~m}$

26

47

$14 \mathrm{~m} \quad 22$

$19 \mathrm{~m} \quad 64$

$9 \mathrm{~m} \quad 83$

$8 \quad 38$

$2 \mathrm{~m} \quad 26$

7

36

$19 \mathrm{~m}$

65
Nickel sulfide, millerite, Nis ... Nickel titanium oxide, $\mathrm{NiTiO}_{3} \ldots \ldots$ $\mathrm{Nickel}$ tungsten oxide, $\mathrm{NiWO}_{4} \ldots \ldots$ Nickel yttrium, $\mathrm{Ni}_{3} \mathrm{Y} \ldots \ldots \ldots \ldots$

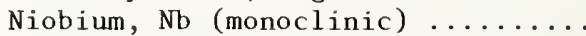
Niobium boride, $\zeta-\mathrm{NbB} \ldots \ldots \ldots \ldots$ Niobium chloride oxide, $\mathrm{NbCl}_{3} \mathrm{O} \ldots$ Niobium osmium, $\mathrm{Nb}_{3} \mathrm{Os} \ldots \ldots \ldots \ldots \ldots$ Niobium platinum, $\mathrm{Nb}_{3} \mathrm{Pt} \ldots \ldots \ldots \ldots$ Niobium silicide, $\mathrm{NbSi}_{2} \ldots \ldots \ldots \ldots$ Niobium silicide, $\alpha-\mathrm{Nb}_{5} \mathrm{Si}_{3} \ldots \ldots \ldots$ Niobium silicide, $\beta-\mathrm{Nb}_{5} \mathrm{Si}_{3} \ldots \ldots \ldots$

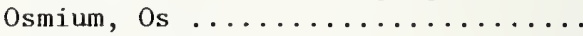

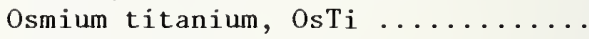

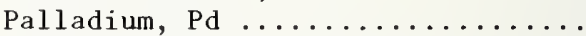
Palladium hydride, $\mathrm{PdH}_{0}, 706 \ldots \ldots$

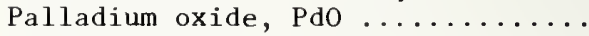
Palladium selenium (palladseite),

$\mathrm{Pd}_{17} \mathrm{Se}_{15} \ldots \ldots \ldots \ldots \ldots \ldots \ldots \ldots$ Palladium vanadium, $\operatorname{PdV}_{3} \ldots \ldots \ldots$

Phosphorus bromide, $\mathrm{PBr}_{7} \ldots \ldots \ldots$. Phosphorus oxide (stable form I),

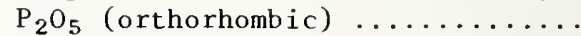
Phosphorus oxide (stable form II),

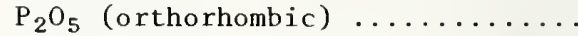
Phosphorus oxide (metastable form),

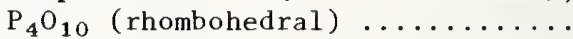

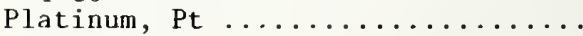

Platinum titanium, $\mathrm{PtTi}_{3} \ldots \ldots \ldots$

Platinum vanadium, $\mathrm{PtV}_{3} \ldots \ldots \ldots \ldots$

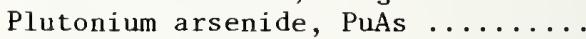

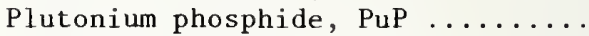

Plutonium telluride, PuTe ........

Potassium aluminum sulfate,

$\mathrm{KAl}\left(\mathrm{SO}_{4}\right)_{2} \ldots \ldots \ldots \ldots \ldots \ldots \ldots \ldots$

Potassium aluminum sulfate hydrate

(potash alum), $\mathrm{KAl}\left(\mathrm{SO}_{4}\right)_{2} \cdot 12 \mathrm{H}_{2} \mathrm{O} \ldots$

Potassium arsenic fluoride,

$\mathrm{KAsF}_{6} \ldots \ldots \ldots \ldots \ldots \ldots \ldots \ldots$

Potassium barium chromium oxide,

$\mathrm{K}_{2} \mathrm{Ba}\left(\mathrm{CrO}_{4}\right)_{2} \ldots \ldots \ldots \ldots \ldots \ldots$

Potassium barium iron titanium

oxide, $\mathrm{K}_{1.16} \mathrm{Ba}_{0.72} \mathrm{Fe}_{0.36} \mathrm{Ti}_{5.58} \mathrm{O}_{13}$

Potassium barium molybdenum oxide,

$\mathrm{K}_{2} \mathrm{Ba}\left(\mathrm{MoO}_{4}\right)_{2} \ldots \ldots \ldots \ldots \ldots \ldots$

Potassium barium nickel nitrite,

$\mathrm{K}_{2} \mathrm{BaNi}\left(\mathrm{NO}_{2}\right)_{6} \ldots \ldots \ldots \ldots \ldots \ldots$

Potassium barium niobium oxide,

$\mathrm{KBa}_{2}\left(\mathrm{NbO}_{3}\right)_{5} \ldots \ldots \ldots \ldots \ldots \ldots \ldots$

Potassium barium phosphate,

$\mathrm{KBaPO}_{4} \ldots \ldots \ldots \ldots \ldots \ldots \ldots \ldots$

Potassium borate hydroxide hydrate,

$\mathrm{K}_{2} \mathrm{~B}_{4} \mathrm{O}_{5}(\mathrm{OH})_{4} \cdot 2 \mathrm{H}_{2} \mathrm{O} \ldots \ldots \ldots \ldots$

Potassium calcium phosphate,

$\mathrm{KCaPO}_{4} \ldots \ldots \ldots \ldots \ldots \ldots \ldots \ldots \ldots \ldots \ldots \ldots \ldots$

Potassium boron hydride, $\mathrm{KBH}_{4} \ldots \ldots$

Potassium bromate, $\mathrm{KBrO}_{3} \ldots \ldots \ldots \ldots$

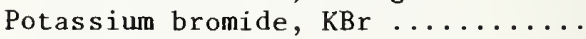

Potassium bromide chloride,

$\mathrm{KBr}_{0.5} \mathrm{Cl}_{0,5} \ldots \ldots \ldots \ldots \ldots \ldots$

Potassium bromide iodide,

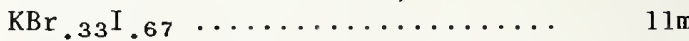

Potassium bromide iodide, $\mathrm{KBr}_{6} \mathrm{I}_{73} \mathrm{I}_{33}$

Potassium cadmium chloride, $\mathrm{KCdCl}_{3}$

Potassium cadmium fluoride, $\mathrm{KCdF}_{3}$

Potassium cadmium sulfate,

$\mathrm{K}_{2} \mathrm{Cd}_{2}\left(\mathrm{SO}_{4}\right)_{3} \ldots \ldots \ldots \ldots \ldots \ldots \ldots \ldots \ldots$

Potassium calcium carbonate

(fairchildite), $\mathrm{K}_{2} \mathrm{Ca}\left(\mathrm{CO}_{3}\right)_{2}$

Sec. Page

$1 \mathrm{~m}$

$18 \mathrm{~m} \quad 54$

$2 \mathrm{~m} \quad 2.7$

$10 \mathrm{~m} \quad 123$

$19 \mathrm{~m} \quad 67$

$17 \mathrm{~m} \quad 54$

$7 \mathrm{~m} \quad 148$

$6 \mathrm{~m} \quad 30$

$6 \mathrm{~m} \quad 31$

$8 \quad 39$

$15 \mathrm{~m} \quad 43$

$15 \mathrm{~m} \quad 44$

48

$6 \mathrm{~m} \quad 85$

$\begin{array}{ll}1 & 21\end{array}$

$5 \mathrm{~m} \quad 72$

$4 \quad 27$

$16 \mathrm{~m} \quad 139$

$6 \mathrm{~m} \quad 32$

$7 \mathrm{~m} \quad 150$

$9 \mathrm{~m} \quad 86$

$9 \mathrm{~m} \quad 88$

$9 \mathrm{~m} \quad 91$

$\begin{array}{ll}1 & 31\end{array}$

$6 \mathrm{~m} \quad 33$

$6 \mathrm{~m} \quad 34$

$4 \mathrm{~m} \quad 65$

$4 \mathrm{~m} \quad 65$

$4 \mathrm{~m} \quad 66$

$9 \mathrm{~m} \quad 31$

$6 \quad 36$

$17 \mathrm{~m} \quad 57$

$14 \mathrm{~m} \quad 23$

$16 \mathrm{~m} \quad 147$

$14 \mathrm{~m}$

$20 m$

73

$19 \mathrm{~m}$

68

$15 \mathrm{~m}$

46

$19 m$

g

7
1

166

$8 \mathrm{~m}$

46

$11 \mathrm{~m}$

$5 \mathrm{~m}$

$8 \mathrm{~m}$

34

西

48

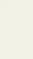

6

8

(1)


Potassium calcium chloride, $\mathrm{KCaCl}_{3}$ Potassium calcium fluoride, $\mathrm{KCaF}_{3}$

Potassium calcium magnesium sulfate,

$\mathrm{K}_{2} \mathrm{CaMg}\left(\mathrm{SO}_{4}\right)_{3} \ldots \ldots \ldots \ldots \ldots \ldots$

Potassium calcium nickel nitrite,

$\mathrm{K}_{2} \mathrm{CaNi}\left(\mathrm{NO}_{2}\right)_{6} \ldots \ldots \ldots \ldots \ldots \ldots \ldots$

Potassium calcium sulfate,

$\mathrm{K}_{2} \mathrm{Ca}_{2}\left(\mathrm{SO}_{4}\right)_{3} \ldots \ldots \ldots \ldots \ldots \ldots \ldots$

Potassium calcium sulfate hydrate

(syngenite), $\mathrm{K}_{2} \mathrm{Ca}\left(\mathrm{SO}_{4}\right)_{2} \cdot \mathrm{H}_{2} \mathrm{O} \ldots \ldots$

Potassium cerium fluoride, $\beta-\mathrm{KCeF}_{4}$

Potassium chlorate, $\mathrm{KClO}_{3} \ldots \ldots \ldots$

Potassium chlorate, $\mathrm{KClO}_{4} \ldots \ldots \ldots$

Potassium chloride (sylvite), $\mathrm{KCl}$

Potassium chromium oxide, $\mathrm{K}_{3} \mathrm{CrO}_{8}$..

Potassium chromium oxide (lopezite),

$\mathrm{K}_{2} \mathrm{Cr}_{2} \mathrm{O}_{7} \ldots \ldots \ldots \ldots \ldots \ldots \ldots \ldots$

Potassium chromium oxide sulfate,

$\mathrm{K}_{2}\left(\mathrm{CrO}_{4}\right){ }_{.33}\left(\mathrm{SO}_{4}\right) .67 \ldots \ldots \ldots \ldots \ldots$

Potassium chromium oxide sulfate,

$\mathrm{K}_{2}\left(\mathrm{CrO}_{4}\right)_{.67}\left(\mathrm{SO}_{4}\right)_{.33} \ldots \ldots \ldots \ldots \ldots$

Potassium chromium sulfate,

$\mathrm{KCr}\left(\mathrm{SO}_{4}\right)_{2} \ldots \ldots \ldots \ldots \ldots \ldots \ldots \ldots$

Potassium chromium sulfate hydrate,

$\mathrm{KCr}\left(\mathrm{SO}_{4}\right)_{2} \cdot 12 \mathrm{H}_{2} \mathrm{O} \ldots \ldots \ldots \ldots \ldots$

Potassium cobalt(II) fluoride,

$\mathrm{KCoF}_{3} \ldots \ldots \ldots \ldots \ldots \ldots \ldots$

Potassium cobalt fluoride, $\mathrm{K}_{2} \mathrm{CoF}_{4}$

Potassium cobalt nitrite,

$\mathrm{K}_{3} \mathrm{Co}\left(\mathrm{NO}_{2}\right)_{6} \ldots \ldots \ldots \ldots \ldots \ldots \ldots$

Potassium cobalt(II) sulfate,

$\mathrm{K}_{2} \mathrm{Co}_{2}\left(\mathrm{SO}_{4}\right)_{3} \ldots \ldots \ldots \ldots \ldots \ldots$

Potassium copper chloride, $\mathrm{KCuCl}_{3}$

Potassium copper chloride hydrate

(mitscherlichite), $\mathrm{K}_{2} \mathrm{CuCl}_{4} \cdot 2 \mathrm{H}_{2} \mathrm{O} \ldots$

Potassium copper(II) fluoride,

$\mathrm{KCuF}_{3} \ldots \ldots \ldots \ldots \ldots \ldots \ldots$

Potassium cyanate, KCNO ..........

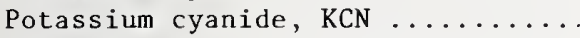

Potassium fluoride, KF ..........

Potassium fluoride hydrate, $\mathrm{KF} \cdot 2 \mathrm{H}_{2} \mathrm{O}$

Potassium germanium fluoride,

$\mathrm{K}_{2} \mathrm{GeF}_{6} \ldots \ldots \ldots \ldots \ldots \ldots \ldots \ldots \ldots \ldots \ldots \ldots$

Potassium hydrogen arsenate,

$\mathrm{KH}_{2} \mathrm{AsO}_{4} \ldots \ldots \ldots \ldots \ldots \ldots \ldots \ldots \ldots \ldots \ldots \ldots \ldots$

Potassium hydrogen iodate,

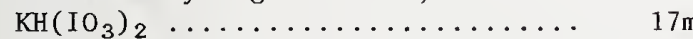

Potassium hydrogen phosphate,

$\mathrm{KH}_{2} \mathrm{PO}_{4} \ldots \ldots \ldots \ldots \ldots \ldots \ldots \ldots$

Potassium hydroxide, $\mathrm{KOH}$ at $300{ }^{\circ}{ }_{\mathrm{C}}$

Potassium iodate, $\mathrm{KIO}_{3} \ldots \ldots \ldots \ldots$

Potassium iodate, $\mathrm{KIO}_{4} \ldots \ldots \ldots \ldots$

Potassium iodide, KI ............

Potassium iron chloride hydrate

(erythrosiderite), $\mathrm{K}_{2} \mathrm{FeCl}_{5} \cdot \mathrm{H}_{2} \mathrm{O} \ldots$

Potassium iron cyanide, $\mathrm{K}_{3} \mathrm{Fe}(\mathrm{CN})_{6}$

Potassium iror cyanide, $\mathrm{K}_{4} \mathrm{Fe}(\mathrm{CN})_{6}$

Potassium iron(II) fluoride, $\mathrm{KFeF}_{3}$

Potassium iron fluoride, $\mathrm{K}_{3} \mathrm{FeF}_{6} \ldots$

Potassium iron sulfate (yavapaite),

$\mathrm{KFe}\left(\mathrm{SO}_{4}\right)_{2} \ldots \ldots \ldots \ldots \ldots \ldots \ldots$

Potassium lead chloride, $\mathrm{KPb}_{2} \mathrm{Cl}_{5} \ldots$

Potassium lead chromium oxide,

$\mathrm{K}_{2} \mathrm{~Pb}\left(\mathrm{CrO}_{4}\right)_{2} \ldots \ldots \ldots \ldots \ldots \ldots \ldots$

Potassium lead molybdenum oxide,

$\mathrm{K}_{2} \mathrm{~Pb}\left(\mathrm{MoO}_{4}\right)_{2} \ldots \ldots \ldots \ldots \ldots \ldots \ldots \ldots$

Potassium lead phosphate,

$\mathrm{K}_{2} \mathrm{~Pb}\left(\mathrm{PO}_{3}\right)_{4}$
$7 \mathrm{~m} \quad 36 \quad$ Potassium lead selenate,

$8 \mathrm{~m} \quad 49$

$7 m$

$9 m$

$7 \mathrm{~m}$

$14 \mathrm{~m}$

$2 m \quad 59$

$3 \mathrm{~m} \quad 42$

$6 \quad 43$

165

$3 \mathrm{~m}$

$15 \mathrm{~m}$

$12 \mathrm{~m}$

28

$12 \mathrm{~m}$

$16 \mathrm{~m}$

58

$6 m$

37

$11 \mathrm{~m}$

46

$6 \mathrm{~m}$

35

$7 \mathrm{~m}$

$9 m$

34

$6 \mathrm{~m}$

38

39

77

64

$18 \mathrm{~m}$
Potassium lead sulfate (palmierite),

$\mathrm{K}_{2} \mathrm{~Pb}\left(\mathrm{SO}_{4}\right)_{2} \ldots \ldots \ldots \ldots \ldots \ldots \ldots \ldots \ldots$

Potassium magnesium chloride

hydrate (carnallite), $\mathrm{KMgCl}_{3} \cdot 6 \mathrm{H}_{2} \mathrm{O}$

Potassium magnesium chromium oxide,

$\mathrm{K}_{2} \mathrm{Mg}_{2}\left(\mathrm{CrO}_{4}\right)_{3} \ldots \ldots \ldots \ldots \ldots \ldots$

Potassium magnesium fluoride, $\mathrm{KMgF}_{3}$
Potassium magnesium fluoride, $\mathrm{K}_{2} \mathrm{MgF}_{4}$

Potassium magnesium selenate

hydrate, $\mathrm{K}_{2} \mathrm{Mg}\left(\mathrm{SeO}_{4}\right)_{2} \cdot 6 \mathrm{H}_{2} \mathrm{O} \ldots \ldots .10 \mathrm{~m}$

Potassium magnesium sulfate

(langbeinite), $\mathrm{K}_{2} \mathrm{Mg}_{2}\left(\mathrm{SO}_{4}\right)_{3} \ldots \ldots .6 \mathrm{~m}$

Potassium magnesium sulfate hydrate

(picromerite), $\mathrm{K}_{2} \mathrm{Mg}\left(\mathrm{SO}_{4}\right)_{2} \cdot 6 \mathrm{H}_{2} \mathrm{O}$

Potassium manganese(II) fluoride,

$\mathrm{KMnF}_{3} \ldots \ldots \ldots \ldots \ldots \ldots \ldots$

Potassium manganese oxide, $\mathrm{KMnO}_{4}$

Potassium manganese(II) sulfate

(manganolangbeinite), $\mathrm{K}_{2} \mathrm{Mn}_{2}\left(\mathrm{SO}_{4}\right)_{3}$

Potassium molybdenum oxide, $\mathrm{K}_{2} \mathrm{MoO}_{4}$

Potassium molybdenum oxide phos-

phate hydrate, $\mathrm{K}_{3}\left(\mathrm{MoO}_{3}\right)_{12} \mathrm{PO}_{4} \cdot 4 \mathrm{H}_{2} \mathrm{O}$

Potassium nickel fluoride, $\mathrm{KNiF}_{3}$

Potassium nickel fluoride, $\mathrm{K}_{2} \mathrm{NiF}_{4}$

Potassium nickel(II) sulfate,

$\mathrm{K}_{2} \mathrm{Ni}_{2}\left(\mathrm{SO}_{4}\right)_{3} \ldots \ldots \ldots \ldots \ldots \ldots \ldots$

Potassium niobium fluoride, $\mathrm{K}_{2} \mathrm{NbF}_{7}$

Potassium niobium oxide, $\mathrm{KNbO}_{3} \ldots$

Potassium nitrate (niter), $\mathrm{KNO}_{3} \ldots$

Potassium nitrite, $\mathrm{KNO}_{2} \ldots \ldots \ldots$

Potassium nitrosyl ruthenium

chloride, $\mathrm{K}_{2} \mathrm{NORuCl}_{5} \ldots \ldots \ldots \ldots \ldots$

Potassium oxide, $\mathrm{K}_{2} \mathrm{O} \ldots \ldots \ldots \ldots$

Potassium platinum bromide, $\mathrm{K}_{2} \mathrm{PtBr}_{6}$

Potassium platinum chloride,

$\mathrm{K}_{2} \mathrm{PtCl}_{6} \ldots \ldots \ldots \ldots \ldots \ldots \ldots \ldots$

Potassium platinum fluoride, $\mathrm{K}_{2} \mathrm{PtF}_{6}$

Potassium rhenium chloride, $\mathrm{K}_{2} \operatorname{ReCl}_{6}$

Potassium rhenium oxide, $\mathrm{KReO}_{4} \ldots$.

Potassium rubidium chloride,

$\mathrm{K}_{0.5} \mathrm{Rb}_{0.5} \mathrm{Cl} \ldots \ldots \ldots \ldots \ldots \ldots \ldots$

Potassium rubidium chromium oxide,

$\mathrm{KRbCrO}_{4} \ldots \ldots \ldots \ldots \ldots \ldots \ldots$

Potassium ruthenium chloride,

$\mathrm{K}_{2} \mathrm{RuCl}_{6} \ldots \ldots \ldots \ldots \ldots \ldots \ldots \ldots \ldots$

Potassium ruthenium oxide chloride

hydrate, $\mathrm{K}_{4} \mathrm{Ru}_{2} \mathrm{OCl}_{10} \cdot \mathrm{H}_{2} \mathrm{O} \ldots \ldots \ldots$

Potassium selenate, $\mathrm{K}_{2} \mathrm{SeO}_{4} \ldots \ldots \ldots$

Potassium selenide, $\mathrm{K}_{2} \mathrm{Se} \ldots \ldots \ldots$

Potassium selenium bromide, $\mathrm{K}_{2} \mathrm{SeBr}_{6}$

Potassium silicon fluoride

(hieratite), $\mathrm{K}_{2} \mathrm{SiF}_{6} \ldots \ldots \ldots \ldots$

Potassium silver cyanide, $\mathrm{KAg}(\mathrm{CN})_{2}$

Potassium sodium aluminum fluoride

(elpasolite), $\mathrm{K}_{2} \mathrm{NaAlF}_{6} \ldots \ldots \ldots$

Potassium sodium bromide,

$\mathrm{K},{ }_{2} \mathrm{Na}{ }_{8} \mathrm{Br} \ldots \ldots \ldots \ldots \ldots \ldots \ldots$

Potassium sodium bromide,

$\mathrm{K} .{ }_{4} \mathrm{Na}{ }_{6} \mathrm{Br} \ldots \ldots \ldots \ldots \ldots \ldots \ldots$

Potassium sodium bromide,

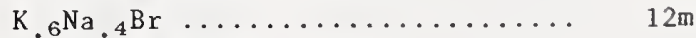

$12 \mathrm{~m}$

Potassium sodium bromide,

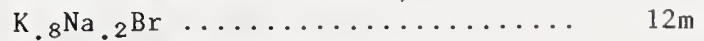

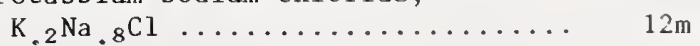

Potassium sodium chle, 
Potassium sodium chloride,

$\mathrm{K},{ }_{4} \mathrm{Na} .{ }_{6} \mathrm{Cl} \ldots \ldots \ldots \ldots \ldots$

$12 \mathrm{~m}$

Potassium sodium chloride,

$\mathrm{K} .{ }_{6} \mathrm{Na}{ }_{4} \mathrm{Cl} \ldots \ldots \ldots \ldots \ldots \ldots$.

$\mathrm{K},{ }_{8} \mathrm{Na}{ }_{2} \mathrm{Cl} \ldots \ldots \ldots \ldots \ldots \ldots \ldots$

Potassium sodium sulfate,

$\mathrm{K} .6{ }_{7} \mathrm{Na}_{1.33} \mathrm{SO}_{4} \ldots \ldots \ldots \ldots \ldots \ldots \ldots$

Potassium sodium sulfate, $\mathrm{KNaSO}_{4} \ldots$

Potassium sodium sulfate

(aphthitalite), $\mathrm{K}_{3} \mathrm{Na}\left(\mathrm{SO}_{4}\right)_{2} \ldots \ldots$

Potassium strontium chromium oxide,

$\mathrm{K}_{2} \mathrm{Sr}\left(\mathrm{CrO}_{4}\right)_{2} \ldots \ldots \ldots \ldots \ldots \ldots \ldots$

Potassium strontium niobium oxide,

$\mathrm{KSr}_{2}\left(\mathrm{NbO}_{3}\right)_{5} \ldots \ldots \ldots \ldots \ldots \ldots$

Potassium strontium phosphate,

$\mathrm{KSrPO}_{4} \ldots \ldots \ldots \ldots \ldots \ldots \ldots \ldots \ldots$

Potassium strontium selenate,

$\mathrm{K}_{2} \mathrm{Sr}\left(\mathrm{SeO}_{4}\right)_{2} \ldots \ldots \ldots \ldots \ldots \ldots$

Potassium strontium sulfate

(kalistrontite), $\mathrm{K}_{2} \mathrm{Sr}\left(\mathrm{SO}_{4}\right)_{2} \ldots \ldots$

Potassium sulfate, $\mathrm{K}_{2} \mathrm{~S}_{2} \mathrm{O}_{7} \ldots \ldots \ldots$

Potassium sulfate, $\mathrm{K}_{2} \mathrm{~S}_{2} \mathrm{O}_{8} \ldots \ldots \ldots$

Potassium sulfate (arcanite), $\mathrm{K}_{2} \mathrm{SO}_{4}$

Potassium sulfide, $\mathrm{K}_{2} \mathrm{~S} \ldots \ldots \ldots \ldots$.

Potassium telluride, $\mathrm{K}_{2} \mathrm{Te} \ldots \ldots \ldots$

Potassium thiocyanate, KCNS .......

Potassium tin chloride, $\mathrm{K}_{2} \mathrm{SnCl}_{6} \ldots$

Potassium titanium fluoride, $\mathrm{K}_{2} \mathrm{TiF}_{6}$

Potassium titanium phosphate,

$\mathrm{KTi}_{2}\left(\mathrm{PO}_{4}\right)_{3} \ldots \ldots \ldots \ldots \ldots \ldots$

Potassium tungsten oxide, $\mathrm{K}_{2} \mathrm{WO}_{4} \ldots$

Potassium vanadium oxide, $\mathrm{KVO}_{3} \ldots$

Potassium vanadium oxide, $\mathrm{KV}_{3} \mathrm{O}_{8} \ldots$

Potassium zinc bromide hydrate,

$\mathrm{KZnBr}_{3} \cdot 2 \mathrm{H}_{2} \mathrm{O} \ldots \ldots \ldots \ldots \ldots \ldots$

Potassium zinc fluoride, $\mathrm{KZnF}_{3} \ldots$

Potassium zinc fluoride, $\mathrm{K}_{2} \mathrm{ZnF}_{4} \ldots$

Potassium zinc iodide hydrate,

$\mathrm{KZnI}_{3} \cdot 2 \mathrm{H}_{2} \mathrm{O} \ldots \ldots \ldots \ldots \ldots \ldots$

Potassium zinc phosphate, $\mathrm{KZnPO}_{4}$.

Potassium zinc sulfate, $\mathrm{K}_{2} \mathrm{Zn}_{2}\left(\mathrm{SO}_{4}\right)_{3}$

Potassium zinc sulfate hydrate,

$\mathrm{K}_{2} \mathrm{Zn}\left(\mathrm{SO}_{4}\right)_{2} \cdot 6 \mathrm{H}_{2} \mathrm{O} \ldots \ldots \ldots \ldots \ldots$

$6 \mathrm{~m}$

$15 m$

57

$20 m$

74

$19 \mathrm{~m}$

$15 \mathrm{~m}$

$10 \mathrm{~m}$

$10 \mathrm{~m}$

\section{8}

6

7

$20 m$

$11 \mathrm{~m}$

$18 \mathrm{~m}$

$8 m$

$11 \mathrm{~m}$

5

$10 \mathrm{~m}$

$11 \mathrm{~m}$

$20 \mathrm{~m}$

$6 \mathrm{~m}$

$7 m$

Potassium zinc vanadium oxide

hydrate, $\mathrm{K}_{2} \mathrm{Zn}_{2} \mathrm{~V}_{10} \mathrm{O}_{28} \cdot 16 \mathrm{H}_{2} \mathrm{O} \ldots \ldots$

Potassium zirconium fluoride,

$\mathrm{K}_{3} \mathrm{ZrF}_{7} \ldots \ldots \ldots \ldots \ldots \ldots \ldots$

Praseodymium arsenate, $\mathrm{PrAsO}_{4} \ldots \ldots$

Praseodymium arsenide, PrAs .......

Praseodymium chloride, $\mathrm{PrCl}_{3} \ldots \ldots$.

Praseodymium chloride oxide, Procl

Praseodymium fluoride, $\operatorname{PrF}_{3} \ldots \ldots$.

Praseodymium sulfide, $\operatorname{PrS} \ldots . . .$.

Praseodymium vanadium oxide, $\mathrm{PrVO}_{4}$

Praseodymium zinc, $\operatorname{PrZn} \ldots . . \ldots \ldots$.

Rhenium, $\operatorname{Re} \ldots \ldots \ldots \ldots \ldots \ldots$

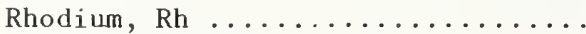

Rhodium vanadium, $\operatorname{RhV}_{3} \ldots \ldots \ldots \ldots$

Rubidium aluminum sulfate

hydrate, $\operatorname{RbAl}\left(\mathrm{SO}_{4}\right)_{2} \cdot 12 \mathrm{H}_{2} \mathrm{O} \ldots \ldots$.

Rubidium amide, $\mathrm{RbNH}_{2} \ldots \ldots \ldots \ldots$

Rubidium barium chromium oxide,

$\mathrm{Rb}_{2} \mathrm{Ba}\left(\mathrm{CrO}_{4}\right)_{2} \ldots \ldots \ldots \ldots \ldots \ldots$

Rubidium barium molybdenum oxide,

$\mathrm{Rb}_{2} \mathrm{Ba}\left(\mathrm{MoO}_{4}\right)_{2} \ldots \ldots \ldots \ldots \ldots \ldots$

Rubidium bromate, $\mathrm{RbBrO}_{3} \ldots \ldots \ldots$.

Rubidium bromide, $\mathrm{RbBr} \ldots \ldots \ldots \ldots$
Rubidium cadmium chloride, high

form, $\mathrm{RbCdCl}_{3}$ (tetragonal) ..... $5 \mathrm{~m}$

Rubidium cadmium chloride,

low form, $\mathrm{RbCdCl}_{3}$ (orthorhombic)

Rubidium cadmium sulfate,

$\mathrm{Rb}_{2} \mathrm{Cd}_{2}\left(\mathrm{SO}_{4}\right)_{3} \ldots \ldots \ldots \ldots \ldots \ldots$

Rubidium calcium chloride, $\mathrm{RbCaCl}_{3}$

Rubidium calcium fluoride, $\mathrm{RbCaF}_{3}$

Rubidium calcium sulfate,

$\mathrm{Rb}_{2} \mathrm{Ca}_{2}\left(\mathrm{SO}_{4}\right)_{3} \ldots \ldots \ldots \ldots \ldots \ldots$

Rubidium chlorate, $\mathrm{RbClO}_{3} \ldots \ldots \ldots$

Rubidium chlorate, $\mathrm{RbClO}_{4} \ldots \ldots \ldots$

Rubidium chloride, $\mathrm{RbCl} \ldots . . \ldots \ldots$

Rubidium chromium oxide, $\mathrm{Rb}_{2} \mathrm{CrO}_{4} \ldots$

Rubidium chromium oxide, $\mathrm{Rb}_{2} \mathrm{Cr}_{2} \mathrm{O}_{7}$

Rubidium chromium sulfate hydrate,

$\operatorname{RbCr}\left(\mathrm{SO}_{4}\right)_{2} \cdot 12 \mathrm{H}_{2} \mathrm{O} \ldots \ldots \ldots \ldots$

Rubidium cobalt(II) chloride,

$\mathrm{RbCoCl}_{3} \ldots \ldots \ldots \ldots \ldots \ldots \ldots$

Rubidium cobalt fluoride, $\mathrm{RbCoF}_{3} \ldots$

Rubidium cobalt sulfate,

$\mathrm{Rb}_{2} \mathrm{Co}_{2}\left(\mathrm{SO}_{4}\right)_{3} \ldots \ldots \ldots \ldots \ldots \ldots$

Rubidium copper chloride hydrate,

$\mathrm{Rb}_{2} \mathrm{CuCl}_{4} \cdot 2 \mathrm{H}_{2} \mathrm{O} \ldots \ldots \ldots \ldots \ldots \ldots$

$\mathrm{Rb}_{2} \mathrm{Cu}\left(\mathrm{SO}_{4}\right)_{2} \cdot 6 \mathrm{H}_{2} \mathrm{O} \ldots \ldots \ldots \ldots \ldots$

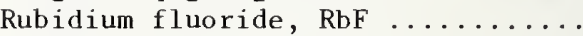

Rubidium hydrogen phosphate,

$\mathrm{RbH}_{2} \mathrm{PO}_{4} \ldots \ldots \ldots \ldots \ldots \ldots \ldots$

Rubidium iodate, $\mathrm{RbIO}_{3} \ldots \ldots \ldots \ldots$

Rubidium iodate, $\mathrm{RbIO}_{4} \ldots \ldots \ldots \ldots$

Rubidium iodide, RbI ...........

Rubidium iron chloride hydrate,

$\mathrm{Rb}_{2} \mathrm{FeCl}_{5} \cdot \mathrm{H}_{2} \mathrm{O} \ldots \ldots \ldots \ldots \ldots \ldots \ldots \ldots \quad 14 \mathrm{~m} \quad 33$

Rubidium iron sulfate hydrate,

$\mathrm{Rb}_{2} \mathrm{Fe}\left(\mathrm{SO}_{4}\right)_{2} \cdot 6 \mathrm{H}_{2} \mathrm{O} \ldots \ldots \ldots \ldots \ldots$. $8 \mathrm{~m}$ 64

Rubidium lead chromium oxide,

$\mathrm{Rb}_{2} \mathrm{~Pb}\left(\mathrm{CrO}_{4}\right)_{2} \ldots \ldots \ldots \ldots \ldots \ldots \ldots \ldots$

$14 \mathrm{~m} \quad 34$

Rubidium lead molybdenum oxide,

$\mathrm{Rb}_{2} \mathrm{~Pb}\left(\mathrm{MoO}_{4}\right)_{2} \ldots \ldots \ldots \ldots \ldots \ldots$

$15 m$

Rubidium magnesium chromium oxide,

$\mathrm{Rb}_{2} \mathrm{Mg}_{2}\left(\mathrm{CrO}_{4}\right)_{3} \ldots \ldots \ldots \ldots \ldots \ldots$

Rubidium magnesium chromium oxide

hydrate, $\mathrm{Rb}_{2} \mathrm{Mg}\left(\mathrm{CrO}_{4}\right)_{2} \cdot 6 \mathrm{H}_{2} \mathrm{O} \ldots \ldots$.

Rubidium magnesium sulfate,

$\mathrm{Rb}_{2} \mathrm{Mg}_{2}\left(\mathrm{SO}_{4}\right)_{3} \ldots \ldots \ldots \ldots \ldots$

Rubidium magnesium sulfate

hydrate, $\mathrm{Rb}_{2} \mathrm{Mg}\left(\mathrm{SO}_{4}\right)_{2} \cdot 6 \mathrm{H}_{2} \mathrm{O} \ldots \ldots$.

Rubidium manganese(II) fluoride,

$\mathrm{RbMnF}_{3} \ldots \ldots \ldots \ldots \ldots \ldots \ldots \ldots \ldots \ldots \ldots$

Rubidium manganese sulfate,

$\mathrm{Rb}_{2} \mathrm{Mn}_{2}\left(\mathrm{SO}_{4}\right)_{3} \ldots \ldots \ldots \ldots \ldots \ldots \ldots \ldots$

Rubidium nickel(II) chloride,

$\mathrm{RbNiCl}_{3} \ldots \ldots \ldots \ldots \ldots \ldots \ldots \ldots$

Rubidium nickel sulfate,

$\mathrm{Rb}_{2} \mathrm{Ni}_{2}\left(\mathrm{SO}_{4}\right)_{3} \ldots \ldots \ldots \ldots \ldots \ldots \ldots$

Rubidium nickel sulfate hydrate,

$\mathrm{Rb}_{2} \mathrm{Ni}\left(\mathrm{SO}_{4}\right)_{2} \cdot 6 \mathrm{H}_{2} \mathrm{O} \ldots \ldots \ldots \ldots \ldots$

Rubidium nitrate, $\mathrm{RbNO}_{3}$ (trigonal)

Rubidium phosphate, $\mathrm{RbPO}_{3} \ldots \ldots \ldots$

Rubidium platinum chloride,

$\mathrm{Rb}_{2} \mathrm{PtCl}_{6} \ldots \ldots \ldots \ldots \ldots \ldots \ldots \ldots \ldots$

Rubidium platinum fluoride, $\mathrm{Rb}_{2} \mathrm{PtF}_{6}$

Rubidium selenate, $\mathrm{Rb}_{2} \mathrm{SeO}_{4} \ldots \ldots \ldots$

Rubidium silicon fluoride, $\mathrm{Rb}_{2} \mathrm{SiF}_{6}$

Rubidium strontium chloride,

$\mathrm{RbSrCl}_{3} \ldots \ldots \ldots \ldots \ldots \ldots$

Rubidium strontium chromium oxide,

$\mathrm{Rb}_{2} \mathrm{Sr}\left(\mathrm{CrO}_{4}\right)_{2} \ldots \ldots \ldots \ldots \ldots \ldots \ldots \ldots \ldots$
$8 \mathrm{~m}$

$5 m$

$20 m$

5

6
$9 \mathrm{~m}$

6

$7 m$

54 
Rubidium strontium niobium oxide,

$\mathrm{RbSr}_{2}\left(\mathrm{NbO}_{3}\right)_{5} \ldots \ldots \ldots \ldots \ldots \ldots$

Rubidium strontium sulfate,

$\mathrm{Rb}_{2} \mathrm{Sr}\left(\mathrm{SO}_{4}\right)_{2} \ldots \ldots \ldots \ldots \ldots \ldots$

Rubidium sulfate, $\mathrm{Rb}_{2} \mathrm{SO}_{4} \ldots \ldots \ldots$

Rubidium tellurium bromide,

$\mathrm{Rb}_{2} \mathrm{TeBr}_{6} \ldots \ldots \ldots \ldots \ldots \ldots \ldots$

Rubidium tellurium chloride,

$\mathrm{Rb}_{2} \mathrm{TeCl}_{6} \ldots \ldots \ldots \ldots \ldots \ldots \ldots$

Rubidium tin chloride, $\mathrm{Rb}_{2} \mathrm{SnCl}_{6} \ldots$

Rubidium zinc fluoride, $\mathrm{RbZnF}_{3} \ldots$

Rubidium zinc sulfate hydrate,

$\mathrm{Rb}_{2} \mathrm{Zn}\left(\mathrm{SO}_{4}\right)_{2} \cdot 6 \mathrm{H}_{2} \mathrm{O} \ldots \ldots \ldots \ldots \ldots$

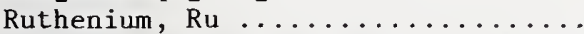

Ruthenium titanium, RuTi ........

Samarium arsenate, $\mathrm{SmAsO}_{4} \ldots \ldots \ldots$

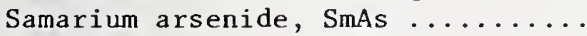

Samarium chloride, $\mathrm{SmCl}_{3} \ldots \ldots \ldots$

Samarium chloride oxide, SmOCl ....

Samarium fluoride, $\mathrm{SmF}_{3} \ldots \ldots \ldots \ldots$

Samarium oxide, $\mathrm{Sm}_{2} \mathrm{O}_{3}$ (cubic) .....

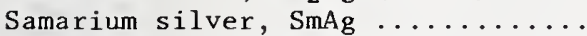

Samarium tin oxide, $\mathrm{Sm}_{2} \mathrm{Sn}_{2} \mathrm{O}_{7} \ldots \ldots$.

Sama rium vanadium oxide, $\mathrm{SmVO}_{4} \ldots$.

Scandium arsenate, $\mathrm{ScAsO}_{4} \ldots \ldots \ldots$.

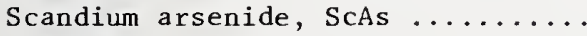

Scandium boride, $\mathrm{ScB}_{2} \ldots \ldots \ldots \ldots$

Scandium oxide, $\mathrm{Sc}_{2} \mathrm{O}_{3} \ldots \ldots \ldots \ldots$

Scandium phosphate, $\mathrm{ScPO}_{4} \ldots \ldots \ldots$

Scandium silicate (thortveitite),

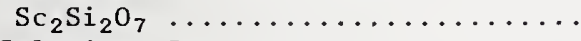

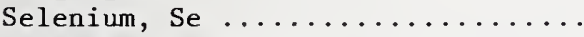

Selenium oxide (selenolite), $\mathrm{SeO}_{2}$

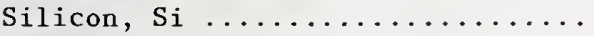

Silicon, Si (reference standard) ..

Silicon nitride, $\beta-\mathrm{Si}_{3} \mathrm{~N}_{4} \ldots \ldots \ldots$

Silicon nitride, $\beta-\mathrm{Si}_{3} \mathrm{~N}_{4}$

(calculated pattern)...

Silicon oxide ( $\alpha$ or low

cristobalite), $\mathrm{SiO}_{2}$ (tetragonal)

Silicon oxide ( $\alpha$ or low

cristobalite), $\mathrm{SiO}_{2}$ (tetragonal)

(calculated pattern) .............................

Silicon oxide (quartz, low), $\alpha-\mathrm{SiO}_{2}$

Silicon oxide ( $\beta$ or high

cristobalite), $\mathrm{SiO}_{2}$ (cubic) .....

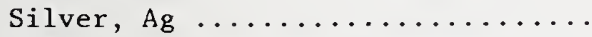

Silver, Ag (reference standard) ...

Silver arsenate, $\mathrm{Ag}_{3} \mathrm{AsO}_{4} \ldots \ldots \ldots$.

Silver arsenic sulfide,

xanthoconite, $\mathrm{Ag}_{3} \mathrm{AsS}_{3} \ldots \ldots \ldots \ldots$

Silver bromate, $\mathrm{AgBrO}_{3} \ldots \ldots \ldots \ldots$

Silver bromide (bromargyrite), $\mathrm{AgBr}$

Silver carbonate, $\mathrm{Ag}_{2} \mathrm{CO}_{3} \ldots \ldots \ldots$.

Silver chlorate, $\mathrm{AgClO}_{3} \ldots \ldots \ldots$.

Silver chloride (chlorargyrite),

$\mathrm{AgCl} \ldots \ldots \ldots \ldots \ldots \ldots \ldots \ldots \ldots \ldots \ldots \ldots \ldots$

Silver chromium oxide, $\mathrm{Ag}_{2} \mathrm{CrO}_{4} \ldots$

Silver cyanide, $\mathrm{AgCN} \ldots \ldots \ldots \ldots \ldots$

Silver fluoride, $\mathrm{Ag}_{2} \mathrm{~F} \ldots \ldots \ldots \ldots$

Silver iodate, $\mathrm{AgIO}_{4} \ldots \ldots \ldots \ldots$.

Silver iodide (iodargyrite), AgI

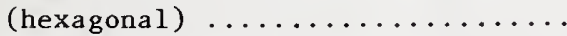

Silver iodide, $\gamma-\mathrm{AgI}$ (cubic) ......

Silver manganese oxide, $\mathrm{AgMnO}_{4} \ldots$. Silver mercury iodide, $\beta-\mathrm{Ag}_{2} \mathrm{HgI}_{4} \ldots$

Silver molybdenum oxide, $\mathrm{Ag}_{2} \mathrm{MoO}_{4} \ldots$

Silver nitrate, $\mathrm{AgNO}_{3}$
$20 \mathrm{~m} \quad 8$

$15 \mathrm{~m} \quad 65$

848

846

$8 \quad 48$

646

$7 \mathrm{~m} \quad 57$

$7 \mathrm{~m} \quad 55$

$4 \quad 5$

$6 \mathrm{~m} \quad 86$

$4 \mathrm{~m} \quad 33$

$4 \mathrm{~m} \quad 68$

$1 \mathrm{~m} \quad 40$

$1 \mathrm{~m} \quad 43$

$1 \mathrm{~m} \quad 41$

$4 \mathrm{~m} \quad 34$

$5 \mathrm{~m} \quad 73$

$8 \mathrm{~m} \quad 77$

$5 \mathrm{~m} \quad 47$

$4 \mathrm{~m} \quad 35$

$4 \mathrm{~m} \quad 68$

$17 \mathrm{~m} \quad 66$

$3 \quad 27$

850

$7 \mathrm{~m} \quad 58$

$5 \quad 54$

$7 \mathrm{~m} \quad 60$

$13 \mathrm{~m} \quad 35$

$12 \mathrm{~m} \quad 2$

$18 \mathrm{~m} \quad 59$

$14 \mathrm{~m} \quad 116$

10

48

$15 \mathrm{~m} \quad 180$

$18 \mathrm{~m} \quad 61$

142

123

$8 \mathrm{~m} \quad 2$

$5 \quad 56$

$8 \mathrm{~m} \quad 126$

$5 \quad 57$

$4 \quad 46$

$13 \mathrm{~m} \quad 36$

$7 \quad 44$

$4 \quad 44$

$12 \mathrm{~m} \quad 30$

$9 \mathrm{~m} \quad 48$

$5 \mathrm{~m} \quad 53$

$9 \quad 49$

$8 \quad 51$

948

$7 \mathrm{~m} \quad 155$

$17 \mathrm{~m} \quad 67$

$7 \quad 45$

$5 \quad 59$
Silver nitrite, $\mathrm{AgNO}_{2} \ldots \ldots \ldots \ldots \ldots . \ldots$

Silver oxide, $\mathrm{Ag}_{2} \mathrm{O} \ldots \ldots \ldots \ldots \ldots \ldots$ 1m 45

Silver(II) oxide nitrate, $\mathrm{Ag}_{7} \mathrm{O}_{8} \mathrm{NO}_{3} \quad 4 \quad 61$

Silver phosphate, $\mathrm{Ag}_{3} \mathrm{PO}_{4} \ldots \ldots \ldots \ldots .62$

Silver rhenium oxide, $\mathrm{AgReO}_{4} \ldots \ldots . \quad 8 \quad 53$

Silver selenate, $\mathrm{Ag}_{2} \mathrm{SeO}_{4} \ldots \ldots \ldots . .2 \mathrm{~m} \quad 32$

Silver sodium chloride,

$\mathrm{Ag}_{0 .} \mathrm{Na}_{0.5} \mathrm{Cl} \ldots \ldots \ldots \ldots \ldots \ldots \ldots$ 8m $\quad$ m 79

Silver sulfate, $\mathrm{Ag}_{2} \mathrm{SO}_{4} \ldots \ldots \ldots \ldots . . .13 \mathrm{~m} \quad 37$

Silver sulfide (acanthite), $\mathrm{Ag}_{2} \mathrm{~S} \ldots \quad 10 \quad 51$

Silver telluride (hessite),

$\mathrm{Ag}_{2} \mathrm{Te} \ldots \ldots \ldots \ldots \ldots \ldots \ldots$ 19m 73

Silver terbium, AgTb .......... 5m 74

Silver thiocyanate, AgCNS ....... 16m 62

Silver thulium, AgTm ......... 5m 74

Silver yttrium, AgY .......... 5m 75

Sodium, Na ............... 9m 105

Sodium aluminum chloride silicate,

sodalite, $\mathrm{Na}_{8} \mathrm{Al}_{6} \mathrm{Cl}_{2}\left(\mathrm{SiO}_{4}\right)_{6} \ldots \ldots$.

Sodium aluminum fluoride (chiolite),

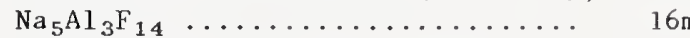

$7 \mathrm{~m} \quad 158$

Sodium aluminum oxide, $\beta-\mathrm{NaAlO}_{2} \ldots$

Sodium aluminum sulfate hydrate

(soda alum), $\mathrm{NaAl}\left(\mathrm{SO}_{4}\right)_{2} \cdot 12 \mathrm{H}_{2} \mathrm{O} \ldots . \quad 15 \mathrm{~m}$

Sodium antimony fluoride, $\mathrm{NaSbF}_{4} \quad 20 \mathrm{~m}$

Sodium azide, $\alpha-\mathrm{NaN}_{3}$, at -90 to

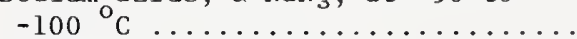

Sodium azide, $\beta-\mathrm{NaN}_{3} \ldots \ldots \ldots \ldots$

Sodium barium niobium oxide,

$\mathrm{NaBa}_{2}\left(\mathrm{NbO}_{3}\right)_{5} \ldots \ldots \ldots \ldots \ldots \ldots$

Sodium barium phosphate, $\mathrm{NaBaPO}_{4} \ldots \quad 19 \mathrm{~m}$

Sodium beryllium calcium aluminum

fluoride oxide silicate, meliphanite,

$\left(\mathrm{Na}_{0.63} \mathrm{Ca}_{1.37}\right) \mathrm{Be}\left(\mathrm{Al}_{0.13} \mathrm{Si}_{1.87}\right)$

$\left(\mathrm{F}_{\left.0.75^{0} \mathrm{O}_{6.25}\right) \ldots \ldots \ldots \ldots \ldots \ldots \ldots \ldots \ldots \ldots \ldots}\right.$

Sodium beryllium calcium fluoride

silicate, leucophanite,

$\mathrm{NaBeCaFS} i_{2} \mathrm{O}_{6} \ldots \ldots \ldots \ldots \ldots \ldots \ldots$

Sodium borate, $\mathrm{NaBO}_{2} \ldots \ldots \ldots \ldots \ldots \quad 18 \mathrm{~m}$

Sodium borate, $\mathrm{Na}_{2} \mathrm{~B}_{4} \mathrm{O}_{7} \ldots \ldots \ldots \ldots \quad 16 \mathrm{~m}$

Sodium borate, $\mathrm{Na}_{2} \mathrm{~B}_{8} \mathrm{O}_{13} \ldots \ldots \ldots \ldots \quad 7 \mathrm{~m}$

Sodium borate hydroxide hydrate

(borax), $\mathrm{Na}_{2} \mathrm{~B}_{4} \mathrm{O}_{5}(\mathrm{OH})_{4} \cdot 8 \mathrm{H}_{2} \mathrm{O} \ldots \ldots . . .6 \mathrm{~m}$

Sodium boron hydride, $\mathrm{NaBH}_{4} \ldots \ldots \ldots$

Sodium bromate, $\mathrm{NaBrO}_{3} \ldots \ldots \ldots \ldots$.

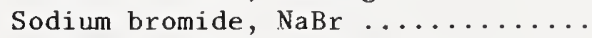

Sodium bromide chloride,

$\mathrm{NaBr}{ }_{33}{ }^{\mathrm{Cl}}{ }_{.67} \ldots \ldots \ldots \ldots \ldots \ldots \ldots$

Sodium bromide chloride,

$\mathrm{NaBr}{ }_{67}{ }_{7} \mathrm{Cl} .33 \ldots \ldots \ldots \ldots \ldots \ldots .11 \mathrm{~m}$

Sodium calcium aluminum fluoride

hydrate, thomsenolite,

$\mathrm{NaCaAlF}_{6} \cdot \mathrm{H}_{2} \mathrm{O} \ldots \ldots \ldots \ldots . \ldots . \ldots . . . . .6 \mathrm{~m}$

Sodium calcium carbonate hydrate,

pirssonite, $\mathrm{Na}_{2} \mathrm{Ca}\left(\mathrm{CO}_{3}\right)_{2} \cdot 2 \mathrm{H}_{2} \mathrm{O} \ldots . .9 \mathrm{~m} \quad 106$

Sodium calcium phosphate, $\beta-\mathrm{NaCaPO}_{4} \quad 15 \mathrm{~m} \quad 69$

Sodium calcium silicate, $\mathrm{Na}_{2} \mathrm{CaSiO}_{4} \quad 10 \mathrm{~m} \quad 48$

Sodium calcium sulfate (glauberite),

$\mathrm{Na}_{2} \mathrm{Ca}\left(\mathrm{SO}_{4}\right)_{2} \ldots \ldots \ldots \ldots \ldots \ldots \ldots \ldots$

Sodium carbonate hydrate (thermo-

natrite), $\mathrm{Na}_{2} \mathrm{CO}_{3} \cdot \mathrm{H}_{2} \mathrm{O} \ldots \ldots \ldots \ldots$

Sodium carbonate sulfate, $\mathrm{Na}_{4} \mathrm{CO}_{3} \mathrm{SO}_{4}$

Sodium carbonate sulfate (burkeite),

$\mathrm{Na}_{6} \mathrm{CO}_{3}\left(\mathrm{SO}_{4}\right)_{2} \ldots \ldots \ldots \ldots \ldots \ldots$

Sodium carbonate sulfate,

$\mathrm{Na}_{6} \mathrm{CO}_{3}\left(\mathrm{SO}_{4}\right)_{2} \ldots \ldots \ldots \ldots \ldots \ldots$

Sodium carbonate sulfate,

$\mathrm{Na}_{6}\left(\mathrm{CO}_{3}\right)_{2} \mathrm{SO}_{4}$
63

62

68

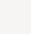

130

83

75

135

138

63

160

66

51

65

47

49

50

132

6
69
48
59

8

54

$11 \mathrm{~m} \quad 51$

$11 \mathrm{~m}$

52

53

54 
Vol. or

Sec. Page

Sodium chlorate, $\mathrm{NaClO}_{3} \ldots \ldots \ldots \ldots$

Sodium chlorate, $\mathrm{NaClO}_{4}$

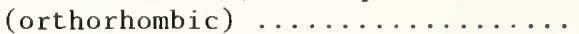

Sodium chlorate hydrate,

$\mathrm{NaClO}_{4} \cdot \mathrm{H}_{2} \mathrm{O} \ldots \ldots \ldots \ldots \ldots \ldots \ldots$

Sodium chloride (halite), $\mathrm{NaCl}$....

Sodium chromium oxide, $\mathrm{Na}_{2} \mathrm{CrO}_{4} \ldots$.

Sodium chromium oxide hydrate,

$\mathrm{Na}_{2} \mathrm{CrO}_{4} \cdot 4 \mathrm{H}_{2} \mathrm{O} \ldots \ldots \ldots \ldots \ldots \ldots$

Sodium chromium oxide hydrate,

$\mathrm{Na}_{2} \mathrm{Cr}_{2} \mathrm{O}_{7} \cdot 2 \mathrm{H}_{2} \mathrm{O} \ldots \ldots \ldots \ldots \ldots \ldots$

Sodium chromium oxide sulfate,

$\mathrm{Na}_{4}\left(\mathrm{CrO}_{4}\right)\left(\mathrm{SO}_{4}\right) \ldots \ldots \ldots \ldots \ldots \ldots$

Sodium cobalt nitrite, $\mathrm{Na}_{3} \mathrm{Co}\left(\mathrm{NO}_{2}\right)_{6}$

Sodium cobalt(II) sulfate hydrate,

$\mathrm{Na}_{2} \mathrm{Co}\left(\mathrm{SO}_{4}\right)_{2} \cdot 4 \mathrm{H}_{2} \mathrm{O} \ldots \ldots \ldots \ldots \ldots$

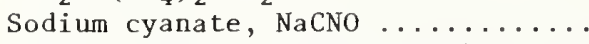

Sodium cyanide, $\mathrm{NaCN}$ (cubic) ......

Sodium cyanide, $\mathrm{NaCN}$ (orthorhombic) at $6{ }^{\circ} \mathrm{C} \ldots \ldots \ldots \ldots \ldots \ldots \ldots \ldots \ldots \ldots$

Sodium fluoride (villiaumite), NaF

Sodium germanium phosphate,

$\mathrm{NaGe}_{2}\left(\mathrm{PO}_{4}\right)_{3} \ldots \ldots \ldots \ldots \ldots \ldots$

Sodium hydrogen carbonate hydrate,

trona, $\mathrm{Na}_{3} \mathrm{H}\left(\mathrm{CO}_{3}\right)_{2} \cdot 2 \mathrm{H}_{2} \mathrm{O} \ldots \ldots \ldots$.

Sodium hydrogen fluoride, $\mathrm{NaHF}_{2} \ldots$

Sodium hydrogen phosphate,

$\mathrm{Na}_{3} \mathrm{H}\left(\mathrm{PO}_{3}\right)_{4} \ldots \ldots \ldots \ldots \ldots \ldots$

Sodium hydrogen silicate hydrate,

$\mathrm{Na}_{2} \mathrm{H}_{2} \mathrm{SiO}_{4} \cdot 4 \mathrm{H}_{2} \mathrm{O} \ldots \ldots \ldots \ldots \ldots$

Sodium hydrogen sulfate hydrate,

$\mathrm{NaHSO}_{4} \cdot \mathrm{H}_{2} \mathrm{O} \ldots \ldots \ldots \ldots \ldots \ldots$

Sodium hydroxide, $\mathrm{NaOH}$ at $300{ }_{\mathrm{C}} \ldots$

Sodium iodate, $\mathrm{NaIO}_{3} \ldots \ldots \ldots \ldots \ldots$

Sodiun iodate, $\mathrm{NaIO}_{4} \ldots \ldots \ldots \ldots \ldots$

Sodium iodate hydrate, $\mathrm{NaIO}_{3} \cdot \mathrm{H}_{2} \mathrm{O} \ldots$

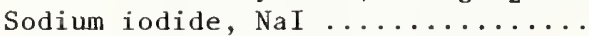

Sodium iron fluoride, $\mathrm{Na}_{3} \mathrm{FeF}_{6} \ldots .$.

Sodium iron silicate (acmite),

$\mathrm{NaFe}\left(\mathrm{SiO}_{3}\right)_{2} \ldots \ldots \ldots \ldots \ldots \ldots \ldots$

Sodium lanthanum fluoride silicate,

$\left(\mathrm{Na}_{2} \mathrm{La}_{8}\right) \mathrm{F}_{2}\left(\mathrm{SiO}_{4}\right)_{6} \ldots \ldots \ldots \ldots \ldots$

Sodium lanthanum molybdenum oxide,

$\mathrm{NaLa}\left(\mathrm{MoO}_{4}\right)_{2} \ldots \ldots \ldots \ldots \ldots \ldots \ldots \ldots$

Sodium magnesium aluminum boron

hydroxide silicate, dravite,

$\mathrm{NaMg}_{3} \mathrm{Al}_{6} \mathrm{~B}_{3}(\mathrm{OH})_{4} \mathrm{Si}_{6} \mathrm{O}_{27} \ldots \ldots \ldots \ldots$ 3m

Sodium magnesium carbonate

(eitelite), $\mathrm{Na}_{2} \mathrm{Mg}\left(\mathrm{CO}_{3}\right)_{2} \ldots \ldots \ldots$

Sodium magnesium sulfate

(vanthoffite), $\mathrm{Na}_{6} \mathrm{Mg}\left(\mathrm{SO}_{4}\right)_{4} \ldots \ldots$.

Sodium magnesium sulfate hydrate,

bloedite, $\mathrm{Na}_{2} \mathrm{Mg}\left(\mathrm{SO}_{4}\right)_{2} \cdot 4 \mathrm{H}_{2} \mathrm{O} \ldots \ldots$.

Sodium magnesium sulfate hydrate

(loeweite), $\mathrm{Na}_{12} \mathrm{Mg}_{7}\left(\mathrm{SO}_{4}\right)_{13} \cdot 15 \mathrm{H}_{2} \mathrm{O}$

Sodium manganese(II) fluoride,

$\mathrm{NaMnF}_{3} \ldots \ldots \ldots \ldots \ldots \ldots \ldots \ldots \ldots \ldots$. . . . . . .

$\mathrm{Na}_{12} \mathrm{Mn}_{7}\left(\mathrm{SO}_{4}\right)_{13} \cdot 15 \mathrm{H}_{2} \mathrm{O} \ldots \ldots \ldots \ldots$

Sodium mercury(II) chloride hydrate,

$\mathrm{NaHgCl}_{3} \cdot 2 \mathrm{H}_{2} \mathrm{O} \ldots \ldots \ldots \ldots \ldots$

Sodium molybdenum oxide, $\mathrm{Na}_{2} \mathrm{MoO}_{4} \ldots$

Sodium molybdenum oxide, $\mathrm{Na}_{2} \mathrm{Mo}_{2} \mathrm{O}_{7}$

Sodium molybdenum oxide hydrate,

$\mathrm{Na}_{2} \mathrm{MoO}_{4} \cdot 2 \mathrm{H}_{2} \mathrm{O} \ldots \ldots \ldots \ldots \ldots \ldots$

Sodium neodymium fluoride silicate,

$\left(\mathrm{Na}_{2} \mathrm{Nd}_{8}\right) \mathrm{F}_{2}\left(\mathrm{SiO}_{4}\right)_{6}$
$17 \mathrm{~m}$
Sodium nickel(II) sulfate hydrate,

$\mathrm{Na}_{2} \mathrm{Ni}\left(\mathrm{SO}_{4}\right)_{2} \cdot 4 \mathrm{H}_{2} \mathrm{O} \ldots \ldots \ldots \ldots$

Sodium niobium oxide (lueshite),

$\mathrm{NaNbO}_{3} \ldots \ldots \ldots \ldots \ldots \ldots \ldots$

Sodium nitrate (soda niter), $\mathrm{NaNO}_{3}$

Sodium nitrite, $\mathrm{NaNO}_{2} \ldots \ldots \ldots \ldots$

Sodium nitrosyl iron cyanide

hydrate, $\mathrm{Na}_{2}(\mathrm{NO}) \mathrm{Fe}(\mathrm{CN})_{5} \cdot 2 \mathrm{H}_{2} \mathrm{O} \ldots \ldots$

Sodium oxide, $\mathrm{Na}_{2} \mathrm{O} \ldots \ldots \ldots \ldots \ldots$

Sodium phosphate, $\mathrm{Na}_{3} \mathrm{P}_{3} \mathrm{O}_{9} \ldots \ldots \ldots$

Sodium phosphate hydrate,

$\mathrm{Na}_{3} \mathrm{P}_{3} \mathrm{O}_{9} \cdot \mathrm{H}_{2} \mathrm{O} \ldots \ldots \ldots \ldots$

Sodium phosphate hydrate,

$\alpha-\mathrm{Na}_{4} \mathrm{P}_{4} \mathrm{O}_{12} \cdot 4 \mathrm{H}_{2} \mathrm{O}$ (monoclinic) $\ldots . \quad 13 \mathrm{~m} \quad 39$

Sodium phosphate hydrate,

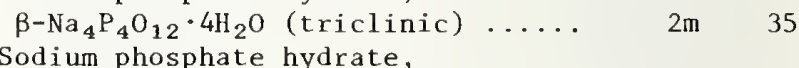

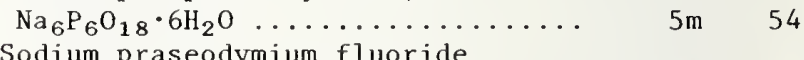

silicate, $\left(\mathrm{Na}_{2} \mathrm{Pr}_{8}\right) \mathrm{F}_{2}\left(\mathrm{SiO}_{4}\right)_{6} \ldots \ldots$

Sodium selenate, $\mathrm{Na}_{2} \mathrm{SeO}_{4} \ldots \ldots \ldots \ldots$

Sodium selenide, $\mathrm{Na}_{2} \mathrm{Se} \ldots \ldots \ldots \ldots$

Sodium silicate, $\alpha$ (III), $\mathrm{Na}_{2} \mathrm{Si}_{2} \mathrm{O}_{5}$.

Sodium silicate, $\beta-\mathrm{Na}_{2} \mathrm{Si}_{2} \mathrm{O}_{5} \ldots \ldots$.

Sodium silicon fluoride

(malladrite), $\mathrm{Na}_{2} \mathrm{SiF}_{6} \ldots \ldots \ldots$

$\mathrm{NaSr}_{2}\left(\mathrm{NbO}_{3}\right)_{5} \ldots \ldots \ldots \ldots \ldots \ldots$

Sodium strontium phosphate,

$\mathrm{NaSrPO}_{4} \ldots \ldots \ldots \ldots \ldots \ldots \ldots \ldots$

Sodium sulfate, $\mathrm{Na}_{2} \mathrm{SO}_{4} \ldots \ldots \ldots \ldots$

Sodium sulfate (thenardite), $\mathrm{Na}_{2} \mathrm{SO}_{4}$

Sodium sulfate hydrate,

$\mathrm{Na}_{2} \mathrm{~S}_{2} \mathrm{O}_{3} \cdot 5 \mathrm{H}_{2} \mathrm{O} \ldots \ldots \ldots \ldots \ldots \ldots \ldots$

Sodium sulfide, $\mathrm{Na}_{2} \mathrm{~S} \ldots \ldots \ldots \ldots \ldots$

Sodium telluride, $\mathrm{Na}_{2} \mathrm{Te} \ldots \ldots \ldots \ldots$

Sodium tin fluoride, $\mathrm{NaSn}_{2} \mathrm{~F}_{5} \ldots \ldots$

Sodium titanium oxide, $\mathrm{Na}_{2} \mathrm{Ti}_{3} \mathrm{O}_{7} \ldots$

Sodium titanium phosphate,

$\mathrm{NaTi}_{2}\left(\mathrm{PO}_{4}\right)_{3} \ldots \ldots \ldots \ldots \ldots \ldots$

Sodium tungsten oxide, $\mathrm{Na}_{2} \mathrm{WO}_{4} \ldots \ldots$

Sodium tungsten(VI) oxide hydrate,

$\mathrm{Na}_{2} \mathrm{WO}_{4} \cdot 2 \mathrm{H}_{2} \mathrm{O} \ldots \ldots \ldots \ldots \ldots \ldots \ldots$

Sodium vanadium oxide, $\beta-\mathrm{NaVO}_{3} \ldots$

Sodium zinc fluoride, $\mathrm{NaZnF}_{3} \ldots \ldots$

Sodium zinc sulfate hydrate,

$\mathrm{Na}_{2} \mathrm{Zn}\left(\mathrm{SO}_{4}\right)_{2} \cdot 4 \mathrm{H}_{2} \mathrm{O} \ldots \ldots \ldots \ldots \ldots \ldots 6.6 \mathrm{~m}$

Sodium zirconium fluoride,

$\mathrm{Na}_{7} \mathrm{Zr}_{6} \mathrm{~F}_{31} \ldots \ldots \ldots \ldots \ldots \ldots \ldots \ldots$

Sodium zirconium phosphate,

$\mathrm{NaZr}_{2}\left(\mathrm{PO}_{4}\right)_{3} \ldots \ldots \ldots \ldots \ldots \ldots$

Strontium aluminum hydroxide,

$\mathrm{Sr}_{3} \mathrm{Al}_{2}(\mathrm{OH})_{12} \ldots \ldots \ldots \ldots \ldots \ldots$

Strontium a luminum oxide, $\mathrm{SrAl}_{2} \mathrm{O}_{4}$.

Strontium aluminum oxide, $\mathrm{Sr}_{3} \mathrm{Al}_{2} \mathrm{O}_{6}$

Strontium arsenate, $\mathrm{Sr}_{3}\left(\mathrm{AsO}_{4}\right)_{2} \ldots$.

Strontium azide, $\operatorname{Sr}\left(\mathrm{N}_{3}\right)_{2} \ldots \ldots \ldots$

Strontium borate, $\mathrm{SrB}_{2} \mathrm{O}_{4} \ldots \ldots \ldots$

Strontium borate, $\mathrm{SrB}_{4} \mathrm{O}_{7} \ldots \ldots \ldots$

Strontium bromate hydrate,

$\operatorname{Sr}\left(\mathrm{BrO}_{3}\right)_{2} \cdot \mathrm{H}_{2} \mathrm{O} \ldots \ldots \ldots \ldots \ldots \ldots$

Strontium bromide fluoride, $\mathrm{SrBrF}$

Strontium bromide hydrate,

$\mathrm{SrBr}_{2} \cdot 6 \mathrm{H}_{2} \mathrm{O} \ldots \ldots \ldots \ldots \ldots \ldots$

Strontium carbonate (strontianite),

$\mathrm{SrCO}_{3} \ldots \ldots \ldots \ldots \ldots \ldots \ldots \ldots \ldots \ldots \ldots \ldots$ ol. or

Sec. Page

$\begin{array}{rr}6 \mathrm{~m} & 68 \\ 18 \mathrm{~m} & 64 \\ 6 & 50 \\ 4 & 62 \\ & \\ 18 \mathrm{~m} & 66 \\ 10 \mathrm{~m} & 134 \\ 3 \mathrm{~m} & 49 \\ & \\ 3 \mathrm{~m} & 50 \\ & \\ 13 \mathrm{~m} & 39 \\ & \\ 2 \mathrm{~m} & 35 \\ & \\ 5 \mathrm{~m} & 54 \\ & \\ 7 \mathrm{~m} & 68 \\ 9 \mathrm{~m} & 55 \\ 10 \mathrm{~m} & 135 \\ 8 \mathrm{~m} & 141 \\ 10 \mathrm{~m} & 136 \\ & \\ 16 \mathrm{~m} & 68\end{array}$

$16 \mathrm{~m} \quad 68$

$20 m \quad 89$

$19 \mathrm{~m} \quad 77$

$11 \mathrm{~m} \quad 57$

$2 \quad 59$

$17 \mathrm{~m} \quad 74$

$10 \mathrm{~m} \quad 140$

360

$10 \mathrm{~m} \quad 141$

$7 \mathrm{~m} \quad 166$

$16 \mathrm{~m} \quad 69$

$19 \mathrm{~m} \quad 79$

$1 \mathrm{~m} \quad 47$

$2 \mathrm{~m} \quad 33$

$18 \mathrm{~m} \quad 67$

$18 \mathrm{~m} \quad 68$

$6 \mathrm{~m} \quad 74$

$6 \mathrm{~m} \quad 72$

$8 m \quad 144$

$19 m \quad 81$

$10 \mathrm{~m} \quad 50$

$20 \mathrm{~m} \quad 91$

$10 \mathrm{~m} \quad 52$

$8 \mathrm{~m} \quad 146$

$3 \mathrm{~m} \quad 53$

$17 \mathrm{~m} \quad 76$

$10 \mathrm{~m} \quad 54$

460
$2 \mathrm{~m} \quad 36$

$4 \mathrm{~m} \quad 36$

$3 \quad 56$ 
Strontium chloride, $\mathrm{SrCl}_{2} \ldots \ldots \ldots$ Strontium chloride fluoride, SrClF Strontium chloride hydrate,

$\mathrm{SrCl}_{2} \cdot 2 \mathrm{H}_{2} \mathrm{O} \ldots \ldots \ldots \ldots \ldots \ldots$

Strontium chloride hydrate,

$\mathrm{SrCl}_{2} \cdot 6 \mathrm{H}_{2} \mathrm{O} \ldots \ldots \ldots \ldots \ldots \ldots$

Strontium chloride hydroxide

phosphate, $\mathrm{Sr}_{5} \mathrm{Cl}{ }_{65}(\mathrm{OH}){ }_{35}\left(\mathrm{PO}_{4}\right)_{3}$

Strontium chromium oxide, $\mathrm{SrCr}_{2} \mathrm{O}_{7}$

Strontium chromium oxide, $\mathrm{Sr}_{2} \mathrm{CrO}_{4}$

Strontium chromium oxide hydrate,

$\mathrm{SrCr}_{2} \mathrm{O}_{7} \cdot 3 \mathrm{H}_{2} 0 \ldots \ldots \ldots \ldots \ldots$

Strontium fluoride, $\mathrm{SrF}_{2} \ldots \ldots \ldots$

Strontium hydroxide, $\mathrm{Sr}(\mathrm{OH})_{2} \ldots \ldots$

Strontium hydroxide hydrate,

$\mathrm{Sr}(\mathrm{OH})_{2} \cdot \mathrm{H}_{2} \mathrm{O} \ldots \ldots \ldots \ldots \ldots \ldots$

Strontium hydroxide hydrate,

$\mathrm{Sr}(\mathrm{OH})_{2} \cdot 8 \mathrm{H}_{2} \mathrm{O} \ldots \ldots \ldots \ldots$.

Strontium indium hydroxide,

$\mathrm{Sr}_{3} \mathrm{In}_{2}(\mathrm{OH})_{12} \ldots \ldots \ldots \ldots \ldots$

Strontium iodide hydrate,

$\mathrm{SrI}_{2} \cdot 6 \mathrm{H}_{2} \mathrm{O} \ldots \ldots \ldots \ldots \ldots \ldots \ldots \ldots$

Strontium iron oxide, $\mathrm{SrFe}_{12} \mathrm{O}_{19} \ldots \mathrm{C}_{19} \quad 18 \mathrm{~m} \quad 69$

Strontium manganese oxide,

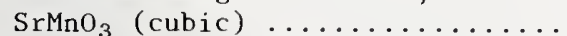

Strontium manganese oxide,

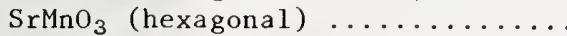

Strontium molybdenum oxide, $\mathrm{SrMoO}_{4}$

Strontium nitrate, $\operatorname{Sr}\left(\mathrm{NO}_{3}\right)_{2} \ldots \ldots$

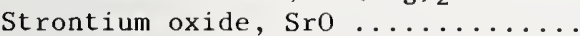

Strontium oxide, $\mathrm{SrO}_{2} \ldots \ldots \ldots \ldots$

Strontium oxide hydrate, $\mathrm{SrO}_{2} \cdot 8 \mathrm{H}_{2} \mathrm{O}$

Strontium phosphate, $\alpha-\mathrm{Sr}_{2} \mathrm{P}_{2} \mathrm{O}_{7} \ldots$...

Strontium phosphate, $\alpha-\mathrm{Sr}_{3}\left(\mathrm{PO}_{4}\right)_{2} \ldots$

Strontium scandium oxide hydrate,

$\mathrm{Sr}_{3} \mathrm{Sc}_{2} \mathrm{O}_{6} \cdot 6 \mathrm{H}_{2} \mathrm{O} \ldots \ldots \ldots \ldots \ldots \ldots$

Strontium silicate, $\alpha-\mathrm{SrSiO}_{3} \ldots \ldots$

Strontium silicate, $\mathrm{Sr}_{3} \mathrm{SiO}_{5} \ldots \ldots$

Strontium sulfate (celestite), $\mathrm{SrSO}_{4}$

Strontium sulfide, $\operatorname{SrS} \ldots \ldots \ldots \ldots$

Strontium telluride, SrTe ........

Strontium tin oxide, $\mathrm{SrSnO}_{3} \ldots \ldots$.

Strontium titanium oxide, $\mathrm{SrTiO}_{3} \ldots$

Strontium tungsten oxide, $\mathrm{SrWO}_{4} \ldots$

Strontium tungsten oxide, $\mathrm{Sr}_{2} \mathrm{WO}_{5} \ldots$

Strontium vanadium oxide, $\mathrm{Sr}_{3}\left(\mathrm{VO}_{4}\right)_{2}$

Strontium zirconium oxide, $\mathrm{SrZrO}_{3}$.

Strontium zirconium phosphate,

$\mathrm{SrZr}_{4}\left(\mathrm{PO}_{4}\right)_{6} \ldots \ldots \ldots \ldots \ldots \ldots \ldots$

Sulfamic acid, $\mathrm{H}_{2} \mathrm{NSO}_{3} \mathrm{H} \ldots \ldots \ldots \ldots$

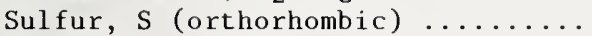

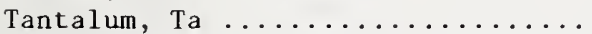

Tantalum silicide, $\mathrm{TaSi}_{2} \ldots . . . .$.

Tantalum tungsten oxide, $\mathrm{Ta}_{2} \mathrm{WO}_{8} \ldots$

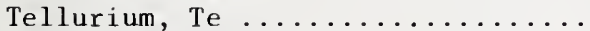

Tellurium(IV) oxide (paratellurite),

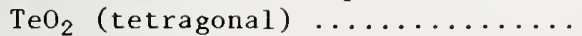

Tellurium(IV) oxide, paratellurite,

$\mathrm{TeO}_{2}$ (tetragonal) .............

Tellurium(IV) oxide, tellurite,

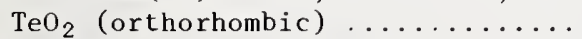

Terbium arsenate, $\mathrm{TbAsO}_{4} \ldots \ldots \ldots \ldots$

Terbium arsenide, TbAs ..........

Terbium nitride, TbN ............

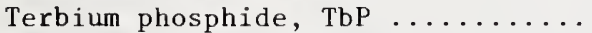

Terbium selenide, TbSe ..........

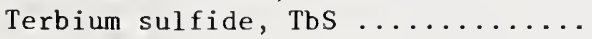

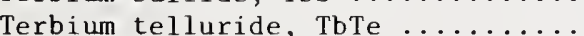

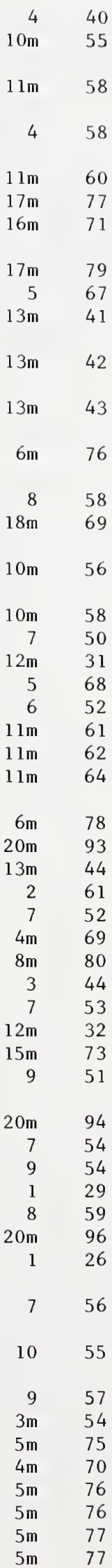

Terbium vanadium oxide, $\mathrm{TbVO}_{4} \ldots .$.

Thallium, $\alpha-\mathrm{T} l \ldots \ldots \ldots \ldots . . .$.

Thallium aluminum sulfate hydrate,

$\mathrm{TlAl}\left(\mathrm{SO}_{4}\right)_{2} \cdot 12 \mathrm{H}_{2} \mathrm{O} \ldots \ldots \ldots \ldots$

Thallium(I) arsenate, $\mathrm{Tl}_{3} \mathrm{AsO}_{4} \ldots$.

Thallium azide, $\mathrm{T}_{1 N_{3}} \ldots \ldots \ldots \ldots$

Thallium(I) bromate, $\mathrm{TlBrO}_{3} \ldots .$.

Thallium bromide, TlBr ..........

Thallium cadmium sulfate,

$\mathrm{Tl}_{2} \mathrm{Cd}_{2}\left(\mathrm{SO}_{4}\right)_{3} \ldots \ldots \ldots \ldots \ldots$

Thallium(I) chlorate, $\mathrm{TlClO}_{4} \ldots \ldots$

Thallium(1) chlorate, $\mathrm{TlClO}_{3} \ldots \ldots$

Thallium(I) chloride, TlCl .......

Thallium chromium oxide, $\mathrm{Tl}_{2} \mathrm{CrO}_{4} \ldots$

Thallium chromium sulfate hydrate,

$\mathrm{TlCr}\left(\mathrm{SO}_{4}\right)_{2} \cdot 12 \mathrm{H}_{2} \mathrm{O} \ldots \ldots \ldots \ldots \ldots$

Thallium cobalt sulfate,

$\mathrm{Tl}_{2} \mathrm{Co}_{2}\left(\mathrm{SO}_{4}\right)_{3} \ldots \ldots \ldots \ldots \ldots$

Thallium cobalt sulfate hydrate,

$\mathrm{Tl}_{2} \mathrm{Co}\left(\mathrm{SO}_{4}\right)_{2} \cdot 6 \mathrm{H}_{2} \mathrm{O} \ldots \ldots \ldots \ldots \ldots$

Thallium copper sulfate hydrate,

$\mathrm{Tl}_{2} \mathrm{Cu}\left(\mathrm{SO}_{4}\right)_{2} \cdot 6 \mathrm{H}_{2} \mathrm{O} \ldots \ldots \ldots \ldots \ldots$

Thallium fluoride, TlF ..........

Thallium gallium sulfate hydrate,

$\mathrm{TlGa}\left(\mathrm{SO}_{4}\right)_{2} \cdot 12 \mathrm{H}_{2} \mathrm{O} \ldots \ldots \ldots \ldots$

Thallium(I) iodate, $\mathrm{TlIO}_{3} \ldots \ldots \ldots$

Thallium(I) iodide, TII

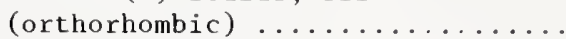

Thallium iron sulfate hydrate,

$\mathrm{Tl}_{2} \mathrm{Fe}\left(\mathrm{SO}_{4}\right)_{2} \cdot 6 \mathrm{H}_{2} \mathrm{O} \ldots \ldots \ldots \ldots$

Thallium lead sulfate,

$\mathrm{Tl}_{2} \mathrm{~Pb}\left(\mathrm{SO}_{4}\right)_{2}$

Thallium magnesium chromium oxide,

$\mathrm{Tl}_{2} \mathrm{Mg}_{2}\left(\mathrm{CrO}_{4}\right)_{3} \ldots \ldots \ldots \ldots \ldots \ldots$

Thallium magnesium sulfate hydrate,

$\mathrm{Tl}_{2} \mathrm{Mg}\left(\mathrm{SO}_{4}\right)_{2} \cdot 6 \mathrm{H}_{2} \mathrm{O} \ldots \ldots \ldots \ldots$

Thallium manganese sulfate,

$\mathrm{Tl}_{2} \mathrm{Mn}_{2}\left(\mathrm{SO}_{4}\right)_{3} \ldots \ldots \ldots \ldots \ldots$

Thallium nickel sulfate hydrate,

$\mathrm{Tl}_{2} \mathrm{Ni}\left(\mathrm{SO}_{4}\right)_{2} \cdot 6 \mathrm{H}_{2} \mathrm{O} \ldots \ldots \ldots \ldots \ldots$

Thallium(I) nitrate, $\mathrm{T}^{\mathrm{N} \mathrm{NO}_{3}} \ldots \ldots \ldots$

Thallium oxide (avicennite), $\mathrm{Tl}_{2} \mathrm{O}_{3}$

Thallium(III) oxide, $\mathrm{Tl}_{2} \mathrm{O}_{3} \ldots \ldots$.

Thallium(I) phosphate, $\mathrm{Tl}_{3} \mathrm{PO}_{4} \ldots$.

Thallium(III) phosphate, $\mathrm{T} \mathrm{PO}_{4} \ldots$.

Thallium platinum chloride,

$\mathrm{Tl}_{2} \mathrm{PtCl}_{6} \ldots \ldots \ldots \ldots \ldots \ldots \ldots$

Thallium silicon fluoride, $\mathrm{Tl}_{2} \mathrm{SiF}_{6}$

Thallium strontium sulfate,

$\mathrm{Tl}_{2} \mathrm{Sr}\left(\mathrm{SO}_{4}\right)_{2} \ldots \ldots \ldots \ldots \ldots \ldots$

Thallium(I) sulfate, $\mathrm{Tl}_{2} \mathrm{SO}_{4} \ldots \ldots$.

Thallium(I) thiocyanate, TlCNS ....

Thallium tin chloride, $\mathrm{Tl}_{2} \mathrm{SnCl}_{6} \ldots$

Thallium(I) tungsten oxide, $\mathrm{Tl}_{2} \mathrm{WO}_{4}$

Thallium zinc sulfate hydrate,

$\mathrm{Tl}_{2} \mathrm{Zn}\left(\mathrm{SO}_{4}\right)_{2} \cdot 6 \mathrm{H}_{2} \mathrm{O} \ldots \ldots \ldots \ldots$

Thorium arsenide, ThAs ..........

Thorium carbide, ThC ...........

Thorium molybdenum oxide,

$\alpha-\mathrm{Th}\left(\mathrm{MoO}_{4}\right)_{2} \ldots \ldots \ldots \ldots \ldots \ldots . \ldots \ldots$

Thorium molybdenum oxide,

$\beta-\mathrm{Th}\left(\mathrm{MoO}_{4}\right)_{2} \ldots \ldots \ldots \ldots \ldots \ldots \ldots$ 20m

Thorium nitrate hydrate,

$\mathrm{Th}\left(\mathrm{NO}_{3}\right)_{4} \cdot 5 \mathrm{H}_{2} \mathrm{O} \ldots \ldots \ldots \ldots \ldots$

Thoriun oxide (thorianite), $\mathrm{ThO}_{2} \ldots$

Thorium silicate (huttonite),

$\beta-\mathrm{ThSiO}_{4} \ldots \ldots \ldots \ldots \ldots \ldots \ldots$

Thorium tantalum oxide, $\mathrm{Th}_{2} \mathrm{Ta}_{2} \mathrm{O}_{9} \ldots$
100

$\begin{array}{rr}5 \mathrm{~m} & 56 \\ 16 \mathrm{~m} & 7\end{array}$

$\begin{array}{rr}6 & 53 \\ 2 m & 37 \\ 8 m & 82 \\ 8 & 60 \\ 7 & 57 \\ & \\ 8 m & 83 \\ 2 m & 38 \\ 8 & 61 \\ 4 & 51 \\ 3 m & 54 \\ & \\ 6 & 55 \\ 8 m & 85\end{array}$

$7 \mathrm{~m}$

$7 \mathrm{~m}$

$16 \mathrm{~m}$

6
8

4

$8 \mathrm{~m}$

$15 m$

$8 m$

$7 m$

$7 m$

$7 \mathrm{~m}$

6
$16 \mathrm{~m}$

2

7

7

5
6

$15 \mathrm{~m}$

6

8

$1 \mathrm{~m}$

$7 m$

$4 \mathrm{~m}$

$18 \mathrm{~m}$

70

71

98

$18 \mathrm{~m}$

72

157

$20 \mathrm{~m}$

102

$20 \mathrm{~m} \quad 104$ 
Thorium tungsten oxide, $\alpha-\mathrm{Th}\left(\mathrm{WO}_{4}\right)_{2}$.

Thulium arsenate, $\mathrm{TmAsO}_{4} \ldots \ldots \ldots$

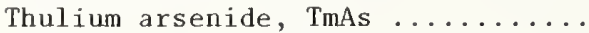

Thulium nitride, $\mathrm{TmN} \ldots \ldots \ldots \ldots \ldots$

Thulium oxide, $\operatorname{Tm}_{2} \mathrm{O}_{3} \ldots \ldots \ldots \ldots$

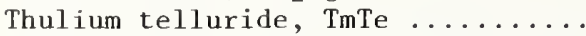

Thulium vanadium oxide, $\mathrm{TmVO}_{4} \ldots$.

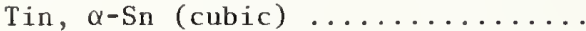

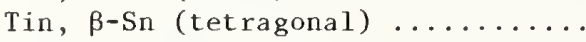

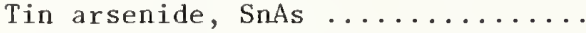

Tin a rsenide, $\mathrm{Sn}_{3 .}{ }_{8} \mathrm{As}_{3} \ldots \ldots \ldots \ldots$

Tin chloride hydrate, $\mathrm{SnCl}_{2} \cdot 2 \mathrm{H}_{2} \mathrm{O} \ldots$

Tin(II) fluoride, $\mathrm{SnF}_{2} \ldots \ldots \ldots \ldots$

Tin hydrogen phosphate, $\mathrm{SnHPO}_{4} \ldots$.

Tin(IV) iodide, $\mathrm{Snl}_{4} \ldots \ldots \ldots \ldots \ldots$

Tin(11) oxide (romarchite), SnO ...

Tin(1V) oxide (cassiterite), $\mathrm{SnO}_{2}$

Tin sulfide (berndtite), $\beta-\mathrm{SnS}_{2} \ldots$

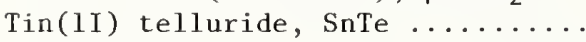

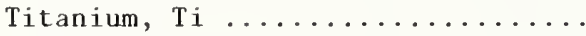

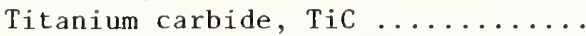

Titanium(111) oxide, $\mathrm{TiO}_{1.515} \ldots$.

Titanium oxide (anatase), $\mathrm{TiO}_{2} \ldots$.

Titanium oxide, brookite, $\mathrm{TiO}_{2}$

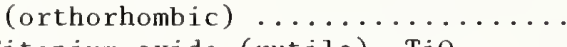

Titanium oxide (rutile), $\mathrm{TiO}_{2} \ldots .$.

Titanium silicide, $\mathrm{Ti}_{5} \mathrm{Si}_{3} \ldots \ldots \ldots$

Titanium sulfide, $\mathrm{TiS}_{2} \ldots \ldots \ldots \ldots$

Titanium sulfide, $\mathrm{Ti}_{2} \mathrm{~S} \ldots \ldots \ldots \ldots$

Tungsten, $W \ldots \ldots \ldots \ldots \ldots \ldots \ldots$

Tungsten, $W$ (reference standard) ..

Tungsten oxide, $\mathrm{WO}_{2} \ldots \ldots \ldots \ldots$.

Tungsten sulfide (tungstenite), $W_{2}$

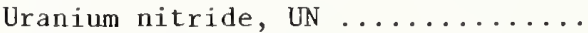

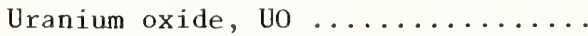

Uranium oxide (uraninite), $\mathrm{UO}_{2} \ldots$

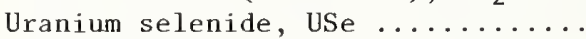

Uranium telluride, UTe ..........

Vanadium, $V \ldots \ldots \ldots \ldots \ldots \ldots . . . .$.

Vanadium oxide (karelianite), $\mathrm{V}_{2} \mathrm{O}_{3}$.

Vanadium(V) oxide (shcherbinaite),

$\mathrm{v}_{2} \mathrm{O}_{5} \ldots \ldots \ldots \ldots \ldots \ldots \ldots$

Vanadium sulfide, $\alpha-V_{3} S \ldots \ldots \ldots$

Vanadium sulfide, $\beta-V_{3} S \ldots \ldots \ldots$

Ytterbium arsenate, $\mathrm{YbAsO}_{4} \ldots \ldots \ldots$

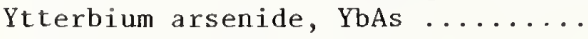

Ytterbium fluoride, $\mathrm{YbF}_{3} \ldots \ldots \ldots$

Ytterbium nitride, $\mathrm{YbN} \ldots \ldots \ldots \ldots$

Ytterbium oxide, $\mathrm{Yb}_{2} \mathrm{O}_{3} \ldots \ldots \ldots \ldots$

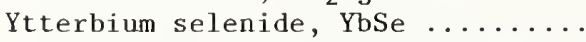

Ytterbium telluride, YbTe ........

Ytterbium(III) vanadium oxide, $\mathrm{YbVO}_{4}$

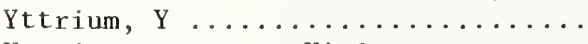

Yttrium arsenate, $\mathrm{YAsO}_{4} \ldots \ldots \ldots \ldots$

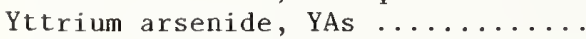

Yttrium chloride oxide, YC10......

Yttrium chromium oxide, $\mathrm{YCrO}_{3} \ldots \ldots$

Yttrium oxide, $\mathrm{Y}_{2} \mathrm{O}_{3} \ldots \ldots \ldots \ldots \ldots$

Yttrium phosphate (xenotime), $\mathrm{YPO}_{4}$

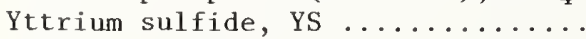

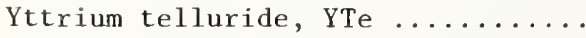

Yttrium titanium oxide, $\mathrm{Y}_{2} \mathrm{TiO}_{5} \ldots$.

Yttrium vanadium oxide, $\mathrm{YVO}_{4} \ldots \ldots$.

Zinc, $\mathrm{Zn}_{n} \ldots \ldots \ldots \ldots \ldots \ldots \ldots \ldots$

Zinc aluminum oxide (gahnite),

$\mathrm{ZnAl}_{2} \mathrm{O}_{4} \ldots \ldots \ldots \ldots \ldots \ldots \ldots \ldots$

Zinc ammine bromide, $\mathrm{Zn}\left(\mathrm{NH}_{3}\right)_{2} \mathrm{Br}_{2}$

Zinc ammine chloride, $\mathrm{Zn}\left(\mathrm{NH}_{3}\right)_{2} \mathrm{Cl}_{2}$
$20 \mathrm{~m} \quad 106$

$3 \mathrm{~m} \quad 56$

$4 \mathrm{~m} \quad 71$

$4 \mathrm{~m} \quad 71$

$9 \quad 58$

$4 \mathrm{~m} \quad 72$

$5 \mathrm{~m} \quad 57$

$2 \quad 12$

124

$4 \mathrm{~m} \quad 37$

$15 \mathrm{~m} \quad 76$

$17 \mathrm{~m} \quad 84$

$3 \mathrm{~m} \quad 51$

$13 \mathrm{~m} \quad 46$

$5 \quad 71$

$4 \quad 28$

$1 \quad 54$

$\begin{array}{ll}7 & 51\end{array}$

$\begin{array}{rr}3 & 4 \\ 18 \mathrm{~m} & 73\end{array}$

959

$7 \mathrm{~m} \quad 82$

$3 \mathrm{~m} \quad 57$

$7 \mathrm{~m} \quad 83$

$8 \quad 64$

$4 \mathrm{~m} \quad 72$

$8 \mathrm{~m} \quad 149$

$\begin{array}{rr}1 & 28\end{array}$

$18 \mathrm{~m} \quad 74$

865

$18 \mathrm{~m} \quad 75$

$5 \mathrm{~m} \quad 78$

$\begin{array}{rr}2 & 33 \\ 5 \mathrm{~m} & 78\end{array}$

$4 \mathrm{~m} \quad 73$

$9 \mathrm{~m} \quad 58$

$20 \mathrm{~m} \quad 108$

$\begin{array}{rr}8 & 66 \\ 14 \mathrm{~m} & 118\end{array}$

$14 \mathrm{~m} \quad 120$

$4 \mathrm{~m} \quad 38$

$4 \mathrm{~m} \quad 73$

$20 \mathrm{~m} \quad 109$

$4 \mathrm{~m} \quad 74$

$6 \mathrm{~m} \quad 80$

$5 \mathrm{~m} \quad 79$

$5 \mathrm{~m} \quad 79$

$5 \mathrm{~m} \quad 58$

$18 \mathrm{~m} \quad 77$

2m $\quad 39$

$4 \mathrm{~m} \quad 74$

$1 \mathrm{~m} \quad 51$

$19 \mathrm{~m} \quad 83$

$3 \quad 28$

$\begin{array}{ll}8 & 67\end{array}$

$5 \mathrm{~m} \quad 80$

$4 \mathrm{~m} \quad 75$

$11 \mathrm{~m} \quad 113$

$5 \mathrm{~m} \quad 59$

116

$\begin{array}{rr}2 & 38 \\ 11 \mathrm{~m} & 68\end{array}$

$10 \mathrm{~m} \quad 59$
Zinc antimony oxide, $\mathrm{ZnSb}_{2} \mathrm{O}_{4} \ldots \ldots$

Zinc Arsenate Hydrate (koettigite),

$\mathrm{Zn}_{3}\left(\mathrm{AsO}_{4}\right)_{2} \cdot 8 \mathrm{H}_{2} \mathrm{O} \ldots \ldots \ldots \ldots \ldots$

Zinc borate, $\mathrm{Zn}_{4} \mathrm{~B}_{6} \mathrm{O}_{13} \ldots \ldots \ldots \ldots$

Zinc carbonate, smithsonite, $\mathrm{ZnCO}_{3}$

Zinc chlorate hydrate,

$\mathrm{Zn}\left(\mathrm{ClO}_{4}\right)_{2} \cdot 6 \mathrm{H}_{2} \mathrm{O} \ldots \ldots \ldots \ldots \ldots$

Zinc chromium oxide, $\mathrm{ZnCr}_{2} \mathrm{O}_{4} \ldots \ldots$

Zinc cobalt oxide, $\mathrm{ZnCo}_{2} \mathrm{O}_{4} \ldots \ldots \ldots$

Zinc cyanide, $\mathrm{Zn}(\mathrm{CN})_{2} \ldots \ldots \ldots \ldots$

Zinc fluoride, $\mathrm{ZnF}_{2} \ldots \ldots \ldots \ldots$

Zinc fluoride hydrate, $\mathrm{ZnF}_{2} \cdot 4 \mathrm{H}_{2} \mathrm{O}$..

Zinc germanium oxide, $\mathrm{Zn}_{2} \mathrm{GeO}_{4} \ldots \ldots$

Zinc hydroxide silicate hydrate,

hemimorphite, $\mathrm{Zn}_{4}(\mathrm{OH})_{2} \mathrm{Si}_{2} \mathrm{O}_{7} \cdot \mathrm{H}_{2} \mathrm{O} \ldots$

Zinc iodide, $\mathrm{Znl}_{2} \ldots \ldots \ldots \ldots \ldots$

Zinc iron oxide (franklinite),

$\mathrm{ZnFe}_{2} \mathrm{O}_{4} \ldots \ldots \ldots \ldots \ldots \ldots \ldots$

Zinc manganese oxide (hetaerolite),

$\mathrm{ZnMn}_{2} \mathrm{O}_{4} \ldots \ldots \ldots \ldots \ldots \ldots$

Zinc molybdenum oxide, $\mathrm{Zn}_{2} \mathrm{Mo}_{3} \mathrm{O}_{8} \ldots$

Zinc nitrate hydrate,

$\alpha-\mathrm{Zn}\left(\mathrm{NO}_{3}\right)_{2} \cdot 6 \mathrm{H}_{2} \mathrm{O} \ldots \ldots \ldots \ldots$

Zinc oxide (zincite), $\mathrm{ZnO} \ldots \ldots \ldots$

Zinc phosphate, $\alpha-\mathrm{Zn}_{3}\left(\mathrm{PO}_{4}\right)_{2} \ldots \ldots$

Zinc phosphate, $\beta-\mathrm{Zn}_{3}\left(\mathrm{PO}_{4}\right)_{2} \ldots \ldots$

Zinc phosphate, $\gamma-\mathrm{Zn}_{3}\left(\mathrm{PO}_{4}\right)_{2} \ldots \ldots$

Zinc phosphate hydrate (hopeite),

$\mathrm{Zn}_{3}\left(\mathrm{PO}_{4}\right)_{2} \cdot 4 \mathrm{H}_{2} \mathrm{O} \ldots \ldots \ldots \ldots \ldots \ldots \ldots$

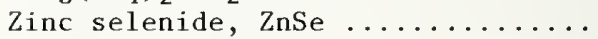

Zinc silicate (willemite), $\mathrm{Zn}_{2} \mathrm{SiO}_{4}$

Zinc silicon fluoride hydrate,

$\mathrm{ZnSiF}_{6} \cdot 6 \mathrm{H}_{2} \mathrm{O} \ldots \ldots \ldots \ldots \ldots \ldots$

Zinc sulfate (zinkosite), $\mathrm{ZnSO}_{4} \ldots$

Zinc sulfate hydrate (gunningite),

$\mathrm{ZnSO}_{4} \cdot \mathrm{H}_{2} \mathrm{O} \ldots \ldots \ldots \ldots \ldots \ldots$

Zinc sulfate hydrate (goslarite),

$\mathrm{ZnSO}_{4} \cdot 7 \mathrm{H}_{2} \mathrm{O} \ldots \ldots \ldots \ldots \ldots \ldots \ldots$

Zinc sulfide (wurtzite), $\alpha$-ZnS

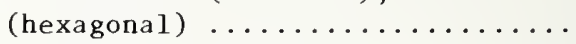

Zinc sulfide (sphaelerite), $\beta-\mathrm{ZnS}$

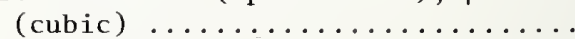

Zinc telluride, $\mathrm{ZnTe} \ldots \ldots \ldots \ldots \ldots$

Zinc tin oxide, $\mathrm{Zn}_{2} \mathrm{SnO}_{4} \ldots \ldots \ldots \ldots$

Zinc titanium oxide, $\mathrm{ZnTiO}_{3} \ldots \ldots$

Zinc titanium oxide, $\mathrm{Zn}_{2} \mathrm{TiO}_{4} \ldots \ldots$

Zinc tungsten oxide (sanmartinite),

$\mathrm{ZnWO}_{4} \ldots \ldots \ldots \ldots \ldots \ldots \ldots \ldots$

Zinc vanadium oxide, $\mathrm{Zn}_{3}\left(\mathrm{VO}_{4}\right)_{2} \ldots$

Zirconium, $\alpha-Z_{r} \ldots \ldots \ldots \ldots \ldots$

Zirconium boride, $\mathrm{ZrB}_{2} \ldots \ldots \ldots \ldots$

Zirconium fluoride, $\mathrm{ZrF}_{4} \ldots \ldots \ldots$

Zirconium hydride, $\mathrm{ZrH}_{2} \ldots \ldots \ldots$

Zirconium hydrogen phosphate hydrate,

$\mathrm{Zr}\left(\mathrm{HPO}_{4}\right)_{2} \cdot \mathrm{H}_{2} \mathrm{O} \ldots \ldots \ldots \ldots \ldots$

Zirconium iodate, $\operatorname{Zr}\left(10_{3}\right)_{4} \ldots \ldots \ldots$

Zirconium nitride, $\mathrm{ZrN} \ldots \ldots \ldots \ldots$

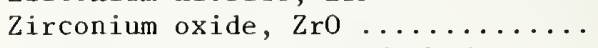

Zirconium oxide chloride hydrate,

$\mathrm{ZrOCl}_{2} \cdot 8 \mathrm{H}_{2} \mathrm{O} \ldots \ldots \ldots \ldots \ldots \ldots$

Zirconium phosphide, $\mathrm{ZrP} \ldots \ldots \ldots$

Zirconium silicate, zircon, $\mathrm{ZrSiO}_{4}$

Zirconium silicide, $\mathrm{ZrSi}_{2} \ldots \ldots \ldots$

Zirconium sulfate hydrate

(zircosulfate), $\mathrm{Zr}\left(\mathrm{SO}_{4}\right)_{2} \cdot 4 \mathrm{H}_{2} \mathrm{O} \ldots$.

Zirconium titanium oxide, $\mathrm{ZrTiO}_{4} \ldots$

Zirconium titanium oxide,

$\mathrm{Zr}_{5} \mathrm{Ti}_{7} \mathrm{O}_{24} \ldots \ldots \ldots \ldots \ldots \ldots$
$4 \mathrm{~m}$

39

$19 \mathrm{~m}$

$13 \mathrm{~m}$

8

85

48

69

$16 \mathrm{~m} \quad 79$

$9 \mathrm{~m} \quad 59$

$10 \mathrm{~m} \quad 60$

$5 \quad 73$

$6 \quad 60$

$11 \mathrm{~m} \quad 69$

$10 \quad 56$

262

$9 \quad 60$

$9 m \quad 60$

$10 \mathrm{~m} \quad 61$

$7 \mathrm{~m} \quad 173$

$12 \mathrm{~m} \quad 36$

225

$16 \mathrm{~m} \quad 80$

$16 \mathrm{~m} \quad 81$

$16 \mathrm{~m} \quad 83$

$16 \mathrm{~m} \quad 85$

323

$7 \quad 62$

$8 \quad 70$

$7 \quad 64$

$19 m \quad 86$

$8 \quad 71$

2

2

$3 \mathrm{~m}$

$10 \mathrm{~m}$

$13 \mathrm{~m}$

$12 \mathrm{~m}$

$2 \mathrm{~m}$

$20 \mathrm{~m}$

2

$20 \mathrm{~m}$

$18 \mathrm{~m}$

$5 \mathrm{~m}$

$20 \mathrm{~m}$

$1 \mathrm{~m}$

$5 \mathrm{~m}$

$5 \mathrm{~m}$

$18 \mathrm{~m}$

$4 \mathrm{~m}$
4

$17 \mathrm{~m}$

7

$20 \mathrm{~m}$

$20 \mathrm{~m}$

86

59
73
69
56

2

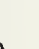

1

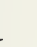

80

3

3

62

70
64


$\mathrm{CHI}_{3}$

$\mathrm{CH}_{4} \mathrm{~N}_{2} \mathrm{O}$

$\mathrm{CH}_{4} \mathrm{~N}_{2} \mathrm{~S}$

$\mathrm{CH}_{5} \mathrm{NO}_{2}$

$\mathrm{CH}_{5} \mathrm{~N}_{3} \cdot \mathrm{HCl}$

$\mathrm{CH}_{5} \mathrm{~N}_{3} \mathrm{~S}$

$\mathrm{C}_{2} \mathrm{Ag}_{2} \mathrm{O}_{4}$

$\mathrm{C}_{2} \mathrm{FeO}_{4} \cdot 2 \mathrm{H}_{2} \mathrm{O}$

$\mathrm{C}_{2} \mathrm{HNaO}_{4} \cdot \mathrm{H}_{2} \mathrm{O}$

$\mathrm{C}_{2} \mathrm{H}_{2} \mathrm{CaO}_{4}$

$\mathrm{C}_{2} \mathrm{H}_{2} \mathrm{O}_{4} \cdot 2 \mathrm{H}_{2} \mathrm{O}$

$\mathrm{C}_{2} \mathrm{H}_{2} \mathrm{O}_{4} \mathrm{~Pb}$

$\mathrm{C}_{2} \mathrm{H}_{2} \mathrm{O}_{4} \mathrm{Sr}$

$\mathrm{C}_{2} \mathrm{H}_{2} \mathrm{O}_{4} \mathrm{Sr} \cdot 2 \mathrm{H}_{2} \mathrm{O}$

$\mathrm{C}_{2} \mathrm{H}_{3} \mathrm{KO}_{4}$

$\mathrm{C}_{2} \mathrm{H}_{3} \mathrm{NaO}_{2} \cdot 3 \mathrm{H}_{2} \mathrm{O}$

$\mathrm{C}_{2} \mathrm{H}_{4} \mathrm{~N}_{2} \mathrm{O}_{2}$

$\mathrm{C}_{2} \mathrm{H}_{5} \mathrm{NO}_{2}$

$\mathrm{C}_{2} \mathrm{H}_{7} \mathrm{NO}_{2}$

$\mathrm{C}_{2} \mathrm{H}_{8} \mathrm{~N}_{2} \cdot 2 \mathrm{HC} 1$

$\mathrm{C}_{2} \mathrm{H}_{8} \mathrm{~N}_{2} \mathrm{O}_{4} \cdot \mathrm{H}_{2} \mathrm{O}$

$\mathrm{C}_{2} \mathrm{~K}_{2} \mathrm{O}_{4} \cdot \mathrm{H}_{2} \mathrm{O}$

$\mathrm{C}_{2} \mathrm{Li}_{2} \mathrm{O}_{4}$

$\mathrm{C}_{2} \mathrm{Na}_{2} \mathrm{O}_{4}$

$\mathrm{C}_{2} \mathrm{O}_{4} \mathrm{Rb}_{2} \cdot \mathrm{H}_{2} \mathrm{O}_{2}$

$\mathrm{C}_{3} \mathrm{H}_{7} \mathrm{NO}_{2}$

$\mathrm{C}_{3} \mathrm{H}_{7} \mathrm{NO}_{2} \mathrm{~S}$

$\mathrm{C}_{3} \mathrm{H}_{9} \mathrm{~N} \cdot \mathrm{HC} 1$

$\mathrm{C}_{4} \mathrm{H}_{3} \mathrm{KO}_{8} \cdot 2 \mathrm{H}_{2} \mathrm{O}$

$\mathrm{C}_{4} \mathrm{H}_{4} \mathrm{CaO}_{5} \cdot 2 \mathrm{H}_{2} \mathrm{O}$

$\mathrm{C}_{4} \mathrm{H}_{4} \mathrm{KNaO}_{6} \cdot 4 \mathrm{H}_{2} \mathrm{O}$

$\mathrm{C}_{4} \mathrm{H}_{4} \mathrm{MnO}_{6}$

$\mathrm{C}_{4} \mathrm{H}_{4} \mathrm{NO}_{8} \mathrm{Y} \cdot \mathrm{H}_{2} \mathrm{O}$

$\mathrm{C}_{4} \mathrm{H}_{4} \mathrm{Na}_{2} \mathrm{O}_{6} \cdot 2 \mathrm{H}_{2} \mathrm{O}$

$\mathrm{C}_{4} \mathrm{H}_{6} \mathrm{CoO}_{4} \cdot 4 \mathrm{H}_{2} \mathrm{O}$

$\mathrm{C}_{4} \mathrm{H}_{6} \mathrm{Hg}_{2} \mathrm{O}_{4}$

$\mathrm{C}_{4} \mathrm{H}_{6} \mathrm{NiO}_{4} \cdot 4 \mathrm{H}_{2} \mathrm{O}$

$\mathrm{C}_{4} \mathrm{H}_{6} \mathrm{O}_{4} \mathrm{Zn} \cdot 2 \mathrm{H}_{2} \mathrm{O}$

$\mathrm{C}_{4} \mathrm{H}_{6} \mathrm{O}_{6}$

$\mathrm{C}_{4} \mathrm{H}_{6} \mathrm{O}_{6} \mathrm{U} \cdot 2 \mathrm{H}_{2} \mathrm{O}$

$\mathrm{C}_{4} \mathrm{H}_{7} \mathrm{~N}_{3} \mathrm{O}$

$\mathrm{C}_{4} \mathrm{H}_{8} \mathrm{~N}_{8} \mathrm{O}_{8}$

$\mathrm{C}_{4} \mathrm{H}_{8} \mathrm{~N}_{8} \mathrm{O}_{8}$

$\mathrm{C}_{4} \mathrm{H}_{8} \mathrm{~N}_{8} \mathrm{O}_{8}$

$\mathrm{C}_{4} \mathrm{H}_{8} \mathrm{~N}_{8} \mathrm{O}_{8}$

$\mathrm{C}_{4} \mathrm{H}_{22} \mathrm{~B}_{20}$

$\mathrm{C}_{5} \mathrm{H}_{4} \mathrm{~N}_{4} \mathrm{O}_{3}$

$\mathrm{C}_{5} \mathrm{H}_{4} \mathrm{~N}_{4} \mathrm{O}_{3}$

$\mathrm{C}_{5} \mathrm{H}_{7} \mathrm{CuNO}_{4} \cdot 2 \mathrm{H}_{2} \mathrm{O}$

$\mathrm{C}_{5} \mathrm{H}_{7} \mathrm{NO}_{4} \mathrm{Zn} \cdot 2 \mathrm{H}_{2} \mathrm{O}$

$\mathrm{C}_{5} \mathrm{H}_{8} \mathrm{NNaO}_{4} \cdot \mathrm{H}_{2} \mathrm{O}$

$\mathrm{C}_{5} \mathrm{H}_{9} \mathrm{NO}_{4}$

$\mathrm{C}_{5} \mathrm{H}_{12} \mathrm{O}_{4}$

$\mathrm{C}_{6} \mathrm{H}_{3} \mathrm{~N}_{3} \mathrm{O}_{7}$

$\mathrm{C}_{6} \mathrm{H}_{5} \mathrm{NO}_{2}$

$\mathrm{C}_{6} \mathrm{H}_{6} \mathrm{O}_{2}$

$\mathrm{C}_{6} \mathrm{H}_{8} \mathrm{Cl}_{2} \mathrm{~N}_{4} \mathrm{Zn}$

$\mathrm{C}_{6} \mathrm{H}_{8} \mathrm{~N}_{2} \cdot \mathrm{HCl}$

$\mathrm{C}_{6} \mathrm{H}_{8} \mathrm{O}_{6}$

$\mathrm{C}_{6} \mathrm{H}_{12} \mathrm{~N}_{4}$

$\mathrm{C}_{6} \mathrm{H}_{12} \mathrm{O}_{6}$
Iodo form

Urea

Thiourea

Ammonium formate

Guanidinium chloride

Thiosemicarbazide

Silver oxalate

I ron oxalate hydrate (humboldtine)

Sodium hydrogen oxalate hydrate

Calcium formate

Oxalic acid hydrate

Lead formate

Strontium formate

Strontium formate hydrate (orthorhombic)

Potassium formate-formic acid complex

Sodium acetate hydrate

Glyoxime

$\alpha$-Glycine

Ammonium acetate

Ethylenediamine Hydrochloride

Ammonium oxalate hydrate (oxammite)

Potassium oxalate hydrate

Lithium oxalate

Sodium oxalate

Rubidium oxalate perhydrate

L-Alanine

L-Cysteine

Trimethylammonium chloride

Potassium hydrogen oxalate hydrate

Calcium malate hydrate

Potassium sodium tartrate hydrate

Manganese Tartrate

Ammonium yttrium oxalate hydrate

Sodium D-tartrate hydrate

Cobalt acetate hydrate

Mercury acetate

Nickel acetate hydrate

Zinc acetate hydrate

D-Tartaric acid

Uranyl acetate hydrate

Creatinine

$\alpha-\mathrm{HMX}$

$\beta-H M X$

0ctahydro-1, 3, 5, 7-tetranitro-

$1,3,5,7$-tetrazocine, alpha-

octahydro-1, 3, 5, 7-tetranitro-

$1,3,5,7$-tetrazocine, beta-

bis-(o-Dodecacarborane)

Uric acid, phase 1 (calc. pattern)

Uric acid, phase 1

Copper glutamate hydrate

Zinc glutamate hydrate

Sodium glutamate hydrate

$\beta$-L-Glutamic acid

Pentaerythritol

Picric acid

Nicotinic acid

$\gamma$-Hydroquinone

Zinc diimidazole chloride

Phenylhydrazine hydrochloride

L-Ascorbic acid

Hexame thylenetetramine

Dextrose
Vol. or

Sec.

Page

$18 \mathrm{~m}$

34

$7 \quad 61$

$17 \mathrm{~m} \quad 83$

$11 \mathrm{~m}$

$17 \mathrm{~m} \quad 35$

$17 \mathrm{~m} \quad 81$

$9 \mathrm{~m} \quad 47$

$10 \mathrm{~m} \quad 24$

$17 \mathrm{~m}$

$8 \quad 16$

$16 \mathrm{~m} \quad 55$

$8-30$

$8 \quad 55$

$8 \quad 56$

$9 \mathrm{~m}$

$15 \mathrm{~m} \quad 66$

$8 \mathrm{~m} \quad 102$

$17 \mathrm{~m} \quad 34$

$8 \mathrm{~m} \quad 95$

19m 43

$7 \quad 5$

$9 \mathrm{~m} \quad 39$

$10 \mathrm{~m} \quad 34$

$6 \mathrm{~m} \quad 70$

$9 \mathrm{~m} \quad 102$

$8 \mathrm{~m} \quad 93$

$11 \mathrm{~m} \quad 86$

$9 \mathrm{~m} \quad 113$

$17 \mathrm{~m} \quad 60$

$10 \mathrm{~m} \quad 76$

$15 \mathrm{~m}$

$19 \mathrm{~m} \quad 57$

$8 \mathrm{~m} \quad 97$

$11 \mathrm{~m} \quad 110$

$12 \mathrm{~m} \quad 19$

$17 \mathrm{~m} \quad 51$

$13 \mathrm{~m} \quad 31$

$18 \mathrm{~m} \quad 78$

$7 \mathrm{~m} \quad 168$

$18 \mathrm{~m} \quad 76$

$15 \mathrm{~m} \quad 31$

$11 \mathrm{~m} \quad 100$

$11 \mathrm{~m} \quad 102$

$11 \mathrm{~m} \quad 100$

$11 \mathrm{~m} \quad 102$

$6 \mathrm{~m} \quad 7$

$8 \mathrm{~m} \quad 154$

$16 \mathrm{~m} \quad 78$

$7 \mathrm{~m} \quad 110$

$7 \mathrm{~m} \quad 170$

$17 \mathrm{~m} \quad 70$

$17 \mathrm{~m} \quad 32$

$17 \mathrm{~m} \quad 55$

$16 \mathrm{~m} \quad 56$

$16 \mathrm{~m}$

$8 \mathrm{~m} \quad 107$

$7 \mathrm{~m} \quad 123$

$17 \mathrm{~m} \quad 56$

$8 \mathrm{~m} \quad 99$

$17 \mathrm{~m} \quad 37$

$11 \mathrm{~m} \quad 28$ 
Vol. or

Sec.

Page

$\mathrm{C}_{6} \mathrm{H}_{12} \mathrm{O}_{6}$

$\mathrm{C}_{6} \mathrm{H}_{15} \mathrm{HoO}_{12} \mathrm{~S}_{3} \cdot 9 \mathrm{H}_{2} \mathrm{O}$

$\alpha-\mathrm{D}-\mathrm{Glucose}$

Holmium ethylsulfate hydrate

Neodymium ethylsulfate hydrate

o-Bromobenzoic acid

m-Chlorobenzoic acid

p-Fluorobenzoic acid

p-Iodobenzoic Acid

Methyl sulfonanilide

Pimelic acid

Mercury o-phthalate

Potassium hydrogen o-phthalate

Thallium hydrogen phthalate

2,4,6-Trinitrophenetole

p-Anisic acid

Acetanilide (calc, pattern)

Acetanilide

Sodium barbital

Barbital, form I

Barbital, form II

Barbital, form IV

Metharbital

Allobarbital

Ethylenediaminetetraacetic Acid

(-)-Ephedrine hydrochloride

Vinbarbital, form I

Amobarbital, form I

Amobarbital, form II

Azobenzene

Benzidine hydrochloride

Phenobarbital, form III

Phenobarbital Hydrate

Copper tetrapyrazole chloride

Nickel tetrapyrazole chloride

Copper tetraimidazole nitrate

$(\mathrm{N}, \mathrm{N})$-Dimet hyl tryptamine

Bufotenine

Psilocin

Sucrose

Hexamethylenediammonium adipate

Procaine hydrochloride

Psilocybin methanolate

4-Acety1-2'-fluorodipheny1

Methapyrilene hydrochloride

$\beta$-Carbamazepine

Dibenzoylmethane

Diazepam

N-Pheny1-2-naphthylamine

Chlorpromazine

Morphine hydrochloride hydrate

L-Cocaine hydrochloride

Phencyclidine hydrochloride

Codeine hydrobromide hydrate

Cadmium hexaimidazole nitrate

Nickel hexaimidazole nitrate

(t)-Amphetamine sulfate

Naloxone hydrochloride hydrate

Cinchonine

Imipramine hydrochloride

Benactyzine hydrochloride

$11 \mathrm{~m} \quad 28$

$1 \mathrm{~m} \quad 18$

941

$16 \mathrm{~m} \quad 22$

$16 \mathrm{~m} \quad 30$

$16 \mathrm{~m} \quad 36$

$19 \mathrm{~m} \quad 47$

$9 \mathrm{~m} \quad 78$

$7 \mathrm{~m} \quad 153$

$10 \mathrm{~m} \quad 113$

$4 \mathrm{~m} \quad 30$

$16 \mathrm{~m} \quad 75$

$8 \mathrm{~m} \quad 152$

$16 \mathrm{~m} \quad 11$

$14 \mathrm{~m} \quad 38$

$16 \mathrm{~m} \quad 7$

$16 \mathrm{~m} \quad 157$

$15 \mathrm{~m} \quad 126$

$15 \mathrm{~m} \quad 128$

$15 \mathrm{~m} \quad 130$

$15 \mathrm{~m} \quad 177$

$14 \mathrm{~m} \quad 41$

$19 \mathrm{~m} \quad 45$

$16 \mathrm{~m} \quad 124$

$16 \mathrm{~m} \quad 162$

$15 \mathrm{~m} \quad 114$

$15 \mathrm{~m} \quad 117$

$7 \mathrm{~m} \quad 86$

$18 \mathrm{~m} \quad 14$

$16 \mathrm{~m} \quad 144$

$19 \mathrm{~m} \quad 88$

$8 \mathrm{~m} \quad 31$

$8 \mathrm{~m} \quad 44$

$13 \mathrm{~m} \quad 24$

$14 \mathrm{~m} \quad 109$

$15 \mathrm{~m} \quad 133$

$16 \mathrm{~m} \quad 152$

$11 \mathrm{~m} \quad 66$

$7 \mathrm{~m} \quad 121$

$16 \mathrm{~m} \quad 149$

$16 \mathrm{~m} \quad 154$

$8 \mathrm{~m} \quad 91$

$14 \mathrm{~m} \quad 112$

$18 \mathrm{~m} \quad 24$

$7 \mathrm{~m} \quad 115$

$14 \mathrm{~m} \quad 106$

$6 \mathrm{~m} \quad 29$

$14 \mathrm{~m} \quad 60$

$16 \mathrm{~m} \quad 133$

$16 \mathrm{~m} \quad 114$

$16 \mathrm{~m} \quad 141$

$16 \mathrm{~m} \quad 117$

$8 \mathrm{~m} \quad 23$

$7 \mathrm{~m} \quad 27$

$15 \mathrm{~m} \quad 119$

$16 \mathrm{~m} \quad 136$

$17 \mathrm{~m} \quad 26$

$16 \mathrm{~m} \quad 129$

$16 \mathrm{~m} \quad 92$

$\alpha$-Dihydrophyllocladene, hartite

(or bombiccite)

Haloperidol

Cannabidiol

Clopenthixol hydrate

$\Delta^{9}$-Tetrahydrocannabinolic acid $\mathrm{B}$
$16 \mathrm{~m} \quad 122$

$16 \mathrm{~m} \quad 127$

$16 \mathrm{~m} \quad 111$

$17 \mathrm{~m} \quad 28$

$16 \mathrm{~m} \quad 160$ 
Vol. or

Sec.

Page

$\mathrm{C}_{24} \mathrm{H}_{32} \mathrm{~N}_{2} \mathrm{O}_{2} \mathrm{Pd}$

Palladium bis-(N-isopropyl-3-

ethylsalicylaldiminate)

$\mathrm{C}_{25} \mathrm{H}_{15} \mathrm{~N}_{6}$

$\mathrm{C}_{33} \mathrm{H}_{40} \mathrm{~N}_{2} \mathrm{O}_{9}$

$\mathrm{N}$-Methylphenazinium- $7,7,8,8-$

tet racyanoquinodimethanide

$7 m$

144

Reserpine 
Acetanilide

Acetanilide

4-Acetyl-2' -fluorodiphenyl

Alanine, L-

Allobarbital

Amobarbital, form I

Amobarbital, form II

Ammonium acetate

Ammonium formate

Ammonium oxalate hydrate (oxammite)

Ammonium yttrium oxalate hydrate

Amphetamine sulfate, (+)-

p-Anisic acid

Ascorbic acid, L-

Azobenzene

Barbital, form I

Barbital, form II

Barbital, form IV

Benactyzine hydrochloride

Benzidine hydrochloride

o-Bromobenzoic acid

Bufotenine

Cadmium hexaimidazole nitrate

Calcium formate

Calcium malate hydrate

Cannabidiol

Carbamazepine, $\beta$ -

m-Chlorobenzoic acid

Chlorpromazine

Cinchonine

Clopenthixol hydrate

Cobalt acetate hydrate

Cocaine hydrochloride, L-

Codeine hydrobromide hydrate

Copper glutamate hydrate

Copper tetraimidazole nitrate

Copper tetrapyrazole chloride

Creatinine

Cysteine, L-

Dextrose

Diazepam

Dibenzoylmethane

Dihydrophyllocladene, $\alpha$-, hartite (or bombiccite)

(N,N) -Dimethylt ryptamine

bis-(o-Dodecacarborane)

Ephedrine hydrochloride, (-)-

Ethylenediamine hydrochloride

Ethylenediaminitetraacetic acid

p-Fluorobenzoic acid

Glucose, $\alpha-\mathrm{D}-$

Glutamic acid, $\beta-\mathrm{L}-$

Glycine, $\alpha$ -

Glyoxime

Guanidinium chloride

Haloperidol

Hexamethylenediammonium adipate

Hexame thy lenet et ramine

HMX, $\alpha-$

HMX, $\beta-$

Holmium ethylsulfate hydrate

Hydroquinone, $\gamma-$

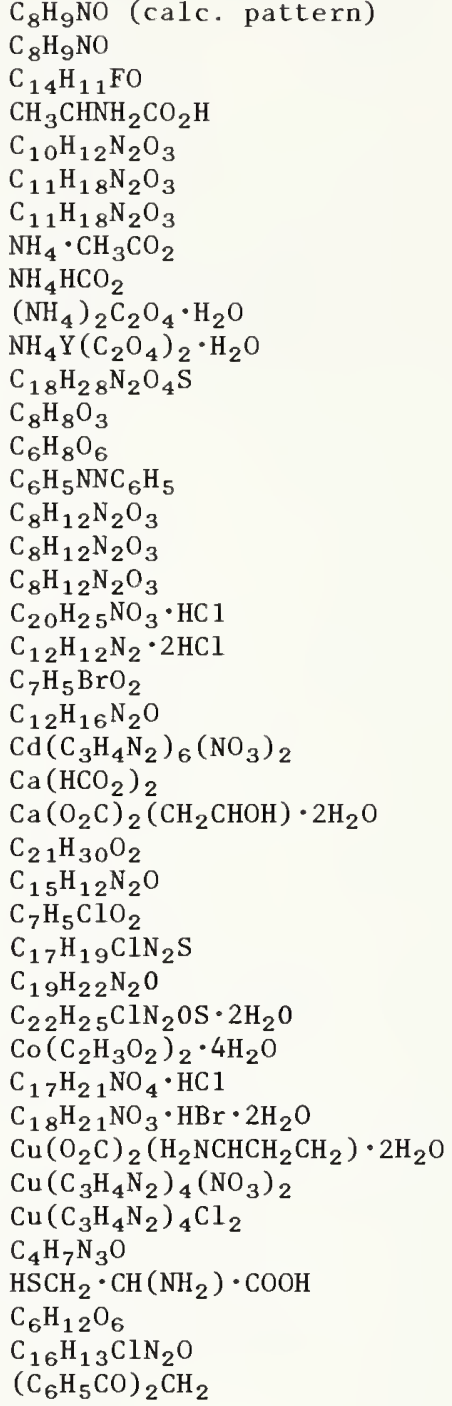

Vol. or

Sec.

Page

$14 \mathrm{~m}$

$16 \mathrm{~m}$

$8 \mathrm{~m} \quad 91$

$8 m-93$

$14 \mathrm{~m} \quad 41$

$15 \mathrm{~m} \quad 114$

$15 \mathrm{~m} \quad 117$

$8 \mathrm{~m} \quad 95$

$11 \mathrm{~m}$

$7-5$

$8 \mathrm{~m}-97$

$15 \mathrm{~m} \quad 119$

$16 \mathrm{~m} \quad 11$

$8 \mathrm{~m} \quad 99$

$7 \mathrm{~m} \quad 86$

$15 \mathrm{~m} \quad 126$

$15 \mathrm{~m} \quad 128$

$15 \mathrm{~m} \quad 130$

$16 \mathrm{~m}$

$18 \mathrm{~m} \quad 14$

$16 \mathrm{~m} \quad 22$

$15 \mathrm{~m}$

$8 \mathrm{~m} \quad 23$

76

$16 \mathrm{~m} \quad 111$

$18 \mathrm{~m}-24$

$16 \mathrm{~m} \quad 30$

$14 \mathrm{~m} \quad 60$

$17 \mathrm{~m} \quad 26$

$17 \mathrm{~m} \quad 28$

$12 \mathrm{~m} \quad 19$

$16 \mathrm{~m} \quad 114$

$16 \mathrm{~m} \quad 117$

$7 \mathrm{~m} \quad 110$

$13 \mathrm{~m} \quad 24$

$8 \mathrm{~m}$

$15 \mathrm{~m} \quad 31$

$11 \mathrm{~m} \quad 86$

$11 \mathrm{~m} \quad 28$

$14 \mathrm{~m} \quad 106$

$7 \mathrm{~m} \quad 115$

$\mathrm{C}_{20} \mathrm{H}_{34}$

$\mathrm{C}_{12} \mathrm{H}_{16} \mathrm{~N}_{2}$

$\mathrm{C}_{4} \mathrm{~B}_{20} \mathrm{H}_{22}$

$\mathrm{C}_{10} \mathrm{H}_{15} \mathrm{NO} \cdot \mathrm{HC} 1$

$\mathrm{C}_{2} \mathrm{H}_{8} \mathrm{~N}_{2} \cdot 2 \mathrm{HC} 1$

$\mathrm{C}_{10} \mathrm{H}_{12} \mathrm{~N}_{2} \mathrm{O}_{8}$

$\mathrm{C}_{7} \mathrm{H}_{5} \mathrm{FO}_{2}$

$\mathrm{C}_{6} \mathrm{H}_{12} \mathrm{O}_{6}$

$\mathrm{C}_{5} \mathrm{H}_{9} \mathrm{NO}_{4}$

$\mathrm{C}_{2} \mathrm{H}_{5} \mathrm{NO}_{2}$,

$\mathrm{H}_{2} \mathrm{C}_{2}(\mathrm{NOH})_{2}$

$\mathrm{CH}_{5} \mathrm{~N}_{3} \cdot \mathrm{HCl}$

$\mathrm{C}_{21} \mathrm{H}_{23} \mathrm{ClFNO}_{2}$

$\left(\mathrm{CH}_{2}\right)_{4}\left(\mathrm{CO}_{2} \mathrm{H}_{3} \mathrm{~N}\right)_{2}\left(\mathrm{CH}_{2}\right)_{6}$

$\mathrm{C}_{6} \mathrm{H}_{12} \mathrm{~N}_{4}$

$\mathrm{C}_{4} \mathrm{H}_{8} \mathrm{~N}_{8} \mathrm{O}_{8}$

$\mathrm{C}_{4} \mathrm{H}_{8} \mathrm{~N}_{8} \mathrm{O}_{8}$

$\mathrm{Ho}\left[\left(\mathrm{C}_{2} \mathrm{H}_{5}\right) \mathrm{SO}_{4}\right]_{3} \cdot 9 \mathrm{H}_{2} \mathrm{O}$

$\mathrm{HOC}_{6} \mathrm{H}_{4} \mathrm{OH}$
$16 \mathrm{~m} \quad 122$

$14 \mathrm{~m} \quad 109$

$6 \mathrm{~m} \quad 7$

$16 \mathrm{~m} \quad 124$

$19 \mathrm{~m} \quad 43$

$19 \mathrm{~m} \quad 45$

$16 \mathrm{~m} \quad 36$

$11 \mathrm{~m} \quad 28$

$17 \mathrm{~m} \quad 32$

$17 \mathrm{~m} \quad 34$

$8 \mathrm{~m} \quad 102$

$17 \mathrm{~m} \quad 35$

$16 \mathrm{~m} \quad 127$

$7 \mathrm{~m} \quad 121$

$17 \mathrm{~m} \quad 37$

$11 \mathrm{~m} \quad 100$

$11 \mathrm{~m} \quad 102$

$1 \mathrm{~m} \quad 18$

$8 \mathrm{~m} \quad 107$ 
Imipramine hydrochloride p-Iodobenzoic acid

Iodo form

Iron oxalate hydrate (humboldtine)

Lead formate

Lithium oxalate

Manganese tartrate

Mercury acetate

Mercury o-phthalate

Methapyrilene hydrochloride

Metharbital

Methyl sulfonanilide

N-Methylphenazinium-7, 7,8,8-tetra-

cyanoquinodimethanide

Morphine hydrochloride hydrate

Naloxone hydrochloride hydrate

2-Naphthylamine, N-phenyl-

Neodymium ethylsulfate hydrate

Nickel acetate hydrate

Nickel hexaimidazole nitrate

Nickel tetrapyrazole chloride

Nicotinic acid

Octahydro-1, 3, 5, 7-tetranitro-

$1,3,5,7$-tetrazocine $(\alpha$-HMX)

0ctahydro- $1,3,5,7$-tetranitro-

$1,3,5,7$-tetrazocine ( $\beta$-HMX)

Oxalic acid hydrate

Palladium bis-(N-isopropyl-3-

ethylsalicylaldiminate)

Pentaerythritol

Phencyclidine hydrochloride

Phenobarbital, form III

Phenobarbital hydrate

Phenylhydrazine hydrochloride

Picric acid

Pimelic acid

Potassium formate-formic acid complex

Potassium hydrogen o-phthalate

Potassium hydrogen oxalate hydrate

Potassium oxalate hydrate

Potassium oxalate perhydrate

Potassium sodium tartrate hydrate

Procaine hydrochloride

Psilocin

Psilocybin methanolate

Reserpine

Rubidium oxalate perhydrate

Silver oxalate

Sodium acetate hydrate

Sodium barbital

Sodium glutamate hydrate

Sodium hydrogen oxalate hydrate

Sodium oxalate

Sodium D-tartrate hydrate

Strontium formate

Strontium formate hydrate

Sucrose

Tartaric acid, D-

$\Delta^{9}$-Tetrahydrocannabinolic acid $B$

Thallium hydrogen phthalate

Thiosemicarbazide

Thiourea

Trimethylammonium chloride

2,4,6-Trinitrophenetole

Uranyl acetate hydrate

Urea

Uric acid, phase 1, (calc. pattern)

Uric acid (phase 1)

Vinbarbital, form I
$\mathrm{C}_{19} \mathrm{H}_{24} \mathrm{~N}_{2} \cdot \mathrm{HCl}$

$\mathrm{C}_{7} \mathrm{H}_{5} \mathrm{IO}_{2}$

$\mathrm{CHI}_{3}$

$\mathrm{FeC}_{2} \mathrm{O}_{4} \cdot 2 \mathrm{H}_{2} \mathrm{O}$

$\mathrm{Pb}\left(\mathrm{HCO}_{2}\right)_{2}$

$\mathrm{Li}_{2} \mathrm{C}_{2} \mathrm{O}_{4}$

$\mathrm{C}_{4} \mathrm{H}_{4} \mathrm{MnO}_{6}$

$\mathrm{C}_{4} \mathrm{H}_{6} \mathrm{Hg}_{2} \mathrm{O}_{4}$

$\mathrm{C}_{6} \mathrm{H}_{4}\left(\mathrm{CO}_{2} \mathrm{Hg}\right)_{2}$

$\mathrm{C}_{14} \mathrm{H}_{19} \mathrm{~N}_{3} \mathrm{~S} \cdot \mathrm{HC} 1$

$\mathrm{C}_{9} \mathrm{H}_{14} \mathrm{~N}_{2} \mathrm{O}_{3}$

$\mathrm{C}_{6} \mathrm{H}_{5} \mathrm{NHSO}_{2} \mathrm{CH}_{3}$

$\mathrm{C}_{25} \mathrm{H}_{15} \mathrm{~N}_{6}$

$\mathrm{C}_{17} \mathrm{H}_{19} \mathrm{NO}_{3} \cdot \mathrm{HCl} \cdot 3 \mathrm{H}_{2} \mathrm{O}$

$7 \mathrm{~m}=146$

$\mathrm{C}_{19} \mathrm{H}_{21} \mathrm{NO}_{4} \cdot \mathrm{HCl} \cdot 2 \mathrm{H}_{2} \mathrm{O}$

$\mathrm{C}_{10} \mathrm{H}_{7} \mathrm{NHC}_{6} \mathrm{H}_{5}$

$\mathrm{Nd}\left[\left(\mathrm{C}_{2} \mathrm{H}_{5}\right) \mathrm{SO}_{4}\right]_{3} \cdot 9 \mathrm{H}_{2} \mathrm{O}$

$\mathrm{Ni}\left(\mathrm{C}_{2} \mathrm{H}_{3} \mathrm{O}_{2}\right)_{2} \cdot 4 \mathrm{H}_{2} \mathrm{O}$

$\mathrm{Ni}\left(\mathrm{C}_{3} \mathrm{H}_{4} \mathrm{~N}_{2}\right)_{6}\left(\mathrm{NO}_{3}\right)_{2}$

$\mathrm{Ni}\left(\mathrm{C}_{3} \mathrm{H}_{4} \mathrm{~N}_{2}\right){ }_{4} \mathrm{Cl}_{2}$

$\mathrm{C}_{6} \mathrm{H}_{5} \mathrm{NO}_{2}$

$\mathrm{C}_{4} \mathrm{H}_{8} \mathrm{~N}_{8} \mathrm{O}_{8}$

$\mathrm{C}_{4} \mathrm{H}_{8} \mathrm{~N}_{8} \mathrm{O}_{8}$

$\mathrm{C}_{2} \mathrm{H}_{2} \mathrm{O}_{4} \cdot 2 \mathrm{H}_{2} \mathrm{O}$

$\mathrm{Pd}\left(\mathrm{C}_{12} \mathrm{H}_{16} \mathrm{NO}\right)_{2}$

$\mathrm{C}_{5} \mathrm{H}_{12} \mathrm{O}_{4}$

$\mathrm{C}_{17} \mathrm{H}_{25} \mathrm{~N} \cdot \mathrm{HCl}$

$\mathrm{C}_{12} \mathrm{H}_{12} \mathrm{~N}_{2} \mathrm{O}_{3}$

$\mathrm{C}_{12} \mathrm{H}_{12} \mathrm{~N}_{2} \mathrm{O}_{3} \cdot \mathrm{H}_{2} \mathrm{O}$

$\mathrm{C}_{6} \mathrm{H}_{8} \mathrm{~N}_{2} \cdot \mathrm{HCl}$

$\mathrm{C}_{6} \mathrm{H}_{3} \mathrm{~N}_{3} \mathrm{O}_{7}$

$\left(\mathrm{CH}_{2}\right)_{5}\left(\mathrm{CO}_{2} \mathrm{H}\right)_{2}$

$\mathrm{KO}_{2} \mathrm{CH} \cdot \mathrm{HO}_{2} \mathrm{CH}$

$\mathrm{C}_{6} \mathrm{H}_{4}(\mathrm{COOH})(\mathrm{COOK})$

$\mathrm{C}_{4} \mathrm{H}_{3} \mathrm{KO}_{8} \cdot 2 \mathrm{H}_{2} \mathrm{O}$

$\mathrm{K}_{2} \mathrm{C}_{2} \mathrm{O}_{4} \cdot \mathrm{H}_{2} \mathrm{O}$

$\mathrm{K}_{2} \mathrm{C}_{2} \mathrm{O}_{4} \cdot \mathrm{H}_{2} \mathrm{O}_{2}$

$\mathrm{C}_{4} \mathrm{H}_{4} \mathrm{KNaO}_{6} \cdot 4 \mathrm{H}_{2} \mathrm{O}$

$\mathrm{C}_{13} \mathrm{H}_{20} \mathrm{~N}_{2} \mathrm{O}_{2} \cdot \mathrm{HCl}$

$\mathrm{C}_{12} \mathrm{H}_{16} \mathrm{~N}_{2} \mathrm{O}$

$\mathrm{C}_{13} \mathrm{H}_{21} \mathrm{~N}_{2} \mathrm{O}_{4} \mathrm{P}$

$\mathrm{C}_{33} \mathrm{H}_{40} \mathrm{~N}_{2} \mathrm{O}_{9}$

$\mathrm{Rb}_{2} \mathrm{C}_{2} \mathrm{O}_{4} \cdot \mathrm{H}_{2} \mathrm{O}_{2}$

$\mathrm{Ag}_{2} \mathrm{C}_{2} \mathrm{O}_{4}$

$\mathrm{C}_{2} \mathrm{H}_{3} \mathrm{NaO}_{2} \cdot 3 \mathrm{H}_{2} \mathrm{O}$

$\mathrm{C}_{8} \mathrm{H}_{11} \mathrm{~N}_{2} \mathrm{NaO}_{3}$

$\mathrm{C}_{5} \mathrm{H}_{8} \mathrm{NNaO}_{4} \cdot \mathrm{H}_{2} \mathrm{O}$

$\mathrm{C}_{2} \mathrm{HNaO}_{4} \cdot \mathrm{H}_{2} \mathrm{O}$

$\mathrm{Na}_{2} \mathrm{C}_{2} \mathrm{O}_{4}$

$\left(\mathrm{CHOH}-\mathrm{CO}_{2} \mathrm{Na}\right)_{2} \cdot 2 \mathrm{H}_{2} \mathrm{O}$

$\mathrm{Sr}\left(\mathrm{CHO}_{2}\right)_{2}$

$\mathrm{Sr}\left(\mathrm{CHO}_{2}\right)_{2} \cdot 2 \mathrm{H}_{2} \mathrm{O}$ (orthorhombic)

$\mathrm{C}_{12} \mathrm{H}_{22} \mathrm{O}_{11}$

$\left(\mathrm{CHOHCO}_{2} \mathrm{H}\right)_{2}$

$\mathrm{C}_{22} \mathrm{H}_{30} \mathrm{O}_{4}$

$\mathrm{C}_{8} \mathrm{H}_{5} \mathrm{O}_{4} \mathrm{Tl}$

$\mathrm{CH}_{5} \mathrm{~N}_{3} \mathrm{~S}$

$\mathrm{CH}_{4} \mathrm{~N}_{2} \mathrm{~S}$

$\mathrm{C}_{3} \mathrm{H}_{9} \mathrm{~N} \cdot \mathrm{HC} 1$

$\mathrm{C}_{8} \mathrm{H}_{7} \mathrm{~N}_{3} \mathrm{O}_{7}$

$\mathrm{C}_{4} \mathrm{H}_{6} \mathrm{O}_{6} \mathrm{U} \cdot 2 \mathrm{H}_{2} \mathrm{O}$

$\mathrm{CO}\left(\mathrm{NH}_{2}\right)_{2}$

$\mathrm{C}_{5} \mathrm{H}_{4} \mathrm{~N}_{4} \mathrm{O}_{3}$

$\mathrm{C}_{5} \mathrm{H}_{4} \mathrm{~N}_{4} \mathrm{O}_{3}$

$\mathrm{C}_{11} \mathrm{H}_{16} \mathrm{~N}_{2} \mathrm{O}_{3}$
$19 \mathrm{~m}$

$18 \mathrm{~m}$

$10 \mathrm{~m}$

8

$10 \mathrm{~m}$

$19 \mathrm{~m}$

$17 m$

$10 \mathrm{~m}$

$14 \mathrm{~m}$

$15 \mathrm{~m}$

$9 \mathrm{~m}$

11

$16 \mathrm{~m}-\quad 55$

129

47

34

24

30

34

57

51

113

112

177

78

146

133

136

$17 \mathrm{~m} \quad 60$

$9 \mathrm{~m} \quad 39$

$9 \mathrm{~m} \quad 96$

$15 \mathrm{~m} \quad 55$

$16 \mathrm{~m} \quad 149$

$16 \mathrm{~m} \quad 152$

$16 \mathrm{~m} \quad 154$

$8 \mathrm{~m} \quad 123$

$9 \mathrm{~m} \quad 102$

$15 \mathrm{~m} \quad 66$

$16 \mathrm{~m} \quad 157$

$17 \mathrm{~m} \quad 70$

$17 \mathrm{~m} \quad 72$

$6 \mathrm{~m} \quad 70$

$11 \mathrm{~m} \quad 110$

$8 \quad 55$

$8 \quad 56$

$11 \mathrm{~m} \quad 66$

$16 \mathrm{~m} \quad 160$

$16 \mathrm{~m} \quad 75$

$17 \mathrm{~m} \quad 81$

$17 \mathrm{~m} \quad 83$

$9 \mathrm{~m} \quad 113$

$8 \mathrm{~m} \quad 152$

$18 \mathrm{~m} \quad 76$

$7 \quad 61$

8m $\quad 154$

$16 \mathrm{~m}$

$16 \mathrm{~m} \quad 162$
$9 \mathrm{~m} \quad 47$

$7 \mathrm{~m} \quad 168$ 
Zinc acetate hydrate

Zinc diimidazole chloride

Zinc glutamate hydrate,
$\mathrm{C}_{4} \mathrm{H}_{6} \mathrm{O}_{4} \cdot 2 \mathrm{H}_{2} \mathrm{O}$

$\mathrm{Zn}\left(\mathrm{C}_{3} \mathrm{H}_{4} \mathrm{~N}_{2}\right)_{2} \mathrm{Cl}_{2}$

$\mathrm{Zn}\left(\mathrm{O}_{2} \mathrm{CCHNH}_{2} \mathrm{CH}_{2} \mathrm{CH}_{2} \mathrm{CO}_{2}\right) \cdot 2 \mathrm{H}_{2} \mathrm{O}$
$18 \mathrm{~m}$

$7 \mathrm{~m}$

$7 \mathrm{~m}$

78

123

170 
Vol. or

Vol. or

Sec. Page

Sec. Page

Acanthite, $\mathrm{Ag}_{2} \mathrm{~S} \ldots \ldots \ldots \ldots \ldots$

Acmite, $\mathrm{NaFe}\left(\mathrm{SiO}_{3}\right)_{2} \ldots \ldots \ldots \ldots$

Aeschynite $\mathrm{CeNbTiO}_{6} \ldots \ldots \ldots \ldots \ldots$

Akermanite, $\mathrm{Ca}_{2} \mathrm{MgSi}_{2} \mathrm{O}_{7} \ldots \ldots \ldots \ldots$

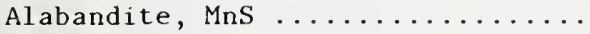

Anatase, $\mathrm{TiO}_{2} \ldots \ldots \ldots \ldots \ldots \ldots$

Andradite, $\mathrm{Ca}_{3} \mathrm{Fe}_{2} \mathrm{Si}_{3} \mathrm{O}_{12} \ldots \ldots \ldots \ldots$

Anglesite, $\mathrm{PbSO}_{4} \ldots \ldots \ldots \ldots \ldots \ldots$

Anhydrite, $\mathrm{CaSO}_{4} \ldots \ldots \ldots \ldots \ldots$

Annabergite, $\mathrm{Ni}_{3}\left(\mathrm{AsO}_{4}\right)_{2} \cdot 8 \mathrm{H}_{2} \mathrm{O} \ldots \ldots$.

Antarcticite, $\mathrm{CaCl}_{2} \cdot 6 \mathrm{H}_{2} \mathrm{O} \ldots \ldots \ldots$

Antimony, $\mathrm{Sb} \ldots \ldots \ldots \ldots \ldots \ldots \ldots$

Aphthitalite, $\mathrm{K}_{3} \mathrm{Na}\left(\mathrm{SO}_{4}\right)_{2} \ldots \ldots \ldots$

Aragonite, $\mathrm{CaCO}_{3} \ldots \ldots \ldots \ldots \ldots$

Aragonite, $\mathrm{CaCO}_{3}$ (calculated pattern)

Arcanite, $\mathrm{K}_{2} \mathrm{SO}_{4} \ldots \ldots \ldots \ldots \ldots \ldots$

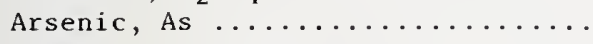

Arsenolite, $\mathrm{As}_{2} \mathrm{O}_{3} \ldots \ldots \ldots \ldots \ldots$

Aurostibite, $\mathrm{AuSb}_{2} \ldots \ldots \ldots \ldots \ldots$

Avicennite, $\mathrm{Tl}_{2} \mathrm{O}_{3} \ldots \ldots \ldots \ldots \ldots$

*Azurite, $\mathrm{Cu}_{3}(\mathrm{OH})_{2}\left(\mathrm{CO}_{3}\right)_{2} \ldots \ldots \ldots$.

$*$ Bahianite, $\mathrm{Al}_{5.66} \mathrm{Fe}_{0.09} \mathrm{Sb}_{2.95} \mathrm{O}_{16}$.

Barite, $\mathrm{BaSO}_{4} \ldots \ldots \ldots \ldots \ldots \ldots$

Bassanite, $\mathrm{CaSO}_{4} \cdot 0.5 \mathrm{H}_{2} \mathrm{O} \ldots \ldots \ldots$

Berlinite, $\mathrm{AlPO}_{4} \ldots \ldots \ldots \ldots \ldots \ldots$

Berndtite, $\mathrm{SnS}_{2} \ldots \ldots \ldots \ldots \ldots \ldots$

*Beryl, $\mathrm{Be}_{3} \mathrm{Al}_{2} \mathrm{Si}_{6} \mathrm{O}_{18} \ldots \ldots \ldots \ldots$

Bischofite, $\mathrm{MgCl}_{2} \cdot 6 \mathrm{H}_{2} \mathrm{O} \ldots \ldots \ldots \ldots$

Bismite, $\alpha-\mathrm{Bi}_{2} \mathrm{O}_{3} \ldots \ldots \ldots \ldots \ldots$

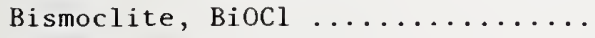

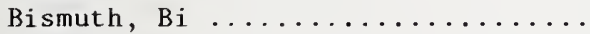

Bismuthinite, $\mathrm{Bi}_{2} \mathrm{~S}_{3} \ldots \ldots \ldots \ldots$

Bixbyite, $\alpha-\mathrm{Mn}_{2} \mathrm{O}_{3} \ldots \ldots \ldots \ldots \ldots$

*Bloedite, $\mathrm{Na}_{2} \mathrm{Mg}\left(\mathrm{SO}_{4}\right)_{2} \cdot 4 \mathrm{H}_{2} \mathrm{O} \ldots \ldots$.

Boehmite, $\mathrm{Al}_{2} \mathrm{O}_{3} \cdot \mathrm{H}_{2} \mathrm{O} \ldots \ldots \ldots \ldots \ldots$

$*$ Bombiccite, $\mathrm{C}_{20} \mathrm{H}_{34} \ldots \ldots \ldots \ldots \ldots$

Borax, $\mathrm{Na}_{2} \mathrm{~B}_{4} \mathrm{O}_{5}(\mathrm{OH})_{4} \cdot 8 \mathrm{H}_{2} \mathrm{O} \ldots \ldots \ldots$

Bromargyrite, $\mathrm{AgBr} \ldots \ldots \ldots \ldots \ldots$

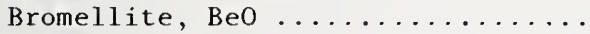

*Brookite, $\mathrm{TiO}_{2} \ldots \ldots \ldots \ldots \ldots$

Brownmillerite, $\mathrm{Ca}_{4} \mathrm{Al}_{2} \mathrm{Fe}_{2} \mathrm{O}_{10} \ldots \ldots$

Brucite, $\mathrm{Mg}(\mathrm{OH})_{2} \ldots \ldots \ldots \ldots$.

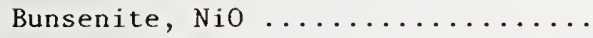

Burkeite, $\mathrm{Na}_{6} \mathrm{CO}_{3}\left(\mathrm{SO}_{4}\right)_{2} \ldots \ldots \ldots \ldots$

*Butlerite, $\mathrm{Fe}(\mathrm{OH}) \mathrm{SO}_{4} \cdot 2 \mathrm{H}_{2} \mathrm{O} \ldots \ldots \ldots$

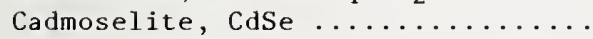

Calcite, $\mathrm{CaCO}_{3} \ldots \ldots \ldots \ldots \ldots \ldots$

Calomel, $\mathrm{Hg}_{2} \mathrm{Cl}_{2} \ldots \ldots \ldots \ldots \ldots$

Carnallite, $\mathrm{KMgCl}_{3} \cdot 6 \mathrm{H}_{2} \mathrm{O} \ldots \ldots \ldots \ldots$

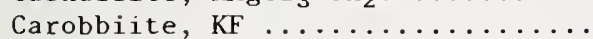

Cassiterite, $\mathrm{SnO}_{2} \ldots \ldots \ldots \ldots \ldots$

Celestite, $\mathrm{SrSO}_{4} \ldots \ldots \ldots \ldots \ldots \ldots$

Cerianite, $\mathrm{CeO}_{2} \ldots \ldots \ldots \ldots \ldots \ldots$

Cerianite, $\mathrm{CeO}_{2} \ldots \ldots \ldots \ldots \ldots \ldots$

Cerussite, $\mathrm{PbCO}_{3} \ldots \ldots \ldots \ldots \ldots$

Cervantite, $\mathrm{Sb}_{2} \mathrm{O}_{4} \ldots \ldots \ldots \ldots \ldots$

*Chabazite, $\mathrm{Ca}_{2} \mathrm{Al}_{4} \mathrm{Si}_{8} \mathrm{O}_{24} \cdot 12 \mathrm{H}_{2} \mathrm{O} \ldots$

Chal cocyanite, $\mathrm{CuSO}_{4} \ldots \ldots \ldots \ldots \ldots$

Chernovite, $\mathrm{YAsO}_{4} \ldots \ldots \ldots \ldots \ldots \ldots$

Chiolite, $\mathrm{Na}_{5} \mathrm{Al}_{3} \mathrm{~F}_{14} \ldots \ldots \ldots \ldots$

Chloraluminite, $\mathrm{AlCl}_{3} \cdot 6 \mathrm{H}_{2} \mathrm{O} \ldots \ldots \ldots$

Chlorargyrite, $\mathrm{AgCl} \ldots \ldots \ldots \ldots \ldots$

Chloromagnesite, $\mathrm{MgCl}_{2} \ldots \ldots \ldots \ldots$

Chromatite, $\mathrm{CaCrO}_{4}$

Chromite, $\mathrm{FeCr}_{2} \mathrm{O}_{4} \ldots \ldots \ldots \ldots \ldots \ldots$

Chrysoberyl, $\mathrm{BeAl}_{2} \mathrm{O}_{4}$

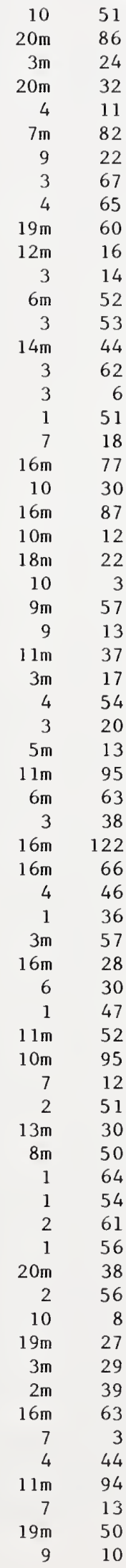

Cinnabar, $\mathrm{HgS} \ldots \ldots \ldots \ldots \ldots \ldots \ldots$

$\mathrm{Cl}$ audetite, $\mathrm{As}_{2} \mathrm{O}_{3}$

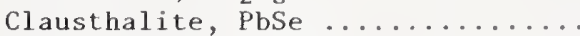

Clinobisvanite, $\mathrm{BiVO}_{4}$

Clinoenstatite, $\mathrm{MgSiO}_{3} \ldots \ldots \ldots \ldots$

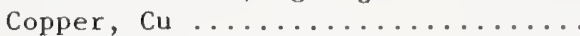

Cordierite, $\mathrm{Mg}_{2} \mathrm{Al}_{4} \mathrm{Si}_{5} \mathrm{O}_{18} \ldots \ldots \ldots$

Corundum, $\mathrm{Al}_{2} \mathrm{O}_{3}$

Cotunnite, $\mathrm{PbCl}_{2} \ldots \ldots \ldots \ldots \ldots \ldots$

Covellite, CuS

Cristobalite ( $\alpha$ or low) $\mathrm{SiO}_{2}$

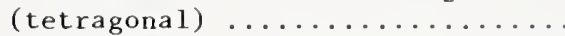

Cristobalite ( $\alpha$ or low) $\mathrm{SiO}_{2}$

(tetragonal, calculated pattern)

Cristobalite ( $\beta$ or high) $\mathrm{SiO}_{2}$ (cubic)

Cryolithionite, $\mathrm{Li}_{3} \mathrm{Na}_{3} \mathrm{Al}_{2} \mathrm{~F}_{12} \ldots \ldots$

Cryptohalite, $\left(\mathrm{NH}_{4}\right)_{2} \mathrm{SiF}_{6} \ldots \ldots \ldots$

Cuprite, $\mathrm{Cu}_{2} \mathrm{O} \ldots \ldots \ldots \ldots \ldots \ldots \ldots \ldots$

*Derbylite, $\mathrm{SbFe}_{4} \mathrm{Ti}_{3} \mathrm{O}_{13}(\mathrm{OH}) \ldots \ldots$.

*Diamond, C

¿Diaspore, $\mathrm{Al}_{2} \mathrm{O}_{3} \cdot \mathrm{H}_{2} \mathrm{O} \ldots \ldots \ldots \ldots$

Diopside, $\mathrm{CaMg}\left(\mathrm{SiO}_{3}\right)_{2} \ldots \ldots \ldots \ldots$

*Dravite, $\mathrm{NaMg}_{3} \mathrm{Al}_{6} \mathrm{~B}_{3} \mathrm{Si}_{6} \mathrm{O}_{27}(\mathrm{OH})_{4} \ldots$

Eitelite, $\mathrm{Na}_{2} \mathrm{Mg}\left(\mathrm{CO}_{3}\right)_{2} \ldots \ldots \ldots \ldots$

Elpasolite, $\mathrm{K}_{2} \mathrm{NaAlF}_{6}$

EEnstatite, $\mathrm{MgSiO}_{3}$

Epsomite, $\mathrm{MgSO}_{4} \cdot 7 \mathrm{H}_{2} \mathrm{O} \ldots \ldots \ldots \ldots \ldots$

Eriochalcite, $\mathrm{CuCl}_{2} \cdot 2 \mathrm{H}_{2} \mathrm{O} \ldots \ldots \ldots$

Erythrite, $\mathrm{Co}_{3}\left(\mathrm{AsO}_{4}\right)_{2} \cdot 8 \mathrm{H}_{2} \mathrm{O} \ldots \ldots$.

Erythrosiderite, $\mathrm{K}_{2} \mathrm{FeCl}_{5} \cdot \mathrm{H}_{2} \mathrm{O} \ldots \ldots$

Eskolaite, $\mathrm{Cr}_{2} \mathrm{O}_{3}$

Ettringite, $\mathrm{Ca}_{6} \mathrm{Al}_{2} \mathrm{~S}_{3} \mathrm{O}_{18} \cdot 31 \mathrm{H}_{2} \mathrm{O} \ldots$.

Fairchildite, $\mathrm{K}_{2} \mathrm{Ca}\left(\mathrm{CO}_{3}\right)_{2} \ldots \ldots \ldots$

Farringtonite, $\mathrm{Mg}_{3}\left(\mathrm{PO}_{4}\right)_{2} \ldots \ldots \ldots$

Fayalite, $\mathrm{Fe}_{2} \mathrm{SiO}_{4} \ldots \ldots \ldots \ldots$

Fluorapatite, $\mathrm{Ca}_{5} \mathrm{~F}\left(\mathrm{PO}_{4}\right)_{3} \ldots \ldots \ldots$

Fluorite, $\mathrm{CaF}_{2} \ldots \ldots \ldots \ldots \ldots \ldots$

Forsterite, $\mathrm{Mg}_{2} \mathrm{SiO}_{4} \ldots \ldots \ldots \ldots \ldots$

Franklinite, $\mathrm{ZnFe}_{2} \mathrm{O}_{4} \ldots \ldots \ldots \ldots$

Fresnoite, $\mathrm{Ba}_{2} \mathrm{TiSi}_{2} \mathrm{O}_{8} \ldots \ldots \ldots \ldots$

Gahnite, $\mathrm{ZnAl}_{2} \mathrm{O}_{4} \ldots \ldots \ldots \ldots \ldots \ldots$

Galaxite, $\mathrm{MnAl}_{2} \mathrm{O}_{4} \ldots \ldots \ldots \ldots \ldots$

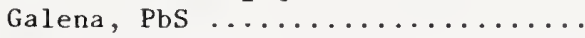

Gaspeite, $\mathrm{NiCO}_{3} \ldots \ldots \ldots \ldots \ldots \ldots$

Geikielite, $\mathrm{MgTiO}_{3} \ldots \ldots \ldots \ldots \ldots$

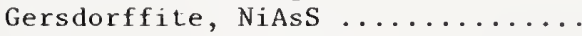

Glauberite, $\mathrm{Na}_{2} \mathrm{Ca}\left(\mathrm{SO}_{4}\right)_{2} \ldots \ldots \ldots \ldots$

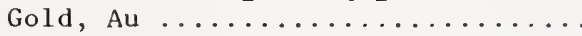

Goslarite, $\mathrm{ZnSO}_{4} \cdot 7 \mathrm{H}_{2} \mathrm{O} \ldots \ldots \ldots \ldots$

Greenockite, $\mathrm{CdS} \ldots \ldots \ldots \ldots \ldots \ldots$

*Groutite, $\mathrm{MnO}(\mathrm{OH}) \ldots \ldots \ldots \ldots \ldots$

Gunningite, $\mathrm{ZnSO}_{4} \cdot \mathrm{H}_{2} \mathrm{O} \ldots \ldots \ldots \ldots$

Gypsum, $\mathrm{CaSO}_{4} \cdot 2 \mathrm{H}_{2} \mathrm{O} \ldots \ldots \ldots \ldots \ldots$

Halite, $\mathrm{NaCl} \ldots \ldots \ldots \ldots \ldots \ldots \ldots$

*Hartite, $\mathrm{C}_{20} \mathrm{H}_{34} \ldots \ldots \ldots \ldots \ldots \ldots$

Hausmannite, $\mathrm{Mn}_{3} \mathrm{O}_{4} \ldots \ldots \ldots \ldots \ldots$

Hematite, $\alpha-\mathrm{Fe}_{2} \mathrm{O}_{3} \ldots \ldots \ldots \ldots \ldots$

*Hemimorphite, $\mathrm{Zn}_{4}(\mathrm{OH})_{2} \mathrm{Si}_{2} \mathrm{O}_{7} \cdot \mathrm{H}_{2} \mathrm{O} \ldots$

Hercynite, $\mathrm{FeAl}_{2} \mathrm{O}_{4} \ldots \ldots \ldots \ldots \ldots$

Hessite, $\mathrm{Ag}_{2} \mathrm{Te} \ldots \ldots \ldots \ldots \ldots$

Hetaerolite, $\mathrm{ZnMn}_{2} \mathrm{O}_{4} \ldots \ldots \ldots \ldots \ldots$

$*$ Hexahydroborite, $\mathrm{Ca} \mathrm{B}(\mathrm{OH})_{4} 2_{2} \cdot 2 \mathrm{H}_{2} \mathrm{O}$

Hieratite, $\mathrm{K}_{2} \mathrm{SiF}_{6} \ldots \ldots \ldots \ldots \ldots$.

Hoernesite, $\mathrm{Mg}_{3}\left(\mathrm{AsO}_{4}\right)_{2} \cdot 8 \mathrm{H}_{2} \mathrm{O} \ldots \ldots$

Hopeite, $\mathrm{Zn}_{3}\left(\mathrm{PO}_{4}\right)_{2} \cdot 4 \mathrm{H}_{2} \mathrm{O} \ldots \ldots$.

$\begin{array}{rr}4 & 17 \\ 3 \mathrm{~m} & 9 \\ 5 & 38 \\ 3 \mathrm{~m} & 14 \\ 20 \mathrm{~m} & 69 \\ 1 & 15 \\ 1 \mathrm{~m} & 28 \\ 9 & 3 \\ 12 \mathrm{~m} & 23 \\ 4 & 13 \\ 10 & 48\end{array}$

$15 \mathrm{~m}$

2

3

$5 \mathrm{~m}$

$3 m$

$11 \mathrm{~m}$

$9 \mathrm{~m}$

7

$18 \mathrm{~m}$

$19 \mathrm{~m}$

$14 \mathrm{~m}$

5

8

$8 \mathrm{~m}$

$19 \mathrm{~m}$

$20 \mathrm{~m}$

$3 m$

1
$20 \mathrm{~m}$

$9 \mathrm{~m}$

$9 \mathrm{~m}$

2

9

2

$1 \mathrm{~m}$

5
$1 \mathrm{~m}$

$6 \mathrm{~m}$

1

8

4
$11 \mathrm{~m}$

$19 \mathrm{~m}$

$17 \mathrm{~m}$

2
$16 \mathrm{~m}$

$10 \mathrm{~m}$

$18 \mathrm{~m}$

2
$19 \mathrm{~m}$

$19 \mathrm{~m}$

$10 \mathrm{~m}$

$16 \mathrm{~m}$

5

$19 \mathrm{~m}$

$16 \mathrm{~m}$
180

42

23

17
9
38
14
69
15
28
3
23
13

48

80
42
23
5
23
89
5

23

89

41

17

47

56

43

32

30

33
39

27

22

3

48

55

59

22

69

71

60

14

38

35

18

36

43

35

59

33

71

15

97

86

16

41

122

38

37

62

48

73

61

104

50

53
85 
Huebnerite, $\mathrm{MnWO}_{4} \ldots \ldots \ldots \ldots \ldots$

Humboldtine, $\mathrm{FeC}_{2} \mathrm{O}_{4} \cdot 2 \mathrm{H}_{2} \mathrm{O} \ldots \ldots \ldots$

Humite, $\mathrm{Mg}_{7} \mathrm{~F}_{2} \mathrm{Si}_{3} \mathrm{O}_{12} \ldots \ldots \ldots \ldots$

Hut tonite, $\beta$-ThSiO $\mathrm{Th}_{4} \ldots \ldots \ldots \ldots$

Hydromolysite, $\mathrm{FeCl}_{3} \cdot 6 \mathrm{H}_{2} \mathrm{O} \ldots \ldots \ldots$

Hydrophilite, $\mathrm{CaCl}_{2} \ldots \ldots \ldots \ldots \ldots$

Ilmenite, $\mathrm{FeTiO}_{3} \ldots \ldots \ldots \ldots \ldots$

Indialite, $\mathrm{Mg}_{2} \mathrm{Al}_{4} \mathrm{Si}_{5} \mathrm{O}_{18} \ldots \ldots \ldots \ldots$

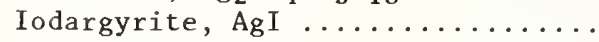

Iron, $\alpha-\mathrm{Fe} \ldots \ldots \ldots \ldots \ldots \ldots$

Jacobsite, $\mathrm{MnFe}_{2} \mathrm{O}_{4} \ldots \ldots \ldots \ldots \ldots \ldots$

$*$ Julgoldite, $\mathrm{Ca}_{2} \mathrm{Fe}_{3} \mathrm{Si}_{3} \mathrm{O}_{10}(\mathrm{OH}, \mathrm{O})_{2}(\mathrm{OH})_{2}$
Kalistrontite, $\mathrm{K}_{2} \mathrm{Sr}\left(\mathrm{SO}_{4}\right)_{2} \ldots \ldots \ldots \ldots$

Karelianite, $\mathrm{V}_{2} \mathrm{O}_{3} \ldots \ldots \ldots \ldots \ldots \ldots$

Kieserite, $\mathrm{MgSO}_{4} \cdot \mathrm{H}_{2} \mathrm{O} \ldots \ldots \ldots \ldots \ldots$

Kirschsteinite, $\mathrm{CaFeSiO}_{4} \ldots \ldots \ldots \ldots$

Koettigite, $\mathrm{Zn}_{3}\left(\mathrm{AsO}_{4}\right)_{2} \cdot 8 \mathrm{H}_{2} \mathrm{O} \ldots \ldots$.

Kremersite, $\left(\mathrm{NH}_{4}, \mathrm{~K}\right)_{2} \mathrm{FeCl}_{5} \cdot \mathrm{H}_{2} \mathrm{O} \ldots \ldots$

Langbeinite, $\mathrm{K}_{2} \mathrm{Mg}_{2}\left(\mathrm{SO}_{4}\right)_{3} \ldots \ldots \ldots \ldots$
Larnite, $\beta-\mathrm{Ca}_{2} \mathrm{SiO}_{4} \ldots \ldots \ldots \ldots \ldots$

Lautarite, $\mathrm{Ca}\left(\mathrm{IO}_{3}\right)_{2} \ldots \ldots \ldots \ldots \ldots$

Lead, $\mathrm{Pb} \ldots \ldots \ldots \ldots \ldots \ldots . \ldots . \ldots$

*Leucophanite, $\mathrm{NaCaBeFSi}{ }_{2} \mathrm{O}_{6} \ldots \ldots \ldots$

Libethenite, $\mathrm{Cu}_{2}(\mathrm{OH}) \mathrm{PO}_{4} \ldots \ldots \ldots \ldots$

*Liddicoatite, $\mathrm{Ca}(\mathrm{Li}, \mathrm{Al})_{3} \mathrm{Al}_{6} \mathrm{~B}_{3} \mathrm{Si}_{6} \mathrm{O}_{27}$

$(\mathrm{O}, \mathrm{OH})_{3}(\mathrm{OH}, \mathrm{F}) \ldots \ldots \ldots \ldots \ldots \ldots$

Lime, $\mathrm{CaO} \ldots \ldots \ldots \ldots \ldots \ldots \ldots \ldots$

Lime, $\mathrm{CaO}$ (calculated pattern) ....

*Linarite, $\mathrm{CuPb}(\mathrm{OH})_{2}\left(\mathrm{SO}_{4}\right) \ldots \ldots \ldots$

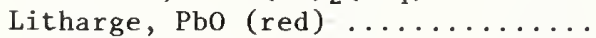

Lithiophosphate, $\mathrm{Li}_{3} \mathrm{PO}_{4} \ldots \ldots \ldots \ldots$

Loellingite, FeAs $_{2} \ldots \ldots \ldots \ldots \ldots$

Loeweite, $\mathrm{Na}_{12} \mathrm{Mg}_{7}\left(\mathrm{SO}_{4}\right)_{13} \cdot 15 \mathrm{H}_{2} \mathrm{O} \ldots$

Lopezite, $\mathrm{K}_{2} \mathrm{Cr}_{2} \mathrm{O}_{7} \ldots \ldots \ldots \ldots \ldots$

*Loveringite, $\mathrm{Ca}_{{ }_{7}{ }_{2} \mathrm{RE}}{ }_{33}(\mathrm{Y}, \mathrm{Th}, \mathrm{U}$,

$\mathrm{Pb}) .05 \mathrm{Ti}_{12.48} \mathrm{Fe}_{3.38} \dot{\mathrm{Cr}}_{2.24} \mathrm{Mg} .92$

$\mathrm{Zr}{ }_{58} \mathrm{Al} .{ }_{39} \dot{\mathrm{V}}_{22} \mathrm{Mn} .04 \mathrm{O}_{.38} \ldots \ldots \ldots \ldots$

Lueshite, $\mathrm{NaNbO}_{3} \ldots \ldots \ldots \ldots \ldots \ldots$

Macedonite, $\mathrm{PbTiO}_{3} \ldots \ldots \ldots \ldots \ldots$

Magnesiochromite, $\mathrm{MgCr}_{2} \mathrm{O}_{4} \ldots \ldots \ldots$

Magnesite, $\mathrm{MgCO}_{3} \ldots \ldots \ldots \ldots \ldots$

Magnetite, $\mathrm{Fe}_{3} \mathrm{O}_{4} \ldots \ldots \ldots \ldots \ldots$

Malachite, $\mathrm{Cu}_{2}(\mathrm{OH})_{2} \mathrm{CO}_{3} \ldots \ldots \ldots \ldots$

Malladrite, $\mathrm{Na}_{2} \mathrm{SiF}_{6} \ldots \ldots \ldots \ldots$

Manganolangbeinite, $\mathrm{K}_{2} \mathrm{Mn}_{2}\left(\mathrm{SO}_{4}\right)_{3} \ldots$

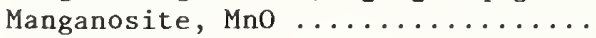

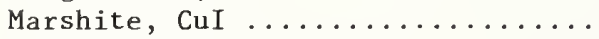

Mascagnite, $\left(\mathrm{NH}_{4}\right)_{2} \mathrm{SO}_{4} \ldots \ldots \ldots \ldots$

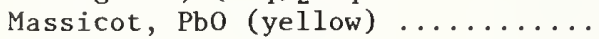

Matlockite, $\mathrm{PbFCl} \ldots \ldots \ldots \ldots \ldots \ldots$

Matteuccite, $\mathrm{NaHSO}_{4} \cdot \mathrm{H}_{2} \mathrm{O} \ldots \ldots \ldots \ldots$

Mayenite, $\mathrm{Ca}_{12} \mathrm{Al}_{14} \mathrm{O}_{33} \ldots \ldots \ldots \ldots$

Melanterite, $\mathrm{FeSO}_{4} \cdot 7 \mathrm{H}_{2} \mathrm{O} \ldots \ldots \ldots$

*Meliphanite,

$\mathrm{Na} .63 \mathrm{Ca}_{1.37} \mathrm{BeAl}{ }_{13} \mathrm{Si}_{1.87} \mathrm{O}_{6.25} \mathrm{~F} .75$

Merwinite, $\mathrm{Ca}_{3} \mathrm{Mg}\left(\mathrm{SiO}_{4}\right)_{2} \ldots \ldots \ldots \ldots$

Metaborite, $\mathrm{HBO}_{2} \ldots \ldots \ldots \ldots \ldots \ldots$

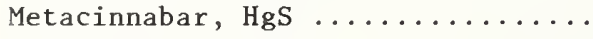

Miargyrite, $\operatorname{AgSbS}_{2} \ldots \ldots \ldots \ldots \ldots$

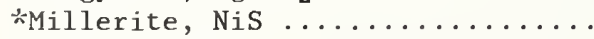

Minium, $\mathrm{Pb}_{3} \mathrm{O}_{4} \ldots \ldots \ldots \ldots \ldots \ldots$

Mitscherlichite, $\mathrm{K}_{2} \mathrm{CuCl}_{4} \cdot 2 \mathrm{H}_{2} \mathrm{O} \ldots \ldots$

Molybdenite, $\mathrm{MoS}_{2} \ldots \ldots \ldots \ldots \ldots \ldots$

Molybdite, $\mathrm{MoO}_{3} \ldots \ldots \ldots \ldots \ldots$

Molybdite, $\mathrm{MoO}_{3}$ (calculated

pattern)

$\begin{array}{rr}2 \mathrm{~m} & 24 \\ 10 \mathrm{~m} & 24 \\ 1 \mathrm{~m} & 30 \\ 20 \mathrm{~m} & 102 \\ 17 \mathrm{~m} & 40 \\ 11 \mathrm{~m} & 18 \\ 15 \mathrm{~m} & 34 \\ 1 \mathrm{~m} & 29 \\ 8 & 51 \\ 4 & 3 \\ 9 & 36 \\ 10 \mathrm{~m} & 72 \\ 14 \mathrm{~m} & 31 \\ 20 \mathrm{~m} & 108 \\ 16 \mathrm{~m} & 46 \\ 20 \mathrm{~m} & 28 \\ 19 \mathrm{~m} & 85 \\ 14 \mathrm{~m} & 8 \\ 6 \mathrm{~m} & 40 \\ 19 \mathrm{~m} & 29 \\ 14 \mathrm{~m} & 12 \\ 1 & 34 \\ 8 \mathrm{~m} & 138 \\ 17 \mathrm{~m} & 30 \\ & \\ 16 \mathrm{~m} & 42 \\ 1 & 43 \\ 14 \mathrm{~m} & 49 \\ 16 \mathrm{~m} & 34 \\ 2 & 30 \\ 4 \mathrm{~m} & 21 \\ 10 & 34 \\ 14 \mathrm{~m} & 35 \\ 15 \mathrm{~m} & 47\end{array}$

$16 \mathrm{~m}$

106

$18 \mathrm{~m} \quad 64$

$5 \quad 39$

$9 \quad 34$

$\begin{array}{ll}7 & 28\end{array}$

$5 \mathrm{~m} \quad 31$

$10 \quad 31$

$16 \mathrm{~m} \quad 68$

$6 \mathrm{~m} \quad 43$

545

$4 \quad 38$

$\begin{array}{rr}9 & 8 \\ 2 & 32\end{array}$

$13 \mathrm{~m} \quad 25$

$9 \mathrm{~m} \quad 52$

920

$8 \mathrm{~m} \quad 38$

$8 \mathrm{~m} \quad 135$

$20 \mathrm{~m} \quad 34$

$4 \mathrm{~m} \quad 27$

421

$5 \mathrm{~m} \quad 49$

$1 \mathrm{~m} \quad 37$

$8 \quad 32$

$9 \mathrm{~m} \quad 34$

$5 \quad 47$

330

$20 m$
Monteponite, Cdo .............

Monticellite, $\mathrm{CaMgSiO}_{4} \ldots \ldots \ldots \ldots$

Mont roydite, $\mathrm{HgO} \ldots \ldots \ldots \ldots \ldots$

Mullite, $\mathrm{Al}_{6} \mathrm{Si}_{2} \mathrm{O}_{13} \ldots \ldots \ldots \ldots \ldots$

Nantokite, $\mathrm{CuCl} \ldots \ldots \ldots \ldots \ldots \ldots$

*Newberyite, $\mathrm{MgHPO}_{4} \cdot 3 \mathrm{H}_{2} \mathrm{O} \ldots \ldots \ldots \ldots$

Nickel-hexahydrite, $\beta$ - $\mathrm{NiSO}_{4} \cdot 6 \mathrm{H}_{2} \mathrm{O} \ldots$

Niter, $\mathrm{KNO}_{3} \ldots \ldots \ldots \ldots \ldots \ldots \ldots \ldots$

Nitrammite, $\mathrm{NH}_{4} \mathrm{NO}_{3} \ldots \ldots \ldots \ldots \ldots$

Nitrobarite, $\mathrm{Ba}\left(\mathrm{NO}_{3}\right)_{2} \ldots \ldots \ldots \ldots$

Norbergite, $\mathrm{Mg}_{3} \mathrm{~F}_{2} \mathrm{SiO}_{4} \ldots \ldots \ldots \ldots \ldots$

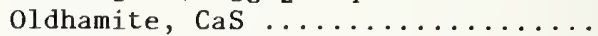

Otavite, $\mathrm{CdCO}_{3} \ldots \ldots \ldots \ldots \ldots \ldots$

Oxammite, $\left(\mathrm{NH}_{4}\right)_{2} \mathrm{C}_{2} \mathrm{O}_{4} \cdot \mathrm{H}_{2} \mathrm{O} \ldots \ldots \ldots \ldots$

Palladium, $\mathrm{Pd} \ldots \ldots \ldots \ldots \ldots \ldots$

Palladseite, $\mathrm{Pd}_{17} \mathrm{Se}_{15} \ldots \ldots \ldots \ldots$

Palmierite, $\mathrm{K}_{2} \mathrm{~Pb}\left(\mathrm{SO}_{4}\right)_{2} \ldots \ldots \ldots \ldots$

Paraguanajuatite, $\mathrm{Bi}_{2} \mathrm{Se}_{3} \ldots \ldots \ldots$

$\because$ Paratellurite, $\mathrm{TeO}_{2} \ldots \ldots \ldots \ldots$

Paratellurite, $\mathrm{TeO}_{2} \ldots \ldots \ldots \ldots \ldots$

Periclase, $\mathrm{MgO} \ldots \ldots \ldots \ldots \ldots \ldots \ldots$

Perovskite, $\mathrm{CaTiO}_{3} \ldots \ldots \ldots \ldots \ldots$

*Phenakite, $\mathrm{Be}_{2} \mathrm{SiO}_{4} \ldots \ldots \ldots \ldots \ldots$

Picromerite, $\mathrm{K}_{2} \mathrm{Mg}\left(\mathrm{SO}_{4}\right)_{2} \cdot 6 \mathrm{H}_{2} \mathrm{O} \ldots \ldots$

*Pirssonite, $\mathrm{Na}_{2} \mathrm{Ca}\left(\mathrm{CO}_{3}\right)_{2} \cdot 2 \mathrm{H}_{2} \mathrm{O} \ldots \ldots$

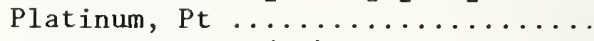

Portlandite, $\mathrm{Ca}(\mathrm{OH})_{2} \ldots \ldots \ldots \ldots$

Potash alum, $\mathrm{KAl}\left(\mathrm{SO}_{4}\right)_{2} \cdot 12 \mathrm{H}_{2} \mathrm{O} \ldots \ldots$

Powellite, $\mathrm{CaMoO}_{4} \ldots \ldots \ldots \ldots \ldots \ldots$

Pyrargyrite, $\mathrm{Ag}_{3} \mathrm{SbS}_{3} \ldots \ldots \ldots \ldots$

Pyrite, $\mathrm{FeS}_{2} \ldots \ldots \ldots \ldots \ldots \ldots$

*Pyroaurite, $\mathrm{Mg}_{6} \mathrm{Fe}_{2} \mathrm{CO}_{3}(\mathrm{OH})_{16} \cdot 4 \mathrm{H}_{2} \mathrm{O}$

Pyrolusite, $\beta-\mathrm{MnO}_{2} \ldots \ldots \ldots \ldots \ldots$

Py rope, $\mathrm{Mg}_{3} \mathrm{Al}_{2}\left(\mathrm{SiO}_{4}\right)_{3} \ldots \ldots \ldots \ldots$

Pyrophanite, $\mathrm{MnTiO}_{3} \ldots \ldots \ldots \ldots \ldots$

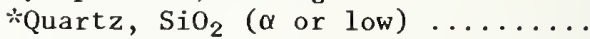

Quartz, low, $\alpha-\mathrm{SiO}_{2} \ldots \ldots \ldots \ldots \ldots$

Rammelsbergite, $\mathrm{NiAs}_{2} \ldots \ldots \ldots \ldots$

Retgersite, $\mathrm{NiSO}_{4} \cdot 6 \mathrm{H}_{2} \mathrm{O} \ldots \ldots \ldots \ldots$

Rhodochrosite, $\mathrm{MnCO}_{3} \ldots \ldots \ldots \ldots$

Rokuhnite, $\mathrm{FeCl}_{2} \cdot 2 \mathrm{H}_{2} \mathrm{O} \ldots \ldots \ldots \ldots$

Romarchite, $\mathrm{SnO} \ldots \ldots \ldots \ldots \ldots \ldots$

*Roscherite, (monoclinic),

$\mathrm{Be}_{2} \mathrm{Ca}\left(\mathrm{Fe} .{ }_{3} \mathrm{Mg} ._{7}\right)_{2} \mathrm{Al} .67_{6}\left(\mathrm{PO}_{4}\right)_{3}(\mathrm{OH})_{3}$.

$2 \mathrm{H}_{2} \mathrm{O} \ldots \ldots \ldots \ldots \ldots \ldots \ldots \ldots \ldots \ldots \ldots \ldots$

*Roscherite, (triclinic), $\mathrm{Be}_{4} \mathrm{Ca}_{2}$

$\left(\mathrm{Mn}_{3} ._{91} \mathrm{Mg} .{ }_{04} \mathrm{Ca} .05\right)\left(\mathrm{Al} .{ }_{13} \mathrm{Fe} .42^{\mathrm{Mn}}{ }_{12}\right)$

$\left(\mathrm{PO}_{4} \mathrm{j}_{6}(\mathrm{OH})_{4} \cdot 6 \mathrm{H}_{2} \mathrm{O} \ldots \ldots \ldots \ldots \ldots\right.$

Rutile, $\mathrm{TiO}_{2} \ldots \ldots \ldots \ldots \ldots \ldots$

Safflorite, $\mathrm{CoFeAs}_{4} \ldots \ldots \ldots \ldots \ldots$

Salammoniac, $\mathrm{NH}_{4} \mathrm{Cl} \ldots \ldots \ldots \ldots \ldots$

Sanbornite, $\beta-\mathrm{BaSi}_{2} \mathrm{O}_{5} \ldots \ldots \ldots \ldots$

Sanmartinite, $\mathrm{ZnWO}_{4} \ldots \ldots \ldots \ldots \ldots$

Scacchite, $\mathrm{MnCl}_{2} \ldots \ldots \ldots \ldots \ldots \ldots$

*Scheelite, $\mathrm{CaWO}_{4} \ldots \ldots \ldots \ldots \ldots \ldots$

Schultenite, $\mathrm{PbHAsO}_{4} \ldots \ldots \ldots \ldots \ldots$

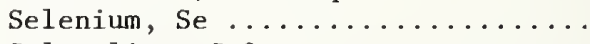

Selenolite, $\mathrm{SeO}_{2} \ldots \ldots \ldots \ldots \ldots \ldots$

Sellaite, $\mathrm{MgF}_{2} \ldots \ldots \ldots \ldots \ldots$

Senarmontite, $\mathrm{Sb}_{2} \mathrm{O}_{3} \ldots \ldots \ldots \ldots \ldots$

Shcherbinaite, $\mathrm{V}_{2} \mathrm{O}_{5} \ldots \ldots \ldots \ldots \ldots$

*Siderite, $\mathrm{FeCO}_{3} \ldots \ldots \ldots \ldots \ldots \ldots$

Silver, $\mathrm{Ag} \ldots \ldots \ldots \ldots \ldots \ldots \ldots \ldots$

Silver, Ag (reference standard) ... *Sjögrenite, $\mathrm{Mg}_{6} \mathrm{Fe}_{2} \mathrm{CO}_{3}(\mathrm{OH})_{16} \cdot 4 \mathrm{H}_{2} \mathrm{O}$

Skutterudite, $\mathrm{CoAs}_{3} \ldots \ldots \ldots \ldots$.
Sec. Page

$\begin{array}{rr}2 & 27 \\ 20 \mathrm{~m} & 30 \\ 9 & 39 \\ 3 \mathrm{~m} & 3 \\ 4 & 35 \\ 7 \mathrm{~m} & 139 \\ 19 \mathrm{~m} & 65 \\ 3 & 58 \\ 7 & 4 \\ 11 \mathrm{~m} & 14 \\ 10 & 39 \\ 7 & 15 \\ 7 & 11 \\ 7 & 5 \\ 1 & 21 \\ 16 \mathrm{~m} & 139 \\ 14 \mathrm{~m} & 30 \\ 18 \mathrm{~m} & 16 \\ 10 & 55 \\ 7 & 56 \\ 1 & 37 \\ 9 \mathrm{~m} & 17 \\ 8 & 11 \\ 8 \mathrm{~m} & 54 \\ 9 \mathrm{~m} & 106 \\ 1 & 31 \\ 1 & 58 \\ 6 & 36 \\ 6 & 22 \\ 5 \mathrm{~m} & 51 \\ 5 & 29 \\ 10 \mathrm{~m} & 104 \\ 10 \mathrm{~m} & 39 \\ 4 \mathrm{~m} & 24 \\ 15 \mathrm{~m} & 42 \\ 3 & 24 \\ 18 \mathrm{~m} & 61 \\ 10 & 42 \\ 7 & 36 \\ 7 & 32 \\ 11 \mathrm{~m} & 32 \\ 4 & 28\end{array}$

$16 \mathrm{~m}$

96

$16 \mathrm{~m} \quad 100$

$7 \mathrm{~m} \quad 83$

$10 \quad 28$

159

$13 \mathrm{~m} \quad 10$

$2 \mathrm{~m} \quad 40$

$8 \mathrm{~m} \quad 43$

$\begin{array}{rr}6 & 23 \\ 14 \mathrm{~m} & 18\end{array}$

554

$7 \mathrm{~m} \quad 60$

433

$3 \quad 31$

866

$15 \mathrm{~m} \quad 32$

$\begin{array}{rr}1 & 23 \\ 8 \mathrm{~m} & 2\end{array}$

$10 \mathrm{~m} \quad 103$

$10 \quad 21$ 
Vol. or

Sec. Page

*Smithsonite, $\mathrm{ZnCO}_{3} \ldots \ldots \ldots \ldots \ldots$

Soda alum, $\mathrm{NaAl}\left(\mathrm{SO}_{4}\right)_{2} \cdot 12 \mathrm{H}_{2} \mathrm{O} \ldots \ldots$

*Sodalite, $\mathrm{Na}_{8} \mathrm{Si}_{6} \mathrm{Al}_{6} \mathrm{O}_{2}{ }_{4} \mathrm{Cl}_{2} \ldots \ldots \ldots$

Soda niter, $\mathrm{NaNO}_{3} \ldots \ldots \ldots \ldots \ldots$

Sphaerocobaltite, $\mathrm{CoCO}_{3} \ldots \ldots \ldots \ldots$

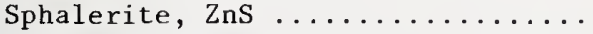

Spinel, $\mathrm{MgAl}_{2} \mathrm{O}_{4} \ldots \ldots \ldots \ldots \ldots \ldots$

Stibnite, $\mathrm{Sb}_{2} \mathrm{~S}_{3} \ldots \ldots \ldots \ldots \ldots \ldots$

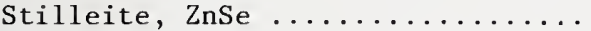

Stolzite, $\mathrm{PbWO}_{4} \ldots \ldots \ldots \ldots \ldots \ldots$

Strontianite, $\mathrm{SrCO}_{3} \ldots \ldots \ldots \ldots \ldots$

Struvite, $\mathrm{MgNH}_{4} \mathrm{PO}_{4} \cdot 6 \mathrm{H}_{2} \mathrm{O} \ldots \ldots \ldots \ldots$

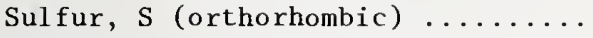

Sylvite, $\mathrm{KCl} \ldots \ldots \ldots \ldots \ldots \ldots \ldots$

Syngenite, $\mathrm{K}_{2} \mathrm{Ca}\left(\mathrm{SO}_{4}\right)_{2} \cdot \mathrm{H}_{2} \mathrm{O} \ldots \ldots \ldots$

Szmikite, $\mathrm{MnSO}_{4} \cdot \mathrm{H}_{2} \mathrm{O} \ldots \ldots \ldots \ldots \ldots$

Tellurantimony, $\mathrm{Sb}_{2} \mathrm{Te}_{3} \ldots \ldots \ldots \ldots$

*Tellurite, $\mathrm{TeO}_{2} \ldots \ldots \ldots \ldots \ldots \ldots$

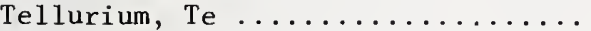

Tellurobismuthite, $\mathrm{Bi}_{2} \mathrm{Te}_{3} \ldots \ldots \ldots$

Tenorite, $\mathrm{CuO} \ldots \ldots \ldots \ldots \ldots \ldots$

Teschemacherite, $\mathrm{NH}_{4} \mathrm{HCO}_{3} \ldots \ldots \ldots$

Thenardite, $\mathrm{Na}_{2} \mathrm{SO}_{4} \ldots \ldots \ldots \ldots \ldots$

Thermonatrite, $\mathrm{Na}_{2} \mathrm{CO}_{3} \cdot \mathrm{H}_{2} \mathrm{O} \ldots \ldots \ldots$

*Thomsenolite, $\mathrm{NaCaAlF}_{6} \cdot \mathrm{H}_{2} \mathrm{O} \ldots \ldots$.

Thorianite, $\mathrm{ThO}_{2} \ldots \ldots \ldots \ldots \ldots \ldots$

Thortveitite, $\mathrm{Sc}_{2} \mathrm{Si}_{2} \mathrm{O}_{7} \ldots \ldots \ldots \ldots$

Tiemannite, $\mathrm{HgSe} \ldots \ldots \ldots \ldots \ldots \ldots$

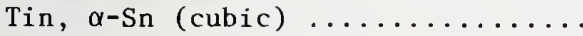

Tin, $\beta-S n$ (tetragonal) ..........

*Topaz, $\mathrm{Al}_{2} \mathrm{SiO}_{4}(\mathrm{~F}, \mathrm{OH})_{2} \ldots \ldots \ldots \ldots$

Trevorite, $\mathrm{NiFe}_{2} \mathrm{O}_{4} \ldots \ldots \ldots \ldots \ldots \ldots$

Trippkeite, $\mathrm{CuAs}_{2} \mathrm{O}_{4} \ldots \ldots \ldots \ldots \ldots$

*Trona, $\mathrm{Na}_{3} \mathrm{H}\left(\mathrm{CO}_{3}\right)_{2} \cdot 2 \mathrm{H}_{2} \mathrm{O} \ldots \ldots \ldots \ldots$

Ts chermigite, $\mathrm{NH}_{4} \mathrm{Al}\left(\mathrm{SO}_{4}\right)_{2} \cdot 12 \mathrm{H}_{2} \mathrm{O} \ldots$

Tungstenite, $\mathrm{WS}_{2} \ldots \ldots \ldots \ldots \ldots$

Ulvöspinel, $\mathrm{Fe}_{2} \mathrm{TiO}_{4} \ldots \ldots \ldots \ldots \ldots$

Unnamed mineral,

$\mathrm{K}_{1.16} \mathrm{Ba}{ }_{.72} \mathrm{Fe} .36 \mathrm{Ti}_{5.58} \mathrm{O}_{13} \ldots \ldots \ldots$

Uraninite, $\mathrm{UO}_{2} \ldots \ldots \ldots \ldots \ldots \ldots$

Uva rovite, $\mathrm{Ca}_{3} \mathrm{Cr}_{2}\left(\mathrm{SiO}_{4}\right)_{3} \ldots \ldots \ldots$

*Valentinite, $\mathrm{Sb}_{2} \mathrm{O}_{3} \ldots \ldots \ldots \ldots \ldots$

Vanthoffite, $\mathrm{Na}_{6} \mathrm{Mg}\left(\mathrm{SO}_{4}\right)_{4} \ldots \ldots \ldots$

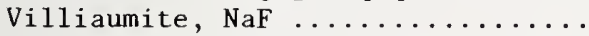

Vivianite, $\mathrm{Fe}_{3}\left(\mathrm{PO}_{4}\right)_{2} \cdot 8 \mathrm{H}_{2} \mathrm{O} \ldots \ldots \ldots$

Wakefieldite, $\mathrm{YVO}_{4} \ldots \ldots \ldots \ldots \ldots$

Willemite, $\mathrm{Zn}_{2} \mathrm{SiO}_{4} \ldots \ldots \ldots \ldots \ldots$

Witherite, $\mathrm{BaCO}_{3} \ldots \ldots \ldots \ldots \ldots$

Wulfenite, $\mathrm{PbMoO}_{4} \ldots \ldots \ldots \ldots \ldots$

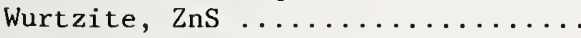

*Xanthoconite, $\mathrm{Ag}_{3} \mathrm{AsS}_{3} \ldots \ldots \ldots \ldots$

Xenotime, $\mathrm{YPO}_{4} \ldots \ldots \ldots \ldots \ldots \ldots$

Yavapaiite, $\mathrm{KFe}\left(\mathrm{SO}_{4}\right)_{2} \ldots \ldots \ldots \ldots$

Zinc, $\mathrm{Zn} \ldots \ldots \ldots \ldots \ldots \ldots \ldots \ldots$

Zincite, $\mathrm{Zno} \ldots \ldots \ldots \ldots \ldots \ldots$

$*$ Zincobotryogen, $(\mathrm{Zn}, \mathrm{Mg}, \mathrm{Mn}) \mathrm{Fe}\left(\mathrm{SO}_{4}\right)_{2}$

$(\mathrm{OH}) \cdot 7 \mathrm{H}_{2} \mathrm{O} \ldots \ldots \ldots \ldots \ldots \ldots \ldots \ldots$

Zinkosite, $\mathrm{ZnSO}_{4} \ldots \ldots \ldots \ldots \ldots \ldots$

$*$ Zircon, $\mathrm{ZrSiO}_{4} \ldots \ldots \ldots \ldots \ldots \ldots$

$\mathrm{Zircosulfate,}, \mathrm{Zr}\left(\mathrm{SO}_{4}\right)_{2} \cdot 4 \mathrm{H}_{2} \mathrm{O} \ldots \ldots$

\begin{tabular}{|c|c|}
\hline 8 & 69 \\
\hline $15 \mathrm{~m}$ & 68 \\
\hline $7 \mathrm{~m}$ & 158 \\
\hline 6 & 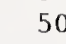 \\
\hline 10 & 2 \\
\hline 2 & \\
\hline $9 m$ & \\
\hline 5 & \\
\hline 3 & 23 \\
\hline $5 m$ & 34 \\
\hline 3 & 56 \\
\hline $3 m$ & 41 \\
\hline 9 & 54 \\
\hline 1 & 00 \\
\hline $14 \mathrm{~m}$ & \\
\hline $16 \mathrm{~m}$ & \\
\hline $3 \mathrm{~m}$ & \\
\hline 9 & 57 \\
\hline 1 & 26 \\
\hline $3 m$ & 16 \\
\hline 1 & $(0$ \\
\hline 9 & \\
\hline 2 & 59 \\
\hline 8 & 54 \\
\hline $8 m$ & 132 \\
\hline 1 & 7 \\
\hline $7 \mathrm{~m}$ & 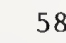 \\
\hline 7 & 35 \\
\hline 2 & 12 \\
\hline 1 & 24 \\
\hline $1 \mathrm{~m}$ & \\
\hline 10 & 44 \\
\hline $16 \mathrm{~m}$ & 120 \\
\hline $15 \mathrm{~m}$ & 71 \\
\hline 6 & \\
\hline 8 & 65 \\
\hline $20 \mathrm{~m}$ & 6 \\
\hline $16 \mathrm{~m}$ & 147 \\
\hline 2 & 33 \\
\hline 10 & 1 \\
\hline 10 & \\
\hline $15 \mathrm{~m}$ & 72 \\
\hline 1 & 63 \\
\hline $16 \mathrm{~m}$ & 38 \\
\hline $5 m$ & 5 \\
\hline 7 & 62 \\
\hline 2 & 5 \\
\hline 7 & 23 \\
\hline 2 & 1 \\
\hline $8 \mathrm{~m}$ & 126 \\
\hline 8 & 67 \\
\hline $16 \mathrm{~m}$ & 5 \\
\hline 1 & 16 \\
\hline 2 & 25 \\
\hline $20 \mathrm{~m}$ & 0 \\
\hline 7 & 0 \\
\hline 4 & 6 \\
\hline 7 & \\
\hline
\end{tabular}


NBS-114A (REV. 2-8C)

U.S. DEPT. OF COMM.

BIBLIOGRAPHIC DATA

SHEET (See instructions)

\begin{tabular}{l|l|l}
\hline 1. PUBLICATION OR & 2. Performing Organ. Report No. & 3. Publication Date \\
REPORT NO. & January 1984 \\
NBS Monogr. 25, Sec. & 0 &
\end{tabular}

4. TITLE AND SUBTITLE

Standard X-ray Diffraction Powder Patterns

Section 20 - Data for 71 Substances

5. AUThOR(S) M. C. Morris, H. F. MCMurdie, E. H. Evans, B. Paretzkin, H. S. Parker, N. P. Pyrros, and C. R. Hubbard

\begin{tabular}{l|l} 
6. PERFORMING ORGANIZATION (If joint or other than NBS, see instructions) & 7. Contract Grant No.
\end{tabular}

NATIONAL BUREAU OF STANDARDS

DEPARTMENT OF COMMERCE

WASHINGTON, D.C. 20234

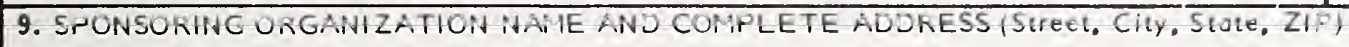

8. Type of Report \& Period Covered

Final

10. SUPPLEMENTARY NOTES

Library of Congress Catalog Card Number: 53-61386

[D Document describes a computer program; SF-185, FIPS Software Summary, is attached.

11. ABSTRACT (A 200-word or less foctual summory of most significant information. If document includes a significant bibliogrophy or literoture survey. mention it here)

Standard x-ray powder diffraction patterns are presented for 71 substances. These patterns, useful for identification, were obtained by manual or automated diffractometer methods or were calculated from published crystal structure data. The lattice constants from the experimental work were refined by least-squares methods, and reflections were assigned Miller indices consistent with space group extinctions. Relative intensities, calculated densities, literature references, and other relevant data are included.

12. KEY WORDS (Six to twelve entries; alphabetical order; capitalize only proper names; and separate key words by semicolons) crystal structure; densities; lattice constants; powder patterns; reference intensities; standard; $x$-ray diffraction.

13. AVAILABILITY

X] Unlimited

$\square$ For Official Distribution. Do Not Release to NTIS

[X] Order From Superintendent of Documents, U.S. Government Printing Office, Washıngton, D.C. 20402.

[ Order From National Technical Information Service (NTIS), Springfield, VA. 2216I
14. NO. OF PRINTED PAGES

\section{9}

15. Price 
, 


\section{NBS TECHNICAL PUBLICATIONS}

\section{PERIODICALS}

JOURNAL OF RESEARCH-The Journal of Research of the National Bureau of Standards reports NBS research and development in those disciplines of the physical and engineering sciences in which the Bureau is active. These include physics, chemistry, engineering, mathematics, and computer sciences. Papers cover a broad range of subjects, with major emphasis on measurement methodology and the basic technology underlying standardization. Also included from time to time are survey articles on topics closely related to the Bureau's technical and scientific programs. As a special service to subscribers each issue contains complete citations to all recent Bureau publications in both NBS and nonNBS media. Issued six times a year. Annual subscription: domestic $\$ 18$; foreign $\$ 22.50$. Single copy, $\$ 4.25$ domestic; $\$ 5.35$ foreign.

\section{NONPERIODICALS}

Monographs-Major contributions to the technical literature on various subjects related to the Bureau's scientific and technical activities.

Handbooks-Recommended codes of engineering and industrial practice (including safety codes) developed in couperation with interested industries, professional organizations, and regulatory bodies.

Special Publications-Include proceedings of conferences sponsored by NBS, NBS annual reports, and other special publications appropriate to this grouping such as wall charts, pocket cards, and bibliographies.

Applied Mathematics Series-Mathematical tables, manuals, and studies of special interest to physicists, engineers, chemists, biologists, mathematicians, computer programmers, and others engaged in scientific and technical work.

National Standard Reference Data Series-Provides quantitative data on the physical and chemical properties of materials, compiled from the world's literature and critically evaluated. Developed under a worldwide program coordinated by NBS under the authority of the National Standard Data Act (Public Law 90-396).

NOTE: The principal publication outlet for the foregoing data is the Journal of Physical and Chemical Reference Data (JPCRD) published quarterly for NBS by the American Chemical Society (ACS) and the American Institute of Physics (AIP). Suhscriptions, reprints, and supplements available from ACS, 1155 Sixteenth St., NW, Washington, DC 20056.
Building Science Series-Disseminates iechnical information developed at the Bureau on building materials, components, systems, and whole structures. The series presents research results, test methods, and performance criteria related to the structural and environmental functions and the durahility and safety characteristics of building elements and systems.

Technical Notes-Studies or reports which are complete in themselves but restrictive in their treatment of a subject. Analogous to monographs but not so comprehensive in scope or definitive in treatment of the subject area. Often serve as a vehicle for linal reports of work performed at NBS under the sponsorship of other government agencies.

Voluntary Product Standards-Developed under procedures published by the Department of Commerce in Part 10. Title 15, of the Code of Federal Regulations. The standards establish nationally recognized requirements for products, and provide all concerned interests with a basis for common understanding of the characteristics of the products. NBS administers this program as a supplement to the activities of the private sector standardizing organizations.

Consumer Information Series-Practical information, based on NBS research and experience, covering areas of interest to the consumer. Easily understandable language and illustrations provide useful background knowledge for shopping in today's technological marketplace

Order the above NBS publications from: Superimtendent of Documents. Government Printing Office, Washington, DC 20402

Order the following NBS publications-FIPS and NBSIR s-from the National Technical Information Services, Springfield, VA 22161.

Federal Information Processing Standards Publications (FIPS PUB)-Publications in this series collectively constitute the Federal Information Processing Standards Register. The Register serves as the official source of information in the Federal Government regarding standards issued by NBS pursuant to the Federal Property and Administrative Services Act of 1949 as amended, Public Law 89-306 (79 Stat. 1127), and as implemented by Executive Order 11717 (38 FR 12315, dated May 11, 1973) and Part 6 of Title 15 CFR (Code of Federal Regulations).

NBS Interagency Reports (NBSIR)-A special series of interim or final reports on work performed by NBS for outside sponsors (both government and non-government). In general, initial distribution is handled by the sponsor; public distribution is by the National Technical Information Services, Springfield, VA 22161 , in paper copy or microfiche form. 
U.S. Department of Commerce

National Bureau of Stanoards

Washington, D.C. 20234

Official Business

Penalty for Private Use $\$ 300$

POSTAGE AND FEES PAID US DEPARTMENT OF COMMERCE COM-215

SPECIAL FOURTH-CLASS RATE BOOK 